THYROID

Volume 27, Supplement 1, 2017

(C) Mary Ann Liebert, Inc.

DOI: 10.1089/thy.2017.29046.abstracts

\title{
Abstracts
}

\section{$87^{\text {th }}$ Annual Meeting of the American Thyroid Association October 18-22, $2017 \quad$ Victoria, BC, Canada}

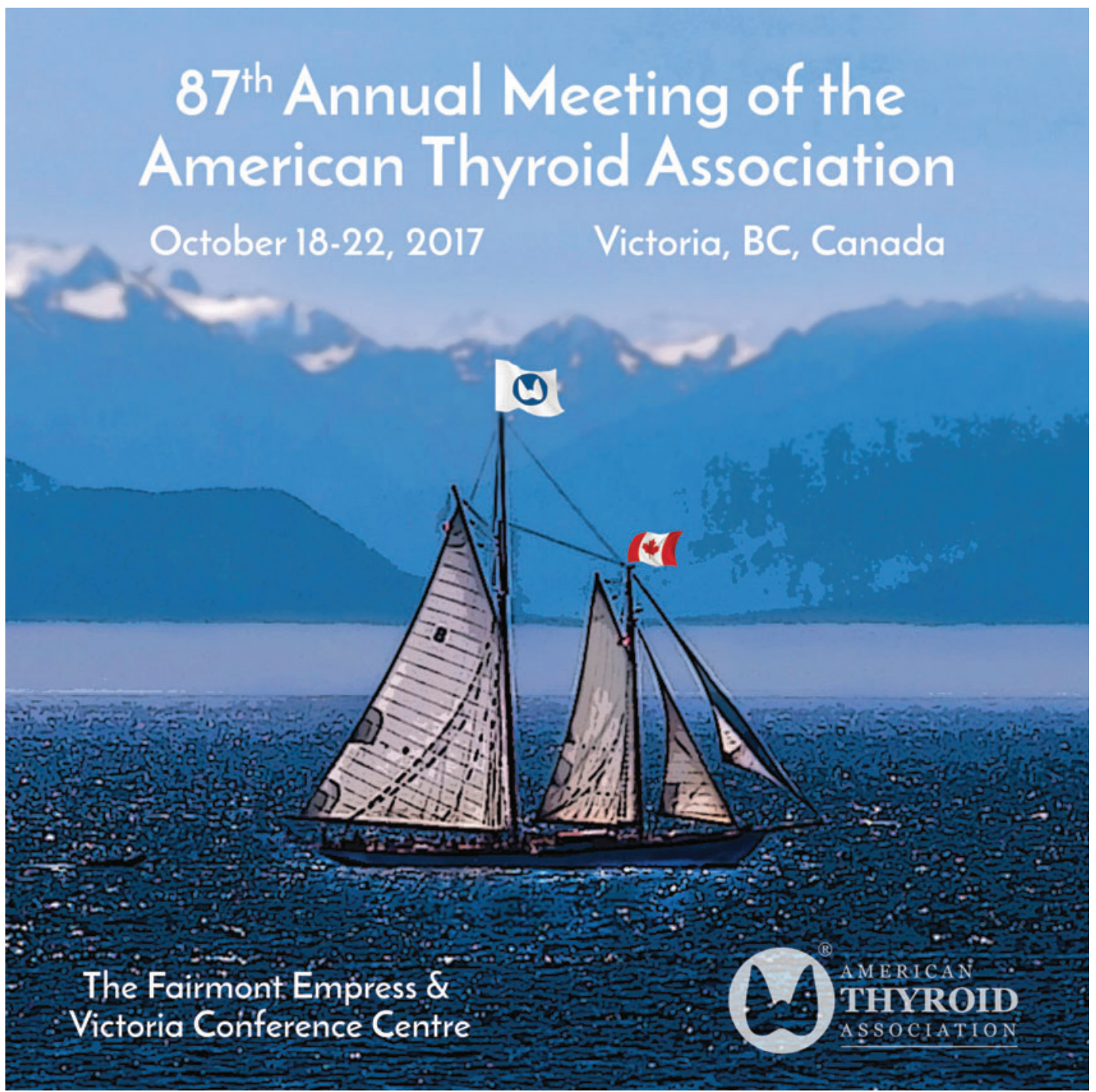




\section{$87^{\text {th }}$ ANNUAL MEETING OF THE AMERICAN THYROID ASSOCIATION WEDNESDAY, OCTOBER 18, 2017}

\author{
6:30 AM - 8:30 PM \\ VCC, Registration \\ 6:30 AM - 6:30 PM \\ VCC, West Coast \\ 7:00 AM - 3:00 PM \\ VCC, Saanich \\ 8:00 AM - 4:00 PM \\ Fairmont, Crystal Ballroom \\ 8:00 AM - 6:00 PM \\ VCC, Crystal Garden \\ 7:30 AM - 1:30 PM \\ Fairmont, Library \\ 1:00 PM - 4:00 PM \\ VCC, Lecture Theatre
}

2:00 PM - 2:30 PM

VCC, Langford

2:00 PM - 5:00 PM

VCC, Metchosin

2:45 PM - 4:00 PM

VCC, Salon C

5:30 PM - 5:45 PM

VCC, Salon AB

5:45 PM - 7:30 PM

VCC, Salon AB

7:30 PM - 9:00 PM

VCC, Crystal Garden
ATA Registration Open

ATA Speaker Ready Room Open (Open to meeting faculty only)

ATA Endocrine Neck Ultrasound Course

(Satellite Program, separate registration required, additional fees apply, ECNU credits available)

Co-Chairs: Kevin T. Brumund and Stephanie L. Lee

E. Chester Ridgway (Ridgway) Trainee Conference: Clinical

Trainees Symposium (Pre-registration required)

ATA Exhibit and Poster Hall Open (Open for exhibitor setup only)

ATA Board of Directors' Meeting (By invitation only)

Ridgway Trainee Conference: Basic Trainees Symposium

- Single Cell and Genomic Technologies (Open to all attendees;

Pre-registration required)

Douglas Forrest

Yun-Bo Shi

Linus Tsai

Hong Liu

Changyu Liu

Veerle M. Darras

International Coordinating Committee Meeting (by invitation only)

$1^{\text {th }}$ International Thyroid Congress Program Organizing

Committee Meeting (by invitation only)

ATA Committee and Task Force Meetings

(Open to current serving committee or task force members only)

Welcome to the 87th Annual Meeting of the American Thyroid Association

John C. Morris, ATA President

Victor J. Bernet, ATA Secretary/COO

Angela M. Leung, Program Committee Co-Chair

Mingzhao Xing, Program Committee Co-Chair

Opening Session: Year in Thyroidology Review

Chair: Antonio C. Bianco

Year in Basic Thyroidology

Year in Clinical Thyroidology

Year in Surgical Thyroidology

ATA Welcome Reception in the Exhibit Hall

(Meeting registration/badge required for entry; hall open to exhibitors and attendees)
Carmelo Nucera Gregory A. Brent Julie Ann Sosa 


\section{$8^{\text {th }}$ ANNUAL MEETING OF THE AMERICAN THYROID ASSOCIATION THURSDAY, OCTOBER 19, 2017}

6:00 AM - 7:45 AM

VCC, Lecture Theatre

6:45 AM - 5:15 PM

VCC, Registration

7:00 AM - 5:30 PM

VCC, West Coast

7:00 AM - 8:00 AM

VCC, Salon C

8:00 AM - 8:05 AM

VCC, Salon AB

8:05 AM - 8:50 AM

VCC, Salon AB

8:50 AM - 9:50 AM

VCC, Salon AB

8:50 AM - 11:45 AM

Fairmont, Crystal Ballroom

9:30 AM - 4:30 PM

VCC, Crystal Garden

9:50 AM - 10:45 AM

VCC, Crystal Garden

10:45 AM - 12:00 PM

VCC, Lecture Theatre
Early Riser Satellite Program: Diagnosis and Management of Advanced Thyroid Cancer (Limited seating; first-come, first served; light breakfast offered)

ATA Registration Open

ATA Speaker Ready Room Open (Open to meeting faculty only)

ATA Women in Thyroidology: Women and the Future of Endocrinology (Pre-registration required; ticket required for entry)

Chair: Honey V. Reddi

David S. Cooper

Stephanie L. Lee

Welcome to the 87th Annual Meeting of the American Thyroid Association

John C. Morris, ATA President

Victor J. Bernet, ATA Secretary/COO

Angela M. Leung, Program Committee Co-Chair

Mingzhao Xing, Program Committee Co-Chair

ATA Van Meter Lecture (announced onsite at time of lecture)

Highlighted Oral Abstracts Session (4 total abstracts)

Co-Chairs: Angela M. Leung and Mingzhao Xing

Oral Abstract \#1: Incident Hypothyroidism During Tyrosine

Kinase Inhibitor Therapy Predicts Significantly Longer

Survival in Patients with Advanced Non-Thyroid Cancers

Oral Abstract \#2: Sumoylation of TR $\beta$ Plays an Essential

Role in Regulation of the Hypothalamic-Pituitary-Thyroid (HPT) Axis

Oral Abstract \#3: TERT Mutations Increase Risk of Treatment Failure in BRAF Positive Papillary Thyroid Cancer

Oral Abstract \#4: Combined Mycophenolate With Intravenous Methylprednisolone Therapy Is More Effective Than Intravenous Methylprednisolone Alone in Active and Moderate-to-Severe Graves' Orbitopathy - A Randomized, Observer Blind, Multicenter Trial

Ridgway Trainee Conference: Surgical Considerations in Management of the Thyroid (Open to all attendees; Preregistration required)

Moderator: Elizabeth G. Grubbs

Exhibit and Poster Hall Open

(Open at 8:00 AM to poster presenters and exhibitors only)

Poster Review Session and Break in the Exhibit Hall

Posters 35-173; Short Call Posters 9-25

Basic Symposium: Thyroid Hormone Transporters

Chair: Peter A. Kopp

Thyroid Hormone Transport in The Brain

Triac for the Treatment of MCT8 Deficiency

Virus 9-Based Gene Therapy Delivery of MCT8 in the Brain
Trevor E. Angell

Sujie Ke

Daria Handkiewicz-Junak

George J. Kahaly

Juan Bernal W. Edward Visser Samuel Refetoff 
10:45 AM - 12:00 PM

VCC, Salon AB

12:00 PM - 1:00 PM

VCC, Crystal Garden

12:00 PM - 1:00 PM

VCC, Saanich

12:00 PM - 1:00 PM

Fairmont, Crystal Ballroom

12:00 PM - 1:00 PM

VCC, Oak Bay

1:00 PM - 1:45 PM

VCC, Salon AB

1:45 PM - 3:15 PM

Fairmont, Crystal Ballroom

1:50 PM - 3:05 PM

VCC, Lecture Theatre

1:50 PM - 3:05 PM

VCC, Salon AB
Clinical Symposium: New Directions in Thyroid Nodules and Thyroid Cancer

Chair: Bryan R. Haugen

An Update: The NEW Bethesda Classification System for Reporting Thyroid Cytopathology

AJCC 8th Edition Update on Differentiated Thyroid Cancer

AJCC 8th Edition with Focus on Patient Age: Is There Actually an Age Cutoff?

Moving Towards Perfection: Avoiding Unnecessary Surgery, Eliminating Complications, and Reducing Costs

Exhibit Hall Closed (Lunch on your own)

Ridgway Trainees Conference: University of Southern California Thyroid Research Group Fellows Fund Basic Trainees Lunch Session - Selling Your Science (Open to all trainees; Lunch ticket required for meal)

Moderator: David S. Sharlin

Ridgway Trainees Conference: Arthur Bauman Clinical Trainees Lunch Session - Setting Up a Thyroid Practice (Open to all trainees; Lunch ticket required for meal)

ATA Expo Theatre Presented by CBLPath/UPMC: Molecular Markers - The New Era

(Limited seating; first-come, first served; lunch offered)

Lewis E. Braverman Distinguished Award Lectureship

Re-Telling the Story About Thyroid Cancer - Rising Incidence, Mortality and Maybe an Explanation Introduction by Tracy S. Wang

Ridgway Trainee Conference: Trainees Thyroid Ultrasound Course (Open to all trainees)

Introduction to Thyroid Ultrasound

Sonographic Assessment of Thyroid Nodules

Neck Ultrasound: Beyond Nodules

Basic/Translational Oral Abstracts Session: Autoimmune

Thyroid Diseases (5 total)

Co-Chairs: Chao Liu and Laura Fugazzola

Oral Abstract \#5: Interferon Alpha Triggers Autoimmune Thyroiditis via Degradation of Thyroglobulin by Cysteine Cathepsins into Immunogenic Peptides

Oral Abstract \#6: First Report of Tree Shrew Graves' Disease Model

Oral Abstract \#7: Identification of Novel Sodium Iodide Symporter (NIS) Interactors which Modulate Iodide Uptake

Oral Abstract \#8: Performance and Specificity of Six

Immunoassays for TSH Receptor Antibodies - A Multicenter Study

Oral Abstract \#9: Functional AIM2 Inflammasome Expressed in Thyrocytes Are Associated with Autoimmune Thyroiditis

Clinical Oral Abstracts Session: Iodine Status and Thyroid Function in Pregnancy (5 total)

Co-Chairs: Sun Y. Lee and Jorge H. Mestman

Oral Abstract \#10: Development and Validation of a Novel Iodine-129 Tracer Method for the Safe Assessment of Iodine Absorption and Thyroidal Uptake from Oral Iodine Supplements and Foods in Humans
Jeffrey $F$. Krane

R. Michael Tuttle

Sanziana A. Roman

Michael W. Yeh

Honey V. Reddi

David S. Sharlin

J. Woody Sistrunk

Katarzyna Piotrowska

Steven P. Hodak

Yuri Nikiforov

Bryan R. Haugen

Julie Ann Sosa

Tracey S. Tylee

Steven P. Weitzman

Alex Tessnow

Larissa C. Faustino

Liping $W u$

Alice Fletcher

Tanja Diana

Qingling Guo

Michael B. Zimmermann 


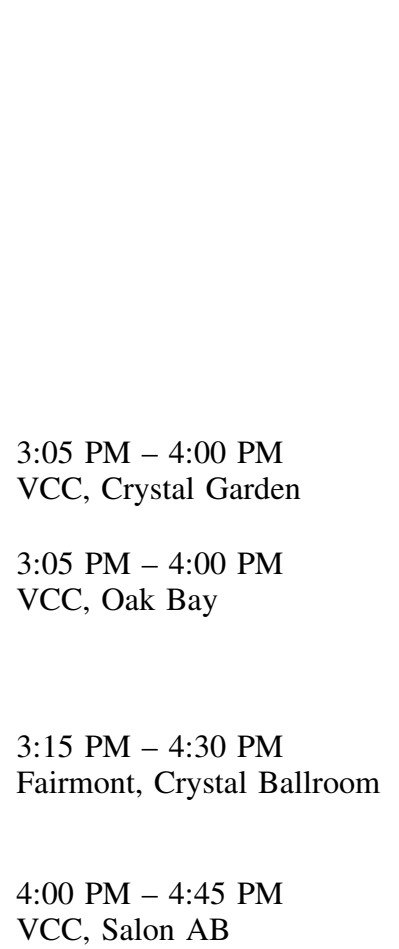

4:50 PM - 5:45 PM

VCC, Salon C

VCC, Colwood

VCC, Salon AB

VCC, Sidney

VCC, Esquimalt
Oral Abstract \#11: Incidence of Neonatal Hyperthyroidism Among Newborns of Graves' Disease Patients Treated With Radioiodine Therapy Within Two Years Before Conception Oral Abstract \#12: Different FT4 Response to HCG Under TPOAb or TgAb During First- Half Trimester

Oral Abstract \#13: Different Trajectories of Thyroid Function During Pregnancy: A New Approach to Look at

Physiological Changes of Thyroid Function During Gestation Oral Abstract \#14: The Association of Phthalate Exposure With Thyroid Function During Pregnancy: A Mixture Analysis

Poster Review Session and Break in the Exhibit Hall

ATA Expo Theatre Presented by Veracyte, Inc.: Leveraging Enriched Genomic Data to Develop a Next-Generation Afirma Gene Expression Classifier (Limited seating; first-come, first served)

Ridgway Trainee Conference: Case Discussions - Thyroid Nodules (Open to all trainees)

Moderators: Mara Y. Roth and Steven P. Weitzman

ATA Research Grants Session

Chair: Motoyasu Saji

Exploring Genetics of Advanced Thyroid Cancer

Cancer Progression and Therapeutic Response in a Mouse

Model of Medullary Thyroid Carcinoma

The Role of the Renal Na+/I- Symporter (NIS) in Iodide Metabolism and Thyroid Function

Discussion-Debates/ Meet the Professor Workshops (6 total)

- DM 1: Mechanisms in Immune Pathogenesis of Thyroid Diseases

Moderator: Matthew D. Ringel

IDO1 and RET/PTC in Immune Dysregulation in Thyroid Cancer

Genetic-Epigenetic Dysregulation in Autoimmune Thyroid Diseases

\section{- DM 2: Deiodinases}

Moderator: Anthony N. Hollenberg

Type 3 Deiodinase in the Regulation of the Leptin-Melanocortin System and Circadian Activity

Pathophysiological Relevance of Deiodinases Polymorphisms

- DM 3: Effects of Drugs and Medications on TFTs Moderator: Leonard Wartofsky Medication Effects

Biotin Effects

- DM 4: Ethics in Thyroid Research

Moderator: M. Sara Rosenthal

Ethical dilemmas in Clinical Thyroid Research

Ethical dilemmas in Basic Thyroid Research

- DM 5: Consequences of Environmental Thyroid Disruptors: Knowns and Unknowns

Moderator: Angela M. Leung

The Burden of Low-Level Environmental Thyroid Toxicants: Potential Impacts on Health

Exposure to Thyroid Disruptors in Pregnancy and Infancy
Ai Yoshihara

Yuanyuan Hou

Victor Pop

Tim Korevaar

Richard T. Kloos

Giulia C. Kennedy

Stephanie L. Lee Gregory W. Randolph

Jennifer A. Sipos

Ming $\mathrm{Li}$

Sarah C. Oltmann

Marcela Andrea

Reyna-Neyra

Efisio Puxeddu

Yaron Tomer

Arturo Hernandez.

Antonio C. Bianco

David H. Sarne

Alicia Algeciras-Schimnich

Peter Angelos

Rebecca E. Schweppe

Tim Korevaar

Robin P. Peeters 
VCC, Saanich

6:00 PM - 7:15 PM

VCC, Lecture Theatre
- DM 6: Intraoperative Consultation of Thyroid Nodules: An Interactive Approach Between a Surgeon and a Pathologist

Moderator: Robert C. Smallridge

The Surgeon's Perspective

The Pathologist's Perspective

John D. Casler

Qihui Jim Zhai

ATA Annual Business Meeting

(for Active and Senior ATA members only)

The annual business meeting features election results, committee reports, discussion and voting on important member issues. 


\section{$87^{\text {th }}$ ANNUAL MEETING OF THE ATA FRIDAY, OCTOBER 20, 2017}

6:00 AM - 7:45 AM

VCC, Lecture Theatre

7:30 AM - 5:15 PM

VCC, Registration

7:30 AM - 5:30 PM

VCC, West Coast

8:00 AM - 8:05 AM

VCC, Salon AB

8:05 AM - 8:50 AM

VCC, Salon AB

8:45 AM - 10:30 AM

Fairmont, Crystal

Ballroom

8:50 AM - 9:50 AM

VCC, Lecture Theatre

8:50 AM - 9:50 AM

VCC, Salon AB

9:30 AM - 4:30 PM

VCC, Crystal Garden

9:50 AM - 10:45 AM

VCC, Crystal Garden
Early Riser Satellite Symposium Presented by Quidel

Corporation:

TSH Receptor Antibodies - Methodology and Clinical Relevance

(Limited seating; first-come, first served; light breakfast offered)

ATA Registration Open

ATA Speaker Ready Room Open (Open to meeting faculty only)

Morning Announcements

Plenary Lecture: Iodine Intake and Thyroid Diseases - Learnings

in China

Introduction by Mingzhao Xing

Ridgway Trainee Conference: Case Discussions - Thyroid Cancer (Open to all trainees)

Moderators: Andrew G. Gianoukakis and Alex Tessnow

Basic/Translational Oral Abstracts Session: Thyroid Cancer

(4 total)

Co-Chairs: Jason D. Prescott and Yufei Shi

Oral Abstract \#15: Berberine Could Inhibit Thyroid Carcinoma Cells by Inducing Mitochondrial Apoptosis, G0/G1 Cell Cycle Arrest and Suppressing Migration Via PI3K-AKT and MAPK Signaling Pathways

Oral Abstract \#16: Large Oncosomes of Anaplastic and Papillary Thyroid Cancer Cells

Oral Abstract \#17: Co-Evolution of Met Amplification and Hgf Overexpression Mediate Resistance to BRAFV600E Inactivation in Murine Anaplastic Thyroid Cancers and Sensitizes Them to MET Kinase Inhibitors

Oral Abstract \#18: TGF $\beta$ 1-Induced Radioiodine Therapy of Hepatocellular Cancer After Mesenchymal Stem Cell (MSC)Mediated Sodium Iodide Symporter (NIS) Gene Delivery

Clinical Oral Abstracts Session: Thyroid Dysfunction and

Pediatric Thyroid Disease (4 total)

Co-Chairs: Yaron Tomer and Catherine A. Dinauer

Oral Abstract \#19: Graves' Disease and Its Effect on Medial Temporal Lobe Structures

Oral Abstract \#20: Higher Remission Rate After Long-Term Methimazole Therapy in Patients with Graves' Disease: A Randomized Clinical Trial

Oral Abstract \#21: Effects of Altering Levothyroxine (L-T4) Doses on Quality of Life, Mood and Cognition in L-T4 Treated Subjects: A Double-Blinded, Randomized, Controlled Trial

Oral Abstract \#22: Accuracy of Adult ATA Ultrasound Risk Stratification in Children

Exhibit and Poster Hall Open

(Open at 8:00 AM to poster presenters and exhibitors only)

Poster Review Session and Break in the Exhibit Hall

Posters 174-311; Short Call Posters 26-43
George J. Kahaly

J. Woody Sistrunk

Tanja Diana

Angela M. Leung

Mingzhao Xing

Weiping Teng

James D. Brierley Virginia A. LiVolsi R. Michael Tuttle Lori J. Worth

Liang $\mathrm{Li}$

Kenneth W. Witwer

Jeffrey A. Knauf

Christina Schug

Mats Holmberg

Fereidoun Azizi

Mary H. Samuels

Ana L. Creo 
9:50 AM - 10:35 AM

VCC, Lecture Theatre

10:45 AM - 12:00 PM

VCC, Lecture Theatre

10:45 AM - 12:00 PM

VCC, Salon AB
Highlights of ATA VideoEndocrinology

Moderator: Gerard M. Doherty

Clinical Symposium: Thyroid Dysfunction Grand Rounds - Case

12:00 PM - 1:00 PM

VCC, Oak Bay

1:00 PM - 1:45 PM

VCC, Salon AB

1:50 PM - 3:05 PM

VCC, Lecture Theatre

1:50 PM - 3:05 PM

VCC, Salon AB

1:50 PM - 3:20 PM

Fairmont, Crystal

Ballroom

3:05 PM - 4:00 PM

VCC, Crystal Garden

Poster Review Session and Break in the Exhibit Hall
Lateral Neck Dissection for Thyroid Cancer

Central Neck Dissection for Thyroid Cancer

Remote Access Thyroid Surgery

Basic Symposium: Modulation of TSHR in Thyroid Disease

Moderator: Antonio Di Cristofano

TSHR and IGF1-R Crosstalk

TSHR Neutral Antibodies

Central Tolerance to the TSHR and Graves' Disease

Discussions

Moderator: Mary Samuels

Panelists: Anne R. Cappola, Stephanie L. Lee, John C. Morris, Paul W. Ladenson and Douglas S. Ross

Exhibit Hall Closed (Lunch on your own)

Ridgway Trainee Conference: All Trainees Lunch Session - How to Get Your Work Published (Open to all trainees; Lunch ticket required for meal)

Maisie L. Shindo

Ralph P. Tufano

William Barry Inabnet

Marvin C. Gershengorn

Syed Morshed

Ricardo Pujol-Borrell

Peter A. Kopp

Leonard Wartofsky

ATA Expo Theatre Presented by Sanofi Genzyme: Employing

Thyroglobulin Assay for Risk Stratification in the Management of Differentiated Thyroid Cancer

(Limited seating; first-come, first served; lunch offered)

Paul Starr Award Lecture

Technical and Technological Advances that Changed Thyroid Surgery

Introduction by Gerard M. Doherty

Basic Symposium: MicroRNAs in Thyroid Cancer

Moderator: Electron Kebebew

Integrated Genomic Analysis in Thyroid FNA

MicroRNA in Thyroid Follicular Cell Differentiation and Carcinogenesis

MicroRNA-LOX-SLUG Axis in Thyroid Cancer Progression

Thomas Giordano

Pilar Santisteban

Myriem Boufraqech

Clinical Symposium: Thyroid and Pregnancy

Chair: John H. Lazarus

Subclinical Hypothyroidism in Pregnancy: Implications and Controversies

Is Pregnancy a Risk Factor for Thyroid Cancer Progression? 2017 ATA Thyroid in Pregnancy Guidelines

Ridgway Trainee Conference: Case Discussions - Thyroid Function (Open to all trainees)

Moderators: Tracey S. Tylee and Jennifer A. Morrison

Spyridoula Maraka

Luba Rakhlin

Elizabeth N. Pearce

Catherine A. Dinauer

Peter A. Singer

Leonard Wartofsky 
3:05 PM - 4:00 PM

VCC, Oak Bay

4:00 PM - 4:15 PM

VCC, Salon AB

4:15 PM - 5:00 PM

VCC, Salon AB

5:05 PM - 6:00 PM

VCC, Salon AB

VCC, Sidney

VCC, Esquimalt

VCC, Colwood

VCC, Saanich

VCC, Lecture Theatre

7:30 PM - 11:00 PM

Royal BC Museum

675 Belleville Street
ATA Expo Theatre Presented by Interpace Diagnostics: Advances in Molecular Testing of Indeterminate Thyroid Nodules - ThyGenX ${ }^{\circledR}$ and ThyraMIR ${ }^{\circledR}$

(Limited seating; first-come, first served)

Presentation of ATA Awards

Presented by John C. Morris, ATA President

2017 ATA Distinguished Service Award

2017 John B. Stanbury Thyroid Pathophysiology Medal

Clark T. Sawin Historical Vignette

1967-2017: From Resistance to Thyroid Hormone to Thyroid

Hormone Receptors

Moderator: Peter Kopp; Introduction by Donald St. Germain

Discussion-Debates/ Meet the Professor Workshops (6 total)

- DM 7: Radioiodine-Refractory Thyroid Cancer

Moderator: Naifa Lamki Busaidy

Genetic Backgrounds and Mechanisms of Radioioidine-Refractory Thyroid Cancer

Targeting Signaling Pathways to Restore Radioiodine Avidity of Thyroid Cancer

- DM 8: Stem Cells in Thyroidology

Moderator: Sheue-yann Cheng

Differentiation of Human Embryonic Stem Cells into Thyrocytes

Thyroid Hormone Regulates Intestine Stem Cell Development during Xenopus Laevis Metamorphosis

- DM 9: Autoimmune Thyroiditis

Moderator: Salvatore Benvenga

The Role of Iodine

The Role of Selenium

- DM 10: Epidemiology of Thyroid Function and Common Diseases

Moderator: David S. Cooper

Thyroid Function and Cardiovascular Disease: Underlying Mechanisms

Hypothyroidism in Chronic Kidney Disease: Physiologic Adaptation or Physiologic Entity?

- DM 11: Pediatric Thyroid Cancer

Moderator: Steven G. Waguespack

Recent Progress in Genetics of Pediatric Thyroid Cancer

The Role of FNAB in Pediatric Thyroid Nodules

- DM 12: Thyroid Cancer Management - Novel Therapeutics and Management Options (Workshop co-sponsored by SNMMI) Moderator: James $V$. Hennessey

Treatment of Distant Metastatic Follicular Thyroid Cancer:

Radioactive Iodine Therapy and Beyond

Evaluation and Management of Patients with Differentiated Thyroid Cancer (DTC), Positive Thyroglobulin Levels $(+\mathrm{Tg}$ ), and Negative Diagnostic Radioiodine Scans (-DxSc)

ATA Annual Banquet at the Royal BC Museum

(Ticketed event, pre-registration required; comfortable shoes encouraged)

The Royal BC Museum is located across the street from the Fairmont and VCC. Tour the First Nations and Modern History museum galleries, enjoy great food along the way at various food stations and socialize with colleagues and friends.
Leonidas Duntas

Josef Koehrle

Thomas J. Fahey

David Lieu

Bryan R. Haugen

James A. Fagin

Samuel Refetoff

Gilbert H. Daniels

Douglas Forrest

Carla Moran

Yan-Song Lin

Sissy M. Jhiang

Terry F. Davies

Yun-Bo Shi

Layal Chaker

Connie M. Rhee

Ali S. Alzahrani

Andrew J. Bauer

Mona M. Sabra

Douglas Van Nostrand 
$87^{\text {th }}$ ANNUAL MEETING OF THE ATA SATURDAY, OCTOBER 21, 2017

6:00 AM - 7:45 AM

VCC, Lecture Theatre

7:30 AM - 5:15 PM

VCC, Registration

7:30 AM - 5:30 PM

VCC, West Coast

8:00 AM - 8:05 AM

VCC, Salon AB

8:00 AM - 12:15 PM

VCC, Salon C

8:05 AM - 8:50 AM

VCC, Salon AB

8:45 AM - 9:15 AM

Fairmont, Crystal

Ballroom

8:50 AM - 9:50 AM

VCC, Lecture Theatre

8:50 AM - 9:50 AM

VCC, Salon AB
Early Riser Satellite Symposium Presented by AACE: Current

Landscape in Medullary Thyroid Cancer - Update for Clinicians

(Limited seating; first-come, first served; light breakfast offered)

\section{ATA Registration Open}

ATA Speaker Ready Room Open (Open to meeting faculty only)

Morning Announcements

ATA Pediatric Thyroid Forum Satellite Program: Update on Pediatric Thyroid Disease 2017 - Current State and Future Directions

Chair: Andrew J. Bauer

Plenary Lecture: Understanding How Breaches in Immune Tolerance Lead to Autoimmune Thyroid Disease

Introduction by Angela M. Leung

Ridgway Trainee Conference: Highlighted Trainee Abstracts

Short Oral Presentations (Open to all trainees)

Moderator: Tracey S. Tylee

Basic - De Novo Tri-iodothyronine (T3) Formation

Clinical - Differential Growth Rates of Benign Versus Malignant Thyroid Nodules

Case Report - The First Report of a Homozygous HSA Gene Mutation Causing Familial Dysalbuminemic Hyperthyroxinemia

Basic/Translational Short Call Oral Abstracts Session (4 total)

Chair: Mabel M. Ryder

Short Call Oral Abstract \#1: The Nuclear and Mitochondrial Genomes of Hürthle Cell Carcinoma and Its Metastases

Short Call Oral Abstract \#2: Modulation of Cholesterol and Sex Steroid Metabolism by 3,5-T2 in Lipid-Loaded Primary Mouse Hepatocytes

Short Call Oral Abstract \#3: Challenging the Adipocyte Color Barrier - TR Activation Elicits a White to Beige Transdifferentiation Independent of Beta-Adrenergic Signaling

Short Call Oral Abstract \#4: Radioiodine Refractoriness Is a Mechanism in the Increased Recurrence of Papillary Thyroid Cancer Driven by Coexisting BRAF V600E and TERT Promoter Mutations

Clinical Short Call Oral Abstracts Session (4 total)

Chair: Gregory W. Randolph

Short Call Oral Abstract \#5: Clinical Validation of ThyroSeq v3® Performance in Thyroid Nodules with Indeterminate Cytology: A Prospective Blinded Multi-Institutional Validation Study

Short Call Oral Abstract \#6: Development and Validation of Classifiers to Enhance the Afirma Genomic Sequencing Classifier Performance Among Hürthle Cell Specimens
Angela M. Leung

Mingzhao Xing

Gregory W. Randolph

Naifa Lamki Busaidy

Amy Y. Chen

Matthew D. Ringel

John Cambier

Cintia E. Citterio

Chirag M. Vyas

Mizuho Mimoto

Raj Gopal

Josef Koehrle

Kevin J. Phillips

Rengyun Liu

Yuri Nikiforov

Quan-Yang Duh 


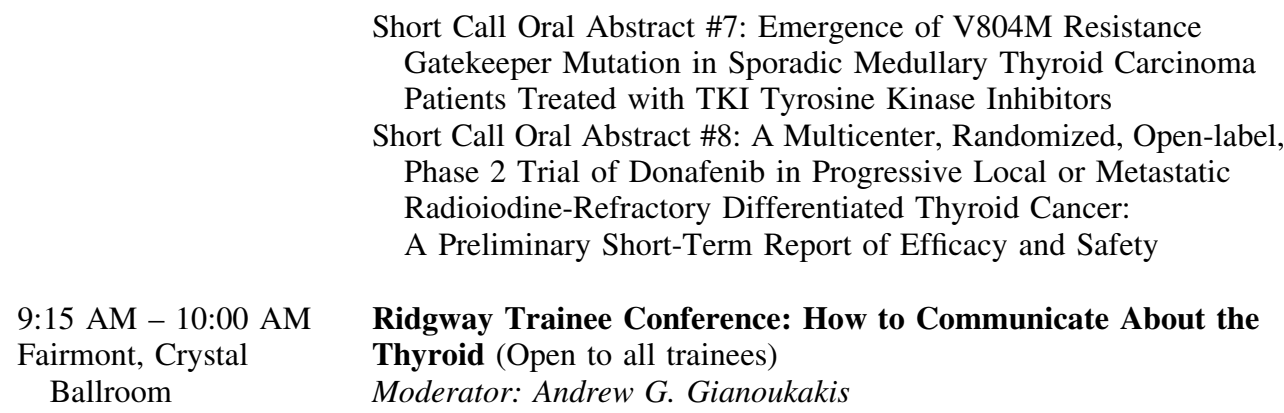

Short Call Oral Abstract \#7: Emergence of V804M Resistance Gatekeeper Mutation in Sporadic Medullary Thyroid Carcinoma Patients Treated with TKI Tyrosine Kinase Inhibitors

Short Call Oral Abstract \#8: A Multicenter, Randomized, Open-label, Phase 2 Trial of Donafenib in Progressive Local or Metastatic Radioiodine-Refractory Differentiated Thyroid Cancer: A Preliminary Short-Term Report of Efficacy and Safety

9:15 AM - 10:00 AM

Fairmont, Crystal

Ballroom

Ridgway Trainee Conference: How to Communicate About the Thyroid (Open to all trainees)

Moderator: Andrew G. Gianoukakis

9:30 AM - 1:30 PM

VCC, Crystal Garden

9:50 AM - 10:45 AM

VCC, Crystal Garden

9:50 AM - 10:45 AM

VCC, Crystal Garden

10:45 AM - 12:00 PM

VCC, Lecture Theatre

10:45 AM - 12:00 PM

VCC, Salon AB

12:00 PM - 1:00 PM

VCC, Crystal Garden

12:00 PM - 1:00 PM

VCC, Saanich

12:00 PM - 1:00 PM

Fairmont, Crystal

Ballroom

12:00 PM - 1:00 PM

VCC, Oak Bay

1:00 PM - 1:45 PM

VCC, Salon AB

1:30 PM

VCC, Crystal Garden

\section{Exhibit and Poster Hall Open}

(Open at 8:00 AM to poster presenters and exhibitors only)

Poster Review Session and Break in the Exhibit Hall

Posters 312-450; Short Call Posters 44-57

Trainee Poster Contest in the Exhibit Hall

(For ATA Trainee Poster Contest judges)

Basic Symposium: Novel Molecular Mechanisms of Thyroid

Cancer

Chair: Young Kee Shong

Nuclear Receptor Signaling Abnormalities in Thyroid Cancer

Synergistic Oncogene Interactions in Thyroid Cancer Progression and Clinical Outcomes

Novel Mutations in Benign Thyroid Neoplasms: Results from Whole Exome Sequencing Fine Arts

Chair: Emad Kandil

Subtleties in Surgical Management of Recurrent Differentiated Thyroid Cancer

Intraoperative Neuromonitoring in Thyroidectomy for Thyroid Cancer Trans-Oral Robotic Thyroid Surgery for Papillary Thyroid Cancer

Poster Review and Break in the Exhibit Hall

Ridgway Trainee Conference: Basic Trainees Lunch Session Career Tracks (Open to all trainees; Lunch ticket required for meal) Moderator: Salma Khan

Ridgway Trainee Conference: Clinical Trainees Lunch Session Career Tracks (Open to all trainees; Lunch ticket required for meal) Moderator: Steven P. Weitzman

ATA Expo Theatre Presented by Eisai, Inc.: LENVIVA Product Theatre (Limited seating; first-come, first served; lunch offered)

Sidney H. Ingbar Distinguished Award Lectureship Genomic Evolution of Thyroid Nodules and Cancer - New Answers to Old Questions Introduction: Sally E. Carty

Exhibit and Poster Hall Closes
Clinical Symposium: Surgical Treatment of Thyroid Cancer - The
Naifa Lamki Busaidy

Hui $\mathrm{Li}$

Gerard M. Doherty

Virginia A. LiVolsi

Andrew G. Gianoukakis

Frances E. Carr

Young Shin Song

Guang Ning

Ralph P. Tufano

Gaosong $W u$

Hoon Yub Kim

Aimee T. Franco

Veerle Darras

Jay Watsky

Joshua P. Klopper

Stephanie A. Fish

Richard T. Kloos

Bryan McIver

Yuri Nikiforov 
1:50 PM - 3:05 PM

VCC, Lecture Theatre

1:50 PM - 3:05 PM

VCC, Salon AB

2:00 PM - 4:00 PM

Fairmont, Shaughnessy

3:05 PM - 3:20 PM

VCC, Salon AB Foyer

3:20 PM - 4:50 PM

VCC, Lecture Theatre

3:20 PM - 4:50 PM

VCC, Salon AB
Basic Symposium: Novel Drug Treatments in Thyroid Oncology An Update

Chair: Steven I. Sherman

Novel Drug Treatments of Medullary Thyroid Cancer

Novel Drug Treatments of Differentiated Thyroid Cancer

Novel Drug Treatments of Anaplastic Thyroid Cancer

\section{Arthur Bauman Clinical Symposium: Thyroid Autoimmunity \\ Chair: Terry F. Davies}

Thyroid Autoimmunity: Overlap with Other Autoimmune Diseases

Thyroid Autoimmunity in the Elderly

Current Treatment Standards and Future Perspectives of Graves'

Disease

ATA Alliance for Patient Education Free Public Forum

(Open to the general public and all meeting attendees)

Sponsored by the ATA Alliance members: American Thyroid

Association, Bite Me Cancer, Graves' Disease and Thyroid

Foundation, Light of Life Foundation, ThyCa: Thyroid Cancer

Survivors' Association, Inc., Thyroid Cancer Canada and Thyroid

Federation International

\section{Refreshment Break}

Basic/Translational Oral Abstracts Session: Thyroid Hormone and Receptor (6 total)

Co-Chairs: Vasyl V. Vasko and Linwah Yip

Oral Abstract \#23: Thyromimetics Lower Cerebral Levels of Very Long Chain Fatty Acids in Mouse Model of X-Linked Adrenoleukodystrophy

Oral Abstract \#24: MCT8-Dependent Thyroid Hormone Uptake Promotes Neurogenesis and Radial Cell Migration Crucial for Proper Cortical Development

Oral Abstract \#25: Molecular and Genetic Analyses of the Function of Thyroid Hormone Receptor Coactivator SRC3 During Xenopus Postembryonic Development

Oral Abstract \#26: Thyroid Hormone Regulates KiSS1 Gene Expression Through PI3K/Akt-mTOR Signaling Pathway and Specificity Protein-1

Oral Abstract \#27: Non-Genomic Effects of Thyroid Hormones on Mesenchymal Stem Cells and Angiogenesis

Oral Abstract \#28: Probing the Thyroid Phenotype of Sbp2 Deficiency

Clinical Oral Abstracts Session: Thyroid Cancer (6 total)

Chair: Masha Livhits and Douglas Van Nostrand

Oral Abstract \#29: Clinical Validation of the Afirma Genomic Sequencing Classifier for Medullary Thyroid Cancer

Oral Abstract \#30: Ultrasound-Guided Radiofrequency Ablation of Low- Risk Papillary Thyroid Microcarcinoma: A Prospective Study on 421 Patients

Oral Abstract \#31: Thyroglobulin Does Not Correlate With Response in Radioactive Iodine Refractory (RAIR) BRAF Mutated Papillary Thyroid Cancer (PTC) Treated With Dabrafenib Alone or in Combination with Trametinib

Oral Abstract \#32: Age and Gender Distribution of Thyroid Nodular Lesions Found in Thyroid Ultrasound Examination Within Three Years After the Nuclear Accident: The Fukushima Health Management Survey
Rossella Elisei

Douglas W. Ball

Keith C. Bible

George J. Kahaly Kristien Boelaert Marius Stan
Meredith D. Hartley

Veerle M. Darras

Lingyu Bao

Gang Chen

Kathrin A. Schmohl

Haruki Fujisawa

Gregory W. Randolph

Mingbo Zhang

Bhavana Konda

Hiroki Shimura 


\begin{abstract}
4:50 PM - 5:45 PM
VCC, Saanich
\end{abstract}

VCC, Salon AB

VCC, Salon C

VCC, Esquimalt

VCC, Colwood

VCC, Lecture Theatre
Oral Abstract \#33: US Prediction of Highly Aggressive TERT Promoter- Mutated Papillary Thyroid Cancer

Oral Abstract \#34: Patient Age-Associated Mortality Risk Is Differentiated by the BRAF V600E Status in Papillary Thyroid

Cancer

Discussion-Debates/ Meet the Professor Workshops (6 total)

- DM 13: Novel Genetic Mouse Models for Basic Thyroid Research

Moderator: James A. Fagin

Genetic Mouse Models on Thyroid Hormones in Hearing

Transgenic Mouse Models in the Thyroid Cancer Microenvironment

- DM 14: Low-Risk Thyroid Cancer

Moderator: Anna M. Sawka

Cancers That Don't Require Surgery at All: Micro-PTC

Observation Cancers that are not Cancers (NIFTP)

- DM 15: Updates in the Understanding of T3, T4, and Thyroid Hormone Derivatives

Moderator: Brian W. Kim

Combination T3 and T4: What's New?

Thyronamines - Thyroid Hormone Derivatives: Biological Actions and Clinical Implications

- DM 16: Obesity and the Thyroid

Moderator: Lewis E. Braverman

Thyroid Function in Weight Gain: Underlying Mechanisms

Thyroid Function and the Metabolic Syndrome

- DM 17: Quality of Life in Thyroid Disease

Moderator: Jeffrey R. Garber

The ThyPRO Quality of Life Questionnaire

Quality of Life in Thyroid Cancer Patients

- DM 18: Use of PET Imaging in Thyroid Nodules and Thyroid Cancer

Moderator: Kenneth D. Burman

Evaluation of Incidental PET-Positive Thyroid Lesions

Use of PET Imaging in Thyroid Cancer: Indications and Interpretation
Tae Hyuk Kim

Xiaopei Shen

Douglas Forrest

Aime T. Franco

Allen S. Ho

Yuri Nikiforov

Jacqueline Jonklaas

Riccardo Zucchi

Shen $Q u$

Sun Y. Lee

Torquil Watt

Brisa Aschebrook-Kilfoy and Raymon H. Grogan

Jacques How Mehrbod Javadi 


\section{$87^{\text {th }}$ ANNUAL MEETING OF THE ATA SUNDAY, OCTOBER 22, 2017}

7:45 AM - 11:15 AM

Fairmont, Palm Court

8:00 AM - 8:05 AM

Fairmont, Crystal Ballroom

8:05 AM - 9:20 AM

Fairmont, Crystal Ballroom

9:25 AM - 11:00 AM

Fairmont, Crystal Ballroom

11:00 AM

ATA Registration Open

\section{Morning/Closing Announcements}

Iodine Global Network Symposium

Chair: Elizabeth N. Pearce

Understanding Iodine Sources in National Diets Status and Source of Iodine in U.S. Diets

The Current Status of Iodine Nutrition in Canada

Thyroid Cancer Tumor Board

Moderator: Manisha H. Shah

Endocrinology/Molecular Markers

Oncology

Surgery

Surgery

Pathology/Molecular Markers

Thyroid Sonography

Radiology

87th Annual Meeting of the ATA Concludes
Angela M. Leung Mingzhao Xing

Jonathan Gorstein Cria G. Perrine Mary L'Abbe

Bryan McIver Lori J. Wirth Gregory W. Randolph David J. Terris Ronald A. Ghossein Jennifer A. Sipos James D. Brierley 


\section{Thursday, October 19, 2017}

\section{Highlighted Oral 1}

Disorders of Thyroid Function Thursday Highlighted Oral Clinical

INCIDENT HYPOTHYROIDISM DURING TYROSINE KINASE INHIBITOR THERAPY PREDICTS SIGNIFICANTLY LONGER SURVIVAL IN PATIENTS WITH ADVANCED NON-THYROID CANCERS

M.G. Lechner ${ }^{1}$, C.M. Vyas ${ }^{2}$, O.R. Hamnvik ${ }^{3}$, E.K. Alexander ${ }^{2}$, P. Larsen ${ }^{2}$, T.E. Angell ${ }^{2}$

${ }^{1}$ Department of Medicine, Brigham and Women's Hospital, Boston, MA; ${ }^{2}$ Thyroid Section, Division of Endocrinology, Diabetes and Hypertension, Brigham and Women's Hospital, Boston, MA; ${ }^{3}$ Division of Endocrinology, Diabetes and Hypertension, Brigham and Women's Hospital, Boston, MA

Tyrosine kinase inhibitor (TKI)-induced thyroid dysfunction is attributed to multiple mechanisms, including immune destruction. Autoimmune thyroid disease can be associated with treatment response in cancer immunotherapy trials. The importance of thyroid dysfunction during TKI therapy to cancer prognosis is unclear. This study analyzes the pattern and significance of hypothyroidism during TKI therapy. A retrospective cohort study of adult patients with advanced non-thyroid cancer treated with TKI and available thyroid function testing (TFT), at three affiliated academic hospitals from 2000-2017. Exclusion: preexisting thyroid disease or insufficient follow-up. Demographic, clinical, and cancer treatment data were collected. Thyroid status with TKI treatment was determined from TFT and initiation of thyroid medication, and classified as euthyroid (TSH normal), subclinical hypothyroidism [(SCH), TSH 5-10 mIU/L, or higher TSH if FT4 normal), or overt hypothyroidism $[(\mathrm{OH}), \mathrm{TSH}>10 \mathrm{mIU} / \mathrm{L}$, low FT4, or requiring replacement). Multivariate modes were used to evaluate the risk factors for incident hypothyroidism during TKI therapy and its effect in an overall survival analysis. From 1120 initial patients, 538 remained after exclusion criteria. Incident SCH occurred in 72 (13\%), and $\mathrm{OH}$ in 147 patients (27\%) with TKI therapy. Multivariate analysis of risk, with adjustment for time, showed increased odds of incident hypothyroidism with female sex (OR 1.98, $\mathrm{p}=0.001$ ), greater TKI exposure (OR compared to $<1 \mathrm{yr}$ : 4.2 for $1-2 \mathrm{yr}, 6.9$ for $2-3 \mathrm{yr}, 41.1$ for $>3 \mathrm{yr}$, all $\mathrm{p}<0.0001$ ), or checkpoint inhibitor therapy (OR 3.6, $\mathrm{p}=0.04$ ). Number of TKIs received, other VEGF inhibitor therapy, race, and age were not associated with risk.

Patients with incident hypothyroidism had significantly longer overall survival (OS). Median survival in $\mathrm{OH}$ was $1643 \mathrm{~d}$ (95\% CI 1215-1991), SCH was 1005d (634-1528), and with euthyroid was 685d (523-851), p <0.001. After adjustment for clinical parameters, $\mathrm{OH}$ remained a significant predictor of OS (HR 0.51, $\mathrm{p}<0.001)$, whereas SCH did not (HR 0.74, p=0.06). New hypothyroidism in cancer patients treated with TKI is associated with improved overall survival and may reflect underlying changes in immune activation.

\section{Highlighted Oral 2}

Thyroid Hormone Action Thursday Highlighted Oral Basic SUMOYLATION OF TRß PLAYS AN ESSENTIAL ROLE IN REGULATION OF THE HYPOTHALAMIC-PITUITARY-THYROID (HPT) AXIS

S. Ke, F. Li, Y. Liu, G.A. Brent

Medicine, UCLA, Los Angeles, CA

Thyroid hormone acts through the thyroid hormone receptor beta $(\mathrm{TR} \beta)$ to regulate the HPT axis. We previously identified sumoylation sites in the $\operatorname{TR} \beta$ protein and demonstrated that posttranslational modification of TR by small unbiquitin-like modifier (SUMO) influences TR/T3-mediated transcription, including negative regulation of the TSHb gene. We introduced a mutation at TR $\beta$ sumoylation site (K146Q) and generated a TR $\beta$ sumoylation mutant mouse model. Here, we present HPT axis phenotype of the mutant mice.

1. Generation a TR $\beta$ K146Q sumoylation site-mutant mice.

2. T3 suppression of TSH Test: wild type (Wt) and mutant mice were rendered hypothyroid with $0.15 \%$ PTU and a low iodine diet for 4 weeks. T3 was administrated daily for 3 weeks with increasing dose for each week $(0.25 \mathrm{mg}, 0.5 \mathrm{mg}$, and $1.0 \mathrm{mg} / 100 \mathrm{~g}$ bw/day $)$. Serum TSH was determined after one week of T3 treatment at each dose.

3. Detection of TSH $\beta$ in pituitary using IF staining WB.

4. Histology analysis of pituitary and thyroid gland. TR $\beta$ sumoylation site-mutant mice (K146Q) had slightly elevated serum T4 (increased 9.5\%, $>>0.05$ ) and elevated serum T3 (increased 33\%, $p<0.05)$ concentrations, compared to Wt mice. In contrast to these modest elevations, the serum TSH in the mutant mice was increased 80fold compared to Wt mice. Histology and immunostaining indicated anterior pituitary enlargement and significant thyrotroph hyperplasia in mutant mice. To determine the dynamic effects of exogenous T3, we performed a TSH suppression test with progressive doses of T3. Although TSH was significantly suppressed by T3 in the mutant mice it remained significantly greater than Wt mice at each $\mathrm{T} 3$ dose level. Mutant mice had thyroid gland enlargement. Histological analysis was consistent with diffuse hyperplasia, indicating TSH bioactivity. These data indicate that the TR $\beta$ sumoylation site-mutant (K146Q) impairs TR/T3-mediated feedback regulation, and leads to secretion of excess TSH. Additionally, T4 levels did not increase proportionately to the elevated TSH, indicating an impairment of TSHdependent thyroid hormone synthesis and release. We conclude that $\operatorname{TR} \beta$ sumoylation is important for HPT axis function and possibly for normal thyroid gland development.

\section{Highlighted Oral 3}

Thyroid Cancer Thursday Highlighted Oral Translational TERT MUTATIONS INCREASE RISK OF TREATMENT FAILURE IN BRAF POSITIVE PAPILLARY THYROID

\section{CANCER}

D. Rusinek, A. Czarniecka, J. Krajewska,

M. Oczko-Wojciechowska, J. Zebracka-Gala,

E. Zebala-Nozynska, E. Chmielik, A. Pawlaczek, R. Cyplinska,

D. Handkiewicz-Junak, B. Jarzab

Nuclear Medicine and Endocrine Oncology Department, Oncology

Centre - MSC Memorial Institute, Gliwice, Poland

Papillary thyroid cancer (PTC) shows excellent prognosis in majority of patients, however, about $15-20 \%$ of them suffers from recurrence or progressive disease. Several molecular markers have been studied to identify poor prognostic factors in PTC. Although association between $\mathrm{BRAF}^{\mathrm{V} 600 \mathrm{E}}$ mutation and poor prognosis was demonstrated, its high prevalence limits clinical application. Recently, a telomerase reverse transcriptase (TERT) promotor mutation has been suggested as a strong prognostic marker in PTC.

The aim of our study was to check the prevalence and prognostic significance of $B R A F / T E R T$ mutation in tertiary referral center for thyroid cancer. Two hundreds PTC paraffin embedded samples (with $B R A F$ mutation already confirmed) were selected for TERT mutation analysis. DNA was extracted with QIamp DNA FFPE Tissue Kit (QIAGEN) and then proceeded with direct DNA sequencing (3130xl Genome Analyser, Life Technologies) with Big Dye 1.1. reagents. 
Up to now 61 samples from PTC patients (88\% women; $49 \%$ classical variant of PTC, median age at diagnosis 63) were analyzed. In 10 (16\%) out of 61 analyzed samples, TERT promotor mutations were found ( 9 in C228T codon and 1 in C250T). In patients with early stage of diseases at diagnosis (pT1-2; N0) rate of TERT mutation was significantly lower than in patients with more advanced disease (6\% vs.28\%; $\mathrm{p}=0.04$ ). When association of TERT promoter mutations with factors of poor prognosis was analyzed, larger tumor diameter and more advanced age at the moment of diagnosis were observed in patients with BRAF and TERT promoter mutations comparing to patients with only BRAFV600E alteration (with the p value 0.002 and 0.025 respectively).

After the median follow-up of 33 (range: 4-49) treatment failure was observed in $9(15 \%)$ patients (4 with disease recurrence and 5 with persistent disease). Rate of TERT mutation was significantly higher in patients with disease failure $(55 \%$ vs $9 \% ; \mathrm{p}<0.05)$. This preliminary results confirm that TERT mutations are found in less than $20 \%$ of $B R A F$ mutated PTC and that coexistence of BRAF/TERT mutation is related to worse PTC outcome than BRAF mutation alone.

\section{Highlighted Oral 4}

Autoimmunity Thursday Highlighted Oral Clinical
COMBINED MYCOPHENOLATE WITH INTRAVENOUS
METHYLPREDNISOLONE THERAPY IS MORE
EFFECTIVE THAN INTRAVENOUS
METHYL-PREDNISOLONE ALONE IN ACTIVE
AND MODERATE-TO-SEVERE GRAVES'
ORBITOPATHY - A RANDOMIZED, OBSERVER
BLIND, MULTICENTER TRIAL

G. Kahaly ${ }^{1}$, M. Riedl ${ }^{1}$, J. Koenig ${ }^{1}$, T. Diana ${ }^{1}$, S. Pitz ${ }^{1}$, A. Eckstein ${ }^{2}$, M. Salvi ${ }^{3}$, M. Marino ${ }^{4}$, C. Marcocci ${ }^{4}$, L. Bartalena ${ }^{5}$, P. Perros ${ }^{6}$, W. Wiersinga ${ }^{7}$

${ }^{1}$ Johannes Gutenberg University Medical Center, Mainz, Germany; ${ }^{2}$ Essen University, Essen, Germany; ${ }^{3}$ University of Milan, Milan, Italy; ${ }^{4}$ Pisa University, Pisa, Italy; ${ }^{5}$ University of Varese, Varese, Italy; ${ }^{6}$ University of Newcastle, Newcastle, United Kingdom;

${ }^{7}$ Academic Medical Center, Amsterdam, Netherlands

The guidelines of the European Thyroid Association/European Group on Graves' Orbitopathy (EUGOGO) recommend intravenous methylprednisolone $(\mathrm{P})$ as first-line treatment for active and severe Graves' orbitopathy (GO), however lack of response or relapse after discontinuation of treatment are frequent encounters. Therefore, EUGOGO compared the efficacy and safety of combination intravenous $\mathrm{P}$ and mycophenolate sodium ( $\mathrm{M}$, a lymphocyte proliferation inhibiting agent), therapy to $\mathrm{P}$ alone in GO. Within an observer-blind, randomized, multicenter trial (EUDRACT number 2008-002123-93), a total of 164 patients with active and moderate-to-severe GO randomly received intravenous $\mathrm{P}$ alone $(0.5 \mathrm{~g} /$ week for 6 weeks followed by $0.25 \mathrm{~g} /$ week for 6 weeks) or the same P protocol with $0.720 \mathrm{~g}$ oral $\mathrm{M} /$ day for 24 weeks $(\mathrm{P}+\mathrm{M})$. Primary endpoints were the response rates at (12 and) 24 weeks defined as reduction of at least two parameters of a composite ophthalmic index without simultaneous deterioration in any parameter. $36 / 73(49.3 \%)$ and $48 / 75$ (64\%) patients receiving $\mathrm{P}$ and $\mathrm{P}+\mathrm{M}$, respectively responded at week 12 , odds ratio 1.83 (95\% CI 0.95-3.53, $\mathrm{p}=0.071)$ whereas $37 / 71(52.1 \%)$ and $52 / 75(69.3 \%)$ responded at week 24 (OR 2.08, 95\%CI 1.06-4.09, p=0.033). Quality of life, clinical activity (CAS) and severity scores, diplopia score, ocular adduction and down gaze duction, as well as serum levels of TSH receptor autoantibodies significantly improved within the groups with significant differences of overall ophthalmic improvement, CAS, down gaze duction, eyelid and caruncle swelling between the groups. During the 12 week follow-up, nine (14\%) and $13(20 \%)$ patients improved while three $(8.6 \%)$ and three $(5.8 \%)$ relapsed in the $\mathrm{P}$ and $\mathrm{P}+\mathrm{M}$ groups, respectively. Overall improvement was noted in 34/65 (52.3\%) and
$49 / 66(74.2 \%), p=0.020$, respectively at week 36 . Mild/moderate drugrelated side effects occurred in 16/81 patients (20\%) and 21/83 (25\%), $\mathrm{p}=0.479$ in the $\mathrm{P}$ and $\mathrm{P}+\mathrm{M}$ groups, respectively. Addition of mycophenolate significantly improves the efficacy of methylprednisolone pulse therapy in patients with active/moderate-to-severe GO.

\section{Basic/Translational Oral 5}

Autoimmunity Thursday Basic/Translational Basic

INTERFERON ALPHA TRIGGERS AUTOIMMUNE THYROIDITIS VIA DEGRADATION OF THYROGLOBULIN BY CYSTEINE CATHEPSINS INTO IMMUNOGENIC PEPTIDES

L.C. Faustino, Y. Tomer

Medicine, Albert Einstein College of Medicine, New York, NY

Autoimmune thyroid diseases (AITD) arise from interactions between genetic, epigenetic and environmental factors. Interferon-alpha (IFN $\alpha$ ), secreted during viral infections, is a key cytokine that triggers AITD. We have previously shown that IFN $\alpha$ interacts epigenetically with a variant in TG promoter that is strongly associated with AITD. The risk $(\mathrm{G})$ allele created an IRF-1 binding site causing upregulation of TG by IFN $\alpha$. Surprisingly, the upregulation of TG mRNA was associated with decreased TG protein levels, which we previously showed was due to IFN $\alpha$-induced TG degradation via specific cathepsins. We therefore hypothesized that IFN $\alpha$ triggers thyroiditis by inducing TG degradation resulting in release of immunogenic TG peptides such as Tg.2098 (a major T-cell epitope that is a product of cathepsin L cleavage). To test this hypothesis, we immunized CBA-J and DR3 "humanized" mice with human thyroid cell lysates that were either untreated or treated with IFN $\alpha$. We observed higher T-cell proliferative responses to TG and Tg.2098 in mice immunized with IFN $\alpha$-treated lysates, suggesting that IFN $\alpha$ triggered TG degradation into immunogenic peptides, specifically Tg.2098. Similarly, cytokine responses to TG and Tg.2098 were higher in both strains immunized with IFN $\alpha$-treated lysate. Mice immunized with IFN $\alpha$-treated lysate also showed higher levels of TG antibodies again supporting the production of immunogenic TG peptides in thyroid cells treated with IFN $\alpha$. To confirm that TG degradation by cathepsins into immunogenic peptides was responsible for the increased autoimmunity in immunized mice, we immunized mice with lysates that were co-treated with IFN $\alpha$ and chymostatin (inhibitor of cysteine cathepsins). Confirming our hypothesis we found lower T-cell proliferative and cytokine responses to TG and $\mathrm{Tg}$.2098, and reduced sera reactivity against TG in mice immunized with lysates from cells incubated with IFN $\alpha$ and chymostatin, which suppressed production of immunogenic TG peptides. Our data suggest a new model for the development of AITD whereby IFN $\alpha$ triggers AITD by inducing degradation of TG via cysteine cathepsins into immunogenic peptides, specifically Tg.2098, leading to activation of self-reactive T-cells.

\section{Basic/Translational Oral 6}

Thyroid \& Development Thursday Basic/Translational Basic FIRST REPORT OF TREE SHREW GRAVES' DISEASE MODEL

L. Wu, B.Y. Shi, X. Guan, F. Zhao, Y. Wang, P. Chen, S. Hu, R. Cui The First Affiliated Hospital of Xi' an Jiaotong University, Xi'an, China

Graves' disease (GD) model was successfully established in mice and nonhuman Primates immuned with recombinant adenovirus expressing the human thyrotropin receptor A subunit (A-Sub-Ad). Tree shrews are climbing animals between insectivores and primates. Evolutionarily closer to primates than rodents, tree shrews are being considered as an alternative to primates for study. The aim of the present study is to 
establish a GD model in female tree shrews to explore its underlying mechanisms. 5-6 months female tree shrews were immunized intramuscularly with A-Sub-Ad or control adenovirus eight times at 3 weeks intervals. Animals were monitored for weight loss, heart rate and fasting blood glucose changes. Blood, thyroid and orbital tissues were collected for detecting the related indicators of the disease. After eight immunization, compared with controls, all the tree shrews injected with A-subAd generated a strong antibody response to TSHR $(\mathrm{P}<0.001)$, and $80 \%$ animal had increased total thyroxine $(\mathrm{T} 4)(\mathrm{P}<0.01)$. Hyperthyroid tree shrews developed goiter and throid tissue hyperplasia. Tachyrhythmia and weight loss was also observed in hyperthyroid tree shrews. Part of the hyperthyroid tree shrews showed obvious orbital changes and elevated fasting plasma glucose. All GD tree shrews exhibited more homologous features to human GD than previous rodent models. To further understand the pathogenesis of the disease, quantitative analysis on $\mathrm{T}$ helper cells cytokine in sera was carried out. The results displayed that hyperthyroid tree shrews produced higher level of IFN- $\gamma$, IL-2, IL-12p70 and IL-10 than controls $(\mathrm{P}<0.05)$. In addition, declined regulatory T cell and dendritic cell population was also observed in hyperthyroid animals. Taken together, these data demonstrated that we had successfully established an animal model manifesting GD in tree shrews, which involved a mixed Th1 and Th 2 response. This model can provide a more effective and practical tool for study on the Graves' disease.

\section{Basic/Translational Oral 7}

Iodine Uptake \& Metabolism Thursday Basic

IDENTIFICATION OF NOVEL SODIUM IODIDE SYMPORTER (NIS) INTERACTORS WHICH MODULATE IODIDE UPTAKE

A. Fletcher ${ }^{1,3}$, V. Poole ${ }^{1,3}$, B. Modasia ${ }^{1,3}$, W. Imruetaicharoenchoke ${ }^{1,3}$, R.J. Thompson ${ }^{1,3}$, N. Sharma ${ }^{1,3}$, H. Nieto ${ }^{1,3}$, M.L. Read ${ }^{1,3}$, A. Turnell ${ }^{2}$, K. Boelaert ${ }^{1,3}$, V.E. Smith ${ }^{1,3}$, C.J. McCabe $\mathrm{M}^{1,3}$

${ }^{1}$ Institute of Metabolism and Systems Research, University of Birmingham, Birmingham, West Midlands, United Kingdom;

${ }^{2}$ Institute of Cancer and Genomic Sciences, University of Birmingham, Birmingham, United Kingdom; ${ }^{3}$ Centre for Endocrinology, Diabetes and Metabolism, Birmingham Health Partners, Birmingham, United Kingdom

By exploiting the canonical function of the sodium iodide symporter (NIS), ablative radioiodide therapy is an effective treatment for thyroid cancer. However, a subset of patients are unable to accumulate sufficient radioiodide due to decreased expression and/or plasma membrane localization of NIS. Radioiodide therapy has been proposed as a viable treatment for breast cancer, but is hampered by low levels of NIS membrane localization. Currently, the regulation of NIS trafficking to the plasma membrane is ill-defined. Mass spectrometry was performed on proteins co-immunoprecipitating with lentivirally expressed NIS in whole cell and plasma membrane extracts. NIS function was assessed following knockdown, overexpression and inhibition of shortlisted interactors using radioiodide uptake assays. Interactors were validated by co-immunoprecipitation and proximity ligation assays. NIS activity was significantly altered by ADP-ribosylation factor 4 (ARF4) and valosin containing protein (VCP) in TPC1 thyroid and MDA-MB231 breast cancer cells lentivirally-expressing NIS. ARF4 downregulation decreased radioiodide uptake by $75 \%$ and $44 \%$, and VCP downregulation increased radioiodide uptake by $71 \%$ and $56 \%$, in thyroid and breast cells, respectively. Transient overexpression of these genes significantly reversed siRNA effects on NIS function. Coimmunoprecipitation assays confirmed NIS interacts with ARF4 and $\mathrm{VCP}$ in vitro, and proximity ligation assays revealed the subcellular sites of interaction. TCGA data analysis of 58 matched papillary thyroid cancers revealed ARF4 was significantly repressed and VCP highly upregulated in thyroid cancer, providing a putative explanation for repressed NIS function. Pharmacological inhibitor studies demonstrated Eeyarestatin-1 and NMS-873 could overcome VCP inhibition of NIS function in both thyroid and breast cells, implicating the endoplasmic reticulum-associated degradation pathway as critical to NIS processing. Further, we identified that NIS is ubiquitylated in vitro, and suggest this as the possible mechanism through which VCP alters NIS function. These studies thus identify two new potential therapeutic targets for enhancing radioiodide uptake in radioiodide-refractory patients.

\section{Basic/Translational Oral 8}

Autoimmunity Thursday Translational

PERFORMANCE AND SPECIFICITY OF SIX

IMMUNOASSAYS FOR TSH RECEPTOR ANTIBODIES - A MULTICENTER STUDY

T. Diana ${ }^{1}$, C. Wuester ${ }^{2}$, P. Olivo ${ }^{3}$, M. Kanitz ${ }^{1}$, A. Bossowski ${ }^{4}$, B. Decallonne ${ }^{5}$, G. Kahaly ${ }^{1}$

${ }^{1}$ Johannes Gutenberg University Medical Center, Mainz, Germany; ${ }^{2}$ Endocrine Laboratory, Mainz, Germany; ${ }^{3}$ Washington University St. Louis, Saint Louis, MO; ${ }^{4}$ Medical University in Bialystok, Bialystok, Poland; ${ }^{5}$ UZ Leuven, Leuven, Belgium

The guidelines of the American Thyroid Association recommend the measurement of TSH receptor (TSHR) autoantibodies for the diagnosis of autoimmune thyroid disease (AITD). The performance, detection sensitivity, and specificity of six TSHR immunoassays were prospectively compared.

Two stimulatory and blocking TSHR bioassays and four automated and/or ELISA binding assays (Kronus, Immulite, Kryptor, and Dynex) were compared in a dilution study performed in serum samples of patients with AITD collected from four European academic referral centers. Both bioassays were compared to two binding assays using stimulatory (M22) and blocking (K1-70) monoclonal antibody (MAb) mixtures. Thirty well-characterized, untreated, hyperthyroid stimulatory (TSAb)-positive/locking (TBAb)-negative patients with Graves' disease (GD) were included as well as ten, euthyroid (on levothyroxine) TBAb-positive/TSAb-negative patients with either GD or Hashimoto's thyroiditis (HT). Thirty samples from TSAb-positive/TBAb-negative patients with GD were diluted serially and measured in all assays. Samples were positive until dilution 1:2187 in the TSAb bioassay; 1:81 in the Immulite $(\mathrm{P}<0.002$ vs. bioassay) and Kronus ELISA $(\mathrm{P}=0.039)$ assays; and 1:27 in the Kryptor and Dynex ELISA ( $\mathrm{P}<0.001$ vs bioassay). Ten samples from TBAb-positive/TSAb negative patients with GD or HT were positive in all binding assays. None of the binding assays differentiated between TSAb and TBAb. Mixtures of 100\% K1-70 $(200 \mathrm{ng} / \mathrm{ml}), 80 \% \mathrm{~K} 1-70+20 \% \mathrm{M} 22,60 \% \mathrm{~K} 1-70+40 \% \mathrm{M} 22,40 \%$ $\mathrm{K} 1-70+60 \% \mathrm{M} 22,20 \% \mathrm{~K} 1-70+80 \% \mathrm{M} 22,100 \% \mathrm{M} 22(20 \mathrm{ng} / \mathrm{ml})$ tested positive in both Immulite $(26.4,20.2,15.2,10.5,6.3,2.00 \mathrm{IU} / \mathrm{L})$, and Kronus assays (27.1, 23.3, 19.3, 12.0, 5.7, 2.2 IU/L). These MAb mixtures were tested in the TBAb bioassay and showed 82, 61, 24 (neg.), -26 (neg.), -77 (neg.), and $-95 \%$ (neg.) percent inhibition, respectively. The sample dilution study showed higher detection sensitivity for the TSAb bioassay and the antibody mixture study demonstrated exclusive specificity of the bioassays over all automated and ELISA binding assays.

\section{Basic/Translational Oral 9}

Autoimmunity Thursday Clinical

FUNCTIONAL AIM2 INFLAMMASOME EXPRESSED IN THYROCYTES ARE ASSOCIATED WITH AUTOIMMUNE THYROIDITIS

Q. Guo, Z. Shan, W. Teng 
Department of Endocrinology and Metabolism, The First Affiliated Hospital of China Medical University, Institute of Endocrinology, Liaoning Provincial Key Laboratory of Endocrine Diseases, Shenyang, Liaoning, China

Absent in melanoma 2 (AIM2) is a double-stranded DNA receptor, and its activation initiates an inflammasome assembly, which leads to cleavage-induced maturation of pro-inflammatory cytokines, IL- $1 \beta$ and IL-18, and also mediates a programmed cell death, pyroptosis. AIM2 inflammasome is an important member of the innate immune system and has been reported to contribute to a variety of autoimmune disorders. The present work aimed to investigate whether AIM2 inflammasome is involved in autoimmune thyroiditis (AIT). Expressions of pro- and active IL- $1 \beta$ and IL-18 and inflammasome components (AIM2, ASC, CASP1) at the mRNA and protein levels in the thyroid tissues from 50 patients with AIT and 50 controls were investigated by quantitative real-time PCR and Western-blot, respectively. Immunohistochemistry and TUNEL immunofluorescence were used to locate the AIM2 and dsDNA expressions. AIM2 inflammasome activation in cultured Nthy-ori 3-1 cells was determined as the co-localization of ASC spots and AIM2 by confocal microscopy. Apoptosis and pyroptosis were analysed via flow cytometry. Active IL$1 \beta, \mathrm{IL}-18, \mathrm{P} 20$ and GASDMD in cell lysates were measured by Westernblot while cytokines in the supernatants were determined by ELISA. Active IL-1 $\beta$ and IL18 proteins were detectible in AIT thyroid glands and significantly higher than that in the controls $(\mathrm{P}<0.05)$. The mRNA and protein levels of AIM2, ASC, CASP1, pro-IL-18 and pro-IL- $1 \beta$ in AIT thyroid tissues were statistically higher than that in the controls $(\mathrm{P}<0.05)$, and positively correlated with serum TPOAb and $\mathrm{TgAb}$ levels. The AIM2 expression was mainly located in the thyroid follicles adjacent to inflammatory infiltration. And we also observed enhanced TUNEL staining in the AIT thyroid tissues. IFN- $\gamma$ promoted about 100 times expression of AIM2 in the cultured thyroid cells. Ploy(dA:dT) stimulation could lead to AIM2 inflammasome activation and active IL18 release accompanied by pyroptosis of thyrocytes. We show for the first time that functional AIM2 inflammasome is present in the thyrocytes and overexpression may be involved in AIT pathology. Our work has provided insights on the organ-specific tissue damage in AIT.

\section{Clinical Oral 10}

\section{Iodine Uptake \& Metabolism Thursday Clinical \\ DEVELOPMENT AND VALIDATION OF A NOVEL IODINE-129 TRACER METHOD FOR THE SAFE ASSESSMENT OF IODINE ABSORPTION AND THYROIDAL UPTAKE FROM ORAL IODINE SUPPLEMENTS AND FOODS IN HUMANS \\ M. Zimmermann, V. Galetti \\ ETH Zurich, Zurich, Switzerland}

Thyroidal iodine uptake and turnover has been measured in adults using radioactive iodine tracers, but these cannot be used safely in women of reproductive age, pregnant women or children. Iodine-129 (I-129) is a semi-stable isotope that is considered safe for use in human trials. Our objective was to assess 129I as a novel tracer for the assessment of fractional iodine absorption and thyroidal uptake. We administered an oral physiological dose of 129I (129I Dose) to euthyroid young adults with adequate iodine intake. We collected baseline complete urines, complete feces and spot plasma samples. After tracer consumption, we collected complete urines (over seven days), complete feces (three days), and spot plasma (five days, with eight withdrawals on first day). The subjects followed an iodine-standardized diet from three days before until three days after tracer consumption. We measured 129I in alkalineextracted plasma (129IP), urine (129IU) and fecal (129IF) samples by a multicollector ICP-MS by use of isotope dilution analysis with 127 I.
129IFA was calculated as 129I dose minus 129IF; 129ITU as129IFA minus 129IU; 129IP kinetics allows the calculation of thyroid uptake patterns. Four male and four female euthyroid (mean \pm SD TSH $1.3 \pm 0.5 \mathrm{mU} / \mathrm{L}$; T4 $110.0 \pm 19.7 \mathrm{nmol} / \mathrm{L}$ ) participants with a median (IQR) urinary iodine concentration of 118 (101-140) $\mu \mathrm{g} / \mathrm{L}$ consumed $12.42 \pm 0.05 \mu \mathrm{g} 129 \mathrm{I}$. 129IU was first detected $\approx 1$ hour after administration and a median cumulative 129IU of $6.9(6.4-7.4) \mu \mathrm{g}$ was excreted within 48 hours; this corresponds to $54.8 \pm 10.6 \%$ of the 129I Dose. Results on 129IU and 129IF will characterise partitioning and kinetic patterns of the remaining 45.2\% 129I. A novel 129I-tracer based method successfully quantified 129I renal excretion patterns, showing that $\approx 55 \%$ of ingested oral iodine is excreted in the subsequent 48 hours. Quantification of 129IFA and 129ITU thyroidal uptake patterns are pending. This promising and safe tracer methodology can safely be used in all age groups to investigate a variety of research questions on thyroid health and iodine nutrition, including iodine requirements in pregnancy, lactation and infancy.

\section{Clinical Oral 11}

Disorders of Thyroid Function Thursday Clinical

INCIDENCE OF NEONATAL HYPERTHYROIDISM AMONG NEWBORNS OF GRAVES' DISEASE PATIENTS TREATED WITH RADIOIODINE THERAPY WITHIN 2 YEARS BEFORE CONCEPTION

A. Yoshihara, J. Noh, N. Watanabe, K. Iwaku, Y. Kunii, H. Ohye, M. Suzuki, M. Matsumoto, N. Suzuki, C. Sekiyama, R. Tadokoro, K. Sugino, K. Ito

Ito Hospital, Tokyo, Japan

The serum TSH receptor antibody (TRAb) titer of Graves' disease (GD) patients is known to increase after radioiodine therapy (RIT) and remain high for years. We investigated the incidence of neonatal hyperthyroidism (NH) among the newborns born to mothers who conceived within 2 years after RIT, and we attempted to identify predictors of NH. The cases of 145 GD patients who conceived within 2 years after RIT were retrospectively reviewed, and information regarding their newborns was collected. Median age at the time of RIT was 28 years old, and mean thyroid volume at the time was $48.8 \mathrm{ml}$ (range 13.1-203.8). There were 7 newborns with $\mathrm{NH}$, and its incidence was $4.8 \%$. The group of patients whose newborns exhibited NH (NH group) had higher TRAb titers at the time of RIT, higher TRAb titers in the first trimester, and higher TRAb titers in the third trimester than the group whose newborns did not develop $\mathrm{NH}$ (non-NH group). Thyroid volume at the time of RIT, radioiodine dose, and number of months between RIT and conception did not differ significantly between the two groups. Multivariate analysis revealed the TRAb titer in the third trimester to be the only risk factor for $\mathrm{NH}$. The receiver operating characteristic curve analysis showed that the cut-off TRAb titer in the third trimester for predicting NH was 9.7 IU/L. The incidence of NH among the newborns born to mothers with GD who conceived within 2 years after RIT was $4.8 \%$. The sole risk factor for $\mathrm{NH}$ was a high TRAb titer in the third trimester of pregnancy. The fetuses of pregnant GD patients whose TRAb titer is 9.7 IU/L or above in the third trimester should be carefully followed up by an obstetrician during pregnancy, and the newborns should be carefully followed up by a pediatrician after birth.

\section{Clinical Oral 12}

Autoimmunity Thursday Clinical

\section{DIFFERENT FT4 RESPONSE TO HCG UNDER TPOAB} OR TGAB DURING FIRST-HALF TRIMESTER

Y. Hou, A. Liu, C. Fan, Y. Li, H. Zhang, W. Hong, S. Ding, W. Teng, Z. Shan 
Department of Endocrinology and Metabolism, The First Affiliated Hospital of China Medical University, Shenyang City,

LiaoningProvince, China

It was reported that thyroperoxidase antibody (TPOAb) positivity might interfere with FT4 stimulation induced by human chorionic gonadotropin (hCG). However, the effects of thyroglobulin antibody $(\mathrm{TgAb})$ on thyroid hormone stimulated by hCG are unknown. The aim of our study was to explore thyroidal response to hCG in different conditions of thyroid autoantibodies during pregnancy. In this cross-sectional study, we screened 677 women at 7 to 20 weeks' gestation within pregnancy-specific reference range for TSH. Serum thyrotropin (TSH), free thyroxine (FT4), TPOAb, TgAb and hCG were measured using the electrochemiluminescence immunoassay (Roche, Basel, Switzerland). According to thyroid autoantibodies, these women were divided into the four groups: co-positive for TPOAb and TgAb, isolated positive TPOAb, isolated positive $\mathrm{TgAb}$, and conegative for TPOAb and $\mathrm{TgAb}$. Nonparametric tests, chi-square tests and linear regression models were performed to assess the reaction of FT4 to hCG using Statistical Package of Social Sciences version 20.0 for Windows (SPSS Inc. Chicago, IL, USA). TSH was negatively correlated with hCG in co-positive for $\mathrm{TPOAb}$ and $\mathrm{TgAb}$ group, isolated positive TPOAb group, isolated positive $\mathrm{TgAb}$ group and co-negative for $\mathrm{TPOAb}$ and $\mathrm{TgAb}$ $\operatorname{group}(\mathrm{P}<0.05)$. FT4 was positively associates with $\mathrm{hCG}$ in isolated positive $\mathrm{TgAb}$ group and co-negative for TPOAb and $\mathrm{TgAb}$ group $(\mathrm{P}<0.05)$. However there were no correlations between FT4 and hCG in co-positive for TPOAb and TgAb group and isolated positive TPOAb group $(\mathrm{P}>0.05)$. When TSH was within pregnancy-specific reference range, the pregnant women with positive TPOAb did not have an adequate FT4 response to hCG regardless of TgAb. In contrast, $\mathrm{TgAb}$ did not interfere FT4 response to hCG. This suggest that TPOAb might play a more significant role in responsive impairment of FT4 to hCG.

\section{Clinical Oral 13}

Thyroid Hormone Metabolism \& Regulation Thursday Clinical DIFFERENT TRAJECTORIES OF THYROID FUNCTION DURING PREGNANCY: A NEW APPROACH TO LOOK AT PHYSIOLOGICAL CHANGES OF THYROID FUNCTION DURING GESTATION

V. Pop

Medical Health Psychology, Tilburg University, Tilburg, Brabant, Netherlands

Latent class growth analysis (LCGA) is a sophisticated statistical approach that can accommodate temporal changes within groups rather than between groups. We evaluated physiological changes of thyroid function during gestation in an iodine sufficient area using LCGA.

Thyroid function (TSH and fT4) was assessed in 1123 healthy pregnant women at 12, 24 and 36 weeks gestation. Thyroid dysfunction was defined at each trimester, using $2.5^{\text {th }}$ and $97.5^{\text {th }} \mathrm{TSH} /$ fT4 percentiles as lower and upper reference limits in TPO-Ab negative women. LCGA discriminated three different trajectories differing longitudinal TSH and T4 changes. The first, $(\mathrm{n}=52$, 4.6\%) was labeled: 'Highest - decreasing TSH along the lowest fT4 trajectory'. Women in this class showed the highest TSH levels (mean of $3.2 \mathrm{IU} / \mathrm{l}$ ) and the lowest fT4 (mean of $15.3 \mathrm{pmol} / \mathrm{l}$ ) levels at 12 weeks. The second trajectory $(\mathrm{n}=32,2.8 \%)$ was labeled: 'Intermediate-increasing TSH/intermediate decreasing fT4 trajectory'; these women showed the highest increase of TSH to- wards term (mean TSH at 36 weeks of $3.3 \mathrm{IU} / \mathrm{l})$. The third group ( $\mathrm{n}=1039,92.4 \%$, reference group) was labeled 'Low-increasing TSH/high decreasing fT4 trajectory'.

(Sub)clinical hypothyroidism at 12 weeks predominantly occurred in the first trajectory (up to 60\%), as did elevated TPO-Ab titres $(50 \%)$ and a parental history of thyroid dysfunction (23\%). Hypothyroxinemia almost exclusively occurred in the reference group. At 12 weeks gestation, $85 \%$ of the TSH $>97.5^{\text {th }}$ percentile cases were found in the first trajectory with only $13 \%$ in the second trajectory, while at 34 weeks this was the opposite: $31 \%$ versus $69 \%$, respectively. LCGA shows different trajectories of thyroid-function (TSH and fT4) change during pregnancy with different characteristics. Future research should compare the obstetric outcome and the offspring's developmental outcome between these different trajectories rather than focusing on crosssectional associations.

\section{Clinical Oral 14}

Thyroid Hormone Metabolism \& Regulation Thursday Translational

THE ASSOCIATION OF PHTHALATE EXPOSURE WITH THYROID FUNCTION DURING PREGNANCY: A MIXTURE ANALYSIS

T. Korevaar ${ }^{1}$, L. Chaker ${ }^{1}$, E. Philips ${ }^{1}$, V. Jaddoe ${ }^{1}$, A. Ghassabian ${ }^{2}$, L. Trasande ${ }^{2}$, T. Visser ${ }^{1}$, R. Peeters ${ }^{1}$

${ }^{1}$ Erasmus University Medical Center, Rotterdam, Netherlands;

${ }^{2}$ NYO MC, New York, NY

Optimal maternal thyroid function is important for an uncomplicated pregnancy and child development. Pregnant women are ubiquitously exposed to phthalates, a group of chemicals used as plasticizers in food processing materials, medical devices and personal care products. Although phthalates have been shown to disrupt thyroid hormone action and regulation in in vitro and animal studies, efforts to translate these effects to a clinical setting are scarce. Furthermore, no studies have used a mixture approach to take into account the simultaneous exposure to multiple phthalates as is likely to occur in pregnant women. Serum TSH, FT4, T4, TPOAbs and hCG) and 15 urinary phthalate metabolites were measured in 1,117 pregnant women from a Dutch prospective birth cohort during the same early pregnancy visit (median week $13 ; 95 \%$ range $8-18$ ). We used elastic net modelling to identify a mixture of phthalates associated with TSH, FT4, T4 or TPOAb positivity. Analyses were adjusted for hCG, age, smoking, BMI, parity, ethnicity, education, gestational age and urinary creatinine. Three phthalate metabolites (reflecting parent compounds: di-iso-butyl, butyl benzyl and di-isononyl phthalate (DiP)) were associated with TSH concentrations (analyzed as mixture: $P=0.020$; change in mean TSH: $+0.34 \mathrm{mU} / 1$ from lowest to highest exposure). Two phthalate metabolites (reflecting dimethyl- (DMP) and di-ethyl phthtalate (DEP)) were associated with FT4 concentrations (analyzed as mixture: $P=0.007$; change in mean FT4 $+1.2 \mathrm{pmol} / 1$ from lowest to highest exposure). Seven phthalate metabolites (reflecting parent compounds: DiP, DMP, DEP, di-ethyl, dicyclohexyl and di-n/iso-butyl phthalate) were associated with T4 (analyzed as mixture: $P=0.001$; change in mean T4: $20 \mathrm{nmol} / \mathrm{l})$. Phthalate metabolites were not associated with TPOAb positivity. This is the first study to investigate the effects of simultaneous exposure to various phthalates on thyroid function. We demonstrate that a higher exposure to phthalates is associated with a higher TSH, FT4 and $\mathrm{T} 4$ concentrations. These findings are in line with experimental studies showing that phthalates can inhibit cellular T3 uptake as well as inhibit THRB gene expression. 


\section{Poster 35 \\ Autoimmunity Thursday Poster Basic \\ NOVEL PREVENTIVE STRATEGY FOR GRAVES' DISEASE IN NONHUMAN PRIMATES: NEONATAL TOLERANCE INDUCTION}

L. Wu, B.Y. Shi, X. Guan, F. Zhao, Y. wang, P. Chen, S. Hu,

R. Cui, P. Hou

The First Affiliated Hospital of Xi' an Jiaotong University, Xi' an, China

Immune tolerance induction is one of effective measures for preventing autoimmune diseases. The neonatal window for induction of tolerance to antigen existed in nonhuman primates appears promising for translation to the clinic. The aims of this study were to obtain preventative effects in adult nonhuman primates by inducing persistent and antigen-specific immune tolerance to GD in neonate. Newborn female rhesus monkeys were administered i.m. with different doses of adenovirus expressing human TSHR A subunit (A-Sub-Ad). A part of them were reinjected after three weeks with high or low dose A-Sub-Ad respectively. Blood was collected at 1, 4 weeks and 3, 6, 12, 24 months. GD induction was followed at 36 months. Rhesus monkeys receiving neonatal antigen-treatment failed to develop antibody response to TSHR at 1, 4weeks and 3, 12, 24 months. After the last injection of disease induction in adults, only 1 of 7 rhesus monkeys immunized by single or booster administration with high dose A-Sub-Ad in neonate were elicited weak anti-TSHR antibody. Slightly elevated TT4 and mild thyroid hyperplasia were found in the positive TRAb animal. Although the difference of positive rate of GD was not significant, the low dose A-Sub-Ad treatment alleviated the severity of the disease. Compared with control animals, the high dose tolerance induction could reduce $\mathrm{T}$ cells immune response to TSHR A subunit without affecting T cell proliferation response to blank and ConA in adults. The number of regulatory $\mathrm{T}$ cell (Treg) and plasmacytoid dendritic cell (pDC) was significantly higher in rhesus monkeys treated with high dose A-Sub-Ad than in controls $(\mathrm{P}<0.05)$. High dose Ad-A-Sub treatment not only significantly promoted the secretion of IL-4, IL-6, IL-10, IL-13, TGF- $\beta(\mathrm{P}<0.05)$, but also inhibited the production of GM-CSF, IL-12p70 compared to controls $(\mathrm{P}<0.05)$. The findings indicated that persistent tolerance to GD can be induced by high dose A-Sub-Ad injection in neonatal rhesus monkeys, and the DC, Treg, T cell cytokine is involved in the regulation of immune responses. These data strongly suggested that the development of anti-Graves' disease vaccine is very promising.

\section{Poster 36 \\ Autoimmunity Thursday Poster Translational \\ T CELL RECEPTOR CLASSIFIER IS POTENTIALLY A USEFUL PREDICTOR FOR THE DISEASE PROGRESSION OF OPHTHALMOPATHY IN GRAVES' DISEASE \\ Y. Wang, Y. Liu, B.Y. Shi \\ Xi' an Jiaotong University, Xi'an, China}

Thyroid-associated ophthalmopathy (TAO) is the most common and serious manifestation in Graves' disease (GD), however the progression from GD to TAO is unpredictable under current diagnosis. We sequenced CDR3 of TCR V $\beta$ of peripheral blood from GD patients with or without TAO from disease onset to remission. We analyzed 75 blood samples in total, including 19 GD, 16 TAO, and 20 healthy control. TCR repertoires from 6 TAO and 9 GD patients were initially used to construct classifiers, and the most important features included TCR V-J subfamily genes, their frequencies in individual patients and speed of expansion.20 additional clinical diagnosed GD and TAO patients (10 GD and 10 TAO) were enrolled in July 2016 to determine whether classifiers built upon TCR could aid distinguish between GD and TAO. GD classifier and TAO classifier agreed on the TCR diagnosis of 17/20 patients. However, Patients GD007, GD011 and GD014 were diagnosed as GD when they were enrolled, whereas both GD TCR classifier and TAO TCR classifier agreed on their diagnosis of TAO. With the follow-up visits, this TCR diagnosis turned out to be predictive in TAO development. Patients GD007 and GD011 had dry eye, ocular discomfort, and eyelid swelling from September 2016, and further diagnosed by computed tomography (CT) of the orbit in October and November 2016, respectively. Patient GD014 developed chemosis and diplopia from February 2017, and CT scan confirmed TAO in April 2017. Thus, TCR prediction were $4.3 \pm 1.5$ months earlier than typical symptoms and signs of ophthalmopathy, and none of the other 7 GD patients developed TAO after 10 months follow-up.

The TCR screening may be required in GD patients to predict the subset of individuals developing TAO, and early interventions could be applied to improve TAO prognosis.

\section{Poster 37}

\section{Autoimmunity Thursday Poster Translational}

CREATING A RODENT MODEL OF GRAVES' DISEASE USING FUNCTIONALLY ACTIVE TSH RECEPTOR ANTIBODY

A. Hsieh ${ }^{1,2}$, S.V. McLennan ${ }^{1,2}$, S. Adelstein ${ }^{3,4}$, A. Rezaeizadeh ${ }^{2,4}$, E.L. Chua ${ }^{1,2}$, P.F. Williams ${ }^{1,2}$, S.M. Twigg ${ }^{1,2}$

${ }^{1}$ Department of Endocrinology, Royal Prince Alfred Hospital, Camperdown, New South Wales, Australia; ${ }^{2}$ Greg Brown Diabetes and Endocrinology Research Laboratory, Charles Perkins Centre, University of Sydney, Sydney, New South Wales, Australia; ${ }^{3}$ Department of Clinical Immunology, Royal Prince Alfred Hospital, Sydney, New South Wales, Australia; ${ }^{4}$ Sydney Medical School, University of Sydney, Sydney, New South Wales, Australia

Graves' disease (GD) is an auto-immune disorder mediated by Thyroid Stimulating Immunoglobulin (TSI). The main animal models use human TSH receptor gene sequences to generate the autoimmune response in mice, rather than the GD human autoantibody.

We report a pilot study of GD induced in an animal model using C57BL6 mice injected with human TSI. The human TSI was harvested using Thermo Scientific NAb ${ }^{\mathrm{TM}}$ Protein A Plus Spin Columns to isolate the IgG fraction from serum of a patient whose TSH receptor antibody (TRAb) measured 491IU/L on the BRAHMS TRAK RIA and high TSI activity was confirmed on the Thyretain ${ }^{\mathrm{TM}}$ TSI Reporter BioAssay and an in-house TSI cAMP assay. The treatment group $(\mathrm{n}=20)$ received intra-peritoneal injection of $1.14 \mathrm{mg}$ of purified immunoglobulin (Ig) containing TSI, every $24 \mathrm{~h}$ for 3 consecutive days. Four mice were euthanized at days 3, 8, 15, 21 , and 28 and TRAb transfer efficiency and evidence of hyperthyroidism was checked. The control group $(n=20)$ received the same total IgG of commercially prepared, TSI negative, human immunoglobulin using the same regimen. Mouse T4 and T3 were measured using Genway mouse/RatT4 ELISA and Sigma-Aldrich Mouse/Rat T3 ELISA. Animal serum at day 3, 8, 15, 21, and 28 contained TRAb levels of 18, 5.5, 3, 2.5, and 0 IU/L respectively with detectable in-vitro TSI activities by the Thyretain ${ }^{\mathrm{TM}}$ assay between days 3 to 21 . At day 3, there was a significantly higher serum T4 level in the treatment group vs the control group (5.0 vs $2.8 \mu \mathrm{g} / \mathrm{dL}, \mathrm{P}=0.0007)$. No difference in the serum $\mathrm{T} 3$ was found, nor in body weight or other biological parameters of thyroid overactivity. 
Immuno-histochemistry staining of the excised thyroid tissue showed that greater numbers of thyroid follicular cells were positive for the proliferation marker Ki-67 in the day 3 treatment groups than in controls $\left(12.2\right.$ vs $\left.4.2 / 0.0625 \mu \mathrm{m}^{2}, \mathrm{P}=0.001\right)$. This animal model of GD showed transient thyroid overactivity biochemically and histologically. As the human IgG was rapidly cleared in the mice, additional approaches may be required to create more persistent biologically relevant models of GD and to identify additional factors that may potentiate thyroid overactivity.

\section{Poster 38}

Autoimmunity Thursday Poster Case Report

HASHIMOTO'S THYROIDITIS, GRAVE'S DISEASE, SICK EUTHYROID SYNDROME AND STEROID RESPONSIVE ENCEPHALOPATHY ASSOCIATED WITH AUTOIMMUNE THYROIDITIS IN AN HIV POSITIVE PATIENT

F. Shanaz, O. Alzohaili, C. Arsene, G. Krishnamoorthy

Internal Medicine, DMC/Sinai Grace Hospital, Dearborn, MI

Thyroid dysfunction is common in HIV patients. Subclinical hypothyroidism is frequent and Grave's Disease can occur during immune reconstitution. We present a case of Hashimoto's Thyroiditis (HT), Sick Euthyroid Syndrome (SES), Grave's Disease (GD) and Steroid Responsive Encephalopathy Associated with Autoimmune Thyroiditis (SREAT) in a patient with HIV.

A 42 year old male with history of 'thyroid disorder', type I diabetes and HIV presented with seizures and encephalopathy, was intubated and treated for seizures and pneumonia. He was on anti-retroviral therapy and insulin and not on any thyroid medications. Work up showed TSH $<0.008 \mathrm{IU} / \mathrm{ml}(0.2-4.78)$, Free T4 (FT4) $=1.3 \mathrm{ng} / \mathrm{dl}(0.8-$ 1.8), Total T3 $=177 \mathrm{mic} / \mathrm{dl}(60-180)$ and $\mathrm{rT} 3=687 \mathrm{pg} / \mathrm{ml}(90-350) . \mathrm{A}$ diagnosis of SES was made. Four years prior, he had high TSH and low FT4, with elevated thyroid microsomal antibody (1:400) consistent with HT and was on levothyroxine. He remained encephalopathic, intubated with normal neuroimaging and CSF cultures, and a diagnosis of SREAT was considered (antithyroid microsomal antibody $=927.7$ $\mathrm{IU} / \mathrm{ml}$, thyroglobulin antibody $=816.8 \mathrm{IU} / \mathrm{ml}$, EEG showed diffuse slowing). He was given methylprednisolone and became responsive, was extubated and steroid was tapered. One month later, he developed tremor, tachycardia and exophthalmos and had suppressed TSH $(0.009$ $\mathrm{IU} / \mathrm{ml})$ and elevated FT4 (3.0 ng/dl) with a Thyroid Stimulating Immunoglobulin of $188 \%$. Diagnosis of GD was made and he continues to be on methimazole. This patient had an interesting course of autoimmune thyroid disease with HT, GD, and SREAT, a rare autoimmune encephalopathy associated with high titer anti-thyroid antibodies. Encephalopathy is a consistent feature, and can be associated with seizures, myoclonus, neuropsychiatric and stroke like symptoms. SREAT should be considered in the differential diagnosis of un resolving encephalopathy in patients with antithyroid antibodies as it responds to steroids. Ours is a unique case of HT, GD, and SREAT occurring in the same patient at various times. Careful clinical diagnosis and work up of his various presentations led to the successful treatment of all the manifestations of autoimmune thyroid disease.

\section{Poster 39}

Autoimmunity Thursday Poster Clinical

\section{THE CORRELATION BETWEEN SEROLOGY AND THE HISTOPATHOLOGIC DIAGNOSIS OF CHRONIC LYMPHOCYTIC THYROIDITIS}

J.S. Erdman ${ }^{1}$, M. Endo ${ }^{2,3}$, M. Nguyen ${ }^{1}$, C. Dosiou ${ }^{2}$, B.J. Holmes ${ }^{4}$, L.A. Orloff ${ }^{5}$

${ }^{1}$ Stanford University School of Medicine, Stanford, CA; ${ }^{2}$ Division of Endocrinology, Gerontology and Metabolism, Department of
Medicine, Stanford University School of Medicine, Stanford, CA; ${ }^{3}$ Division of Endocrinology, Diabetes and Metabolism, Department of Internal Medicine, The Ohio State University Wexner Medical Center, Columbus, OH; ${ }^{4}$ Department of Pathology, Stanford University School of Medicine, Stanford, CA; ${ }^{5}$ Department of Otolaryngology - Head \& Neck Surgery, Stanford University School of Medicine, Stanford, CA

The diagnosis of Hashimoto's thyroiditis (HT) is based on a combination of clinical findings, thyroid hormone levels, serologic markers, and ultrasound features. Although the presence of antibodies to the thyroid enzyme thyroperoxidase (TPO) is used frequently to support the diagnosis, the prior studies that established the association between anti-TPO antibodies and HT confirmed the presence of the disease using clinical or cytological evidence, neither of which has been shown to consistently predict a positive tissue diagnosis. The aim of this retrospective clinical study was to evaluate the correlation between anti-TPO antibodies and the presence of chronic lymphocytic thyroiditis (CLT), the histopathologic finding supportive of HT, in thyroid tissue specimens. Patients who had undergone thyroid surgery between 1999 and 2015 at Stanford University Medical Center and who had both a preoperative anti-TPO antibody titer and a subsequent thyroid pathology report documented in their medical records were included in the study. Of the 81 patients whose thyroid pathology showed CLT, 54 patients had a positive anti-TPO antibody titer, for a sensitivity of $66.7 \%$. The positive predictive value of an elevated anti-TPO antibody titer was $93.1 \%$. Among patients with a positive anti-TPO antibody titer, patients with CLT were found to have a higher average titer $(494.6 \mathrm{IU} / \mathrm{mL})$ than patients without CLT (162.5 IU/mL), although the difference did not reach statistical significance $(p=0.13)$. Furthermore, among patients with CLT, patients with positive anti-TPO antibody titers were more likely to have hypothyroidism, as evidenced by an elevated TSH or the use of thyroid hormone supplementation, compared to patients with negative titers (OR 13.0; 95\% CI 1.6-103.6). This study demonstrates that a positive anti-TPO antibody titer is strongly predictive of CLT, and a higher titer may further increase the positive predictive value of the test. However, given the relatively low sensitivity of antiTPO antibodies, a negative titer cannot definitely rule out the diagnosis of HT or its underlying pathology finding of CLT.

\section{Poster 40}

Autoimmunity Thursday Poster Clinical

\section{DEXAMETHASONE FOR GRAVES' THYROTOXICOSIS} IN THE SETTING OF MOYAMOYA VASCULITIS: A REASONABLE APPROACH

H. Goel, H. Bakeri, K. Szczepanczyk, B. Tendler

University Of Connecticut, Hartford, CT

Moyamoya vasculitis is characterized by abnormal blood vessels in the cerebral circulation and occasionally in extra-cranial, for example, renal arteries. Genetic, inflammatory and autoimmune factors have been described in the pathogenesis of this disorder. It has been seen in association with other autoimmune disorders including Graves' disease. Management of patients with thyrotoxicosis in the setting of moyamoya vasculitis may be challenging given the risk of vasospasm, stroke and cerebral hemorrhage.

A 51 year old lady with moyamoya vasculitis and Graves' disease presented to our clinic for definitive management of hyperthyroidism. She had been doing well on methimazole; however, was interested in radioactive iodine. She had a cerebrovascular accident in 2012. On examination, she had no exophthalmos. Thyroid gland was non tender, with a known subcentimeter nodule and no overlying 
bruit. Thyroid ultrasound showed a 9 mm nodule in right lobe without any suspicious characteristics.

We had previously treated two similar cases: One patient had presented with an acute ischemic stroke after RAI (before the diagnosis of Moyamoya was made). The other patient treated with thyroidectomy and peri-operative dexamethasone had no neurological complications. Currently, our patient elected to stay on methimazole. Dexamethasone appears to be a reasonable intervention to prevent acute inflammation. Should she decide in the favour of thyroidectomy, she will need an arterial line to closely monitor the blood pressure to prevent both hypo and hypertension, as both appear to be equally important in cerebral perfusion in this patient.

Hyperthyroxinemia may alter cerebral perfusion in patients with moyamoya disease. Sudden surges of thyroid hormone should be avoided. If the patient has treatment with RAI, a close follow-up is recommended for early detection and management of thyroiditis. Steroid therapy before definitive treatment to induce normalization of the thyroid function has been found to be effective. In our experience, dexamethasone has shown to cause less edema and hypertension and has better inflammatory action compared to hydrocortisone.

\section{Poster 41}

Autoimmunity Thursday Poster Clinical

\section{HASHIMOTO'S ENCEPHALOPATHY PRESENTING AS STATUS EPILEPTICUS: A RARE CASE UNRESPONSIVE TO STEROIDS}

K. Szczepanczyk, H. Goel, P. Taxel

University of Connecticut, Farmington, CT

Hashimoto's encephalopathy (HE) is a rare condition defined as steroid responsive encephalopathy associated with autoimmune thyroiditis and increased levels of anti-thyroid antibodies in serum and/or cerebrospinal fluid (CSF). We present a case of HE unresponsive to steroids, but who had partial response plasmapheresis.

A 72 year old female with hypertension and end stage renal stage on haemodialysis presented with altered mental status and difficulty with speech after missing 1 hemodialysis treatment. On admission, she was uremic (BUN of $73 \mathrm{mg} / \mathrm{dL}$ ) and hypertensive with blood pressure of $240 / 90 \mathrm{~mm}$ hg, which normalized with hydralazine. Mentation did not improve after dialysis treatments. CVA work-up was negative. Per family, patient's son passed away 2 months prior and she was depressed since his death. She was transferred to the ICU and intubated because of difficulty protecting her airway and worsening encephalopathy symptoms. She was in status epilepticus, which resolved on EEG monitoring within 24 hours of initiating 3 anti-epileptics. Lumbar puncture and CSF analysis showed increased protein, normal glucose and elevated TPO antibodies (36.4 IU/ml, normal 0-9). Serum TPO and thyroglobulin antibodies were elevated at $4275 \mathrm{IU} / \mathrm{ml}$ (normal, 0-9) and $>2200 \mathrm{IU} / \mathrm{mL}$ (normal, 0-4), respectively. Patient had no known underlying thyroid disease or autoimmune disorders. Thyroid function tests were normal. The diagnosis of Hashimoto's encephalopathy was made. Patient was started on pulse dose methylprednisolone for 5 days with little improvement and subsequently received 5 treatments of plasmapheresis. After the $5^{\text {th }}$ treatment, serum antibodies decreased and mental status improved as she was more alert, responding to questions and following commands. HE should be considered in encephalopathic patients with unexplained episodes of status epilepticus. The triad of encephalopathy, EEG slowing, and increased CSF protein should prompt testing for anti-thyroid antibodies in blood and CSF, regardless of thyroid function. Patients are often responsive to steroids, but some warrant $2^{\text {nd }}$ line treatment for resolution of symptoms, as in this patient.

\section{Poster 42}

Autoimmunity Thursday Poster Clinical

GRAVES' DISEASE AND TRENDS IN ANTIBODY TITRES AFTER TREATMENT OF TOXIC MULTINODULAR GOITRE WITH 131 RADIOACTIVE IODINE

C.R. Barreiros ${ }^{1}$, T. Pilli ${ }^{2}$, F. Pacini ${ }^{2}$

${ }^{1}$ Endocrinology and Metabolism, Hospital de Egas Moniz, C.H.L. O-E.P.E., Lisbon, Lisbon, Portugal; ${ }^{2}$ Endocrinology and Metabolism Section, Dpt. of Internal Medicine, Endocrinology and Metabolism and Biochemistry, University Hospital of Siena, Siena, Italy

Radioiodine (131I) is a safe definite treatment option for toxic goiter (TMG). Graves'-like reactions and post-treatment hyperthyroidism recurrences have been linked to autoimmunity arising after treatment. This study describes the post-treatment trends in antibody (Ab) titres, the incidence of Grave's Disease (GD) and investigates associations with pre-treatment autoimunity and post-treatment outcomes of thyroid function. TMG patients treated with $15 \mathrm{mCi}$, with serial measurements of $\mathrm{Ab}$ were included. The clinical records were retrospectively analysed to collect TSH and fT4 before treatment and at the end of follow-up (FU). Anti-thyroglobulin (TGL), anti-thyroid-peroxidase (TPO) and anti-TSH-receptor $\mathrm{Ab}$ (TRAb) titres were analysed 0-3 months before treatment, at 3 month intervals during the first year post-treatment, yearly thereafter up to 5 years. The total 72 patients, $77.8 \%$ females, had a mean FU of $2.5(0.25-6.4)$ years. At the time of treatment, $90 \%$ had subclinical hyperthyroidism and $60 \%$ detectable Abs.

Development of new anti-thyroid Abs was identified in 5 patients $(6.9 \%)$, but in none were positive. Peak increments in TGL titres relative to pre-treatment levels reached 55\% at 6-12 months with progressive decrease in the following years. TPO titres were maximal at $0-3$ months (54\% relative rise) and decreased after $1-2$ years.

New TRAb positivity developed in $4(5.5 \%)$ patients $0.5-3$ years post-treatment and 3 had concomitant hyperthyroidism recurrence. The 3/4 with borderline result did not show titre increments.

Pre-treatment $\mathrm{Ab}$ titres did not associate with specific patterns of thyroid dysfunction at the end of FU. There was no significant association of post-treatment anti-thyroid Ab titres with thyroid dysfunction but TRAb titres were associated with hyperthyroidism at the end of FU. None of the patients with negative TPO developed post131I TRAbs but patients with detectable TPO did so. Pre-treatment autoimunity does not seem to relate with post-131I outcomes on thyroid function, neither does the increments in titres after treatment. Post-treatment TRAbs associate with hyperthyroidism, the incidence of 131I induced GD was $4.2 \%$ and pre-treatment TPO increased the risk for this phenomenon by 4 fold.

\section{Poster 43}

Thyroid Cancer Thursday Poster Clinical

\section{NON-FOLLICULAR AND NON-PARAFOLLICULAR} MALIGNANT THYROID TUMOURS, A REVIEW

\section{OF 4 YEARS}

C.R. Barreiros, M. Carriço, C. Saraiva, C. Limbert, C. Vasconcelos Endocrinology and Metabolism, Hospital de Egas Moniz, C.H.L. O-E.P.E., Lisbon, Lisbon, Portugal

This study describes the characteristics of malignant thyroid tumours of non-follicular and non-parafollicular origin, diagnosed during a period of 4 years.

All fine-needle-aspiration cytologies (FNAC) performed from 2013 to 2017 were reviewed. Those suggesting non-follicular and nonparafollicular cell origin, that were histologically confirmed, were included. The clinical files provided age, sex, symptoms and signs, thyroid function and autoimmunity. Ultrasound and tomography scans, 
histological and surgical reports were evaluated. From 4928 FNAC, 6 were histologically proven anaplastic carcinomas (AC), 2 were primary thyroid lymphomas (TL) and 1 was a metastatic lesion (ML). The patients were mostly females 7/9, with mean age of 74 (56-87) years. Only 3 had thyroid dysfunction (1 hyperthyroid) and none had antithyroid antibody positivity. Out of the malignant FNAC, 4/6 suggested $\mathrm{AC}, 1 / 2 \mathrm{TL}$ and $1 / 1$ metastatic disease. Immunohistochemistry (IHC) was diagnostic in 9/9. All patients but 2, presented with local symptoms and previously undiagnosed goiter, with 20-120 days of duration, the shortest duration corresponded to a TL. Reported symptoms were dysphagia 5/9, dysphonia 3/9 and pain 2/9. Pain was exclusive to AC. Palpation revealed stony-hard consistency in $4 / 6$ cases of AC and in 1/2 cases of TL; fixation in $2 / 6 \mathrm{AC}$ and in $1 / 2 \mathrm{TL}$; lymphadenopathy in $1 / 6$ $\mathrm{AC}$ and in $2 / 2 \mathrm{TL}$, findings absent in the ML. On US, 6/9 had multinodularity. A dominant suspicious node was found in AC only, with mean diameter of 65 (47-96) mm, significantly different from the 18 (14-20) $\mathrm{mm}$ of the remainder. All were hypoechoic and heterogeneous, except for 1 hyperechoic TL. Calcifications were exclusive to AC but extra-thyroidal invasion (4/9) and necrosis (3/9) were also found in 1 TL, features absent in the ML. CT showed cervical lymphadenopathy in $8 / 9$ patients and extrathyroidal extension in $7 / 9$.

These very rare lesions that typically afflict elderly females, may show characteristic clinical-radiological features that should raise suspicion of the specific diagnosis. FNAC with IHC was as accurate as histology. CT showed superiority on cervical and mediastinal disease characterization with important surgical implications.

\section{Poster 44}

Disorders of Thyroid Function Thursday Poster Basic

\section{EFFECTS OF HYPERTHYROIDISM ON CONSTITUTIVE ACTIVE ACETYLCHOLINE DEPENDENT $\mathrm{K}^{+}$CURRENTS IN MURINE ATRIA}

\section{K. $\mathrm{Cho}^{2}$, B. Kim ${ }^{1}$}

${ }^{1}$ Internal Medicine, Pusan National University Hospital, Busan, Korea (the Republic of ); ${ }^{2}$ Internal Medicine, Kosin University College of Medicine, Busan, Korea (the Republic of)

Although hyperthyroidism is known etiology of atrial fibrillation (AF), the ionic mechanisms in hyperthyroidism remain unclear. We aimed to investigate the association of thyroid hormone and acetylcholine-activated $\mathrm{K}^{+}$current $\left(I_{\mathrm{KACh}}\right)$ in atrial cardiomyocytes, which is important for AF initiation and maintenance. Hyperthyroidism was induced in ICR mice by daily intraperitoneal injection of 1-thyroxine ( $\mathrm{T}_{4}, 100 \mathrm{mg} / \mathrm{kg}$ per day). $100 \mu \mathrm{M}$ acetylcholine induced $I_{\mathrm{KACh}}$ was recorded in hyperthyroidism mouse atrial cardiomyocytes by nystatin-perforated patch clamp technique at the baseline, 2 weeks, 4 weeks, and 6 weeks. Peak and quasi-steady-state $I_{\mathrm{KACh}}$ and action potential duration was compared. A rat model of hyperthyroidism established by daily intraperitoneal injections of L-thyroxine for 4 weeks underwent echocardiography, which was compared with controls and the recovery state (after 2 weeks washout period). Fourweek-thyroxine treatment induced significant tachycardia, increased left atrial (LA) dimension and decreased peak systolic LA stain, which were improved after 2 weeks washout period. Specifically, $I_{\mathrm{KACh}}$ was steeply increased at the 2-weak-thyroxine treatment, which was gradually declined at the 4-week and 6-week-throxine treatment. Interestingly, shortened action potential duration by acetylcholine was more aggregated by the times (control: $4.66 \mathrm{~ms}$ vs. 2 week-T4: $3.26 \mathrm{~ms}$ vs. 4 week-T4: $2.63 \mathrm{~ms}$ vs. 6 week-T4: $1.13 \mathrm{~ms}$ ) $(\mathrm{P}<0.001)$ These findings provide that thyroid hormone may have a role in modulating $I_{\mathrm{KACh}}$ in atrial cardiomyocites via a time dependent manner and it establish important background information for the relationship between hyperthyroidism and AF.

\section{Poster 45}

Thyroid Cancer Thursday Poster Clinical

BRAF $^{\text {V600E }}$ MUTATION IN FOLLICULAR VARIANT

OF PAPILLARY THYROID CARCINOMA AND

NON-INVASIVE FOLLICULAR TUMOR WITH

PAPILLARY-LIKE NUCLEAR FEATURES (NIFTP)

IN BRAF ${ }^{\mathrm{V} 600 \mathrm{E}}$-PREVALENT AREA

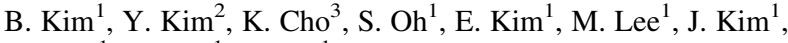
Y. Jeon ${ }^{1}, \mathrm{~S} . \mathrm{Kim}^{1}$, I. Kim${ }^{1}$

${ }^{1}$ Internal Medicine, Pusan National University Hospital, Busan, Korea (the Republic of); ${ }^{2}$ Department of Pathology, Pusan National University Hospital, Busan, Korea (the Republic of); ${ }^{3}$ Department of Internal Medicine, Kosin University College of Medicine, Busan, Korea (the Republic of)

The aim of this study was to investigate the $B R A F^{\mathrm{V} 600 \mathrm{E}}$ mutation status of follicular variant of papillary thyroid carcinoma (FVPTC) and to determine prevalence of non-invasive follicular thyroid neoplasm with papillary-like nuclear features (NIFTP) after reclassifying non-invasive encapsulated FVPTC as NIFTP by careful histopathological review based on diagnostic criteria of NIFTP in Korean patients. This study included 1411 consecutive patients who underwent total thyroidectomy with routine prophylactic central lymph node dissection for PTC. All PTC cases were classified according to histological variant. $B R A F^{\mathrm{V} 600 \mathrm{E}}$ mutation analysis was performed routinely. Clinico-pathological characteristics and ultrasonography images were compared between $\mathrm{BRAF}^{\mathrm{V} 600 \mathrm{E}}$ mutation-positive and -negative groups for FVPTC. Two cytopathologists specializing in thyroid pathology reviewed and interpreted the pathology slides according to consensus diagnostic criteria for the encapsulated FVPTC and NIFTP. The FVPTC represented $22(1.6 \%)$ patients and prevalence of the $B R A F^{\mathrm{V} 600 \mathrm{E}}$ mutation in FVPTC is $27.3 \%$. BRAF $F^{\mathrm{V} 600 \mathrm{E}}$ mutation-positive FVPTC was more highly associated with old age than $B R A F^{\mathrm{V} 600 \mathrm{E}}$ mutationnegative FVPTC $(\mathrm{p}=0.021)$. Encapsulated FVPTC $(13.6 \%, 3 / 22)$ was marginally associated with large tumor size $(\mathrm{p}=0.065)$ and positive halo sign $(\mathrm{p}=0.055)$ compared with infiltrative FVPTC $(86.4 \%, 19 / 22)$. The prevalence of NIFTP was $0.18 \%$ among all PTC patients $(2 / 1411)$ and proportion of NIFTP among FVPTC was $9.1 \%(2 / 22)$. The $B R A F$ ${ }^{\mathrm{V} 600 \mathrm{E}}$ mutation is prevalent in Korean patients with FVPTC in a region with high frequency of the $B R A F^{\mathrm{V} 600 \mathrm{E}}$ mutation. However, the prevalence of FVPTC and NIFTP was lower than that of western countries. These findings need to be confirmed in further multicenter studies to define the prevalence of NIFTP in Korea.

\section{Poster 46}

Disorders of Thyroid Function Thursday Poster Case Report A RARE ACTIVATION CAUSING A CLASSIC SYNDROME S.A. Avadhanula ${ }^{1}$, K. Araque $^{2}$, S. Chittimoju ${ }^{3}$, P. Kundra ${ }^{3}$

${ }^{1}$ Endocrinology, Georgetown University Hospital, Washington, DC; ${ }^{2}$ Endocrinology, National Institute of Health, Bethesda, MD;

${ }^{3}$ Internal Medicine, Washington Hospital Center, Washington, DC

Bartter syndrome type $\mathrm{V}$, secondary to an activating mutation of the Calcium sensing receptor (CaSR) gene is a rare occurrence (1-4) and has been infrequently described in the literature (1-4). Pathophysiology of the disorder has been described extensively (5-9) with various genetic mutations (10-11). The majority of patients who are described have an entity entitled "autosomal dominant hypocalcemia" and are often asymptomatic. Here, we describe a mild phenotypic presentation of the Bartter syndrome. Our patient is a 58 year old female with a past medical history of osteoarthritis, rheumatoid arthritis (requiring the use of steroids) and hypertension who had 
numerous presentations to the emergency department for symptomatic hypocalcemia with tingling, numbness of fingertips, palpitations and chest pain. At each evaluation has been found to have a normal total calcium, but ionized calcium was reported to be at its' lowest, 0.6. She was treated with infusions of intravenous calcium gluconate. At presentation, she was also found to be hypomagnesemic and hypokalemic. On further work up, ACE levels were normal, Renin plasma levels were $1.49 \mathrm{ng} / \mathrm{ml} / \mathrm{hr}$, serum cortisol $0.8 \mathrm{ug} / \mathrm{dl}$ with urinary cartiosol $<1 \mathrm{ug} / 24 \mathrm{hr}$. Patient's parathyroid hormone (PTH) levels were noted to be in the lower limit of normal.

Ultimately, our patient underwent genetic testing which showed 3 activating mutations of the calcium sensing receptor. She was treated with Forteo (recombinant PTH), Calcium, potassium and magnesium supplements with resolution of her symptoms, but required frequent monitoring of her electrolyte levels and adjustments in the doses of her medications. In this case, we present a rare constellation of electrolyte abnormalities associated with an activating mutation of the calcium sensing receptor gene causing classic Bartter syndrome type V. Our intention is that this will add to the highly variable spectrum of phenotypes of this genetic mutation.

\section{Poster 47}

Disorders of Thyroid Function Thursday Poster Case Report THYROTOXIC PERIODIC PARALYSIS WITH URINARY INCONTINENCE

G. Gupta, J. Kung

Endocrinology, Tufts Medical Center, Boston, MA

Thyrotoxic periodic paralysis (TPP) is a potentially life-threatening complication of thyrotoxicosis, characterized by hypokalemia and muscle paralysis. Asian males are most often affected (McFadzeon et al. BMJ 1967). Patients of African descent rarely present with this disease. Here we present a case report of an African American male presenting with TPP as an initial presentation of Grave's disease with an atypical feature of urinary incontinence. 46 year old African-American male who presented with sudden onset bilateral leg weakness and subsequent fall. He had also been experiencing intermittent weakness and tightness in his thighs for 1.5 months. No pain, tingling or numbness sensation but a single episode of urinary incontinence during sleep. Weight loss was present. He denied any recent illness or use of over-the-counter thyroxinecontaining supplements. On examination, BP was normal but he was tachycardiac to 115 . He had mild proptosis with stare gaze and diffuse thyromegaly along with profound weakness of the proximal muscles of bilateral lower extremities. Initial labs were positive for hypokalemia with a K of 2.9, CPK 318 and TSH $<0.03$, free T4 2.85 , total T3 515, TSI $497(<140)$. EMG was performed and the results were consistent with myopathy. He was given intravenous potassium supplementation along with beta blockers and methimazole which improved his clinical symptoms. TPP is a type of muscle disease in the family of channelopathies, manifested by episodes of painless muscle weakness. These episodes may be precipitated by heavy exercise, fasting, high carbohydrate meals, emotional stress and steroids. The presence of both hypokalemia and elevated levels of thyroxine (T4) and triiodothyronine (T3) are important diagnostic features during the acute episode. Treatment of TPP involves two steps, immediate action to reverse the paralysis by correction of hypokalemia followed by measures to prevent future attacks by restoration of a euthyroid state. There has been no case report of TPP presenting with urinary incontinence which might be an associated feature, possibly due to smooth muscle dysfunctionTPP is a rare but important clinical entity which needs to be recognized and treated early.

\section{Poster 48}

Disorders of Thyroid Function Thursday Poster Case Report

THE "SKINNY" ON TYPE II AMIODARONE-INDUCED THYROTOXICOSIS: A THOUGHT-PROVOKING CASE IN A CACHECTIC PATIENT

S. Shaik, M. Averneni, A. Drake, C. Houston, F. Cook

Endocrinology, East Carolina University/Vidant Medical center, Greenville, NC

Amiodarone may cause a destructive thyroiditis many months after discontinuation. Its long half-life has been attributed to its lipophilicity and large stores within adipose tissue. We present a thought-provoking case of suspected type II amiodarone-induced thyrotoxicosis (AIT-II) in a patient with cachexia of malignancy and a remote history of amiodarone use. A 55 year old Caucasian male with a history of arrhythmias, treated with amiodarone until one year prior, underwent extensive neck surgery for metastatic squamous cell carcinoma of the tongue, previously treated with cisplatin and radiation. Three days postoperatively, he developed rapid atrial fibrillation and was found to be thyrotoxic with TSH $<0.01 \mathrm{uIU} / \mathrm{mL}(0.35-4.94)$ and free T4 (FT4) > $6.00 \mathrm{ng} / \mathrm{mL}(0.70-1.48)$. Two months earlier, his TSH had been 0.05 $\mathrm{uIU} / \mathrm{mL}$ with concomitant symptoms of heat intolerance and insomnia. He had no prior personal history or family history of thyroid disease. He was cachectic, having lost $20 \mathrm{~kg}$ since the diagnosis of cancer 9 months before. His thyroid was not enlarged, nodular, or tender. Thyrotropin receptor antibody level was normal. Urinary iodine, measured 46 days after last IV contrast exposure, was markedly elevated at $2504 \mathrm{mcg} /$ $24 \mathrm{hrs}(75-500)$. He was started on methimazole, prednisone, potassium iodide, and metoprolol. He was ultimately discharged on methimazole $20 \mathrm{mg}$ BID and prednisone $40 \mathrm{mg}$ daily. His FT4 improved rapidly, and methimazole was readily tapered off by 7 weeks postop. At 11 weeks post-op, his TSH was $5.27 \mathrm{uIU} / \mathrm{mL}$ and a prednisone taper was begun. Our patient's rapid response to prednisone and taper off methimazole was consistent with a diagnosis of thyroiditis. His very high urinary iodine could not be explained solely by IV contrast exposure and was suggestive of residual amiodarone, despite his cachexia and remoteness of amiodarone use. This thought-provoking case raises the question of whether residual amiodarone may be released from adipose tissue in the setting of rapid weight loss. We postulate that our patient's loss of adiposity outpaced his elimination of amiodarone, leading to increased release of drug into the circulation, uptake by the thyroid, and consequent destructive thyroiditis.

\section{Poster 49}

Disorders of Thyroid Function Thursday Poster Case Report IMMEDIATE LIVER TOXICITY WITH METHIMAZOLE USE

E. Al Zaghal

Endocrinology, George Washington University, Washington, DC

Methimazole is the thianomide of choice in hyperthyroidism, due to its faster action, and less frequent side effects compared to PTU. Methimazole is rarely associated with liver toxicity, usually manifested as cholestatic jaundice. Here we report a case of hepatotoxicity that manifested within 24 hours of methimazole initiation. A 43-year-old woman presented to the hospital with a fever to $103 \mathrm{~F}$, generalized malaise, palpitations, and insomnia. On physical exam, she was tachycardic, diaphoretic, and anxious. She had a diffusely enlarged thyroid, about 30 grams in size.

She was admitted for treatment of severe hyperthyroidism. Initial labs were TSH 0.01, FT4 3.35 (0.76-1.46), and FT3 6.4 (2.8-5.3). Liver enzymes on presentation were within normal limits. Methimazole was initiated for hyperthyroidism and metoprolol for heart rate control. 
Within 24 hours, the patient started to complain of nausea and vomiting. Liver enzymes were checked: AST 1060U/L (10-40), ALT 1449U/L (7-56), Alkaline phosphatase 81IU/L (44-147) and total bilirubin $2.1 \mathrm{mg} / \mathrm{dL}(0.3-1.9)$. Suspicion was for immediate hepatotoxicity induced by methimazole. Viral hepatitis markers were negative.

Upon discontinuation of methimazole, liver function tests improved within 24 hours as did her nausea and vomiting. Liver function tests were: AST 698U/L, ALT 1176U/L, alkaline phosphatase 71IU/L and total bilirubin $1.0 \mathrm{mg} / \mathrm{dL}$. Her liver enzymes continued to decrease in 48 hours: AST 265U/L, ALT 779U/L, alkaline phosphatase 72IU/L and total bilirubin $1.0 \mathrm{mg} / \mathrm{dL}$.

Graves' disease was diagnosed after work up. The patient underwent total thyroidectomy within one month of her presentation. Her liver enzymes normalized. This case demonstrates a rapid hepatotoxicity with methimazole treatment within 24 hours of initiation. To date, there is no published report of this side effect occurring so rapidly to this degree. The exact mechanism of methimazole-induced hepatotoxicity is still far from clear and no protective agent has been developed for this toxicity.

\section{Poster 50 \\ Disorders of Thyroid Function Thursday Poster Case Report THIONAMIDE RESISTANCE AND THE USE OF CHOLESTYRAMINE IN ACHIEVING A EUTHRYOID STATE \\ K.N. Jackson, T. Kouvatsos \\ Internal Medicine, Thomas Jefferson University Hospital, Philadelphia, PA}

Conventional treatment for Graves' thyrotoxicosis includes antithyroid medications, radioactive iodine therapy, and thyroidectomy. Thyroidectomy is considered definitive treatment when other treatment modalities fail. To minimize complications, a euthyroid state should be achieved preoperatively. Thionamides are often used preoperatively, in addition to beta blockers and corticosteroids for symptom management. Rarely, patients are resistant to traditional therapy and require additional agents such as cholestyramine, high-dose steroids, and/or short term iodine to normalize thyroid function. A 36-year-old female with no significant past medical history was diagnosed with Graves' disease and atrial flutter after presenting with 3 months of 50 pound weight loss, diarrhea, tremor, and palpitations. Methimazole $30 \mathrm{mg}$ daily and propranolol $40 \mathrm{mg}$ twice daily were initiated. Due to poor compliance, the patient re-presented 15 months after diagnosis with persistent hyperthyroidism. Labs at that time were significant for TSH $<0.005 \mathrm{uIU} / \mathrm{mL}$, FT3 $>32.6 \mathrm{pg} / \mathrm{mL}$, FT4 $>7.77 \mathrm{ng} / \mathrm{dL}$, and severe neutropenia with an absolute neutrophil count of 1000 cells/uL (0 absolute basophils/uL, 0 absolute eosinophils/uL, 0 immature granulocytes/uL). Methimazole was discontinued given concern for pending agranulocytosis. Prednisone $1 \mathrm{mg} / \mathrm{kg} /$ day and PTU, titrated to a dose of $200 \mathrm{mg}$ TID, were started with frequent CBC checks. Despite resolution of neutropenia, hyperthyroidism persisted (TSH $<0.005$ $\mathrm{uIU} / \mathrm{mL}$, FT3 $8.0 \mathrm{pg} / \mathrm{mL}$, FT4 $3.46 \mathrm{ng} / \mathrm{dL}$ ) for several months and cholestyramine $4 \mathrm{mg}$ BID was added preoperatively. Within 3 weeks of treatment, TFTs normalized (FT4 $1.77 \mathrm{ng} / \mathrm{dL}$, FT3 $3.0 \mathrm{pg} / \mathrm{mL}$, TSH $<0.005 \mathrm{uIU} / \mathrm{mL}$, TSI 542\%) and the patient underwent uncomplicated total thyroidectomy. Cholestyramine is used to lower cholesterol by forming insoluble complexes with bile acids preventing their reabsorption. Similarly, cholestyramine binds iodothyronines and enhances their excretion. Studies have demonstrated that adding cholestyramine to methimazole or PTU causes a more rapid decline in serum thyroid hormone. While use of cholestyramine for thyroid storm is widely accepted, this case report supports its use in thionamide resistant Graves' disease as well.

\section{Poster 51}

Disorders of Thyroid Function Thursday Poster Case Report MYXEDEMA COMA MASQUERADING AS PSYCHOSIS?

E. Valenzuela Scheker, C. Edwards, N. Singh Ospina

Department of Endocrinology Division of Medicine, University of Florida, Gainesville, FL

Myxedema coma (MC) is a life-threatening condition that results from severe hypothyroidism. The diagnosis of $\mathrm{MC}$ is a clinical challenge due to rarity of the condition, in addition to poor characterization of atypical presentations. A 59-year-old woman without any past medical history was brought to the emergency department when she was found wandering around her neighborhood. She was complaining of visual hallucinations for about a week.

On exam, she was alert, appeared tired and unkempt, and was actively having visual hallucinations. Her vital signs revealed normal blood pressure, pulse, respiratory rate and temperature. She had evidence of palpebral edema and no neck scar. She had distant heart sounds and lower extremity edema with scattered ecchymosis.

Her laboratory evaluation revealed a sodium of $128 \mathrm{mmol} / \mathrm{L}$ and normal glucose, kidney and liver function. Head CT scan, UA and tox screen for evaluation of psychosis were normal. Her TSH was $31.4 \mathrm{mIU} / \mathrm{L}$. Chest $\mathrm{x}$-ray showed an enlarged cardiac silhouette and the EKG had low voltage.

Upon arrival to the medical floor, she had worsening mental status, decreased respiratory rate and laboratory evaluation showing $\mathrm{CO}_{2}$ retention. MC was suspected. Her free T4 was $<0.02 \mathrm{ng} / \mathrm{dL}$ and TPO antibodies were positive $(28.8 \mathrm{U} / \mathrm{ml})$. She was intubated and started on IV levothyroxine $(350 \mathrm{mcg}$ ) followed by $50 \mathrm{mcg}$ daily in addition to stress dose steroids.

After 3 days of IV levothyroxine therapy, she was extubated. She no longer had evidence of psychosis. She was discharged on $125 \mathrm{mcg}$ of levothyroxine with follow up TSH of 1.70 mIU/L 6 weeks after her initial presentation. Although diagnostic tools for MC are available, they can fail to identify atypical cases, underscoring the importance of high clinical suspicion. Our case highlights psychosis as an unusual initial presentation of MC, which might not be expected by clinicians. Although psychosis has been reported in cases of uncontrolled hypothyroidism without $\mathrm{MC}$, it is rarely the initial manifestation of MC. In fact, our literature review identified only a few cases in which psychosis was the primary presentation for MC. Clinicians should be aware of this atypical presentation as identification of hypothyroidism can be lifesaving.

\section{Poster 52}

Disorders of Thyroid Function Thursday Poster Case Report

PEDIATRIC PULMONARY ARTERIAL HYPERTENSION AND HYPERTHYROIDISM: TWO CASE REPORTS OF TOTAL THYROIDECTOMY AND REVIEW OF LITERATURE

F. Saito ${ }^{1}$, Y. Kubota ${ }^{1}$, K. Tsuboi ${ }^{2}$, H. Ogata ${ }^{1}$

${ }^{1}$ Department of Surgery Toho University, Division of Breast and Endocrine Surgery, Tokyo, Ota-ku, Japan; ${ }^{2}$ Department of Medicine, Toho University School of Medicine, Division of Diabetes, Metabolism ans Endocrinology, Tokyo, Japan

Pulmonary arterial hypertension (PAH) is rare and has poor prognosis. However, with the development of epoprosterol, prognosis has dramatically improved. On the other hand, chronic epoprosterol therapy may be connected with induction of thyroid disease.

Patients with PAH who develop hyperthyroidism are at risk for acute cardiopulmonary decompensation and death. We describe two cases of total thyroidectomy due to hyperthyroidism with primary PAH. 
Case 1 involved a 22-year-old man diagnosed with primary $\mathrm{PAH}$ at 10 years old and Grave's disease 10 years later. Epoprestenorol was administered for 20 months. Initially, he was treated with thiamazole. Two years later, control of thyroid function became difficult and total thyroidectomy was performed.

Case 2 involved a 21-year-old woman. She was diagnosed with Grave's disease 8 years after primary PAH diagnosis. Because of agranulocytosis due to thiamazole treatment, potassium iodide had been administered for 8 years. After that, total thyroidectomy was performed.

These two cases successfully underwent total thyroidectomy without major perioperative complications, because the operation was performed while cardiac function remained satisfactory.

Patients with PAH show markedly increased rates of both morbidity and mortality with anesthesia and surgery. In particular, the risks associated with primary PAH seem to be even more frequent than those related to secondary PAH.

Detailed preoperative clinical evaluations of cardiac and thyroid function lead to successful total thyroidectomy.

\section{Poster 53-WITHDRAWN}

\section{Poster 54}

Disorders of Thyroid Function Thursday Poster Case Report A RARE CAUSE OF REFRACTORY GRAVES' DISEASE A. George ${ }^{1}$, R.J. Anderson ${ }^{2,1}$, S. Yousefian ${ }^{3}$

${ }^{1}$ Endocrinology, Diabetes and Metabolism, Creighton University Medical Center, Omaha, NE; ${ }^{2}$ Endocrinology, Diabetes and Metabolism, VA-Nebraska Western Iowa Healthcare System, Omaha, NE; ${ }^{3}$ Endocrinology, Diabetes and Metabolism, Santa Clara Valley Medical Center, San Jose, CA

Refractory Graves' disease (GD) requiring high doses of thionamides and beta-blockers has been reported. Conventional treatment of GD may not be effective due to simultaneous occurrence of thyroiditis. We present a case of refractory GD with likely simultaneous silent thyroiditis responsive to decadron.60 year old female presented on 10/2014 with atrial fibrillation, cardiac decompensation and Graves' Orbitopathy. Laboratory values included: TSI $>500 \%(\mathrm{~N}<122 \%)$, TSH $0.006 \mathrm{uIU} / \mathrm{ml}$ (N:0.400-5.0), FT4 6.2 ng/dL (N:0.7-1.6) FT3 17.9pg/mL (N: 2.3-4.2). TPO antibodies $>1300(\mathrm{~N}<59 \mathrm{u} / \mathrm{mL})$. Thyroid ultrasound showed diffusely enlarged bilateral thyroid lobes without increased blood flow. RAIU and scan were not feasible. She was started on Propylthiouracil(PTU) $200 \mathrm{mgTID}$, Decadron $1 \mathrm{mg}$ TID, SSKI 3 drops TID, propranolol $30 \mathrm{mg}$ TID. Three weeks later, given minimal improvement, propranolol, PTU and SSKI doses were increased. Medication adherence was confirmed. A month later, FT4 remained elevated; concomitant silent thyroiditis was suspected. An increase in decadron to $4 \mathrm{mg}$ daily resulted in symptom improvement after 4 weeks. Labs on 2/2015:TSH <0.005 uIU/ml, FT4 $1.9 \mathrm{ng} / \mathrm{dL}$, FT3 $9.3 \mathrm{pg} / \mathrm{mL}$. However, she developed overt side effects from the decadron therefore the dose was decreased to $2 \mathrm{mg}$ daily with subsequent results of TSH $0.001 \mathrm{uIU} / \mathrm{ml}$, FT4 $2.3 \mathrm{ng} / \mathrm{dL}$, FT3 $7.4 \mathrm{pg} / \mathrm{mL}$. PTU was increased to $300 \mathrm{mg}$ TID. One month later her labs showed no change and PTU was switched to Methimazole $30 \mathrm{mg}$ BID. In 7/2015, FT4 $1.3 \mathrm{ng} / \mathrm{dL}$, TSH $0.000 \mathrm{uIU} / \mathrm{mL}, \mathrm{FT} 33.8 \mathrm{pg} / \mathrm{mL}$ and Decadron was discontinued. Despite beta-blockade, iodine, decadron and high doses of PTU, the patient failed to respond to therapy. This may be due to resistance to PTU or a simultaneous occurrence of silent thyroiditis. Her symptoms improved with an increased decadron dose, a result consistent with thyroiditis.

A FT3/FT4 ratio <4.4, as was seen initially in our patient, was more consistent with thyroiditis than with GD.

Resistance to anti-thyroid medicines or simultaneous occurrence of thyroiditis should be considered in difficult to treat GD patients. Both are rare, but their identification will enhance treatment.

\section{Poster 55}

Thyroid Cancer Thursday Poster Case Report

\section{MEN2A WITHOUT MEDULLARY THYROID CANCER} (MTC): A RARE EVENT OR A MISNOMER?

A. George ${ }^{1}$, R.J. Anderson ${ }^{2,1}$

${ }^{1}$ Endocrinology, Diabetes and Metabolism, Creighton University

Medical Center, Omaha, NE; ${ }^{2}$ Endocrinology, Diabetes and

Metabolism, VA-Nebraska Western Iowa Healthcare System,

Omaha, NE

The occurrence of MTC or its precursor, C-cell hyperplasia, has been reported as a constant feature in MEN2A syndrome. We report two cases of patients with Pheochromocytoma and Primary Hyperparathyroidism (PHPT), without MTC and without a RET mutation. A 75 year old male with no family history was diagnosed with a Pheochromocytoma in 2001, which was laparoscopically removed. Complications occurred during the surgery resulting in a nephrectomy. He was later found to have an elevated calcium at $11.2 \mathrm{mg} / \mathrm{dL}$ $(\mathrm{N}: 8.4-10.2 \mathrm{mg} / \mathrm{dL})$ and PTH $80 \mathrm{pg} / \mathrm{mL}(\mathrm{N}: 8-53 \mathrm{pg} / \mathrm{mL})$ with a negative sestamibi scan. Calcitonin was low $<9 \mathrm{pg} / \mathrm{mL}(\mathrm{N}<10 \mathrm{pg} / \mathrm{mL})$. His MEN2 and FMTC mutations (RET) were negative. Thyroid ultrasound showed multiple bilateral nodules, all less than $9 \mathrm{~mm}$. His PHPT was managed without surgical intervention. Our second case is a 43 year old female who initially presented with flank pain, palpitations and headaches. She was found to have a left adrenal mass that was resected in 2009, and proved to be a Pheochromocytoma. She also had hypercalcemia due to a parathyroid adenoma which was removed. Her Calcitonin levels were $<2 \mathrm{pg} / \mathrm{mL}$ and her family history of RET mutation was negative. Her thyroid ultrasound showed thyroid nodules, all less than $6 \mathrm{~mm}$ in size. Neither patient has had evidence of MTC during follow-up. MTC or its precursor, are thought to be a constant feature of germline MEN2. Approximately 2-5\% of MEN2 families do not have RET mutations. Our patients most likely represent sporadic MEN2A without expression of MTC as they fit the clinical definition of MEN2A: the manifestation of any two of the three primary components, (pheochromocytoma, PHPT and MTC). Further confirms, MTC expression can vary in patients with MEN2A without the RET mutation despite different genders and age groups. Somatic RET mutations are not detected in tumor tissue of 40-60\% of sporadic MTC patients and these have better prognosis. Careful monitoring of these patients is required since the absence of RET mutations may reflect the existence of unidentified mutations that predispose to variable expression of MEN2A components. A negative RET mutation may predict a better prognosis, however, there may continue to be an ongoing risk of developing MTC.

\section{Poster 56}

Disorders of Thyroid Function Thursday Poster Clinical IDENTIFICATION OF NOVEL MUTATIONS IN SLC26A7 AS A CAUSATIVE DEFECT IN CONGENITAL HYPOTHYROIDISM DYSHOMONOGENESIS BY NEXT-GENERATION EXOME SEQUENCING M. Zou ${ }^{1}$, A. Alzahrani ${ }^{2}$, A. Al-Odaib ${ }^{1}$, R. Al-Rijjal ${ }^{1}$, H. BinEssa $^{1}$, A. Al-Enazi ${ }^{1}$, M.S. Al-Faham ${ }^{1}$, B. Meyer ${ }^{1}$, Y. Shi ${ }^{1}$

${ }^{1}$ Genetics, King Faisal Specialist Hospital and Research Centre, Riyadh, Saudi Arabia; ${ }^{2}$ Medicine, King Faisal Specialist Hospital and Research Centre, Riyadh, Saudi Arabia

Congenital hypothyroidism $(\mathrm{CH})$ is the most common neonatal endocrine disorder affecting one in 3000 to 4000 newborn babies. The present study aims to identify novel $\mathrm{CH}$-causing genes. Two girls aged 5 and 10 were diagnosed as $\mathrm{CH}$ since childhood. The elder sister developed a goiter at the age of 7 due to persistent and fluctuating 
high serum TSH even though she was given $75-100 \mu \mathrm{g} /$ day levothyroxine. Her two brothers and parents are normal.

Mutational analysis was first performed by PCR-based sequencing of 7 known dyshomonogenesis-causing genes: TG, TPO, DUOX2, DUOXA2, SLC26A4, SLC5A5 and IYD. The entire family was also subjected to exome sequencing. PCR-based sequencing analysis identified a heterozygous mutation in $T G(\mathrm{c} .95 \mathrm{G}>\mathrm{A}, \mathrm{p}$. R32H) from mother and the two patients and SLC26A4 (c.1363A >T, p. I455F) from mother, one normal brother, and the elder patient. Since these two mutations are present in the normal mother, they are unlikely disease-causing genes. Exome sequencing was performed to search for disease-causing genes. Based on the following criteria: 1. allele frequency $<0.01 ; 2$. predicted to be damaging by at least two of 3 prediction programs: Mutation Taster, PolyPhen-2, and SIFT; 3. homozygous variants present only in the patients, 3 candidate genes were identified: VPS13B (c.4247G >A, p. R1416Q), SLC26A7 (c.926_ 929delCACT, and c.932_933delAA), and C20orf202 (c.317_318 insGTCT). VPS13B is involved in vesicle-mediated transport and sorting of proteins in the cell. Its mutations are associated with Cohen syndrome. C20orf202 has unknown function. SLC26A7 is an anion transporter and is in the same family as $S L C 26 A 4$. Given that Slc26a7 deletion mice display hypothyroidism with hyperplastic thyroid histologic changes, SLC26A7 is likely the disease-causing gene in our patients. The two unusual tandem homozygous deletions in SLC26A7 would result in the complete loss of its function, resembling Slc26a7 knockout mice. We have reported the first SLC26A7 mutation in $\mathrm{CH}$ patients. SLC26A7 may be involved in the iodide transport different from the $\mathrm{Na}^{+} / \mathrm{I}^{-}$symporter SLC5A5 and its defect cannot be compensated by the wild-type SLC5A5.

The study is supported by KACST grant P-L-10-0051

\section{Poster 57}

Disorders of Thyroid Function Thursday Poster Clinical

\section{GASTRIC PH AND ORAL THYROXINE REQUIREMENT:} THE IN VIVO STUDY

C. Virili ${ }^{1}$, G. Bruno ${ }^{2}$, M. Santaguida ${ }^{1}$, B. Porowska ${ }^{3}$, C. De Vito ${ }^{4}$, C. Severi ${ }^{2}$, M. Centanni ${ }^{1}$

${ }^{1}$ Medico-Surgical Sciences and Biotechnologies, "Sapienza" University of Rome, Latina, Italy; ${ }^{2}$ Department of Internal Medicine and Medical Specialties, "Sapienza" University of Rome, Rome, Italy; ${ }^{3}$ Department of Cardio-Thoracic-Vascular Surgery and Organ Transplantation, "Sapienza" University of Rome, Roma, Italy; ${ }^{4}$ Department of Public Health and Infectious Diseases, "Sapienza" University of Rome, Rome, Italy

An increased need for thyroxine has been observed in patients with $\mathrm{H}$ pylori related gastritis and gastric atrophy, disorders that may impair gastric acid secretion. In vitro evidence supports the notion that the variations of gastric $\mathrm{pH}$ may also interfere with $\mathrm{T} 4$ dissolution profile. This study was, therefore, designed to obtain the in vivo evidence of a direct correlation between the therapeutic dose of $\mathrm{T} 4$ and the gastric $\mathrm{pH}$, directly measured during endoscopy. A total of $43 \mathrm{~T} 4-$ treated hypothyroid patients $(36 \mathrm{~W} / 7 \mathrm{M}$; median age $=51 \mathrm{yrs})$ have pledged to take thyroxine in fasting conditions, abstaining from eating or drinking for one hour. All patients underwent endoscopy with multiple biopsy specimens while gastric juice was collected for $\mathrm{pH}$ evaluation. To calculate the excess of $\mathrm{T} 4$ required in each patient, the individual dose of T4 was compared to the one observed in ageand BMI-matched patients but without gastrointestinal and/or pharmacological interference. The results were plotted against the actual $\mathrm{pH}$ and the $\mathrm{H}^{+}$concentration titrated with $\mathrm{NaOH}^{-}$. To obtain a similar serum TSH (median $=1.11 \mathrm{mU} / \mathrm{l})$, the dose of thyroxine was progressively higher along with the increase of the gastric $\mathrm{pH}\left(\mathrm{r}^{2}=0.1209 ; \mathrm{p}<\right.$ $0.0223)$ and the actual $\mathrm{H}^{+}$concentration $\left(\mathrm{r}^{2}=0.1275 ; \mathrm{p}<0.0219\right)$. A multivariate analysis revealed that $\mathrm{pH}$ act as an independent variable in determining the dose of T4 $(\mathrm{p}<0.029)$. Also, the excess of T4 dose (ED) required, plotted with gastric acidity in each patient, was highly correlated with the increased $\mathrm{pH}(\mathrm{p}<0.001)$ and decreased $\mathrm{H}^{+}$concentration $(\mathrm{p}<0.0001)$. Based on their $\mathrm{pH}$, patients were then subdivided in two groups using as cut-off the median $\mathrm{pH}$ of the whole sample (2.4; IQ1-3 = 1.5-6.1). Only 6 out of 24 patients (25\%) in group A (median $\mathrm{pH}=1.52)$ needed a high $\mathrm{T} 4$ dose vs 17 out of 19 $(89 \%$; $<<0.0001)$ in group $\mathrm{B}$ (median $\mathrm{pH}=6.47)$. Overall, the median $\mathrm{T} 4$ dose was increased by $15 \%$ in group $\mathrm{A}$ and by $48 \%$ in group $\mathrm{B}$. The ED of T4 was also differently distributed in patients with atrophic gastritis (90\%), with pangastritis $(73 \%)$ and with antritis $(40 \%)$. This is the first in vivo study showing a positive correlation between gastric $\mathrm{pH}$ and the need for thyroxine in humans.

\section{Poster 58}

Disorders of Thyroid Function Thursday Poster Clinical THYROID HORMONE THERAPY DOES NOT IMPROVE HYPOTHYROID SYMPTOMS IN OLDER ADULTS WITH SUBCLINICAL HYPOTHYROIDISM EVEN AMONG THOSE WITH MORE SYMPTOMS AT BASELINE: THE TRUST RANDOMIZED TRIAL

D. Bauer ${ }^{7}$, N. Rodondi ${ }^{1}$, P. Kearney ${ }^{2}$, I. Westendorp ${ }^{3}$, J. Gussekloo ${ }^{6}$, I. Ford ${ }^{4}$, S. Mooijaart ${ }^{6}$, T. Watt ${ }^{5}$, A. McConnachie ${ }^{4}$, D. Stott ${ }^{4}$ ${ }^{1}$ University of Bern, Bern, Switzerland; ${ }^{2}$ University College Cork, Cork, Ireland; ${ }^{3}$ University of Copenhagen, Copenhagen, Denmark; ${ }^{4}$ University of Glasgow, Glasgow, United Kingdom; ${ }^{5}$ Copenhagen University Hospital Herlev, Copenhagen, Netherlands; ${ }^{6}$ Leiden University Medical Center, Leiden, Netherlands; ${ }^{7}$ University of California, San Francisco, San Francisco, CA

The recently published TRUST trial (NEJM, April 3, 2017) was a placebo-controlled randomized trial of thyroid hormone replacement among 737 older adults with subclinical hypothyroidism. After 1-3 yr of follow-up, the overall results found no beneficial effects on self-reported hypothyroid symptoms or tiredness. The effects of thyroid replacement among TRUST participants with more baseline hypothyroid symptoms and tiredness, who might benefit to a greater extent than those with fewer symptoms, have not been reported. TRUST randomized adults $\geq 65$ years old with untreated persistent subclinical hypothyroidism (TSH 4.6 to 19.0 $\mathrm{mIU} / \mathrm{L}$ ) to either levothyroxine or matching placebo. 368 were assigned to a levothyroxine starting dose of $50 \mu \mathrm{g} / \mathrm{d}$ (or $25 \mu \mathrm{g}$ if weight $<50 \mathrm{Kg}$ or coronary heart disease) with blinded dose titration to reach a TSH $<4.6 \mathrm{mU} / \mathrm{L}$, and 369 received matching placebo with mock dose titration. The 2 primary outcomes were 1 yr. change in Hypothyroid Symptoms and Tiredness scores (range for each scale 0-100 with 9 point difference considered clinically significant). In this analysis we examine the effects of treatment vs. placebo on the primary outcomes among participants with higher (i.e. worse) baseline symptom scores. Mean $( \pm \mathrm{SD}) \mathrm{Hy}-$ pothyroid Symptom scores at baseline were $18 \pm 19$ and $17 \pm 18$ in the thyroxine and placebo groups, respectively, and over 1 yr. mean symptom scores fell by 0.2 in both groups (between group difference $0.0,95 \%$ confidence interval: -2.0 to 2.1). Similar to the overall results, we found no evidence that baseline Hypothyroid Symptom scores were associated with the effects of thyroxine vs. placebo on symptom scores (interaction $\mathrm{p}$-value $=0.85$ ). For example, among those with baseline Hypothyroid Symptom scores $>30$ (approximately the upper quintile, $N=132$ ), mean Hypothyroid Symptom scores fell from $45 \pm 14$ to $33 \pm 21$ after 1 yr. in the thyroxine group, and fell from $45 \pm 15$ to $35 \pm 21$ in the placebo group. Results were similar among those with greater baseline Tiredness scores (interaction $\mathrm{p}$-value $=0.65$ ) Levothyroxine replacement did not improve Hypothyroid Symptoms or Tiredness in older persons with subclinical hypothyroidism, including those with higher symptom scores at baseline. 


\section{Poster 59}

Disorders of Thyroid Function Thursday Poster Clinical

CENTRAL HYPOTHYROIDISM IN PATIENTS WITH INTRACRANIAL PITUITARY AND NON-PITUITARY LESIONS IN CHILD- OR ADULTHOOD TREATED WITH OR WITHOUT IRRADIATION

\section{U. Feldt-Rasmussen ${ }^{1,2}$, A. Astradson ${ }^{3}$, E. Hansen Moeller ${ }^{1}$,} L. Poulsgaard ${ }^{3}$, M. Klose ${ }^{1}$, D. Marina ${ }^{1}$, H. Roed ${ }^{3}$, A. Rasmussen ${ }^{1}$, M. Juhler ${ }^{3,2}$

${ }^{1}$ Endocrinology, National University Hospital, Copenhagen, Denmark; ${ }^{2}$ Copenhagen University, Copenhagen, Denmark;

${ }^{3}$ Neurosurgery, National University Hospital, Copenhagen, Denmark

Introduction and Aims: Hypopituitarism is frequent in patients with pituitary diseases, but possibly also in patients with other intracranial lesions, probably dependent on treatment. The aim of the present study was to compare the incidence of central hypothyroidism $(\mathrm{CH})$ in several patient groups eligible to having pituitary insufficiency.

Material and Methods: Sixteen patients operated for craniopharyngeoma and subsequently treated by fractionated stereotactic radiotherapy (FSRT) from 1999-2014 with a follow up time of median 3.7 years (0.8-15.2); 54 patients operated for acromegaly and subsequently treated with FSRT from 1999-2014; 31 adult patients (81\% females) receiving FSRT for meningeomas. Median age was 57.8 years at FSRT (range 39.4 to 77.9 ). The total irradiation dose was 54 Gy per patient in either 27 or 30 fractions. 71 adults treated for childhood cancers (age $<15$ years): 29 received craniospinal and 42 only cranial irradiation; 208 patients ( 98 women, 110 men) with various hypothalamo-pituitary diseases, all with growth hormone deficiency and starting growth hormone replacement.

Results: $13 / 16$ of the patients with craniopharyngeomas had $\mathrm{CH}$ after their surgery, and no further insufficiency after FRST; the patients with acromegaly had $88 \% \mathrm{CH}$, most of them also present before FSRT; In hypopituitary patients with growth hormone insufficiency, $78 \%$ had $\mathrm{CH}$ at baseline and follow up, while in 71 childhood irradiated intracranial processes only $6 \%$ had $\mathrm{CH}$ (while $71 \%$ primary hypothyroidism due to spinal irradiation). Irradiation of adult meningeomas showed a progressive decrease of normal thyroid function with time from $88-62 \%$ from 2-7 years of observation.

Conclusion: Direct pituitary lesions are more and highly prone to $\mathrm{CH}$ with or without cranial irradiation, compared to other intracranial lesions independent of treatment. $\mathrm{CH}$ however progresses with time after cranial irradiation of both adult and childhood intracranial processes. In the meningioma group the mass lesion seemed to have more influence than the irradiation. However, $\mathrm{CH}$ is more prevalent than anticipated in some patient groups and need long term surveillance.

\section{Poster 60}

Disorders of Thyroid Function Thursday Poster Clinical MYOCARDIAL BIOMARKER IS ELEVATED IN THYROTOXICOSIS: A PROSPECTIVE OBSERVATIONAL STUDY USING HIGH-SENSITIVITY TROPONIN I

N. Watanabe, J. Yoshimura Noh, N. Hattori, K. Iwaku, N. Suzuki, A. Yoshihara, H. Ohye, M. Suzuki, M. Matsumoto, Y. Kunii, C. Sekiyama, R. Tadokoro, K. Sugino, K. Ito Ito Hospital, Tokyo, Japan

In addition to the early diagnosis of myocardial infarction, highsensitivity troponin I (hsTnI) has been reported to reflect micromyocardial injury and to allow evaluation of prognosis and treatment effects in heart failure. A residential health cohort study also reported that hsTnI predicts the future risk of cardiovascular death and pro- posed very low cut-offs of $\geq 4.7 \mathrm{pg} / \mathrm{ml}$ for women and $\geq 7.0 \mathrm{pg} / \mathrm{ml}$ for men (Eur Heart J. 35:271, 2014). However, hsTnI level has not been studied in detail among patients with thyrotoxicosis.

A prospective observational study was performed to evaluate hsTnI in patients with thyrotoxicosis, and to clarify the frequency of elevated hsTnI and clinical features. A total of 143 patients (median age, 42 years; 32 men and 111 women) with untreated thyrotoxicosis who first visited our hospital between March 2015 and May 2016, excluding those on infertility treatment or who were pregnant, were analyzed. Measurement of thyroid function, hsTnI, and BNP, clinically used as a marker of heart failure, was performed at the time of first visit, then every 3 months for a year. At the first visit, median FT4, TBII, BNP and thyroid weight were as follows: $4.6 \mathrm{ng} / \mathrm{dl}, 9.1 \mathrm{U} /$ $\mathrm{L}, 24 \mathrm{pg} / \mathrm{ml}$, and $31 \mathrm{~g}$. Four patients $(2.7 \%)$ showed atrial fibrillation. Median hsTnI was 1.9 (range, 0-69.6 pg/ml). Five patients (4\%) showed a high level (>26.2 pg/ml), and 21 patients $(15 \%)$ showed a mildly high level (female $\geq 4.7 \mathrm{pg} / \mathrm{ml}$ or male $\geq 7.0 \mathrm{pg} / \mathrm{ml}$ ). Multivariate analysis showed $\mathrm{Hb}$ (per linear $1 \mathrm{~g} / \mathrm{dl}$ ) as a risk factor for mildly high hsTnI (odds ratio; 0.65, $\mathrm{p}=0.029$ ). Correlations were seen between hsTnI and BNP at first visit $(\rho=0.42 ; \mathrm{p}<0.0001)$ and, also, of the first 3 months decrease $(\rho=0.49, \mathrm{p}<0.0001)$. And the first 3 months decrease of FT4 correlated weakly with that of both hsTnI $(\rho=0.32, \mathrm{p}=0.0004)$ and BNP $(\rho=0.32 ; \mathrm{P}=0.0003)$.

Elevated hsTnI concentrations may reflect micro-myocardial injury associated with heart failure conditions due to thyrotoxicosis, and $\mathrm{Hb}$ reductions may be adversely affected by relative hypoxia. Concentrations of hsTnI increased to a high level in $4 \%$ and to a mildly high level in $15 \%$ of patients with thyrotoxicosis and decreased correlating with improvements in thyroid function and BNP level.

\section{Poster 61}

Disorders of Thyroid Function Thursday Poster Clinical INCREASED PARATHYROID HORMONE ASSAY USE HAS NOT IMPROVED SURGICAL OUTCOMES

A.J. Ferrara ${ }^{1}$, C.H. Frenkel ${ }^{1}$, J. Yang ${ }^{2}$, J. Pak ${ }^{2}$, G. Samara ${ }^{3}$

${ }^{1}$ General Surgery, Stony Brook University, Stony Brook, NY; ${ }^{2}$ Family, Population, and Preventive Medicine, Stony Brook University, Stony Brook, NY; ${ }^{3}$ Otolaryngology - Head and Neck Surgery, Stony Brook University, Stony Brook, NY

Hypocalcemia is a common complication of total thyroidectomy. The optimal algorithm to minimize postoperative hypocalcemia is unknown. The purpose of this study is to determine whether increased utilization of parathyroid hormone (PTH) assays has led to improvements in surgical outcomes related to calcium homeostasis. Patient-level data on adults undergoing total thyroidectomy from 2009-2013 was extracted from the Cerner Health Facts ${ }^{\circledR}$ database. All patients had at least two serum calcium levels, at least one of which was postoperative. Multivariable logistic regression analysis was used to compare postoperative outcomes, including length of stay (LOS) and need for intravenous (IV) calcium or vitamin D, between patients with and without PTH levels. There were 589 and 2,190 patients with and without PTH levels, respectively. Frequency of PTH increased over time (11.6 to 31.5\%). Demographics were similar with respect to age, gender, presence of malignancy, thyroid disorder, vitamin D deficiency, and serum levels of albumin $(n=916)$, ionized calcium $(n=594)$ and TSH $(n=139)$. PTH was more common in patients undergoing parathyroid autotransplantation ( 11.4 vs. $8.5 \%, \mathrm{p}=0.03)$, limited (5.8 vs. $1.7 \%, \mathrm{p}<0.0001)$, and radical neck dissection ( 1.7 vs. $0.2 \%, \mathrm{p}<0.0001)$. PTH was inversely associated with LOS $(r=-0.15, p=0.0004)$. PTH $<10$ was associated with longer LOS $(\mathrm{p}<0.001)$. Average postoperative calcium did not differ between groups $(8.49 \pm 0.6 \mathrm{mg} / \mathrm{dL}, \mathrm{p}=0.89)$. Median PTH was $20.1 \mathrm{pg} / \mathrm{ml}$ (IQR 27.1). After risk-adjustment, patients without PTH 
had a $12.1 \%$ shorter average LOS $(\mathrm{p}<0.0001, \mathrm{OR}=0.60,95 \% \mathrm{CI}$ : 0.49-0.74). Patients without PTH were less likely to require IV calcium and vitamin D ( $\mathrm{p}<0.0001$, OR $0.49,95 \% \mathrm{CI}$ : $0.38-0.64$ and OR $0.48,95 \%$ CI $0.39-0.58$, respectively). Increased use of PTH assays has not translated into decreased LOS or reduced the number of patients requiring IV calcium or vitamin D during initial inpatient hospital admission. Improved standardization in managing PTH results or routine supplementation with calcium and/or vitamin D may be required to significantly improve post-thyroidectomy perioperative hypocalcemia rates.

\section{Poster 62}

Disorders of Thyroid Function Thursday Poster Clinical

ASSOCIATION BETWEEN DIETHYLHEXYL PHTHALATE EXPOSURE AND THYROID FUNCTION: A META-ANALYSIS

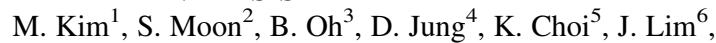
S. Cho ${ }^{1}$, Y. Park ${ }^{1}$

${ }^{1}$ Department of Internal Medicine, Seoul National University College of Medicine, Seoul, Korea (the Republic of); ${ }^{2}$ Division of Endocrinology and Metabolism, Department of Internal Medicine, Hallym Universigy College of Medicine, Seoul, Korea (the Republic of); ${ }^{3}$ Department of Physiology, Lee Gil Ya Cancer and Diabetes Institute, Gachon University College of Medicine, Incheon, Korea (the Republic of); ${ }^{4}$ Division of Environmental Health, Korea Environment Institute, Sejong, Korea (the Republic of); ${ }^{5}$ School of Public Health, Seoul National University, Seoul, Korea (the Republic of); ${ }^{6}$ Department of Internal Medicine, National Medical Center, Seoul, Korea (the Republic of)

Diethylhexyl phthalate (DEHP) has been widely used as industrial products and are detected in humans worldwide. Although epidemiological studies suggest that DEHP can disrupt thyroid hormones, the association between DEHP exposure and thyroid function remains inconclusive. To investigate the association between DEHP exposure and thyroid hormones in humans, we performed a comprehensive meta-analysis. Studies with the correlation coefficient values or regression coefficient values between three major DEHP metabolites - MEHP, MEHHP, and MEOHP - and thyroid stimulating hormone (TSH), free T4, or total T4 were included from PubMed databases. Ten eligible articles were included. Urinary concentrations of DEHP metabolites were negatively correlated with free $\mathrm{T} 4$ and total T4. The pooed $\mathrm{z}$ values between MEHP and free T4/ total T4 were -0.05 (95\% confidence interval $(\mathrm{CI}):-0.08,-0.03)$ and -0.03 (95\% CI: $-0.06,0.00$ ), respectively. The pooled $\mathrm{z}$ values between MEHHP and free T4/total T4 were -0.05 (95\% CI: -0.08 , $-0.02)$ and -0.04 (95\% CI: $-0.06,-0.02)$, respectively. The pooled $z$ values between MEOHP and free T4/total T4 were -0.03 (95\% CI: $-0.06,-0.01)$ and -0.02 (95\% CI: $-0.04,0.00)$, respectively. However, there was no significant association between DEHP metabolites and TSH. The findings in this meta-analysis suggested DEHP exposure could reduce thyroid hormone.

\section{Poster 63}

Disorders of Thyroid Function Thursday Poster Clinical

ANALYSIS OF CURRENT THYROID FUNCTION TESTING PRACTICES

J. Kluesner, D. Beckman, J. Tate, M. True

San Antonio Military Medical Center, Cibolo, TX

Current guidelines recommend thyroid stimulating hormone (TSH) alone as the best test to detect thyroid dysfunction, yet free thyroxine (FT4) and free triiodothyronine (FT3) are commonly ordered when not clinically indicated. Excessive testing can lead to added economic burden in an era of rising healthcare costs, while rarely contributing to the evaluation or management of thyroid disease.

Our knowledge of current patterns of thyroid function testing within the United States remains limited. This study seeks to evaluate our institution's providers' practice in ordering thyroid function tests (TFTs) to determine if changes are needed to reduce excessive laboratory testing.

All outpatient TFT ordering patterns at the San Antonio Military Health System (SAMHS) during a three month period in 2016 were analyzed. TFTs of interest were: TSH, FT4, thyroid panel (TSH + FT4), FT3, total thyroxine (T4), and total triiodothyronine (T3). Between 1 August and 31 October 2016 there were 38,214 individual thyroid labs ordered via 28,597 total lab requests. 11,486 of these requests were in patients with a history of hypothyroidism. The number (percent) of lab requests fell into these patterns: TSH alone 14,919 (52.14\%), TSH + FT4 7,641 (26.72\%), FT3 alone 3,039 (10.63\%), FT4 alone 1,219 (4.26\%), TSH + FT4 + FT3 $783(2.74 \%)$, and others $996(3.48 \%) .35 .96 \%$ of all thyroid function tests ordered were free thyroid hormones. Projected out to a year, using Department of Defense laboratory costs, $\$ 317,429$ worth of TFTs would be ordered, with free thyroid hormone testing accounting for $\$ 107,720$.

Free thyroid hormone tests are ordered excessively. In an era of rising healthcare costs, inappropriate thyroid function testing is an ideal target for efforts to reduce laboratory overutilization. Further research is needed to determine strategies that can reduce excessive thyroid hormone testing.

\section{Poster 64}

Disorders of Thyroid Function Thursday Poster Clinical

\section{QUALITY OF LIFE IN INDIAN PATIENTS WITH GRAVES'} OPHTHALMOPATHY (GO)

S.C. Yadav, K. Delampady, V. Reddy

Endocrinology, Sanjay Gandhi Post Graduate Institute of Medical Sciences, Lucknow, UP, India

GO is severely disabling due to ocular discomfort, effect on vision and effect on appearance and may pose cosmetic problems and may hamper social relationships.

Objectives: To study the quality of life in GO among Indian patients and to determine the correlation of GO-specific QOL scores (visual functioning and appearance) with disease severity and activity. We recruited 98 consecutive patients with GO attending Endocrinology Clinic over a period of 1.5 years. Detailed eye examination was performed and quality of life was assessed by questionnaire (GO-QOL), as per EUGOGO recommendations. The mean age of patients with GO were $37.3 \pm 12.7$ years (females $(65.3 \%)$ ). GO was mild in $87.7 \%$, moderate-severe in $11.2 \%$ and sight-threatening in only $1 \%$ of cases. Ophthalmopathy was clinically active in only $8(8.2 \%)$ cases. Exophthalmos $(83.7 \%)$ was the most common manifestation, followed by upper eyelid retraction (79.6\%). Extra-ocular muscle involvement (9.2\%) and optic nerve dysfunction (2\%) were uncommon. The GOQOL scores were significantly decreased in patients with GO as compared to GD without ophthalmopathy $(\mathrm{p}<0.001)$. In visual functioning, patients with GO had a lower median score of 87.0 (IQR 74.1-100). Similarly, psycho-social effects score was also low in those with GO (median 75.0, IQR 50-87.5). Patients with moderate-severe and sightthreatening GO had lower GO-QOL scores compared to mild disease in both visual $(60.8 \pm 24.3$ vs. $83.5 \pm 20.2, \mathrm{p}<0.01)$ and psycho-social functioning $(50.0 \pm 28.2$ vs. $69.7 \pm 22.6, \mathrm{p}<0.01)$. Subjects with exophthalmos had lower GO-QOL scores as compared to those without exophthalmos, in both visual $(78.1 \pm 22.4$ vs. $94.3 \pm 15.6, \mathrm{p}<0.01)$ and psycho-social functioning $(62.5 \pm 23.0$ vs. $92.0 \pm 10.9, \mathrm{p}<0.001)$. Similarly subjects with clinically active ophthalmopathy had lower GO- 
QOL scores as compared to those with inactive GO, in both visual $(47.2 \pm 30.3$ vs. $83.7 \pm 18.8, \mathrm{p}<0.001)$ and psycho-social functioning $(48.9 \pm 28.7$ vs. $69.0 \pm 23.2, \mathrm{p}<0.05)$. The GO-QOL scores were not associated with TRAb, TPO antibody, total T4 and T3. GO-QOL scores were significantly reduced in patients with GO despite the fact that most had mild and inactive disease. Hence such patients also require counseling and education.

\section{Poster 65}

Disorders of Thyroid Function Thursday Poster Clinical

AN ALTERNATIVE APPROACH IN THE MANAGEMENT OF HYPOTHYROIDISM AFTER FAILURE OF ORAL LEVOTHYROXINE

M.E. Sevilla Alsina ${ }^{1}$, M. Candelario ${ }^{1}$, A. Ramirez ${ }^{1,2}$,

J. Gomez-Daspet ${ }^{1,2}$

${ }^{1}$ Endocrinology, University Of South Florida, Tampa, FL;

${ }^{2}$ Endocrinology, James A. Haley Veterans' Hospital, Tampa, FL

We present a case of Primary Hypothyroidism refractory to oral levothyroxine, successfully treated with weekly intramuscular (IM) levothyroxine (L-T4). 38-year-old woman with a history of benign goiter, underwent resection with subsequent hypothyroidism. She also has morbid obesity, type 2 diabetes mellitus complicated with severe gastroparesis and episodes of daily emesis, often occurring after taking L-T4.

During the last 2 years, Thyroid Stimulating Hormone (TSH) level fluctuated between $28-65 \mathrm{mIU} / \mathrm{L}$. Dose was titrated to $300 \mathrm{mcg}$ daily, later moved to bedtime. Afterwards, $1,000 \mathrm{mcg}$ weekly L-T4 replacement was attempted. Supervised L-T4 intake through a home health nurse assistant was tried, and failed as well. Thyroid function studies demonstrated persistent severe hypothyroidism. Extensive work up for malabsorption was negative.

References suggest starting IM dose that is 50 to $80 \%$ of oral dose. A trial of weekly IM $500 \mathrm{mcg}$ L-T4 was started in March 2017. Baseline TSH, $40.7 \mathrm{mIU} / \mathrm{L}$; free T4, $0.4 \mathrm{ng} / \mathrm{dL}$. After two weeks, both TSH and free-T4 improved to $25.3 \mathrm{mIU} / \mathrm{L}$ and $0.7 \mathrm{ng} / \mathrm{dL}$, respectively. Her dose was raised to $750 \mathrm{mcg}$ IM weekly. After four weeks, TSH was $13 \mathrm{mIU} / \mathrm{L}$ and f-T4 normalized to $0.9 \mathrm{ng} / \mathrm{dL}$. At the time of this report, her symptoms of hypothyroidism resolved without adverse events. She is scheduled to obtain follow up thyroid function studies. It is rare for subjects to fail therapy with oral levothyroxine (L-T4) despite progressive dose adjustments. In this subject, vomiting of LT4 tablets is the apparent only cause for lack of response. Although, the U.S. Food \& Drug Administration does not approve the use of intramuscular levothyroxine, in this case the clinical and biochemical response has been successful. All patients are unique and will benefit by individualized management. This case represents an opportunity to review an effective and so far, safe alternative in cases where patients do not respond to oral therapy in a long-term basis.

\section{Poster 66}

Disorders of Thyroid Function Thursday Poster Clinical

A CASE OF GRAVES' DISEASE OCCURRED AFTER PERCUTANEOUS ETHANOL INJECTION THERAPY FOR A NON-TOXIC FUNCTIONING THYROID NODULE

T. Michigishi ${ }^{1}$, S. Minami ${ }^{4}, \mathrm{~S}$. Tsuji ${ }^{1}$, T. Yoneyama ${ }^{1}$,

K. Yokoyama ${ }^{1}$, N. Ueda ${ }^{4}$, T. Tsukatani ${ }^{2}$, H. Takeda ${ }^{3}$, M. Akimoto ${ }^{5}$, Y. Imamura ${ }^{7}$, Y. Mizukami ${ }^{6}$

${ }^{1}$ Thyroidology, Public Central Hospital of Matto-Ishikawa, Hakusan, Japan; ${ }^{2}$ Otolaryngology, Public Central Hospital of Matto-Ishikawa, Hakusan, Japan; ${ }^{3}$ Ophthalmology, Public Central Hospital of MattoIshikawa, Hakusan, Japan; ${ }^{4}$ Pediatrics, Public Central Hospital of Matto-Ishikawa, Hakusan, Japan; ${ }^{5}$ Radiology, Public Central
Hospital of Matto-Ishikawa, Hakusan, Japan; ${ }^{6}$ Rehabilitation, Japanese Red Cross Kanazawa Hospital, Kanazawa, Japan; ${ }^{7}$ Surgical Pathology, University of Fukui Hospital, Fukui, Japan

Co-existence of Graves' disease and Plummer's disease is known as Marine-Lenhart syndrome. We present a case of Graves' disease occurred after percutaneous ethanol injection therapy (PEIT) for a functioning thyroid nodule. A 14-year-old girl with a nodular goiter was referred from pediatrics in August, 2014. She had a palpable nodule in the left lobe. She was euthyroid with a low TSH value, and negative for $\mathrm{TgAb}, \mathrm{TPOAb}, \mathrm{TRAb}$ and TSAb. Laboratory data revealed that TSH $0.145 \mu \mathrm{IU} / \mathrm{mL}$, FT3 $2.83 \mathrm{pg} / \mathrm{mL}$, FT4 $1.06 \mathrm{ng} / \mathrm{dL}, \mathrm{TgAb} 16 \mathrm{IU} / \mathrm{mL}$, TPOAb $7 \mathrm{IU} / \mathrm{mL}$, TRAb human $<1.0 \mathrm{IU} / \mathrm{L}$, and TSAb $113 \%$. Ultrasound showed a mixed nodule $(42 \times 21 \times 30 \mathrm{~mm})$ in the left lobe. Tc- $99 \mathrm{~m}$ pertechnetate $(\mathrm{Tc}-99 \mathrm{~m})$ thyroid scan showed a hot nodule corresponding to the left nodule and mild tracer uptake into the extra-nodal parenchyma. Cytological examinations revealed benign features. She received PEIT on the nodule 3 times every 2 months. No relevant adverse effects were observed. Although the nodule shrunk (19x14x15 mm), TSH remained to be suppressed. In October, 2015, she was thyrotoxic, and, surprisingly, both TRAb and TSAb became positive. Laboratory data showed that TSH $<0.003 \mu \mathrm{IU} / \mathrm{mL}$, FT3 $5.91 \mathrm{pg} / \mathrm{mL}$, FT4 $2.17 \mathrm{ng} / \mathrm{dL}$, TRAb human $6.0 \mathrm{IU} / \mathrm{L}$, and TSAb 388\%. Thyroid scans with Tc-99m and I-123, respectively, revealed uniformly diffuse tracer uptake into the extra-nodal parenchyma and the hot nodule disappeared. I-123 uptake at 24 hours was $66.6 \%$. She had no Graves' ophthalmopathy (Heltel: Right $15 \mathrm{~mm}$, Left $15 \mathrm{~mm})$. She received daily methimazole $(10 \mathrm{mg})$ and potassium iodide $(50 \mathrm{mg})$. Potassium iodide was withdrawn 2 months later as she complained of thyroid enlargement. Then, she has been euthyroid with methimazole $(5 \mathrm{mg})$. Both TRAb and TSAb became negative in March, 2017. Graves' disease may occur several months after I-131 therapy for Plummer's disease, as temporary increase of TRAb exacerbates pre-existing subclinical Graves' disease. Like this phenomenon, PEIT may make Graves' disease overt in our case of a non-toxic functioning thyroid nodule. This presentation may be the first case report of Graves' disease developed after PEIT for a functioning thyroid nodule.

\section{Poster 67}

\section{Disorders of Thyroid Function Thursday Poster Clinical}

\section{SUBCLINICAL HYPOTHYROIDISM AND DYSLIPEMIA:} FREQUENCY, TYPES AND ASSOCIATED FACTORS

\section{M.G. Jimenez ${ }^{1}$, D. Fernandez ${ }^{1}$, P. Aguilera ${ }^{1}$, J. Roldan ${ }^{2}$, N. Bautista ${ }^{3}$}

${ }^{1}$ Internal Medicine, Universidad de Cuenca, Cuenca, Ecuador;

${ }^{2}$ Research, Universidad de Cuenca, Cuenca, Ecuador;

${ }^{3}$ Endocrinology, SOLCA, Guayaquil, Ecuador

Subclinical hypothyroidism ( $\mathrm{SCH}$ ) is defined as an elevated thyroid stimulating hormone (TSH) level with normal free thyroxine (FT4) and triiodothyronine (FT3). The interaction between thyroid hormones and lipid metabolism is well known, however, the role of SCH in dyslipemia remain controversial. There are several studies that analyze association between SCH and dyslipidemia with different outcomes. The discrepancies might be related to different age cut points, ethnicity, gender and geographical location.

Objectives: To determine the frequency and types of lipid profile abnormalities seen in patients with $\mathrm{SCH}$ at university hospital in Cuenca - Ecuador.

Methods: We performed a retrospective chart review of all the patients seen in the Endocrinology Department at Hospital Vicente Corral Moscoso, either as a new patient or follow visit, with thyroid function test that meets criteria for $\mathrm{SCH}$. Patients were classified according to age, gender, nutritional status, and smoking. The following levels in serum were measured: TSH, FT3, FT4, total 
cholesterol, triglycerides, high density lipoproteins (HDL) and low density lipoproteins (LDL).

Results: 89 patients with SCH were analyzed, 42 (47.2\%) presented elevated total cholesterol levels, $55.1 \%$ (49) low HDLs levels; 9 patients $(10.1 \%)$ had an elevated LDL levels and 44 patients $(49.4 \%)$ had hypertriglyceridemia. Elevated total cholesterol were associated with age ( $\geq 40$ years old) $p=0,020$; low HDL levels were present in obese patients (IMC $\geq 25$ ) $p=0,005$. None of variables were significant for an association with high LDL levels or hypertriglyceridemia.

Conclusion: Dyslipidemia is frequently seen in patients with $\mathrm{SCH}$. Age above 40 and obesity are important risk factors.

\section{Poster 68}

Iodine Uptake \& Metabolism Thursday Poster Clinical STUDY OF EFFECT OF IODINATED CONTRAST AGENTS ON THYROID FUNCTIONS

S. Kumar, S.K. Jain, R. Aggarwal

Department of Medicine, Lady Hardinge Medical College, New Delhi, New Delhi, India

Exposure of high doses of iodine can lead to both hypothyroidism and hyperthyroidism. Contrast agents are one of the major sources of high iodine exposure. We studied the effect of iodinated contrast agents (omnipaque 350) on the thyroid functions in patients undergoing contrast enhanced computed tomography (CECT) scan. Forty patients (26 males and 14 females) with no personal or family history of thyroid disease and normal thyroid on examination undergoing CECT scan were studied prospectively. $\mathrm{FT}_{3}, \mathrm{FT}_{4}, \mathrm{TSH}$ were assessed at baseline and then weekly from week 1 to week 6 after administration of contrast agent. Anti TPO antibody estimation was done at baseline. Age of the patients ranged from 18 to 64 years $($ mean $=37)$. All patients were euthyroid at the baseline. No significant change in thyroid function was noted at the end of 1 week. Mean values of $\mathrm{FT}_{3}$ $(\mathrm{p}=0.039)$ and $\mathrm{FT}_{4}(\mathrm{p}=0.026)$ increased significantly at week 2 in comparison to baseline but TSH values showed no significant change. Beyond $2^{\text {nd }}$ week, $\mathrm{FT}_{3}$ increased significantly at week 3 $(\mathrm{p}=0.001)$, week $4(\mathrm{p}=0.001)$, week $5(\mathrm{p}<0.01)$ and week 6 $(\mathrm{p}<0.01)$, without any significant changes in $\mathrm{FT}_{4}$ and $\mathrm{TSH}$, indicating isolated high $\mathrm{FT}_{3}$ from week 3 onwards, although all mean values were within the normal range. $27 / 40(67.5 \%)$ cases showed increased $\mathrm{FT}_{3}$ or $\mathrm{FT}_{4}$ or both beyond the normal range at any point of time after contrast administration, but none of the patients were symptomatic. From these 27 cases, 20 (74\%) had their deranged values of $\mathrm{FT}_{3} / \mathrm{FT}_{4} /$ both returned within normal range by the end of $6^{\text {th }}$ week. TSH was within normal range in all cases throughout the study. Anti TPO antibody was within normal range in all patients. Administration of iodinated contrast agents can cause significant changes in thyroid functions which are self limiting in most cases. Nonetheless, history of contrast use in the recent past must be considered while advising and interpreting thyroid function tests.

\section{Poster 69}

Iodine Uptake \& Metabolism Thursday Poster Clinical AN UPDATE ON URINARY IODINE CONCENTRATIONS IN BOSTON PREGNANT WOMEN

S.Y. Lee ${ }^{1}$, E. Zhabjaku ${ }^{2}$, D. Willard ${ }^{1}$, X. He ${ }^{1}$, L. Braverman $^{1}$, E.N. Pearce ${ }^{1}$

${ }^{1}$ Endocrinology, Boston University School of Medicine, Boston, MA; ${ }^{2}$ Boston University School of Medicine, Boston, MA

Iodine is an essential micronutrient for thyroid hormone production. Adequate thyroid hormone is critical for normal fetal neurodeve- lopment. Although the general U.S. population is deemed iodinesufficient, the median urinary iodine concentration (UIC) for U.S pregnant women has fallen below the World Health Organizationrecommended cutoff of $150 \mathrm{mcg} / \mathrm{L}$. A previous study of 100 pregnant women at Boston Medical Center (BMC) in 2004 showed their median UIC was $149 \mathrm{mcg} / \mathrm{L}$ and $9 \%$ had UIC $<50 \mathrm{mcg} / \mathrm{L}$. We conducted a cross-sectional study at BMC to assess the current iodine nutrition status of Boston-area pregnant women. Pregnant women seen for prenatal care at BMC from 6/2015 to 9/2015 were recruited. Demographic information, use of iodine-containing dietary supplements, recent dietary intake of iodine-rich foods, and knowledge regarding iodine were ascertained by questionnaire. Spot urine samples were collected to measure UIC. Spearman correlation or ANOVA was used as appropriate to assess correlations between UIC and individual covariates. A multivariable regression model was used to assess predictors of UIC. A total of 61 pregnant women (mean age $31 \mathrm{yr}, 21 \%$ white, $39 \%$ black, median gestational age 26wks6d) were included. Most women (80\%) reported using a multivitamin (MVI) and 38 (62\%) used an iodine-containing MVI. 24 women used iodized salt. Only $10(16 \%)$ women reported that a medical provider discussed iodine nutrition in pregnancy and only $20(33 \%)$ looked for iodine when purchasing household salt. The median (range) for UIC in our cohort was $146(17.5-886) \mathrm{mcg} / \mathrm{L}$. The amount of iodine in MVIs, daily servings of milk, and daily servings of cheese were positively correlated with UIC ( $p<0.001$ for each). These variables and gestational age were included in the multivariable analysis. Although the overall multivariable model was statistically significant $(p=0.002)$, none of the individual covariates were significant predictors of UIC. The median UIC of 61 pregnant women in Boston was $146 \mathrm{mcg} / \mathrm{L}$, marginally low and similar to that seen in 2004 . However, the range of UIC remains wide, with some women with UIC as low as $17.5 \mathrm{mcg} / \mathrm{L}$. Ingestion of iodine-containing MVIs and dairy foods were significantly correlated with UIC.

\section{Poster 70}

Iodine Uptake \& Metabolism Thursday Poster Clinical IODINE DEFICIENCY EMERGES AMONG PREGNANT WOMEN IN SWEDEN DESPITE SALT IODINE FORTIFICATION AT 50 PPM - A NATIONAL, CROSS-SECTIONAL STUDY S. Manousou ${ }^{2,3}$, M. Andersson ${ }^{4}$, R. Eggertsen ${ }^{2,5}$, L. Hulthén ${ }^{6,2}$, H. Filipsson Nyström ${ }^{1,2}$

${ }^{1}$ Deparmtent of Endocrinology, Sahlgrenska University Hospital, Göteborg, Sweden; ${ }^{2}$ Sahlgrenska Academy, University of Gothenburg, Institute of Medicien, Göteborg, Sweden; ${ }^{3}$ Kungälv's Hospital, Department of Medicine, Kungälv, Sweden; ${ }^{4}$ ETH, Lab of human Nutrition, Institute of Food, Nutrition, and Health, Zurich, Switzerland; ${ }^{5}$ Mölnlycke Health care center, Mölnlycke, Sweden; ${ }^{6}$ Sahlgrenska Academy, University of Gothenburg, Department of Clinical Nutrition, Göteborg, Sweden

Voluntary salt iodization at $50 \mathrm{mg} / \mathrm{kg}$ ensures adequate iodine nutrition in Swedish school-aged children, but the iodine status in pregnant women is uncertain. Our aim was to assess iodine status, thyroid function and the consumption of iodine containing prenatal vitamin supplements in pregnant women. We measured urinary iodine concentration (UIC), dried blood spot (DBS) thyroglobuline (Tg) and DBS thyroxine (T4) and thyroid stimulating hormone (TSH) in a national, cross sectional study of 750 pregnant women. All Swedish maternal health care centers $(\mathrm{MCH})$ were classified according to nativity rate; the number of $\mathrm{MCH}$ from each nativity group was determined statistically to get a proportional representative randomly selected, subsample of 25 MHC. From each MHC, mid-wives recruited 10 women from each trimester. We obtained 
information on gestational age and consumption of prenatal vitamin supplements. Women were divided into supplement users (using multivitamins containing $\geq 75 \mu \mathrm{g}$ iodine/day) and non-supplement users (eating $<75 \mu \mathrm{g}$ iodine/day from multivitamin supplements). The overall geometric mean (95\% confidence interval) UIC was $102(96-108) \mu \mathrm{g} / \mathrm{L}(\mathrm{n}=737)$ that is below the recommended UIC threshold during pregnancy of $150 \mu \mathrm{g} / \mathrm{L}$. UIC did not differ between trimesters $(p=0.570)$. The geometric mean UIC in women taking supplements was $142(130-155) \mu \mathrm{g} / \mathrm{L}(\mathrm{n}=470)$, higher than in nonsupplement users 84 (79-91) $\mu \mathrm{g} / \mathrm{L}, \mathrm{n}=267, \mathrm{p}<0.001$. The geometric mean DBS-Tg in the supplement group was 22.7 (20.0-25.9) $\mu \mathrm{g} / \mathrm{L}$ compared to 26.9 (24.4-29.6) $\mu \mathrm{g} / \mathrm{L}$ in the non-supplement group, $\mathrm{p}=0.044$. Thyroid hormones did not differ between supplement and non-supplement users. Pregnant women in Sweden have inadequate iodine intake and elevated $\mathrm{Tg}$ concentrations, particularly the third of women not taking iodine containing multivitamin supplements. This study highlights that adequate UIC of $125 \mu \mathrm{g} / \mathrm{L}$ in school children 10 years ago, does not guarantee adequate intake in risk populations, even in a country with a high level of iodine fortification.

\section{Poster 71}

Iodine Uptake \& Metabolism Thursday Poster Clinical

THE EFFECTS OF IRON AND SELENIUM IN IODINE CONTAINING MULTIVITAMINS ON THYROID RELATED COMPOUNDS DURING PREGNANCY IN SWEDEN: A RANDOMIZED PLACEBO-CONTROLLED TRIAL

J. Eriksson ${ }^{2}$, S. Manousou ${ }^{2,3}$, R. Eggertsen ${ }^{2,5}$, L. Hulthén ${ }^{2,4}$, H. Filipsson Nyström ${ }^{1,2}$

${ }^{1}$ Deparmtent of Endocrinology, Sahlgrenska University Hospital, Göteborg, Sweden; ${ }^{2}$ Sahlgrenska Academy, University of Gothenburg, Institute of Medicine, Göteborg, Sweden; ${ }^{3}$ Kungälv's Hopsital, Department of Medicine, Kungälv, Sweden; ${ }^{4}$ Sahlgrenska Academy, University of Gothenburg, Department of Clinical Nutrition, Göteborg, Sweden; ${ }^{5}$ Mölnlycke Health Care center, Göteborg, Sweden

Multivitamins with iodine are advocated to pregnant women to avoid iodine deficiency, as iodine may be beneficial for brain development in the child. Multivitamins also contain iron and selenium that may affect thyroid hormone metabolism. Iron is included in the tyreoperoxidase enzyme promoting the coupling of iodine to thyroglobulin ( $\mathrm{Tg}$ ) and selenium is incorporated in deiodinases that regulates levels of thyroxine (T4) and triiodothyronine (T3). There is no previous studies on the effects of iodine containing multivitamins on iron and selenium levels in pregnant women and the relation to thyroid hormone levels. This was a randomized, double-blinded controlled trial of 200 pregnant women, who were randomized to multivitamins containing $150 \mu \mathrm{g}$ iodine, $12 \mathrm{mg}$ iron and $50 \mu \mathrm{g}$ selenium/day or multivitamins without iodine, iron and selenium in pregnancy week 7-12 until delivery, besides iron supplements on usual routines. Thyroid hormones, Tg, selenium (ref 0.7$1.2 \mu \mathrm{mol} / \mathrm{L}$ ) and iron measurements (ferritin (ref $15-150 \mathrm{mg} / \mathrm{L}$ ), transferrin saturation (ref $0.1-0.5)$ ) were collected in the third trimester. Urinary iodine concentration confirmed mild ID in the control group with a $\mathrm{Tg}$ increase. In the third trimester, 139 patients were left for sampling. In the active group $(n=67)$ median (interquartile range (IQR)) selenium levels were $0.72(0.16)$ vs $0.61(0.14)$ in the control group $(n=72), p<0.001$. Low selenium values were noted in $70.0 \%$ of participants and it was more common in the control group (81.9\%), $\mathrm{p}<0.001$. Median (IQR) Tg levels was higher in those with low selenium 30.0 (30.5) than in those with normal selenium 20.5 (21.5), $\mathrm{p}=0.037$. Thyroid hormones did not differ between active/control groups or low/normal selenium groups, but FT3/FT4 ratio was higher in the low selenium group than in the normal selenium group $(0.35(0.08)$ vs $0.33(0.08)), p=0.025$. Ferritin in the active group was $22.0(21.0)$ and
20.0 (21.5) in the control group, $\mathrm{p}=0.393$ and transferrin saturation 0.20 (0.11) and $0.18(0.18), p=0.802$, respectively. Multivitamins used during pregnancy to increase iodine levels also increase selenium levels where effects on thyroid metabolism needs further evaluation.

\section{Poster 72}

Thyroid \& Development Thursday Poster Case Report

ISOSEXUAL PRECOCITY AND BLEEDING DIATHESIS AS THE PRESENTING FEATURE OF SEVERE PRIMARY HYPOTHYROIDISM IN CHILDHOOD

I. Maisnam ${ }^{1}$, N. Karjyi ${ }^{2}$

${ }^{1}$ Endocrinology, RG Kar Medical College, Kolkata, West Bengal, India; ${ }^{2}$ General Medicine, R G Kar Medical College, Kolkata, India

Typical presentations of childhood primary hypothyroidism are declining height velocity, poor school performance, delayed puberty, lethargy, constipation and dry hair and skin. Isosexual precocity can rarely occur due to activity of TSH on FSH receptors. Thyroid hormone has permissive effects on clotting factor synthesis. Severe hypothyroidism can lead to bleeding diathesis. We describe a case of severe primary hypothyroidism in a 7 year old girl presenting with isosexual precocity and bleeding diathesis that responded promptly to levothyroxine.7 year old girl was referred for irregular vaginal bleeding, breast development and spontaneous bruising. Height was $111 \mathrm{~cm}$ (<3rd percentile), weight was $19 \mathrm{Kg}$ (50th percentile). She had moderate anaemia, dry skin and hair, bruises all over her body and lanugo hair over her back. There was no goitre, lymphadenopathy or organomegaly. Breast was B4 (Tanner) with no axillary or pubic hair.

Biochemistry: TSH $>150 \mu \mathrm{IU} / \mathrm{ml}$, FT4 $0.11 \mathrm{ng} / \mathrm{dl}$, FSH $8.8 \mu \mathrm{IU} / \mathrm{ml}$, LH $0.18 \mathrm{miu} / \mathrm{ml}$, prolactin $28 \mathrm{ng} / \mathrm{ml}$, estradiol (E2) $49.2 \mathrm{pg} / \mathrm{ml}$. AntiTPO antibody was strongly positive. There was normocytic anaemia and adequate platelets. Bleeding time was normal but prothrombin and activated partial thromboplastin time were elevated. Bone age was 4 years. USG abdomen: bilateral large ovaries with multicystic appearance. MRI: pituitary macroadenoma abutting on optic chiasma. Diagnosis of Van Wyk Grumbach syndrome with bleeding diathesis due to severe primary autoimmune hypothyroidism was made.

She was immediately treated with levothyroxine at $4 \mu \mathrm{g} / \mathrm{kg} /$ day. Parents were assured that symptoms would improve over time. She has been on our treatment for 7 months. She is euthyroid, vaginal bleeding has stopped, breasts has regressed and bruises have disappeared. Recent ultrasonography showed persistence but reduction of the size of ovarian cysts. Pituitary MRI also shows a smaller yet still enlarged pituitary. This case highlights the atypical and rare presentations of severe untreated hypothyroidism in childhood. Awareness of these rare presentations ensures that levothyroxine replacement is promptly instituted and precious time and energy is not wasted on expensive and invasive investigations and treatment.

\section{Poster 73}

Thyroid \& Development Thursday Poster Clinical

CLINICAL UTILITY OF INTRAOPERATIVE PARATHYROID SCORES SYSTEM (IPSS) IN THYROIDECTOMY

B. Wang ${ }^{1,3}$, W. Zhao ${ }^{1,2}$, S. Yang ${ }^{1}$, L. Zhang ${ }^{1}$

${ }^{1}$ Thyroid Surgery, Fujian Medical University Union Hospital, FuZhou, FuJian, China; ${ }^{2}$ Standing Committee, Chinese Thyroid Association, BeiJing, BeiJing, China; ${ }^{3}$ Youth Committee, Chinese Thyroid Association, BeiJing, China

Establish the Intraoperative Parathyroid Scores System (IPSS) for thyroidectomy and study the clinical value of Intraoperative Parathyroid Scores System for thyroidectomy. IPSS include transplantation 
Parathyroid Scores and in situ Parathyroid Scores, from 2014, Totally 266 cases of thyroid tumor patients received bilateral thyroidectomy in Fujian Medical university union Hospital. The Intraoperative Parathyroid Scores were recorded, parathyroid hormone and calcium were test in $\mathrm{d} 0, \mathrm{~d} 1, \mathrm{~d} 14, \mathrm{~d} 60$ post operation. The correlation between Intraoperative Parathyroid Scores and PTH calcium were calculated. And ROC curve and logistic regression were tested for the prediction value. transplantation Parathyroid Scores were positively correlated with transplanted parathyroid hormone, the Pearson relation value is 0.563 and $p=0.00$. Intraoperative Parathyroid Scores and in situ Parathyroid Scores were positively correlated with PTH postoperative, the correlation value is 0.404 and 0.301 . Intraoperative Parathyroid Scores is predicted factors for long-term PTH function, the sensitivity was $49.0 \%$, specificity of $81.0 \%$. Intraoperative Parathyroid Scores system can clear record every state and the function of the parathyroid glands, Intraoperative Parathyroid Scores system is a predicted factor for long-term PTH function, it can reduce hospital stay and make a dissection making in patients with recurrent surgery, This system has great clinical significance and social benefits. it Can be easy promote in thyroid surgery.

\section{Poster 74}

Thyroid Cancer Thursday Poster Basic

\section{MOUSE MODELS OF SPORADIC THYROID CANCER WITH LUNG METASTASIS DERIVED FROM BRAF ${ }^{\text {V600E }}$ ALONE OR IN COMBINATION WITH PTEN LOSS}

Y. Nagayama ${ }^{1}$, M. Shimamura ${ }^{1}$, N. Shibusawa ${ }^{2}$, T. Kurashige ${ }^{1}$, H. Matsuzaki ${ }^{1}$, M. Nakashima ${ }^{3}$, M. Yamada ${ }^{2}$

${ }^{1}$ Dept Mol Med, Nagasaki University, Nagasaki, Japan; ${ }^{2}$ Dept Internal Med, Gunma University, Maebashi, Japan; ${ }^{3}$ Dept Pathol, Nagasaki University, Nagasaki, Japan

The mutant $\mathrm{BRAF}\left(\mathrm{BRAF}^{\mathrm{V} 600 \mathrm{E}}\right)$ is the most prevalent driver mutation in sporadic PTC. Although prenatal expression of $\mathrm{BRAF}^{\mathrm{V} 600 \mathrm{E}}$ in $T g-B R A F^{V 600 E}$ mice had induced thyroid cancer, we have previously shown, by injecting Ad-TgP-Cre into the thyroids of the conditional transgenic $T g\left(L N L-B R A F^{V 600 E}\right)$ mice, that the postnatal expression of $\mathrm{BRAF}^{\mathrm{V} 600 \mathrm{E}}$ by itself was insufficient for thyroid cancer development. We first compared Cre-mediated DNA recombination efficiency between $T g\left(L N L-B R A F^{V 600 E}\right)$ and $L S L-B R A F^{V 600 E}\left(B R A F^{C A}\right)$ mice, and then $B R A F^{C A}$ mice as well as $B R A F^{C A / w} ; P T E N^{f / w}$ and $P T E N^{f / w}$ mice were used for our thyroid cancer model with Ad-TgPCre. $B R A F^{C A}$ mice exhibited more efficient Cre-mediated DNA recombination than $T g\left(L N L-B R A F^{V 600 E}\right)$ mice and, as a result, most of $B R A F^{C A}$ mice injected with Ad-TgP-Cre developed thyroid cancers in 1 year. Histological examination showed follicular or cribriformlike structure with positive Tg staining and no colloid formation. 2/8 tumors also had a component of papillary structure with lower levels of Tg expression, and were accompanied by lung metastases. All AdTgP-Cre injected $B R A F^{C A / w} ; P T E N^{f / w}$ mice developed tumors of predominantly papillary structures and, occasionally, solid pattern with absent $\mathrm{Tg}$ expression; lung metastases were observed in 6/9. Typical nuclear features of human PTC and extrathyroidal invasion were also observed. The percentages of $\mathrm{Ki}^{+} 7^{+}$cells and $\mathrm{TUNEL}^{+}$cells were both higher in tumors of $B R A F^{C A / w} ; P T E N^{f / w}$ mice than $B R A F^{C A / w}$ mice. Conversely, $P T E N^{f / w}$ mice injected with Ad-TgP-Cre did not develop thyroid lesions, while the prenatal PTEN loss in $P T E N^{+/}$mice induced thyroid hyperplasia/adenomas. We established a novel thyroid cancer mouse model in which postnatal expression of $\mathrm{BRAF}^{\mathrm{V} 600 \mathrm{E}}$ alone under the physiological TSH levels induces metastatic PTC. Simultaneous PTEN loss tends to promote tumor growth, de-differentiation and metastasis. Additionally, the significance of $\mathrm{BRAF}^{\mathrm{V} 600 \mathrm{E}}$ expression and PTEN loss differs between the prenatal versus postnatal periods in terms of tumorigenicity, and prenatal loss is critical for the tumorigenic effect of PTEN.

\section{Poster 75}

Thyroid Cancer Thursday Poster Basic

MICRORNA CLUSTER MIR-17-92 PROMOTES THYROID FOLLICULAR CELLS LOSS OF DIFFERENTIATION AND MIGRATION

C.S. Fuziwara, K.C. Saito, E.T. Kimura

Cell and developmental Biology, Institute of Biomedical Sciences, University of Sao Paulo, Sao Paulo, Sao Paulo, Brazil

BRAF oncogene activation, highly prevalent in papillary thyroid cancer, impairs thyroid cell differentiation and induces microRNA miR-17-92. The miR-17-92 cluster transcribes seven mature miRNAs (miR-17-5p/3p, -18a, -19a, -20a, -19b, -92a) overexpressed in aggressive thyroid carcinoma usually refractory to radioiodine therapy. In this study, we aim to investigate the influence of miR-1792 in thyroid growth and differentiation once understanding thyroid follicular cells loss of differentiation in cancer is an important step to improve thyroid cancer management. We constructed cell lines derived from $\mathrm{PCCl} 3$ that overexpress: 1) the whole cluster miR-17-92; 2) miR-17; 3) miR-19b; 4) miR-20a; 5) miR-92a and 6) miR-19a/ $\mathrm{miR} 20 \mathrm{a} / \mathrm{miR}-19 \mathrm{~b}$. Also, we generated miR-17-92 deficient anaplastic cell line KTC2 using CRISPR/CAS9n. In order to evaluate cell biology, we performed gene and protein expression, cell counting, migration and reporter assays for TGF $\beta$ signaling and Nis promoter. Overexpression of whole cluster led to thyroid differentiation impairment marked by Nis protein reduction and downregulation of Nis, Tpo, Ttf1 and Pax8 mRNAs, while enhanced cell migration. Overexpression of cluster components showed differential effects over Nis protein being miR-17 and miR-19 the most effective in inhibiting Nis. This effect was observed at transcriptional levels by Nis promoter luciferase assay pointing to a role of transcription factor modulation. Indeed, Pax- 8 and Ttf 1 protein levels were also reduced in these cells. Meanwhile, CRISPR/CAS9n guided edition of pre-mir-17 of KTC2 cells led to overall reduction of miR17-92 miRNAs, particularly in miR-17. miR-17-CRISPR-edited clones showed reduced cell counting and migration, while TTF1 and PAX8 proteins were enhanced. Moreover, SMAD4 and TGFBR2 proteins were increased, both are miR-17-92 targets and TGF $\beta$ signaling components, leading to partial recovery of anti-mitogenic response to rTGF $\beta 1$. The effects of miR-17-92 overexpression in the follicular cells as a potent inhibitor iodine metabolism gene-related, enhancing migration and impairing the antimitogenic effect of TGF $\beta$ could contribute to thyroid cancer refractoriness to radioiodine and progression to aggressive behavior.

\section{Poster 76}

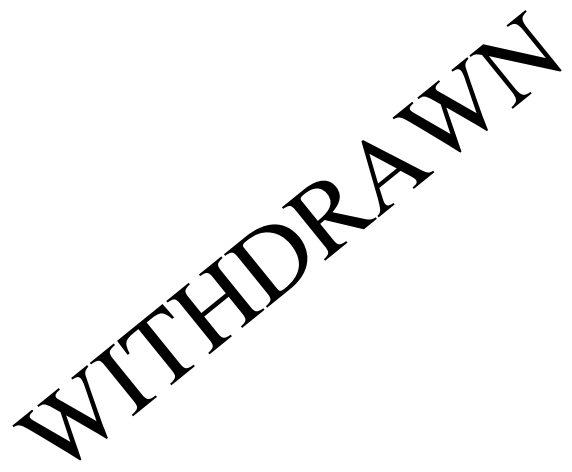




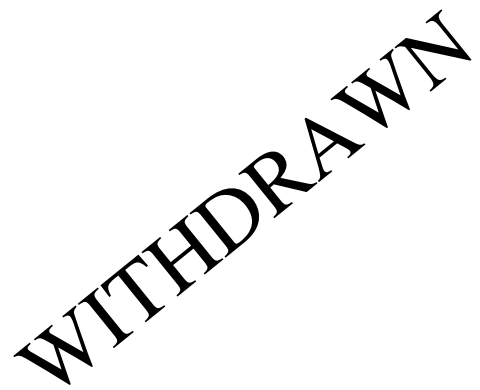

Poster 77

Thyroid Cancer Thursday Poster Basic

CHEMOSENSITIZING EFFECT OF BAICALEIN WITH DOCETAXEL ON 8505C ANAPLASTIC THYROID CANCER CELLS

E. Kim, C. Park, I. Nam-Goong, Y. Kim

Internal Medicine, Ulsan University Hospital, College of Medicine University of Ulsan, Ulsan, Korea (the Republic of)

Cancer cell resistance to chemotherapy is one of the problems for better cancer treatment. Natural bioactive compounds may exert their synergistic effects by different cell cycle pathways, including different ones to those responsible of resistance phenotypes such as transcription factors, membrane receptors, adhesion and structural molecules, cell cycle regulatory components, and apoptosis pathways. Docetaxel is anticancer microtubule-stabilizing agent that severely doselimited because of its adverse side effects, including neutropenia, diarrhea, nausea, and fatigue. Baicaelin (5,6,7-trihydroxyflavone) is a type of flavoid, originally isolated from the roots of Scutellaria baicalensis and Scutellaria lateriflora, and has been reported antiinflammatory and anticancer activities. In this study, we aim to identify a new approach for overcoming ATC chemoresistance by using natural compound, baicalein. $8505 \mathrm{C}$ cells were used. After treatment of baicalein with or without docetaxel, cell survival was investigated by MTT assay. The effect of baicalein in combination with docetaxel on the expression of apoptotic proteins (Bax, Bcl-2, and cleaved caspase-3), invasion/ metastasis-regulating proteins (VEGF, TGF- $\beta, \mathrm{N}$-cadherin, and Ecadherin), and the phosphorylation of Akt/mTOR/ERK MAPK was determined by Western blot, respectively. Baicalein sensitized docetaxel-induced cell death in $8505 \mathrm{C}$ cells through the induction of apoptosis. Along with apoptosis induction, the expression of apoptotic proteins, Bax and cleaved caspase- 3 was synergistically increased in $8505 \mathrm{C}$ cells. Baicalein induced synergic inhibition of the expression of VEGF, TGF- $\beta$, E-cadherin, and N-cadherin proteins with docetaxel in the cells. We also observed an inhibition of the phosphorylation of Akt, mTOR, and ERK MAPK after the co-treatment with baicalein and docetaxel. In our results, baicalein showed a sensitizing effect in the combination with an clinical anticancer drug, docetaxel in anaplastic thyroid cancer, suggesting that baicalein can be an attractive chemosensitizing adjuvant for enhancing the clinical effect of cancer chemotherapy.

\section{Poster 78}

Thyroid Cancer Thursday Poster Basic

\section{LAMB3 MEDIATES METASTATIC TUMOR BEHAVIOR IN PAPILLARY THYROID CANCER VIA REGULATING C-MET/AKT SIGNAL}

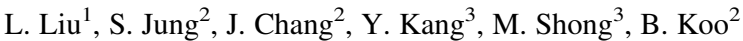

${ }^{1}$ Department of Medical Science, College of Medicine, Chungnam

National University, Daejeon, Korea (the Republic of); ${ }^{2}$ Department of Otolaryngology-Head and Neck Surgery, Research Institute for Medical Science, Chungnam National University College of Medicine, Daejeon, Korea (the Republic of); ${ }^{3}$ Department of Endocrinology and Metabolism, College of Medicine, Chungnam National University College of Medicine, Daejeon, Korea (the Republic of)

The LAMB3 (Laminin subunit beta-3) encodes one of the trimeric proteins constituting Laminin-5, a protein of the extracellular matrix secreted by cultured human keratinocytes. LAMB3 is deeply involved with the invasive and metastatic abilities of several tumor types, including the colon, pancreas, lung, cervical, stomach and prostate. However, the function and mechanism of LAMB3 in thyroid cancer has not yet been studied. By doing the proliferation assay, matrigel invasion assay, western blotting. Our results show that LAMB3 is upregulated in papillary thyroid cancer and LAMB3 suppression reduces cell migration/invasion via EMT-associated proteins (vimentin, slug) down-regulation and MMP-9 inhibition. LAMB3 suppression is also significantly decreases AKT phosphorylation and inhibits the transcription of c-MET, leading to reduction of its activation. These results suggest that LAMB3 leads to tumor invasion through AKT activation induced by the HGF/c-MET axis in papillary thyroid cancer cells. Our findings suggest a novel mechanism of LAMB3 in papillary thyroid cancer and support the synergistic application of LAMB3 inhibition with anti-cancer drugs in cancer therapy.

\section{Poster 79}

\section{Thyroid Cancer Thursday Poster Basic}

\section{MICRORNA EXPRESSION IN THE RISK STRATIFICATION} OF PAPILLARY THYROID CARCINOMA (PTC)

\section{IN AN AUSTRALIAN COHORT}

L.F. Olaya ${ }^{1}$, B. Hookins ${ }^{1}$, J. Malek ${ }^{1}$, A. Gargya ${ }^{2}$, R. Gupta ${ }^{3}$, M. Elliott ${ }^{1,4}$, E.L. Chua ${ }^{1,2}$, S.V. McLennan ${ }^{1,2}$

${ }^{1}$ Sydney Medical School, Charles Perkins Centre, University of Sydney, Sydney, New South Wales, Australia; ${ }^{2}$ Department of Endocrinology, Royal Prince Alfred Hospital, Sydney, New South Wales, Australia; ${ }^{3}$ Department of Diagnostic Oncology and Tissue Pathology, Royal Prince Alfred Hospital, Sydney, New South Wales, Australia; ${ }^{4}$ Sydney Head and Neck Cancer Institute, Royal Prince Alfred Hospital, Sydney, New South Wales, Australia

Some miRNAs are differentially expressed in PTC compared to noncancerous (NC) and multi-nodular goitre (MNG) tissue. The objective of this study was to identify new markers that could be useful in the management and risk stratification of patients diagnosed with PTC. Thyroid tissue was micro-dissected from Formalin Fixed Paraffin Embedded (FFPE) samples ( $n=4 /$ group) from patients with PTC diagnosed as either high $(\mathrm{H})$ or low $(\mathrm{L})$ risk of progression based on the ATA 2009 Risk Stratification System. Tissue from adjacent $\mathrm{NC}$ and tissue from MNG acted as control. miRNAs were extracted and analysed by OpenArray.

By OpenArray, 210 of 748 miRNAs amplified in all four groups, but as the miRNA profile in MNG was different to all other groups only miRNAs expressed in $\mathrm{H}-\mathrm{L}-\mathrm{NC}$ groups were further studied. 54 were expressed in the H-L-NC groups, 11 in the H-L groups, one in the $\mathrm{H}$ group alone and 36 in the $\mathrm{L}$ group. Of these 18 miRNAs, including miRNAs (miR-146b, miR-221 and miR-222), known to be highly expressed in PTC, were chosen for validation by qRT-PCR based on their level of expression and known role in cancer and thyroid function. Results were calculated as $\Delta \Delta \mathrm{Ct}$ relative to miR-16 which was not altered by PTC. Six of the 18 selected miRNAs were differentially expressed between $\mathrm{H}$ and $\mathrm{L}$ and $\mathrm{miR}-146 \mathrm{~b}$, miR-221 and miR-222 were differentially expressed in PTC vs normal tissue. These results in this small cohort confirmed that miR-146b, miR-221 and miR-222 are highly 
expressed in PTC compared to NC, but this expression was not significantly different between the $\mathrm{H}$ and $\mathrm{L}$ risk groups. This study identified 6 novel miRNAs with potential utility for the stratification of patients into those with $\mathrm{H}$ or $\mathrm{L}$ risk of progression of disease. Further studies in larger cohorts are required to validate the utility of these miRNAs.

\section{Poster 80}

Thyroid Cancer Thursday Poster Basic

\section{ASSOCIATION OF MIRNA WITH BRAF VGOOE MUTATION} AND HISTOPATHOLOGY FEATURES IN PAPILLARY THYROID CARCINOMA IN NEW CALEDONIA

L.F. Olaya ${ }^{4}$, T. Lee ${ }^{4}$, T. Lie ${ }^{4}$, C. Woolnough ${ }^{2}$, D. Baron-Dubourdieu, V. Damiens ${ }^{5}$, S.V. McLennan ${ }^{4,1}$, E.L. Chua ${ }^{4,3}$

${ }^{1}$ Department of Endocrinology, Royal Prince Alfred Hospital, Sydney, New South Wales, Australia; ${ }^{2}$ Chemical Pathology, Royal Prince Alfred Hospital, Sydney, New South Wales, Australia; ${ }^{3}$ Department of Endocrinology, Royal Prince Alfred Hospital, Sydney, New South Wales, Australia; ${ }^{4}$ Sydney Medical School, Charles Perkins Centre, University of Sydney, Sydney, New South Wales, Australia; ${ }^{5}$ Anatomie Pathologique, Nouméa, New Caledonia

Some miRNAs have been previously described to be de-regulated in Papillary Thyroid Cancer (PTC), and some are highly expressed in those with BRAF mutation. New Caledonia (NC), a French territory in the Pacific, has the highest incidence of thyroid cancer ${ }^{(1)}$. Our group have previously shown BRAF V600E prevalence was $64 \%$ in this population and this mutation was significantly more common in multifocal bilateral tumours ${ }^{(2)}$. The objective of this study is to analyse the expression of miRNAs in this cohort, the relationship with BRAF V600E status and histopathology features. Twenty samples were randomly selected from the Formalin Fixed Paraffin Embedded (FFPE) PTC tumour tissue obtained from Laboratoire d'Anatomie et Cytopathologie, Nouméa that was used for our previous study. Ten were $\operatorname{BRAF}(+)$ and 10 were $\operatorname{BRAF}(-)$. Four of the $10 \mathrm{BRAF}(+)$ were multifocal bilateral. Micro-dissected cancer tissues were used for miRNA extraction. The expression of three miRNAs known to be highly expressed in PTC (miR-146b, miR-221 and miR-222) were measured by qRT-PCR. Results were calculated by the $\Delta \Delta \mathrm{Ct}$ method using a reference sample and cel miR-39 as an exogenous control. Pathological data were obtained from histopathology reports and patients' medical records. The relative expression of the three miRNAs was not significantly different between the BRAF(+) and BRAF(-) samples. Of the BRAF(+) group, there was a trend toward a decrease in relative expression of the three miRNAs in the multifocal bilateral group. Interestingly in this small NC cohort, despite the higher prevalence of $\mathrm{BRAF}(+)$, we show no difference in the relative expression of these miRNAs. Furthermore, each of these miRNAs tends to be decreased in those with multifocal lesions. The lower expression of these non-coding RNAs may affect post transcriptional regulation and indicate more aggressive tumours in these individuals. There is a need to continue these studies with larger cohorts to gain a more complete picture and draw stronger comparisons in the NC population.

\section{Poster 81}

Thyroid Cancer Thursday Poster Translational

GROWTH ARREST BY SYNERGISTIC EFFECTS OF BET BROMODOMAIN AND MEK-ERK INHIBITORS IN ANAPLASTIC THYROID CANCER

X. Zhu, E. Holmsen, S. Cheng

Gene Regulation Section, LMB, CCR, NCI, NIH, Bethesda, MD

Anaplastic thyroid cancer (ATC) is an aggressive malignancy with limited treatment. Over-activation of the MEK-ERK pathway often drives ATC progression. Recent studies showed that ATC exhibits complex molecular aberrations, including epigenetic alterations in tumors, that render single-targeted therapeutics ineffective. The bromodomain and extra-terminal domain (BET) proteins interact with acetylated histones on the chromatin to globally impact gene transcription. We recently showed that a BET bromodomain inhibitor, JQ1, is effective for ATC in both xenograft and genetically engineered mouse models through preferential suppression of oncogenic MYC transcription and its downstream signaling to inhibit tumor growth. Here we tested whether combined treatment with a MEK-ERK inhibitor, trametinib, and JQ1 could be more effective than singletargeted treatment in 2 human ATC cell lines, THJ-11T and THJ-16. Cell-based studies showed that either trametinib or JQ1 alone partially inhibited cell proliferation, while the combination totally blocked proliferation. Molecular analysis demonstrated that trametinib and JQ1 acted synergistically to suppress the MYC expression at the mRNA and protein levels, leading to the decreased cell cycle regulators (e.g., p-Rb, E2F3) and increased pro-apoptotic proteins (e.g., BIM, cleaved caspase 3) to corroboratively block tumor cell proliferation. In mouse xenograft studies, trametinib or JQ1 alone only partially inhibited tumor growth (66\%-68\% for THJ-16T-induced tumors), but the combined treatment led to $90 \%$ inhibition of THJ-16T-induced tumor growth. The virtually total inhibition of tumor growth was mediated by synergistic actions of trametinib and JQ1 on the inhibition of MYC expression at the protein level, leading to increased pro-apoptotic BIM to arrest tumor growth. Our studies showed that combined treatment of MEK-ERK and BET inhibitors was more effective than single target treatment in blocking tumor growth. Moreover, the synergistic suppression of the $M Y C$ transcription via trametinib-induced sensitization of JQ1 actions suggested that epigenetic modifications on the chromatin could provide novel treatment opportunities for ATC.

\section{Poster 82}

Thyroid Cancer Thursday Poster Translational A SELECTIVE CYCLIN-DEPENDENT KINASE 4/6 INHIBITOR, RIBOCICLIB (LEE011) INHIBITS CELL PROLIFERATION AND INDUCES APOPTOSIS IN AGGRESSIVE THYROID CANCER

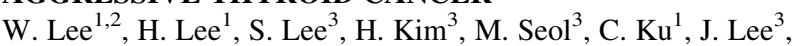
Y. Jo ${ }^{1}$, E. Lee ${ }^{1,2}$

${ }^{1}$ Division of Endocrinology and Metabolism, Department of Internal Medicine, Yonsei University College of Medicine, Seoul, Korea (the Republic of); ${ }^{2}$ Brain Korea 21 PLUS Project for Medical Science, Yonsei University, Seoul, Korea (the Republic of); ${ }^{3}$ Division of Endocrinology and Metabolism, Department of Surgery, Yonsei University College of Medicine, Seoul, Korea (the Republic of)

The retinoblastoma protein (RB)-E2F1 pathway is an important mechanism of cell-cycle control, and deregulation of this pathway is one of the key factors contributing to tumorigenesis. Cyclin-dependent kinases (CDKs) and Cyclin D have also known to increase in aggressive thyroid cancer. However, there has been no study to investigate the effect of a selective cyclin-dependent kinase 4/6 inhibitor, Ribociclib (LEE011), in thyroid cancer. Therefore, we aimed to investigate the effect of LEE011 on thyroid cancer cells. LEE011 was provided by Novartis pharmaceuticals. We performed database analysis using The Cancer Genome Atlas (TCGA) data, Immunoblotting, qRT-PCR, Immunohistochemical study and flow cytometric analysis. To verify the results, we performed animal experiments using xenograft tumor mice models. Comparing TPM (Transcripts per Million) between normal and tumor mRNAs using the TCGA data, CDK4, Cyclin D1, D2, D3 and E2F1 were significantly higher in papillary thyroid cancer (PTC) than in normal (all $P<0.0001$ ). Performing western blotting and qRT-PCR using thyroid cancer cell lines, we 
found that RB phosphorylation on serine 795 and serine 807/811, cyclin D1 and cyclin D2 is significantly higher in PTC cell lines (TPC1, BCPAP and K1) as well as Anaplastic thyroid cancer (ATC) cell lines (8505C, FRO, CAL62 and C643), compared with normal thyroid epithelial cell lines and other types of thyroid cancer cell lines. LEE011 dose-dependently inhibited RB phosphorylation and also decreased the expressions of its target genes such as FOXM1, Cyclin A1 and Myc in ATC cell lines. Furthermore, LEE011 induced cell cycle arrest in G0G1 phase and cell apoptosis, and inhibited cell proliferation in ATC cell lines. Consistently, Oral administration of LEE011 to the FRO xenograft tumor models significantly inhibited tumor growth with decreased expressions of RB phosphorylation and Ki-67. Taken together, our data show that LEE011 is active in aggressive thyroid cancer and support the rationale for clinical development of the CDK4/ 6 inhibitor as a therapy for patients with this disease.

\section{Poster 83}

Thyroid Cancer Thursday Poster Translational

\section{DIGITAL PCR IS A POWERFUL TECHNIQUE TO DETECT BRAF MUTATION IN PATIENTS WITH THYROID CANCER}

K. Jensen ${ }^{1}$, A. Patel ${ }^{1}$, J. Costello ${ }^{1}$, J. Klubo-Gwiezdzinska ${ }^{2}$, A. Bikas ${ }^{3}$, K.D. Burman ${ }^{3}$, V. Vasko ${ }^{1}$

${ }^{1}$ Pediatrics, USUHS, Bethesda, MD; ${ }^{2} \mathrm{NIH}$ NIDDK, Bethesda, MD; ${ }^{3} \mathrm{GU}$, Washington, DC

Digital PCR (dPCR) incorporates exceptional sensitivity with low DNA template requirement. dPCR allows accurate quantification of mutant alleles and was shown to improve mutation analysis in patients with melanomas, pancreatic and colon cancers. Our aim was to determine the utility of dPCR for detection and quantification of BRAFV600 mutations in DNA extracted from patients with thyroid tumors.

DNA was extracted by magnetic bead technology from thyroid cancer cell lines (BCPAP and FTC133), cytological (6 cases), surgical (10 cases) and human plasma samples (10 cases). dPCR was performed using a QuantStudio 3D dPCR platform. QuantStudio software was used for relative and quantitative data analysis.

BRAFV600 mutant alleles were detected in BCPAP, but only wild type BRAF was present in FTC133 cells. In serial dilution experiments, dPCR, but not Sanger sequencing, allowed detection of mutant copies in a mixture of 9.99 ng of DNA from FTC133 cells with $0.01 \mathrm{ng}$ of DNA from BCPAP cells. The sensitivity for detection of mutant alleles was $0.15 \%$. In cytological and FFPE samples, dPCR results correlated with sequencing data. BRAFV600 was detected in $7 / 16$ cases (3/6 cytological and 4/10 FFPE samples); and the ratio of mutant/wild type alleles ranged from $17.1 \%$ to $75 \%$. In plasma from patients with BRAF-negative primary tumors (7 cases) only wild type BRAF alleles were detected. In plasma from patients with BRAF-positive primary tumors (3 cases), mutant alleles were detected in 2 cases, and only wild type alleles were detected in the remaining sample. Results of dPCR were generated within 6 hours.

dPCR performs sensitive, accurate and rapid analysis of BRAFV600. This method is useful for: (1) analysis of cytological samples (especially with limited cellularity); (2) examination of thyroid surgical specimens, and; (3) analysis of cell-free DNA extracted from the blood of patients with thyroid cancer.

\section{Poster 84}

Thyroid Cancer Thursday Poster Translational

\section{ALTERED METALLOPROTEINASES IN AGGRESSIVE HURTHLE CELL CARCINOMAS}

M.D. Williams, M.E. Cabanillas, M. Zafereo, G.J. Cote, B. Liu UT MD Anderson Cancer Center, Houston, TX
Hurthle cell carcinomas (HCC) are distinct tumors derived from the thyroid follicular cells notable for an abundance of mitochondria. There is limited knowledge regarding pathways altered in HCC or biomarkers of aggressiveness. As matrix metalloproteases (MMPs) are matrix-degrading enzymes that are pivotal in cancer progression and have been implicated in local tumor invasion, angiogenesis and distant metastases, the status of MMPs was evaluated in HCCs with metastatic disease. Transcriptional gene expression was performed by RNA-seq from fresh-frozen tissue on a cohort of 4 normal thyroid tissues and 15 Hurthle cell carcinomas (HCC) with metastases ( 3 with positive lymph nodes and 12 with distant metastases. Comparative gene expression analysis for genes associated with metalloproteinases were evaluated between HCC and normal controls for genes with significant (p-value $<0.05$ ) $\log 2$ fold change and pathways were visualized by Ingenuity analysis (Qiagen, Redwood City, CA). Eight MMPs reached significant increased transcription in HCC over controls, for which four (MMPs 7, 9, 11 and 14) showed a based mean expression over 70 copies (range 71-1703). MMP14 had the highest copy number in this group. Fold change ranged from 1.6 (MMP14) to 3.0-3.6 in MMPs 7, 9, and 11. TIMP3 an inhibitor of MMPs showed a -1.6 fold decrease in HCC over normal tissues. Ingenuity analysis placed "inhibition of MMP" pathway as a top altered canonical pathway (p-value $=0.00003)$. Downstream genes including TGFB1 and SERPINA were also notably activated ( $1.9 \& 2.6$ fold respectively). MMP 9 and 11 have also been associated with increased TGFB 1 in the literature and secondary suppression of T- lymphocyte reactions. MMPs are transcriptionally dysregulated in aggressive HCC; Specifically MMPs which have been associated with cancer cell invasion, angiogenesis, and immune evasion. Reversing MMP overexpression through drug targeting is under investigation and may also allow for translation in HCC.

\section{Poster 85}

Thyroid Cancer Thursday Poster Translational

EXPANDED WHOLE-EXOME SEQUENCING IN A FAMILY WITH SUSPECTED NONMEDULLARY FAMILIAR THYROID CANCER

E.U. Lima $^{1,2}$, F.O. Rego ${ }^{3}$, P. Galante ${ }^{3}$, F. Koyama ${ }^{3}$, A. Camargo ${ }^{3}$, G. Medeiros-Neto ${ }^{2}$, I.G. Rubió ${ }^{4,2}$

${ }^{1}$ Postgraduate program in structural and functional biology, Universidade Federal de São Paulo, São Paulo, São Paulo/SP, Brazil; ${ }^{2}$ Laboratório de Ciências Moleculares da Tireoide (LCMT), Universidade Federal de São Paulo, São Paulo, São Paulo, Brazil; ${ }^{3}$ Centro de Oncologia Molecular, Instituto Sírio-Libanês de Ensino e Pesquisa, São Paulo, São Paulo, Brazil; ${ }^{4}$ Ciências Biológicas, Universidade Federal de São Paulo, São Paulo, São Paulo, Brazil

Approximately $90 \%$ of thyroid cancers originate from follicular cells are called Non-medullary thyroid cancer (NMTC). Despite most of the cases are sporadic, familial or hereditary forms (FNMTC) represent 3$6 \%$ of the cases. Several candidate chromosomal loci (TCO, fPTC/ PRN, FTEN, NMTC1, MNG1, 6q22, 8q24) and genes of susceptibility (TERT, NKX2-1, SRGAP1, FOXE1 and HABP2) have been reported, but the genetic causes for FNMTC remain largely unknown.

Objectives: To identify genetic alterations involved in the predisposition to FNMTC in a Brazilian kindred through Whole-Exome Sequencing (WES).

Methods: The kindred had 3 patients with papillary thyoid cancer (PTC) and 6 with multinodular goiter (MNG). Expanded WES was performed in the blood DNA from four patients DNA (2 PTC, 2 MNG) as well as in DNA from two MNG tissues using SureSelectXT Human All Exon V6+UTR Capture Library kit in Illumina NextSeq 500. The single nucleotide variants (SNVs) and insertion/deletion 
(indels) selection criteria were: present in the genomic sequences of the four patients or in both MNG tissue, non-synonymous, located in exons, 3'UTR and 5'UTR, not present in the exome of 30 brazilian controls individuals, absent in databases and being damaging through SIFT, Polyphen2, MutationAssessor, Mutation Taster, FATHMM programs. Interactions and involved pathways were analysed with STRING, GO, KEGG, DAVID databases. No variants (SNVs, indels) in loci and genes previously associated with FNMTC were found. New SNVs in CYTH2, LILRA1 and PIM1 genes were identified however did not segregate with the disease in this kindred. Other new genomic SNVs in KIR2DL4, MDH2, SMCHD1, EPPK1, PDE4DIP and PCDHA9 genes are on investigations. No somatic variants were identified as strong candidates for association with the disease. With the preliminary data it was not yet possible to identify one or a set of candidate genes for the FNMTC predisposition in this kindred. Further analysis and validation of new variants are beeing caried out.

\section{Poster 86}

Thyroid Cancer Thursday Poster Case Report

LEUKOCYTOSIS ASSOCIATED WITH ANAPLASTIC
THYROID CANCER HAS AN ESPECIALLY POOR
PROGNOSIS. A CASE REPORT AND SERIES FROM
PUBLISHED LITERATURE

N. Chandhok, H. Deshpande

Medical Oncology, Yale Cancer Center, New Haven, CT

Anaplastic thyroid cancer (ATC) has a poor prognosis with median survival rates estimated at around 5 months and $20 \%$ one year survival. It is important to identify the few patients who are likely to have a long term survival, as well as those who have a prognosis of a few weeks or less, to allow optimal access to aggressive management or hospice care. We describe a case of a patient with ATC associated with leukocytosis and compared the survival with reports of similar patients from the literature.

A 54 year old man was noted to have paresthesia in his left hand in January 2017. Physical exam was unrevealing. An MRI of the cervical spine demonstrated an expansile lesion replacing the marrow in C7, T1, C6 as well as an enlarged thyroid gland. A CT scan of the chest abdomen and pelvis confirmed the thyroid enlargement, lymphadenopathy in the mediastinum and multiple pulmonary nodules. A fine needle aspirate of the left thyroid confirmed high grade carcinoma. A decompressive cervical laminectomy was performed on $2 / 17 / 17$. He received concurrent chemoradiotherapy from $3 / 28 / 17$ $-5 / 15 / 17,70$ cGy with weekly doxorubicin, $15 \mathrm{mg} / \mathrm{m} 2$. He was noted to have leukocytosis $20.5 \times 10-9$ cells/ 1 on $3 / 24 / 17$. An extensive work up revealed no evidence of infection. He received empiric antibiotics. His leukocytosis increased to $43.2 \times 10-9$ cells/l on 5/15/ 17 and $90.2 \times 10-9$ cells/1 on 5/25/17. CT scans of the chest abdomen and pelvis demonstrated progressive metastatic disease. He expired on $5 / 28 / 17$.

This represents the $4^{\text {th }}$ published case of leukocytosis associated with anaplastic thyroid carcinoma. From the time of leukocytosis greater than $20 \times 10-9$ cells/1, the survival was 62 days and was only 13 days from a level of $43 \times 10-9$ cells/l. This correlates well with other published reports where survivals ranged from 26 days with a leukocytosis of $30.9 \times 10-9$ cells/ 1 to 3 days associated with a white blood cell count of $51 \times 10-9$ cells/1. In these cases, leukocytosis was associated with elevated colony stimulating factor levels. Leukocytosis in the absence of an infectious cause represents an especially poor prognosis despite aggressive treatment in patients with high grade or anaplastic thyroid cancers.

\section{Poster 87}

Thyroid Cancer Thursday Poster Case Report

ACCELERATED PROGRESSION OF METASTATIC DISEASE AFTER THERAPEUTIC OR DIAGNOSTIC TSH STIMULATION: A CASE SERIES

J. Chiang, Z. Quandt, S. Patzek, C. Liu

Medicine, Division of Diabetes, Endocrine, and Metabolism,

University of California, San Francisco, San Francisco, CA

TSH stimulation is important in the management of differentiated thyroid cancer and is required for radioactive iodine treatment (RAI). Stimulated thyroglobulin ( $\mathrm{Tg}$ ) is the most sensitive test in detecting recurrences, and stimulated PET-CT has been used to detect additional RAI negative metastases. While Tg is expected to rise during TSH stimulation, worsening or rapid progression after TSH stimulation is very unusual. We report 3 cases of progression soon after TSH stimulation, 2 after RAI and 1 after stimulated PET-CT. Case 1: 58 year-old man with papillary thyroid cancer (PTC), tall cell variant (pT3N1bMx), received 50mCi of I-131 after recombinant human TSH (rhTSH) stimulation at another hospital with thyroid bed uptake. Tg was $4.7 \mathrm{ug} / \mathrm{L}$ at RAI. After RAI, Tg was 8.21, 27.7, 105, $539.8 \mathrm{ug} / \mathrm{L}$ at $1,5,8$ and 10 months, respectively. PET-CT showed a large hypermetabolic sternal metastasis.

Case 2: 77 year-old man with PTC (pT3N1bMx) received $153 \mathrm{mCi}$ of I-131 after rhTSH with thyroid bed uptake. Tg was $1.1 \mathrm{ug} / \mathrm{L}$ at 2 months post-operatively and $2.5 \mathrm{ug} / \mathrm{L}$ at RAI (3 months post-op). After RAI, Tg was 2.9, 9.7, $28.7 \mathrm{ug} / \mathrm{L}$ at 2, 5, and 11 months, respectively. PET-CT showed progressive hypermetabolic pulmonary metastases.

Case 3: 70 year-old man with widely invasive follicular thyroid cancer (pT3NxM1). He received $190 \mathrm{mCi}$ of I-131 after rhTSH with metastatic uptake in the sternum and lungs. Prior to RAI, Tg was $449.6 \mathrm{ug} / \mathrm{L}$. After RAI, Tg was between 200 to $300 \mathrm{ug} / \mathrm{L}$ for 6 months. Tg doubled 2 months after rhTSH stimulated PET-CT scan ordered by another physician and rose to $1899 \mathrm{ug} / \mathrm{L}$ in another 4 months. Follow up PET-CT showed progressive hypermetabolic metastases. The timing and speed of $\mathrm{Tg}$ rising after TSH stimulation with structural progression in these cases raise the concern that TSH stimulation may potentially lead to progression in some patients. These three patients had aggressive histologies and RAI refractory metastases, and all received rhTSH. These cases serve as a reminder that diagnostic TSH stimulation should be used judiciously and illustrate the importance of understanding the genomic bases of these tumors to allow for effective individualized treatment.

\section{Poster 88}

Thyroid Cancer Thursday Poster Case Report

\section{SHORT TERM MAPK INHIBITOR TREATMENT INCREASES RADIOIODINE UPTAKE IN THYROID CANCER LUNG METASTASES}

K. Lithgow ${ }^{1}$, P. Grundy ${ }^{1}$, S. Ghaznavi ${ }^{1}$, C.J. Symonds ${ }^{1}$, D. Van Nostrand ${ }^{2}$, R. Paschke ${ }^{1}$

${ }^{1}$ Internal Medicine, University of Calgary, Calgary, Alberta, Canada; ${ }^{2}$ Nuclear Medicine, Georgetown University School of Medicine, Washington, DC

Metastatic thyroid cancer that is resistant to radioactive iodine (RI) is associated with poor prognosis compared to RI avid disease. In a clinical trial the MAPK inhibitor selumetinib produced clinically meaningful improvements in RI uptake in $8 / 20$ patients with RI resistant metastatic thyroid cancer. A 29-year-old female with a history of Hodgkin lymphoma diagnosed in 2008 received treatment with chemotherapy, involved field radiation, and bleomycin. In November 
2013 she underwent total thyroidectomy with partial neck dissection and upper mediastinal dissection for a $4.5 \mathrm{~cm}$ papillary thyroid carcinoma with extrathyroidal extension, positive surgical margins, and vascular invasion. Molecular analysis was negative for RAS and BRAF mutations and PAX8 PPRg and RET/PTC rearrangements. 2 months later, $100 \mathrm{mCi}$ of RI was administered. 12 months from her initial surgery, recurrent disease was found in the neck. Complete neck dissection was performed, revealing multiple metastatic lymph nodes. PET CT 9 months later showed multiple new lung nodules, and subsequently a further $100 \mathrm{mCi}$ of RI was given. Follow-up over the next 14 months demonstrated progression of lung metastases, and rising thyroglobulin $(\mathrm{Tg})$ from $48.9 \mathrm{ug} / \mathrm{L}$ to $136.6 \mathrm{ug} / \mathrm{L}$; features suggesting RI resistant disease or underdosing of RI. The patient subsequently received a 4-week course of selumetinib and dosimetry with I-124 and I-131 followed by treatment with $325 \mathrm{mCi}$ of RI (reduced from the maximum tolerated dose of $375 \mathrm{mCi}$ due to previous lung radiation and bleomycin). 28 days after treatment start with selumetinib Tg increased from $136.6 \mathrm{ug} / \mathrm{L}$ to $5538 \mathrm{ug} / \mathrm{L}$. She experienced minor dermatologic side effects with exacerbation of facial acne and rash on the face and chest. Post treatment I-131 scan demonstrated marked uptake in both lungs, bilateral cervical lymph node chains, and thyroid resection bed. Pulmonary function tests were stable immediately pre and post treatment. Tg levels since the last RI therapy have been declining, and ultrasound has shown stability or regression of cervical lymph nodes. Our patient has shown a promising initial response to this MAPK inhibitor induced increased radioiodine uptake without any significant toxic effects.

\section{Poster 89}

Thyroid Cancer Thursday Poster Case Report

\section{DON'T FORGET THE THYROID: CONCURRENCE OF PARATHYROID ADENOMA WITH MULTIFOCAL PAPILLARY THYROID CARCINOMA}

V. Alaigh, B. Tendler

Internal Medicine Residency, University of Connecticut, Stamford, CT

A component of pre-operative work-up for surgical intervention for hyperparathyroidism is neck ultrasound for discovery and assessment of a parathyroid adenoma. However, ultrasound also serves the purpose to look for additional lesions. Concurrent thyroid lesions with parathyroid adenomas are a rare finding, especially in patients without familial syndromes. The following is a case of a patient who, upon evaluation for hyperparathyroidism, was also found to have multiple thyroid lesions. The patient is a 61-year-old lady with a prior history of Vitamin D deficiency who presented for evaluation of asymptomatic hypercalcemia with a serum calcium level of $10.8 \mathrm{mg} /$ $\mathrm{dL}$. She does not have any family history of familiar calcium disorders. Additionally, multiple endocrine neoplasia syndromes were excluded. The patient had an elevated parathyroid hormone level of $293 \mathrm{pg} / \mathrm{mL}$ as well as a parathyroid scan consistent with adenoma. She was initially scheduled for neck exploration surgery for treatment of her hyperparathyroidism at an outside institution. Prior to surgery, she underwent a thyroid ultrasound which incidentally revealed multiple nodules in the right lobe and isthmus of the thyroid. Fine-needle aspiration biopsy confirmed multifocal papillary thyroid carcinoma including a $1.5-\mathrm{cm}$ follicular variant lesion in the right lobe with infiltrative growth and suspected vascular invasion as well as a $1.1-\mathrm{cm}$ classic variant lesion in the right lobe with suspected lymphatic invasion. The patient's surgery was revised to include a total thyroidectomy. This case highlights a relatively unusual presentation of both hyperparathyroidism in the presence of a parathyroid adenoma with subsequent discovery of a multifocal papillary thyroid carcinoma. A review of literature has shown it is uncommon to see a parathyroid adenoma with concurrent thyroid cancer especially in a patient with no familial history of parathyroid disorders or multiple endocrine neoplasia syndromes. A link between parathyroid and thyroid tumors is currently a rising topic in endocrinologyThe case emphasizes the importance of pre-operative imaging and comprehensive thyroid assessment in patients with hyperparathyroidism to prevent repeat surgery.

\section{Poster 90}

Thyroid Cancer Thursday Poster Case Report

\section{UPPER RESPIRATORY INFECTION AS PRESENTATION} FOR ANAPLASTIC THYROID CANCER

\section{L.E. Arzeno ${ }^{1}$, O. Mahmoud ${ }^{1}$, E. Nunez ${ }^{2}$}

${ }^{1}$ Internal Medicine, Morristown Medical Center, Morristown, NJ;

${ }^{2}$ Diabetes and Endocrinology, Morristown Medical Center,

Morristown, NJ

Cough is a common symptom, and when combined with fever and chills the diagnosis of malignancy is low in your differential especially without risk factors and/or history. Although there is a broad differential associated with these symptoms, anaplastic thyroid cancer (ATC) will be low in the differential if present at all. ATC affects approximately 1-2 people per million, and without risk factors is rarely suspected as the cause of non-specific upper respiratory infection. Here we describe a case of a 67-year-old healthy woman who presented with subjective fevers, cough, and chills, noted to have a right-sided neck mass thought to be a swollen lymph node, initially treated with a course of antibiotics. The patient's symptoms did not resolve and her neck mass continued to enlarge, resulting in hoarseness and dysphagia. She presented to the Emergency Department here weeks later. A computed tomography (CT) scan of the neck done revealed a right-sided thyroid mass measuring $6.1 \times 3.6 \times 6.4 \mathrm{~cm}$, and extending into the chest cavity. Imaging for staging revealed diffuse lymphadenopathy, nodularity, and heterogeneity concerning for lung and liver metastasis. A fine needle aspiration of the neck mass was consistent with adenocarcinoma. Given the patient's history of secondhand smoke exposure it was unclear if the patient had primary lung adenocarcinoma with metastasis to the thyroid and liver or if it was primary thyroid cancer with metastasis to lung and liver. A core needle biopsy, from the neck mass revealed a poorly differentiated carcinoma consistent with ATC. The patient was offered palliative chemotherapy and radiation but expired within 2 weeks of her cytological diagnosis. ATC is a rare, rapidly evolving, highly aggressive malignancy that has nearly always spread at the time of initial diagnosis. As such, early detection is paramount in improving patient outcomes. This case highlights the importance of physical examination, including that of the thyroid in patients with respiratory symptoms. Furthermore, when a rapidly evolving neck mass is identified, aggresive forms of thyroid malignancies should be considered.

\section{Poster 91 \\ Thyroid Cancer Thursday Poster Case Report \\ WEAKNESS AND APHASIA AS A PRESENTATION OF HURTHLE CELL CARCINOMA OF THE THYROID L.E. Arzeno, K. Capaccione}

Internal Medicine, Morristown Medical Center, Morristown, NJ

Thyroid cancer is a common malignancy and is divided into several common subtypes: however, rare variants also exist, creating a clinical challenge for practitioners managing these variants. Hurthle cell carcinoma is a very rare type of follicular thyroid cancer representing only 3-5 percent of all thyroid cancers. Although the incidence of malignant potential varies in the literature, approximately $1 / 3$ of these 
tumors metastasize. Here, we present a rare case of Hurthle cell carcinoma which failed multiple systemic therapeutic agents, and ultimately metastasized to multiple sites including the brain, leading to the development of neurological symptoms. The patient was an $80-$ year-old female with a history of a $1.8 \mathrm{~cm}$ Hurthle cell carcinoma who underwent total thyroidectomy and radioactive iodine treatment in 2009, who presented to our hospital with right-sided weakness for 2 days. The patient reported onset of progressive right upper and lower extremity weakness, which progressed to slurred speech and then aphasia. An initial Computed Tomography (CT) showed multiple lucent intra- and extra-cranial lesions with soft tissue components, which were confirmed on subsequent Magnetic Resonance Imaging (MRI). The patient was started on steroids, which improved her symptoms almost to resolution. She was evaluated by oncology for possible referral to radiation oncology, however neurosurgery evaluation was recommended instead. The patient was discharged on a steroid tapper with the recommendation to follow up as an outpatient. Here we present a case of an aggressive malignant Hurthle cell carcinoma that failed multiple medical treatments. Hurthle cell has been shown to frequently metastasize to places such as lymph nodes and bone. This case was unique for its metastases to the inner part of the calvarium with brain lesions producing neurological symptoms. Although Hurthle Cell carcinoma has generally not been classified as an aggressive malignancy, this case demonstrates the disease's very aggressive potential with multiple metastatic sites.

\section{Poster 92}

Thyroid Cancer Thursday Poster Case Report

A CASE REPORT ILLUSTRATING THE USEFULNESS OF MOLECULAR MARKER IN ECTOPIC THYROID TISSUE N.R. Amatya ${ }^{1}$, V. Piziak ${ }^{1}$, G. Chune ${ }^{3}$, S. Challagulla ${ }^{1}$, L. Lopez ${ }^{2}$, N. Tachamo

${ }^{1}$ Endocrinology, Texas A\&M Baylor Scott \& White, Belton, TX;

${ }^{2}$ Anatomic Pathology, Texas A\&M Baylor Scott \& White, Temple, TX; ${ }^{3}$ Endocrinology, Olin E. Teague Veterans' Medical Center,

Temple, TX; ${ }^{4}$ Reading Hospital and Medical Center,

West Reading, PA

Molecular profiling is not routinely done in most FNA biopsies of thyroid nodules, and management is usually dictated by histological diagnosis. We present a challenging case with initial normal histology of thyroid and biopsied lung nodule but with molecular mutation in both. A 68 year old female was referred for evaluation of incidental thyroid nodules seen in CT chest in work up for dyspnea. It showed left thyroid nodule with mass effect and right tracheal deviation and left lower lobe pulmonary nodule with smaller bilateral pulmonary nodules. PET CT was negative for hypermetabolic activity. Thyroid USG showed heterogeneous $3.6 \mathrm{~cm}$ right and $4.8 \mathrm{~cm}$ complex left thyroid nodules. She denied symptoms of compression or of hypo/ hyperthyroidism, history of radiation or family history of thyroid cancer. She had thyromegaly with bilateral palpable nodules without lymphadenopathy.

USG guided FNA biopsy of both thyroid nodules were reported as benign follicular nodules. She had elective total thyroidectomy. Surgical histology was consistent with multinodular goiter with adenomatous nodules. Biopsy of the lung nodule showed thyroid tissue positive for TTF1 and thyroglobulin. Molecular genetics revealed negative BRAF and KRAS mutation. However, both thyroid tissues in the lung and thyroid nodules were positive for NRAS mutation in codon 61. Based on these findings, the left lung nodule is consistent with metastatic follicular thyroid carcinoma (FTC). With plans of subsequent I-131 ablation of remnant thyroid tissue, she underwent Video-assisted thoracoscopic lower lobe wedge resection and mediastinal lymph node dissection. We were then able to make a microscopic diagnosis of metastatic FTC with 2 foci of $1.2 \mathrm{~cm}$ and $0.4 \mathrm{~cm}$ in the lung. Metastatic FTC with positive molecular marker and negative histology has not been reported. Even in the presence of normal thyroid gland, especially with ectopic thyroid tissue, thyroid cancer should be considered as a differential diagnosis and molecular profiling may be crucial to substantiate the diagnosis as in our case. Management is surgical resection of tumor and metastases followed by I-131 ablation and TSH suppression. Monitoring for recurrence is according to ATA guidelines.

\section{Poster 93}

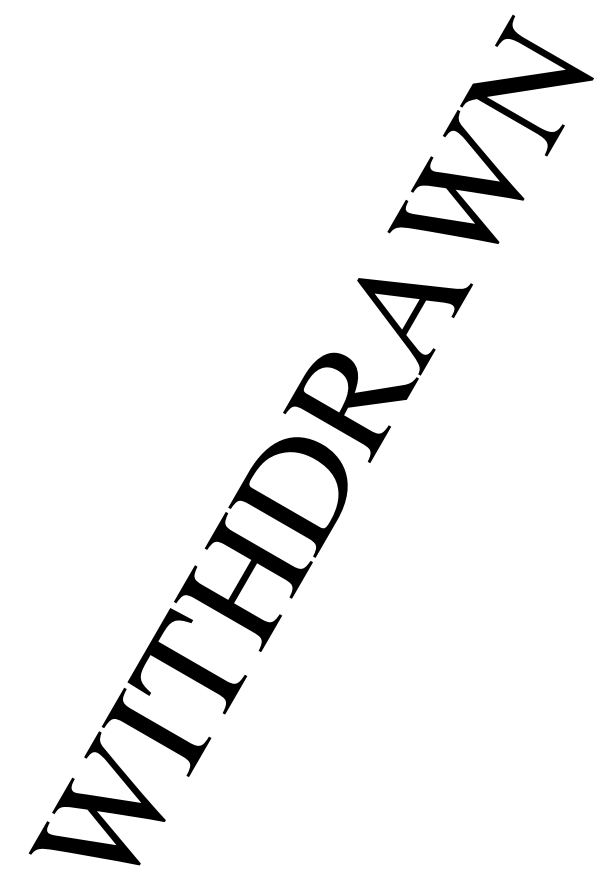

\section{Poster 94}

Thyroid Cancer Thursday Poster Case Report

METASTASIS IN THYROID GLAND DERIVATED FROM DIFFERENT ORIGINS

A. Orlandi, A.G. Puscar, G. Frascaroli

Endocrinology, Hospital Teodoro Alvarez, Buenos Aires, Buenos

Aires, Argentina 
The thyroid is a rare site for metastasis (MTS). The porpouse of this presentation is comunicate 3 cases of thyroid MTS derivated of other organs. Case 1: $\$ 41$ year old with thyrotoxicosis, goiter with compressive symptoms. Left mastectomy for breast carcinoma was done in 2009. Ultrasonography:thyroid gland enlargement, without nodular image and bilateral cervical nodes whit suspicious characteristics. FNA results of thyroid and lymph nodes were compatible with undifferentiated carcinoma. Needle wash out(-) for Tg. She was sent to thyroidectomy. Histology confirm MTS of mammary carcinoma by morphology and immunohistochemistry GCDFP 15(+) RE(+) $10 \%, \mathrm{RP}(-), \mathrm{CK} 7(+), \mathrm{CK} 20(-)$. Case 2: 966 years old with a nodular goiter. Left nephrectomy for clear cell renal carcinoma in 2007 and left mastectomy for breast cancer in 2008 were done. Thyroid ultrasound showed nodules with suspicious features in right lobe and isthmus. FNA: Atypical epithelial cells with hyperchromatic nuclei and clear cytoplasm arranged in groups. Immunostaining: CK7 (+), Tg (-), GCDFP15 (-), CD10(+), compatible with MTS of renal carcinoma of clear cells. Case 3: 054 years old with thyroid enlargement and multiple cervical lymph nodes. Serology: (-) for infectious diseases. Tumoral markers(-). MRI:multiple images at brain, protuberance and cerebellum compatible with MTS due to unknown disease. CT-Scan:adenomegalic conglomerates in cervical, supraclavicular and mediastinum regions. Nodular image of $13 \mathrm{~mm}$ in thyroid right lobe. Nodular image of $14 \mathrm{~mm}$ in apical region of upper lobe of left lung. Focal lesions in liver, pancreas and retroperitoneum compatible with metastatic disease. FNA of right and left thyroid lobes and bilateral nodes: atypical cell proliferation. Immunostaining of lymph node biopsy (+) for CK AE1/AE3, CK17 patched, TTF-1, Chromogranin, CD56 and EMA.(-) for Vimentin, Calcitonin, Tg, CK19, P53. Compatible with MTS of small and intermediate cell lung carcinoma (Oat cell). Thyroid nodules in a patient with history of malignancy can pose a diagnostic challengeThyroid lesions in patients with history of malignancy, regardless the time elapsed since initial diagnosis, recurrence or malignant tumor progression should be considered until proven otherwise.

\section{Poster 95}

Thyroid Cancer Thursday Poster Clinical

PRACTICAL INITIAL RISK STRATIFICATION BASED ON LYMPH NODE METASTASES IN PEDIATRIC DIFFERENTIATED THYROID CANCER

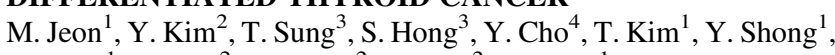
W. Kim ${ }^{1}$, S. Kim ${ }^{2}$, J. Chung ${ }^{2}$, T. Kim ${ }^{2}$, W. Kim ${ }^{1}$

${ }^{1}$ Internal Medicine, Asan Medical Center, Seoul, Seoul, Korea (the Republic of); ${ }^{2}$ Samsung Medical Center, Seoul, Korea (the Republic of); ${ }^{3}$ Surgery, Asan Medical Center, Seoul, Korea (the Republic of); ${ }^{4}$ Gyeongsang National University School of Medicine, Jinju, Korea (the Republic of)

Data on the risk stratification of pediatric differentiated thyroid cancer (DTC) remain scarce. We aimed to evaluate the risk factors of structural persistent/recurrent disease and propose an initial risk stratification system in pediatric DTC patients. This retrospective cohort study included 203 patients from two tertiary referral centers in Korea. We classified the extent of cervical lymph node (LN) metastasis based on the location or number of metastatic LNs. During a median follow-up duration of 5.5 years, structural persistent/recurrent disease was observed in 51 patients $(25 \%)$, including 22 $(11 \%)$ with distant metastases. The presence of extrathyroidal extension (ETE) and lateral cervical LN metastases or $>5$ metastatic LNs were independent risk factors for structural persistent/recurrent disease. The presence of bilateral lateral cervical LN metastases or $>10$ metastatic LNs were independent risk factors for distant metastasis. A total of 67 (33\%), $72(35 \%)$, and 64 (32\%) patients were classified into the low-risk, intermediate-risk, and high-risk groups, respectively, based on the presence of ETE and the extent of cervical LN metastases. Compared to the low-risk group, the intermediateand high-risk groups had a significantly greater risk of structural persistent/recurrent disease (hazard ratio [HR] 7.32, $p$-value $=0.008$ and HR 24.28, $p$-value $<0.001$, respectively). Our initial risk stratification system based on the presence of ETE and the extent of cervical LN metastasis is useful for predicting the clinical outcomes of pediatric DTC patients. Our findings could facilitate the practical use of a risk stratification system.

\section{Poster 96}

Thyroid Cancer Thursday Poster Clinical

RE-VISITING THE ATA 2015 SONOGRAPHIC GUIDELINES: WHO ARE WE MISSING? K. Gong ${ }^{1}$, D. Chan Chun Kong ${ }^{2}$, M. Roskies ${ }^{2}$, R. Payne ${ }^{2}$ ${ }^{1}$ Faculty of Medicine, McGill University, Montreal, Quebec, Canada; ${ }^{2}$ Department of Otolaryngology, McGill University, Montreal, Quebec, Canada

The American Thyroid Association published revised guidelines in 2015 on the management of adults with thyroid nodules. One of the key changes made in this most recent revision concerns the decision to perform FNA based on sonographic findings and the size of the nodule. The overall effect of these changes results in fewer nodules requiring biopsy. Given the imperfect sensitivities of sonographic characteristics for predicting malignancies, this study was conducted to determine if the use of the described sonographic patterns alone would result in overlooked thyroid cancers, especially malignancies with aggressive characteristics measuring between $1-1.5 \mathrm{~cm}$. Patients $(n=2083)$ with thyroid nodules who underwent total or subtotal/hemi thyroidectomy with or without neck dissection by a single surgeon between 2006 and 2016 were retrospectively enrolled. Demographic information and nodule characteristics were collected for all patients. Ultrasonography and final pathology reports were reviewed for patients with thyroid nodules between the sizes of $1-1.5 \mathrm{~cm}(n=155)$. $45 \%(n=70)$ of patients with nodules between $1-1.5 \mathrm{~cm}$ had "low suspicion" nodules according to ultrasound. 47 of these nodules contained malignancies on final histopathological examination, $100 \%$ of which were papillary. $21 \%(n=10)$ of these malignant nodules demonstrated extrathyroidal extension and $34 \%(n=16)$ were associated with positive lymph nodes. Reliance on sonographic patterns alone could result in missed cancer diagnoses in patients with thyroid nodules measuring between $1-1.5 \mathrm{~cm}$. Moreover, a significant portion of these malignancies may be associated with aggressive features. The effect of this finding on long-term outcomes is unclear.

\section{Poster 97}

Thyroid Cancer Thursday Poster Clinical

\section{SECOND PRIMARY MALIGNANCY RISK IN THYROID} CANCER SURVIVORS TREATED WITH RADIOACTIVE IODINE: AN UPDATED SYSTEMATIC REVIEW AND META-ANALYSIS

C. $\mathrm{Yu}^{1}$, O. Saeed ${ }^{1}$, A.S. Goldberg ${ }^{4}$, S. Farooq ${ }^{5}$, R. Fazelzad ${ }^{2}$, D.P. Goldstein ${ }^{6}$, R.W. Tsang ${ }^{2}$, J.D. Brierley ${ }^{2}$, S. Ezzat ${ }^{2}$, L. Thabane ${ }^{3}$, A.M. Sawka ${ }^{6}$

${ }^{1}$ University of Toronto, Toronto, Ontario, Canada; ${ }^{2}$ Princess Margaret Cancer Centre, Toronto, Ontario, Canada; ${ }^{3}$ McMaster University, Hamilton, Ontario, Canada; ${ }^{4}$ Sunnybrook Health Sciences Centre, Toronto, Ontario, Canada; ${ }^{5}$ University Health Network, Toronto, Ontario, Canada; ${ }^{6}$ University Health Network and University of Toronto, Toronto, Ontario, Canada 
In surgically treated differentiated thyroid cancer (TC), radioactive iodine (RAI) may be utilized for ablation of the thyroid remnant as adjuvant treatment, or for treatment of persistent disease. A common concern of patients and healthcare providers is whether RAI treatment is associated with an increased risk of second primary malignancy (SPM). We performed a systematic review and meta-analysis of the published English language literature, examining whether the risk of SPM was different in TC patients treated with RAI, compared to those not treated with RAI. We searched 6 electronic databases (2008-2016), supplemented with a hand search. There was independent duplicate execution of reviews of citations and full-text papers, data abstraction, and study quality appraisal. Random effects meta-analyses were performed. We examined 2797 citations and 84 full-text papers. We included 14 studies (3 systematic reviews and 11 original studies). Two systematic reviews, suggested increased SPM risk after RAI, with methodologic limitations acknowledged in the primary data. A published meta-analysis of 6 studies suggested slightly reduced breast cancer risk with RAI. In our updated metaanalysis, the risk difference (RD) for SPM in RAI group was -4.9 SPM cases per 1000 TC patients, 95\% CI -26.5 to $16.8, \mathrm{p}=0.658$, $\mathrm{n}=63,908$ from 9 studies). The RD for breast cancer after RAI was -6.1 per 1000 TC patients, $95 \% \mathrm{CI}-16.4$ to $4.2, \mathrm{p}=0.246, \mathrm{n}=66,400$ from 6 studies). The RD for leukemia (any type) was 2 per 10,000 TC patients, 95\% CI 0 to $4.0, \mathrm{p}=0.0134, \mathrm{n}=264,179$ from 3 studies). There was significant heterogeneity in the pooled analyses for any SPM and breast cancer. There is conflicting observational evidence on SPM risk of TC patients with RAI, underscoring the need for more large, long-term, prospective studies. Nevertheless, this data suggests lack of a clinically significant increased risk of SPM with RAI.

\section{Poster 98}

Thyroid Cancer Thursday Poster Clinical

MOLECULAR TESTING AND ITS CLINICAL SIGNIFICANCE REGARDING MALIGNANT THYROID NODULES

M. Sasson ${ }^{1}$, E. Kay-Rivest ${ }^{2}$, M. Hier ${ }^{3}$, V. Forest ${ }^{3,4}$, R. Payne ${ }^{3,4}$

${ }^{1}$ Medicine, McGill University, Montreal, Quebec, Canada;

${ }^{2}$ Otolaryngology, McGill University, Montreal, Quebec, Canada;

${ }^{3}$ Otolaryngology - Head and Neck Surgery, Jewish General Hospital, Montreal, Quebec, Canada; ${ }^{4}$ Otolaryngology - Head and Neck Surgery, McGill University Health Center, Montreal, Quebec, Canada

The incidence of thyroid nodules is increasing among patients in North America. In 2015 the American Thyroid Association published revised guidelines regarding the management of thyroid nodules. This included recommendations on molecular testing. This study sets out to understand the potential role of molecular testing in the preoperative period on patients with thyroid nodules $<4 \mathrm{~cm}$ categorized as either Bethesda V or Bethesda VI. A case-control study was conducted with 36 cases and 144 controls from the McGill University Teaching Hospitals. Patients with thyroid nodules $<4 \mathrm{~cm}$, categorized as Bethesda V or Bethesda VI, and underwent molecular testing using the ThyGenX panel (BRAF, HRAS, NRAS, KRAS, PIK3CA, RET/PTC1, RET/PTC3, PAX8/PPARy) were incuded. Cases were defined as patients with molecular testing, and controls were defined as patients who did not undergo molecular testing. Patients were evaluated based on whether they had the ideal surgery, based on what the surgical team would have recommended if the final pathology result would have been known prior to surgery. For example, a total thyroidectomy for patients with extrathyroidal extension or lymph node metastasis, or hemi/subtotal thyroidectomy for patients with a nodule $<4 \mathrm{~cm}$ without extrathyroidal extension or lymph node metastasis. Patients in the case group were more likely to have the ideal surgery when compared to patients in the control group, $86.11 \pm 11.87 \%$ vs $53.55 \pm 7.93 \%$ (p-value 0.0034 ). Amongst cases, the mean number of lymph nodes removed was $6.0 \pm 3.2$, and the mean number of metastatic lymph nodes was $2.9 \pm 2.4$. Amongst controls, the mean amount of lymph nodes removed was $2.3 \pm 0.8$, and the mean amount of metastatic lymph nodes was $0.04 \pm 0.09$. In this study, patients with molecular testing had the ideal surgery $86 \%$ of the time compared with patients without molecular testing (53\%). This study demonstrates that molecular testing may have a role in the management of Bethesda V and Bethesda VI thyroid nodules that measure less than $4 \mathrm{~cm}$.

\section{Poster 99}

Thyroid Cancer Thursday Poster Clinical

\section{THE RISK OF THYROID CANCER AND OBESITY:} A NATIONWIDE POPULATION-BASED STUDY USING THE KOREA NATIONAL HEALTH INSURANCE CORPORATION COHORT DATABASE

H. Son ${ }^{1,2}$

${ }^{1}$ Surgery, Catholic Kwandong University International St. Mary's Hospital, Incheon, Seo-gu, Korea (the Republic of); ${ }^{2}$ Surgery, Graduate School, Yonsei University College of Medicine, Seoul, Korea (the Republic of)

In recent years, the incidence of thyroid cancer and obesity has increased rapidly worldwide. Many studies have been conducted on the relationship between thyroid cancer and obesity; however, the potential mechanisms are not well understood, and few studies have been performed in Asia. This study aimed to identify the correlation between the risk of thyroid cancer and obesity in the Korean population based on a large cohort of data. We analyzed clinical data from a total of 351,402 individuals (males: 181,709, females: 169,693) aged over 20 years who received medical examinations arranged by the national insurance program from 2003 to 2008. Newly diagnosed thyroid cancer was identified using insurance claims data. The median follow-up duration was 7.01 years. A total of 3,308 individuals $(0.94 \%)$ developed thyroid cancer during the study period. The mean body mass index (BMI) of the subjects was $23.6 \pm 3.2 \mathrm{~kg} / \mathrm{m}^{2}$ (males, $24 \pm 3$; females, $23.1 \pm 3.3$ ). The risk of thyroid cancer was higher with increasing BMI in both men and women. Hazard ratios $(95 \%$ confidence interval) for obese $\left(25-29.9 \mathrm{~kg} / \mathrm{m}^{2}\right)$ and extremely obese $\left(\geq 30 \mathrm{~kg} / \mathrm{m}^{2}\right)$ groups were $1.23(1.13-1.344)$ and $1.26(1.023-1.504)$, respectively, compared to the normal group. In particular, there was a more significant correlation between increased risk of thyroid cancer and increased BMI in the male group ( $\beta=1.07$ vs 1.03 ). In the young age group (aged $\leq 45$ ), there was a significant correlation between increased risk for thyroid cancer as BMI increased ( $\beta=1.03$ vs 1.02). Higher BMI is correlated to the risk of thyroid cancer as an independent risk factor. These results suggest that obesity is associated with biological effects that contribute to the development of thyroid cancer.

\section{Poster 100}

Thyroid Cancer Thursday Poster Clinical

\section{MANAGEMENT OF ADVANCED THYROID CANCER} WITH MEDIASTINAL METASTASIS

Y. Lee, H. Chang, C. Park, S. Kim

Department of Surgery, Yonsei University College of Medicine, Seoul, Korea (the Republic of)

In thyroid cancer, mediastinal lymph node metastasis are extremely rare. But the invasiveness of the disease leads often to significant morbidity and also to frequent cause of death. The aim of this study is 
to evaluate the effectiveness of surgical management and safety of mediastinal lymph node dissection. From October 2000 to November 2016, 195 patients who underwent mediastinal dissection for thyroid cancer metastasis were retrospectively analyzed. Clinical characteristics including extent of surgery, pathologic features and prognosis were reviewed. There were 80 male $(41.0 \%)$ and 115 female $(59.0 \%)$ patients with a mean age of $49.9 \pm 15.1$ years.

Synchronous mediastinal metastasis was found in 118 patients $(60.5 \%)$. The most common thyroid cancer type was papillary thyroid cancer (165 patients, $84.6 \%)$. In 35 patients (18.3\%) there was distant metastasis observed at time of surgery, whereas $13(6.7 \%)$ developed distant metastasis during follow-up. In 46 cases, combined resection of either trachea $(8,4.1 \%)$, lung $(5,2.6 \%)$, major vessel $(12$, $6.2 \%)$ or nerve $(21,10.8 \%)$ was performed. Transcervical approach was the most common (126 cases, 64.6\%), followed by full sternotomy $(40,20.5 \%)$ and partial sternotomy $(24,12.3 \%)$. Median follow-up was 40.6 months $[18.8 ; 64.9]$ with overall survival of $91.3 \%$ of the patients. In $17(8.7 \%)$ patients, disease related death occurred. Recurrence was observed in $47(24.1 \%)$ patients with median time to recurrence of 19 month $[8.4 ; 31.7]$. Aggressive surgical treatment for locally advanced thyroid carcinoma may offer improved local control of disease, prolonged palliation and opportunity for cure in selected patients.

\section{Poster 101}

Thyroid Cancer Thursday Poster Clinical

\section{LONG-TERM RESULTS AND PROGNOSTIC FACTORS IN PATIENTS WITH PAPILLARY THYROID MICROCARCINOMA}

J. Ruan, W. Wang, X. Su, Y. Chen, Y. Ding, Z. Mao, S. Chen, L. Teng

Department of Surgical Oncology, First Affiliated Hospital, Zhejiang University School of Medicine, Hangzhou, Zhejiang, China

The incidence of papillary thyroid microcarcinomas (PTMCs) has increased sharply and the treatment for patients with PTMC is still controversial. Our aim is to identify clinical and pathologic factors that are associated with recurrence and survival, and ultimately to guide therapy for clinically unfavorable PTMCs. This study includes 2693 patients with papillary thyroid cancer, including 1688 patients with PTMC (1688/2693, 62.7\%), who were treated at First Affiliated Hospital of Zhejiang University School of Medicine (Hangzhou, China) from 1997 to 2011. Data points includingage, sex, extrathyroidal extension and lymph nodal metastasis were analyzed. The mean follow-up time was 6.6 years, ranging from 1 to 19.3 years. The 10-year disease-free survival(DFS) rates and over-all survival(OS) rates were calculated to compare the prognosis. Prognostic factors were evaluated by uni- and multi-variate statistical analysis. Patients with PTMCs were less likely to have cervical lymph nodal metastasis and extrathyroidal extension, and earlier clinical stagein comparison with non-PTMC(diameter $>1 \mathrm{~cm}, \mathrm{P}<0.01$ ). The 10 -year DFS rate for patients with PTMC was much higher than that for those with nonPTMC (93.4\% vs $85.6 \%$; $p<0.001)$. In our cohort, no PTMC patient had died of disease (10-year OS rate, $100 \%$ vs $97.9 \%$; $\mathrm{P}<0.001)$. The univariate analysis found aged $<45$ years and cervical lymph node metastasis were two factors that significantly associated with recurrence in PTMCs $(\mathrm{P}<0.05)$, while lymph node metastasis is the only independent risk factor for PTMC recurrence which was identified by multivariate analysis. The current study provides evidence with longterm results and outcomes that papillary thyroid microcarcinoma usually has benign clinical courses with good prognosis. Only those PMTC patients with lymph node metastasis need careful treatment and more frequent follow-up, as these patients may be at an increased risk of recurrence.

\section{Poster 102}

Thyroid Cancer Thursday Poster Clinical

\section{LOCOREGIONAL DISEASE CONTROL AFTER} EXTERNAL BEAM RADIOTHERAPY IN 93 PATIENTS WITH DIFFERENTIATED THYROID CARCINOMA AND PT4 TUMOR STAGE - A SINGLE INSTITUTION EXPERIENCE

N. Besic ${ }^{1}$, M. Dremelj ${ }^{2}$, N. Glumac ${ }^{1}$

${ }^{1}$ Surgical Oncology, Institute of Oncology, Ljubljana, Slovenia;

${ }^{2}$ Radiotherapy, Institute of Oncology, Ljubljana, Slovenia

Locoregional recurrence is common in patients with locally advanced differentiated thyroid carcinoma (DTC). Our aim was to find out the rate of locoregional control of the disease after external beam radiotherapy (EBRT) of the neck and mediastinum in patients with DTC and pT4 tumor. Altogether 93 patients (48 males, 45 females, median age 61 years) with DTC had EBRT of the neck and mediastinum as part of multimodal treatment of pT4 tumor (65 cases pT4a, 28 cases pT4b) from year 1973 to 2015. Data on clinical factors, histopathology and recurrence were collected. Disease-free, disease-specific and overall survival was calculated. Median tumor size was $5 \mathrm{~cm}$ (range 1-30 cm). Out of 93 patients, 24 had distant and 40 regional metastases. A total or near-total thyroidectomy, lobectomy, subtotal thyroidectomy and lymph node dissection was performed in $70 \%, 14 \%, 2 \%$ and $32 \%$ of cases, respectively. Thirteen percent of patients were not treated with surgery. All patients had EBRT and 40 had chemotherapy. Radioiodine (RAI) ablation of thyroid remnant and RAI therapy was applied in $90 \%$ and $41 \%$ of cases, respectively. Follicular, papillary and Hürthle cell carcinoma was diagnosed in 27, 54 and 12 cases, respectively. Follow-up period was 6 to 308 (median 61) months. Recurrence was diagnosed in 29/65 patients without a persistent disease: locoregional and distant in 16 and 13 cases, respectively. Five-year and ten-year disease-free survival rate was $64 \%$ and $48 \%$, respectively. Median survival of patients with follicular, papillary and Hürthle cell carcinoma was 62,131 and 240 months, respectively. By the end of the study, 46 patients were still alive (29 no evidence of the disease, 17 alive with the disease), 34 patients died of thyroid carcinoma (29 of distant metastases, 1 of locoregional disease and 4 of locoregional disease and distant metastases), 10 patients died of causes unrelated to the primary disease, while 3 patients were lost to follow-up. The majority of patients with DTC and pT4 tumors who were treated with EBRT of the neck and mediastinum region as part of multimodal treatment have long-lasting locoregional control of the disease.

\section{Poster 103}

Thyroid Cancer Thursday Poster Clinical

\section{[F-18] FLUORODEOXYGLUCOSE POSITRON EMISSION} TOMOGRAPHY ACCURATELY PREDICTS BENIGN PATHOLOGY IN THYROID NODULES WITH INDETERMINATE FINDINGS ON FINE-NEEDLE ASPIRATION

M. McGilvray ${ }^{1}$, F. Dehdashti ${ }^{2}$, A.L. Traugott ${ }^{4}$, K. Trinkaus ${ }^{1}$, M.S. Cohen ${ }^{5}$, E.A. Fialkowski ${ }^{6}$, F. Quayle ${ }^{7}$, H. Hussain ${ }^{1}$, R. Davila ${ }^{8}$, L. Ylagan', J.F. Moley ${ }^{1,3}$

${ }^{1}$ Department of Surgery, Washington University School of Medicine, St Louis, MO; ${ }^{2}$ Department of Radiology, Washington University School of Medicine, St Louis, MO; ${ }^{3}$ Surgical Service, St. Louis Veterans Affairs Medical Center, John Cochran Division, St Louis, MO; ${ }^{4}$ The Ohio State University, Columbus, $\mathrm{OH} ;{ }^{5}$ University of Michigan Medical School, Ann Arbor, MI; ${ }^{6}$ Oregon Health \& Science University, Portland, OR; ${ }^{7}$ Mercy Surgical Associates, Durango, CO; ${ }^{8}$ Missouri Baptist Medical Center, St Louis, MO; ${ }^{9}$ Roswell Park Cancer Institute, Buffalo, NY 
Thyroid nodules with indeterminate fine-needle aspiration (FNA) results present a diagnostic dilemma - while a minority of patients will have malignant disease, the majority will have benign disease and will not benefit from surgery. This large, government-funded prospective study correlates the results of pre-operative [F-18] fluorodeoxyglucose positron emission tomography (FDG-PET) with pathology of excised indeterminate thyroid nodules. Patients were $\geq 18$ years of age with a solitary or dominant FNA-indeterminate thyroid nodule (follicular lesion) $\geq 1 \mathrm{~cm}$ by ultrasound (US). All patients underwent FDG-PET imaging. FDG uptake was blindly evaluated both qualitatively on an ordinal scale and quantitatively using maximum standardized uptake values $\left(\mathrm{SUV}_{\max }\right)$. Uptake was compared to final pathologic diagnosis after thyroidectomy. Malignant disease was found histopathologically in $17 \%$ (16/94) of patients. An US size cut-off of $\geq 1.5 \mathrm{~cm}$ was found to exclude all excised lesions $<1 \mathrm{~cm}$ on final pathology measurement (the approximate size threshold for lesions detectable by PET) via sensitivity analysis. When compared to histopathologic diagnosis in patients with lesions $\geq 1.5 \mathrm{~cm}$ on US, FDG-PET had a $100 \%$ negative predictive value (NPV). These data suggest that FDG-PET may be used to safely and reliably rule out malignancy in patients with FNA-indeterminate thyroid nodules $\geq 1.5 \mathrm{~cm}$ on US. We propose a modified treatment algorithm for FNA-indeterminate thyroid nodules incorporating use of this non-invasive and $100 \%$ sensitive diagnostic tool.

\section{Poster 104}

Thyroid Cancer Thursday Poster Clinical

\section{CYTOPLASMIC EXPRESSION OF GALECTIN-3 CAN DISTINGUISH MALIGNANT FROM BENIGN ENCAPSULATED PAPILLARY-FOLLICULAR THYROID TUMORS}

O. Polyakova ${ }^{1}$, G. Fu ${ }^{1}$, R.S. Chazen ${ }^{1}$, R. Ralhan ${ }^{1,3}$, P.G. Walfish ${ }^{1,2}$ ${ }^{1}$ Alex and Simona Shnaider Research Laboratory in Molecular Oncology, Mount Sinai Hospital, Toronto, Ontario, Canada; ${ }^{2}$ Department of Medicine, Endocrine Division, Mount Sinai Hospital and University of Toronto Medical School, Toronto, Ontario, Canada; ${ }^{3}$ Department of Pathology and Laboratory Medicine, Mount Sinai Hospital, Toronto, Ontario, Canada

Improved discrimination of benign NonInvasive Follicular Thyroid neoplasms with Papillary-like nuclear features (NIFTP) from malignant invasive Encapsulated Follicular Variant of Papillary Thyroid Carcinoma (EFVPTC) by using a molecular biomarker test as an ancillary aid to traditional pathology could provide useful insights into post-surgical management. The aim of this study was to determine whether cytoplasmic and/or nuclear expression of Galectin-3 (Gal-3) could distinguish EFVPTC from NIFTP and other causes of benign tumors. Immunohistochemical analysis of Gal-3 expression was performed using Vectastain ABC system in 174 cases of Formalin-fixed paraffin-embedded (FFPE) sections with four specific diagnostic subgroups- 41 benign nodules, 51 NIFTPs, 45 EFVPTCs, and 37 FVPTCs with coexisting significant lymphocytic thyroiditis and no capsule invasion (LT). Statistical analyses were performed using a 1-way ANOVA and paired 2 -tailed $t$ tests were considered significant for $p<0.05$. Cytoplasmic Gal-3 expression (mean \pm SEM) was significantly increased in EFVPTC $(4.8 \pm 0.24)$ compared to NIFTPs $(2.8 \pm 0.22, \mathrm{p}<0.001)$ and benign neoplasms $(2.1 \pm 0.19, \mathrm{p}<0.001)$ with no significant difference between NIFTPs and benign lesions $(p=0.064)$. The presence of lymphocytic thyroiditis also enhanced cytoplasmic Gal-3 expression in LT (3.8 \pm 0.22$)$. Cytoplasmic Gal-3 expression in EFVPTC had an area under the curve of 0.90 with a sensitivity of $84 \%$, specificity $80 \%$, positive predictive value $83 \%$ and negative predictive value $83 \%$. However, no significant difference in nuclear Gal-3 expression was detected between EFVPTC (1.8 \pm 0.19$)$ and benign nodules $(1.4 \pm 0.17, \mathrm{p}=0.215)$. Our observations have indicated that cytoplasmic expression of Gal-3: i) can distinguish EFVPTC from NIFTP and other benign lesions and ii) suggests that it can serve as an ancillary aid to $\mathrm{H} \& \mathrm{E}$ diagnostic criteria in confirming EFVPTC and NIFTP among FVPTC when there is no LT interference. [PGW is shareholder in Proteocyte Diagnostics Inc. All the other authors $(G F, O P, R C$ and $R R)$ have nothing to disclose.]

\section{Poster 105}

Thyroid Cancer Thursday Poster Clinical

\section{PERCUTANEOUS ULTRASOUND GUIDED} RADIOFREQUENCY ABLATION IS EFFECTIVE AND SAFE FOR TREATING METASTATIC LYMPH NODES IN THE NECK FROM PAPILLARY THYROID CARCINOMA

Y. Luo, Y. Guang, Y. Zhang, M. Zhang, N. Li, J. Tang

Ultrasound, Chinese PLA General Hospital, Beijing, China

Our objective was to assess the effectiveness and safety of ultrasound guided percutaneous radiofrequency ablation (RFA) for nonsurgical treatment of metastatic lymph nodes (LNs) in the neck from papillary thyroid carcinoma (PTC).45 patients (mean age, 41.5 years; range 21-67 years) with previous total thyroidectomy and radioiodine therapy were enrolled in this retrospective study. A total of 71 metastatic LNs in the neck from PTC confirmed by percutaneous biopsy. Contrast-enhanced ultrasound (CEUS) examinations were performed to rapidly evaluate treatment responses before and shortly after ablation. Follow-up consisted of conventional US, CEUS, thyroglobulin (Tg) level at 1, 3, 6, and 12 months and every 6 months thereafter. All 45 patients were successfully treated without immediate or later major complications occurred. With a mean follow-up of $23 \pm 5$ months (range 12-30 months), there were no evidence of recurrence at ablated sites. The mean volume reduction ratio (VRR) was $31.6 \pm 7.4 \%$ (range $20.5-60.5 \%$ ), $47.3 \pm 8.6 \%$ (range 33.6$69.6 \%$ ), $63.7 \pm 11.8 \%$ (range $41.8-95.4 \%$ ), $78.3 \pm 9.7 \%$ (range $53.7-100.0 \%$ ), $88.7 \pm 10.3 \%$ (range $65.4-100.0 \%$ ), $95.2 \pm 4.9 \%$ (range $78.2-100.0 \%$ ) and $97.1 \pm 4.3 \%$ (range $83.7-100.0 \%$ ) at 1,3 , $6,12,18,24$ and 30 months after RFA respectively. Significant differences in the VRR were found between every two follow-up visits $(\mathrm{P}<0.001)$. After RFA, mean serum $\mathrm{Tg}$ level decreased from $11.3 \pm 6.3 \mathrm{ng} / \mathrm{ml}$ (range $0.8-19.4 \mathrm{ng} / \mathrm{ml}$ ) to $1.3 \pm 0.9 \mathrm{ng} / \mathrm{ml}$ (range $0.2-3.9 \mathrm{ng} / \mathrm{ml})$ at the last follow-up visit $(\mathrm{P}<0.001) .46$ metastatic LNs completely disappeared $(46 / 71,64.8 \%)$ and 25 metastatic lymph nodes remained as small scarlike lesions $(25 / 71,35.2 \%)$ at the last follow-up visit. Ultrasound guided percutaneous RFA is a feasible, effective and safe therapy for metastatic LNs in the neck from PTC. This procedure may reduce or delay a large number of highly invasive repeat neck dissections.

\section{Poster 106}

Thyroid Cancer Thursday Poster Clinical

\section{IDENTIFICATION OF ETV6 GENE REARRANGEMENT IN PAPILLARY THYROID CANCER}

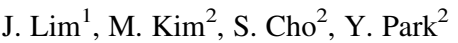

${ }^{1}$ Internal Medicine, National Medical Center, Seoul, Korea (the Republic of ); ${ }^{2}$ Internal Medicine, Seoul National University Hospital, Seoul, Korea (the Republic of)

The reported incidence of BRAF mutation in papillary thyroid cancer (PTC) is around $70-80 \%$ in Korea, showing the highest rate all over the world. On the other hand, the frequency of other genetic mutation 
such as RAS, RET/PTC or PAX8/PPAR $\gamma$ was considered to be relatively low. The purpose of this study was to elucidate the prevalence of ETV6 rearrangement in this BRAF-V600E-prevalent area and its clinical implication

We reviewed 606 BRAF-wild type PTC cases who underwent total thyroidectomy at Seoul National University Hospital between 1997 and 2012. ETV6 rearrangement in the postoperative formalinfixed paraffin-embedded samples were assessed by fluorescence in situ hybridization.

Two of thirty-four cases (5.9\%) had ETV6 gene rearrangement among BRAF-wild type PTC. Both two patients were middle-aged woman, and denied having a history of neck irradiation. Tumor size was 1.2 and $0.9 \mathrm{~cm}$, respectively. Both two cases are classic type of PTC. One patient got radioactive iodine treatment due to microscopic extrathyroidal extension and lymph node metastases. However, both two patients with rearrangement of ETV6 showed no evidence of recurrence or metastases so far. It was impossible to compare the prognosis of cancer between two groups with or without ETV6 rearrangement due to small number of cases.

We report the existence of ETV6 rearrangements in thyroid cancer but cannot show the association with exposure to radiation. The prevalence of ETV6 rearrangement was relatively low in BRAF-V600E-prevalent area, suggesting different etiologic factors in Korea.

\section{Poster 107}

Thyroid Cancer Thursday Poster Clinical

\section{ARE PREGNANCY, PARITY, MENSTRUATION AND BREAST FEEDING A RISK FACTOR FOR THYROID CANCER?}

H. Kim, J. Baek, K. Kim, J. Jung

Internal medicine, Gyeongsang National University Changwon Hospital, Changwon, Korea (the Republic of)

It is unclear that whether reproductive factors, including pregnancy, parity, menstruation and breast feeding, are risk factors for thyroid cancer. The aim of this study is to evaluate whether reproductive factors are risk factors for thyroid cancer. Large-scale nation-wide cross-sectional data were analyzed from a representative sample of the people as part of the Korea National Health and Nutrition Examination Survey V-VI (2010 to 2015). In this study, 38,086 people over the age of 18 were enrolled, and 241 were diagnosed as thyroid cancer. Of the 21,543 female, 210 had thyroid cancer. In the logistic regression analysis, experience of pregnancy, parity, duration of reproductive years (period between menarche to menopause) and breast feeding were independent risk factors for thyroid cancer after adjusted age, BMI and smoking [Odd ratio $(\mathrm{OR})=7.60$ for pregnancy, $\mathrm{p}<0.001$; OR $=3.79$ for parity, $\mathrm{OR}=1.08$ for duration of reproductive years, $\mathrm{P}<0.001$; $\mathrm{P}=0.010 ; \mathrm{OR}=1.74$ for breast feeding, $\mathrm{P}=0.038]$. However, when pregnancy divided into categorical variables, prevalence of thyroid cancer increased only 3 or 4 times of pregnancies, and decreased when numbers of pregnancies were more than 6. Among breast feeding women, total duration of breast feeding and number of breast feeding baby was significantly decreased risk for thyroid cancer $(\mathrm{OR}=0.98$ and $\mathrm{OR}=0.69$, respectively. $\mathrm{P}<0.001)$. In subgroup analysis, there was no significant difference in the prevalence of thyroid cancer among men and never-pregnant women $(0.19 \%$ for men and $0.12 \%$ for nulliparous women, $\mathrm{p}=0.712$ ). Reproductive factors including pregnancy, menstruation and breast feeding have a complex impact on the thyroid cancer. Understanding the effects of reproductive factors on thyroid cancer can present new directions for the development and treatment of thyroid cancer.

\section{Poster 108}

Thyroid Cancer Thursday Poster Clinical

GPR48/LGR4 IS A NEW POTENTIAL TARGET FOR THYROID CARCINOGENESIS BY REGULATING MEK/ ERK AND WNT/ $\beta$-CATENIN PATHWAYS

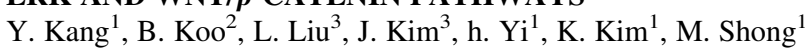

${ }^{1}$ Department of Endocrionology and Metabolism, Chungnam

National University Hospital, Daejeon, Korea (the Republic of);

${ }^{2}$ Department of Otolaryngology-Head and Neck Surgery, Research Institute for Medical Science, Chungnam National University College of Medicine, Daejeon, Korea (the Republic of ); ${ }^{3}$ Department of Medical Science, College of Medicine, Chungnam National University, Daejeon, Korea (the Republic of)

The signaling pathway involving the R-spondins (RSPOs) and GPR48/LGR4 pathway plays crucial roles in development and carcinogenesis. However, the functional implications of GPR48/LGR4 in thyroid epithelial cells and thyroid carcinogenesis remained to be identified. The aim of this work was to investigate the functional role of the RSPOs and GPR48/LGR4 signaling in differentiated papillary thyroid carcinomas (PTCs). Retrospectively, a total of 214 patients who underwent total thyroidectomy and cervical lymph node dissection for PTC at Chungnam National University Hospital reviewed. We compared clinicopathologic features of PTC patients according to the expression levels of RSPOs and GPR48/LGR4. The role of GPR48/LGR4 on cell proliferation and migration was examined in thyroid cancer cell lines. RSPO2, and GPR48/LGR4 were expressed at significantly higher levels in thyroid cancer than in normal controls. Elevated GPR48/LGR4 expression was significantly associated with tumor size $(\mathrm{p}=0.050)$, lymph node metastasis $(p=0.004)$, recurrence $(p=0.037)$, and the BRAFV600E mutation $(\mathrm{p}=0.003)$. Multivariate analysis revealed that high GPR48/LGR4 expression was an independent risk factor for lymph node metastasis $(\mathrm{p}=0.027)$ and the BRAFV600E mutation $(\mathrm{p}=0.009)$ in PTC patients. In vitro assays demonstrated that elevated expression of GPR48/LGR4 promoted proliferation and migration of thyroid cancer cells, whereas downregulation of GPR48/LGR4 decreased proliferation and migration by regulating $\beta$-catenin pathway. Moreover, PTC cells with treatment of MEK inhibitor or RAF inhibitor revealed decrease of the expression of GPR48/LGR4 and induced the downregulation of $\beta$-catenin pathway. In conclusion, this work demonstrated that R-spondin family proteins and their receptor, GPR48/ LGR4, which activated $\mathrm{Wnt} / \beta$-catenin pathway regulated by MAPK/ ERK1/2, are functional in thyroid cancer cells and expressed at higher levels in PTC.

\section{Poster 109}

Thyroid Cancer Thursday Poster Clinical

PROGNOSIS AND PROGNOSTIC FACTORS FOR CANCER MORTALITY IN PATIENTS WITH LYMPH NODE METASTASES FROM PAPILLARY THYROID CARCINOMA AS A FIRST-TIME RECURRENCE

E. Nagai, H. Kamio, Y. Yoshida, E. Noguchi, A. Sakamoto, K. Horiuchi, T. Okamoto

Breast and Endocrine Surgery, Tokyo Women's Medical University, Tokyo, Japan

Little is known about the clinical course of patients with papillary thyroid carcinoma(PTC) who underwent surgery for lymph node metastases as a first time recurrence. A retrospective observational study was conducted. Patients who underwent surgery for lymph node metastases from PTC as a first-time recurrence from April 1981 to December 2014 after curative treatment were enrolled. Those with 
previous history of recurrent disease, who had recurrent tumors other than lymph nodes or distant metastases were excluded. Cause-specific survival (CSS) from re-do surgery for lymph node metastases were estimated using the Kaplan-Meier method. Following patient or tumor characteristics at initial surgery were examined for relative impact on the prognosis after reoperation: gender, age, presence or absence of high-risk features (tumor size larger than $4 \mathrm{~cm}$, palpable lymph nodes, and invasion of primary tumor or metastatic lymph nodes to the adjacent structures) as well as TNM classification. A hundred and twelve patients met the inclusion criteria. Female/male ratio was 69/43. Median (range) age at initial surgery was 54(17-85) years old. Median (range) time from initial treatment to re-do surgery was $3.6(0.2-23)$ years. With a median followup of 6.1 years, the CSS for the entire population was $82 \%$ (95\% CI: 70$90 \%)$ at 10 years after reoperation.

The CSS at 10 years for patients with at least one of the high-risk features and those without ones were 78\% (95\%CI: 64-88\%), 97\% (95\%CI: 78-99\%), respectively. The 10-year CSS for aged $>=45$ years was 73\% (95\%CI: 56-85\%)while that for aged $<45$ years was 94\% (95\%CI: 63-99\%). Prognostic features of PTC at initial surgery were also associated with clinical course after reoperation for lymph node metastases as a first-time recurrence.

\section{Poster 110}

Thyroid Cancer Thursday Poster Clinical

NON-INVASIVE FOLLICULAR TUMOR WITH PAPILLARY-LIKE NUCLEAR FEATURES (NIFTP): A CLINICAL-PATHOLOGICAL STUDY OF 28 CASES WITH FOLLOW UP

N. Al-Brahim ${ }^{1}$, M. Gilani ${ }^{2}$, K. Al-Saleh ${ }^{3}$, F. Al-Hamdan ${ }^{4}$

${ }^{1}$ Pathology, Farwaniya Hospital, Kuwait, Kuwait;

${ }^{2}$ Otorhinolaryngology, Farwaniya Hospital, Kuwait, Kuwait,

Kuwait; ${ }^{3}$ Radiation Oncology, Kuwait Cancer Control Center, Kuwait, Kuwait; ${ }^{4}$ Faculty of Medicine, Kuwait University, Kuwait, Kuwait

Differentiating between aggressive thyroid cancer and indolent ones is an ongoing effort to avoid over treatment. Recently, a study demonstrated that a category of (FVPTC) with certain histological features had an excellent prognosis. Therefore, the study proposed to change the nomenclature of these carcinomas into (NIFTP). However, ATA guidelines committee considered this as a weak recommendation and advised to see more studies. Therefore, we conducted this study in our hospital to see the outcome of these patients. Files of Department of Pathology, Farwaniya Hospital were reviewed for (FVPTC) between 2008 and 2015. The criteria of the recently published index paper were applied in this study including: well circumscribed tumor, follicular growth pattern and nuclear morphology of papillary carcinoma. The exclusion criteria included: papillae in $>1 \%$, psammoma bodies, infiltrative border, tumor necrosis, high mitotic activity and other variants of papillary carcinoma. In cases of multifocal tumors, the previous criteria were applied in all tumors. The treatment and follow up were retrieved from patients' files. Fifty five cases of (FVPTC) were identified. Twenty eight $(51 \%)$ cases fulfilled the inclusion criteria of which $23(82 \%)$ female and 5 males. Age ranged 23-66 years. Nineteen cases (19) were unifocal tumors and (9) cases multifocal; 6 of which associated with papillary microcarcinomas. The tumor sizes ranged 1.2$10.0 \mathrm{~cm}$. Nineteen cases $(68 \%)$ had a documented follow up. Eighteen patients received RAI and (1) did not receive the treatment. RAI doses were $100 \mathrm{mci}$ in 11 patients and $30 \mathrm{mci}$ in 7 patients. The follow up of the patients ranged between 1 and 103 months with an average of 40 months. Eighteen patients (95\%) were alive with no recurrence or systemic metastasis. One patient died due to metastatic breast carcinoma. The data presented herein in concordance with the previous study that the selected histological criteria were able to predict tumor with indolent behavior. $51 \%$ of cases of (FVPTC) were reclassified to
(NFITP) indicating a high percentage of patients could avoid over treatment. Our limitations include having a small number of patients and some patients that lost to followup were expatriates.

\section{Poster 111 \\ Thyroid Cancer Thursday Poster Clinical}

DIFFERENTIATED THYROID CANCER IN CHILDREN: A UK MULTICENTRE REVIEW

K. Lee ${ }^{1}$, M. Taghavi Azar Sharabiani ${ }^{2}$, J. Wadsley ${ }^{3}$, V. Gill ${ }^{4}$, S. Retnabai ${ }^{5}$, G. Genev ${ }^{5}$, L. Moss ${ }^{6}$, K.L. Newbold ${ }^{1}$

${ }^{1}$ Thyroid Unit, The Royal Marsden NHS Foundation Trust, London, United Kingdom; ${ }^{2}$ Statistics, The Royal Marsden NHS Foundation Trust, Sutton, United Kingdom; ${ }^{3}$ Sheffield Teaching Hospitals NHS Foundation Trust, Sheffield, United Kingdom; ${ }^{4}$ Leeds Teaching Hospitals NHS Trust, Leeds, United Kingdom; ${ }^{5}$ The Christie NHS Foundation Trust, Manchester, United Kingdom; ${ }^{6}$ Velindre NHS Trust, Cardiff, United Kingdom

Aim to retrospectively collect data from UK centres that treat paediatric differentiated thyroid cancer (DTC) in order for a better understanding of the presentation and outcomes in this patient populationA retrospective observational study of patients diagnosed with DTC aged $\leq 18$ years old. The study was open to all UK centres; data was received from five. Monte-Carlo simulations were employed to generate background (control) population using age, gender and yearmatched probabilities of survival taken from the Office of National Statistics. Data on 150 patients was collected across five UK centres. Patients received treatment between 1964 and 2016. Mean age at diagnosis was 14.1yrs (3-18yrs). Forty-two (28\%) were male, 108 (72\%) were female. All patients were treated surgically with thyroid lobectomy \pm completion thyroidectomy. 139 (93\%) underwent Iodine-131 ablation with $50(33 \%)$ receiving more than one treatment. $60 \%$ had N1 disease at diagnosis. $11 \%$ had M1 disease at diagnosis.

Mean follow up was 7.5yrs.

At most recent follow up, of 144 known outcomes ( 6 lost to follow up) $104(69 \%)$ have no evidence of disease: thyroglobulin $(\mathrm{Tg})<1$, no structural disease; 28 (19\%) have biochemical residual disease: Tg $>1$ with no structural disease and $9(6 \%)$ have structural disease. Three patients have died of disease (all had brain metastases) at 12.5, 5.5 and 7.5 years post diagnosis.

Female children with DTC had significantly worse survival than their background population $(\mathrm{p}=0.000015)$. In contrast, no statistically significant difference was observed between survivor functions of male patients and of their background population $(\mathrm{p}=0.85)$.

Cumulative radiation activity, age at diagnosis and histology do not predict progression free survival according to multivariate Cox regression model. Despite presence of nodal and metastatic disease at presentation, the UK data confirm an excellent long-term prognosis for children diagnosed with DTC. Prospective data should be collected to understand long-term impact of diagnosis and treatment.

Funded by Thyroid Cancer Support Group Wales Charity

\section{Poster 112 \\ Thyroid Cancer Thursday Poster Clinical \\ IS PROPHYLACTIC CENTRAL-COMPARTMENT NECK DISSECTION NECESSARY FOR THE PATIENTS WITH PAPILLARY THYROID MICROCARCINOMA WITHOUT SUSPICIOUS METASTASIS BY PREOPERATIVE ULTRASONOGRAPHY?}

Y. Kim

Surgery, Chosun University Hospital, Gwangju, Korea (the Republic of) 
Recently, incidence of papillary thyroid microcarcinoma(PTMC) has been increased due to an increase in the detection of subclinical disease such as small and low-risk carcinomas with ultrasonography and fine needle aspiration cytology. However, there is central neck lymph node metastasis in patients with PTMC without clinical evidence of metastasis by preoperative ultrasonography. We tried to identify the predictive factors for central lymph node metastasis in PTMC although there was no clinical evidence of metastasis by preoperative ultrasonography. We analyzed retrospectively 625 patients with PTMC underwent thyroid surgery at Chosun University Hospital from January 2002 to December 2012. Finally, we included 575 patients who had no evidence of lymph node metastasis by preoperative ultrasonography. We reviewed medical records including clinical information and pathologic report. Central lymph node metastasis was found in 81 patients $(14.1 \%)$ among total 575 patients. A lymph node metastasis occurred frequently according to univariate analysis in patients with following factors; more than $0.5 \mathrm{~cm}$ in largest tumor size by preoperative sonography and pathologic reports $(\mathrm{p}=0.048$ and $\mathrm{p}=0.001$, respectively) and lymphovascular invasion $(\mathrm{p}<0.001)$. Multivariate analysis revealed that the gender (Female vs Male), pathologic tumor size $(0.5 \sim 1 \mathrm{~cm}$ vs $<0.5 \mathrm{~cm}$ ) and lymphovascular invasion (Yes vs No) were significant associated factors for lymph node metastasis [Odds ratio $(\mathrm{OR})=0.498$, $95 \%$ confidence interval $(\mathrm{CI})=0.250-0.992, P=0.047 ; \mathrm{OR}=2.450$, $95 \% \mathrm{CI}=1.313-4.570, P=0.005$; and $\mathrm{OR}=24.954,95 \% \mathrm{CI}=2.430$ 256.217, $P=0.007$, respectively]. Male gender, larger tumor size $(\geq 0.5 \mathrm{~cm})$ and lymphovascularinvasion were associated with central neck lymph node metastasis in patients with PTMC without suspicious clinical evidence of node metastasis. We concluded that prophylactic central neck lymph node dissection might be required in these cases of PTMC.

\section{Poster 113 \\ Thyroid Cancer Thursday Poster Clinical \\ METASTASIS TO THE THYROID GLAND FROM CANCER OF THE CERVIX: EXPERIENCE OF A COMPREHENSIVE CANCER CENTER AND REVIEW OF THE LITERATURE} T. Jaber ${ }^{1}$, K. Pandit ${ }^{1,2}$, M.D. Williams ${ }^{3}$, D. Bodurka ${ }^{4}$, M.I. Hu ${ }^{1}$

${ }^{1}$ Endocrine Neoplasia and Hormonal Disorders, The University of Texas MD Anderson Cancer Center, Houston, TX; ${ }^{2}$ Endocrinology, Diabetes and Metabolism, Baylor College of Medicine, Houston, TX; ${ }^{3}$ Pathology, The University of Texas MD Anderson Cancer Center, Houston, TX; ${ }^{4}$ Gynecologic Oncology and Reproductive Medicine, The University of Texas MD Anderson Cancer Center, Houston, TX

Thyroid metastases (mets) represent $1.4-3 \%$ of thyroid malignancies with higher prevalence noted on autopsy. Reported literature suggests cancers of the cervix (CC) rarely metastasize to the thyroid. We describe a case of neuroendocrine carcinoma (NEC) of the cervix (NECC) presenting with thyroid mets, mimicking a medullary thyroid cancer. To evaluate the frequency of such presentations, we reviewed our center's experience and published reports of $\mathrm{CC}$ with thyroid mets. This is a retrospective analysis of patients (pts) with CC metastatic to the thyroid from the tumor registry at MD Anderson Cancer Center (MDA) from 1944-2017. The literature review included search words primary cervical cancer and mets to the thyroid.

A 64-year-old woman presented to MDA for evaluation of a NEC identified by total thyroidectomy for as a $5 \mathrm{~cm}$ right thyroid mass. Pathology revealed high-grade NEC positive for CEA but not for calcitonin by immunohistochemistry. Two weeks postoperatively, serum calcitonin was undetectable and CEA was elevated at $74.7(0-3) \mathrm{ng} / \mathrm{mL}$. Staging scans at MDA revealed widely metastatic disease in the lungs, liver and a $5 \mathrm{~cm}$ cervical mass. The cervix was biopsied identifying a high-grade small cell NECC and chemotherapy was initiated. A review of our tumor registry identified 5 historical pts with $\mathrm{CC}$ and thyroid mets: squamous cell carcinoma of the cervix (SCCC) $(n=4)$, NECC $(n=1)$. Thyroid mets were identified at a median of 1.1 $(0.6-2.7)$ years after diagnosing CC. Thyroid mets were identified in. 4 of the 5 patients at autopsy. A review of the literature identified 5 additional pts with thyroid mets from CC ( 4 with SCCC and 1 with cervical adenocarcinoma) at a median of $2(0.5-12)$ years from CC diagnosis. All 10 patients had widely metastatic disease. Median overall survival (OS) from time of diagnosis of thyroid mets of the 4 patients identified pre-mortem was 6.8 (4-16) months. Thyroid mets from $\mathrm{CC}$ are rare and may be historically underreported given the frequency of identification at autopsy at MDA prior to 1970. The most common subtype represented is SCCC. The presence of thyroid mets is usually indicative of widespread mets and short OS.

\section{Poster 114}

\section{Thyroid Cancer Thursday Poster Clinical}

\section{ROBOTIC TRANSORAL THYROIDECTOMY:} EXPERIENCE OF 100CASES OVER 1YEAR

H. Kim ${ }^{1}$, Y. Chai ${ }^{5}$, G. Dionigi ${ }^{3}$, A. Anuwong ${ }^{4}$, R.P. Tufano ${ }^{2}$, H. $\mathrm{Kim}^{1}$

${ }^{1}$ Breast Endocrine, Korea university, college of medicine, Seoul, Korea (the Republic of); ${ }^{2}$ Department of Otolaryngology - Head and Neck Surgery, The Johns Hopkins University School of Medicine, Baltimore, MD; ${ }^{3}$ Department of Surgical Sciences and Human Morphology, Research Center for Endocrine Surgery, Varese, Italy; ${ }^{4}$ Department of Surgery, Police General Hospital, Faculty of Medicine, Siam University, Bangkok, Thailand; ${ }^{5}$ Department of Surgery, Seoul National University Boramae Medical Center, Seoul, Korea (the Republic of)

Transoral robotic thyroidectomy (TORT) through oral vestibule draws attention recently because it requires minimal flap dissection and postoperative scar disappears in several weeks. In this study, we evaluated surgical outcomes of a single surgeon's initial 100 cases of TORT. From March 2016, to February 2017, 100 patients underwent TORT with the da Vinci surgical system at Korea University Hospital. Patient's clinicopathologic characteristics, operation times, the learning curve, postoperative hospital stays and complications were evaluated. Eight patients underwent total thyroidectomy, one patient underwent bilateral subtotal thyroidectomy, ninety-one patient underwent unilateral thyroid lobectomy. All procedures were successfully performed without conversion. Ninety-five patients $(95 \%)$ had papillary thyroid carcinoma (PTC) and four (4\%) had benign nodules. The mean operation time was $210 \mathrm{~min}$, and the mean hospital stay was 3.1days. The number of retrieved central lymph nodes in the PTC patients was 4.8. There were no reports of transient or permanent vocal cord palsy. There was one postoperative bleeding and bleeding was controlled with endoscopic transoral approach. There were no seroma, chyle leakage and infection. In regard to TORT specific complications, there were one bruising in the zygomatic region, one chin flap perforation, three oral commissure tearing and three skin dimpling. However, no further TORT specific complications were recorded from the $50^{\text {th }}$ cases. There were no paresthesias of the lower lip and the chin from mental nerve injury after the location of intraoral incisions were modified. TORT is feasible and safe for patients with benign or malignant thyroid disease. TORT might be a potential alternative approach for a surgeon who is experienced in remote-access thyroid surgery. 


\section{Poster 115}

Thyroid Cancer Thursday Poster Clinical

SMALL GLANDS IN EXPERIENCED HANDS: EARLY THYROIDECTOMY IN PEDIATRIC PATIENTS WITH MULTIPLE ENDOCRINE NEOPLASIA TYPE 2 (MEN2) OVER 24 YEAR PERIOD

M.A. Romero Arenas ${ }^{1}$, R.L. Bassett ${ }^{3}$, E.G. Grubbs ${ }^{1}$, R. Gagel ${ }^{2}$, S.E. Waguespack ${ }^{2}$, J.E. Lee ${ }^{1}$, N.D. Perrier ${ }^{1}$

${ }^{1}$ Surgical Oncology, UT MD Anderson Cancer Center, Houston, TX; ${ }^{2}$ Endocrine Neoplasias, UT MD Anderson Cancer Center, Houston, TX; ${ }^{3}$ Biostatistics, UT MD Anderson Cancer Center, Houston, TX

Discovery of the RET proto-oncogene as the cause of multiple endocrine neoplasia type 2 (MEN2) revolutionized management of medullary thyroid cancer (MTC) by establishing an indication for early thyroidectomy aimed prevention of MTC. We reviewed institutional databases to identify patients with MEN2 who underwent early thyroidectomy before age 18. Inclusion criteria were: confirmed MEN2 diagnosis, no preoperative sonographic suspicion of MTC, grossly normal thyroid intraoperatively, and no concurrent therapeutic lymphadenectomy. Clinical data were abstracted from the electronic medical record. Descriptive analyses were performed.71 pediatric patients underwent initial thyroidectomy at our institution from 1992-2016. Nine patients were excluded, thus 62 patients underwent early thyroidectomy for MTC prevention. All patients had preoperative genetic testing confirming RET mutations; most had MEN2A (58/62, 93.5\%) and 4/62 (6.5\%) had MEN2B. Median age at thyroidectomy was 7.3 years (3-17.5y). Median time from genetic testing to thyroidectomy was 0.7 years $(0-11.6 y)$. Experienced, senior endocrine surgeons performed most operations (58/ $62,93.5 \%$ ). Pathologic review demonstrated MTC in 25/62 (38.9\%), all were pT1; C-cell hyperplasia 23/62 (37.3\%); and no histological cancer or precursor lesions in $14 / 62(23.7 \%)$. Preoperative calcitonin ranged from undetectable to $33 \mathrm{pg} / \mathrm{ml}$. One (1.6\%) patient had locoregional recurrence prompting lymphadenectomy within 1 year. Nine (14.5\%) patients required post-operative calcium supplementation; none experienced permanent hypocalcemia. There were no transient or permanent recurrent laryngeal nerve injuries documented. One (1.6\%) patient had a postoperative pneumonia requiring hospitalization. Median follow-up was 2.4 years (0-19.1y). Early thyroidectomy was preventative in the majority of pediatric MEN2 patients, and those diagnosed with MTC had early-stage disease. Early recurrence rate was low. A minority required post-operative calcium supplementation; and none long-term. There were no documented recurrent laryngeal nerve injuries. Early thyroidectomy for pediatric patients can be safely performed with low morbidity in experienced hands.

\section{Poster 116 \\ Thyroid Cancer Thursday Poster Clinical 25 YEARS OF THERAPY FOR PAPILLARY THYROID CANCER \\ B.C. James ${ }^{1}$, L. Timsina ${ }^{1}$, G. Welch ${ }^{2}$, D. Haggstrom ${ }^{3}$ \\ ${ }^{1}$ Surgery, Indiana University, Indianapolis, IN; ${ }^{2}$ Medicine, The Dartmouth Institute, Lebanon, NH; ${ }^{3}$ Medicine, Indiana University, Indianapolis, IN}

The substantial rise in papillary thyroid cancer incidence over the past 25 years mostly reflects increased detection of small, nonlethal cancers - not an increase in the true underlying burden of disease. Although multiple studies suggest that less aggressive surgical intervention is possible, the treatment strategies currently employed in practice have not been well described. We used the National Cancer Institute Surveillance, Epidemiology, and End Results (SEER) 9 database to determine time trends in surgical intervention for papillary thyroid cancer diagnosed between 1998 and 2014. From 1998 to 2014, the incidence of papillary thyroid cancer has tripled: from 3.5 [CI 3.3-3.8] to 13.5 [13.01-13.9] per 100,000. The rate of total thyroidectomy increased from 4.35 [4.10-4.61] to 11.35 [CI 10.97-11.74] per 100,000 with an annual percent change of 6.34 $(\mathrm{p}<0.001)$. The rate of thyroid lobectomy increased from $0.85[0.74$ 0.97 ] to 1.45 [1.32-1.59] per 100,000 with an annual percent change of $3.66(\mathrm{p}<0.001)$. Despite studies suggesting that a more conservative surgical treatment for papillary thyroid cancer may result in a similar prognosis, the proportion of patients treated conservatively has not increased over the past 25 years. Further reserach is needed to determine whether this reflects patient preference or whether surgeons are not offering the option of a more conservative approach to the surgical treatment of thyroid cancer.

\section{Poster 117}

Thyroid Cancer Thursday Poster Clinical

TSH VARIABILITY IN PATIENTS AFFECTED BY

DIFFERENTIATED THYROID CANCER TREATED WITH LEVOTHYROXINE LIQUID SOLUTION OR TABLET

I. Pirola, E. Gandossi, C. Casella, D. Lombardi, F. Marini, A. Cristiano, A. Delbarba, E. Muraca, B. Agosti, C. Cappelli University of Brescia, Brescia, Italy

Recent guidelines from the American Thyroid Association (ATA) indicate that in many patients affected by differentiated thyroid cancer (DTC) the serum TSH should be maintained between 0.1 and $0.5 \mathrm{mU} / \mathrm{L}$ taking into account the ATA risk score and the risks of TSH suppression. Considering this small therapeutic window is therefore important to reduce the TSH variability during the L-T4 treatment. The purpose of this study was to evaluate the TSH variability of DTC patients treated with liquid L-T4 formulation or in tablet form. Patients were eligible if: a) submitted to a total-thyroidectomy and ${ }^{131}$ I remnant ablation for DTC in our Institution; b) low-risk patients according to ATA guidelines 2009. The 102 elegible patients ( 80 females and 22 males, aged 57.7 \pm 11.2 years) were randomized $(1: 1)$ to receive treatment of hypothyroidism with liquid L-T4 or tablet form since the day after radiometabolic treatment and with a standard dosage of $1.9 \mathrm{mcg} / \mathrm{kg} / \mathrm{day}$. Liquid L-T4 was taken with breakfast, whereas tablet 60 minutes prior to food ingestion. TSH values were established firstly from 8 to 12 months after the radio-ablation, than again after a further 12 months. All participants had to maintain the same dosage of LT4 therapy for the entire period of the study. A significant increase in TSH values (median) was observed in patients taking tablets [TSH (min-max): $0.28(0.10-0.45)$ vs. 0.34 (0.01$0.78), \mathrm{mU} / \mathrm{L}, p=0.041]$ as compared to those taking liquid formulation [TSH (min-max): $0.28(0.10-0.47)$ vs. $0.30(0.10-0.55), \mathrm{mU} / \mathrm{L}$, $p=0.345]$. In detail, $8 / 51(15.7 \%)$ subjects on tablets vs. $2 / 51$ (3.9\%) on liquid formulation did not reach TSH values on target $[\mathrm{p}<0.05$, OR 4.9 , $95 \%$ CI 1.0-24.7]. No changes in body weight were observed among our patients, both for those ingesting tablets $(p=0.569)$ and for those on liquid LT4 formulation $(p=0.968)$ from recruitmentThe use of LT4 liquid formulation, compared to tablets, resulted in a significantly higher number of DTC patients maintaining TSH values in range according to the ATA risk score with an attendant reduction in TSH variability.

\section{Poster 118}

Thyroid Cancer Thursday Poster Clinical

FINE NEEDLE ASPIRATION OF THYROID NODULES : DIAGNOSTIC YIELD AND IMPACT OF THE AMERICAN THYROID ASSOCIATION GUIDELINES IN A TERTIARY MEDICAL CENTER

C. Guertin, M. Desrochers, C. Pelletier, N. Van Rossum,

D. Bellabarba, R. Temmar, M. Massicotte

Endocrinology, University of Sherbrooke, Sherbrooke, Quebec, Canada 
Prevalence of thyroid nodules is $4-7 \%$ in the general population and close to $50 \%$ in patients over 60 years old, but only 5-10\% are malignant. Fine needle aspiration (FNA) is the recommended method of investigation. The 2015 American Thyroid Association (ATA) guidelines for adult patients with differentiated thyroid cancer and thyroid nodules redefined indication criteria for FNA. The aim of this study is to compare, with a four-year interval, our FNA diagnostic yield and to evaluate the impact of the 2015 ATA guidelines on our FNA results. From 01/01/2016 to 31/03/2017, all patients of 18 years old or more who had a FNA performed in our tertiary medical center were included. Results were compared to previous data colligated from $01 / 01 / 2012$ to $31 / 10 / 2013$. We included 380 patients $(75.3 \%$ women, mean age: 58) and analyzed 419 first FNAs. All FNAs were ultrasound-guided and interpreted according to Bethesda classification. Sampling was performed according to ATA recommendations for $95.2 \%$ of nodules, the remaining being purely cystic nodules punctured for evacuation $(1.7 \%)$ and infracentimetric nodules (2.9\%). Nondiagnostic was reported for $26.0 \%$ of FNAs (vs $34.4 \%$ in 2012-2013, $\mathrm{p}=0.0056$ ) and, when excluding purely cystic nodules punctured for evacuation, $25.2 \%$ of our samples were nondiagnostic. Nodules were diagnosed as benign in $56.6 \%$ of cases (vs $48 \%$ ), $9.5 \%$ were follicular lesion of undetermined significance (FLUS) (vs $9.6 \%$ ), $1.9 \%$ were follicular neoplasms (vs $4.8 \%$ ), $1.9 \%$ were suspicious for malignancy (vs $1.0 \%$ ), and $4.1 \%$ were malignant (vs $2.2 \%$ ). When combining the two last Bethesda categories, which confer the greatest risk of malignancy, a higher frequency was observed $(6.0 \%$ vs $3.2 \%, \mathrm{p}=0.0550)$ and the pathologic correlation was consistent with cytologies, showing $95 \%$ of malignancy. We improved our diagnostic yield compared to 2012-2013 by reducing significantly our nondiagnostic samples. Our proportion of nondiagnostic nodules still remains higher than in the literature (2-16\%). Finally, the vast majority of the nodules were sampled according to the new ATA recommendations, which possibly led to a better selection of nodules, with an increased rate of detected malignancy.

\section{Poster 119}

Thyroid Cancer Thursday Poster Clinical

EXTRA-THYROIDAL ANAPLASTIC THYROID CANCER: A SINGLE INSTITUTION EXPERIENCE

N.M. Iniguez Ariza ${ }^{1}$, M. Ryder ${ }^{1}$, J. Morris ${ }^{1}$, C. Hilger ${ }^{2}$, C. Dalpiaz ${ }^{2}$, M.E. Menefee ${ }^{3}$, R. Smallridge ${ }^{4}$, T. Sebo ${ }^{5}$, M. Rivera ${ }^{5}$,

A. Chintakuntlawar ${ }^{2}, \mathrm{~K}$. Bible ${ }^{2}$

${ }^{1}$ Division of Endocrinology, Diabetes, Metabolism, and Nutrition, Mayo Clinic, Rochester, MN; ${ }^{2}$ Division of Medical Oncology, Mayo Clinic, Rochester, MN; ${ }^{3}$ Hematology Oncology, Mayo Clinic, Jacksonville, FL; ${ }^{4}$ Endocrinology, Mayo Clinic, Jacksonville, FL; ${ }^{5}$ Anatomic Pathology, Mayo Clinic, Rochester, MN

Anaplastic thyroid cancer (ATC) is rare and highly lethal, often coexisting with differentiated thyroid cancer (DTC), suggesting dedifferentiation from DTC. Herein we summarize our experience with ATC arising from metastases among DTC patients previously subject to thyroidectomy. Institutional Review Board-approved single institution retrospective case series. Seven patients with extra-thyroidal ATC were identified, all arising in the setting of preceding papillary histology DTC, 2 with tall cell features/variant. In 3/7, PDTC or PTC was coexistent in the ATC diagnostic material. Median time from DTC to ATC diagnosis was 6 years (range: $0.25-18$ years). Five presented with clinically symptomatic disease; 4/7 were female; median age at ATC diagnosis was 59 years (range 48 to 67). Sites of ATC origin included: lymph nodes (3), soft tissue (3), and pleural space (1). Of 5 with tumor interrogation, mutations in TP53 (4), TERT promoter (4), and or BRAF V600E (5) were most common. Five of 7 had locoregionally-confined ATC, mimicking stage IVB disease; accordingly, they were treated with definitive-intention chemo-radiation (1/5 alive at $19 \mathrm{mos} ; 4 / 5$ dead from recurrence 9,12 , 12 , and 65 mos from ATC diagnosis). Two of seven instead presented with disseminated (IVC) disease ( 1 alive at 2 mos; another, presenting with pleural ATC while on lenvatinib therapy, dead 1 mo after ATC diagnosis).

ATC arising from extra-thyroidal sites should be considered in the differential diagnosis of any patient with unexpectedly rapidly progressing DTC, but occurs uncommonly. In this disease entity, ATC is clearly linked to dedifferentiation of underlying metastatic DTC. Such patients sometimes present with locoregionally-confined disease, but universally recur even in the setting of definitive-intention chemo-radiation; prognosis is overall poor.

\section{Poster 120 \\ Thyroid Cancer Thursday Poster Clinical \\ NEXT GENERATION SEQUENCING AND MIRNA EXPRESSION PROFILING OF THYROID MALIGNANCY USING MICRO DISSECTED CYTOLOGY SMEARS BY COMBINED THYGENX ${ }^{\mathrm{TM}}$ AND THYRAMIR ${ }^{\mathrm{TM}}$ TESTING $^{-}$}

\section{G. Kumar}

Interpace Diagnostics Inc., New Haven, CT

Previously, we reported the suitability and advantages of carrying out the combined ThyGenX + ThyraMIR test using fine needle aspirates (FNA) in preservative buffer (1-2). We also demonstrated that cytology smears were an adequate sample type for the molecular profiling, for both NGS and microRNA testing, giving comparative performance to the FNA in preservative buffer (3). In this study, we investigated the cell enrichment approach of microdissection applied to cytology smears. Twenty two cases of thyroid fine need aspirate biopsy smears with known cytopathology diagnosis were retrospectively collected for process validation. Regions of interest from cytology smears were selectively micro dissected to enrich tumor cellularity and molecular analysis on these samples was carried out using the next gen sequencing based mutation detection (ThyGenX) and miRNA expression classifier profiling (ThyraMIR). Molecular testing was successfully carried out from all twenty-two cases of micro dissected cytology smears. Correlation of molecular testing results with clinical diagnosis showed that the combined microdissection-based ThyGenX + ThyraMIR test was $82 \%$ accurate, $78 \%$ sensitive, and $85 \%$ specific in detecting risk of malignancy in micro dissected cytology smears. These results clearly demonstrate the suitability of the microdissection enrichment sampling approach to analyze for combined mutation (ThyGenX) and microRNA classifier status (ThyraMIR) in accordance with CAP/CLIA guidelines. Combined molecular testing is thus validated for needle aspirates/rinses as well as all types of micro dissected slide format specimens.

References:

(1) Kumar G. et al. (2015) Thyroid. 25, S1: 1-337.

(2) Labourier E. et al, JCEM (2015) 100: 2743-2750.

(3) Kumar G., et. al. (2016) Thyroid. 26, S1 A138-A163.

\section{Poster 121}

Thyroid Cancer Thursday Poster Clinical

FINE-NEEDLE ASPIRATION CYTOPATHOLOGY

ASSOCIATED WITH NON-INVASIVE FOLLICULAR THYROID NEOPLASM WITH PAPILLARY-LIKE NUCLEAR FEATURES: HOW TO COUNSEL PATIENTS ABOUT OPERATIVE RISK?

B. Lindeman, M.A. Nehs, A. Gawande, F.D. Moore, G.M. Doherty, N.L. Cho

Surgery, Brigham and Women's Hospital, Boston, MA 
In April 2016, encapsulated follicular variant of papillary thyroid carcinoma underwent a nomenclature change to "noninvasive follicular thyroid neoplasm with papillary-like nuclear features" (NIFTP). We investigated the reported malignancy rates associated with thyroid nodule fine-needle aspiration (FNA) results after this change.

A retrospective cohort study of patients undergoing resection of a thyroid nodule sampled pre-operatively with FNA at one tertiarycare hospital was conducted from 4/2016-2/2017. Surgical procedure, anatomic pathology, thyroid cytopathology classification and demographic characteristics were recorded. Outcome variables were change in malignancy rate for each thyroid cytopathology category and number of patients that could have undergone thyroid lobectomy (TL) versus total thyroidectomy (TT).

Thyroidectomy was performed in 427 patients from 4/2016-2/ 2017. Twenty-six patients $(6.1 \%)$ met criteria for NIFTP on final pathology. Among this group, preoperative FNA demonstrated atypia/follicular lesions of undetermined significance (AUS/FLUS) in $13(50 \%)$, suspicious for malignancy in $6(23 \%)$, suspicious for follicular neoplasm (SFN) in $4(15 \%)$, benign in $2(8 \%)$, and malignant in $1(4 \%)$. Change in malignancy rates across cytologic categories due to nomenclature change were as follows: benign $(n=74)$ from $4 \%$ to $1.3 \%$, AUS/FLUS $(n=85)$ from $33 \%$ to $17.6 \%$, SFN $(\mathrm{n}=58)$ from $29 \%$ to $22.4 \%$, suspicious for malignancy $(\mathrm{n}=33)$ from $91 \%$ to $72.7 \%$, and malignant $(\mathrm{n}=99)$ from $99 \%$ to $98 \%$. Among the 26 NIFTP patients, 17 had TL and 9 TT. Eight patients who underwent TT for Bethesda category III - VI nodules could have been definitively treated with TL, an $89 \%$ decrease. NIFTP is an increasingly common pathologic finding and is often associated with indeterminate Bethesda classifications on FNA. This nomenclature change led to an overall decrease in the malignancy rate at our institution. Thyroidectomy will remain the treatment modality of choice, but more patients could be considered for thyroid lobectomy with suspicious for malignancy FNA results.

\section{Poster 122}

Thyroid Cancer Thursday Poster Clinical

URINARY IODINE/CREATININE RATIO

MEASUREMENTS PRIOR TO RADIOACTIVE IODINE ABLATION MAY PREDICT SHORT-TERM DISEASE OUTCOMES IN PATIENTS WITH DIFFERENTIATED THYROID CANCER

J.H. $\mathrm{Li}^{1}$, Z.H. $\mathrm{He}^{2}$, J.V. Hennessey ${ }^{1}$

${ }^{1}$ Division of Endocrinology, Diabetes \& Metabolism, Department of Medicine, Beth Israel Deaconess Medical Center, Boston, MA;

${ }^{2}$ Department of Medicine, Cambridge Health Alliance,

Cambridge, MA

The goal of radioactive iodine ablation (RAIA) in the treatment of differentiated thyroid cancer (DTC) is to destroy remnant thyroid and microscopic cancerous tissue after thyroidectomy. Radioactive iodine uptake is enhanced by elevating TSH levels and initiating a low iodine diet (LID). Studies examining the effect of LID on outcomes are largely inconsistent and lack measurements of iodine status. We investigated whether pre-ablation iodine levels predict disease recurrence/persistence in patients with DTC. We retrospectively reviewed 207 patients with DTC who underwent thyroidectomy and initial RAIA at Beth Israel Deaconess Medical Center from 20072015. All were directed to adhere to a 2 week LID. A urinary iodine/ creatinine ratio (UICR) was calculated based on spot samples obtained prior to ablation. The primary endpoint was disease recurrence/persistence at 18 months post-ablation, defined as the presence of any one of the following: 1) basal serum thyroglobulin $\geq 1 \mathrm{ng} / \mathrm{mL}$ or stimulated thyroglobulin $\geq 2 \mathrm{ng} / \mathrm{mL}, 2$ ) a positive neck ultrasound,
3) positive whole body scintigraphy. Logistic regression tested the association between UICR (as a continuous variable) and disease outcome. Survival analysis was performed using a Cox proportional hazards model. Our cohort had a mean age of 49 years and $72 \%$ were female. 151 patients $(73 \%)$ underwent thyroid hormone withdrawal (THW) and $56(27 \%)$ received recombinant human TSH (rhTSH) prior to ablation. $11 \%$ had disease recurrent/persistence at 18 months post-ablation. In univariate analysis, there was a trend toward association of higher pre-ablation UICR with higher rates of persistence/ recurrence $(\mathrm{p}=0.12)$. In multivariate analysis adjusting for age, sex, tumor size, and type of preparation, there was a $21.9 \%$ absolute risk increase in disease recurrence/persistence per $50 \mathrm{mcg} / \mathrm{g}$ increase of UICR $(p=0.02)$. This was further confirmed in multivariate survival analysis (HR 1.003, 95\% CI 1.001-1.006, $\mathrm{p}=0.006$ ). In our study, higher UICR was associated with disease persistence/recurrence at 18 months. Pre-ablation UICR may be a useful marker for predicting outcomes of DTC and should be further evaluated in longitudinal studies.

\section{Poster 123}

Thyroid Cancer Thursday Poster Clinical

\section{THE UTILITY OF INTACT PARATHYROID HORMONE LEVEL IN PREDICTING HYPOCALCEMIA AFTER THYROIDECTOMY IN CHILDREN \\ W. Jiang ${ }^{1,2}$, R. Newfield ${ }^{3,4}$}

${ }^{1}$ Pediatric Otolaryngology, Rady Children's Hospital in San Diego, San Diego, CA; ${ }^{2}$ Surgery, University of California, San Diego, San Diego, CA; ${ }^{3}$ Pediatric Endocrinology, Rady Children's Hospital, San Diego, San Diego, CA; ${ }^{4}$ Pediatrics, University of California, San Diego, San Diego, CA

Hypocalccemia is a common complication after thyroidectomy. Many adult centers have used postoperative intact parathyroid hormone (PTH) level in the recovery room as a predictive indicator for postop hypocalcemia, with the aim for early discharge if the value is within normal range. However, very few studies have validated this approach in children. After obtaining IRB approval, we retrospectively reviewed all patients who underwent thyroidectomy at risk of hypocalcemia at our tertiary pediatric hospital with available PTH level from 1/1/2010 to 12/31/2016. Patient demographics, pathology, postop PTH, calcium level, and length of hospital stay were analyzed. Total of 40 patients were included in the study period with total thyroidectomy or completion thyroidectomy. The average age was 13.3 years $(4.3-19.2)$.

Majority of the patients, $28 / 40(70 \%)$ had normal PTH level $(>8.5 \mathrm{pg} / \mathrm{ml})$ postoperatively. $12 / 40(30 \%)$ patients had transient hypoparathyroidism during the postoperative period, and 2 of those (5\%) had permanent hypoparathyroidism at follow-up.

Of those with normal PTH, 13/28 (46.4\%) patients had no transient hypocalcemia, and the PTH ranged from $27.5-65.5 \mathrm{pg} / \mathrm{mL}$ in this group. The remaining 15 patients all required inpatient calcium replacement with or without calcitriol and 11 were discharged home on oral replacement and tapered off on an outpatient basis. The PTH range in this group was $10-41.8 \mathrm{pg} / \mathrm{mL}$. In pediatric patients, there does not appear to be a clear "cut-off " level of PTH that is predictive of postop normocalcemia. Among the patients with normal postop $\mathrm{PTH}$, there is a significant overlap in value between those who required calcium replacement or not. Adult guideline or pathway that advocate for outpatient thyroidectomy surgery based on normal PTH in the recovery room may not apply to children.

Low postop PTH level $<10 \mathrm{pg} / \mathrm{ml}$ is highly sensitive in predicting high-risk patients with anticipated decline in calcium level, and can provide helpful guidance on early replacement, and possibly teriparatide injection to prevent symptomatic hypocalcemia. However, 
a "normal" PTH level alone does not always predict a normal calcium level postoperatively, and cannot guide replacement or early discharge.

\section{Poster 124}

Thyroid Cancer Thursday Poster Clinical

FOLLOW UP OUTCOMES OF THYROID NODULES WITH FOLLICULAR NEOPLASM/SUSPICIOUS FOR FOLLICULAR NEOPLASM IN AN ERA OF NONINVASIVE FOLLICULAR THYROID NEOPLASM WITH PAPILLARY-LIKE NUCLEAR FEATURES

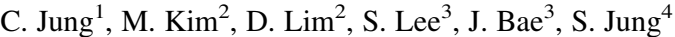

${ }^{1}$ Department of Hospital Pathology, College of Medicine, The Catholic University of Korea, Seoul, Korea (the Republic of); ${ }^{2}$ Department of Internal Medicine, The Catholic University of Korea, Seoul, Korea (the Republic of); ${ }^{3}$ Department of Surgery, The Catholic University of Korea, Seoul, Korea (the Republic of); ${ }^{4}$ Department of Radiology, The Catholic University of Korea, Seoul, Korea (the Republic of)

Follicular neoplasm/suspicious for follicular neoplasm (FN/SFN) is a triage category for surgical excision because capsular or vascular invasion of the lesion cannot be evaluated on cytology or biopsy specimen. Noninvasive follicular thyroid neoplasm with papillarylike nuclear features (NIFTP) formerly classified as papillary carcinoma is now considered to be a borderline tumor and has introduced significant diagnostic challenges in thyroid cytology. We reviewed all patients who were preoperatively diagnosed with FN/SFN between August 2012 and December 2014. Of these, 289 cases with the diagnosis of FN/SFN were confirmed histologically after surgical resection and enrolled for this study. All histologic slides were reviewed. Among resected thyroid nodules with FN/SFN cytology/biopsy diagnosis, follicular adenoma $(41.9 \%, 121 / 289)$ was the most common subtype, followed by 63 NIFTP (21.8\%), 34 follicular carcinoma $(11.8 \%), 33$ nodular hyperplasia $(11.4 \%), 31$ papillary carcinoma $(10.7 \%), 3$ poorly differentiated carcinoma (1.0\%), 3 Hashimoto thyroiditis, and 1 parathyroid adenoma. Overall rates of malignant and benign lesions were $23.5 \%$ and $54.7 \%$, respectively. NIFTP was found in $61(26.6 \%)$ of 229 conventional FN/SFN cases and in $2(3.3 \%)$ of 60 FN/SFN cases with Hürthle cell type. There was no significant difference in malignancy rate between conventional and Hürthle cell types $(24.0 \%$ vs $23.5 \%, P<0.05)$. About three-fourths of patients with a preoperative diagnosis of FN/SFN were proven to have indolent diseases (benign or NIFTP) after surgery. NIFTP reduced the risk of malignancy by more than half in conventional FN/SFN category when the tumor was not considered malignant.

\section{Poster 125}

Thyroid Cancer Thursday Poster Clinical

OUTCOME OF LOW-RISK MULTIFOCAL PAPILLARY MICROCARCINOMA AFTER HEMITHYROIDECTOMY J. An ${ }^{1,5}$, J. Kim ${ }^{1,5}$, H. Kim ${ }^{1,5}$, H. Kim ${ }^{2,5}$, S. Baek ${ }^{3,5}$, K. Jung ${ }^{3,5}$, Y. Chae ${ }^{4,5}$, S. Kim ${ }^{1,5}$

${ }^{1}$ Internal Medicine, Korea University College of Medicine, Seoul, Korea (the Republic of); ${ }^{2}$ Surgery, Korea University College of Medicine, Seoul, Korea (the Republic of); ${ }^{3}$ Otolaryngology-Head and Neck Surgery, Korea University College of Medicine, Seoul, Korea (the Republic of); ${ }^{4}$ Pathology, Korea University College of Medicine, Seoul, Korea (the Republic of); ${ }^{5}$ Thyroid Center, Korea University Hospital, Seoul, Korea (the Republic of)

Papillary thyroid carcinomas frequently occur with multiple foci in the gland. We investigated the clinical course of patients with mul- tifocal papillary thyorid microcarcinomas (PTMCs) who underwent hemithyroidectomy. We analyzed 937 patients with PTMCs (mean age $45.1 \pm 11.7$ years; $82.2 \%$ female) who underwent hemithyroidectomy from 2000 to 2014 without preoperative evidence of tumor foci in the contralateral lobe or suspicious lymph node (LN) metastasis. Multifocal PTMCs were detected in 144 (15.4\%) of the 935 patients who underwent hemithyroidectomy. Of these PTMCs, $111(11.9 \%)$ had two foci, $20(2.1 \%)$ had three foci, and $13(1.3 \%)$ had four or more foci. Among the patients with multifocal tumors, 73 $(50.7 \%)$ had additional tumor foci (range $1-9$ ) found incidentally in pathological specimens. In patients with multifocal tumors, compared with those with solitary lesions, the size of the largest tumor $(0.55 \pm 0.23$ vs. $0.50 \pm 0.20 \mathrm{~cm}, p=0.031)$ and the prevalence of extrathyroidal extension ( 33.1 vs. $20.2 \%, p=0.002)$ were greater. The incidence of $\mathrm{LN}$ metastasis revealed by prophylactic central LN dissection was slightly higher, albeit not significantly, in patients with multifocal tumors (12.4 vs. $7.8 \%, p=0.069)$. Age, sex, family history, prevalence of combined thyroiditis/nodular hyperplasia, and BRAFV600E mutation were not significantly different between patients with multifocal and those with solitary PTMCs. Among the 144 patients with multifocal PTMCs, 24 (16.7\%) underwent prophylactic complete thyroidectomy within 1 year of initial surgery. Among the 120 patients with multifocal PTMCs who did not receive complete thyroidectomy within 1 year, $7(5.8 \%)$ patients developed tumor recurrence on the contralateral lobe or LNs within an average of 4.1 (range 1-14) years. The number of tumor foci was a significant risk factor for tumor recurrence, after adjusting for age, sex, size of the largest tumor, extrathyroidal extension, LN metastasis, and followup duration (OR 2.23, $p=0.011$ ). Multifocality was a risk factor for tumor recurrence in patients with PTMCs who underwent hemithyroidectomy as an initial treatment. However, the overall tumor recurrence rate was low in clinically low-risk patients with multifocal PTMCs.

\section{Poster 126}

Thyroid Cancer Thursday Poster Clinical

\section{THE LEARNING CURVE OF TRANSORAL ENDOSCOPIC THYROIDECTOMY VESTIBULAR APPROACH (TOETVA) : A COMPARISON WITH BILATERAL AXILLO-BREAST} APPROACH (BABA)

J. Lee ${ }^{1}$, J. Lee ${ }^{2}$, Y. Choi ${ }^{1}$

${ }^{1}$ General surgery, Samsung Changwon Hospital, Sungkyunkwan University School of Medicine, Changwon, Korea (the Republic of); ${ }^{2}$ Ewha Womans University, Seoul, Korea (the Republic of)

Transoral endoscopic thyroidectomy vestibular approach (TOETVA) and bilateral axillo-breast approach (BABA) offers the advantage a good cosmetic outcome. Recently, In the evolution of thyroid surgery, for many endocrine surgeons, the transoral approach seems to be the next step. However, some endocrine surgeons are reluctant to advocate TOETVA due to unacquainted approach. The purpose of this study was to evaluate the learning curve for TOETVA to comparison with BABA. Between July 2015 and May 2017, Each 40 and 22 consecutive patients who underwent endoscopic thyroidectomy via BABA and TOETVA. All of the procedures were performed by the same surgeon. $1^{\text {st }}$ TOETVA was performed after $30^{\text {th }}$ case via BABA. Each patient's operative outcomes were collected and retrospectively analyzed. The cumulative summation (CUSUM) analysis was used to assess the learning curve of TOETVA and BABA. There was no significant difference between TOETVA and BABA in the adverse postoperative outcomes included transient or permanent vocal cord palsy. However, TOETVA showed more satisfaction with the cosmetic result, postoperative pain relief and voice outcome, especially high-pitched tone. Additionally, TOETVA has superior result in 
reducing transient hypocalcemia among total thyroidectomy patients. CUSUM analysis showed a decreasing trend at the $11^{\text {th }}$ patient via TOETVA, suggesting that more than 11 cases were needed for the surgeon who experienced via BABA to gain proficiency. Our results showed that TOETVA has reasonable surgical outcomes compared to BABA. The TOETVA is safe and feasible and has an acceptable learning curve for surgeons who are proficient in conventional endoscopic thyroidectomy, especially BABA procedure.

\section{Poster 127}

Thyroid Cancer Thursday Poster Clinical

\section{LONG TERM PROGNOSIS OF DIFFERENTIATED THYROID CANCER PATIENTS ACCORDING TO EXISTENCE OF THYROIDITIS}

R. Emral, M. Sahin, I. Aydogan, S. Gullu, M. Erdogan,

D. Corapcioglu

Endocrinology and Metabolic Diseases, Ankara University, Faculty of Medicine, Ankara, Turkey

Prognosis of differentiated thyroid cancer in patients with autoimmune thyroiditis is a matter of debate. We aim to evaluate the clinical outcome of differentiated thyroid cancer patients according to antithyroglobulin antibodies and presence of thyroiditis in our patients.

We retrospectively analyse 609 thyroid cancer patients diagnosed and followed in our clinic. We separated these patients according to the presence of thyroiditis at the time of diagnosis.

There are 237 male and 372 female patients with a mean age $47.18 \pm 12$ years. We have followed up our patients up to 372 months. We found lymphocytic thyroiditis in 199 of 609 differentiated thyroid cancer patients. Tumor diameter were smaller in patients with thyroiditis $(13.8$ vs $18.1 \mathrm{~mm}, \mathrm{p}<0.0001)$ and associated with less capsule and vascular invasion. We also found higher remission rate in patients with thyroiditis compared to patients without thyroiditis. But we could not find any association with preoperative antithyroglobulin levels and remission or reccurence rates in subgroup of patients with thyroiditis.

Differantiated thyroid cancer patients with thyroiditis may have better prognosis and clinical outcome. This could be due to smaller tumour size of these patients, at least in our thyroid cancer patient group.

\section{Poster 128}

Thyroid Cancer Thursday Poster Clinical CALCIFICATION PATTERNS ARE ASSOCIATED WITH SERUM FREE T4 LEVELS IN PAPILLARY THYROID CARCINOMA

J. Ha, J. Lee, M. Kim, M. Kang, B. Cha, D. Lim

Endocrinology, Seoul St. Mary's hospital of the Catholic University of Korea, Seoul, Korea (the Republic of)

Papillary thyroid carcinoma (PTC) frequently shows typical calcification pattern like microcalcification which may be related to pathogenesis of PTC. On recent studies thyroid hormone is associated with vascular calcification and may have influences on molecules associated with calcification. Therefore, we investigated the relationship between the calcification pattern of PTC and serum thyroid hormone concentration. We retrospectively reviewed patients with PTC with or without calcification, which were classified as no calcification, microcalcification, macrocalcification, and mixed type by preoperative neck ultrasound and CT scan. Patient demographics, preoperative serum thyroid hormones, thyroid autoantibodies, and postoperative pathological parameters were evaluated according to calcification patterns. Serum T3, TSH, and AntiTPO Ab levels were not related to the presence of calcification or calcification pattern but lower free T4 concentration definitely correlated with increased calcification frequency and microcalcification in PTC. Calcification pattern was not associated with primary tumor size but indicated association with several clinical and pathological prognostic parameters. Thyroid hormone concentration, especially free T4 is closely associated with calcification pattern in PTC and may be related to the pathogenesis and prognosis of PTC.

\section{Poster 129}

Thyroid Cancer Thursday Poster Clinical

LOW-IODINE DIET AND URINE IODINE MEASUREMENTS FOR ${ }^{131}$ I TREATMENTS IN PATIENTS IN DIFFERENTIATED THYROID CANCER: A COMPARISON OF 18 CLINICAL PRACTICE GUIDELINES

D. Turner ${ }^{1,2}$, D. Wu ${ }^{2,4}$, K.D. Burman ${ }^{3}$, L. Wartofsky ${ }^{3}$, D. Van Nostrand ${ }^{2,4}$

${ }^{1}$ Biology, Howard University, Washington, DC; ${ }^{2}$ Medstar Health Research Institute, Washington, DC; ${ }^{3}$ Endocrinology, Medstar Washington Hospital Center, Washington, DC; ${ }^{4}$ Nuclear Medicine, Medstar Washington Hospital Center, Washington, DC

The use of low-iodine diets (LID) in the preparation of ${ }^{131}$ I treatments (tx) for patients (pts) with differentiated thyroid cancer (DTC) is controversial. The objective of this evaluation was to compare the recommendations for the use of LID in various clinical practice guidelines (CPG) around the world that have been published in/ translated into English. A literature search for CPG on DTC management was conducted using Pubmed, Embase and Google Scholar. Search terms included thyroid, cancer, carcinoma, tumor, differentiated, papillary, follicular, and guideline. From 2006-2016, 24 CPG were available in full-text. After exclusion of CPG that did not have translations available and/or were for specific sub-populations, such as children or pregnant women, $18 \mathrm{CPG}$ remained and were compared regarding the following for LID: (1) recommendations, (2) acceptable/ unacceptable foods, (3) duration, (4) measurements of urine iodine (UI) levels, (5) methods of measuring UI levels, and (6) acceptable thresholds for UI levels. Each CPG was evaluated for quality using a checklist of 22 criteria obtained from the World Health Organization's (WHO), the United States Institute of Medicine's (IOM), and various medical societies' guide to CPG development (separate abstract). A total of $18 \mathrm{CPG}$ from 13 countries/regions met the inclusion criteria. Of the $18 \mathrm{CPG}$, only 4 met more than half of the criteria, 3 CPG met $\geq 70 \%$ quality checks. The specific data on the quality checks of the CPG will be presented. Not all of the CPG discussed preparation of the pt with LID for ${ }^{131} \mathrm{I}$ tx or scan, and the typical recommendation for the duration of the LID was 1-2 wks. Most CPG did not discuss acceptable/unacceptable foods, and only 2 included sources for additional information. UI levels (i.e. measuring, method of measuring, and thresholds) were infrequently discussed. Majority of the checklist criteria were met by less than half of the reviewed CPG, and 2 of the criteria checks were not met by any of the selected CPG. Further research on LID is warranted to confirm the value of LID, and UI levels. In addition, further effort is warranted to continue to improve the quality and consistency of the format and process of the CPG.

\section{Poster 130}

Thyroid Cancer Thursday Poster Clinical

\section{ROBOTIC NECK SURGERY IN THE PEDIATRIC} POPULATION: A CASE SERIES

E.L. $\mathrm{Wu}^{1}$, S. Kang ${ }^{2}$, E. Kandil ${ }^{1}$

${ }^{1}$ Division of Endocrine and Oncological Surgery, Department of Surgery, Tulane University, New Orleans, LA; ${ }^{2}$ Department of Surgery, Yonsei University College of Medicine, Seoul, Seodaemun-gu, Korea (the Republic of) 
Thyroid, parathyroid, and thymus surgeries are traditionally performed via transcervical approaches. However, robotic-assisted approaches can provide a safe alternative to avoid visible neck incisions or thoracotomy for thymus surgery. Due to the relatively limited space in the pediatric population, robotic surgery in the neck poses a unique challenge. We report our experiences with robotic-assisted transaxillary and retroauricular approaches in 9 pediatric cases. We conducted a retrospective review of pediatric patients who underwent robotic neck surgery by a single North American surgeon between April 2010 and May 2017. All patients underwent pre- and postoperative vocal cord evaluation. Demographics, surgical outcomes, and length of hospital stay were evaluated. Nine cases in 7 distinct patients were included in the study. All patients were females with a mean age of $16.0 \pm 2.24$ years. Mean body mass index was $22.5 \pm 4.73$. There were 3 thyroid lobectomies, 2 completion thyroidectomies, 1 thyroid lobectomy with thymectomy, 2 subtotal parathyroidectomies with thymectomy, and 1 excision of a dermoid cyst. Two thyroid lobectomies were completed via a retroauricular approach with a mean surgery time of $142.0 \pm 18.38$ minutes. The other 7 cases were completed via a transaxillary approach with a mean surgery time of $146.1 \pm 63.02$ minutes. Five patients (56\%) were discharged home the same day of surgery, $3(33 \%)$ were discharged after 1 day, and $1(11 \%)$ was discharged after 2 days. There were no reported permanent vocal cord paralysis, permanent hypoparathryoidism, hematomas, or seromas. Two cases $(22 \%)$ complained of temporary hypoesthesia after surgery. Two cases (22\%) reported temporary hoarseness. Robotic-assisted approaches are feasible and safe options to operations in the neck for a select group of pediatric patients. Future studies are needed to evaluate the benefits and limitations of these approaches in the pediatric population.

\section{Poster 131 \\ Thyroid Cancer Thursday Poster Clinical \\ OVER-TREATMENT OR UNDER-TREATMENT OF PTC? ANALYSIS OF RE-OPERATION CASES IN PUMCH \\ H. Liu \\ Pekin Union Medical College Hospital, Beijing, China}

Are there over-treatment or under-treatment in PTC therapy? We performed a retrospective analysis to evaluate it. All the PTC reoperation cases in PUMCH included (1998-2015), analyzed the data to find the reason of reoperation and confirm whether over-treatment or under-treatment existed. Re-operations happened in 1-year we affirmed persistent disease and those beyond 1-year we affirmed recurrent disease. 10354 PTC operation cases in total and $665 \mathrm{re}=$ operation cases found, 382 persistent cases and 283 recurrent cases, 209 simple LLND, 217 simple CLND, 239 leaf removal+CLND/LLND. Of the causes, 94 diagnosis factor/54 operation factor, but the rest were difficult to classify so just put them to selection factor. Because of large amount of re-operation cases in PTC patients, and the rich variety of the cause, it's reasonable to believe that not only over-treatment but also under-treatment existed in PTC treatment.

\section{Poster 132}

Thyroid Cancer Thursday Poster Clinical OUTCOME OF 76 GENE CARRIERS OF HEREDITARY MEDULLARY THYROID CARCINOMA AFTER THYROIDECTOMY IN A SINGLE INSTITUTION: IMPACT OF LATE DIAGNOSIS AND RET MUTATION RISK L.A. Castroneves ${ }^{2}$, M.R. Tavares ${ }^{1}$, F.A. Correa ${ }^{2}$, F.A. Vanderlei ${ }^{1}$, J.M. Correia-Deur ${ }^{3}$, A.A. Jorge ${ }^{3}$, A.O. Hoff ${ }^{2}$
${ }^{1}$ Head and Neck Surgery, University of Sao Paulo, Sao paulo, SP, Brazil; ${ }^{2}$ Endocrinology, ICESP, university of sao paulo, Sao paulo, SP, Brazil; ${ }^{3}$ Endocrinology, University of Sao Paulo, Sao paulo, SP, Brazil

Introduction: Hereditary medullary thyroid cancer (HMTC) can be cured by prophylactic thyroidectomy (PT). The recommended timing for PT is based on aggressiveness of HMTC and stratified into 3 risk groups: moderate (MOD), high (H) and highest (HST). The aim of this study was to analyze the outcome of family members diagnosed with HMTC by RET genotyping. To understand the natural history of MTC according to risk group, all patients, regardless of age at total thyroidectomy (TT) were included. From 15 MEN2A families, $5 \mathrm{MOD}$ (RET codon 620 and 804) and $10 \mathrm{H}$ (RET codon 634 and 634/ 791 ) and 1 MEN2B family (HST, RET M918T) followed at our clinic, 76 gene carriers underwent TT after gene testing. Median age at TT was 16 yrs (2-62) (MOD) and 22 yrs (3-61) (H). Histology: normal $(\mathrm{n}=10)$, c-cell hyperplasia $(\mathrm{CCH})(\mathrm{n}=6)$, T1a tumors $(\mathrm{n}=26)$, T1b-T3 $(\mathrm{n}=22)$; LN involvement in $20(26.3 \%)$ and metastatic disease in 1 patient. Serum calcitonin (Ctn) 1 -yr postthyroidectomy was $<2 \mathrm{pg} / \mathrm{mL}$ in 49 patients $(64.4 \%)$, measurable in 21 patients with median Ctn $12 \mathrm{pg} / \mathrm{mL}$ (5.2-872). Median follow-up was 8.5 yrs (1-21) (MOD), 7 yrs (1-19) (H) and 3 yrs (HST). At last follow-up, 35 patients $(46 \%)$ had persistent or recurrent disease, median Ctn $116 \mathrm{pg} / \mathrm{mL}(2.9-74,421) ; 40$ patients remained with Ctn $<2 \mathrm{pg} / \mathrm{mL}(52.6 \%)$ and defined as cured MOD $(\mathrm{n}=23 / 40), \mathrm{H}(\mathrm{n}=17 /$ 34), HST $(n=0 / 2) .7$ patients have distant metastasis (4 MOD, $2 \mathrm{H}, 1$ HST) and 1 died (MOD). Median age at TT in cured patients was 16.5 yrs (2-62) in MOD and 17 yrs (3-53) in H. Both MEN2B carriers were not cured, ages at TT 6 and 48 yrs. Further analysis showed that cure rate in carriers of codons 634 and 620 RET mutations were similar (42\% and $51 \%)$ and lower than cure rate observed in codon 804 and the double 634/791 mutation (80\% and 67\%). The earliest age of $\mathrm{CCH}$ and T1a tumors in codons 634 and 620 were 8 and 7 yrs and 3 and 5 yrs, respectively. The low cure rate in our cohort supports the risk-based recommendations for timing of PT. However, the similar cure rate and onset of disease between carriers of RET codons 620 and 634 mutations suggests that codon 620 mutations should be stratified as a high risk level.

\section{Poster 133}

\section{Thyroid Cancer Thursday Poster Clinical}

\section{DISTINCT PHYSICAL FEATURES OF MEN 2B ASSOCIATED WITH RET A833F MUTATION}

T.C. Freitas ${ }^{2}$, F.S. Souza ${ }^{2}$, M.A. Pereira ${ }^{2}$, L.A. Castroneves ${ }^{1}$, D.M. Lourenço $\mathrm{Jr}^{1,2}$, F.A. Vanderlei ${ }^{3}$, A.A. Jorge ${ }^{2}$, M.R. Tavares ${ }^{3}$, A.O. Hoff ${ }^{1}$

${ }^{1}$ Endocrinology, ICESP, Sao Paulo, Sao paulo, Brazil;

${ }^{2}$ Endocrinology, University of Sao Paulo, Sao paulo, SP, Brazil;

${ }^{3}$ Head and Neck Surgery, University of Sao Paulo, Sao paulo, SP, Brazil

Multiple Endocrine Neoplasia Type 2B (MEN2B) is a rare syndrome associated with medullary thyroid cancer (MTC), pheochromocytoma and a distinct phenotype including mucosal neuromas, elongated facies and marfanoid habitus. More than 95\% of MEN2B patients harbor a RET M918T mutation (exon 16), while only a few cases harbor a RET A883F mutation (exon 15). The aim of this study is to report a new case of MEN2B with a A883F mutation and compare the phenotype with MEN2B M918T mutation patients. A 22-year-old female with a 12-month history of intermittent hypertension and headache presented to the ER with severe left leg pain which investigation revealed femoral artery thrombosis requiring thrombectomy. During this evaluation, a $5.3 \mathrm{~cm}$ right adrenal mass 
was identified; urinary metanephrines were elevated and a right adrenalectomy confirmed a pheochromocytoma. With the suspicion of a genetic syndrome, she was referred to our Institution. Physical examination revealed palpable thyroid nodules, elongated facies, oral mucosa neuromas, nanophtalmos, palpebral ptosis, retinal detachment, pes cavus. She reported a history of a bowel surgery at age 3. Work-up revealed a serum calcitonin $1790 \mathrm{pg} / \mathrm{mL}(<5 \mathrm{pg} / \mathrm{mL})$, CEA $19.2 \mathrm{ng} / \mathrm{mL}(<5 \mathrm{ng} / \mathrm{mL}), 1.5 \mathrm{~cm}$ right lobe and $1.0 \mathrm{~cm}$ left lobe thyroid nodules on ultrasound and no evidence of distant metastases on chest, abdomen CT and bone scan. Genetic analysis revealed a RET A883F mutation. She underwent a total thyroidectomy with lymph node dissection, pathology revealed a multifocal MTC T1bN1b; post-operative calcitonin at 6 months remained elevated at $1447 \mathrm{pg} / \mathrm{mL}$. Compared to other MEN2B patients harboring a RET M918T mutation ( $\mathrm{N}=7)$, we could observe differences in physical features and in MTC presentation. MTC was more aggressive in M918T carriers with a greater median primary tumor size and presence of distant metastatic disease at diagnosis. Regarding physical features, while lacking marfanoid habitus, the most prominent features included pes cavus and eye abnormalities not observed in M918T carriers. We report a patient with MEN2B due to a RET codon A883F mutation with a less aggressive MTC and with physical features distinct from MEN2B due to a RET codon M918T mutation.

\section{Poster 134}

Thyroid Cancer Thursday Poster Clinical

THE UTILITY OF COMBINED MUTATIONS AND MICRORNA EXPRESSION PROFILING IN ASSESSING CANCER RISK IN THYROID NODULES

A. Banizs ${ }^{2}$, N. Toney ${ }^{1}$, K. Haugh ${ }^{1}$, C.M. Narick ${ }^{1}$, S. Jackson ${ }^{1}$, J.F. Silverman ${ }^{2}$, S. Finkelstein ${ }^{1}$

${ }^{1}$ Interpace Diagnostics, Pittsburgh, PA; ${ }^{2}$ Department of Pathology, Allegheny General Hospital, Pittsburgh, PA

We correlated real-world results of i) Bethesda (BDC) classification, ii) mutational analysis, and iii) a two-tiered (positive, negative) miRNA classifier ${ }^{1}$ incorporating two additional miRNA thresholds.3,472 patients who had fine needle aspirates (FNA) of thyroid nodules that underwent both mutation and microRNA (miRNA) classifier ${ }^{1}$ testing were examined. Two additional miRNA thresholds, defining a four-tiered approach (Tier 1-4), were determined based on the miRNA score in nodules with known disease status. Projected risk of cancer at variable malignancy prevalence was based on the performance of molecular test categories in nodules with known disease and calculated using Bayes theorem. PIK3CA, PAX8/ $P P A R$ and RET/PTC mutations each occurred in $<1 \%$ of all nodules. RAS mutation occurred in $16 \%$ of both BDC III and IV nodules, $8 \%$ of BDC II and only $3 \%$ of BDC VI. $43 \%$ of BDC VI nodules had BRAF mutation; 55\% lacked any detectable mutational change. BRAF was $100 \%$ specific for BDC II and $43 \%$ sensitive for BDC VI. Cancer risk for BDC III and IV nodules that were BRAF negative was 3-9\% and 9-20\%, respectively. Positive miRNA status (Tier 3-4) escalated risk. Both BDC III and IV nodules with Tier-4 status had $100 \%$ risk, while Tier-3 status did not provide any incremental change to risk. Negative miRNA status (Tier 1-2) de-escalated risk. Both BDC III and IV nodules with Tier-1 status had $0 \%$ risk. Tier-2 status de-escalated risk (1-4\%) in BDC III nodules to a level of BDC II nodules. For BDC IV nodules, risk was de-escalated to $4-9 \%$, a level similar to that of BDC III nodules. Tier-1 status occurred in $21 \%$ of BRAF negative nodules; Tier- 2 occurred in $66 \%$, Tier-3 in $9 \%$ and Tier-4 in $4 \%$. Mutational change is not sufficient to risk stratify thyroid nodular disease unless accompanied by attention to clinical, imaging and additional molecular findings. miRNA classi- fication complements cytology and mutation analysis with the capacity to better predict biological aggressiveness. A four-tier miRNA classifier approach can provide additional opportunities to impact patient management.

References: 1. Labourier, et al. doi: 10.1210/jc.2015-1158.

\section{Poster 135}

Thyroid Cancer Thursday Poster Clinical

\section{PERFORMANCE ATTRIBUTES OF MICRODISSECTED SLIDE-FORMAT AND DEDICATED NEEDLE ASPIRATION BIOPSY ANALYSIS OF INDETERMINATE THYROID} NODULES

S. Finkelstein, S. Jackson, G. Kumar, C.M. Narick, N. Toney

Interpace Diagnostics, Pittsburgh, PA

Molecular analysis of cytology indeterminate thyroid nodules can lead to definite diagnosis and predict biological aggressiveness (benign versus low grade malignancy versus high grade malignancy). Two general testing formats are available to choose from consisting of 1) separate dedicated needle biopsy/rinse and 2) material removed from cytology/surgical pathology microscopic slides. We previously reported combination molecular testing performance (mutation panel and microRNA classifier) with needle aspirate/rinse specimens. Here we extend the performance review to the different slide formats that are available incorporating microdissection to enrich for cells of interest. Molecular analysis included $B R A F, N, H, K R A S, P I K 3 C A$, $P A X 8 / P P A R \gamma$ and $R E T / P T C$ translocations on next generation sequencing platform (ThyGenX). The microRNA (miRNA) classifier utilized a 10 miRNA panel (ThyraMIR). Microdissection under stereoscopic guidance was used to select cellular material from slide. Slide formats (total $\mathrm{n}=206$ ) included Pap stained smears (PS, $\mathrm{n}=64$ ), Diff Quik smears (DS, $n=53$ ), unstained FFPE cell block sections $(\mathrm{CB}, \mathrm{n}=38)$, Pap stained monolayer preps (MP, n-35) and unstained FFPE core biopsy section (Core, $n=16$ ). The five slide formats all performed well with low insufficiency rates equivalent to or lower than that seen for dedicated needle aspirates/rinses (Asp) previously reported $(\mathrm{n}=4503)$. The insufficiency rate of mutation analysis was $1.6 \%$ (PS), $1.9 \%$ (DS), $2.6 \%$ (CB), $0 \%$ (MP), $0 \%$ (Core), and $1.4 \%$ (Asp). That of the miRNA classifier was $4.7 \%$ (PS), 3.7\% (DS), $2.6 \%$ (CB), $5.7 \%$ (MP), $6.3 \%$ (Core), and $4.7 \%$ (Asp). Recourse to slide format testing for insufficient needle aspirate samples enabled full molecular combination testing to be achieved in virtually all cases. Ancillary molecular testing combining mutational analysis and an miRNA classifier can be accomplished with equivalent effectiveness on dedicated needle aspirates/rinses or by microdissection of all cytology/histopathology slide formats. The ability to evaluate both aspirates and slides enables a definitive result in nearly all patients with indeterminate thyroid nodules.

\section{Poster 136}

Thyroid Cancer Thursday Poster Clinical

DIAGNOSTIC MOLECULAR PROFILING DIFFERENTIATING NONINVASIVE FOLLICULAR THYROID NEOPLASM WITH PAPILLARY-LIKE NUCLEAR FEATURES (NIFTP) FROM CLOSELY RELATED ENTITIES

S. Finkelstein ${ }^{1}$, S. Jackson ${ }^{1}$, N. Toney ${ }^{1}$, C.M. Narick ${ }^{1}$, L. Thompson ${ }^{2}$ ${ }^{1}$ Interpace Diagnostics, Pittsburgh, PA; ${ }^{2}$ Southern California Permanente Medical Group, Woodland Hills, CA

Follicular patterned thyroid neoplasms with papillary carcinoma nuclear features with or without invasion are increasingly recognized as a diverse group of neoplastic processes. While sharing similar 
microscopic features, the biological behavior of these entities appears to be distinct from that of usual type papillary thyroid carcinoma (uPTC). Combined molecular analysis (mutational, microRNA classifier) was applied to cohorts of closely related lesions and known positive (uPTC) \& negative (benign) nodules in search of discriminating diagnostic markers. Unstained FFPE tissue sections of known NIFTP $(n=10)$, encapsulated follicular variant of PTC with invasion (iEFVPTC) $(\mathrm{n}=10)$, encapsulated non-invasive usual PTC (ePTC) $(\mathrm{n}=7)$, positive control uPTC $(\mathrm{n}=12)$ and negative control adenomatoid nodules $(\mathrm{n}=21)$ were microdissected. Mutational analysis included $B R A F, N, H$, and $K R A S, P I K 3 C A, P A X 8 / P P A R \gamma$ and RET/PTC translocations on next generation sequencing platform. miRNA classifier utilized a 10 miRNA panel. NIFTP, iEFVPTC and ePTC lacked $B R A F$ V600E mutation and had benign/less aggressive miRNA classifier status distinctly different from uPTC. RAS mutations or PAX8/ PPAR fusions were detected in subsets in NIFTP, iEFVPTC and ePTC tumors and was non-discriminating. 8 of 10 miRNAs showed expression differences in NIFTP compared to iEFVPTC and ePTC suitable for classifier differentiation. Combining follicular variant neoplasms (NIFTP and iEFVPTC), 5 miRNAs showed significant expression differences with ePTC suitable for classifier differentiation. Despite all tumors showing PTC nuclear features, NIFTP, iEFVPTC and ePTC showed distinct mutational and microRNA classifier differences from uPTC. Despite cytomorphology overlap with uPTC, these entities manifested molecular changes closer to benign than malignant disease supporting less aggressive biological behavior. Mutational changes typical of follicular neoplasia were present in all three closely related entities and could not discriminate between them.

\section{Poster 137 \\ Thyroid Cancer Thursday Poster Clinical \\ SIGNIFICANCE AND MANAGEMENT OF PLEURAL DISEASE IN THYROID MALIGNANCY \\ F. Kazzaz ${ }^{1}$, T. Jaber², M.E. Cabanillas ${ }^{2}$, N. Busaidy ${ }^{2}$, S. Faiz ${ }^{3}$ \\ ${ }^{1}$ Internal Medicine, University of Texas Health Science Center at \\ Houston. McGovern Medical School, Houston, TX; ${ }^{2}$ Department of Endocrine Neoplasia and Hormonal Disorders, The University of Texas MD Anderson Cancer Center, Houston, TX; ${ }^{3}$ Department of Pulmonary Medicine, The University of Texas MD Anderson Cancer Center, Houston, TX}

Pleural disease in thyroid cancer is rare, and the prognosis with malignant pleural effusion (MPE) is thought to be dismal. Our purpose was to evaluate the characteristics and management of pleural effusion in thyroid cancer and evaluate overall survival (OS) with MPE. Patients with thyroid malignancy that underwent pleural intervention from $1 / 2000$ to $12 / 2015$ were identified. Clinical history and laboratory data were reviewed retrospectively. Seventy-three patients underwent pleural interventions. Median age at time of pleural intervention was 65 , and $49 \%$ were male. Tumor histology was predominantly differentiated (71\%). Many (47\%) were receiving active chemotherapy, and pulmonary metastases were present in $18 \%$. Respiratory symptoms were present in $96 \%$ with dyspnea (85\%) and cough (56\%). Effusions were mostly unilateral (70\%). Thoracentesis (93\%) was the most frequent initial intervention, but many $(71 \%)$ required multiple pleural interventions. Etiology of pleural effusion included malignancy (59\%), infection (5\%), volume $(4 \%)$ and other $(23 \%)$. Forty patients underwent indwelling pleural catheter (IPC) placements for symptom control.

MPE was identified in 43 patients, and tumor histology included: $69 \%$ differentiated, $23 \%$ poorly differentiated/anaplastic, $5 \%$ medullary pathology. Pulmonary metastases were present in $19 \%$. Most $(83 \%)$ required multiple pleural interventions, and $81 \%$ underwent indwelling pleural catheter placement. Median OS from time of pleural intervention was $4.9(0.3-20)$ months for differentiated, 1.5 (0.5-5.9) months for poorly differentiated/anaplastic, and 4.1 (1.86.4) months for medullary. Median OS from cancer diagnosis was 6.6 (0.3-58) years for differentiated, $0.54(0.3-2.8)$ years for poorly differentiated/anaplastic, and 12.8 (4.9-20.7) years for medullary. Although rare, pleural effusion in thyroid cancer may create significant symptom burden. Pulmonary metastases are not frequently concomitant with pleural disease. Management with indwelling pleural catheter may alleviate respiratory symptoms and improve quality of life. In those with MPE, survival may be limited, but congruent to survival in other solid organ malignancies.

\section{Poster 138}

Thyroid Cancer Thursday Poster Case Report

\section{LENVATINIB ASSOCIATED BILATERAL SPONTANEOUS PNEUMOTHORACES}

\section{F. Kazzaz ${ }^{1}$, M.E. Cabanillas ${ }^{2}$, S. Faiz ${ }^{3}$}

${ }^{1}$ Internal Medicine, University of Texas Health Science Center at Houston. McGovern Medical School, Houston, TX; ${ }^{2}$ Department of Endocrine Neoplasia and Hormonal Disorders, The University of Texas MD Anderson Cancer Center, Houston, TX; ${ }^{3}$ Department of Pulmonary Medicine, The University of Texas MD Anderson Cancer Center, Houston, TX

Anaplastic thyroid cancer has limited response to cytotoxic chemotherapy and poor prognosis. Recent reports show that targeted therapy with the VEGFR inhibitor, lenvatinib, may have efficacy in this disease. We report a rare complication of lenvatinib. A 73 year old man with p53 mutated anaplastic thyroid cancer underwent total thyroidectomy, and repeat imaging 3 weeks after surgery revealed recurrent tumor in the right neck, mediastinal lymphadenopathy, and bilateral pulmonary nodules. Lenvatinib was initiated. Restaging studies 2 months later revealed bilateral pneumothoraces, increased pulmonary metastases with cavitation, and progression of large necrotic recurrence in the right neck. He was hemodynamically stable with saturations of $94 \%$ on room air. He reported development of dyspnea 4 days prior without cough, wheezing, chest pain or recent trauma. He underwent an emergent left-sided chest tube. Lenvatinib was held. A right-sided chest tube was placed, and subsequent pleurodesis was performed on the left hemithorax. Radiation therapy to the tumor bed was performed. Lenvatinib was resumed at a lower dose after chemical pleurodesis. Due to progressive malignancy, he was discharged to hospice. Simultaneous bilateral spontaneous pneumothorax is extremely rare, and in our patient may have been related to his targeted therapy and malignancy. Lenvatinib is a multiple receptor tyrosine kinase (RTK) inhibitor that impedes the kinase activities of various RTKs to impede pathogenic angiogenesis, tumor growth, and cancer progression. Other agents such as bevacizumab, which binds VEGF, notoriously may lead to gastrointestinal or mediastinal fistulas. Pazopanib is another angiogenesis inhibitor agent that has been associated with pneumothorax in those with pulmonary metastases. Necrosis of metastatic lesions leading to a pleural defect has been postulated with pazopanib. In our patient, the development of bilateral pneumothoraces was likely due to the location of pulmonary metastases and lenvatinib. Pneumothorax in patients with pulmonary metastases is a rare complication with lenvatinib.

\section{Poster 139}

Thyroid Cancer Thursday Poster Clinical

RISK STRATIFICATION OF DIFFERENTIATED THYROID CARCINOMA IN PEDIATRIC PATIENTS

K. Kim ${ }^{1}$, w. kim ${ }^{1}$, S. Lee ${ }^{2}$, J. Jeong ${ }^{1}$, K. Nam ${ }^{1}$

${ }^{1}$ general surgery, Yonsei university, Seoul, Korea (the Republic of); ${ }^{2}$ yeungnam university, Seoul, Korea (the Republic of) 
Recently ATA initial and dynamic risk stratification have been found to be more valuable than static anatomic staging system in differentiated thyroid cancer and these strategies have been accepted for treatment in adult DTC patients. But there is not a standard guideline for treatment in pediatric DTC patients. The present study was designed to compare the clinical usefulness of response to initial therapy stratifications with ATA initial risk stratification. From August 1982 to December 2013, a total of 151 pediatric patients underwent thyroidectomy in Yonsei University Hospital. Among them, $144(95.3 \%)$ patients with complete clinical data and sustained follow-up were enrolled in this study. Clinicopathological features and surgical outcomes were analyzed by retrospective medical chart review. Mean follow up duration was $94.47 \pm 69.25$ monthsIn this study, mean tumor size was $2.15 \pm 1.24 \mathrm{~cm}$ and $82.9 \%$ patients had conventional PTC, $3.4 \%$ patients had FTC. Low risk patients had highest probability of excellent response to initial therapy $(50 \%)$. High risk patients had highest probability of biochemical and structural incomplete response to initial therapy (30.7\% and 10.5\%) and lowest probability of excellent response to initial therapy (26.9\%). The ATA initial system and response to initial therapy systems provided risk stratification regarding to not only disease free survival (DFS), disease specific survival (DSS) and the probability of having no evidence of disease at final outcome but also the probability of having biochemical persistent/recurrence disease at final outcome. The ATA initial system and response to initial therapy systems were independent risk factors for DFS $(p=0.001$ and $\mathrm{p}=0.002$, respectively). In this study, we demonstrated that dynamic risk stratification with adjusted response to initial therapy system can offer more useful prognostic information with ATA risk stratification in pediatric patients with DTC.

\section{Poster 140}

Thyroid Cancer Thursday Poster Clinical

\section{DYNAMIC RISK STRATIFICATION IN MEDULLARY THYROID CARCINOMA: SINGLE INSTITUTION EXPERIENCES}

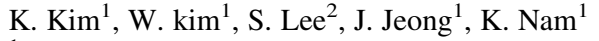

${ }^{1}$ General surgery, Yonsei university, Seoul, Korea (the Republic of);

${ }^{2}$ Yeungnam university, Seoul, Korea (the Republic of)

Recently dynamic risk stratification has been found to be more valuable than static anatomic staging system in non-medullary thyroid cancer and this strategy has also been accepted in medullary thyroid cancer (MTC). The present study was designed to compare the clinical usefulness of response to initial therapy stratification with a traditional anatomic staging system. From August 1982 to December 2012, a total of 144 MTC patients underwent thyroidectomy in Yonsei University Hospital. Among them, 117 (82.2\%) patients with complete clinical data and sustained follow-up were enrolled in this study. Clinicopathological features and surgical outcomes were analyzed by retrospective medical chart review. Mean follow up duration was $85.78 \pm 62.51$ months. In this study, mean tumor size was $1.94 \pm 1.40 \mathrm{~cm}$ and $22(18.9 \%)$ patients had hereditary MTC, 95 $(81.1 \%)$ patients had sporadic MTC. Stage I patients had highest probability of excellent response to initial therapy (92.1\%). Stage IV patients had highest probability of biochemical and structural incomplete response to initial therapy $(57.5 \%$ and $30.3 \%)$ and lowest probability of excellent response to initial therapy (12.1\%). Response to initial therapy stratification and TNM staging system were significantly difference in statistically $(p<0.001)$. The TNM staging system provided risk stratification regarding to disease free survival (DFS), disease specific survival (DSS) and the probability of having no evidence of disease at final outcome, but did not provide risk stratification regarding to the probability of having biochemical persistent/recurrence disease at final outcome. However response to initial therapy stratification provided risk stratification regarding to not only DFS, DSS and the probability of having no evidence of disease at final outcome but also the probability of having biochemical persistent/recurrence disease at final outcome. In this study, we demonstrated that dynamic risk stratification with adjusted response to initial therapy system can offer more useful prognostic information than anatomic staging system in MTC.

\section{Poster 141}

Thyroid Cancer Thursday Poster Clinical

\section{SHIFTING TRENDS IN THE CLINICOPATHOLOGICAL FEATURES AND SURGICAL OUTCOMES OF MTC}

K. Kim ${ }^{1}$, W. Kim ${ }^{1}$, S. Lee ${ }^{2}$, J. Jeong ${ }^{1}$, K. Nam ${ }^{1}$

${ }^{1}$ General surgery, Yonsei university, Seoul, Korea (the Republic of); ${ }^{2}$ Yeungnam university, Seoul, Korea (the Republic of)

Recently the early diagnosis of PTC has resulted in the improved clinical outcomes. But shifting trends of MTC over time remain controversial. The present study was designed to evaluate the changes of the clinicopathological features and surgical outcomes in patients with MTC in recent years. From August 1982 to December 2013, a total of 150 MTC patients underwent thyroidectomy in Yonsei University Hospital. Among them, 123 (82\%) patients with complete clinical data and sustained follow-up were enrolled in this study. Clinicopathologic features and surgical outcomes were analyzed by retrospective medical chart review. Mean follow up duration was $81.57 \pm 58.30$ months. In this study, the primary tumor size significantly decreased over time $(p<0.001)$ and the proportions of extrathyroidal invasion, central $\mathrm{LN}$ metastasis and lateral $\mathrm{LN}$ metastasis significantly decreased over time $(p<0.001, p<0.001$ and $p=0.035$, respectively). The level of postoperative serum calcitonin dramatically decreased over time $(p=0.002)$. Regarding primary tumor size, tumors sized $<=1.0 \mathrm{~cm}$ were seen in $41(33.3 \%)$ patients and tumors sized $>1.0 \mathrm{~cm}$ in $82(66.6 \%)$ patients. Patients with tumor sized $<=1.0 \mathrm{~cm}$ significantly increased over time $(p=0.003)$ and showed better clinicopathological features regarding to extrathyroidal invasion, central LN metastasis, lateral LN metastasis and postoperative serum calcitonin level compared to patients with tumor sized $>1.0 \mathrm{~cm}(p=0.001, p<0.001, p<0.001$ and $p<0.001$, respectively). Recurrence rates and disease-specific mortality rates were significantly decreased over time $(p<0.001$ and $p=0.001$, respectively) and disease-free survival rate of patients was improved over time $(p<0.001)$. In conclusion, we demonstrated that primary tumor size and extent of cervical LN metastasis in patients of MTC decreased significantly in recent years. Furthermore, the prognosis of MTC significantly improved over time.

\section{Poster 142}

Thyroid Cancer Thursday Poster Clinical

\section{PROGNOSTIC VALUE OF THE EIGHTH EDITION AJCC TNM CLASSIFICATION FOR DIFFERENTIATED THYROID CARCINOMA}

T. Kim, Y. Kim, H. Kim, S. Park, M. Choi, S. Park, S. Kim, J. Chung Samsung Medical Center, Seoul, Korea (the Republic of)

The prognostic value of the proposed eighth edition of the American Joint Committee on Cancer (AJCC) tumor, node, and metastasis (TNM) classification is currently unclear. The aim of the study was to evaluate the prognostic value of the eighth edition of the AJCC TNM classification. We retrospectively assessed 3,176 patients with differentiated thyroid carcinoma (DTC) who underwent thyroidectomy at a tertiary Korean hospital from 1996 to 2005. Cancer-specific 
survival (CSS) was analyzed using the Kaplan-Meier method and compared using the log-rank test. Performance of the eighth edition TNM with respect to prediction of CSS was assessed against the current seventh edition. Upon reclassification according to the eighth edition, $37.6 \%$ of patients were down-staged. The proportions of stage I and II tumors increased from $61.9 \%$ to $81.1 \%$ and from $1.7 \%$ to $16.0 \%$, respectively, whereas those of stage III and IVB (formerly IVC in the seventh edition) decreased from $27.6 \%$ to $2.3 \%$ and $0.8 \%$ to $0.5 \%$, respectively. The proportions of variance explained (PVEs) for the ability of the eighth and the seventh edition to predict CSS were $3.9 \%$ and $2.9 \%$, respectively. The C-index values were 0.765 (95\% confidence interval $0.764-0.766$ ) for the eighth edition and $0.736(0.735-0.737)$ for the seventh edition. Our results demonstrate that the eighth edition TNM more accurately predicts CSS for patients with DTC than does the seventh edition.

\section{Poster 143}

Thyroid Cancer Thursday Poster Clinical

\section{REAL CLINICAL EXPERIENCE OF SORAFENIB FOR TREATMENT OF PROGRESSIVE RADIOIODINE REFRACTORY DIFFERENTIATED THYROID CARCINOMA: KOREAN MULTICENTER STUDY}

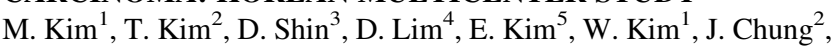
Y. Shong ${ }^{1}$, B. Kim ${ }^{6}$, W. Kim ${ }^{1}$

${ }^{1}$ Asan Medical Center, University of Ulsan College of Medicine, Seoul, Korea (the Republic of); ${ }^{2}$ Samsung Medical Center, Sungkyunkwan University School of Medicine, Seoul, Korea (the Republic of); ${ }^{3}$ Yonsei University College of Medicine, Seoul, Korea (the Republic of); ${ }^{4}$ Seoul St. Mary's Hospital, The Catholic University of Korea, Seoul, Korea (the Republic of); ${ }^{5}$ Dongnam Institute of Radiological and Medical Sciences Cancer Center, Busan, Korea (the Republic of); ${ }^{6}$ Biomedical Research Institute, Pusan National University Hospital, Busan, Korea (the Republic of)

Sorafenib, a multi-kinase inhibitor, has been approved for treatment of patients with radioactive iodine (RAI)-refractory differentiated thyroid cancer (DTC). Real-world studies determine how new drugs perform beyond the scope of clinical trial, because life does not always mirror the idealized world of a clinical trial. In this study, we aimed to evaluate the efficacy and safety of sorafenib in real clinical practice. This multicenter, retrospective cohort study evaluated 98 patients with progressive RAI-refractory DTC who were treated with sorafenib in 6 tertiary hospitals in Korea. The primary objective was the progression-free survival (PFS) according to Response Evaluation Criteria In Solid Tumors (RECIST), version 1.1. The overall survival (OS), response rate (defined as the best objective response according to RECIST), and safety were also evaluated. The median PFS was 9.7 months and median OS has not been reached during the follow-up. Partial responses and stable disease (SD) were achieved in 25 (25\%) and 64 $(65 \%)$ patients, respectively. SD more than 6 months was in 41 patients $(42 \%)$. In subgroup analysis, we identified several prognostic indicators of better PFS: absence of disease-related symptom $(\mathrm{HR}=0.5, \mathrm{P}=0.041)$, lung-only metastasis $(\mathrm{HR}=0.4, \mathrm{P}=0.048)$; thyroglobulin reduction $\geq 60 \%(\mathrm{HR}=0.4, \mathrm{P}=0.012)$; and daily maintenance dose $\geq 600 \mathrm{mg}$ ( $\mathrm{HR}=0.3, \mathrm{P}=0.005)$. The mean daily dose of sorafenib was $666 \pm 114 \mathrm{mg}$ and drug withdrawals due to adverse events (AEs) occurred in $13 \%$ of patients. AEs and serious AEs were reported in $93(95 \%)$ and $40(41 \%)$ patients, respectively. The most frequent AE was hand-foot skin reaction (76\%). Sorafenib improved PFS in real clinical setting, consistent with the results of DECISION trial. AEs were mostly mild and manageable. These results suggest that sorafenib is an effective treatment option for patients with progressive RAI-refractory DTC.

\section{Poster 144}

Thyroid Cancer Thursday Poster Clinical

\section{CLINICOPATHOLOGIC CHARACTERISTICS AND} SURGICAL OUTCOMES OF PTC PATIENTS WITH CYSTIC LATERAL LYMPH NODE METASTASES

W. Kim ${ }^{1}$, K. Kim ${ }^{1}$, M. Kim ${ }^{1}$, J. Choi ${ }^{1}$, S. Lee ${ }^{2}$, C. Lee ${ }^{1}$, S. Kang ${ }^{1}$, J. Lee ${ }^{1}$, J. Jeong ${ }^{1}$, K. Nam ${ }^{1}$, W. Chung ${ }^{1}$

${ }^{1}$ Surgery, Yonsei University College of Medicine, Seoul, Korea (the Republic of ); ${ }^{2}$ surgery, Yeungnam University College of Medicine, Daegu, Korea (the Republic of)

Papillary thyroid carcinoma (PTC) is the most common thyroid malignancy and tends to metastasize rather early to local lymph nodes (LNs). Incidence of cystic LN metastases are relatively rare comparing to solid LN metastases, and the relationship of PTC and cystic LN metastases in the lateral neck has been reported in the literature over the past several decade. Few studies, however, have attempted to assess the clinicopathologic features and surgical outcomes in these patients. This study aimed to compare the clinicopathologic characteristics and surgical outcomes of cystic LN metastases with solid LN metastases.

We performed a retrospective review of 1028 patients with N1b PTC patients who underwent total thyroidectomy (TT) and MRND between January 2005 and September 2011. Of these, 136 patients $(13.2 \%)$ were diagnosed cystic LN metastases, and 892 (86.8\%) had solid LN metastases. Cliniopathologic characteristics and surgical outcomes were compared in these two groups. Comparing to solid LN metastases, cystic LN metastases had significantly more male patients (42/136 [30.9\%] vs. 193/892 [21.7\%]; $\mathrm{p}=0.029)$, tumor multiplicity $(27 / 136$ [19.9\%] vs. $113 /$ 892 [12.7\%]; $p=0.048)$, number of positive lateral LNs $(6.9 \pm 6.3$ vs. $5.5 \pm 4.6 ; \mathrm{p}=0.018)$, extranodal extensions $(78 / 136$ [57.4\%] vs. $406 / 892$ [45.5\%]; $\mathrm{p}=0.017)$, multiple level metastases $(105 / 136$ [77.2\%] vs. 406/892 [64.7\%]; $p=0.002)$, and recurrence $(19 / 136$ [15.2\%] vs. $27 / 892$ [2.8\%]; $\mathrm{p}<0.001)$. Cox-regression analyses revealed cystic LN metastases as a major contributing factor for the short recurrence-free survival (RFS) (Hazard ratio (HR) 5.631, 95\% confidence interval (CI) 3.002-10.565; $<<0.001)$. This study demonstrates that cystic lateral LN metastases are associated with aggressive tumor behavior in PTC patients and its presence is a significant independent prognostic factor for RFS. Thus, surgeons should consider aggressive treatment to minimize local recurrence in these patients

\section{Poster 145}

Thyroid Cancer Thursday Poster Clinical

\section{CURRENT TRENDS IN THE FEATURE OF MALE THYROID CANCER : EVALUATION OF ITS PROGNOSTIC VALUE}

W. Kim ${ }^{1}$, K. Kim ${ }^{1}$, M. Kim ${ }^{1}$, J. Choi ${ }^{1}$, S. Lee ${ }^{2}$, C. Lee ${ }^{1}$, S. Kang ${ }^{1}$, J. Lee ${ }^{1}$, J. Jeong ${ }^{1}$, K. Nam ${ }^{1}$, W. Chung ${ }^{1}$

${ }^{1}$ surgery, yonsei university college of medicine, Seoul, Korea (the Republic of); ${ }^{2}$ surgery, Yeungnam university college of medicine, Daegu, Korea (the Republic of)

Thyroid cancer is the most common endocrine cancer with gender disparity, and male patient with papillary thyroid cancer (PTC) have been shown more aggressive disease and worse survival than female patient in the previous studies. The incidence of thyroid cancer has continuously increased in the last three decades all over the world, and the increase has been shown mainly in papillary microcarcinoma (PMC). This study aimed to review changing trends in male PTCs and suggest treatment strategies to early stage of PTCs in male 
patients by comparing patient characteristics and oncologic outcomes with female PTCs. Between January 2007 and December 2010, 8508 patients with PTC underwent thyroid surgery in the endocrine surgery division of the Severance Hospital, Yonsei University College of Medicine. Of these, 1232 patients (14.5\%) were male patients, and $7276(85.5 \%)$ were female. First, an exact 1:4 matching for operation type was performed to decrease the risk of confounding variables, and the patient characteristics and 5-year oncologic outcomes were compared in the matched groups. Second, we compared 270 male and 1501 female patients who received hemithyroidectomy after applying exact 1:4 matching analysis for age. Male patients had significantly more lymph node (LN) metastases than female, both in total $(612 / 1232$ [49.7\%] vs. $2537 / 7276$ [33.9\%]; $\mathrm{p}<0.0001)$ and hemithyroidectomy $(82 / 270$ [30.4\%] vs. $285 / 1501$ [19.0\%]; $\mathrm{p}<0.0001)$ patients. In hemithyroidectomy patients, tumor size $\leq 2.5 \mathrm{~mm}$ in male would have same nodal status with female. In pN1 hemithyroidectomy patients, male and female had the similar proportion of LN micrometastases (65/82 [79.3\%] vs. $130 / 161$ [81.7\%]; $p=0.962)$ and similar size of metastatic LNs $(5.2 \pm 2.3$ vs. $6.2 \pm 2.5 ; \mathrm{p}=0.203)$. Even with different nodal status, male and female had similar 5-year recurrence-free survivals, both in total $(55.7 \pm 17.8$ vs. $59.0 \pm 17.9 ; \mathrm{p}=0.815)$ and hemithyroidectomy $(54.2 \pm 18.5$ vs. $66.0 \pm 16.9 ; \mathrm{p}=0.148)$ patients. According to our data, more aggressive treatment strategies seems unnecessary to male patients, especially in small PMCs. Prospective randomized clinical trials with long-term follow-up data are needed to validate these results.

\section{Poster 146}

Thyroid Cancer Thursday Poster Clinical

\section{YONSEI EXPERIENCE OF 5,000 TRANSAXILLARY ROBOTIC THYROIDECTOMIES}

W. kim, K. Kim, M. Kim, J. Choi, C. Lee, J. Lee, S. Kang, J. Jeong, K. Nam, W. Chung

Department of Surgery, Severance Hospital, Yonsei Cancer Center, Yonsei University College of Medicine, Seoul, Korea (the Republic of)

Since the use of robot system in the thyroid surgery was first introduced in 2007 , we have advanced a novel method of robotic thyroidectomy (RT) using a gasless transaxillary approach (TAA). The authors herein report our experience with this technique and detail the surgical outcome of 5000 patients. From October 2007 to May 2016, 5000 patients with thyroid tumor underwent gasless transaxillary RT in the Department of Surgery, Yonsei University Health System. Of these, 4804 patients (96\%) had thyroid cancer and 196 patients (4\%) had benign thyroid tumor. Less than total thyroidectomy (LTT) was performed in 3123 patients $(62.5 \%)$ and 1543 (30.9\%) underwent bilateral total thyroidectomy (BTT). Robotic modified radical neck dissection (MRND) was performed in the remaining 334 patients $(6.7 \%)$ who had lateral neck node metastases. The most common subtype of thyroid cancer was papillary histology (98\%). The 196 benign tumors consisted of 104 adenomatous hyperplasia (52\%), 43 follicular adenoma (21\%), 30 Graves' disease (15\%), and so on. The mean operation time was $113.9 \pm 29.7$ minutes in LTT and $142.9 \pm 33.9$ minutes in TT, respectively. In robotic MRND, the mean operation time was $284.2 \pm 52.6$ minutes, and the mean number of retrieved central and lateral node were $6.1 \pm 5.0$ and $31.1 \pm 12.1$, respectively. Among thyroid cancer patients, locoregional recurrence was observed in 26 patients $(0.5 \%)$. The ultimate goals of gasless transaxillary robotic thyroid surgery are to achieve the best possible oncologic outcomes and to enhance patient quality of life after surgery.

\section{Poster 147}

Thyroid Cancer Thursday Poster Clinical

A PRAGMATIC RANDOMIZED CLINICAL TRIAL COMPARING MOLECULAR MARKERS FOR INDETERMINATE THYROID NODULES: AN INTERIM ANALYSIS

M.J. Livhits ${ }^{1}$, E.J. Kuo ${ }^{1}$, K.A. Zanocco ${ }^{1}$, A.M. Leung ${ }^{2}$, D.S. Cheung ${ }^{2}$, Y. Gofnung ${ }^{2}$, M. Yeh ${ }^{1}$

${ }^{1}$ Surgery, UCLA David Geffen School of Medicine, Los Angeles, CA; ${ }^{2}$ Endocrinology, UCLA David Geffen School of Medicine, Los Angeles, CA

Molecular testing for indeterminate thyroid nodules allows more patients with benign nodules to avoid surgery. There are no studies directly comparing different tests, and long-term follow-up is lacking for patients with benign molecular testing who do not undergo surgery. The objective of this study was to compare the value delivered by Afirma Gene Expression Classifier (GEC) and ThyroSeq v.2 gene mutation panel (GMP) in the context of real-world clinical decisionmaking. Patients who underwent thyroid fine-needle aspiration (FNA) throughout the UCLA Health System (5/2016 - 4/2017) were block randomized by month to GEC or GMP. A molecular test sample was collected during the biopsy and reflexively sent if the FNA was indeterminate (Bethesda 3 or 4). Treatment recommendations were made using best practices, incorporating molecular test results. Patients who had surgery had histopathologic evaluation, while the remainder was followed with bi-annual ultrasounds. Of 1,143 thyroid nodules, $74.6 \%$ were benign, $5.7 \%$ were suspicious for malignancy/malignant, and $13.0 \%$ were indeterminate. Of the indeterminate group, 138 nodules $(92.6 \%)$ were included in the study (median patient age 59 years, $82 \%$ female). The median nodule size was $2.0 \mathrm{~cm}$ (interquartile range $1.5-3 \mathrm{~cm}$ ). Of nodules tested with GEC $(\mathrm{N}=63), 39.0 \%$ were suspicious, $49.2 \%$ were benign, and $11.9 \%$ were insufficient for analysis. Of nodules tested with GMP $(\mathrm{N}=67), 19.4 \%$ had a positive mutation, $76.1 \%$ had a negative result (no mutation), and $4.5 \%$ were insufficient. The positive predictive value was $53.9 \%$ for GEC and $54.6 \%$ for GMP. Diagnostic hemithyroidectomy was avoided in 26 patients tested with GEC $(41.3 \%)$ and 41 patients tested with GMP $(61.2 \%)$. Of 11 nodules with a negative molecular test ( 3 GEC and 8 GMP) that were operated on due to patient preference, all had benign histopathology. Follow-up ultrasounds at 6-12 months were available for 30 nodules: 27 were stable, and 3 had growth of 2-3 mm and underwent repeat FNA with benign results. GEC and GMP had a similar PPV, although GMP allowed more patients to avoid surgery based on a negative test result. Long-term ultrasound surveillance is crucial to compare the false-negative rate between the tests.

\section{Poster 148}

Thyroid Cancer Thursday Poster Clinical

\section{DECREASING USE OF RADIOACTIVE IODINE FOR} LOW-RISK THYROID CANCER IN CALIFORNIA, 1999-2012 K. Park ${ }^{1}$, J.X. Wu ${ }^{1}$, L. Du ${ }^{2}$, A.M. Leung ${ }^{3,4}$, M. Yeh ${ }^{1}$, M.J. Livhits ${ }^{1}$ ${ }^{1}$ Surgery, UCLA David Geffen School of Medicine, Los Angeles, CA; ${ }^{2}$ Biostatistics, UCLA Fielding School of Public Health, Los Angeles, CA; ${ }^{3}$ Endocrinology, UCLA David Geffen School of Medicine, Los Angeles, CA; ${ }^{4}$ Endocrinology, VA Greater Los Angeles Healthcare System, Los Angeles, CA

Radioactive iodine (RAI) ablation in patients with low-risk differentiated thyroid cancer (DTC) has not been proven to decrease recurrence. American Thyroid Association (ATA) guidelines have discouraged its routine use since 2009. The objective of this study 
was to analyze trends in RAI ablation over time and to determine whether the rate has decreased in low-risk patients in response to the 2009 guidelines. The California Cancer Registry was used to identify patients with DTC who underwent total thyroidectomy from 19992012. Patient demographics and tumor characteristics (size, disease stage, and histological subtype) were analyzed. Disease stage was defined as local (confined to thyroid), regional (extrathyroidal extension or nodal metastasis), or distant. The rate of RAI ablation was determined annually and dichotomized (1999-2009 vs. 2010-2012) based on the 2009 ATA guidelines. Of 37,848 patients with DTC who underwent surgery (mean age 46.9 \pm 15.4 [SD] years, $76.4 \%$ female), 20,594 (54.4\%) received RAI. Mean tumor size was $2.5 \pm 4.4[\mathrm{SD}] \mathrm{cm}$. The proportion of patients with regional/distant disease who received RAI was $66.4 \%$, and this remained stable over the study period. The rate of RAI for patients with local disease decreased from $53.8 \%$ in 1999 to $36.2 \%$ in 2012 . The most significant change in RAI ablation occurred in tumors $<1 \mathrm{~cm}$ (35.6\% in 1999 to $18.2 \%$ in 2012). The rate also decreased for tumors between $1-2 \mathrm{~cm}$ (61.2\% in 1999 to $46.9 \%$ in 2012$)$ and $2-4 \mathrm{~cm}(66.2 \%$ in 1999 to $53.1 \%$ in 2012), and it remained stable at $55 \%$ for tumors $>4 \mathrm{~cm}$. In multivariable analysis, patients with local disease were less likely to receive RAI if they were $>65$ years (OR $0.76,95 \%$ CI: $0.70-0.84$ ), had tumors $<1 \mathrm{~cm}$ (OR $0.35,95 \%$ CI: $0.32-0.37)$, and were treated in an academic versus non-academic hospital (OR 0.75, 95\% CI: 0.690.79). RAI ablation for patients with low-risk DTC has decreased over time, mostly attributable to a decrease in RAI for localized microcarcinomas. Academic hospitals have adopted recommended treatment guidelines earlier than non-academic centers. Despite ATA guidelines, many patients still receive unnecessary RAI for low-risk DTC (particularly for local tumors between $1-4 \mathrm{~cm}$ ).

\section{Poster 149}

Thyroid Cancer Thursday Poster Clinical

\section{PATIENTS' AND PROVIDERS' REACTIONS TO A PTMC DIAGNOSIS}

M.F. Bates, M.C. Saucke, J.L. Jennings, H.J. Khokhar, S.C. Pitt University of Wisconsin, Madison, Madison, WI

We previously showed that patients with papillary thyroid cancer $>1 \mathrm{~cm}$ (PTC) have an emotional response to their diagnosis and a strong urge to "get it out," but little data currently exists about reactions to a diagnosis of papillary microcarcinoma (PTMC). The aim of this study was to examine patient and provider experiences with the PTMC diagnosis. This qualitative study used semistructured interviews with 10 patients who had PTMC, and 12 endocrinologists and 12 Surgeons who treat PTMC. We performed thematic content analysis of interview transcripts until saturation was reached $(n=21)$. While almost all patients $(90 \%)$ discussed "cancer" as having grave connotations, only $30 \%$ recalled strong emotional reactions to their own PTMC diagnosis. Reactions ranged from "it didn't really affect me that much," to "complete emotional breakdown." When educated about the relatively benign nature of PTMC, most patients still expressed a need to "get it out." Their reasons included the provider's recommendation, prevention of cancer spread, and peace of mind that the cancer was gone. Providers described their patients with PTMC as having strong reactions to their diagnosis. The " $\mathrm{C}$ word" evokes fear and anxiety, and often drives patients to want surgery: "Their gut reaction is that it's cancer, it needs to come out." Providers felt patients still preferred surgery even after being educated about the very low risks of adverse outcomes of PTMC. They also believed patients' reactions were due to misinformation from other sources, past experiences with cancer, and cultural fear of cancer. Most providers also expressed a desire to "get it out" because they feared the rare bad outcome and desired to fix the problem. Many providers felt patients expected thyroidectomy and were better off with a surgical treatment. Therefore, they tailored their recommendation to perceived patient preference. Patients with PTMC and their providers both express a desire to "get it out" regardless of indolence or tumor size. Reactions to the "C word" and its cultural stigma likely play a role in choosing surgery and the potential overtreatment of PTMC. Understanding these responses may facilitate less aggressive treatment.

\section{Poster 150}

Thyroid Cancer Thursday Poster Clinical

\section{SERUM THYROGLOBULIN LEVELS CAN PREDICT THE PRESENCE OF DISEASE BUT NOT THE EXTENT}

M.F. Bates, R. Randle, K.L. Long, S.C. Pitt, D. Schneider, R. Sippel

Surgery, University of Wisconsin, Madison, WI

Serum thyroglobulin (Tg) is used to detect recurrent or residual disease in patients with papillary thyroid carcinoma (PTC), but it is unclear if degree of Tg elevation correlates with disease burden. We aimed to determine if $\mathrm{Tg}$ levels predicted extent of disease in patients with recurrent PTC. We conducted a retrospective review of a prospectively maintained surgical database. Forty-four patients that had a reoperation for nodal disease from 2000-2016 were included. We collected thyroglobulin levels before and after reoperation and calculated the $\Delta \mathrm{Tg}$ (preoperative-postoperative). We then correlated preoperative $\mathrm{Tg}$ and $\Delta \mathrm{Tg}$ with lymph node (LN) pathology. The median unstimulated $\mathrm{Tg}$ prior to reoperation was $5.7 \mathrm{ng} / \mathrm{mL}(0.1-$ 49.9). Over half of patients had preoperative Tg levels $<5 \mathrm{ng} / \mathrm{mL}$ (52.3\%), $23 \%$ had $\mathrm{Tg}<1 \mathrm{ng} / \mathrm{mL}$, and $14 \%$ had $\mathrm{Tg}<0.5 \mathrm{ng} / \mathrm{mL}$. At surgery a median of $4(1-11)$ positive $\mathrm{LNs}$ were removed and the size of the largest node was $1.6 \pm 1.0 \mathrm{~cm}$. Postoperative Tg levels decreased by $71 \%$, mean postoperative $\mathrm{Tg}$ was $3.1 \pm 4.7 \mathrm{ng} / \mathrm{mL}$, and $\Delta \mathrm{Tg}$ was $8.2 \pm 11.8 \mathrm{ng} / \mathrm{mL}$. The preoperative $\mathrm{Tg}$ and $\Delta \mathrm{Tg}$ correlated weakly $\left(\mathrm{r}^{2}=0.315\right.$ and 0.340$)$ with number of positive LNs $(\mathrm{p}<0.005)$ and poorly with size of largest $\mathrm{LN}\left(\mathrm{r}^{2}=0.085\right.$ and 0.096$)$. $\Delta \mathrm{Tg}$ associated with removal of a positive node ranged from $<0.1-$ $16.5 \mathrm{ng} / \mathrm{mL}$. Sixteen percent of patients had preoperative $\mathrm{Tg}<1 \mathrm{ng} / \mathrm{mL}$ but had $\geq 3$ involved LNs. While a positive Tg equates to the presence of disease, it does not correlate with extent of disease. These data suggest there is no reliable cut-off for unstimulated $\mathrm{Tg}$ levels that can be used to eliminate the presence of residual nodal disease.

\section{Poster 151}

Thyroid Hormone Action Thursday Poster Basic

DECREASED THYROID HORMONE SIGNALING IN HIPPOCAMPUS AND PRE-FRONTAL CORTEX OF RATS WITH OBESITY-INDUCED DEPRESSION IS PARTIALLY CORRECTED BY DITPA F.B. Lorena
A.C. ${ }^{1}$, Jianco

${ }^{1}$ Biological Science, Universidade Presbiteriana Mackenzie - UPM, Sao Paulo, Brazil; ${ }^{2}$ Department of Internal Medicine, Division of Endocrinology and Metabolism, Rush University and Medical Center, Chicago, IL

Thyroid hormone (TH) modulates the central nervous system (CNS) by regulating the expression of genes related to brain plasticity. There is a well described relationship between thyroid hormone and depression that may be reversed by treatment with T3. A risk factor for depressive behavior is obesity, and there is substantial overlap between the CNS genes affected by obesity and T3. Here we tested the hypothesis that obesity changes $\mathrm{T} 3$ signaling in the brain that may be involved in the onset of depression observed in these animals. We 
also tested whether the T3 analogue DITPA could revert these alterations. Male Wistar rats were fed a high fat diet (40\%) for 32 weeks. At the end of this period animals were tested for memory acquisition, anxiety and/or depressive behavior. A control group was fed with a high fat diet and also treated with on DITPA $(10 \mathrm{mg} / \mathrm{kgBW} /$ day; i.p. for 21 days). The expression of T3-responsive genes, genes involved in depression and inflammation was measured by RT-qPCR in pre-frontal cortex and hippocampus. Obese rats exhibited an anxiety/depressive behavior but memory acquisition remained intact. We found an increase in mRNA levels for OATP1, $\operatorname{TR} \alpha, \operatorname{TR} \beta$, PPARgama and PPARbeta in the hippocampus and pre-frontal cortex of obese rats. Also, the expression of two positively T3-regulated genes, Aldh1a1 and RBM3, was decreased while Halpln1, a negatively T3-regulated gene, was increased, all suggesting decreased thyroid hormone signaling. While treatment with DITPA normalized the expression of OATP1, TR $\alpha$ and $\operatorname{TR} \beta$, it failed to eliminate the anxiety/depressive behavior within the time-frame of the experiment. Our data indicate that obese rats exhibit decreased thyroid hormone signaling in the brain. Even though it is logical to assume that the local hypothyroidism could contribute with the anxiety/depressive behavior observed in obese rats, normalization of the T3-dependent gene expression was not associated with normalization of the humor phenotype.

\section{Poster 152}

Thyroid Hormone Action Thursday Poster Case Report AN INTRAVAGAL PARATHYROID CYST INDUCED HYPERCALCEMIC CRISIS

J. Lee

Surgery, Gachon University, Incheon, Korea (the Republic of)

Parathryoid cysts (PC) and intravagal parathyroid adenomas are very rare endocrine disease entities with less than 400 and 10 cases reported in the medical literature respectively. To the best of our knowledge, we report the first case of an intravagal PC diagnosed preoperatively and confirmed at operation. An 84 year-old women presented with symptoms of hypercalcemic crisis. Initial lab reports were indicative of primary hyperparathyroidism while parathyroid single-photon emission computed tomography and ultrasonography indicated a $4 \mathrm{~cm}$ cystic lesion at the left level IV compartment. The patient exhibited paroxysmal coughing upon fine needle aspiration and the aspirate demonstrated parathyroid hormone (PTH) level of $1399 \mathrm{pg} / \mathrm{mL}$ (normal range, $10-65 \mathrm{pg} / \mathrm{mL}$ ). During operation a $4 \mathrm{~cm}$ cystic lesion embedded within the vagus nerve was enucleated. The procedure was facilitated by aspirating the cystic contents. Histologic study reported parathyroid tissue within neural fibers. The patient remains symptomless after the operation and both immediate post-operative post 3 month labs are controlled within normal range. The enucleation was difficult because the nerve had to be minimally dissected to preserve vagus nerve functions while leaving the cyst completely intact without perforation. Moreover, the capsule of the cyst was intertwined with the neural fibers. In this case we attempted to reserve as much nerve fasciclse as possible by aspirating the cystic material with a 26 gauge needle and thus facilitating enucleation with minimal nerve dissection. As her post-operative biochemical status and symptoms of hypercalcemic crisis have resolved, aspirating the cyst before enucleation may be a useful method in preserving the vagal fibers while effectively removing the lesion. The enucleation of the intravagal PC, the first to be reported in medical literature, is hindered by the task of resecting the cystic lesion completely while damaging minimal nerve fibers at the same time. Despite the technical difficulties, saving all vagal fibers while completely removing the lesions is of paramount importance.

\section{Poster 153}

Thyroid Hormone Action Thursday Poster Case Report

SEVERE HYPERTRIGLYCERIDEMIA UNLEASHED BY LEVOTHYROXINE WITHDRAWAL IN PATIENT WITH DIFFERENTIATED THYROID CANCER

G. Sigal ${ }^{1}$, T. Tavoni ${ }^{1}$, L. Brandão ${ }^{3}$, R. Maranhão ${ }^{1,2}$

${ }^{1}$ Heart Institute Medical School Hospital University of São Paulo, São- Paulo, Brazil; ${ }^{2}$ Faculty of Pharmaceutical Science, University of São Paulo, Sâo Paulo, Brazil; ${ }^{3}$ Department of Head and Neck Surgery, Medical School Hospital, University of São Paulo, São-Paulo, Brazil

Severe hypertriglyceridemia is associated with acute pancreatitis. Thyroid dysfunctions may be associated with hypertriglyceridemia. We present a case of a 41-years-old female patient diagnosed with differentiated thyroid cancer. Patient denies diabetes, family history of dyslipidemia or use of any medications. BMI was $33,8 \mathrm{~kg} / \mathrm{m}^{2}$. Triglycerides plasma concentrations in the past were $270 \mathrm{mg} / \mathrm{dl}$ and $261 \mathrm{mg} / \mathrm{dl}$. Before thyroidectomy, in euthyroidism, triglycerides were $146 \mathrm{mg} / \mathrm{dl}$.

After thyroidectomy patient was put on levothyroxine (LT4). Three months later, LT4 was withdrawn in preparation for whole body scan (WBS). One week before WBS, 22 days after LT4 withdrawal, patient was in overt hypothyroidism. Triglycerides were $679 \mathrm{mg} / \mathrm{dl}$. Patient was without medications.

On the next day after radioiodine therapy (RIT) patient was restarted on LT4 $125 \mathrm{mcg} /$ day and oriented to continue no sugar, very low carbohydrate diet. Six weeks after RIT, on LT4 regular use, patient was in subclinical hyperthyroidism. Triglycerides concentration was $665 \mathrm{mg} / \mathrm{dl}$. Patient was not taking medications other than LT4. Ciprofibrate $100 \mathrm{mg}$ /day dose was then introduced and after 4 month treatment triglycerides went down to $107 \mathrm{mg} / \mathrm{dl}$. Alteration of triglycerides levels in overt hypothyroidism are reported normal or slightly elevated.

In our patient, the huge elevation of triglycerides was apparently related to LT4 withdrawal. Even after LT4 was restarted and the patient was in subclinical hyperthyroidism triglycerides remained at the same levels.

The current case is one among 18 patients with overt hypothyroidism consequent to thyroidectomy for thyroid cancer we have studied, but the other did not present alterations in triglycerides during follow-up. A genetic covert cause aggravated by overt hypothyroidism could explain the finding in this patient? In this regard, mutations or polymorphism of several genes, such as LPL, APOC2, APOA5(4), GPIHBP1, and LMF1 could have been implicated in the above-described findings. Our finding in this patient highlights the importance of determining plasma triglycerides during the periods subsequent to thyroidectomy, particularly having in mind that this determination is not usually requested.

\section{Poster 154}

Thyroid Hormone Action Thursday Poster Clinical THYROID HORMONE EFFECTS ON NON-INSULIN MEDIATED GLUCOSE DISPOSAL IN PATIENTS WITH INSULIN RECEPTOR MUTATIONS: PRELIMINARY RESULTS OF A SHORT AND LONG-TERM STUDY Y. Kushchayeva ${ }^{1}$, M. Startzell ${ }^{1}$, E. Cochran ${ }^{1}$, W. Dieckmann ${ }^{2}$, M. Skarulis ${ }^{1}$, A. Cypess ${ }^{1}$, T. Lin ${ }^{1}$, S. Kushchayev ${ }^{3}$, P. Gorden ${ }^{1}$, R. Brown ${ }^{1}$

${ }^{1}$ NIDDK/NIH, Bethesda, MD; ${ }^{2} \mathrm{CC} / \mathrm{NIH}$, Bethesda, MD; ${ }^{3}$ Radiology, Mercy Catholic Medical Center, Philadelphia, PA

Patients with insulin receptor mutations (IRM) have extreme insulin resistance and are at risk for early morbidity and mortality from diabetes complications with limited efficacy of available diabetes drugs. 
Skarulis et al reported a patient with homozygous IRM in whom suppressive thyroid hormone $(\mathrm{TH})$ for papillary thyroid cancer led to normalization of $\mathrm{HbA} 1 \mathrm{c}$ and discontinuation of diabetes medications with activation of brown adipose tissue (BAT). Since THs are known to increase non-insulin mediated glucose uptake by BAT and muscle, we hypothesized that $\mathrm{TH}$ might ameliorate hyperglycemia in patients with impaired insulin signaling.

7 patients with homozygous $(n=5)$ or heterozygous $(n=2)$ IRM, $4 / 7$ male, age 15-30y, on stable medications for 10 weeks took liothyronine (T3) q8h for $2 \mathrm{wk}$, with additional $6 \mathrm{mo}$ treatment in those with $\mathrm{HbA} 1 \mathrm{c}>7 \%$. Doses were targeted to T3 peak $25-50 \%$ above the upper normal limit. Outcomes measured at baseline, $2 \mathrm{wk}$ and 6 mo on T3 included glucose uptake in muscle, white adipose tissue (WAT) and BAT by dynamic FDG-PET/CT, thyroid hormones, lipids, SHBG, FFA, HbAlc, fructosamine, glucose, insulin, cpeptide, bone density by DXA, energy expenditure (EE) and respiratory quotient $(\mathrm{RQ})$.

Mean age was $22.2 \pm 6.5 \mathrm{yr}$; mean BMI $21.9 \pm 9.3 \mathrm{~kg} / \mathrm{m} 2$. No significant change in bone density, weight, or vital signs except SBP was found over $2 \mathrm{wk}$ or $6 \mathrm{mo}$. There were significant decreases in total cholesterol, HDL, LDL, LDL particle number and increase in SHBG and FFA as expected. No TH effect on c-peptide, insulin, $\mathrm{RQ}$, or EE was seen. Fructosamine decreased after $2 \mathrm{wk}$ of T3 $(\mathrm{p}=0.03)$ with no change in either fructosamine or HbA1c after $6 \mathrm{mo}$. Average glucose uptake by muscle and WAT assessed by FDG-PET/CT did not change after $2 \mathrm{wk}$, but was significantly higher at $6 \mathrm{mo}$ (muscle: baseline $0.47 \pm 0.04,2 \mathrm{wk}=0.68 \pm 0.03$; $6 \mathrm{mo}=1.6 \pm 0.06$; WAT: baseline $0.9 \pm 0.1 ; 2 \mathrm{wk}=0.7 \pm 0.08$; $6 \mathrm{mo}=1.87 \pm 0.25 \mathrm{umol} / \mathrm{min} / 100 \mathrm{~g}$ with no increase in BAT glucose uptake.

TH treatment was well tolerated with no significant side effects. TH did not improve glycemia in $6 \mathrm{mo}$ of treatment. Longer exposure or higher doses of TH may be needed to improve glycemia in patients with IRM.

\section{Poster 155 \\ Thyroid Hormone Action Thursday Poster Clinical BIOEQUIVALENCE OF A NEW LEVOTHYROXINE SODIUM ORAL SOLUTION IN HEALTHY VOLUNTEERS UNDER FASTING CONDITIONS}

M. Tanguay ${ }^{1}$, J. Girard ${ }^{1}$, C. Scarsi ${ }^{2}$, G. Mautone ${ }^{2}$, R. Larouche ${ }^{1}$ ${ }^{1}$ inVentiv Health, Montreal, Quebec, Canada; ${ }^{2}$ IBSA Institut Biochimique SA, Lugano, Switzerland

A new formulation of levothyroxine (LT4) sodium has been developed in the form of an oral solution contained in unit-dose ampules, with strengths spanning from 13 to $200 \mu \mathrm{g}$. The product has been formulated in agreement with the strict potency guideline set forward by the FDA, requiring $95 \%$ to $105 \%$ potency specification over the entire shelf-life. A study has been conducted to assess the bioequivalence (BE) between LT4 oral solution (by IBSA Institut Biochimique SA, Test) and LT4 capsule (Tirosint ${ }^{\circledR}$, Reference), under fasting conditions. The rate and extent of absorption of the new LT4 solution was also evaluated when administered upon dilution in water or directly into the mouth without water. In each period, according to the randomization scheme, subjects were administered single oral doses of either Test or Reference, as $4 \times 150 \mu \mathrm{g}$ unit-dose ampules, with or without water, or $4 \times 150 \mu \mathrm{g}$ Tirosint ${ }^{\circledR}$ capsules in cross-over. Treatment periods were separated by washout phases of 35 days. In each period, 19 blood samples were drawn, and LT4 was analyzed in serum samples using a validated LC/MS/MS method. Thirty-six (36) subjects were randomized and dosed in this study; of these, 31 completed all study periods. All formulations were well tolerated, with no major side effects. When comparing the solution to the capsule (both products administered with water), the least-squares means (LSM) ratios (90\% geometric confidence intervals (CIs)) of $\mathrm{AUC}_{0-48}$ and $\mathrm{C}_{\max }$ were respectively $98.47 \%(94.97 \%-102.11 \%)$ and $95.33 \%$ (91.97\%-98.82\%) for baseline corrected LT4. Moreover, the administration of LT4 solution without water did not affect its rate and extent of absorption with respect to administration with water, with LSM ratios (90\% geometric CIs) of $\mathrm{AUC}_{0-48}$ and $\mathrm{C}_{\max }$ that were respectively $102.72 \%(98.98 \%-106.60 \%)$ and $99.28 \%(95.70 \%-$ $102.99 \%$ ). All $90 \%$ CIs for AUC and $\mathrm{C}_{\max }$ were contained within 90-111\%, which is the BE acceptance limit defined by EMA for narrow therapeutic index drugs. In conclusion, LT4 oral solution, administered with or without water under fasting conditions, is bioequivalent to Tirosint ${ }^{\circledR}$ capsules. Thanks to the unit-dose containers, the new formulation will allow exact dosing of the oral solution.

\section{Poster 156 \\ Thyroid Hormone Metabolism \& Regulation Thursday Poster Basic}

\section{SIMULTANEOUS MEASUREMENT OF T3, TA3 AND TA4 BY ISOTOPE DILUTION - LIQUID CHROMATOGRAPHY MASS SPECTROMETRY}

R. Jongejan ${ }^{1}$, M. Meima ${ }^{3,4}$, T. Luider ${ }^{2}$, R. Peeters ${ }^{3,4}$, T. Visser $^{3,4}$, Y.B. de Rijke ${ }^{1,4}$

${ }^{1}$ Clinical Chemistry, Erasmus MC, University Medical Center, Rotterdam, Netherlands; ${ }^{2}$ Neurology, Erasmus MC, University Medical Center, Rotterdam, Netherlands; ${ }^{3}$ Internal Medicine, Erasmus MC, University Medical Center, Rotterdam, Netherlands; ${ }^{4}$ Academic Centre for Thyroid Diseases, Erasmus MC, University Medical Center, Rotterdam, Netherlands

Antibody-based routine T3 assays generally show cross-reactivity with T3 metabolites such as 3,3',5-triiodothyroacetic acid (TA3). TA3 is used in the treatment of patients with thyroid cancer, and patients with TR $\beta$ or MCT8 mutations. Hence, we aimed to establish an isotope dilution-liquid chromatography mass spectrometry (ID-LC/MS) method to measure T3 and TA3 without cross-interference. Serum $(150 \mathrm{ul})$ was mixed with internal standard solution $\left(2 \mathrm{nM} \mathrm{T} 3-{ }^{13} \mathrm{C}_{6}, 800 \mathrm{pM}\right.$ TA3 $-{ }^{13} \mathrm{C}_{6}, 800 \mathrm{pM}$ TA4- ${ }^{13} \mathrm{C}_{6}$ ) followed by deproteinization with acetonitrile $+1 \%$ formic acid and a strong anion-exchange solid phase extraction (Waters SPE Oasis MAX). A UPLC-MS/MS system from Sciex ${ }^{\mathrm{TM}}$ QTRAP 5500 was used for chromatographic separation and subsequent detection by electrospray ionization in the negative mode with mass transitions $649.8>126.8 ; 649.8>505.8$ for T3, 576.7>126.9; $576.7>449.8$ for TA3 and $746.6>126.8$; $746.6>702.6$ for TA4. Validation of the lower limit of quantification (LLOQ) and the linearity were performed according to international guidelines (FDA, Clinical and Laboratory Standards Institute). To assess linearity, stripped serum was spiked with 11 different levels of T3, TA3 and TA4 $(0-20 \mathrm{nM})$. Linearity was accepted with a lack-of-fit $<3.29$. Each level was accepted with an imprecision $<15 \%$ (except LLOQ imprecision $<20 \%$ ). The linear correlation coefficient $\left(\mathrm{r}^{2}\right)$ was accepted if $>0.99$. For the LLOQ six levels $(0.4-2 \mathrm{nM})$ were prepared in serum. A signal-to-noise (S/N) of 20:1 and an imprecision $<20 \%$ was considered acceptable as LLOQ. The calibration curve was linear with a lack-of-fit of 0.22 for $\mathrm{T} 3$ and 0.98 for TA 3 and $\mathrm{r}^{2}>0.99$. The imprecision was $<15 \%$ for both T3 and TA3. The LLOQ of both T3 and TA3 was $800 \mathrm{pM}$. We developed an ID-LC/MS method that allows accurate and simultaneous quantification of T3 and TA 3 at concentrations as low as $800 \mathrm{pM}$ in a single run. This method can be used to monitor T3 and TA3 in individuals treated with TA3. 


\section{Poster 157}

Thyroid Hormone Metabolism \& Regulation Thursday

Poster Clinical

ISOLATED THYROID STIMULATING HORMONE ELEVATIONS ARE ASSOCIATED WITH POOR SLEEP: A CROSS-SECTIONAL AND LOGITUDINAL STUDY IN CHENGDU, CHINA

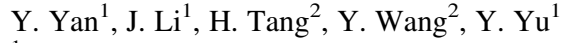

${ }^{1}$ Department of Endocrinology and Metabolism, West China

Hospital of Sichuan University, Chengdu, Sichuan, China; ${ }^{2}$ Health

Management Center, West China Hospital of Sichuan University, Chengdu, Sichuan, China

Isolated thyroid stimulating hormone (TSH) elevations and poor sleep are common in the clinic, and sleep exerts influences on TSH secretion. This study aimed to elucidate the relationship between isolated TSH elevations and poor sleep status and to investigate whether elevated TSH levels could normalize when sleep improved. Subjects with isolated TSH elevations and euthyroidism were included, and sleep status was first assessed by Pittsburgh Sleep Quality Index (PQSI). Patients with isolated TSH elevations were longitudinally followed-up, and TSH levels were re-measured when sleep status had improved. The rates of poor sleep and good sleep to occasional poor sleep among subjects with isolated TSH elevations were significantly higher than those in the euthyroidism group (70.24\% vs $49.58 \%, \mathrm{p}<0.05 ; 9.52 \%$ vs $1.68 \%, \mathrm{p}<0.05)$. Patients with isolated TSH elevations had significantly higher PSQI scores of subjective sleep quality, sleep latency, sleep duration, and habitual sleep efficiency than the euthyroidism subjects $(\mathrm{p}<0.05)$. In the follow-up study, among the patients with isolated TSH elevation at baseline, the ratio of TSH normalization in patients who slept better was significantly higher than in those who still slept poorly $(85.42 \%$ vs $6.45 \%, \mathrm{p}<0.05)$. Poor sleep was revealed in subjects with isolated TSH elevations, and elevated TSH may be more likely to normalize when sleep status improves. We recommend thorough inquiries into sleep history in the clinic and a reexamination of thyroid hormone levels when sleep status improves rather than a subclinical hypothyroidism $(\mathrm{SCH})$ diagnosis or even levothyroxine treatment.

\section{Poster 158}

Thyroid Imaging Thursday Poster Basic

\section{IMPACT OF CERVICAL ULTRASOUND PERFORMED BY THE SURGEON ON THE MANAGEMENT OF PATIENTS WITH THYROID DIDEASE IN BRAZIL}

R.M. Nagato, P.E. Portari

General Surgery, Universidade Federal do Rio de Janeiro, Rio de Janeiro, Rio de Janeiro, Brazil

This study aims to evaluate the impact of the cervical ultrasound examination by the surgeon planning the management of patients with thyroid disease in a country where such heterogeneous exams are performed and interpreted by a radiologist, not necessarily an especialist in thyroid disease. Patients referred to the general surgery service for evaluation of thyroid disease will have their examinations reviewed and repeated by the surgeon.

These will be compared and the change in surgical planning will be expressed as a percentage. Of 51 patients evaluated until now, 44 $(86.3 \%)$ are women. In $19(37.3 \%)$ there was a difference between the two ultrasounds. Of these, 19 cases, $11(21.5 \%)$ was significant enough to change the treatment plans. 3 patients had pseudonodules, characteristic of thyroiditis, and biopsy was avoided. 1 pyramidal lobe was confused with nodule. In 22 patients (43.1\%) there was no description of the lymph nodes in the pre-consultation examination, of these, 2 had suspicious lymph nodes and biopsy was performed. 3 cases that was planning a lobectomy due a single nodule was necessary a total thyroidectomy in a multinodular goiter. This study, like others published, confirms the clear benefit of performing ultrasound by the surgeon. Preventing unnecessary procedures, as well as change in planned surgery. Another important fact was the absence of lymph node investigation in a large percentage of patients.

\section{Poster 159}

Thyroid Cancer Thursday Poster Case Report

\section{CARCINOMA SHOWING THIMUS-LIKE} DIFFERENTIATION (CASTLE) OF THYROID: RARE DISEASE, LOW INDEX OF SUSPICION AND A DIFFICULT DIAGNOSE

R.M. Nagato, R.J. Carvalho, I.M. Compagnoni, P.E. Portari, R.P. Basílio, P.M. Alburquerque

General Surgery, Universidade Federal do Estado do Rio de Janeiro, Rio de Janeiro, Rio de Janeiro, Brazil

Although the majority of thyroid cancers are well-differentiated with an excellent prognosis. Rare tumors occasionally involve the thyroid gland and represent a clinical challenge.

This is a case report of CASTLE, managmented at Gaffree and Guinle University Hospital, highlighting its rarity, low preoperative index of suspicion and lack of standard treatment in the literature. Woman, 49 yo, with right cervical nodule with a fast growth, perceived in 2012. In 2013 she developed dyspnea and gagging. Thyroid ultrasonography evidenced an irregular hypoechoic nodule, with $4 \times 3 \times 3 \mathrm{~cm}$ in the right lobe of the thyroid, without cervical linofonodomegalia and fine needle aspiration suggestive of papillary thyroid carcinoma.

In 2014 she was operated, with a total thyroidectomy intention, However, the right lobe was adhered to the trachea and right carotid artery, resulting in remnants of thyroid tissue in these areas. Histopathologically compatible with mucoepidermoid tumor of the thyroid with angiolymphatic invasion, and questioning the possibility of secondary metastatic tumor. Tomography of skull, chest and abdomen, with no evidence of primary lesions. Immunoreactivity test: CEA, p63 and CD5 positive, CK pool, chromogram A, synaptophysin, calcitonin and TTF-1 negative antigen, confirming CASTLE.

Complementary treatment with chemo and radiotherapy, but without evidence of reduction of remaining tissue. Indicated new surgery, with right cervical exploration and tissue resected involving the cervical vessels and trachea, whose histopathological evidence only fibrous tissue. CASTLE is a rare neoplasm, with incidence between $0.085-0.15 \%$, low relapse rate, good prognosis and difficult preoperative diagnosis. Because of its rarity, it is difficult to define a standard and effective treatment for these patients. In addition to complete resection, adjuvant therapy may be used. This case shows a fast-growing tumor, of greater extension than the average of the literature, treated with adjuvant chemo and radiotherapy and reoperated for resection of tumor remnant. This entity was confirmed only in 2002 with immunohistochemistry. A strict follow-up is necessary, because there is no standard treatment and behavior could be uncertain.

\section{Poster 160}

Thyroid Imaging Thursday Poster Clinical

A MULTICENTER ANALYSIS ON THE EFFICACY OF AUTOFLUORESCENCE IMAGING TO DETECT PARATHYROID GLANDS

B. Kahramangil ${ }^{1}$, F. Benmiloud ${ }^{2}$, F. Dip ${ }^{3,4}$, J. Falco ${ }^{3}$, M. de La Fuente $^{3}$, S.V. Verna ${ }^{3}$, R. Rosenthal ${ }^{4}$, E. Berber ${ }^{1}$ 
${ }^{1}$ Department of Endocrine Surgery, Cleveland Clinic, Cleveland, $\mathrm{OH} ;{ }^{2}$ Department of Endocrine Surgery, Hôpital Européen, Marseille, France; ${ }^{3}$ Department of Endocrine Surgery, Hospital de Clínicas “José de San Martín”, University of Buenos Aires, Buenos Aires, Argentina; ${ }^{4}$ Department of Surgery, Cleveland Clinic Florida, Weston, FL

Identification of parathyroid glands (PGs) is critical during thyroidectomy and parathyroidectomy procedures. This has been conventionally done visually, with new technologies, such as autofluorescence imaging (AF), introduced in the recent years. The aim of this study is to analyze the efficacy of AF to detect PGs intraoperatively and to see if there is concordance between different centers. At 3 different centers, AF was used to localize the PGs during thyroidectomy and parathyroidectomy procedures. IRB-approval and informed consent were obtained. The ability of AF to localize PGs and concordance between 3 centers were analyzed. Regression analysis was performed to determine factors predicting successful localization with AF. A total of 210 (183 female; $\mathrm{n}=70$ per center) patients underwent total thyroidectomy $(\mathrm{n}=95)$, thyroid lobectomy $(\mathrm{n}=41)$, and parathyroidectomy $(\mathrm{n}=74)$. Overall, 98\% (584/594) of PGs detected with naked eye (NE) were localized with $\mathrm{AF}(99 \%, 99 \%, 97 \%$ at each center, respectively). Fortysix percent (272/594) of these PGs were localized with AF prior to detection with NE $(40 \%, 67 \%, 37 \%$, respectively). In the whole series, at least one PG was localized with AF prior to NE identification in $77 \%$ $(161 / 210)$ of patients $(77 \%, 84 \%, 69 \%$, respectively). The median number of PGs localized with AF before detection by NE per patient was 1 (range 0-4) (1[0-4], 2[0-4], 1[0-3], respectively). On regression analysis, gland size was the only parameter affecting detection by $\mathrm{AF}$ $(p=0.007)$. To our knowledge, this is the largest study assessing the utility of AF in localizing PGs intraoperatively. There was concordance between 3 different centers regarding efficacy. The fact that about half of the PGs could be localized with AF prior to identification with NE, quantifies the impact on the conduct of the operation.

\section{Poster 161}

Thyroid Imaging Thursday Poster Clinical

INTRAOPERATIVE NEURAL MONITORING DURING THYROID SURGERY: DIFFERENCES BETWEEN TRANSAXILLARY AND CERVICAL APPROACHES

J. Crowther ${ }^{1}$, D. Bu Ali ${ }^{2}$, K. Mohsin ${ }^{3}$, T. Ahmed ${ }^{1}$, H. Alzahrani ${ }^{1}$, J. Bamford ${ }^{2}$, S. Kang ${ }^{1}$, E. Kandil ${ }^{1}$

${ }^{1}$ Surgery, Tulane University School of Medicine, Baton Rouge, LA; ${ }^{2}$ Neurosurgery, Tulane University School of Medicine, New Orleans, LA; ${ }^{3}$ Tulane University School of Public Health and Tropical Medicine, New Orleans, LA

Intraoperative neural monitoring has become increasingly common in thyroid surgeries as a way of not only locating nerves during surgery but also monitoring their function and detecting injuries. In the current study, we assessed what effect differences of arm positioning during robotic-assisted transaxillary surgeries might have on intraoperative neural monitoring measurements by comparing robotic-assisted transaxillary and cervical cases. We identified 370 thyroid surgery cases ( 290 cervical, 80 transaxillary) performed by a single surgeon in a Northern American institution. We compared intraoperative vagal stimulation between the two groups during surgery prior to any resection. A subset of the transaxillary cases had ultrasound measurements recorded of the distance from the vagus nerve to the trachea both pre-positioning (arm fully adducted at side) and post-positioning (arm abducted and flexed over the head) for surgery, and we compared measurements of pre- and postpositioning data. We found significantly shorter latencies with right vagal stimulation in robotic cases $(2.97 \mathrm{~ms} \pm 1.04 \mathrm{SD})$ compared to cervical cases ( $3.41 \mathrm{~ms} \pm 1.01 \mathrm{SD})$ ( $p=0.03$ ), but no difference was found with left vagal stimulation comparing robotic $(5.12 \mathrm{~ms} \pm 0.77$ $\mathrm{SD})$ and cervical cases $(5.37 \mathrm{~ms} \pm 1.22 \mathrm{SD})(\mathrm{p}=0.14)$. In the robotic cases, we found a significant $(p<0.001)$ decrease in distance of the vagus nerve to the trachea from $2.60 \mathrm{~cm}(0.60 \mathrm{SD})$ pre-positioning to $2.29 \mathrm{~cm}(0.51 \mathrm{SD})$ post-positioning, and the difference was significant for both the right $(2.57$ to $2.32 \mathrm{~cm}, p=0.003)$ and left $(2.62$ to $2.25 \mathrm{~cm}, \mathrm{p}<0.001$ ) vagus nerves. Surgeons should be aware that robotic assissted-transaxillary thyroid surgery contributes to variations in vagal nerve stimulation latencies as compared to the traditional cervical approach. These results suggest that surgical positioning is a factor that can affect intraoperative neural stimulation measurements. Future multi-institutional studies are warranted to further investigate these interesting findings.

\section{Poster 162}

Thyroid Nodules \& Goiter Thursday Poster Basic

\section{SIGNIFICANCE OF VASCULARIZATION OF THYROID GLAND IN DIFFUSE TOXIC GOITER}

E. Gasparyan, O. Goroshko

"Professor" Medical Centre, Saint-Petersburg, Russian Federation

Evaluation of vascularization character with the use of Color Doppler regime (CD) and systolic velocity of blood flow (SVBF) in patients with diffuse toxic goiter (DTG). In medical centre 68 patients with DTG and 66 patients with autoimmune thyroiditis (AIT, the group of comparison) aged 18-73 were observed. In ultrasound examinations with B- and CD regimes, vascularization as well as SBF velocity in upper thyroid artery in $\mathrm{cm} / \mathrm{s}$ were estimated. Out of 68 patients with DTG 26 showed thyrotoxicosis and 42 patients showed euthyroidism (30 of them - medicamental and 12 - after the cancellation of therapy). Out of 66 patients with AIT 37 showed subclinical and overt hypothyroidism; 29 patients were in the state of euthyroidism (medicamental and without therapy). In the process of research in DTG we established the dependence of vascularization character and SVBF on TH function. All patients (100\%) with thyrotoxicosis showed considerable increase or hypervascularization of TH. At the same time, SVBF was in average $68.0 \pm 12.1 \mathrm{~cm} / \mathrm{s}$ (with the normal being $20.0-40.0 \mathrm{~cm} / \mathrm{s}$ ) and reached $80.0 \mathrm{~cm} / \mathrm{s}$. Euthyroidism in patients was characterized with normalization of thyroid vascularization irresrective of the presence or absence of antithyroid therapy. SVBF in them was in average $32.0 \pm 6.4 \mathrm{~cm} / \mathrm{s}$. We noticed the interrelation between $\mathrm{TH}$ vascularization character, SVBF, TSH level and TRAb values. In all patients with AIT in the state of hypothyroidism and euthyroidism with and without of therapy $\mathrm{TH}$ vascularization was normal and SVBF was in average $24.6 \pm 4.7 \mathrm{~cm} / \mathrm{s}$ which is considerably lower than in patients in the state of thyrotoxicosis. Determination of the character of vascularization in CD regime and SVBF is noninvasive available study, permitting to assess thyroid function in patients with DTG within a few minutes

Thyroid vascularization and high indexes of SVBF, observed in all patients on the stage of thyrotoxicosis, have diagnostic significance

Normalization of vascularization and SVBF of TH in patients with DTG, together with normal TSH and TRAb, can serve as in indication for antithyroid therapy cancellation.

\section{Poster 163}

Thyroid Nodules \& Goiter Thursday Poster Case Report

\section{THYROIDITIS SECONDARY TO DISSEMINATED}

\section{BLASTOMYCOSIS}

I. Casimiro, D. Sarne

Endocrinology, University of Chicago, Chicago, IL 
Blastomycosis is a systemic fungal infection caused by Blastomyces dermatitidis endemic along the Mississippi and Ohio rivers. There are only a few reported cases of thyroid involvement by blastomycosis. A 44 year old female with a history of substance abuse presented to an outside hospital for shortness of breath and flu like symptoms and was found to be in septic shock. She was intubated and transferred to our hospital for evaluation of an abdominal wall abscess and a large left sided neck mass. The patient was diagnosed with disseminated blastomycosis with pulmonary, cutaneous and osseous involvement of the spine and ankle (skin nodules and right ankle aspirate showed blastomycosis on biopsy). She was treated with amphotericin and later switched to itraconazole due to acute tubular necrosis. Ultrasound evaluation of the thyroid at the time of her initial presentation showed a large, heterogeneous mass which decreased significantly in size over two weeks with systemic antifungal therapy without aspiration or drainage. Given the size regression and improved clinical picture, FNA was not performed. Her thyroid function tests were TSH of $4.04(0.30-4.00)$, free T4 0.72 (0.9-1.7) and T3 of 112 (80-195). These values were thought to be consistent with mild hypothyroidism from infectious thyroiditis versus nonthyroidal illness. As she was clinically euthyroid, thyroid replacement therapy was not initiated. The patient was followed in Infectious Disease clinic with plans for continuing itraconazole therapy for one year. Repeat evaluation months later showed normalization of thyroid function tests and remission of the thyroid mass. There have been four previous reports documenting blastomycosis infection in the thyroid tissue of patients infected with this organism. Thyroiditis in our patient resolved with only systemic therapy.

\section{Poster 164}

Thyroid Nodules \& Goiter Thursday Poster Clinical

STANDARD THYROID NODULE ULTRASOUND REPORTS PROVIDE INSUFFICIENT INFORMATION TO ESTIMATE RISK OF MALIGNANCY

\section{C.J. Symonds ${ }^{1}$, P. Seal ${ }^{2}$, S. Ghaznavi ${ }^{1}$, R. Paschke ${ }^{1}$}

${ }^{1}$ Endocrinology \& Metabolism, University of Calgary, Calgary, Alberta, Canada; ${ }^{2}$ Diagnostic Imaging, University of Calgary, Calgary, Alberta, Canada

Ultrasonographic characteristics of thyroid nodules play an important role in estimating the risk of malignancy (ROM) and indications for fine needle aspiration biopsy (FNAB). Insufficient information in radiologist reports may impair the estimation of ROM leading to inappropriate selection of nodules to undergo FNAB. Furthermore, ultrasoundderived ROM may provide complementary information to FNAB results. Administrative health care databases provide an opportunity to evaluate differences between practice patterns and guidelines. We reviewed all consecutive diagnostic thyroid ultrasound reports in 2012 from a large community radiology practice in Calgary, Canada. Of the 2037 reports, $1434(70.4 \%)$ identified one or more thyroid nodules. We excluded subsequent reports on the same patient leaving a cohort of 1339 unique patients- mean age 56 years ( $85 \%$ female). Each report was analyzed for 6 key ultrasonographic features: size, internal content, echogenicity, calcification, margin and shape. A utility score was calculated with a single point given for a comment on each feature, with the proviso that pertinent negative features might not be reported. Score range: $0-6$ points. $76 \%$ of the studies reported $>1$ nodule (mean number of nodules $=3.4$ ). Mean maximal diameter of the largest nodule per patient $=1.8 \mathrm{~cm}$ (range $0.2-10 \mathrm{~cm})$. Largest nodules: $<1 \mathrm{~cm}(35.7 \%)$, $1-2 \mathrm{~cm}(31.6 \%)$ and $>2 \mathrm{~cm}(32.7 \%)$. Mean utility score for 1339 unique patients $=2.57$. This score did not change significantly when the largest nodule was $<1 \mathrm{~cm}(2.53), 1-2 \mathrm{~cm}(2.68)$ and $>2 \mathrm{~cm}$ (2.49). Individual utility scores: $\%$ of cohort- $0: 0.1 \%, 1: 8.1 \%, 2: 43.5 \%, 3: 34.5 \%$, 4: $11.2 \%, 5: 2.1 \%, 6: 0.4 \%$. Using validated ultrasound feature algo- rithms, we believe a utility score of 4 or greater is needed to confidently estimate ROM: we found this in $13.7 \%$ of reports. In conclusion, our analysis of a large number of consecutive thyroid ultrasound reports on unique patients suggests that $>86 \%$ of these studies provide insufficient information to allow the clinician to risk stratify the nodules. We hypothesize that this may lead to more patient anxiety and inappropriate utilization of health care resources through repeat imaging, FNAB, and thyroid surgery.

\section{Poster 165}

Thyroid Nodules \& Goiter Thursday Poster Clinical

\section{CLINICAL OUTCOMES, ASSOCIATED RISKS, AND FUTURE LONGEVITY FOR PATIENTS SEEKING THYROID NODULE EVALUATION OVER THE AGE OF 70 YEARS}

Z. Wang, C.M. Vyas, E. Marqusee, M.I. Kim, P. Larsen, E.K. Alexander, T.E. Angell

Thyroid Section, Division of Endocrinology, Diabetes and Hypertension, Brigham and Women's Hospital, Boston, MA

In older patients, thyroid nodules are common though most thyroid cancer remains low risk. As such, management is influenced by the relative risks and benefit of intervention. Few data provide quantitative metrics to guide clinical decisions for ideal management in this population. We performed an analysis of all patients $>70$ years old (yo) consecutively assessed by ultrasound (US) and FNA from 1995-2015. We obtained clinical, US, and histologic data, including patient outcomes during treatment and follow up. High risk cancer was defined as anaplastic, medullary, or poorly differentiated carcinoma, or the presence of distant metastatic disease. We evaluated 1,129 patients $>70$ yo with 2,527 nodules $>1 \mathrm{~cm}$. Cytology was benign in $64.7 \%$ of patients. Only 1 significant FNA complication occurred $(0.1 \%)$. Surgery was performed in 210 patients, with $132(11.7 \%)$ diagnosed with thyroid cancer. There were $17(12.5 \%)$ high risk cancers, all of which were preoperatively identifiable by imaging and/or cytology. Our data demonstrates that 1 of every 66 patients $>70$ yo was helped by identification of high risk cancer. However, 1 of every 13 patients $>70$ yo was exposed to excess morbidity due to surgery for benign disease, and 1 of every 49 patients without high-risk cancer had a major operative complication. During a median follow up of 4 years (interquartile range 2-7 yrs), there were $10(0.9 \%)$ thyroid cancer deaths, all of which occurred in high risk cancers. In patient without high risk cancer, there were no thyroid cancer deaths observed yet 1 of every 7 patients died from other causes during this time. These data suggest US and FNA are safe for patients $>70$ yo and may prove helpful, as identification of benign cytology or high risk US features/malignant cytology likely adds value. However, the surgical management of patients $>70$ yo presenting with lower risk thyroid nodules based on US and cytology, including those with indeterminate or non-diagnostic results, should be questioned given a high morbidity to value ratio. While one cannot exclude a benefit in treating all biopsyconfirmed malignancy, it is notable that $96 \%$ of deaths during follow up evaluation were due to non-thyroid causes.

\section{Poster 166}

Thyroid Nodules \& Goiter Thursday Poster Clinical

\section{CYTOLOGICALLY INDETERMINATE THYROID} NODULES: IMPACT OF SIZE $\geq \mathbf{4 C M}$ ON CANCER RISK J.B. Tourtelot ${ }^{1}$, L. Khazai ${ }^{2}$, K. Otto ${ }^{1}$, C.H. Chung ${ }^{1}$, B. Centeno ${ }^{2}$, B. McIver ${ }^{1}$, P. Valderrabano ${ }^{1}$

${ }^{1}$ Head and Neck-Endocrine Oncology, H. Lee Moffitt Cancer Center and Research Institute, Tampa, FL; ${ }^{2}$ Anatomic Pathology, H. Lee Moffitt Cancer Center and Research Institute, Tampa, FL 
ATA guidelines recommned thyroid lobectomy as initial surgical approach for solitary cytologically indeterminate thyroid nodules (ITNs: categories B-III and B-IV of Bethesda). However, it is suggested that total thyroidectomy may be preferred for ITNs $>4 \mathrm{~cm}$ due to increased cancer risk (recommendation \#20. a). We aimed to characterize the histological features and clinical outcomes of ITNs $\geq 4 \mathrm{~cm}$ to determine whether a total thyroidectomy should be the preferred initial surgical approach in that scenario. We included 589 patients with 652 ITNs (546 [84\%] $<4 \mathrm{~cm}$ [median $2.6 \mathrm{~cm}$ ]; and 106 $[16 \%] \geq 4 \mathrm{~cm}$ [median $5.4 \mathrm{~cm}$ ] by ultrasound measurements; $42 \% \mathrm{~B}$ III and 58\% B-IV) evaluated between 10/2008 and 4/2016; without pre-surgical evidence of malignancy; and with known histology. We compared the histological features of all ITNs $<4 \mathrm{~cm}$ and $\geq 4 \mathrm{~cm}$; and the clinical outcomes of 84 malignancies $(68<4 \mathrm{~cm}$ and $16 \geq 4 \mathrm{~cm})$ with $>6$ months of follow-up, and without any other concurrent or incidental thyroid cancer foci. Patients with ITNs $\geq 4 \mathrm{~cm}$ were more frequently males ( $21 \%$ vs $31 \%, \mathrm{P}=0.04)$. Differences in age, thyroid function status, multinodularity, thyroid cancer risk factors, or cytological diagnosis were not significant. The ATA sonographic patterns' distribution was different $(\mathrm{P}<0.001)$ due to higher rates of heteroechogenicity (classified as Non-ATA pattern) in ITNs $\geq 4 \mathrm{~cm}$ (26\% vs $54 \%$ ). Differences in prevalence of malignancy between nodules $<4$ and $\geq 4 \mathrm{~cm}$ were not significant ( $26 \%$ vs $31 \%$ ). Differences in the proportion of malignancies with extrathyroidal extension (10\% vs $10 \%$ ), positive surgical margins (9\% vs $10 \%)$, lympho-vascular invasion ( $14 \%$ vs $24 \%$ ), or lymph node metastases ( $11 \%$ vs $3 \%$ ) were not significant. There was only one patient with distant metastases, who had a $6.8 \mathrm{~cm}$ tumor ( $0 \%$ vs $3 \%, \mathrm{P}=0.04)$. In both groups, $<10 \%$ of all ITNs were intermediate/high risk cancers. No recurrences were detected during a median follow-up of 32 months; and almost all of the cancer patients had no evidence of disease at last visit (90\% vs $94 \%)$. Histological features and follow-up data do not significantly differ in ITNs $<4$ and $\geq 4 \mathrm{~cm}$. A therapeutic or diagnostic lobectomy seems appropriate for $>90 \%$ of solitary ITNs, regardless of size.

\section{Poster 167}

Thyroid Nodules \& Goiter Thursday Poster Clinical CLINICAL VALIDATION OF THE AFIRMA GENOMIC SEQUENCING BRAF V600E CLASSIFIER

T.E. Angell ${ }^{1}$, J. Babiarz ${ }^{2}$, N. Barth ${ }^{3,4}$, T. Blevins ${ }^{5}$, Q. Duh ${ }^{6}$, R.A. Ghossein ${ }^{7}$, R.M. Harrell ${ }^{8}$, J. Huang ${ }^{2}$, U. Imtiaz ${ }^{4}$, G. Kennedy ${ }^{2}$, S. Kim ${ }^{2}$, R.T. Kloos ${ }^{3}$, V.A. LiVolsi ${ }^{9}$, K.N. Patel ${ }^{10}$, G. Randolph ${ }^{11}$, P.M. Sadow ${ }^{12}$, M.H. Shanik ${ }^{13}$, J. Sosa ${ }^{14}$, S.T. Traweek ${ }^{15}$, P.S. Walsh ${ }^{2}$, D. Whitney ${ }^{2}$, M. Yeh ${ }^{16}$, P.W. Ladenson ${ }^{17}$

${ }^{1}$ Department of Medicine, Division of Endocrinology, Diabetes, and Hypertension, Brigham and Women's Hospital and Harvard Medical School, Boston, MA; ${ }^{2}$ Department of Research \& Development, Veracyte, Inc., South San Francisco, CA; ${ }^{3}$ Department of Medical Affairs, Veracyte, Inc., South San Francisco, CA; ${ }^{4}$ Department of Clinical Affairs, Veracyte, Inc., South San Francisco, CA; ${ }^{5}$ Texas Diabetes and Endocrinology, Austin, TX; ${ }^{6}$ Department of Surgery, Section of Endocrine Surgery, University of California San Francisco, San Francisco, CA; ${ }^{7}$ Department of Pathology, Division of Head and Neck Pathology, Memorial Sloan-Kettering Cancer Center, New York, NY; ${ }^{8}$ The Memorial Center for Integrative Endocrine Surgery, Boca Raton, FL; ${ }^{9}$ Department of Pathology and Laboratory Medicine, Anatomic Pathology Division, University of Pennsylvania School of Medicine, Philadelphia, PA; ${ }^{10}$ Department of Otolaryngology-Head and Neck Surgery, Division of Endocrine Surgery, NYU Langone Medical Center, New York, NY;

${ }^{11}$ Department of Otolaryngology, Division of Thyroid and Parathyroid Endocrine Surgery, Massachusetts Eye and Ear Infirmary and Harvard Medical School, Boston, MA; ${ }^{12}$ Department of Pathology, Head and Neck Pathology Subspecialty, Massachusetts
General Hospital and Harvard Medical School, Boston, MA; ${ }^{13}$ Endocrine Associates of Long Island, Smithtown, NY; ${ }^{14}$ Duke University Medical Center, Department of Surgery, Section of Endocrine Surgery, Durham, NC; ${ }^{15}$ Thyroid Cytopathology Partners, Austin, TX; ${ }^{16}$ Department of Surgery, Endocrine Surgery Program, UCLA David Geffen School of Medicine, Los Angeles, CA;

${ }^{17}$ Department of Medicine; Division of Endocrinology, Diabetes and Metabolism, Johns Hopkins University, Baltimore, MD

Several DNA variants have a high positive predictive value (PPV) for thyroid cancer, including BRAF V600E. The Afirma Genomic Sequencing Classifier (GSC) identifies, by RNA sequencing and machine learning algorithms, genomically benign thyroid nodules among those with indeterminate cytology to prevent unnecessary diagnostic surgery with high NPV, and modest PPV. Additional cassettes within the GSC aim to detect the molecular signatures of specific neoplasms with high PPV that further alter patient care. Here we report the clinical performance of an embedded BRAF V600E classifier. There is no single accepted gold standard test or mutated allele frequency to define BRAF V600E status. We used castPCR to define this "truth". Samples with $<5 \%$ BRAF V600E allele frequency were defined as negative; those with $\geq 5 \%$ as positive. 452 FNA samples were used in algorithm training to build an RNA-based classifier. The classifier is comprised of 12 expression-based submodels and are further improved with variant features from RNAseq. 9,880 genes were included in the classifier with 1,042 having the largest impact on classification performance. The final classifier was blindly tested on 264 independent FNAs. Compared with BRAF status by castPCR DNA sequencing, the classifier had $100 \%$ PPA [62/62 BRAF V600E positive samples called correctly; CI 94.2$100 \%$ ] and $99.0 \%$ NPA [200/202 BRAF V600E negative samples called correctly; CI 96.5-99.9\%]. One "false positive" had a castPCR allele frequency of $4.77 \%$ and was histologically PTC, and the other had a castPCR allele frequency of $0 \%$ with a double mutation on DNA sequencing at nucleotide positions $1798(\mathrm{~T}>\mathrm{A})$ and 1799 ( T > A) with $27 \%$ allele frequency, demonstrating that it is a true positive. The preoperative genomic identification of thyroid nodules with a virtually $100 \%$ chance of malignancy may influence patient and physician decisions regarding the need for surgery, and potentially refine the extent of thyroid surgery prior to the initial operation. This information may improve patient care by avoiding delays in diagnosis and/or the need for a completion thyroidectomy.

\section{Poster 168}

\section{Thyroid Nodules \& Goiter Thursday Poster Clinical}

\section{CLINICAL VALIDATION OF THE AFIRMA GENOMIC SEQUENCING PARATHYROID CLASSIFIER}

J. Sosa ${ }^{1}$, T.E. Angell ${ }^{2}$, J. Babiarz ${ }^{3}$, N. Barth ${ }^{4,5}$, T. Blevins ${ }^{6}$, Q. Duh ${ }^{7}$, R.A. Ghossein ${ }^{8}$, R.M. Harrell ${ }^{9}$, J. Huang ${ }^{3}$, U. Imtiaz ${ }^{5}$, G. Kennedy ${ }^{3}$, S. Kim ${ }^{3}$, R.T. Kloos ${ }^{4}$, V.A. LiVolsi ${ }^{10}$, K.N. Patel ${ }^{11}$, G. Randolph ${ }^{12}$, P.M. Sadow ${ }^{13}$, M.H. Shanik ${ }^{14}$, S.T. Traweek ${ }^{15}$, P.S. Walsh ${ }^{3}$, D. Whitney ${ }^{3}$, M. Yeh ${ }^{16}$, P.W. Ladenson ${ }^{17}$

${ }^{1}$ Department of Surgery, Section of Endocrine Surgery, Duke University Medical Center, Durham, NC; ${ }^{2}$ Department of Medicine, Division of Endocrinology, Diabetes, and Hypertension, Brigham and Women's Hospital and Harvard Medical School, Boston, MA; ${ }^{3}$ Department of Research \& Development, Veracyte, Inc., South San Francisco, CA; ${ }^{4}$ Department of Medical Affairs, Veracyte, Inc., South San Francisco, CA; ${ }^{5}$ Department of Clinical Affairs, Veracyte, Inc., South San Francisco, CA; ${ }^{6}$ Texas Diabetes and Endocrinology, Austin, TX; ${ }^{7}$ Department of Surgery, Section of Endocrine Surgery, University of California San Francisco, San Francisco, CA;

${ }^{8}$ Department of Pathology, Division of Head and Neck Pathology, Memorial Sloan-Kettering Cancer Center, New York, NY; ${ }^{9}$ The 
Memorial Center for Integrative Endocrine Surgery, Boca Raton, FL; ${ }^{10}$ Department of Pathology and Laboratory Medicine, Anatomic Pathology Division, University of Pennsylvania School of Medicine, Philadelphia, PA; ${ }^{11}$ Department of Otolaryngology-Head and Neck Surgery, Division of Endocrine Surgery, NYU Langone Medical Center, New York, NY; ${ }^{12}$ Department of Otolaryngology, Division of Thyroid and Parathyroid Endocrine Surgery, Massachusetts Eye and Ear Infirmary and Harvard Medical School, Boston, MA;

${ }^{13}$ Department of Pathology, Head and Neck Pathology Subspecialty, Massachusetts General Hospital and Harvard Medical School, Boston, MA; ${ }^{14}$ Endocrine Associates of Long Island, Smithtown, NY; ${ }^{15}$ Thyroid Cytopathology Partners, Austin, TX; ${ }^{16}$ Department of Surgery, Endocrine Surgery Program, UCLA David Geffen School of Medicine, Los Angeles, CA; ${ }^{17}$ Department of Medicine; Division of Endocrinology, Diabetes and Metabolism, Johns Hopkins University, Baltimore, MD

The parathyroid glands are located adjacent to the thyroid and occasionally within it. Enlarged and imbedded parathyroid glands can be mistaken as thyroid nodules or suspicious lymph nodes. On fine needle aspiration biopsy (FNAB) of such lesions, cytology is often indeterminate, failing to identify its parathyroid origin and potentially resulting in an unnecessary thyroid surgery. The Afirma Genomic Sequencing Classifier (GSC) identifies genomically benign thyroid nodules among those with indeterminate FNAB to prevent unnecessary diagnostic surgery using RNA sequencing and machine learning algorithms. Integrated cassettes are used to detect the molecular signatures of specific neoplasms that may further alter patient care, such as unsuspected parathyroid tissue. Here we report the clinical performance of the parathyroid classifier cassette used with the GSC. Algorithm training was performed with a set of 476 FNAs -6 parathyroid and 470 thyroid FNAs. An additional 97 tissues were used in feature selection, but not model training. A support vector machine classifier was developed using 109 differentially expressed genes, including the 5 genes in the Afirma GEC parathyroid cassette. The final classifier was blindly tested on an independent test set of 195 FNAs (118 Bethesda III, 77 Bethesda IV). The classifier had $100 \%$ sensitivity [4/4 parathyroid correctly called positive; CI 39.8$100 \%]$ and $100 \%$ specificity [191/191 thyroid correctly called negative; CI 98.1-100\%]. All positive samples had clinical/surgical confirmation of the parathyroid etiology, while all negative samples were negative on surgical pathology. Preoperative genomic identification of parathyroid tissue facilitates appropriate management of parathyroid tissue within and adjacent to the thyroid gland. As a result, inappropriate interventions, costs, and potential complications may be avoided.

\section{Poster 169}

Thyroid Nodules \& Goiter Thursday Poster Clinical THYROID NODULES IN CHILDREN

N.G. Allen ${ }^{1}$, N. Desai ${ }^{2}$, G. Francis ${ }^{1}$

${ }^{1}$ Pediatrics, Virginia Commonwealth University Health System, Richmond, VA; ${ }^{2}$ Virginia Commonwealth University School of Medicine, Richmond, VA

Thyroid nodules (TN) are commonly found in children with autoimmune thyroid disease (AIT) or goiter $(\mathrm{G})$. Some providers obtain thyroid ultrasound (US) on all children with AIT to discover DTC prior to growth and metastasis. It is unclear if earlier detection impacts long-term outcomes because DTC has a favorable prognosis in children regardless of tumor size. Guidelines advise thyroid US on children with a 'suspicious' thyroid gland or palpable cervical lymphadenopathy but 'suspicious' characteristics are not defined.
Objectives:

1. To retrospectively examine the clinical course of AIT and G in children.

2. To determine the frequency and timeline with which children with AIT or G develop "suspicious" features, palpable TN, or DTC.

3. To identify predictive historical and clinical characteristics of children who develop "suspicious" features, palpable TN or DTC. We identified 881 patients $<18$ yrs of age who have been seen in the Pediatric Endocrinology Clinic at VCU with AIT or G. We reviewed charts for diagnosis, age, and our criteria for 'suspicious' thyroid glands. Criteria include family history of TN or thyroid cancer, personal history of cancer or radiation, personal or family history of heritable cancer syndrome, firm, large, or asymmetric gland, and cervical lymphadenopathy. We included data for autoantibodies, US, fine needle aspiration, and pathology results. We report preliminary data for the first 141 subjects. Subjects range in age from $6-18$ years, $72 \%$ are female, and the primary diagnoses are AIT in $34 \%$ and $\mathrm{G}$ in $62 \%$. Twenty-eight had TN, 9 underwent surgery of which, two had DTC (one had family history of thyroid cancer and one had personal history of cancer). Based on the preliminary data, $14.3 \%$ of patients with TN had family history of TN or DTC compared with only $0.9 \%$ of patients without TN. Patients with TN were more likely to have an asymmetric gland on exam with $17.8 \%$ versus $9.7 \%$ of patients without TN. While this did not reach statistical significance at p-value of 0.235 , we have only reviewed 141 of the 881 charts identified. We plan to further analyze the data to draw conclusions regarding what constitutes 'suspicious' features and the rate with which patients with AIT or G develop DTC.

\section{Poster 170}

\section{Thyroid Nodules \& Goiter Thursday Poster Clinical} CORRELATION OF INTRA-OPERATIVE PARATHYROID HORMONE LEVEL AND PATIENT RECOVERY TIME

S. Tsai, S. Mostoufi-Moab, K. Kazahaya, S. Adzick, S. Bauer, A. Bauer

Endocrinology and Diabetes, Children's Hospital of Philadelphia, Philadelphia, PA

Post-surgical hypoparathyroidism is a risk for patients who undergo thyroidectomy. Many patients are discharged on calcium supplementation with serial laboratory surveillance over several weeks to assess for parathyroid gland recovery. The primary objective of our study was to determine what clinical indicators may correlate with predicting parathyroid gland recovery post-thyroidectomy. This retrospective study examined pediatric and young adult patients (age $\leq 23$ years) who presented to our institution for a thyroidectomy between 2013 and 2017. At the start, middle, and end of surgery, 3 intraoperative parathyroid (ioPTH) hormone levels were measured. Time of recovery (TOR) for patients was defined as when their PTH was within normal bounds (between 10 and $55 \mathrm{pg} / \mathrm{mL}$ ) coincident with normal calcium and phosphorus. Patients were excluded if they did not have sufficient bloodwork done, since their TOR could not be accurately determined. Over the study time period, 84 patients (68 female, 14 male) aged 3-22 years (mean 14.7) met inclusion and exclusion criteria. We found that the $2^{\text {nd }}$ and $3^{\text {rd }}$ ioPTH's correlate with parathyroid recovery time: the lower the ioPTH, the greater the recovery time. Subdividing patients into high-risk $\left(2^{\text {nd }}\right.$ ioPTH $\leq 10 \mathrm{mg} / \mathrm{dl}$ ), moderate-risk ( $2^{\text {nd }}$ ioPTH between 10 and $20 \mathrm{mg} / \mathrm{dl}$ ), and low-risk ( $2^{\text {nd }}$ ioPTH $\geq 20 \mathrm{mg} / \mathrm{dl}$ ) pools, the TOR decreased by an order of magnitude from an average of $522 \pm 291$ to $113 \pm 81$ to $42 \pm 22$ hours $(\operatorname{Pr}(>F)=0.0416)$. Furthermore, we also found that intra-op and inpatient phosphate and calcium blood tests correlated with time of recovery, but not ionized calcium blood tests. Our findings demonstrate the usefulness of obtaining intra-operative PTH 
levels to predict patient recovery post-surgery. Parathyroid gland recovery is correlated with intra-op PTH at the middle and end of surgery as well as phosphate and calcium blood tests. This information may be used to optimize the timing and frequency of postsurgical laboratory surveillance.

\section{Poster 171}

Thyroid Nodules \& Goiter Thursday Poster Clinical

\section{EVALUATION OF THE I-131 THERAPY IN PATIENTS WITH AUTONOMOUSLY FUNCTIONING THYROID NODULES AND NORMAL TSH LEVEL}

M. Lacic

Thyroid, Polyclinic Lacic, Zagreb, Croatia

Objectives: The purpose of this study was to evaluate the results of radioactive iodine-131 (I-131) therapy in patients (pts) with autonomously functioning thyroid nodules (AFTNs) and a normal thyroid stimulating hormone (TSH). Up to our knowledge, this is the first study which has scintigraphically evaluated the effect of I-131 therapy in patients with AFTNs and normal TSH level.

Methods: In this study 58 cytological benign AFTNs in 51 pts (45 female and 6 male) with normal TSH level have been treated with a fixed I-131 doses (370 MBq). Clinical exam, ultrasonography with color Doppler (US), fine needle aspiration biopsy (FNAB), TSH, FT4, FT3, anti-TPO, anti-Tg and thyroid scan (scintigraphy) have been performed in all pts before and 6 months after I-131 therapy.

Results: The median age of the pts was 57 (range 37-83) years AFTNs were located more frequently in the right thyroid lobe (33 nodules) than in the left lobe ( 25 nodules). In 11 pts a solitary AFTN has been found on ultrasonography and the other 40 patients had AFTNs in multinodular goiter. Seven pts had two AFTNs. On post I-131 therapy thyroid scan in 41 AFTNs complete therapy effect has been observed, but in 17 AFTNs a scintigraphycally partial effect has been noted. Statistical analysis showed a significant reduction in the thyroid ( $\mathrm{p}=4.7515 \mathrm{E}-15)$ and AFTNs $(\mathrm{p}=0.0018)$ volume after J-131 therapy. TSH value significantly increased $(\mathrm{p}=0.0048)$ and FT4 value significantly decreased $(\mathrm{p}=0.0012)$ after I-131 therapy. FT3 $(\mathrm{p}=0.3508)$, anti-TPO $(\mathrm{p}=0.8701)$ and anti-Tg $(\mathrm{p}=0.6805)$ values did not change significantly.

Conclusion: In conclusion we wish to stress that I-131 therapy in pts with AFTN and normal TSH level is a simple, cheap and very effective modality. The effect of the I-131 therapy on AFTNs can be exactly evaluated with a post I-131 therapy thyroid scan.

\section{Poster 172}

Thyroid Nodules \& Goiter Thursday Poster Clinical A COMPARISON OF INDIAN AND ABROAD THYROID SURGERY WEBSITES

A. Chekavar, S. Mayilvaganan, S. Bothra, a. agrawal

SGPGIMS, Lucknow, Uttar Pradesh, India

At present patients suffering from thyroid diseases gain knowledge through website rather than direct interaction with the physician. We aimed at assessing whether Indian website about Thyroid Surgery matched with their counterpart abroad. We identified the most searched Thyroid Surgery website world wide and 10 endocrine surgery website maintained by trained Endocrine Surgeons (three years training leading to award of Superspeciality degree) from India. The website parameters and Number of hits, demographic data of website, rank and other parameters wereassessed using professional website (www.Alexa.com). An Endocrine surgeon along with a technical website advisorrated the content, presentation and likesfrom a scale of 1to 5. (1-minimum score and 5 maximum score). We analysed 10 Indian and 1website from outside India. Fisher exact test was used to test the association between websites (India/Abroad) and individual variables. Results shows that there was no significant association Website(India/Abroad) and Thyroid facts, Photographs, videos, Publications, Landscape of website, Quality, Presentation of website, Number of views, Gender of person viewing the website and age group of person $(\mathrm{p}>0.05)$, while there was significant association with Post-operative advise as well as complications $(\mathrm{p}<0.05)$. Most parameters were comparable in both groups. But the complication rate was not available in many websites. Post-operative advice was present in only few websites. The advantage of these website is that patient can gather much needed information and also save much of unneeded discussion with physician and also save on second consultation.

\section{Poster 173}

\section{Thyroid Nodules \& Goiter Thursday Poster Clinical}

THYROID SURGERY OUTCOMES IN A LOW-INCOME COUNTRY: ASSESSING THE FEASIBILITY OF SHORT-TERM SPECIALTY SURGERY MODELS K.L. Long ${ }^{1}$, H. Starnes ${ }^{2}$, R.J. Harding ${ }^{3}$, M.R. Starks ${ }^{4}$

${ }^{1}$ Surgery, University of Wisconsin School of Medicine and Public Health, Madison, WI; ${ }^{2}$ Albany Medical Center, Albany, NY;

${ }^{3}$ Arizona Associated Surgeons, Phoenix, AZ; ${ }^{4}$ St Joseph Healthcare, Bangor, ME

Sub-Saharan Africa represents an endemic goiter zone, with massively enlarged thyroid glands commonplace. Access to surgery in rural areas is minimal to non-existent. Best practices to expand specialized health care are currently unknown. We evaluated the safety of focused surgical trips to low-resource settings with an emphasis on minimizing complications using high-volume endocrine surgeons. Over the course of 4 days in January 2017, 4 high-volume thyroid surgeons participated in a dedicated endocrine surgery trip to Migori, Kenya. All patients were screened for symptoms and operative fitness during the first clinical day, and those with prior anterior neck surgery were declined intervention in this setting. Thyroid stimulating hormone (TSH), hematocrit and surgeon-performed ultrasound were available for preoperative assessment. Patients were evaluated on post-operative day 1 by the surgeons, and phone followup with all patients has been attempted by the clinic. During the 3 operative days, 49 procedures were performed. 40 of these procedures were thyroid-related. Patients were mostly female (43/49), with an average age of 40.4 years (range $=13-71$ years). 31 procedures consisted of unilateral lobectomies, 5 were subtotal thyroidectomies, and 4 were near-total thyroidectomies. No total thyroidectomies were performed due to concern for levothyroxine availability and to minimize potential for bilateral complications. One intraoperative recurrent laryngeal nerve injury occurred and was repaired primarily. One patient was re-explored but no expanding hematoma was noted and only excess skin excised. Unlike other thyroid-focused surgical trips, no issues with hypoparathyroidism or bilateral nerve injury/ palsy occurred, and no long-term complications have been reported. Surgical treatment in low-resource settings presents many challenges. Efforts to maximize availability of quality surgery while ensuring excellent outcomes for this vulnerable patient population are imperative. Further efforts to improve access to medication and ancillary services are needed; however, we show that safe surgery can be performed by high-volume surgeons, with minimal resources, in this setting. 


\section{Friday, October 20, 2017}

\section{Basic/Translational Oral 15 \\ Thyroid Cancer Friday Basic \\ BERBERINE COULD INHIBIT THYROID CARCINOMA CELLS BY INDUCING MITOCHONDRIAL APOPTOSIS, G0/G1 CELL CYCLE ARREST AND SUPPRESSING MIGRATION VIA PI3K-AKT AND MAPK SIGNALING PATHWAYS}

L. Li, S. Rampersad, X. Wang, S. Qu

Department of Endocrinology and Metabolism, Shanghai Tenth Hospital, Tongji University, School of Medicine, Shanghai, China, Shanghai, China

Berberine, an important natural isoquinoline alkaloid from traditional Chinese medicine, is reported to exhibit multiple pharmacological properties, including anti-microbial, anti-diabetic, anti-inflammatory and anti-carcinogenic activities. Although studies have shown that a wide range of carcinoma cells could be inhibited by berberine, few studies involved thyroid carcinoma. We therefore examined the effect of berberine on papillary thyroid carcinoma (PTC, the most common subtype, low mortality but high recurrence) and anaplastic thyroid carcinoma (ATC, the most malignant and aggressive subtype with low survival). Also, we investigated the mechanisms of berberine on crucial signaling pathways of thyroid carcinogenesis. Three thyroid carcinoma cell lines with different aberrant genotypes and one normal thyroid cell line were selected, including C643 (ATC, with H-RAS mutation), OCUT1 (ATC, with BRAF ${ }^{\mathrm{V} 600 \mathrm{E}}$ and PIK3CA mutations), TPC1 (PTC, with RET/PTC1 rearrangement) and Htori3 (normal). We assessed the suppressive effects of berberine on cell proliferation by CCK-8 assay; apoptosis, cell cycle and mitochondrial membrane potential by flow cytometry; as well as cell migration by wound healing assay. Western blot was used to examine the protein alterations in various signaling pathways. We found that berberine inhibited the proliferation of C643, OCUT1 and TPC1 thyroid carcinoma cells in a dose- and time-dependent manner, while normal human thyroid cells showed less sensitivity to the cytotoxicity of berberine. Berberineinduced mitochondrial apoptosis, G0/G1 cell cycle arrest and inhibitive migration in thyroid carcinoma cells were also observed. Increased Bax/Bcl-2, cleaved Caspase 3, P21 and decreased Cyclin E1, CDK2 and Vimentin were verified by western blot. Additionally, berberine caused markedly decreased p-AKT1 expression and disturbances in classic ERK MAPK as well as P38 and JNK MAPK pathways in diverse thyroid carcinoma cell lines. Berberine modulates PI3K-AKT and MAPK signaling pathways in thyroid carcinoma cells, which leads to mitochondrial apoptosis, G0/G1 cell cycle arrest and suppressive migration. Berberine may represent a promising chemotherapy for the treatment of thyroid carcinoma.

\section{Basic/Translational Oral 16 \\ Thyroid Cancer Friday Basic}

LARGE ONCOSOMES OF ANAPLASTIC AND PAPILLARY THYROID CANCER CELLS

K.W. Witwer ${ }^{1}$, T. Seale ${ }^{1}$, Y. Wang ${ }^{3}$, B.H. Powell ${ }^{1}$, D. Di Vizio ${ }^{2}$, C.B. Umbricht ${ }^{3}$, M.A. Zeiger ${ }^{3}$

${ }^{1}$ Molecular and Comparative Pathobiology, Johns Hopkins University School of Medicine, Baltimore, MD; ${ }^{2}$ Cedars Sinai, Los Angeles, CA; ${ }^{3}$ Surgery, Johns Hopkins University School of Medicine, Baltimore, MD

Large oncosomes (LOs) are extracellular vesicles produced by nonapoptotic blebbing of cancer cells and are thought to be capable of enhancing metastasis by transfer of oncogenic microRNAs (miRNAs) and other cargo. LOs of 1 to $10 \mu \mathrm{m}$ in diameter have been reported in prostate cancer, glioblastoma, and acute lymphoblastic leukemia, with production enhanced by exposure to epidermal growth factor (EGF) or knockdown of cytoskeletal element DIAPH3. LOs have not yet been described in association with thyroid cancers. Here, we report LO formation by anaplastic and papillary thyroid cancer cell lines and compare the miRNA cargo of LOs and parent cells. Production of LOs by two ATC and two PTC lines (confirmed by sequencing) was measured under control conditions, EGF treatment, and siRNA-mediated DIAPH3 knockdown. Cells and LOs were stained with membrane dyes and visualized by fluorescence microscopy. LOs were measured by flow cytometry (LSR Fortessa or FACSAria II) with sizing beads to gate populations of $>1 \mu \mathrm{m}$. RNA was isolated from parent cells and from LOs separated from conditioned medium by a series of low-speed centrifugations and retention on a $0.2 \mu \mathrm{m}$ membrane. miRNA profiling (triplicates for each cell or LO type) was performed using custom TaqMan low density arrays and small RNA-seq (Illumina). LO formation from all four thyroid cancer lines was observed by fluorescence microscopy and flow cytometry. EGF treatment and DIAPH3 knockdown enhanced ameboid phenotypes as well as LO production (by up to 20 -fold) in all four cell lines, with a more striking change in phenotype occurring in PTC lines. miRNA profiling suggested enrichment of miR-223 and miR-150 in LOs compared with cells, while significant differences in miRNA expression were found in ATC versus PTC, including previously cancer- and senescence-associated miR-34a. All ATC and PTC lines examined produce LOs, and production is enhanced by EGF or DIAPH3 knockdown. miRNA expression distinguished ATC from PTC cells. We observed an apparent and unexpected enrichment of several miRNAs in LOs. We now seek to validate and extend these findings and determine functional significance, if any, of LOs in ATC and PTC.

\section{Basic/Translational Oral 17}

Thyroid Cancer Friday Basic

CO-EVOLUTION OF MET AMPLIFICATION AND HGF OVEREXPRESSION MEDIATE RESISTANCE TO BRAF ${ }^{\text {V60E }}$ INACTIVATION IN MURINE ANAPLASTIC THYROID CANCERS AND SENSITIZES THEM TO MET KINASE INHIBITORS

J.A. Knauf ${ }^{1,2}$, K.A. Luckett ${ }^{1}$, K. Chen $^{1}$, F. Voza ${ }^{1}$, R.A. Ghossein ${ }^{3}$, J. Fagin ${ }^{1,2}$

${ }^{1}$ HOPP, Memorial Sloan Kettering Cancer Center, New York, NY; ${ }^{2}$ Medicine, Memorial Sloan Kettering Cancer Center, New York, NY; ${ }^{3}$ Pathology, Memorial Sloan Kettering Cancer Center, New York, NY

ATCs arising from preexisting PTCs have a high prevalence of $B R A F$ and TP53 mutations. A recent basket trial of vemurafenib in nonmelanoma $\mathrm{BRAF}^{\mathrm{V} 600 \mathrm{E}}$-mutant cancers showed partial responses in 3 of 6 ATCs, and stable disease in the 3 others, suggesting that these virulent tumors retain an addiction to oncogenic BRAF. However, responses were short-lived, with nearly all patients recurring within a year. The mechanism mediating acquired resistance of ATCs to BRAF inhibitors is unknown. We used a mouse model of $\mathrm{BRAF}^{\mathrm{V} 600 \mathrm{E}}$-driven ATC to explore mechanisms of acquired resistance to BRAF ${ }^{\mathrm{V} 600 \mathrm{E}}$ inhibition. Nine weeks after dox treatment $\sim 50 \%$ of mice with thyroid-specific deletion of $p 53$ and dox-dependent expression of $\mathrm{BRAF}^{\mathrm{V} 600 \mathrm{E}}$ developed large tumors that closely phenocopy human 
ATCs, characterized by spindle cells with heavy infiltration of tumor associated macrophages. Discontinuing dox for 3 weeks caused complete regression in all mice studied, although recurrences were detected in $85 \%$ of the animals within 1 year. We used expression arrays and exome sequencing to identify mechanisms of resistance to loss of BRAF ${ }^{\mathrm{V} 600 \mathrm{E}}$. Recurrent tumors had elevated MAPK transcriptional output compared to normal thyroid, suggesting continued dependency on MAPK. Consistent with this, MEK inhibitors suppressed growth in cell lines derived from relapses, and in mice with recurrent tumors in vivo. Whole exome sequencing identified focal amplification of chromosome 6 in $6 / 11$ relapses, whose minimal region of overlap included Met. Recurrences with Met amplification overexpressed the receptor and its ligand Hgf. Growth, viability and Met downstream signaling was potently suppressed by MET kinase inhibitors in cell lines derived from relapses with Met amplification, whereas cell lines from primary ATCs and Met diploid relapses were resistant. $\mathrm{BRAF}^{\mathrm{V} 600 \mathrm{E}}$-driven ATC remain addicted to the oncoprotein. Recurrences are the rule after BRAF suppression, and associated with BRAF-independent reactivation of MAPK. Although multiple mechanisms are likely involved, the most common is due to activation of HGF/MET signaling, which generates exquisite dependency to MET kinase inhibitors.

\section{Basic/Translational Oral 18 \\ Thyroid Cancer Friday \\ TGF $\beta 1$-INDUCED RADIOIODINE THERAPY OF HEPATOCELLULAR CANCER AFTER MESENCHYMAL STEM CELL (MSC)-MEDIATED SODIUM IODIDE SYMPORTER (NIS) GENE DELIVERY \\ C. Schug ${ }^{1}$, S. Urnauer ${ }^{1}$, K. Schmohl ${ }^{1}$, M. Tutter ${ }^{1}$, N. Schwenk ${ }^{1}$, P. Nelson ${ }^{1,2}$, C. Spitzweg ${ }^{1}$ \\ ${ }^{1}$ Medizinische Klinik und Poliklinik IV, Klinikum der Universität München, Munich, Germany; ${ }^{2}$ Clinical Biochemistry Group, Munich, Germany}

Genetically engineered mesenchymal stem cells (MSCs) can be used as tumor-selective gene delivery vehicles into nonthyroidal tumors due to their excellent tumor-homing capacity. Delivery of the sodium iodide symporter (NIS) allows noninvasive imaging of functional NIS expression as well as therapeutic application of ${ }^{131}$ I. Tumorstroma specific promoters show great potential to improve tumor specificity. TGF $\beta 1$ is known to be overexpressed within human hepatocellular carcinomas (HCC) and is also released by cells of the tumor stroma. The essential role of TGF $\beta 1$ in the signaling between tumor and stroma made it a favorable target for the present study to control NIS expression specifically in experimental $\mathrm{HuH} 7$ tumors using MSCs stably transfected with a SMAD-responsive promoter, which is activated upon TGF $\beta 1$ stimulation. Bone marrow-derived MSCs were stably transfected with a NIS expressing plasmid driven by a SMAD-responsive promoter (NIS-MSC). Using iodide uptake assay, stimulation with TGF $\beta 1$ revealed a 4.9 -fold increase in NISmediated perchlorate-sensitive iodide uptake in NIS-MSCs as compared to unstimulated cells. NIS-MSCs were systemically injected in mice harboring subcutaneous $\mathrm{HuH} 7$ tumors and iodide uptake was monitored by ${ }^{123}$ I-scintigraphy showing significant tumor-specific accumulation. NIS immunohistochemistry of resected tumors confirmed selective NIS protein expression within the stroma. Therapeutic response was investigated after systemic injection of NIS-MSCs followed by ${ }^{131} \mathrm{I}$ application demonstrating a significant delay in tumor growth and prolonged survival in ${ }^{131}$ I treated mice. These data demonstrate the successful establishment of MSCs highly expressing NIS using a TGF $\beta 1$-inducible, SMAD-responsive promoter to increase tumor stroma-specificity of NIS expression after MSC-mediated NIS gene delivery in a HCC xenograft mouse model. In vivo studies confirmed high stromal targeting of NIS after systemic application of NIS-MSCs resulting in a significant therapeutic response after ${ }^{131} \mathrm{I}$ application. Using the tumoral TGF $\beta 1$ signaling network is a promising approach to enhance tumor stroma-selectivity of NIS transgene expression in the context of genetically engineered MSCmediated NIS gene therapy.

\section{Clinical Oral 19}

\section{Disorders of Thyroid Function Friday Clinical}

\section{GRAVES DISEASE AND ITS EFFECT ON MEDIAL} TEMPORAL LOBE STRUCTURES

M. Holmberg ${ }^{1,2}$, P. Berglund ${ }^{3}$, R. Heckemann ${ }^{3,4}$, B. Johansson ${ }^{3}$, N. Klasson ${ }^{3}$, E. Olsson ${ }^{2}$, H. Malmgren ${ }^{2}$, H. Filipsson Nyström ${ }^{2,5}$ ${ }^{1}$ ANOVA, Karolinska University Hospital, Stockholm, Sweden; ${ }^{2}$ Institute of medicine, Sahlgrenska Academy, University of Gothenburg, Gothenburg, Sweden; ${ }^{3}$ Institute of Neuroscience and physiology, University of Gothenburg, Gothenburg, Sweden; ${ }^{4}$ MedTech West, Sahlgrenska University Hospital, Gothenburg, Sweden; ${ }^{5}$ Dept of Endocrinology, Sahlgrenska University Hospital, Gothenburg, Sweden

Cognitive symptoms in patients with Graves hyperthyroidism are well-known and usually restores after achieved euthyroidism. Mechanisms are unknown, but similar cognitive impairment in Cushing's disease and chronic corticosteroid therapy are accompanied with reduced volume of brain medial temporal lobe (MTL) structures like hippocampus and amygdala - structures known to play an important role in forming and retrieving new memories. These structures also have high density of thyroid hormone receptors (TR), and data from TR knockout mice indicates that TR are involved in hippocampal structure and function. Hippocampal volume has also been described as reduced in volume in untreated patients with hyperthyroidism. However, longitudinal data is lacking. This project therefore hypothesises that: 1) MTL volumes are reduced in hyperthyroidism 2) After achived euthyroidism, MTL volumes are restored 3) Patients that do not regain MTL volume may persist in cognitive dysfunction. This is a case-control 15-month study recruiting 60 consecutive premenopausal women with newly diagnosed GD that are evaluated at diagnosis and after 15 months in long-term stable euthyroidism. Investigations includes neuropsychological and neuropsychiatric tests, thyroid hormones, thyroid antibodies and MTL volumetry with MRI analyzed manually and automatically with the Freesurfer and MAPER methods. We are now presenting longitudinal MAPER data for the first 30 patients. Results from the MAPER analysis show that both amygdalae are significantly increased in size between inclusion and at 15 months, while the hippocampi show a close-to-significant (left $\mathrm{p}=0.051$, right $\mathrm{p}=0.063$ ) trend towards increased size during the same period. These preliminary data support the hypotheses that MTL structures like amygdala and hippocampus are affected in Graves disease. It also supports the idea that MTL structures regain size when hormone levels are normalized More work will follow to track symptoms in connection to volume changes in order to identify this uncharacterized complication in GD and to compare with a control population to target individualized treatment.

\section{Clinical Oral 20}

Disorders of Thyroid Function Friday Clinical

\section{HIGHER REMISSION RATE AFTER LONG-TERM} METHIMAZOLE THERAPY IN PATIENTS WITH GRAVES DISEASE: A RANDOMIZED CLINICAL TRIAL F. Azizi ${ }^{1}$, A. Amouzegar ${ }^{1}$, M. Tohidi ${ }^{2}$, M. Hedayati ${ }^{2}$, L. Cheraghi ${ }^{2}$, Y. Mehrabi ${ }^{2}$ 
${ }^{1}$ Endocrine Research Center, Research Institute for Endocrine Sciences, Shahid Beheshti University of Medical Sciences, Tehran, Iran, Tehran, Iran (the Islamic Republic of); ${ }^{2}$ Prevention of Metabolic Disorders Research Center, Research Institute for Endocrine Sciences, Shahid Beheshti University of Medical Sciences, Tehran, Iran, Tehran, Tehran, Iran (the Islamic Republic of)

Introduction: Some studies suggest that long-term antithyroid drug treatment may induce high remission rates in patients with Graves' hyperthyroidism. We aimed to compare the rates of and the variables associated with remission of hyperthyroidism in patients with longterm vs. short-term methimazole (MMI) therapy.

Methods: In this randomized, parallel-group trial, 302 consecutive, untreated patients with a first episode of Graves' hyperthyroidism were enrolled. After 18.8 \pm 2.2 months MMI treatment, 258 patients were randomized to continue maintenance dose of MMI $(n=130$, long-term $=$ LT group $)$ or to discontinue treatment $(n=128$, short-term =ST group); 115 patients in the LT group continued MMI treatment for at least 60 months. MMI was withdrawn after a median of 96 months. Patients of both groups were followed for 48 months after discontinuation of MMI treatment.

Results: During the first 18 months of MMI treatment, 14 patients experienced cutaneous reactions and 2 had a rise in liver enzymes; no further side effects related to MMI were observed in LT groups for up to 120 months of MMI therapy. Of 128 patients in the ST group, 43 and $51 \%$ and of 115 patients in LT group, 8 and $16 \%$ had recurrent hyperthyroidism within 12 and 48 months after MMI withdrawal, respectively. Median time of relapse after discontinuation of MMI was 6.0 and 11.5 months in ST and LT groups, respectively. In the ST group, male gender, thyroid volume, serum TSH receptor antibody (TRAb) and HLA polymorphism were associated with the risk of relapse of hyperthyroidism.

Conclusion: Long-term, low dose MMI treatment for 60-120 months is a safe and effective method for treatment of Graves' hyperthyroidism. It is accompanied by much higher remission rates than the conventional 18-24 months MMI therapy.

\section{Clinical Oral 21}

Disorders of Thyroid Function Friday Clinical

\section{EFFECTS OF ALTERING LEVOTHYROXINE (L-T4) DOSES ON QUALITY OF LIFE, MOOD AND COGNITION IN L-T4 TREATED SUBJECTS: A DOUBLE-BLINDED, RANDOMIZED, CONTROLLED TRIAL}

M.H. Samuels ${ }^{1}$, I. Kolobova ${ }^{3}$, M. Niederhausen ${ }^{2}$, J. Janowsky ${ }^{4}$, K. Schuff ${ }^{1}$

${ }^{1}$ Endocrinology, Diabetes and Clinical Nutrition, Oregon Health \& Science University, Portland, OR; ${ }^{2}$ Biostatistics and Design Program, Oregon Health \& Science University, Portland, OR; ${ }^{3}$ Penn State Health St. Joseph, Reading, PA; ${ }^{4}$ Saturday Academy, Portland, OR

The brain is a critical target organ for thyroid hormone, but it is not clear whether variations in thyroid function within and near the laboratory reference range (indicated by high-normal or mildly elevated TSH levels) affect quality of life, mood, or cognitive function.138 subjects with L-T4 treated primary hypothyroidism and normal TSH levels underwent baseline measures of quality of life (SF-36, ThyDQoL), mood (Profile of Mood States, Affective Lability Scale), and cognition (declarative and working memory, motor learning, executive function). They were then randomly assigned to receive their usual L-T4 dose or a higher or lower dose in double blind fashion, targeting one of three TSH ranges (Low-Normal TSH (0.34-2.50 mU/L), High-Normal TSH (2.51-5.60 mU/L), or Mildly Elevated TSH $(5.60-12.0 \mathrm{mU} / \mathrm{L}))$. Doses were adjusted every 6 weeks based on TSH levels. Quality of life, mood and cognitive tests were reassessed at 6 months. Outcomes were compared among the three groups using mixed models or multiple logistic regressions. At end of study, mean L-T4 doses were $1.52 \pm 0.06,1.10 \pm 0.10$, and $0.92 \pm 0.08 \mathrm{mcg} / \mathrm{kg}(\mathrm{p}<.001)$, and mean TSH levels were $1.34 \pm 0.08$, $3.74 \pm 0.12$, and $9.74 \pm 0.63 \mathrm{mU} / \mathrm{L}(\mathrm{p}<.001)$ in the Low-Normal TSH, High-Normal TSH, and Mildly Elevated TSH groups. There were minor differences in a few outcomes among the three groups (SF-36 bodily pain best in the High-Normal TSH group $(\mathrm{p}=.04)$, 1 -Back best in the Low-Normal TSH group $(\mathrm{p}=.004)$ ), but no clinically meaningful effects in any outcomes. Subjects preferred L-T4 doses they perceived to be higher $(\mathrm{p}<.001)$, but not actual higher $\mathrm{L}-\mathrm{T} 4$ doses $(\mathrm{p}=0.54)$. Altering L-T4 doses in L-T4 treated subjects to achieve low-normal TSH levels (vs high-normal or mildly elevated levels) does not significantly improve quality of life, mood, or cognition. L-T4 treated subjects prefer perceived higher L-T4 doses despite a lack of objective benefit from actual higher L-T4 doses. Adequately treated hypothyroid patients' reports of symptoms in these areas should not be used as a basis for adjusting L-T4 doses.

\section{Clinical Oral 22}

\section{Thyroid Cancer Friday Clinical}

\section{ACCURACY OF ADULT ATA ULTRASOUND RISK} STRATIFICATION IN CHILDREN

A.L. Creo ${ }^{1}$, F. Alahdab ${ }^{4}$, A. Al Nofal ${ }^{2}$, A. Kolbe ${ }^{3}$, K. Thomas ${ }^{3}$, S. Pittock ${ }^{1}$

${ }^{1}$ Pediatric Endocrinology, Mayo Clinic, Rochester, MN; ${ }^{2}$ Pediatric Endocrinology, Sanford Health, Souix Falls, SD; ${ }^{3}$ Pediatric Radiology, Mayo Clinic, Rochester, MN; ${ }^{4}$ Mayo Clinic, Rochester, MN

Pediatric thyroid nodules are more likely malignant compared to adults and may have different concerning ultrasound (US) features. Recent adult guidelines stratify malignancy risk by US features. Our aim is to determine if adult American Thyroid Association (ATA) risk stratification guidelines apply in children. We identified 330 children with thyroid nodules on US between 1996 and 2015. Two blinded pediatric radiologists read all US, described multiple features, and reported their overall impression: benign, indeterminate, or malignant. Each nodule was independently assigned to an ATA risk stratification category (benign (B), very low suspicion (VLS), low suspicion (LS), intermediate suspicion (IS), or high suspicion (HS)). Radiologists' impressions and ATA risk stratification were compared to histology and cytology results. Histology or cytology results combined with stable follow-up US were available for 145 nodules, of which $37 \%$ were malignant or had Bethesda V or VI cytology. No nodules were assigned to the ATA risk stratification benign (B) category. Overall, 91/145 nodules had benign histology or cytology (3/4 VLS, 28/31 LS, 19/25 IS, and 41/85 HS). Sensitivity and specificity for ATA risk stratification detecting malignancy in HS nodules was $82 \%$ and $58 \%$ respectively; area under ROC curve was 0.71 (95\% CI 0.63-0.78, P<0.001). ATA risk stratification significantly correlated to the radiologists' overall impression $(\mathrm{P}<0.001)$. All nodules radiologists read as malignant were in the HS category. However, $36 \%$ of nodules categorized as HS, radiologists reported as benign or indeterminate. The radiologists' impression had a higher sensitivity (91\%) and specificity (83\%) at detecting malignancy than the risk stratification tool. ATA risk stratification resulted in many false positives as benign nodules frequently were classified HS or IS (predominantly due to presence of hyperechogenicity, hypoechogenicity, or irregular margins). At our institution, pediatric radiologists' overall impressions detected malignancy more accurately than the risk stratification tool. Further work is needed to determine the usefulness of adult ATA risk stratification in children. 


\section{Poster 174}

Autoimmunity Friday Poster Basic

ENOLASE $\alpha$-IMMUNIZATION CAN INDUCE THE DEVELOPMENT OF AUTOIMMUNE THYROIDITIS IN CBA/J MICE

J. Li, J. Qin, Y. Xiang, Y. Lu, H. Li, R. Sun, Z. Shan, W. Teng

Department of Endocrinology and Metabolism, Institute of

Endocrinology, Liaoning Provincial Key Laboratory of Endocrine

Diseases, The First Affiliated Hospital of China Medical University,

Shenyang, Liaoning, China

$\alpha$-enolase (ENO1) is a conserved protein. Anti-ENO1 autoantibody $(\mathrm{ENO} 1 \mathrm{Ab})$ is an important marker for Hashimoto's Encephalopathy. Our preliminary study has found that ENO1 is expressed on thyroid follicular cells, and serum ENO1Ab was significantly higher in patients with autoimmune thyroiditis (AIT) than heathy controls. This study was to investigate the effects of immune attack against ENO1 on thyroid tissue of CBA/J mice, which can develop typical AIT after thyroglobulin $(\mathrm{Tg})$ immunization. Five-week-old female CBA/J mice were immunized twice with recombinant ENO1 protein in Freund's adjuvant at two-week interval. Some were injected only with Freund's adjuvant as control. Anti-Tg antibody (TgAb), EN$\mathrm{O} 1 \mathrm{Ab}$ and thyroid functions were measured by ELISA. Thyroid tissue was processed for histological examination and immunofluorescent staining of immunocyte phenotype, IgG and Complement3 (C3) deposition and apoptosis. Splenic cytokine expression was assessed by RT-PCR. Serum ENO1-sepcific total IgG level was significantly higher in ENO1-immunized mice than the controls from the $4^{\text {th }}$ to $18^{\text {th }}$ week after the second challenge. The predominant subclass of ENO1 Ab was IgG2a and less IgG1 and IgG2b, consistent with enhanced mRNA expressions of IL-17A and IL-4 in the spleen. Serum $\mathrm{TgAb}$ level had been obviously increased in ENO1 group as compared with controls since the $10^{\text {th }}$ week. Furthermore, there was inflammatory cell infiltration to some extent in the thyroid after ENO1 challenge. Both the incidence of thyroiditis and inflammatory score were significantly increased at $10^{\text {th }}$ and $14^{\text {th }}$ weeks. In those mononuclear infiltrates, $\mathrm{CD} 4^{+}, \mathrm{CD}^{+}$and $\mathrm{CD} 19^{+}$cells were all found. The deposition of IgG but not $\mathrm{C} 3$ was obviously present together with ENO1 on thyroid follicles around which there was $\mathrm{CD}_{16}{ }^{+}$cell aggregation. The expression of intrathyroidal Cleaved Caspase-3 in ENO1 group was significantly up-regulated as compared with controls. Serum TSH showed a significant increase in ENO1 group although TT4 concentration was not changed. ENO1 may act as a nonclassical autoantigen in the pathogenesis of AIT. Its immune responses can induce the damages of thyroid follicles through antibody dependent cellular cytotoxicity.

\section{Poster 175}

Autoimmunity Friday Poster Translational

\section{RNASET2 TAG SNP IS ASSOCIATED WITH GRAVES' DISEASE IN THE JAPANESE POPULATION}

Y. Ban ${ }^{1}$, T. Tozaki ${ }^{2}$, Y. Kiuchi ${ }^{2}$

${ }^{1}$ Third Department of Internal Medicine, Teikyo University Chiba Medical Center, Chiba, Japan; ${ }^{2}$ Division of Medical Pharmacology, Department of Pharmacology, Showa University School of Medicine, Tokyo, Japan

The etiology of autoimmune thyroid diseases (AITDs), such as Graves' disease (GD) and Hashimoto's thyroiditis (HT), is largely unknown. However, genetic susceptibility is believed to play a major role. Recently, we found that the SNP rs9355610 within 6q27 was significantly associated with GD in the Japanese population (Autoimmunity, 46:395-398, 2013). Polymorphisms of the CC chemokine receptor 6 (CCR6) and RNASET2 tag single nucleotide polymorphism (SNP) have been shown to be associated with the susceptibility to several immune-related diseases. Therefore, we conducted a case-control study to determine the genetic association of the three selected SNPs of CCR6 and one tagging SNP within RNASET2 with AITD in a cohort of the Japanese population. We genotyped the three CCR6 SNPs (rs3093023/rs3093024/rs6902119) and one tagging SNP (rs9355610) within RNASET2 gene in 457 Japanese patients with AITD (287 with GD, 170 with HT) and 241 matched Japanese control subjects. Those SNPs were analyzed by the high-resolution melting and unlabeled probe methods. Case-control association studies were performed using the $\mathrm{c}^{2}$ and Fisher's exact tests with Yates correction. GD was significantly associated with rs 9355610 within RNASET2 gene $(\mathrm{P}=0.046, \mathrm{OR}=1.29)$, while HT showed no significant associations. No association was found for the tested CCR6 SNPs. Moreover, when patients with GD were stratified according to Graves' ophthalmopathy $(\mathrm{GO})(\mathrm{n}=96)$, there were no allelic associations with GO. These findings suggest that the rs9355610 tag SNP of the RNASET2 gene, but not rs3093023, rs3093024, or rs6902119 in the CCR6 gene, is associated with susceptibility to GD in the Japanese population.

\section{Poster 176}

Autoimmunity Friday Poster Clinical

\section{CHOROIDAL THICKNESS CHANGES IN GRAVES' OPHTHALMOPATHY}

E. Cagiltay ${ }^{1}$, F. Akay ${ }^{2}$

${ }^{1}$ Endocrinology, Sultan Abdulhamid Khan Education and Research Hospital, Istanbul, Uskudar, Turkey; ${ }^{2}$ T.R. Ministry of Health, Medical Sciences University, Izmir Katip Çelebi University Atatürk Training and Research Hospital, Izmir, Turkey

Graves' ophthalmopathy (GO) may develop nearly $25-50 \%$ in Graves' Disease (GD). Clinical manifestations of GO are caused by the over compression of orbital tissues within the restricted orbital bone cavity. Impaired ocular blood flow may disrupt the retinal microstructure and functions. Early recognition of retinal and choroidal changes may alert the physicians for preventing ocular complications of GO.

We aimed to investigate the choroidal thickness changes in GO compared with healthy subjects. The study group comprised 50 adult patients with previously diagnosed Graves' Disease with ophthalmopathy who were on anti thyroid treatment, compared with controls. For the assessment of GO activity, VISA (vision, inflammation, strabismus, and appearance) inflammatory score was used. When euthryoidism was achieved without side effects, the patients were referred to the ophthalmology clinic for Spectral-domain optical coherence tomography (SD-OCT) evaluation. SD-OCT is a noninvasive method that is used for quantitive assessment of retinal morphology and choroidal thickness. We used the VISA inflammatory score for the assessment of GO activity. Subfoveal, mean and temporal choroidal thicknesses were increased significantly in study group according to the controls. But nasal, peripapillary and choroidal thickness minimal increased in study group and there was no statistical difference. None of our patients had severe GO. The VISA Inflammatory Score was 4.72 \pm 1.27 . However, the mean choroidal thickness was elevated.

Choroidal thickness location. (Study group $\mathrm{n}=50$ \&Control group $\mathrm{n}=50) . \mathrm{P}$ value*

Subfoveal; 304.22 $\pm 36.09 \& 275.54 \pm 34.20, \mathrm{p}<0.001$

Temporal, $500 \mu \mathrm{m} ; 311.06 \pm 38.18 \& 274.22 \pm 34.92, \mathrm{p}<0.001$

Temporal, $1000 \mu \mathrm{m} ; 309.68 \pm 36.83 \& 272.98 \pm 33.24, \mathrm{p}<0.001$

Temporal, $1500 \mu \mathrm{m} ; 302.00 \pm 32.44 \& 267.64 \pm 31.77, \mathrm{p}<0.001$

Mean; $293.08 \pm 34.81 \& 268.24 \pm 33.11, \mathrm{p}<0.001$ 
$*=$ student $\mathrm{T}$ testWe think that this elevation is because of the retroorbital inflammation even in this non-severe GO group. We also suggest that choroidal thickness might be affected from the venous obstruction and congestion in patients with GO. The elevation of the choroidal thickness might be an early sign of venous congestion that occurs before the elevation of IOP.

\section{Poster 177}

Autoimmunity Friday Poster Clinical ASSOCIATION BETWEEN OBESITY PHENOTYPES AND INCIDENT OF THYROID AUTOIMMUNITY: A 10-YEAR FOLLOW-UP

E. Kazemian, F. Azizi, A. Amouzegar, S. Gharibzadeh

Research Institute for Endocrine Sciences, Shahid Beheshti

University of Medical Sciences, Tehran, Iran, Tehran, Iran (the

Islamic Republic of)

Several articles have demonstrated a relation between thyroid autoimmunity and obesity. Some of these articles have concluded that thyroid autoimmunity is a cause of hyperlipidemia and abdominal obesity. Some other articles have indicated that thyroid autoimmunity is a result of obesity. The aim of present study is to compare thyroid autoimmunity incidences among different obesity phenotypes during a median follow-up of about 10 years. This study was conducted in a population-based cohort study, Tehran Thyroid Study (TTS). Body weight, waist circumference (WC) and blood pressure (BP) were measured. Serum concentrations of lipids and lipoproteins, fasting blood glucose (FBG), 2-h blood glucose (2-hBG), Free T4 (FT4) and TSH were after 3, 6 and 9 years of follow-up. Individuals were categorized into different obesity phenotype based on metabolic syndrome component and body mass index (BMI). Follow-up duration and person-years were calculated using the measured survival time. Cox proportional hazards models were used to evaluate association of potential risk factors with incidence of autoimmunity adjusted for age, sex, FT4 and TSH. The highest and lowest incidence rate of TPOAb positivity was observed among metabolically unhealthy non-abdominal obese (MUNAO) [8.78 (7.31 - 10.55) per 1000 person-years of followup] and metabolically unhealthy abdominal obese (MUAO) [4.98 (3.88-6.41) per 1000 person-years of follow-up] respectively. Compared with MHNAO (metabolically healthy non-abdominal obese) phenotype, no significant association was observed between MUNAO, MHAO (metabolically healthy abdominal obese) and MUO phenotypes and risk of developing TPOAb positivity. The incidence rate $(95 \% \mathrm{CI})$ of TPOAb positivity among individuals with normal WC (waist circumference) was higher compared to individuals with high WC 8.44 (7.13-10.0) vs 5.11 (4.01-6.51) per 1000 person-years respectively. Higher WC was not associated with progression of TPOAb positivity neither in sex, age FT4 nor in sex, age TSH model. we did not found significant association between at risk phenotypes and development of TPOAb positivity while the lowest incidence rate of TPOAb positivity was observed among MUAO individuals.

\section{Poster 178}

Autoimmunity Friday Poster Clinical

\section{CLINICAL FEATURES IN UNTREATED HASHIMOTO'S THYROIDITIS WITH ELEVATED SERUM IMMUNOGLOBULIN G4 LEVELS IN TAIWAN}

W. Wu, F. Tseng

Internal Medicine, National Taiwan University Hospital, Taichung, Taiwan

Immunoglobulin G4-related disease (IgG4-RD) is a newly identified syndrome characterized by high serum IgG4 levels and increased
IgG4-positive plasma cells in involved organs. Hashimoto's thyroiditis (HT) was first sub-classified to IgG4-related thyroiditis and non-IgG4 thyroiditis based on the immunohistochemistry of IgG4 in 2009. IgG4-related thyroiditis exhibited histopathological features of IgG4-RD, and its source of serum IgG4 is suggested to be thyroid gland. Although the relationship between IgG4-RD and IgG4-related thyroiditis has been reported, the clinical implication of serum IgG4 level in HT is uncertain. We prospectively evaluated serum IgG4 levels and clinical features of patients with untreated and euthyroid HT. We divided them into two groups by using the serological diagnostic criteria of IgG4-RD: elevated IgG4 HT (serum IgG4 > 135 $\mathrm{mg} / \mathrm{dL}$ ) and non-elevated IgG4 HT (serum IgG4 $\leq 135 \mathrm{mg} / \mathrm{dL}$ ). A total 56 patients with HT were recruited. Median serum IgG4 levels were $50.8 \mathrm{mg} / \mathrm{dL}$ (interquartile range, 34.0-86.8). Six of them had elevated serum IgG4 above $135 \mathrm{mg} / \mathrm{dL}$. The elevated IgG4 HT group had higher proportion of male (female/male $3 / 3$ [50\%] vs. 1/49 [2\%], $p=0.003)$ and exhibited larger thyroid volume $(18.9 \mathrm{~mL}$ [17.6-23.4] vs. $15.2 \mathrm{~mL}$ [12.4-18.6], $p=0.036)$. There were no significant difference in age and body mass index, thyroid function, titer of antithyroperoxidase and anti-thyroglobulin antibody between the two groups. One of the six elevated IgG4 HT patients was diagnosed of monoclonal gammopathy of undetermined significance. There was no significant extra-thyroid organ involvement as seen in IgG4-RD in the other five patients. HT patients with elevated IgG4 levels shared some clinical features of both IgG4-RD and IgG4-related thyroiditis, such as gender and goiter. However, other clinical features including age and titers of anti-thyroid autoantibody were inconsistent with previous studies. Therefore, longer follow-up periods and histopathological assessments are needed to help understand the clinical implication of elevated serum IgG4 levels in untreated HT.

\section{Poster 179}

Autoimmunity Friday Poster Clinical

\section{AUTOIMMUNE THYROID DISEASE IS POSITIVELY ASSOCIATED WITH NON-ALCOHOLIC FATTY LIVER DISEASE IN FEMALE: A NEW INSIGHT} FROM A POPULATION-BASED STUDY SPECT-CHINA

Y. Chen, Y. Chen, N. Wang, Q. Li, B. Han, Y. Lu

Endocrinology and Metabolism, Shanghai Ninth People's Hospital, Shanghai Jiaotong University School of Medicine, Shanghai, China, Shanghai, China

Non-alcoholic fatty liver disease (NAFLD) is caused by many risk factors, including innate immune, but its association with thyroid autoimmunity in a large population was unreported. We explored the association between NAFLD with the prevalence of autoimmune thyroid disease (AITD). The data were obtained from a cross-sectional population-based study, the SPECT-China study, 2014-2015. A total of 8,947 over 18 years of age (3,456 males; 5,491 females) were enrolled in this study. We defined AITD based on the presence of TPOAb and $\operatorname{TgAb}$ levels, and the presence of thyroid ultrasonography (US) findings. The degrees of NAFLD were categorized into mild and moderate to severe hepatic steatosis groups by US as well.

Overall, the prevalence of positivity for TPOAb and/or $\mathrm{TgAb}$ $[\mathrm{TPO} / \mathrm{TgAb}(+)]$ was $19.06 \%$, and the positivity for TPOAb and/or $\mathrm{TgAb}$ together with US [TPO/TgAb(+) \& US(+)] was $9.78 \%$. A total of $21.76 \%, 32.06 \%$ of men and $20.29 \%, 22.87 \%$ of women had mild and moderate to severe hepatic steatosis. The prevalence of moderate-severe hepatic steatosis was significantly higher in the $\mathrm{TPO} / \mathrm{TgAb}(+)$ group $(36.39 \%$ vs. $31.49 \%, P=0.047$ in men and $28.59 \%$ vs. $21.10 \%, P<0.001$ in women) and TPO/TgAb(+)\&US(+) group ( $42.51 \%$ vs. $31.49 \%, P=0.003$ in men and $34.04 \%$ vs. $21.10 \%$, $P<0.001$ in women) when compared with the TPO\&TgAb(-). After full adjustment, multivariate logistic analysis showed that both TPO/ 
$\mathrm{TgAb}(+)$ and $\mathrm{TPO} / \mathrm{TgAb}(+) \& \mathrm{US}(+)$ group were associated with an increased risk of moderate to severe hepatic steatosis in female participants. The ORs were $1.42(95 \%$ CI $1.17,1.72, P<0.001)$ and 1.58 (95\% CI 1.24, 2.00, $P<0.001)$, respectively.

AITD and NAFLD are highly prevalent in China. AITD was positively associated with moderate to severe NAFLD in females. This is the first report suggesting a increased risk of NAFLD in AITD female which suggested a common innate immune pathways in AITD and NALFD.

\section{Poster 180}

Thyroid \& Development Friday Poster Clinical THE STATUS OF THYROID DISEASES IN EAST CHINA: SPECT-CHINA (2014-2017)

Y. Lu, Y. Chen, Y. Chen, N. Wang, Q. Li, H. Zhai

Endocrinology and Metabolism, Shanghai Ninth People's Hospital, Shanghai Jiaotong University School of Medicine, Shanghai, China, Shanghai, China

To investigate the status of thyroid diseases in population in East China. The data were obtained from a cross-sectional study (SPECTChina study, 2014-2017, ChiCTR-ECS-14005052, www.chictr.org) which were based on the population. 12,666 subjects above 18 years old with a standardized questionnaire, physical examinations, completely assays of thyroid function, glucose and lipid profiles, sex related hormones as well as thyroid ultrasound were enrolled.

1. Among all the participants (54.71 \pm 13.01 years old), 5,121 were males and 7,545 were females.

2. Thyroid nodule: $52.7 \%$ of the participants had thyroid nodule(s) Among them, the prevalence of multiple nodules, nodule $>1 \mathrm{~cm}$, nodule with calcification, with microcalcification and with a "taller" than "wider" shape was $85.0 \%, 20.2 \%, 6.3 \%, 1.4 \%$ and $1.9 \%$, respectively. Female had a significantly higher prevalence of thyroid nodule(s), multiple nodules, nodule $>1 \mathrm{~cm}$, and nodules with microcalcifications than men $(58.5 \%$ vs. $44.1 \%, 87.1 \%$ vs. $81.0 \%, 22.3 \%$ vs. $16.0 \%$ and $1.6 \%$ vs. $1.0 \%, P<0.05)$. The prevalence of thyroid nodule, multiple nodules, nodule $>1 \mathrm{~cm}$ and nodule with calcification was increasing along with age $(P<0.05)$. By stepwise logistic regression, thyroid nodule was significantly related to age, sex, thyroid antibody, parameters of obesity, glucose and lipid metabolism, and hypogonadism.

3. Thyroid function distribution: The mean level of TSH was $2.83 \pm 4.37$ (mIU/L), [men: $2.45 \pm 3.79$ (mIU/L), female: $3.09 \pm 4.70$ $(\mathrm{mIU} / \mathrm{L})]$. The prevalence of hypothyroidism, subclinical hypothyroidism, hyperthyroidism and subclinical hyperthyroidism was $0.2 \%, 8.3 \%$, $0.4 \%$ and $1.27 \%$, respectively. TSH increased with age $(P<0.05)$.

3. Thyroid antibody: The prevalence of positivity for TPOAb (>60IU/L) was $13.1 \%$ (male: $8.5 \%$ and female: $16.3 \%$ ). The prevalence of positivity for $\mathrm{TgAb}(>60 \mathrm{IU} / \mathrm{L}$ ) was $13.0 \%$ (male: $5.9 \%$ and female: $17.8 \%$ ). TPOAb and TgAb levels were positively associated with TSH levels. The prevalence of thyroid nodules in East China was $44.1-58.5 \%$, subclinical hypothyroidism is $8.3 \%$. The prevalence of positivity for TPOAb was $13.1 \%$. Thyroid disease were closely related to other endocrine and metabolic diseases.

\section{Poster 181}

Iodine Uptake \& Metabolism Friday Poster Clinical

\section{THE STATUS OF IODIZED SALT INTAKE IN URBAN CHINESE, AND ITS LINK WITH URINARY IODINE, TPOAB AND TGAB}

Y. Lu, C. Chen, Y. Chen, Y. Chen, N. Wang

Endocrinology and Metabolism, Shanghai Ninth People's Hospital, Shanghai Jiaotong University School of Medicine, Shanghai, China, Shanghai, China
Whether iodized salt increases the risk of thyroid diseases has been strongly debated in China, especially in the urban areas of coastal regions, in recent years. We aimed to investigate the status of iodized salt in terms of urinary and serum iodine concentration in urban coastal areas, and to further explore whether iodized salt or noniodized salt was associated with autoimmune thyroid disease (AITD). Our data source was SPECT-China (ChiCTR-ECS-14005052, www.chictr.org. cn). We enrolled 1678 subjects from 12 communities in downtown Shanghai. The type of salt consumed, the urinary iodine concentration (UIC), serum iodine, thyroid peroxidase antibody (TPOAb) and thyroglobulin antibody (TgAb) levels were obtained. AITD was defined as serum TPOAb and/or TgAb $>60 \mathrm{kIU} / \mathrm{L}$ [TPO/TgAb (+)]. The prevalence of AITD was $10.5 \%$ in men, and $21.4 \%$ in women. The median UIC and serum iodine concentration were $106.4 \mu \mathrm{g} / \mathrm{L}$ and 60 . $9 \mu \mathrm{g} / \mathrm{L}$, respectively. Among all the subjects, $46.4 \%$ consumed noniodized salt; the prevalence of iodine deficiency among those subjects was significantly higher than that of the subjects who consumed iodized salt $(54.2 \%$ vs $40.1 \%, P<0.001)$. Consumption of non-iodized salt was positively associated with AITD in all participants (OR 1.49; $95 \%$ CI $1.15-1.95 ; P=0.003)$ and in women (OR 1.63; 95\% CI 1.20-2. $21 ; P<0.01)$ after multivariable adjustment. Additionally, the association between low UIC and AITD was observed among all subjects (OR 1.50; 95\% CI 1.10-2.05; $P=0.01$ ) and in women (OR 1.45; 95\% CI 1.02-2.07; $P=0.038$ ). In coastal areas that are believed rich in iodine, consuming non-iodized salt still led to lower UIC levels and a higher prevalence of iodine deficiency. The consumption of noniodized salt and low UICs might be a risk factor for AITD, especially for women, which should be further confirmed by longitudinal studies.

\section{Poster 182}

Thyroid Imaging Friday Poster Clinical

\section{PREOPERATIVE SONOGRAPHIC FEATURES OF FOLLICULAR THYROID CARCINOMA PREDICT BIOLOGICAL BEHAVIOR}

X. Lai, B. Zhang, Z. Liang, Y. Jiang, J. Li, R. Zhao, X. Yang, X. Zhang, S. Zhu

Peking Union Medical College Hospital, Beijing, Beijing, China

Although some clinicopathological features of follicular thyroid carcinoma (FTC) can predict a more progressive course and suggest poorer prognosis, little is known regarding biological behavior according ultrasound (US) features. We investigated whether there was a difference in biological behavior between benign-looking FTCs (B-FTCs) and malignant-looking FTCs (M-FTCs). A total of 55 cases of FTCs between January 2000 and December 2015 were included in this study and clinical records and histological and US findings were reviewed. B-FTC was defined as showing none of the accepted US criteria for malignancy. M-FTCs and B-FTCs were compared in terms of patients' age, sex, tumor size, multifocality, lymph node metastasis, distant metastasis, extrathyroidal extension, extranodal extension, and degree of invasiveness in pathology (minimally invasive FTC or widely invasive FTC). B-FTCs accounted for $31(56.4 \%)$ of all 55 FTCs. Compared with the patients with BFTCs, the patients with M-FTCs showed a significantly higher prevalence of widely invasive FTCs, central lymph node metastases, lateral lymph node metastases as well as extrathyroidal extension $(\mathrm{p}<0.001$, $\mathrm{p}=0.012, \mathrm{p}=0.031$, and $\mathrm{p}=0.032$, respectively). The common US features of B-FTCs included an ovoid to round shape, a smooth margin, a regular thin halo ring, hypoechoic echogenicity, heterogeneous echotexture, no cystic change, no calcification and hypervascularity. M-FTCs with more than one malignancy features on US showed a significantly higher prevalence of extrathyroidal extension than MFTCs with only one US malignancy feature $(p=0.022)$. Widely invasive FTCs were more common in the patients with more than one 
malignancy features on US than in those with only one US malignancy feature. However, the difference was not statistically significant $(\mathrm{p}=0.089)$. FTCs that did not meet malignant US criteria had better prognostic indicators than FTCs that met malignant US criteria. Therefore, preoperative US features can serve as a useful tool for predicting biological behavior in FTC.

\section{Poster 183}

Autoimmunity Friday Poster Clinical

A META-ANALYSIS OF HASHIMOTO'S THYROIDITIS
AND BREAST CANCER RISK

X. Lai, Q. Zhu, B. Zhang, J. Li, Y. Jiang

Peking Union Medical College Hospital, Beijing, Beijing, China

Whether Hashimoto's thyroiditis (HT) predisposes patients to the development of breast cancer (BC) is a continuing debate. We performed a comprehensive meta-analysis to investigate the possible associations of HT and BC. We identified all relevant published studies by searching PubMed and Embase. Two authors independently extracted the data from each original article and independently assessed the quality of the included studies according to the Newcastle-Ottawa Scale. The pooled odds ratio (OR) with $95 \%$ CIs were calculated by the random-effects models for the association between HT and BC. Heterogeneity among articles was quantitatively assessed using the $\mathrm{Q}$ test and $\mathrm{I}^{2}$ statistic. Two types of studies were included in this meta-analysis. The first type of studies (HT studies) compared the prevalence of BC between patients with HT and patients without HT, including 3 cohort studies. The second type of studies (BC studies) compared the prevalence of HT between patients with $\mathrm{BC}$ and patients without $\mathrm{BC}$, including 2 cohort studies and 18 case-control studies. The pooled OR, based on overall HT studies, was 1.61 (95\% CI: 1.12-2.31). Satisfactory homogeneity was found among the studies $\left(\mathrm{I}^{2}=0.0 \%, \mathrm{P}=0.935\right)$. The sensitivity analyses revealed that no study had a significant influence on the overall estimates. The shape of the funnel plot of HT studies seemed to be symmetrical, and the P value of Begg's test was 1.000 , indicating the absence of publication bias. The pooled OR, based on overall BC studies, was 1.81 (95\% CI: 1.32-2.49). However, there was significant heterogeneity among the studies $\left(\mathrm{I}^{2}=74.3 \%, \mathrm{P}<0.001\right)$. The sensitivity analyses revealed that no study had a significant influence on the overall estimates. The shape of the funnel plot of $\mathrm{BC}$ studies seemed to be symmetrical, and the $\mathrm{P}$ value of Begg's test was 0.381, indicating the absence of publication bias. HT studies confirmed that BC was more often found in patients with HT than in patients without HT. BC studies confirmed that HT was more often found in patients with $\mathrm{BC}$ than in patients without BC. These two types of studies both confirmed the association between $\mathrm{HT}$ and $\mathrm{BC}$.

\section{Poster 184}

Disorders of Thyroid Function Friday Poster Basic THE INFLUENCE OF GESTATIONAL STAGE OF PREGNANCY TO THYROID DYSFUNCTION IN GOITER REPLETE AND NONREPLETE AREA

I. Kusrini, D. Mulyantoro, B. Budiman

Health research And Development, Center Of research and development of Iodine, Magelang, Central Java, Indonesia

Physiological changes during pregnancy and thyroid hormone transfer from mother to the fetus leads to an increase requirement of iodine. Objective. This study was conducted to assess the influence of the gestational stage on thyroid dysfunction in pregnancy in goiter replete and nonreplete area. This is a cross sectional study in four districts in Magelang Indonesia with estimation proportion for stratification for minimal sample size design and gestational stage of pregnancy as strata. Iodine intake was measured and iodine urine concentration $($ UIC) for three days $(3 \times 24$ hours). Thyroid function was measured by Thyroid Stimulating Hormone (TSH) and free thyroxin (fT4) with specific reference for pregnant women from American Thyroid Association. There were 242 pregnant women in the replete and the non replete area were conducted by Juli until November 2015. Mean TSH and free thyroxin (fT4) concentrations in replete and non replete area were normal range in every gestational stage in replete and the non replete. But the results showed that there was a significant difference of mean TSH and ft4 every stage of pregnancy on both areas, with p value; 0,002 (TSH) and 0,001 (ft4). Prevalence of thyroid dysfunction as hypothyroidism in replete area $17,1 \%\left(10,5 \%{ }^{1 s t} ; 17,7 \%^{2 n d} ; 25 \%{ }^{3 r d}\right)$ and $19,2 \%\left(14 \%^{1 s t} ; 26 \%^{2 n d}\right.$; $\left.29,1 \%{ }^{3 r d}\right)$ in non replete area. Discussion hypotiroid dysfunction in pregnancy, such overt hypothyroidism, subclinical hypothyroidism and hypothyroxinemia can adversely effect to neurodevelopment. Diagnosis Thyroid dysfunction with gestational specific as stratification are required because different of physiological change every trimester, this would permit accurate assessment to describe the profiles of thyroid function during pregnancythere was an influence from the gestational stage of pregnancy and thyroid dysfunction in pregnancy because physiological changes during pregnancy.

\section{Poster 185}

\section{Disorders of Thyroid Function Friday Poster Case Report}

\section{SEVERE THYROIDITIS CAUSED BY NIVOLUMAB}

J. Way, S. Smooke

Medicine - Endocrinology, UCLA, Los Angeles, CA

Anti-PD-1 antibodies (PD-1Ab) are effective cancer therapies that stimulate the immune system and may cause immune-related adverse events including endocrinopathies. We report a case of severe thyroiditis caused by the PD-1Ab nivolumab. A 50 year-old male with refractory glioblastoma multiforme on bevacizumab and nivolumab presented with lethargy. Two weeks after his second dose of nivolumab, he developed profound fatigue, heat intolerance, and tremor. Examination was significant for normal vital signs, confusion, leftsided hemiplegia, normal thyroid gland, tremor, and warm skin. TSH was $<0.02 \mathrm{mcIU} / \mathrm{mL}$ (nl 0.3-4.7), $\mathrm{TT}_{3} 340 \mathrm{ng} / \mathrm{dL}$ (nl 85-185), $\mathrm{TT}_{4}$ $>25 \mathrm{mcg} / \mathrm{dL}$ (nl 4.9-11.4), $\mathrm{FT}_{4}>7 \mathrm{ng} / \mathrm{dL}$ (nl 0.8-1.6), TPOAb 387 $\mathrm{IU} / \mathrm{mL}(\mathrm{nl} \leq 20), \operatorname{TgAb} 4.6 \mathrm{IU} / \mathrm{mL}(\mathrm{nl}<4)$. Prior TSH was normal. Nivolumab was held, and he was started on high dose prednisone and propranolol. After one week, $\mathrm{TT}_{4}$ remained $>25 \mathrm{mg} / \mathrm{dL}$, so methimazole was added. TRAb later returned normal. The patient experienced rapid improvement of symptoms. Thyroid function tests improved after one month, thus steroids and methimazole were tapered off. Nivolumab was restarted. Three months after the onset of thyrotoxicosis, he developed overt hypothyroidism. His cancer progressed and he transitioned to hospice. Thyroid dysfunction can occur in $39 \%$ of patients treated with PD-1Ab. Overt hyper- and hypothyroidism occurs in $3 \%$ and $6.5 \%$ of patients, respectively. Onset of thyroid dysfunction ranges from 3 weeks to 3 years after initiation of treatment. A common presentation is transient thyroiditis followed by hypothyroidism, suggesting autoimmune destruction of the thyroid gland. However, TPO and Tg antibodies are not universally present. Glucocorticoids and beta-blockers may be indicated in the thyrotoxic phase, while levothyroxine is used to treat hypothyroidism. Thionamides are typically not indicated in thyrotoxicosis, but were used in this case while awaiting TRAb results. PD-1Ab can usually be safely continued. This is a unique case of nivolumabinduced thyroiditis given the severity of thyrotoxicosis requiring discontinuation of PD-1 Ab, lack of immediate improvement with 
steroids necessitating methimazole, and subsequent rapid conversion to hypothyroidism.

\section{Poster 186}

Disorders of Thyroid Function Friday Poster Case Report THYROID STORM WITH NORMAL TSH SECONDARY TO IPILIMUMAB AND NIVOLUMAB

M. Caldwell ${ }^{1}$, S.L. Blount ${ }^{2}$, V.R. Hudspeth ${ }^{1}$

${ }^{1}$ Endocrinology, University of North Carolina Hospitals, Chapel

Hill, NC; ${ }^{2}$ Internal Medicine, University of North Carolina

Hospitals, Chapel Hill, NC

Checkpoint inhibitor use is increasing in oncologic practice. Diagnosis and management of the endocrinopathies associated with these therapies is evolving as we see more patients with these complications. A 64 year old female with metastatic melanoma and euthyroid goiter presented one week after initiation of ipilimumab and nivolumab with fever, tachycardia, and altered mental status. Thyroid stimulating hormone (TSH) was $0.85 \mathrm{uIU} / \mathrm{mL}$, with negative infectious workup. She returned 4 days later with continued fevers, ten pound weight loss and lower extremity edema. She was tachycardic with an enlarged thirty five gram smooth, firm thyroid and pitting lower extremity edema. TSH was $0.08 \mathrm{uIU} / \mathrm{mL}$, free T3>22.8 pg/ $\mathrm{mL}$, free T4 $>6.99 \mathrm{ng} / \mathrm{dL}$. Prior PET scan showed a mildly enlarged thyroid with increased uptake suggestive of chronic thyroiditis. Ultrasound showed an enlarged, lobulated, and hypervascular thyroid. She was treated for thyrotoxicosis (Burch-Wartofsky Point Scale (BWPS) of 25) in the setting of a goiter and hypervascular ultrasound with PTU, and then methimazole. Antibody testing showed a TSI $<1$, anti-TPO antibody $519.96 \mathrm{IU} / \mathrm{mL}$. Methimazole was stopped after three weeks when TSH increased to $7.97 \mathrm{uIU} / \mathrm{mL}$, and later peaked at $42 \mathrm{uIU} / \mathrm{mL}$. Eleven months later patient is euthyroid on levothyroxine replacement. In retrospect, her initial admission was likely a thyrotoxicosis with BWPS of 75 that was overlooked due to a "normal" TSH that was notably lower than her prior baseline. Her symptoms likely represented thyroiditis secondary to Nivolumab/ Ipilimumab in the setting of underlying subclinical thyroid dysfunction. Ipilimumab, an Anti-CTLA-4, and Nivolumab, an AntiPD-1, cause a variety of endocrinopathies. Generally, thyroiditis associated with immunomodulation therapy is painless, asymptomatic, and transient with mildly elevated T4 and T3 occurring after a mean of 9 weeks of therapy. This case is unique due to the early onset of hyperthyroidism, a larger degree of T4 and T3 elevation, and the presence of a pre-existing goiter. This case represents the diagnostic difficulty with immunotherapy-related thyroid dysfunction in the setting of an abnormal thyroid at baseline.

\section{Poster 187}

\section{Disorders of Thyroid Function Friday Poster Case Report}

\section{CARDIOMYOPATHY AS PRIMARY MANIFESTATION OF} GRAVES DISEASE

K. Araque ${ }^{1}$, S.A. Avadhanula ${ }^{2}$, S. Chittimoju ${ }^{3}$, J. Jonklaas ${ }^{2}$

${ }^{1}$ Endocrinology, National Institute of Health, Bethesda, MD;

${ }^{2}$ Endocrinology, Georgetown University Hospital, Washington, DC;

${ }^{3}$ Internal Medicine, Washington Hospital Center, Washington, DC

The thyroid hormone's effects on the cardiovascular system include adrenergic effects, chronotropic and inotropic stimulation due to T3 actions on the cardiac myocite. [1]

25 year old African American male with past medical history of hypertension, presenting to the emergency department with heart failure developping over 6 months. He had a 20lbs weight gain, muscle loss, cough, shortness of breath and night sweats. He reported no allergies. He was taking multivitamins and ginseng. There was history of alcohol and tobacco exposure socially. There was no report of recreational drug use. Family history was noncontributory. On admission, his blood pressure was $152 / 83$ with sinus tachycardia 123 bpm. He had a cachectic appearance, temporal wasting and exophthalmus. His thyroid gland was diffusely enlarged and soft. There was evidence of jugular venous dissension to the angle of the jaw, laterally displaced point of maximal impulse, S4 gallop, coarse breath sounds without hypoxemia, liver span of $14 \mathrm{~cm}$ and pitting lower extremity edema. He was admitted to the telemetry ward. TSH was low $0.02 \mathrm{uIU} / \mathrm{ml}(0.34-5.6)$, free $\mathrm{t} 4$ was greater than $6.11 \mathrm{ng} / \mathrm{dl}$ (0.58-1.64), total T3 $351.20 \mathrm{ng} / \mathrm{dl}$ (ref range 87-178), TSI Abs were elevated $217(<125)$. Transthoracic Echocardiogram showed decreased left ventricular ejection fraction $20-25 \%$, severe global hypokinesis, dilated left ventricle and mild to moderate mitral regurgitation. Based on our observations this was related to Graves disease. Hospital course was complicated by multiple episodes of asymptomatic ventricular tachycardia. He received metoprolol $150 \mathrm{mg}$ every 12 hours. Electrolytes were repleted. Methimazole was uptitrated. He developed hepatotoxicity and therapy was discontinued. Decision was to start lithium and proceed with subtotal thyroidectomy. Patient became hypertermic after manipulation of the thyroid gland during surgery. He was started on levothyroxine $125 \mathrm{mcg}$ daily. Cardiomyopathy can be the primary manifestation of hyperthyroidism especially in the absence of underlying structural abnormalities. In this context, clinicians should seek endocrine evaluation since the cardiac manifestations are best corrected by treating the underlying hyperthyroid state.

\section{Poster 188}

\section{Disorders of Thyroid Function Friday Poster Case Report}

\section{PSEUDO-HYPERTHYROIDISM FROM HIGH DOSE}

\section{BIOTIN USE}

\section{B. Tchang, S. Ebner, J. Starr}

Endocrinology, New York Presbyterian Columbia University

Medical Center, New York, NY

We describe the case of a patient with no major past medical history who presented for evaluation of what was presumed to be new hyperthyroidism with positive TSH Receptor Antibody (Trab). We evaluated a 95-year-old woman who was referred for hyperthyroidism. Her only symptom was increased irritability. She otherwise denied heat intolerance, insomnia, sweating, eye changes, neck enlargement, weight loss, diarrhea or tremors. Her laboratory testing revealed low but detectable TSH $(0.07$, N1 range $0.41-4.81 \mathrm{mIU} / \mathrm{L})$, elevated free T4 (2.21, Nl range $0.83-1.90 \mathrm{ng} / \mathrm{dL})$, normal TT4 (7.53, $\mathrm{Nl}$ range 5-10.69ug/dL), and elevated TT3 (404.1, Nl range 78$158 \mathrm{ng} / \mathrm{dL})$. Serologies revealed elevated Trab (29.52, Nl range $\leq 1.75 \mathrm{IU} / \mathrm{L})$ and normal TSI $(98, \mathrm{Nl}$ range $<122 \%)$. The patient was started on methimazole 10mg daily for presumed Graves' disease. On medication reconciliation, patient reported taking biotin $10,000 \mathrm{mcg}$ daily for hair, skin, and nail health. There are reports in the literature of patients erroneously diagnosed with hyperthyroidism in the setting of high-dose biotin use. Many laboratories employ a competitive assay using biotinylated antibody complexes, in which the signal is indirectly proportional to the hormone level of interest, or sandwich complexes, in which the signal is directly proportional to the hormone level. Free T4 and Trab assays are competitive assays and TSH is often a sandwich assay. Biotin in the patient's sample competes for binding resulting in low signal readout, which is interpreted as high free T4, high Trab, and low TSH. Fortunately, this laboratory interference normalizes if the biotin is discontinued for 8-16 hours prior to testing. Our patient was asked to stop all supplements and methimazole. Repeat thyroid function tests two weeks later were entirely normal (TSH 3.87, freeT4 1.16, total T4 6.16, total T3 83.6, Trab 
$<0.90$ ). Biotin use is becoming more prevalent among patients. Incorrect diagnoses can occur if the clinician is not aware of biotin ingestion or its interference with laboratory testing. Biotin interference should be on the differential when the hormone profile is not consistent with the clinical picture.

\section{Poster 189}

Disorders of Thyroid Function Friday Poster Case Report

\section{A DIFFERENT STROKE}

S. Chittimoju ${ }^{2}$, S.A. Avadhanula ${ }^{1}$, K. Araque ${ }^{3}$, S. Naidu ${ }^{2}$

${ }^{1}$ Endocrinology, Georgetown University Hospital, Washington, DC;

${ }^{2}$ Internal Medicine, Washington Hospital Center, Washington, DC; ${ }^{3}$ Endocrinology, National Institute of Health, Bethesda, MD

Thrombophilia is a well-known manifestation of hyperthyroidism (1-4) and cerebral venous thrombosis (CVT) is a rare cause of stroke (5). CVT in the setting of hyperthyroidism is rare (6-9). Here, we present a case of the Antiphospholipid syndrome secondary to hyperthyroidism (10) at its' presentation.37 year old man with past medical history of hypertension, obstructive sleep apnea, hyperthyroidism, thymoma status post resection, and thyrotoxic periodic paralysis, presents with two day history of sudden onset left sided severe headache and unsteady gait. This was associated with nausea, vomiting and lightheadedness. Clinically his National Institute of Health Stroke Scale resulted at 1 for mild left nasolabial fold flattening, right horizontal end gaze nystagmus, left pronator drift, wide based gait (considered a minor stoke). Imaging revealed a subacute cerebellar infarct, which was later found to have a hemorrhagic conversion and cerebral venous thrombosis extension into the confluence of sinuses. MRV showed extensive dural venous thrombosis. His labs showed TSH $<0.005$, FT4 1.72, TSI 5, TRAb 35, TPO $>13,000$, Burch Wartofsky score of 30 indicating impending storm. Thyroid Ultrasound showed diffusely enlarged heterogeneous thyroid gland and hyperechoic nodule in the right superior pole. Further work up showed negative Beta 2 GP1 Ab IgM, positive Beta 2 GP1 $\mathrm{Ab}$ IgG, positive DRVVT screen, platelet neutralization tests and abnormal LA Screen (meeting criteria for diagnosis of antiphospholipid antibody syndrome). ANA Titer $<1: 40$, with negative ACA $\mathrm{IgG}$ and IgM. Our patient was treated for impending thyroid storm with hydrocortisone and hyperthyroidism was treated with Methimazole and propranolol. He was started on anticoagulation for CVT and showed steady improvement of his symptoms. Subsequently, our patient presented with worsening shortness of breath, dysphagia and underwent total thyroidectomy for compressive symptomsOur case illustrates the potential association of hyperthyroidism with the APA syndrome and also strengthens the association of thrombophilia with hyperthyroidism. In conclusion, we urge that clinicians keep in mind the importance of recongnizing potential rare associations with hyperthyroidism.

\section{Poster 190}

\section{Disorders of Thyroid Function Friday Poster Case Report} THYROID STORM WITH FATAL MULTI ORGAN FAILURE R. Bhattarai ${ }^{1}$, B. Dhakal ${ }^{2}$, S. Jyothinagaram ${ }^{1}$

${ }^{1}$ Endocrinology, University of Arizona College of Medicine, Phoenix, AZ; ${ }^{2}$ Internal Medicine, University of Arizona College of Medicine, Phoenix, AZ

Thyroid storm is an endocrine emergency with high mortality rate. Cases of fatal multi organ failure due to thyroid storm are rarely reported. A 52 years old female with Graves' disease on methimazole, was switched to Propylthiouracil (PTU) due to severe skin rash. She developed jaundice on PTU, and was referred to us. She had tachypnea, tachycardia, and icterus. Thyroid was enlarged, soft and non-tender. Blood work revealed TSH at $<0.01 \mathrm{mIU} / \mathrm{L}(0.45-4.50)$, Free T4 (FT4) at $>8 \mathrm{ng} / \mathrm{dl}(0.8-1.7)$, positive TSI at $362 \%(<140)$, elevated total bilirubin at $7.1 \mathrm{mg} / \mathrm{dl}(0.2-1.3)$, elevated AST $58 \mathrm{IU} / \mathrm{L}$ (10-41) with normal ALT and ALP, and elevated INR. Liver imaging was unremarkable. PTU induced hepatitis was suspected and PTU was discontinued. Propranolol and potassium iodide were started. Her clinical condition rapidly deteriorated with altered mental status, hypotension, and respiratory distress, requiring intubation and vasopressors support. Echocardiogram showed reduced EF 15-20\%. Total bilirubin elevated at $21.6 \mathrm{mg} / \mathrm{dl}$, AST at $7043 \mathrm{IU} / \mathrm{L}$, ALT at $1802 \mathrm{IU} / \mathrm{L}$. FT4 remained elevated with suppressed TSH. With the diagnosis of thyroid storm and multi organ failure, IV steroids, SSKI and cholestyramine were started. She was clinically unstable for thyroidectomy, and emergent plasmapheresis was done. However, patient developed DIC and GI bleeding. Despite aggressive management, her condition deteriorated, so, end of life care was pursued, and the patient expired. Thyroid storm is an acute, life-threatening condition caused by excessive release of thyroid hormones with an incidence of $1-2 \%$ among patients with hyperthyroidism, and the mortality of 10-30\%. Graves' disease is the most common cause of thyroid storm, and often precipitated by abrupt cessation of antithyroid therapy as in our case. Thyroid storm is a clinical diagnosis based on presence of severe life threatening symptoms in a patient with laboratory evidence of elevated FT4, FT3 and suppressed TSH. Early recognition and treatment can avoid morbidity and mortality. When diagnosis of thyroid storm is strongly suspected, one should rapidly initiate effective life-saving treatment in the ICU. However, despite aggressive treatment thyroid storm can be fatal.

\section{Poster 191}

Disorders of Thyroid Function Friday Poster Case Report ABNORMAL THYROID FUNCTION TESTS IN A PATIENT WITH HISTORY OF AMIODARONE USE: CLINICAL CONSIDERATIONS

\section{Resman}

Endocrinology, UT Houston, Houston, TX

Most patients on amiodarone therapy are euthyroid, with only $3 \%$ developing Amiodarone-induced thyrotoxicosis (AIT). AIT type 1 occurs in patients with underlying thyroid pathology and is due to accelerated thyroid hormone synthesis. In type 2 AIT, the thyrotoxicosis is a destructive thyroiditis typically occurring in patient with no underlying thyroid disease and results in excess release of preformed T4 and T3 into circulation. IL 6 and RAIU scans have been used in differentiating between AIT1 and AIT2. Here, we report a case of amiodarone induced thyrotoxicosis with significantly elevated IL6 level. 55-year-old male with past medical history of CHF with AICD placement and chronic atrial fibrillation on amiodarone therapy for past 3 years presented for failure to thrive. Patient had no prior history of thyroid disease and has had normal thyroid function tests (TFTs) in the past. On admission, patient reported 40-50 lb weight loss in the period of 4 weeks along with decreased appetite, vomiting, fatigue, palpitation and tremors. Labs were significant for $\mathrm{TSH}<0.005$, FT4 $>8.0$, total T4 $>24$. TSI and TPO Ab were negative. Thyroid U/S showed normal thyroid gland without increased vascularity or nodules. Patient was unable to get thyroid uptake scan secondary to iodine load during hospitalization. He was started on MMI $10 \mathrm{mg}$ q8hrs, Propranolol $30 \mathrm{mg}$ q $8 \mathrm{hrs}$ as well as prednisone $20 \mathrm{mg}$ daily. IL6 was found to be elevated at $20.3 \mathrm{pg} / \mathrm{ml}$ (range 2$12 \mathrm{pg} / \mathrm{ml}$ ). Two weeks following treatment with combination therapy, the patient showed an improvement in FT4 to 5.22. He will be followed in Endocrine clinic for further treatment While, given therapeutic implications, it is important to differentiate type 1 and type 2 
AIT, it is often quite difficult to achieve an accurate diagnosis. In the inpatient setting, uptake scans might not be possible given the need for contrast studies. IL6 can be used to help support the diagnosis of AIT2, especially in patients without history of thyroid disease. Addition of glucocorticoids to the treatment regimen can be essential when there is a suspicion of AIT2, or coexistence of Type 1 and Type 2 AIT.

\section{Poster 192}

\section{Disorders of Thyroid Function Friday Poster Case Report SUCCESSFUL TREATMENT OF PRETRIAL MYXEDEMA WITH GLUTEN-FREE DIET}

J. Abayev, I. Bernstein, E. Orlev, N. Goldenberg

Internal Medicine, The Jewish Hospital, Cincinnati, $\mathrm{OH}$

Pretibial myxedema (PTM), or thyroid dermopathy, is a rare complication of hyperthyroidism caused by Graves' disease, resulting from the deposition of glycosaminoglycan into the dermis of tissue. It is characterized by a thickened and indurated skin with the diagnosis supported by elevated Thyroid Stimulating Immunoglobulins (TSIs)/ Thyroid Receptor Antibodies (TRABs). Although pharmacologic treatment with topical medium-high potency corticosteroids and intralesional steroid injections are standard of care, the response is not always optimal. We found one case report that had demonstrated improvement of myxedema with implementation of a gluten-free diet. This approach was attempted on a clinic patient with PTM. Patient is a 65-year-old female with post-ablative hypothyroidism, remote history of Graves' disease, presented with a pruritic rash in the pretibial area and on elbows. Physical examination demonstrated characteristic features of pretibial myxedema. Blood work showed thyroid function panel within normal range with elevated levels of TSI [TSI of 399 (reference range <122)]. She had presented with a similar rash in the past that resolved after treatment with dapsone and doxepin, but recurred approximately one year later. She tried topical steroids with no improvement, and was then placed on a gluten-free diet for 3 months. This led to complete resolution of her dermopathy. This case illustrates a patient with hypothyroidism presenting with a typical case of myxedematous skin rash that completely resolved after she was placed on a gluten-free diet. Patients with prior history of Graves' disease are not immune to developing pretibial myxedema. Topical corticosteroids had very little effect on our patient. Complete resolution of dermopathy within 3 months after implementing only a gluten-free diet was achieved. Mechanism of action remains undetermined but there may be antigenic crossreactivity between gluten and thyroid. The removal of gluten may have resulted in reduction of antibodies toward the thyroid. Implementation of gluten-free diet led to complete resolution of recurrent thyroid dermopathy in a patient who had little success with topical corticoidsteroid therapy.

\section{Poster 193}

Disorders of Thyroid Function Friday Poster Case Report CO-EXISTING GRAVES' DISEASE WITH BENIGN STRUMA OVARII: A CASE REPORT

L.D. Alexander ${ }^{1}$, A. Leckie $^{3}$, K. Burton ${ }^{3}$, L. Jain ${ }^{2}$, M. Geary ${ }^{2,4}$, C.H. $\mathrm{Yu}^{1,5}$

${ }^{1}$ Department of Medicine, University of Toronto, Toronto, Ontario, Canada; ${ }^{2}$ Obstetrics \& Gynecology, University of Toronto, Toronto, Ontario, Canada; ${ }^{3}$ Medical Imaging, University of Toronto, Toronto, Ontario, Canada; ${ }^{4}$ Obstetrics \& Gynecology, St Michael's Hospital, Toronto, Ontario, Canada; ${ }^{5} \mathrm{Li}$ Ka Shing Knowledge Institute of St. Michael's Hospital, Toronto, Ontario, Canada
Struma ovarii $(\mathrm{SO})$ is a rare ovarian teratoma containing thyroid tissue that accounts for $1 \%$ of ovarian tumors. Thyrotoxicosis is seen in 5\% of cases. We present a case of co-existing Graves' disease with a functioning struma ovarii, which has seldom been reported.

A 33 year-old G0 presented with a 3-month history of fatigue and abdominal pain, a recent history of facial sweating, hand tremor, dry eyes, and an unintentional $23 \mathrm{~kg}$ weight loss in the past year. On exam, she had brisk reflexes, mild proptosis with lid lag and a pelvic mass. Blood work showed an elevated thyroid stimulating hormone (TSH)-receptor antibody level, thyroid function tests consistent with thyrotoxicosis and negative ovarian tumor markers. An MRI pelvis showed large, bilateral adnexal lesions with areas of calcification and haemorrhage. Whole body uptake and scan showed homogenous thyroid uptake at $2 \mathrm{~h}$ and $24 \mathrm{~h}$ of $54 \%$ and $77 \%$ respectively and increased activity in the right adnexa. Graves' disease with functioning SO was suspected. She commenced atenolol $25 \mathrm{mg}$ PO daily and methimazole $5 \mathrm{mg}$ twice daily. She was euthyroid prior to laparoscopic cystectomy, which showed bilateral complex cysts. Histology showed benign cystic teratomas and with positive immunohistochemical staining for thyroid transcription factor-1. Coexisting Graves' disease and SO is rare. In previous cases, treatment of Graves' disease typically preceded the diagnosis of SO by several years, with the SO presenting as persistent thyrotoxicosis following thyroidectomy. This is also the first case of co-existing Graves' disease and SO where a laparoscopic, fertility-sparing surgery was used. The diagnosis of SO alone should be considered if there is thyrotoxicosis without cervical radioiodine uptake and: (1) a pelvic mass (2) no history of thyroiditis, or (3) persistent thyrotoxicosis post-thyroidectomy. For imaging, I-123 whole body scintigraphy is preferred, as I-123 has a short half-life and lower beta emission than I-131 and pertechnetate accumulates more rapidly in the bladder, which may obscure ovarian lesions. Co-existing Graves' disease and functioning SO has rarely been reported and may present as persistent thyrotoxicosis post thyroidectomy.

\section{Poster 194}

Disorders of Thyroid Function Friday Poster Clinical

PREVALENCE AND TREATMENT OF

HYPOTHYROIDISM: A RETROSPECTIVE ANALYSIS OF OLDER AMERICANS AGED $\geq 65$ YEARS

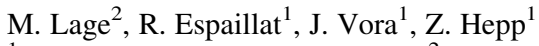

${ }^{1}$ AbbVie Inc., North Chicago, IL; ${ }^{2}$ HealthMetrics Outcomes

Research, Bonita Springs, FL

Hypothyroidism is common in older individuals, but data on prevalence and treatment patterns are lacking. The objective of this analysis was to determine the prevalence of hypothyroidism in adults aged $\geq 65 \mathrm{y}$, the proportion of those diagnosed who receive treatment, and the rates of treatment outcomes. Patients aged 18-64 and $\geq 65 y$ were identified in the Optum Clinformatics ${ }^{\mathrm{TM}}$ Data Mart retrospective claims database from 1/1/2007-12/31/2015. Patients had to have continuous insurance coverage, $\geq 2$ diagnoses of hypothyroidism or 1 diagnosis and $\geq 1$ filled prescription for levothyroxine, 1 recorded thyroid-stimulating hormone (TSH) laboratory value, and no diagnosis of thyroid cancer in a calendar year. Prevalence was calculated annually for 2007-2015. Treatment outcomes were based on the last TSH value within 1 year of the first diagnosis of hypothyroidism and were considered appropriate if TSH levels were 0.45-4.12 mIU/L. Differences in treatment outcome rates for 2014-2015 were assessed by chi-square test. Of 64,708 patients with hypothyroidism, 4025 were $\geq 65 \mathrm{y}$ and 57,235 were $18-64 \mathrm{y} ; 68.4 \%$ and $79.3 \%$ were women, respectively; median TSH levels were 2.28 and $2.32 \mathrm{mIU} / \mathrm{L}$ for patients $>65 \mathrm{y}$ and $18-64 \mathrm{y}$, respectively. Unadjusted prevalence of hypothyroidism steadily increased from $13.1 \%$ in 2007 to $17.8 \%$ in 
2015 in patients $65-74$ y. Prevalence was higher in women than men in all years; for $2015,65-74$ y group, $19.7 \%$ vs $14.2 \%$. Of patients $\geq 65 \mathrm{y}, 72.0 \%$ were treated with levothyroxine. A lower proportion of patients $\geq 65 \mathrm{y}$ versus patients $18-64 \mathrm{y}$ were appropriately treated $(46.0 \%$ vs $48.9 \% ; P=0.003)$ or undertreated (17.4\% vs $20.8 \% ; P<0.0001)$. A higher proportion of patients $\geq 65$ y versus $18-64$ y were not monitored (no TSH test recorded) after treatment ( $32.9 \%$ vs $26.8 \% ; P<0.0001)$. Results of sensitivity analyses using alternative TSH cutoffs and definitions of monitoring were consistent with the main findings. Prevalence of hypothyroidism increased over time in patients $\geq 65 \mathrm{y}$ and was higher in women than men. Appropriate treatment of hypothyroidism in older adults remains an unmet medical need, as less than half of patients receive appropriate treatment and approximately a third are not monitored after treatment.

\section{Poster 195}

Disorders of Thyroid Function Friday Poster Clinical FOUR OUT OF FIVE PATIENTS WITH ACUTE KIDNEY INJURY HAVE THYROID DYSFUNCTION AND A TSH <1.92 MIU/ML IS ASSOCIATED WITH HIGHER IN-HOSPITAL MORTALITY

M.K. Choulia ${ }^{2}$, J.J. Jacob ${ }^{1}$, J. Das ${ }^{3}$, M. Ghosh ${ }^{4}$

${ }^{1}$ Endocrine \& Diabetes Unit, Christian Medical College and Hospital, LUDHIANA, PUNJAB, India; ${ }^{2}$ Department of Medicine, Christian Medical College and Hospital, LUDHIANA, PUNJAB, India; ${ }^{3}$ Department of Nephrology, Christian Medical College and Hospital, LUDHIANA, PUNJAB, India; ${ }^{4}$ Department of Biochemistry, Christian Medical College and Hospital, LUDHIANA, PUNJAB, India

This study was undertaken to ascertain the prevalence of thyroid dysfunction (TD) in patients with Acute Kidney Injury (AKI) and assess the prognostic implications of TD in patients with AKI. Adult patients admitted to the renal $\&$ medical wards were recruited if they met the AKIN classification criteria for diagnosis of AKI (1). Recruited patients were screened for TD with serum sampling of thyroid stimulating hormone, free T3 and free T4 after taking informed consent. Prognosis of TD in patients with AKI was assessed in terms of: 1) In-hospital mortality, 2) Need for Renal Replacement Therapy (RRT) and 3) Duration of hospital stay.147 patients with AKI (93M:54F) with a mean age of 57 years met the inclusion criteria and were recruited after informed consent. TD was noted in 114 patients $(77.5 \%)$. Of these, $74(64.91 \%)$ patients had Non-thyroidal illness (NTI), $10(8.77 \%)$ had primary hypothyroidism,17(14.91\%) had subclinical hypothyroidism, $4(3.51 \%)$ had primary hyperthyroidism, $7(6.14 \%)$ had subclinical hyperthyroidism and $2(1.75 \%)$ had central hyperthyroidism (associated with low stimulated cortisol values). Patients with lower TSH values tended to have poorer prognosis. Patients with AKI having TSH values $<2 \mathrm{IU} / \mathrm{ml}$ were found to have higher mortality as compared to those having TSH value $\geq 2 \mathrm{IU} / \mathrm{ml}$ $(40.7 \%$ v.s $19.7 \%)(p=0.007)$. Using a reciever operated curve (ROC) determination we found that a TSH cut off of $<1.92 \mathrm{mIU} / \mathrm{L}$ provided the best sensitivity $(74.5 \%$ ) with a specificity of $52 \%$ for inhospital mortality estimation in patients admitted with AKI. A trend to longer duration of hospitalisation for AKI patients ( $>7$ days) was seen in $65(75.58 \%)$ patients with TD compared to $17(62.96 \%)$ patients without TD $(\mathrm{p}=0.09)$. Four out of five patients with AKI had some form of thyroid dysfunction, of which majority of them had NTI. Patients admitted with AKI with an admission TSH value of $<1.92 \mathrm{mIU} / \mathrm{L}$ tended to have higher in-hospital mortaliy.

Reference- 1. Lopes JA, Jorge S. The RIFLE and AKIN classification for acute kidney injury: a critical and comprehensive review. Clin Kidney J 2013;6:8-14.

\section{Poster 196}

Disorders of Thyroid Function Friday Poster Clinical

POVERTY FACTORS ARE PRIMARY CAUSE OF ADMISSION FOR HYPERTHYROIDISM AT A UNIVERSITY HOSPITAL IN WEST TEXAS

A.G. Adiga, E. Juarez-Tello, J. Lado-Abeal

Endocrinology- Internal medicine, Texas Tech University Health Sciences Center-School of Medicine, Lubbock, TX

Purpose: West Texas has one endocrinologist for 92,000 inhabitants, far below the USA average, 1 per 46,000. We perceive a high admission rate for hyperthyroidism at our Institution. The aims of the study are 1) To quantify the admission rate for hyperthyroidism in our hospital, and 2) To evaluate the social and clinical factors associated with hospital admissions due to hyperthyroidism. A retrospective study involving 223 patients with a diagnosis of hyperthyroidism. Seventy-two patients admitted to our hospital from January 1, 2011 to January 1, 2017 were included as cases. One hundred and fifty-one patients selected from our clinic during the same period were included as controls. The clinical variables were divided into cardiovascular, neuropsychiatric, gastrointestinal and other physical symptoms. Biochemical variables were serum free T4 and free T3 levels. Other variables were age, gender, ethnicity, education, annual income, marital status, insurance, primary care physician (PCP), and medication compliance. Re-admissions due to hyperthyroidism was also calculated. The admission rate for hyperthyroidism was $0.26 \%$ of total admissions at our hospital during the study period. The number of readmissions among cases ranged 0 to 4: none, $31 \%$; one, $35.2 \%$; two, $24 \%$; three, $5.6 \%$; four, $4.2 \%$. The length of stay during each hospitalization ranged 1 to 34 days. Symptoms responsible for admission were cardiovascular $58 \%$, neuropsychiatric $23.6 \%$, gastrointestinal $6.9 \%$ and other symptoms $11 \%$. Serum free T4 and free T3 levels were higher in the hospitalized cohort. African American and uninsured patients showed higher unadjusted odds of hospitalization. Having PCP, better compliance with medications, married status, Hispanic ethnicity, higher levels of education, and living in higher median income zip code had a protective effect. Age and gender were not significantly associated to the outcome. In West Texas, hospitalization for hyperthyroidism is 26 times higher than average in USA. The high rate of admissions is associated low income, low education, and poor health care, all indicators of poverty.

\section{Poster 197}

Disorders of Thyroid Function Friday Poster Clinical IDENTIFYING ADULT PATIENTS WITH MYXEDEMA COMA: COMPARING TWO SCORING SYSTEMS IN PATIENTS FROM SIX COLOMBIAN HOSPITALS C.A. Gomez Fontalvo ${ }^{1}$, R. Castellanos Bueno ${ }^{1}$, A. Román González ${ }^{3}$, A. Pinzón Tovar ${ }^{2}$, A. Castellanos Pinedo ${ }^{4}$, D. Pantoja Guerrero ${ }^{5}$, D. Alzate Gomez ${ }^{1}$, L.M. Vera-Cala ${ }^{1}$, A. Abreu Lomba ${ }^{6}$, L.P. Pradilla ${ }^{1}$

${ }^{1}$ Santander, Universidad Industrial De Santander, Bucaramanga, Santander, Colombia; ${ }^{2}$ Universidad Surcolombiana, Neiva, Colombia; ${ }^{3}$ Universidad de Antioquia, Medellin, Colombia; ${ }^{4}$ Universidad del Sinú, Monteria, Colombia; ${ }^{5}$ Hospital Departamental de Nariño, Pasto, Colombia; ${ }^{6}$ Universidad Libre, Cali, Colombia

To compare the Popoveniuc's scoring system from Washington (W-score) and the score proposed by Chiong and colleagues in Indianapolis (I-score) to identify patients with myxedema coma in six different Colombian cities. Patients with diagnosis of myxedema 
coma from January 2012 to Jun 2017 were retrospectively included from six colombian cities. Diagnosis (the gold standard) was made independently by two endocrinologists, based on the clinical criteria. The I-score included: Glasgow's scale, TSH, low serum T4 (free or total), hypothermia, bradycardia and precipitant disease. A score $\geq 7$ was diagnostic, 5-6 was likely, and $<5$ unlikely disease. The $\mathrm{W}$ scale included: thermoregulatory dysfunction, central nervous system effects, gastrointestinal findings, precipitating factors, cardiovascular dysfunction, and metabolic disturbances. A score $\geq 60$ was diagnostic, 25-59 was likely, and $<25$ was unlikely for myxedema coma. The statistical analysis was made with STATA v.14. Nineteen patients were diagnosed as myxedema coma. Thirteen patients $(68.4 \%)$ were female. Mean age was 55.8 years-old (SD: 13.6) and 9 (56.9\%) had previous hypothyroidism. Endocrinologists considered 17 as positive myxedema coma. Applying the scores, 14/19 (73.6\%) were positive by $\mathrm{W}$-score, 2 were likely, and 3 were unlikely cases. By I-score 11/19 (57.8\%) were positive and 8 were likely and none was unlikely. For statistical purposes in both scores the "likely" cases were considered as positive for myxedema coma. The kappa-index between the scoring scales was 0.184 (SD 0.182). Comparing with the endocrinologist's diagnosis, the kappa-index was 0.59 (SD 0.207) for W-score and 0,25 (SD 0,193, $\mathrm{p}=0,1015$ ) for I-score. The sensitivity for W-score was $89,5 \%$ (95\%CI: $66.9 \%, 98.7 \%)$ and the specificity was $75.0 \%$ (95\%CI: $19.4 \%, 99.4 \%$ ), and for I-score the sensitivity was $94.7 \%$ (95\% CI: $74.0 \%, 99.9 \%)$ and the specificity was $25.0 \%$ (95\%CI: $0.63 \%$, $80.59 \%)$. The correlation between the Scores for diagnosing Myxedema coma was low. The $\mathrm{W}$-score had a better correlation with clinical specialist's criteria than the I-score to diagnose Myxedema coma. Both score systems have high sensitivity, however the Wscore is better to confirm the diagnosis.

\section{Poster 198}

Disorders of Thyroid Function Friday Poster Clinical INCIDENCES OF TREATMENTS FOR MODERATE-SEVERE AND SEVERE THYROID ASSOCIATED OPHTALMOPATHY IN SWEDEN M. Holmberg ${ }^{1,2}$, R. Kavand ${ }^{2}$, M. Lindqvist ${ }^{3}$, S. Sahlin ${ }^{4}$, H. Filipsson Nyström ${ }^{2,5}$

${ }^{1}$ ANOVA, Karolinska University Hospital, Stockholm, Sweden;

${ }^{2}$ Intitute of Medicine, Sahlgrenska Academy, University of Gothenburg, Gothenburg, Västra Götaland, Sweden; ${ }^{3}$ Dept of Ophtalmopathy, Skaraborgs Hospital, Skövde, Sweden; ${ }^{4}$ Dept of Ophtalmology Surgery, Karolinska University Hospital, Stockholm, Sweden; ${ }^{5}$ Dept of Endocrinology, Sahlgrenska University Hospital, Gothenburg, Sweden

Thyroid associated ophtalmopathy (TAO) may complicate Graves disease (GD). In moderate-severe (MS) and severe TAO intravenous (iv) glucocorticoids (GC) is the treatment of choice and if optic nerve compression persists, subacute orbital decompression (SAOD) is necessary to restore visual acuity. Incidence data for treatments of MS and severe TAO are scares, and risk factors for SAOD need to be determined. All patients in 2011-15 with TAO that had iv GC in Västra Götaland County (VGR) (1.7 million inhabitants) or had SAOD in Sweden (excluding Skåne's County) (8.7 million inhabitants) were collected by a search in the diagnose related registry. After informed consent, information on age, sex, smoking, TSH receptor antibodies (TRab) levels, tyreoperoxidase antibody (TPOab) levels, GD treatment, form of GD (stimulating or blocking TRab) and ophthalmology parameters before and after intervention were collected from patients' files. Incidences were calculated and risk factors were compared between MS-TAO $(n=31)$ (with the 12-week iv GC scheme), and the SAOD group $(n=38)$. In total, 6.08/million/ year had iv GC in VGR. Incidences of the 12-week iv GC scheme for MS-TAO, and the 2 week-high dose scheme for severe TAO were 4.72 , and $1.37 / \mathrm{million} /$ year, respectively. With the 12 -week iv GC treatment $18.4 \%$ deteriorated and needed SAOD, whereas $45 \%$ with severe TAO needed SAOD after the 2-week high dose iv GC treatment. The incidences of SAOD were 1.49 and $0.96 /$ million/year in VGR and Sweden, respectively. Compared to MS-TAO, SAOD was more frequent in females (female: male ratio 18.5:1 vs 3.4, $\mathrm{p}<0.05$ ), those with higher TRab (median (IQR) 23.0 (27.0) vs 6.0 (10.6), $\mathrm{p}<0.01$ ) with a tendency for older age (median (IQR) 68.0 (12.0) vs 58 (15.0), $\mathrm{p}=0.091)$. No difference was seen for TPOab, smoking (25\% smoked at intervention) and treatment for GD (39\% had radioactive iodine treatment). GD from blocking TRab was observed in $17.3 \%$ of cases distributed equally in SAOD and MS-TAO groups.

These new data on treatment frequencies of the most severe forms of TAO can be used for health care planning. The significant proportion of blocking TRab in the TAO aetiology needs further evaluations.

\section{Poster 199}

Disorders of Thyroid Function Friday Poster Clinical

SERUM FAM3B IS ELEVATED IN PATIENTS WITH OVERT HYPOTHYROIDISM AND SUBCLINICAL HYPOTHYROIDISM: A CASE-CONTROL STUDY

H. Wang, Y. Lai, W. Teng, Z. Shan

Department of Endocrinology and Metabolism, Institute of Endocrinology, The First Affiliated Hospital of China Medical University, China Medical University, Shenyang, Liaoning, China

The member B of the family with sequence similarity 3 (FAM3B), as a pancreatic-derived factor, is mainly synthesized in islet $\beta$-cells. Previous studies suggested that FAM3B may have a vital role in inducing apoptosis of $\beta$-cells, and inhibiting secretion of insulin. Hypothyroidism is associated with abnormal glucose metabolism, but the mechanisms remain uncovered. This study aimed to determine the relationship between serum FAM3B levels and thyroid funtion. Study participants were recruited from community residents and patients. Individuals with diabetes mellitus, history of antithyroid drugs, and any other acute or chronic disease were excluded. Age-, gender-, and body mass index-matched subjects were included and individed according to their thyroid function as following: overt hypothyroidism $(\mathrm{OH} ; \mathrm{n}=25)$, subclinical hypothyroidism $(\mathrm{SCH}$; $\mathrm{n}=25$ ), isolated thyroid peroxidase antibody positivity with euthyroid (Isolated TPOAb; $n=25)$ and healthy control $(\mathrm{HC} ; \mathrm{n}=25)$. All subjects underwent oral glucose tolerance test, as well as fasting insulin and lipid profile were also examined. Their demographic and anthropometric parameters were also collected. Circulating FAM3B levels were determined by enzyme-linked immunosorbent assay. Compared with $\mathrm{HC}, \mathrm{OH}$ and $\mathrm{SCH}$ have significantly increased serum FAM3B levels $(36.63 \pm 11.22 \mathrm{ng} / \mathrm{mL}$ vs $30.79 \pm 8.17$ vs $21.06 \pm 3.62$, $P<0.001)$. However, there was no significant difference between Isolated Ab group and $\mathrm{HC}(20.54 \pm 2.84$ vs $21.06 \pm 3.62, P=0.572)$. Correlation analysis suggested that circulating FAM3B levels were positively correlated with thyroid-stimulating hormone (TSH) $(\mathrm{r}=0.75, P<0.001)$, fasting plasm glucose $(\mathrm{r}=0.23, P=0.020)$, 2-hour post-loading plasm glucose $(\mathrm{r}=0.17, P=0.082)$, fasting insulin $(\mathrm{r}=0.36, P<0.001)$, homeostasis model assessment of insulin resistance $(\mathrm{r}=0.40, P<0.001)$, and triglyceride $(\mathrm{r}=0.29, P=0.003)$, but negatively with free thyroxine $(\mathrm{r}=-0.47, P<0.001)$. In addition, it is found that Log TSH was identified as an independent predictor for serum FAM3B levels after for multivariable adjustment $(P<0.001)$. Serum FAM3B levels were significantly elevated in patients with overt and subclinical hypothyroidism, and TSH was an independent risk factor for elevated FAM3B. 


\section{Poster 200}

Disorders of Thyroid Function Friday Poster Clinical

COMPARISON OF INCIDENT CARDIOVASCULAR EVENT RATES BETWEEN GENERIC AND BRAND L-THYROXINE FOR TREATMENT OF HYPOTHYROIDISM: A POPULATION BASED COHORT STUDY

R. Smallridge ${ }^{1}$, L. Sangaralingham ${ }^{2}$, R. Mwangi ${ }^{2}$, F. Kusumoto ${ }^{1}$, H. Van Houten ${ }^{2}$, V. Bernet ${ }^{1}$

${ }^{1}$ Medicine, Mayo Clinic, Jacksonville, FL; ${ }^{2}$ Mayo Clinic, Rochester, $\mathrm{MN}$

Hypothyroidism increases cardiovascular risk. Thyroid hormone may reverse these changes, but overtreatment may increase the risk of cardiovascular events. Cardiovascular events may vary by $\mathrm{T} 4$ preparation. We performed a retrospective analysis using a large administrative claims database, OptumLabs Data Warehouse from 2006 to 2014, creating two well-balanced one-to-one propensity score-matched (matching on age, gender, race, census region, Charlson index, year of index prescription, and 12 baseline comorbidities) cohort of patients initiating on generic or brand L-Thyroxine (T4) for treatment of hypothyroidism. Exclusions were prior use of any thyroid preparation, amiodarone or lithium. The primary outcomes were hospitalizations for acute myocardial infarction, atrial fibrillation, congestive heart failure and stroke. Patients were censored if treatment ended or changed from generic to brand $\mathrm{T} 4$ and vice versa or they disenrolled from their health plan coverage. Cox proportional hazards models were used to compare outcomes in the matched cohorts. Hazard Ratios (HRs) with 95\% confidence intervals (CIs) were reported. A total of 87,902 patients (43,951 patients in each cohort) initiating generic or brand T4 were included. Patients were followed for a mean (SD) of 1.0 (1.2) years (range $=0-9.3$ ). We found no differences in the rates of the 4 outcomes between generic and brand T4 therapy. Event rates (per 1000 person years) were similar in 1:1 matched comparisons for (a) atrial fibrillation (1.82 vs. 2.19 [HR 1.22, p=0.19]); (b) myocardial infarction ( 2.12 vs. 1.83 [HR 0.86, p=0.35]); (c) congestive heart failure ( 2.27 vs. 2.00 [HR $0.88, \mathrm{p}=0.41])$; and (d) stroke (3.10 vs. 2.38 [HR 0.77, $\mathrm{p}=0.05]$ ). Age stratification showed no differences in any outcomes. Study strengths include: a large, diverse real world population, wide range of ages, pharmacy claims documenting continued refills, and propensity score matching. Limitations include: coding and billing variations, unmeasured residual confounding, and lack of TSH and T4 values. In patients with predominantly benign thyroid disease and newly treated hypothyroidism, the short/intermediate term cardiovascular events rates were similar for generic and brand $\mathrm{T} 4$ therapy.

\section{Poster 201}

Disorders of Thyroid Function Friday Poster Clinical

THE CLINICAL PRESENTATION OF PROFOUND HYPOTHYROIDISM IN AN IRISH TERTIARY REFERRAL HOSPITAL (2002-2016)

D.T. O’Keefe, L. Martyn, M. Bell

Endocrinology, University Hospital Galway, Galway, Ireland

Hypothyroidism is a systemic illness with diverse symptoms and signs, often non specific. In undiagnosed infants it can cause mental and physical retardation and in adults it can rarely lead to a medical emergency called myxedma crisis. Therefore Congenital Hypothyroidism screening was introduced in Ireland in 1979. At the University Hospital Galway (UHG) the reference range for Thyroid Stimulating Hormone (TSH) is $0.51-4.3 \mathrm{mIU} / \mathrm{L}$ and Free T4 (fT4) is $12.6-21 \mathrm{pmol} / \mathrm{L}$. The aim of this research was to describe the inci- dence and clinical features of patients presenting with biochemically profound ( $\mathrm{TSH}>400 \mathrm{mIU} / \mathrm{L}$ ) hypothyroidism. A retrospective review of all clinical cases referred to the Endocrinology Department UHG over a fifteen year period with a TSH $>400 \mathrm{mIU} / \mathrm{L}$. Cases with elevated TSH secondary to recombinant TSH stimulation were excluded. The age, sex, race and clinical features of hypothyroidism were recorded for each case. Lab values were recorded for TSH, T4 and anti-thyroperoxidase (anti-TPO) antibodies. In total 18 caucasian patients presented with an initial TSH $>400 \mathrm{mIU} / \mathrm{L}$ during the period of 2002 to 2016 . The average age at presentation was 17.66 years ( \pm 13.28 years). There were 6 male patients and 12 female patients. The average TSH at diagnosis was $640.5( \pm 389.7) \mathrm{mIU} /$. The average free T4 at diagnsis was $3.07( \pm 2.35) \mathrm{pmol} / \mathrm{L}$. Anti-TPO antibodies was measured in 13/19 patients and was positive. Average anti-TPO antibodies were 558.67. Clinical symptoms were only recorded for 13/18 patients and included fatigue (8/13), dry skin (4/13), weight gain (4/ $13)$, cold intolerance (3/13), growth retardation (3/13) and cognitive dysfunction (1/13). There is no biochemical definition for profound hypothyroidism in the literature and therefore we have defined it as $>100$ times upper limit of normal TSH range. We have then described a cohort of relatively young and predominantly asymptomatic patients, presenting in a profound biochemical hypothyroid state. This data suggests that despite a national screening program and often mild symptoms, clinicians should request thyroid function tests in any suspected case, particularly in adolescents.

\section{Poster 202}

\section{Disorders of Thyroid Function Friday Poster Clinical}

ELEVATED SERUM TSH IS ASSOCIATED WITH LOWER ACHIEVEMENT OF LIPID-TREATMENT GOALS IN ELDERLY EUTHYROID WOMEN WITH METABOLIC SYNDROME

G. Brenta ${ }^{1}$, E. Blanc ${ }^{1}$, A. Nepote ${ }^{1}$, A. Barreto ${ }^{1}$, Y. Morosán Allo ${ }^{1}$, C. Parisi ${ }^{1}$, A. Fernández ${ }^{1}$, C. Sedlinsky ${ }^{1}$, C. Frigerio ${ }^{1}$, P. Fossati $^{1,2}$, M. Faingold ${ }^{1}$, T. Meroño ${ }^{3}$, C. Musso ${ }^{1}$

${ }^{1}$ Endocrinology, Milstein Hospital, Buenos Aires, Argentina;

${ }^{2}$ Biochemistry, Milstein Hospital, Buenos Aires, Argentina;

${ }^{3}$ Clinical biochemistry, School of Pharmacy and Biochemistry, University of Buenos Aires, Buenos Aires, Argentina

TSH levels increase as we age. Recently, a role for elevated TSH in the incidence of metabolic syndrome (MetS) was suggested. Moreover, TSH was shown to directly influence hepatocyte hydroxi-metilglutaryl (HMG) CoA reductase activity. Our aim was to explore the relationship between TSH levels and the achievement of lipid treatment goals in elderly euthyroid female patients with MetS. Euthyroid female patients $>65$ years with MetS were prospectively recruited at our Endocrine Unit. TSH and free T4 (fT4) serum levels were determined together with several anthropometric, metabolic and inflammatory markers. Spearman correlation and MannWhitney U test were used for statistical analysis. Subanalyses were performed according to statin treatment and achievement of lipid treatment goals (LDL-cholesterol (C) $<100 \mathrm{mg} / \mathrm{dl}$ or non-HDL$\mathrm{C}<130 \mathrm{mg} / \mathrm{dl}$ ). One hundred women aged: [median (interquartile range) ] 71(68-76) years participated in the study. The most prevalent components of MetS were: abdominal obesity (95\%) and impaired fasting glycemia (85\%). The prevalence of diabetes was $60 \%$ and of hypertension $44 \%$. Almost $80 \%$ of patients were on at least one antihypertensive drug, $42 \%$ on metformin and $40 \%$ on statins. Among statin users, TSH levels were positively correlated with IL-6 $(\mathrm{r}=0.41, \mathrm{p}<0.05)$ and with non-HDL-C $(\mathrm{r}=0.33, \mathrm{p}<0.05) .20$ patients on statins achieved an LDL-C $<100 \mathrm{mg} / \mathrm{dl}$ and 19 a non-HDL$\mathrm{C}<130 \mathrm{mg} / \mathrm{dl}$. Strikingly, when patients were compared according to non-HDL-C target significant increase in TG, TSH, IL-6 and 
decrease in HDL-C levels were observed $(p<0.05)$ in those outside the target, whereas only TG levels were significantly higher when patients were compared according to LDL-C achieved goal. As shown by others the use of a non-HDL-C in a population with MetS might be a better treatment target than LDL-C. Indeed, higher IL-6 values were observed only in the patients who did not achieve the non-HDL-C goal. The relationship between elevated TSH and IL-6 might be associated with impaired catabolism of remnant lipoproteins in statin-treated elderly women with MetS. The impact of elevated TSH in residual cardiovascular risk in patients with MetS merits further studies.

\section{Poster 203}

\section{Disorders of Thyroid Function Friday Poster Clinical EXTERNAL RADIATION THERAPY: A RARE CAUSE OF THYROIDITIS}

M. Cillo ${ }^{1}$, S.N. Thosani ${ }^{2}$, V.R. Lavis ${ }^{2}$, A.S. Mohamed ${ }^{2}$, W.H. Morrison ${ }^{2}$

${ }^{1}$ UT at Houston, McGovern Medical School, Houston, TX; ${ }^{2}$ UT MD Anderson Cancer Center, Houston, TX

Thyroiditis can be caused by a variety of processes including infectious and inflammatory causes, medications and radioactive iodine therapy. Although external beam radiation therapy (EBRT) has usually been associated with hypothyroidism, there are a few published reports of thyroiditis due to EBRT. Our goal was to characterize thyroiditis after EBRT. We reviewed 15 patients who were referred to our Endocrinology Department for abnormal thyroid function tests following EBRT for various malignancies. Thyroiditis was defined as either 1) a combination of thyrotoxicosis with low uptake of radioactive iodine or Technetium-99m, or 2) an initial hyperthyroid phase followed by a euthyroid or hypothyroid phaseOf the 15 patients, 5 demonstrated thyroiditis, 7 had isolated hypothyroidism, and 3 had an initial subclinical hyperthyroidism that later progressed to hypothyroidism. For the thyroiditis cases, the average age was 57.6 (range, 49-62) years. Four of the 5 were male, and 4 of the 5 had squamous cell carcinoma of the head and neck while 1 had lung adenocarcinoma. Two of the thyroiditis patients showed low uptake of radioisotope; the other 3 did not have nuclear studies performed. All 5 thyroiditis cases had negative tests for thyroid stimulating immunoglobulin, TSH receptor antibody, and thyroglobulin and thyroid peroxidase antibodies in various combinations. The biochemical hyperthyroid phase occurred a mean of 3.2 (range, 1.5 -4.5) months after the start of EBRT, and 1.8 (range, 0.03-3) months after radiation was completed. For thyroiditis patients, the mean radiation dose to the thyroid averaged 49.8 (range, 32-63) Gy. Four of these patients developed hypothyroidism after the initial hyperthyroid phase and required levothyroxine therapy, while the fifth patient recovered to a euthyroid state spontaneously. Thyroiditis due to EBRT typically does not warrant clinical evaluation unless the patient is symptomatic. After EBRT, symptoms of anxiety or palpitations should lead a clinician to consider thyroid function testing. Even though hypothyroidism is the most common thyroid complication of EBRT to the neck, we may be missing radiation induced thyroiditis.

\section{Poster 204 \\ Disorders of Thyroid Function Friday Poster Clinical STUDY OF THYROID FUNCTIONS IN PATIENTS ADMITTED IN ICU}

M. Anand, S.K. Jain, R. Aggarwal

Department of Medicine, Lady Hardinge Medical College, New Delhi, Delhi, India
Patients in the ICU typically have low T3, low T4, and normal or decreased TSH. These changes are described as non-thyroidal illness syndrome (NTIS). The extent of NTIS is associated with prognosis, but no proof exists for causality of this association. We studied the thyroid functions in patients admitted in ICU (medical and surgical). Serial estimation of thyroid functions [fT3 (2.5 to $3.9 \mathrm{pg} / \mathrm{ml}), \mathrm{fT} 4(0.6$ to $1.12 \mathrm{ng} / \mathrm{dl})$, and TSH $(0.34$ to $5.6 \mu \mathrm{IU} / \mathrm{mL})]$ was done from Day 1 to Day7 (or mortality) of ICU stay. $(\mathrm{N}=43) 16$ patients $(37.2 \%)$ died, 15 patients $(34.9 \%)$ were discharged and 12 patients $(27.9 \%)$ were continuing in the ICU at the end of followup.

27 of $43(62.8 \%)$ patients had low mean fT3 levels during the 7 day period of observation.

Of these 27 patients 15 died, 7 were discharged and 5 were still continuing in ICU at the end of 7 days. Non survivors had significantly lower mean fT3 levels compared to survivors $(\mathrm{p}=0.001$ significant). Discharged patients had highest mean $\mathrm{ft} 3$ values $(2.57 \mathrm{pg} /$ $\mathrm{ml})$ followed by those still continuing in ICU $(2.39 \mathrm{pg} / \mathrm{ml})$ and deceased patients $(1.87 \mathrm{pg} / \mathrm{ml})(\mathrm{p}$ value $<0.01)$. Decreasing trend of fT3 levels from day $1(1.94 \mathrm{pg} / \mathrm{ml})$ to day6 $(1.49 \mathrm{pg} / \mathrm{ml})$ was observed among patients who died and further fall in fT3 values were noted just prior to death. fT3 levels were low from day 1 and remained subnormal throughout the 7 day period of observation in these patients.

TSH values were highest among discharged $(3.38 \mu \mathrm{IU} / \mathrm{ml})$ followed by deceased $(2.85 \mu \mathrm{IU} / \mathrm{ml})$ and continuing patients $(2.58 \mu \mathrm{IU} /$ $\mathrm{ml}$ ) (p value 0.034$)$. Recovery of patients $(15 / 43)$ was associated with continuously rising TSH levels [3.89 $\mu \mathrm{IU} / \mathrm{ml}$ (day7) vs $2.85 \mu \mathrm{IU} / \mathrm{ml}$ (day1)] with concurrent rise in fT3 values $[2.83 \mathrm{pg} / \mathrm{ml}$ (day7) vs $2.36 \mathrm{pg} / \mathrm{ml}$ (day 1 )] (p value 0.021 ). Low fT3 syndrome was the most common Non Thyroidal Illness in ICU and was associated with poorer outcome. Fall in fT3 levels was associated with higher mortality. Spontaneous normalization of fT3, fT4 and increasing TSH was associated with recovery and better prognosis.

\section{Poster 205}

Disorders of Thyroid Function Friday Poster Clinical

\section{DOES LEVOTHYROXINE MONOTHERAPY MIMIC THE} EFFECTS OF ENDOGENOUS THYROID HORMONES? F. Azizi ${ }^{1}$, A. Amouzegar ${ }^{1}$, M. Tohidi ${ }^{2}$, M. Hedayati ${ }^{2}$, L. Cheraghi ${ }^{2}$ ${ }^{1}$ Endocrine Research Center, Research Institute for Endocrine Sciences, Shahid Beheshti University of Medical Sciences, Tehran, Iran, Tehran, Tehran, Iran (the Islamic Republic of); ${ }^{2}$ Prevention of Metabolic Disorders Research Center, Research Institute for Endocrine Sciences, Shahid Beheshti University of Medical Sciences, Tehran, Iran, Tehran, Tehran, Iran (the Islamic Republic of)

Objective: The belief that levothyroxine (LT4) monotherapy is sufficient to restore euthyroidism in hypothyroid patients has come under question because of certain of psychological and metabolic findings and low T3/T4 ratio in LT4 treated patients. The aim was to compare markers of thyroid status in LT4 users and euthyroid Graves' patients.

Methods: Cross-sectional data from 277 patients, including 140 radioiodine-treated Graves' hypothyroid subjects on LT4 treatment (LT4 group) and 137 euthyroid Graves' patients (Eu-G group): 83 euthyroid on methimazole (MMI) therapy and 54 euthyroid Graves' patients off MMI or radioiodine therapy for $>2$ years. Pearson and Spearman correlation coefficients for assessing relationship between T3:T4 ratio and variables in each group and univariate and multivariate linear regression models were employed.

Results: Mean age and female/male ratio were similar in the 2 groups. Serum FT4 was significantly higher and T3, TSH and $\mathrm{TPOAb}$ were significantly lower in the LT4 group, as compared to 
the Eu-G group ( $p<0.015$ in all cases) which translated to $27 \%$ lower serum T3:T4 ratio in the LT4 group. Higher waist circumference $(\mathrm{p}<0.083)$, BMI $(\mathrm{p}<0.003)$, serum cholesterol $(\mathrm{p}<0.005)$, and LDL cholesterol $(p<0.008)$ and lower HDL cholesterol $(p<0.015)$ were observed in the LT4 group compared to the Eu-G group. Age, BMI, triglycerides, and HOMA-IR in the LT4 group, and FBG, TRAb and TSH in the Eu-G group were significantly associated with T3:T4 ratio. In multivariate regression analysis, $\mathrm{BMI}, \mathrm{FBG}$, triglycerides and TSH entered into a "forward selection" model to compare T3:T4 ratio between 2 groups.

Conclusion: Patients using LT4 exhibited lower T3:T4 ratio despite lower TSH levels and differed in BMI and lipid parameters from those with euthyroid Graves' disease. Levothyroxine monotherapy does not mimic endogenous euthyroidism.

\section{Poster 206}

Iodine Uptake \& Metabolism Friday Poster Clinical

\section{IODINE STATUS IN BREAST AND FORMULA-FED} INFANTS AND THEIR MOTHERS: IS THERE ANY DIFFERENCE?

P. Mirmiran ${ }^{1}$, P. Nazeri ${ }^{1}$, Y. Mehrabi ${ }^{2}$, M. Hedayati ${ }^{3}$, F. Azizi ${ }^{4}$

${ }^{1}$ Nutrition and Endocrine Research Center, Research Institute for Endocrine Sciences, Shahid Beheshti University of Medical Sciences, Tehran, Iran (the Islamic Republic of); ${ }^{2}$ School of Public Health, Shahid Beheshti University of Medical Sciences, Tehran, Iran (the Islamic Republic of); ${ }^{3}$ Cellular and Molecular Research Center, Research Institute for Endocrine Sciences, Shahid Beheshti University of Medical Sciences, Tehran, Iran (the Islamic Republic of); ${ }^{4}$ Endocrine Research Center, Research Institute for Endocrine Sciences, Shahid Beheshti University of Medical Sciences, Tehran, Iran (the Islamic Republic of)

Despite substantial progress in elimination of iodine deficiency globally, lactating mothers and their infants are still susceptible to insufficient iodine intake. Hence, the aim of present study was to compare iodine status of breastfed and formula-fed infants as well as their mothers residing in an iodine sufficient area. In this cross sectional study conducted in Tehran, four health care centers were randomly selected. Healthy infants aged less than 3 months, who were being currently breastfed or formula-fed (exclusively or partially) as well as their mothers were randomly selected to participate. Urine and breast milk samples were collected from each infant and mother. Urinary and breast milk iodine concentrations and iodine content of infant formula were measured using the Sandell-Kolthoff method. A total of 124 postpartum mothers and infants, aged $29.2 \pm 4.9$ years and $2.0 \pm 0.23$ months, respectively completed this study. Iodine level of infant formula ranged between 50 and $184 \mu \mathrm{g} / \mathrm{L}$, while, median breast milk iodine concentration (BMIC) was $100 \mu \mathrm{g} / \mathrm{L}$ (range, $40-320 \mu \mathrm{g} / \mathrm{L}$ ) and $122 \mu \mathrm{g} / \mathrm{L}$ (range, 25-394 $\mu \mathrm{g} / \mathrm{L}$ ) in exclusive and partial breast feeding groups, respectively. Median [interquartile range (IQR)] urinary iodine concentration (UIC) of infants and mothers were 183.0 (76.0-285.2) and 78.0 (42.4-145.1) $\mu \mathrm{g} / \mathrm{L}$ in breastfeeding and 139.8 (68.4-290.3) and 86.6 (43.8-159.2) $\mu \mathrm{g} / \mathrm{L}$ in formula feeding groups, respectively, with no statistically significant difference between two types of feeding. A significant correlation was found between BMIC and UIC in infants $(\mathrm{r}=0.35, P=0.001)$. Analysis of covariance showed after adjustment for iodine concentration of breast milk, different types of feeding method (breastfeeding vs. formula feeding) had no effect on urinary iodine levels of infants and mothers. Findings of the current study reveal that in an area of iodine sufficiency, iodine status in infants as well as their mother did not differ between two types of feeding. It seems that adequate levels of breast milk rather than maternal urinary iodine concentration reflect iodine sufficiency of breastfed infants, due to uptake of iodine by mammary gland during lactation period.

\section{Poster 207}

Thyroid Hormone Metabolism \& Regulation Friday

Poster Clinical

\section{ASSOCIATION BETWEEN THYROID FUNCTION AND DEVELOPMENT OF OBESITY PHENOTYPES IN HEALTHY EUTHYROID SUBJECTS}

F. Azizi, A. Amouzegar, E. Kazemian, H. Abdi

Research Institute for Endocrine Sciences, Shahid Beheshti

University of Medical Sciences, Tehran, Iran, Tehran, Iran (the Islamic Republic of)

Although epidemiological studies have documented associations of thyroid function, within the normal range, with anthropometric measures and metabolic syndrome, however they have documented inconsistent and conflicting results. We aimed to investigate the relationship between thyroid function and different obesity phenotypes over a decade follow-up. This study was conducted on 1990 individuals from an ongoing population-based cohort study, Tehran Thyroid Study. Participants were categorized into four obesity phenotypes based on body mass index and metabolic status. To investigate the association of changes in thyrotropin (TSH) and free thyroxine (FT4) and incidence of different obesity phenotypes across the study period, a multivariate approach based on generalized estimating equation (GEE) method was used. patient with MHO phenotype had higher baseline serum TSH levels compared to others. The results of GEE (Generalized Estimating Equations) analysis for developing different obesity phenotype showed that each single unit increment in serum FT4 levels was accompanied with 1.65 fold increased risk of developing MHNO phenotype [Odds Ratio (OR), $1.65 ; 95 \%$ confidence interval $(\mathrm{CI}), 1.09-2.50$ ] during about 10 years follow up and after adjustment for age, sex, smoking, physical activity, education level, TPOAb and HOMA-IR. Also, results showed that each unit decrement in FT4 was associated with $46 \%$ increased risk of development of MHO over 9 years follow up after adjustment for potential confounder [0.54; 95\% CI, 0.35-0.82]. In addition, a significant positive association was found between serum TSH levels and development of MONW phenotype after adjustment for age, sex, smoking, physical activity, education level, TPOAb and HOMA-IR [OR, 1.22; 95\% CI, 1.01-1.48]. Changes in serum levels of FT4 and TSH in association with development of different obesity phenotypes, even after consideration of potential confounders.

\section{Poster 208}

Disorders of Thyroid Function Friday Poster Clinical

ORAL LIQUID L-THYROXINE (L-T4) SHOWS A BETTER EFFICACY IN COMPARISON TO TABLET FORMULATION IN PATIENTS SUBMITTED TO TOTAL THYROIDECTOMY (WITHOUT MALABSORPTION)

P. Fallahi ${ }^{1}$, S. Ferrari ${ }^{1}$, F. Ragusa $^{1}$, G. Elia ${ }^{1}$, G. Materazzi ${ }^{2}$, P. Miccoli ${ }^{2}$, A. Antonelli ${ }^{1}$

${ }^{1}$ Department of Clinical and Experimental Medicine, University of Pisa, Pisa, Italy; ${ }^{2}$ Department of Surgical, Medical, Molecular Pathology and Critical Area, University of Pisa, Pisa, Italy

The efficacy of levothyroxine (L-T4) in liquid formulation in patients recently subjected to total thyroidectomy is not still well understood. For this reason we evaluated its effectiveness in patients recently submitted to total thyroidectomy for euthyroid benign nodular goiter (without malabsorption or drug interference), with respect to L-T4 tablets. 
One hundred and sixteen patients were enrolled in the study: 56 were administered with L-T4 in tablets, and 60 with liquid L-T4 at the same dosage $(1.5 \mathrm{mcg} / \mathrm{kg} / \mathrm{day}), 30 \mathrm{~min}$ before breakfast, starting the day after thyroidectomy. Serum thyrotropic hormone (TSH), free thyroxine (FT4), and free triiodothyronine (FT3) were assessed in both groups, in a first control after 6 weeks, then in a second control after 12 weeks.

At the first control TSH values were significantly lower in the liquid L-T4 group, than in the tablet L-T4 group $(\mathrm{P}<0.05)$, as well as at the second control $(\mathrm{P}<0.01)$, while FT4 and FT3 levels were not significantly different. The prevalence of patients in the hypothyroid range $(\mathrm{TSH}>3.6 \mathrm{mcU} / \mathrm{ml})$ was significantly higher in the L-T4 tablet group.

Our results show that thyroidectomized patients (without malabsorption, gastric disorders, or drug interference) in a liquid L-T4 therapy have a better control of TSH levels.

\section{Poster 209 \\ Disorders of Thyroid Function Friday Poster Clinical \\ ORAL L-THYROXINE IN GEL CAPSULE VERSUS THE TABLET FORMULATION IN PATIENTS SUBMITTED TO TOTAL THYROIDECTOMY: PRELIMINARY DATA P. Fallahi ${ }^{1}$, S. Ferrari ${ }^{1}$, I. Ruffilli ${ }^{1}$, A. Bonatti ${ }^{1}$, S. Paparo ${ }^{1}$, G. Materazzi ${ }^{2}$, P. Miccoli ${ }^{2}$, A. Antonelli ${ }^{1}$ \\ ${ }^{1}$ Department of Clinical and Experimental Medicine, University of Pisa, Pisa, Italy; ${ }^{2}$ Department of Surgical, Medical, Molecular Pathology and Critical Area, University of Pisa, Pisa, Italy}

The efficacy of levothyroxine (L-T4) in soft gel capsule formulation in patients recently submitted to total thyroidectomy for multinodular goiter has not yet been evaluated. We compared the effectiveness of L-T4 gel capsule with respect to L-T4 tablets, in patients recently submitted to total thyroidectomy for multinodular goiter (not having malabsorption or drug interference). The study involved 58 patients, of whom 28 were administered with L-T4 therapy in tablets, while 30 with gel capsule L-T4 at the same dosage $(1.5 \mathrm{mcg} / \mathrm{kg} / \mathrm{day}), 30 \mathrm{~min}$ before breakfast, beginning from the day after thyroidectomy. Serum thyrotropic hormone (TSH), free thyroxine (FT4), and free triiodothyronine (FT3) were evaluated firstly after 6 weeks and then after 12 weeks, in both groups. At the first control, patients of the L-T4 gel capsule group showed TSH values significantly lower, with respect to the ones belonging to the L-T4 tablet group $(\mathrm{P}<0.05)$, as well as at the second control $(\mathrm{P}<0.01)$, while FT4 and FT3 levels were not significantly different. The prevalence of patients in the hypothyroid range (TSH $>3.5 \mathrm{mcU} / \mathrm{ml}$ ) was significantly higher in the $\mathrm{L}-\mathrm{T} 4$ tablet group. These data suggest a better efficacy of soft L-T4 gel capsule than L-T4 tablets in controlling TSH levels in patients thyroidectomized for thyroid cancer without malabsorption, gastric disorders, or drug interference. Further studies in a larger series of patients are necessary to obtain a definitive result.

\section{Poster 210}

Disorders of Thyroid Function Friday Poster Case Report IDIOPATHIC NECROTIZING GRANULOMAS OF THE THYROID: REPORT OF A RARE CASE A. Kotwal ${ }^{1}$, J. Eckmann ${ }^{2}$, D. Salomao ${ }^{3}$, V. Fatourechi ${ }^{1}$ ${ }^{1}$ Division of Endocrinology, Diabetes, Metabolism, and Nutrition, Mayo Clinic, Rochester, MN; ${ }^{2}$ Department of Medicine, Mayo Clinic, Rochester, MN; ${ }^{3}$ Department of Laboratory Medicine and Pathology, Mayo Clinic, Rochester, MN

Granulomatous disease of the thyroid commonly presents as subacute thyroiditis. Necrotizing granulomas in thyroid have been re- ported in infection (tuberculous and fungal), granulomatosis with polyangiitis, plasma cell granuloma, post-surgical/palpation thyroiditis and rarely in sarcoidosis. We report a case of necrotizing granulomas of thyroid associated with sudden profound hypothyroidism. A 75 year old male presented to his physician with 6 months of fatigue, poor concentration, cold intolerance and generalized weakness, without neck pain, preceding illness or hyperthyroid symptoms. Palpation revealed a non-tender stony hard thyroid. His TSH was $153 \mathrm{mIU} / \mathrm{L}$ (ref 0.3-4.2) as compared to a normal level 6 months prior, and free $\mathrm{T} 4$ was $0.3 \mathrm{ng} / \mathrm{dL}$ (ref 0.9-1.7). TPO and TG Abs were negative. He underwent partial left lobe resection for diagnosis at another institution which on histology showed necrotizing granulomas with multinucleated giant cells on a background of fibrosis and chronic lymphocytic inflammation. 6 months after being treated with levothyroxine $100 \mathrm{mcg} / \mathrm{day}$, he presented to our Endocrinology clinic. He had a small, firm thyroid without nodularity or tenderness, and proximal muscle weakness, with a TSH of $1.9 \mathrm{mIU} / \mathrm{L}$ and free T4 of $1.8 \mathrm{ng} / \mathrm{dL}$. Ultrasound showed a small, diffusely hypoechoic and hypovascular thyroid without nodules. Workup for pertinent autoimmune conditions was negative (ANA, RF, anti-SS, anti-Sm, RNP, Scl, anti-MPO, anti-proteinase 3, IgG4). ESR and CRP were normal. Core biopsy of the right lobe had histologic features similar to the previous specimen. Tissue stains and cultures did not show bacteria, mycobacteria or fungi. 8 months later, strength had improved and he was clinically and biochemically euthyroid on replacement. An extensive search of Mayo Clinic records from 19972017 and available literature did not yield any cases of similar pathology involving the thyroid in the absence of a clear etiology; however, this has been reported in other organs like the lungs. Hence, this case of necrotizing granulomas of thyroid causing sudden debilitating hypothyroidism would be considered idiopathic in etiology, the first such reported case to our knowledge.

\section{Poster 211}

Iodine Uptake \& Metabolism Friday Poster Clinical

\section{AMIODARONE INDUCED THYROTOXICOSIS} REQUIRING THYROIDECTOMY: MAYO CLINIC EXPERIENCE

A. Kotwal ${ }^{1}$, J. Clark ${ }^{2}$, G. Thompson ${ }^{3}$, M. Richards ${ }^{3}$,

\section{T. McKenzie ${ }^{3}$, M. Stan ${ }^{1}$}

${ }^{1}$ Division of Endocrinology, Diabetes, Metabolism, and Nutrition, Mayo Clinic, Rochester, MN; ${ }^{2}$ Department of Medicine, Mayo Clinic, Rochester, MN; ${ }^{3}$ Department of Surgery, Mayo Clinic, Rochester, MN

The management of amiodarone-induced thyrotoxicosis (AIT) is challenging due to the unpredictable and at times suboptimal response to medical therapy. Thyroidectomy is effective but has high perioperative morbidity and $\sim 10 \%$ mortality.

We retrospectively analyzed adults with AIT after $>3$ months of amiodarone (AM) therapy who underwent total or near-total thyroidectomy at Mayo Clinic from 11/2002 through 12/2016. Thyroid size, structure, vascularity and antibody status were used to differentiate types of AIT. Iodine uptake was considered if normal or elevated, which suggested type 1.17 patients $(4.6 \%)$ underwent surgery from a total of approximately 370 AIT cases. $82 \%$ were men with median age 60 yrs (range 45-88). AIT developed after 24 mos (3-72) of AM use and surgery was done after $2 \operatorname{mos}(0.5-24.5)$. All were symptomatic with palpitations and weight loss as the most common symptoms. At diagnosis, median TSH was 0.005 (0.005-0.09) mIU/L (ref 0.3-5), free T4 3.25 (1.8-18) ng/dL (ref 0.8-1.8) and total T3 198.5 (113-675) $\mathrm{ng} / \mathrm{dL}$. We classified AITas type 1 ( 5 cases), type 2 ( 5 cases), mixed ( 2 cases) and 5 were undefined (no US available). $70 \%$ were treated with anti-thyroid drug (ATD) and prednisone preoperatively. Surgical 
indications were medically refractory disease (70\%), worsening cardiac status (47\%), severe thyrotoxicosis requiring prompt resolution (35\%) and intolerance or contraindication to ATD (24\%). Median post-surgical hospital stay was 2 days (1-8). Within 1 wk post-surgery, median TSH was $0.565(0.03-1.1)$ and free T4 was $1.8(0.7-2.6) .7$ patients had a complication $<30 \mathrm{~d}$ post-surgery: re-hospitalization $(n=4)$, cervical hematoma $(n=2)$, recurrent arrhythmia $(n=2)$ and symptomatic hypocalcemia $(\mathrm{n}=1)$. One patient $(4 \%)$ died after developing stroke and cardio-respiratory failure.

Thyroidectomy was required for AIT management in a small proportion of cases, mostly due to medically refractory disease or need for prompt control of severe thyrotoxicosis affecting cardiac status. AIT type did not predict the need for surgery. The rate of complications in this cohort was higher than for other thyrotoxic etiologies but fortunately mortality was less than previously reported.

\section{Poster 212}

Iodine Uptake \& Metabolism Friday Poster Clinical INSTITUTIONAL EXPERIENCE OF COMPARISON BETWEEN SPOT URINARY IODINE CONCENTRATION AND 24-HOUR URINE IODINE CONCENTRATION K.B. Buddhdev ${ }^{1}$, R. Kedia ${ }^{1}$, V. Shostrom ${ }^{2}$, N.R. Hardy ${ }^{3}$, W. Goldner ${ }^{1}$

${ }^{1}$ Department of Internal Medicine, Division of Diabetes, Endocrine and Metabolism, University of Nebraska Medical Center, Omaha, NE; ${ }^{2}$ College of Public Health, Department of Biostatistics, University of Nebraska Medical Center, Omaha, NE; ${ }^{3}$ Cowdery Center Clinic, University of Nebraska Medical Center, Omaha, NE

Prior to treatment with radioactive iodine (RAI) for thyroid cancer, urine iodine concentration (UIC) must be appropriately low. At our institution, we require 24 hour UIC $<100-150 \mathrm{mcg} / \mathrm{L}$ prior to RAI therapy. Studies report 4-6 weeks for UIC to return to baseline post IV contrast, however, in those studies, the baseline is often higher than our UIC goal. In our experience, this is not long enough to achieve goal UIC. At our institution we collect a 24 hour UIC and spot UIC on all patients who have received IV contrast within 5 months of planned RAI therapy to determine if those can be used interchangeably. We performed a QI project to evaluate time to achieve UIC $<100,<150$ and $<200 \mathrm{mcg} / \mathrm{L}$ post IV contrast. We also compared the spot and 24 hour UIC in those who collected both simultaneously. The median time to achieve each endpoint was presented as $95 \%$ confidence interval (CI). Spearman correlation curve was used to determine correlation between spot and 24 hour UIC.27 subjects were evaluated. 20 subjects were $<100 \mathrm{mcg} / \mathrm{L}$ at a median of 93 days post IV contrast (CI: 76,116). 25 subjects were $<150 \mathrm{mcg} / \mathrm{L}$ at a median of 87 days $(\mathrm{CI}: 69,96)$ and 26 were $<200 \mathrm{mcg} / \mathrm{L}$ at a median of 87 days (CI: 69, 96). Twenty three subjects had both spot and 24 hour UIC collected simultaneously. These were not correlated. It took a median of 87-93 days to achieve UIC of $<100-200 \mathrm{mcg} / \mathrm{L}$. This is longer than previously published data reporting 4-6 weeks. We recommend waiting 3 months after IV contrast before considering RAI therapy if target UIC is $<100-200 \mathrm{mcg} / \mathrm{L}$. There was no correlation between the 24 hour and spot UIC at our institution.

\section{Poster 213}

Thyroid \& Development Friday Poster Basic

HUMAN THYROSTIMULIN IS MORE POTENT THAN HUMAN AND BOVINE TSH IN STIMULATING DIFFERENTIATION OF A MODEL PRE-OSTEOBLAST CELL SYSTEM IN VITRO

S. Neumann, E. Eliseeva, S.J. Morgan, A. Boutin, M. Gershengorn NIDDK, National Institutes of Health, Bethesda, MD
TSH has been shown to stimulate bone formation by activating TSH receptors (TSHRs) on bone precursor cells during development and in adult rodents. Thyrostimulin, an ancestral glycoprotein hormone, might play a role in rodent bone differentiation also; e.g. thyrostimulin appears to regulate bone formation during early skeletal development in mice. We demonstrated that thyrostimulin upregulates osteoblast markers osteopontin (OPN) and interleukin (IL)-11 in a pre-osteoblastic cell line derived from a human osteosarcoma made to over-express human TSHRs, U2OS-TSHR cells. To gain further insight into a potential role for thyrostimulin in human bone, we measured TSHRmediated cAMP production and OPN and IL-11 secretion stimulated by thyrostimulin, recombinant human TSH (rhTSH, Thyrogen ${ }^{\circledR}$ ) and bovine TSH (bTSH) in U2OS-TSHR cells. We confirmed that the potency of thyrostimulin to stimulate cAMP production $\left(\mathrm{EC}_{50} 0.07 \mathrm{nM}\right)$ was higher than for rhTSH $\left(\mathrm{EC}_{50} 0.5 \mathrm{nM}\right)$ and for bTSH $\left(\mathrm{EC}_{50} 3.4 \mathrm{nM}\right)$. The concentration-response curves of these three TSHR agonists for OPN secretion showed thyrostimulin was again the most potent ligand with an $\mathrm{EC}_{50}$ of $3.9 \mathrm{nM}$ whereas that for rhTSH was $12.6 \mathrm{nM}$ and for bTSH was 280 -fold lower $\left(\mathrm{EC}_{50} 1100 \mathrm{nM}\right)$. This activity pattern was confirmed by measurement of IL-11 secretion. The $\mathrm{EC}_{50} \mathrm{~s}$ for thyrostimulin, rhTSH and bTSH were $0.8 \mathrm{nM}, 1.3 \mathrm{nM}$, and $400 \mathrm{nM}$, respectively. The consistent high potency for thyrostimulin in human cells was confirmed by measurement of cAMP in primary cultures of human thyrocytes $\left(\mathrm{EC}_{50} 0.2 \mathrm{nM}\right.$ ); however, $\mathrm{EC}_{50}$ s for rhTSH and bTSH were similar, $\mathrm{EC}_{50} \mathrm{~s} 140 \mathrm{nM}$ and $190 \mathrm{nM}$, respectively. Our data confirm that human thyrostimulin is more potent than rhTSH and bTSH in activating human TSHRs. However, in contrast to previous findings, we found that rhTSH is more potent than bTSH in U2OS-TSHR cells or equally potent as bTSH in human thyrocytes, which is in stark contrast to previously reported data. Most importantly, the consistent high potencies (low nM concentration for human TSHR activation) observed for thyrostimulin in both human cell types support a role for thyrostimulin in human bone (and perhaps human thyroid) biology.

\section{Poster 214}

\section{Thyroid \& Development Friday Poster Clinical}

\section{EVOLUTION OF TRAB VALUES DURING PREGNANCIES AFFECTED BY GRAVES' DISEASE}

D. Luton, P. Ceccaldi, M. Banigé

Gynécologie Obstétrique, Université Paris VII APHP, Paris, France

We have recently shown on a restrospective study involving 417 patients that cut off values of TRAb in the maternal blood to predict fetal thyroid hypertrophy or neonatal dysthyroidism were respectively 2,5 and 5,9 IU/l. In order to best organize the follow up of the women, the fetus and the neonate; predicting as scarcely as possible the evolution of TRAb during pregnancy would be of great value.

We retrospectively analysed on 417 pregnant patients with Graves' disease the evolution of their TRAb on serial measurement. TRAb were measured with a "second-generation" human assay with thyrotropin receptor antibodies (TRAK) (Human Recombinant Thyroid-Stimulating Hormone [TSH] receptor h-TBII assay.B.R.A.M.H.S. Diagnostica, Berlin, Germany). TRAb were detected when their concentration was greater than $1 \mathrm{IU} / \mathrm{L}$.

In our study $144(34.7 \%)$ patients had only one TRAb determination, $168(40.3 \%)$ had two determinations, 63(15.1\%) had three, $42(9.9 \%)$ had more than three.

Among them 272 patients were considered for their first and last TRAb measurement, $97 \%$ of the cases displayed the highest value at the first TRAb. In 8 cases where the last value was the highest; the mean difference in these cases was $0,3875( \pm 0,22)$ which has neither clinical nor biochmical impact.

Among our 272 patients 138 had TRAb equal or higher to 2,5. For the remaining below 2,5 none had any variation leading to a level over 2,5. 
$100 \%(n=144)$ the patients with TRAb equal or below 2.7 at the first visit remained under 2.5 at the last TRAb determination. Among them 121 became negative.

73 patients had TRAb above 5.9 at their first visit, among them 30 evolved under 5.9 with a mean initial value of $10.5 \pm 6.2 \mathrm{IU} / \mathrm{l}$. All patients (198) with initial values under 5.9 IU/l remained under that level.

In conclusion, in $97 \%$ of cases TRAb levels will decrease during pregnancy.

A first TRAb value below $2.7 \mathrm{IU} / \mathrm{l}$ predicts in $100 \%$ of cases a final value below $2.5 \mathrm{IU} / 1$ with a negativation in $84 \%$ of cases. In this situation a specific prenatal evaluation might be avoided in most of cases.

A first TRAb value below $5.9 \mathrm{IU} / 1$ predicts in $100 \%$ of cases a final value below $5.9 \mathrm{IU} / \mathrm{l}$ with a negativation in $64 \%$ of cases. In this situation pediatric team will be reassured.

\section{Poster 215}

Thyroid \& Development Friday Poster Clinical

\section{THE IMPACT OF AGE ON POST-THYROIDECTOMY VOICE AND SWALLOWING COMPLAINTS}

Z.T. Sahli ${ }^{1}$, J.K. Canner ${ }^{1}$, O. Najjar ${ }^{1}$, E.D. Schneider ${ }^{1}$, J.D. Prescott ${ }^{1}$, J.O. Russell ${ }^{2}$, R.P. Tufano ${ }^{2}$, M.A. Zeiger ${ }^{1}$, A. Mathur ${ }^{1}$

${ }^{1}$ Endocrine Surgery, Johns Hopkins, Baltimore, MD;

${ }^{2}$ Otolaryngology, Head and Neck Surgery, Johns Hopkins Hospital, Baltimore, MD

Despite intact recurrent laryngeal nerves, voice and swallowing complaints are common after thyroidectomy. The association of patient age and frailty status on these changes is unknown. The aim of this study was to evaluate the impact of age and frailty on the incidence of voice and swallowing changes and determine associated risk factors. We performed an IRB-approved retrospective review of consecutive patients who underwent total thyroidectomy with intraoperative recurrent laryngeal nerve monitoring at a single institution between April 2013 and June 2015. Patients with recurrent laryngeal nerve injury were excluded. After data extraction, a modified frailty index $(\mathrm{mFI})$ was calculated for each patient. Patients with a scores of $0-0.08,0.09-0.18$, and $\geq 0.27$ were considered robust, pre-frail, and frail, respectively. The association among risk factors, including age, $\mathrm{mFI}$, prior history of neck surgery, occupational voice use, presence of malignancy or gastroesophageal reflux disease, and smoking status and voice and/or swallowing complaints was examined. Of 925 patients undergoing thyroidectomy, 148 (16.0\%) had only voice complaints, $52(5.6 \%)$ only swallowing complaints, and $26(2.8 \%)$ patients had both voice and swallowing complaints. We found a significant increase in voice or swallowing complaints up to the age of 50 (5\% increased odds per year) after which complaints plateaued. This association remained significant on multivariate analysis adjusting for covariates. Among our patient cohort, 200 (40.3\%), 272 (54.8\%), $24(4.8 \%)$ patients $\geq 50$ years were robust, prefrail, and frail, respectively. We found that $\mathrm{mFI}$ was not associated with voice or swallowing complaints. Age $\geq 50$ is independently associated with voice or swallowing changes after thyroidectomy despite intact recurrent laryngeal nerves. Additional prospective studies are needed to validate these findings, further define this association, and identify risk factors for developing these changes.

\section{Poster 216}

Thyroid \& Development Friday Poster Clinical HYPOTHYROIDISM SECONDARY TO IODINE DEFICIENCY

S. Ali, J. Samantray, C. Buggs

Endocrinology, Wayne State University, Detroit, MI
Iodine deficiency is reemerging in the US. Particular populations affected are patients with autism spectrum disorder (ASD). ASD patients are fed a gluten-free, casein-free diet in hopes of improving symptoms of autism. As a result of restrictive dietary habits, we report one ASD patient who developed hypothyroidism secondary to iodine deficiency. A 13 year old boy with ASD presented with neck swelling, weight gain, and constipation.

He has a family history of hypothyroidism in a maternal aunt. His diet consisted of turkey, bacon, carrots, muffins, unsalted nuts, and waffles.

Physical exam revealed a non-tender goiter with audible bruit bilaterally. Labs showed elevated TSH $45.3 \mathrm{mIU} / \mathrm{mL}$, low FT4 $0.2 \mathrm{ng} / \mathrm{dL}$, normal TT3 $99 \mathrm{ng} / \mathrm{dL}$, and negative thyroid antibody titers. Thyroid ultrasound showed a diffusely enlarged, hyperemic gland, suggestive of Hashimoto's disease. A random urine iodine revealed undetectable levels. Patient was started on levothyroxine 25 mcg daily which was increased a week later to $37.5 \mathrm{mcg}$ and he was instructed to add iodinated salt to diet.

One month later, his goiter size decreased and symptoms improved. He was started on iodine supplement $225 \mathrm{mcg}$ daily and levothyroxine was decreased to $25 \mathrm{mcg}$ daily. Repeat urine iodine showed transient elevation that normalized along with normalization of his TSH and FT4. Levothyroxine was decreased and then discontinued and iodine was decreased to $107 \mathrm{mcg} / \mathrm{day}$. Shortly after discontinuing levothyroxine, patient had recurrence of symptoms. As a result, levothyroxine was restarted at $25 \mathrm{mcg}$ daily and iodine was reduced to $75 \mathrm{mcg}$ daily. His symptoms resolved, although his TSH returned suppressed with normal FT4. Levothyroxine was decreased to $25 \mathrm{mcg} 6$ days/week and continued on iodine $75 \mathrm{mcg}$ daily. ASD patients have restrictive food behavior that can cause nutritional deficiencies. Considering the increasing rates of ASD among children, hypothyroidism from iodine deficiency is a grave concern. Screening for low urinary iodine along with appropriate iodine supplementation should be recommended for patients with restricted iodine diets, especially in patients found to have primary hypothyroidism with negative thyroid antibodies.

\section{Poster 217}

Thyroid Cancer Friday Poster Basic

\section{THE NOVEL MUTATION OF DMBT1 P. D1667E SHOW ONCOGENIC FUNCTION IN THYROID CARCINOMA CELL LINES}

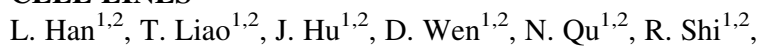

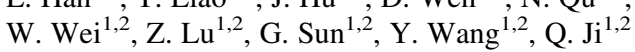

${ }^{1}$ Department of Head and Neck Surgery, Fudan University Shanghai Cancer Center, Shanghai, China; ${ }^{2}$ Department of Oncology, Shanghai Medical College, Fudan University, Shanghai, China

The deleted in malignant brain tumors 1 (DMBT1) is a glycoprotein containing multiple scavenger receptor cysteine-rich (SRCR) domains. The somatic mutation DMBT1 have been reported in gliboblastoma. Here we describe a novel mutation of DMBT1 p. D1667E and its function in thyroid carcinoma cell lines. DMBT1 expression in thyroid tumor and the matched normal thyroid tissue was measured by immunohistochemistry and qRT-PCR. Human papillary thyroid cancer cell line K1, human follicular thyroid cancer cell line FTC-133, human medullary thyroid cancer cell line TT and human anaplastic thyroid cancer cell line Cal62 were tested. Knock down of DMBT1 were conducted by siRNA. DMBT1 wild type and p. D1667E mutation plasmids were constructed successfully to investigate its role in vitro. Cell proliferation were carried out by CCK- 8 assay. Cell 
migration were measured by transwell assay. Apoptosis and cell cycle were evaluated by flow cytometry. DMBT1 expression in thyroid cancer tissue was lower than the match normal thyroid tissue. siRNA-DMBT1 was transfected into DMBT1 high expressed cell lines Cal62 and TT. Inhibition of DMBT1 upregulated the proliferation and migration of Cal62 and TT. Wild type and mutation plasmid were performed in DMBT1 low expressed cell lines K1 and FTC133. Overexpression of DMBT1 inhibited cell proliferation, migration and induced apoptosis of both cell lines, and arrested G1/S phase of cell cycle of FTC133. While p. D1667E variant could enhance cell migration and reverse the effect of DMBT1 on cell cycle in G2 phase. However, no significant effect on cell proliferation and apoptosis were observed in the mutation plasmid p. D1667E. We have identified that DMBT1 is a tumor suppressor in thyroid carcinoma and the distinct mutation of DMBT1 has oncogenic effect on cell cycle and migration. Further exploration will be implemented to figure out the underlying mechanism and feasible clinical scheme of DMBT1 in thyroid cancer.

\section{Poster 218}

Thyroid Cancer Friday Poster Basic

\section{INHIBITION OF CDK 5 BLOCKS GROWTH OF MEDULLARY THYROID TUMORS}

A. Carter ${ }^{2}$, R. Telange ${ }^{2}$, R. Jaskula-Sztul ${ }^{2}$, S. Oltmann ${ }^{1}$, J.A. Bibb ${ }^{2}$ ${ }^{1}$ Surgery, University of Texas Southwestern, Dallas, TX; ${ }^{2}$ Surgery, University of Alabama at Birmingham, Birmingham, $\mathrm{AL}$

Medullary thyroid carcinoma (MTC) is a form of neuroendocrine cancer that arises from C-cells of the thyroid gland. MTC occurs both as a sporadic form, originating from de novo mutations in body cells, as well as a hereditary disease. Hereditary MTC is typically generated due to germ-line mutations in the RET proto-oncogene which encodes a tyrosine kinase (TK) cell surface receptor. Although sporadic MTC may arise from various mutations, aberrant activation of cyclin dependent kinase (Cdk) 5 has been shown to play role in tumorigenesis. Cell lines have been derived from both types of human MTC tumors and are commonly used as screening tools for drug development. Vandetanib is a RET TK inhibitor that blocks growth of the RET mutant TT cell line. Cdk 5 inhibitors have recently been shown decrease growth of the sporadic MTC-SK cell line. The objective of this study is to compare the effects of Vandetanib and Cdk 5 inhibitors on both sporadic and familial disease and explore the therapeutic potential Cdk 5 inhibitors in vivo. Human MTC cell lines were treated with increasing concentrations of Vandetanib and the Cdk 5 inhibitor Indolinone A (Indo A). Cells were monitored for effects on growth using Cyquant Direct from Invitrogen. Bi-transgenic mice that develop MTC within the thyroid were treated with $0,10,20$, and $30 \mathrm{mg} / \mathrm{kg} \mathrm{BW}$ of Indo A by IP injection once every 3 days and tumor growth monitored over a 2 week period via MRI. Human TT cells were injected subcutaneously into Nude mice and tumors allowed establish for 4 weeks. Mice were then treated with 0,10 , and $20 \mathrm{mg} / \mathrm{kg}$ BW Indo A by IP injection once every 3 days and tumor growth monitored throughout 2 weeks by measurement with calipers. Vandetanib inhibits proliferation of TT cells more potently than MTC-SK cells. The Cdk5 inhibitor Indo A blocks growth of both TT and MTC-SK cells at similar IC50 levels. Indo A suppresses tumor growth in both a p25-driven transgenic mouse model of MTC and in human TT cell xenograft models. Inhibition of Cdk5 has the potential to be therapeutic for patients with both familial and sporadic disease. Future work will investigate drug combinations to achieve full tumor clearance.

\section{Poster 219}

Thyroid Cancer Friday Poster Basic

THE EFFECT OF HIGH FAT FEEDING ON THYROID TUMORIGENESIS USING TAMOXIFEN INDUCIBLE THYROCYTE-SPECIFIC BRAF ${ }^{\mathrm{V} 600 \mathrm{E}}$ KNOCK IN MICE J. Kim ${ }^{1,2}$, Y. Kang ${ }^{2}$, H. Yi ${ }^{2}$, H. Chung ${ }^{2}$, J. Lee ${ }^{4}$, B. Koo ${ }^{3}$, M. Shong ${ }^{1,2}$ K. Kim ${ }^{1,2}$

${ }^{1}$ Department of Medical Science, Chungnam National University, Daejeon, Korea (the Republic of); ${ }^{2}$ Research Center for Endocrine and Metabolic Diseases, Chungnam National University, Daejeon, Korea (the Republic of); ${ }^{3}$ Department of Otorhinolaryngology, Chungnam National University, Daejeon, Korea (the Republic of); ${ }^{4}$ Department of pathology, Catholic University of Korea Daejeon St. Mary's hospital, Daejeon, Korea (the Republic of)

A mutation in the Braf gene commonly expressed in approximately $45 \%$ of papillary thyroid carcinoma (PTC), and is enriched in tumors with more aggressive properties. Many studies also have suggested that people with high BMI have large-sized PTC risks and aggressiveness of PTC, however, the precise mechanism of obesity with $\mathrm{Braf}^{\mathrm{V} 600 \mathrm{E}}$ induced thyroid tumorigenesis is remained to identified. In this study, we investigated the effect of excessive fat intake in physiologic thyroid cancer rodent models using tamoxifen inducible thyrocyte-specific Braf ${ }^{\mathrm{V} 600 \mathrm{E}}$ knock in system. We developed mice with a tamoxifen-inducible thyrocyte-specific knock-in of Braf ${ }^{\mathrm{V} 600 \mathrm{E}}$ (LSL-Braf ${ }^{\mathrm{V} 600 \mathrm{E}} /$ TgcreERT2) at 8weeks, and fed these mice a normal chow diet (NCD) and high fat diet (HFD). To investigate whether HFD changes tumor characteristic, we showed the histology. To assess the immune microenvironment, we performed flow cytometry to identify the difference in immune cell composition in regional lymph nodes between NCD-fed and HFD-fed mice. LSL- Braf ${ }^{\mathrm{V} 600 \mathrm{E}}$ mice fed a HFD showed increased body weight compared to LSLBraf $^{\mathrm{V} 600 \mathrm{E}}$ mice fed a NCD. LSL-Braf ${ }^{\mathrm{V} 600 \mathrm{E}}$ mice with HFD presented more increased cellularity and proliferative tumor cells compared to LSL-Braf ${ }^{\mathrm{V} 600 \mathrm{E}}$ mice with NCD. Epithelial cells of thyroid follicles revealed abundant cytoplasm in LSL- Braf ${ }^{\mathrm{V} 600 \mathrm{E}}$ mice with HFD. Totally, the atypical follicular structure revealed in both LSLBraf $^{\mathrm{V} 600 \mathrm{E}}$ mice with $\mathrm{HFD}$ and with $\mathrm{NCD}$, however, papillary growing structures was significantly increased in mice with HFD, compared with in mice NCD. We evaluated the immune cell populations of enlarged lymph nodes, and HFD induced increasing of IFN-r+ CD8 $\mathrm{T}$ cells and reduction of regulatory $\mathrm{T}$ cell population in LSL-Braf $^{\mathrm{V} 600 \mathrm{E}}$ mice. Excessive fat intake aggravates the development of thyroid tumor and alters the population of immune cells in regional lymph nodes of LSL- Braf ${ }^{\mathrm{V} 600 \mathrm{E}}$ mice. These findings support the functional effect of obesity in thyroid tumorigenesis by regulation of tumor microenvironments, and suggest that altered tumor microenvironments by obesity-induced metabolic stress in $\mathrm{Braf}^{\mathrm{V} 600 \mathrm{E}}$ mutation, could be an important therapeutic target for treatment of differentiated thyroid cancer.

\section{Poster 220}

Thyroid Cancer Friday Poster Basic

\section{TREATMENT ALGORITHM FOR INDETERMINATE} THYROID NODULES: AN ANALYSIS OF COST EFFECTIVENESS USING MOLECULAR TESTING E.E. Cottrill ${ }^{1}$, J.S. Kim ${ }^{3}$, A.L. Schilling ${ }^{2}$, T. Shokri ${ }^{1}$, C. Pool ${ }^{1}$, M.M. Boltz ${ }^{2}$, B.D. Saunders ${ }^{2}$, C.S. Hollenbeak ${ }^{2,4}$, D. Goldenberg ${ }^{1}$ ${ }^{1}$ Otorhinolaryngology, Penn State Hershey Medical Center, Philadelphia, PA; ${ }^{2}$ Surgery, Penn State Hershey Medical Center, Hershey, PA; ${ }^{3}$ The Penn State College of Medicine, Hershey, PA; ${ }^{4}$ Public Health Sciences, Penn State Hershey Medical Center, Hershey, PA 
FNA of suspicious thyroid nodules yields indeterminate cytopathology in $10 \%-30 \%$. Of these, $15-35 \%$ prove to be malignant on final histopathology. Options include repeat FNA or diagnostic thyroid lobectomy, however, repeat FNA has low accuracy and diagnostic lobectomy subjects patients to surgical morbidity. Molecular analysis of cytologic samples has emerged to better assess cancer risk for indeterminate nodules. We present a decision tree model for the cost effectiveness of treatment modalities for indeterminate nodules, and analysis of the use of Thyroseq in our institution. A retrospective review was undertaken in a tertiary academic center over 5 years, enrolling adult patients with FNA cytology of Bethesda Category III-AUS/FLUS. Subject demographics include age, sex, race, underlying thyroid diagnosis, family history of thyroid cancer, and radiation exposure. A decision tree was constructed in TreeAge ${ }^{\circledR}$ and a cost-effectiveness analysis performed to compute the incremental costs per case of thyroid cancer correctly managed following an indeterminate FNA result. Costs were from the perspective of Medicare. Three strategies were considered: diagnostic lobectomy, repeat FNA, and ThyroSeq. The tree was parameterized using values from the literature. The study included 116 patients, $79 \%$ female, with a mean (SD) age of 49.5 (17) years. Twenty-five patients underwent repeat FNA, 55 diagnostic surgery, and 36 Thyroseq analysis. Average costs for treatment were $\$ 7,323$ for repeat FNA; \$9,482 for ThyroSeq; \$9,648 for diagnostic surgery. Effectiveness values, defined as the expected proportion of correctly managed cases in which malignancy was removed and benign lesions were not, were $61 \%$ for repeat FNA, 92\% for ThyroSeq, and $20 \%$ for diagnostic surgery. Computation of the Incremental Cost Effectiveness Ratio demonstrated that ThyroSeq is cost effective relative to repeat FNA (ICER $=\$ 6,978$ ) as well as diagnostic surgery. Calculating the ICER allows for consideration of cost as well as the true effectiveness of the management in correctly treating malignancy with surgery and benign disease with observation. Our findings suggest that molecular testing is the most cost effective method of evaluating indeterminate cytology.

\section{Poster 221}

Thyroid Cancer Friday Poster Basic

METFORMIN INHIBITS THE GROWTH OF HTH74RDOX WHICH ENRICHED IN THYROID CANCER STEM CELLS BY UP-REGULATING MIRNAS

C. $\mathrm{Liu}^{1,2}$, G. Chen ${ }^{1,2}, \mathrm{X} . \mathrm{Li}^{1,2}, \mathrm{Y} . \mathrm{Fu}^{1,2}$

${ }^{1}$ Affiliated Hospital of Integrated Traditional Chinese and Western Medicine, Nanjing University of Chinese Medicine, Nanjing, Jiangsu, China; ${ }^{2}$ Jiangsu Province Academy of Traditional Chinese Medicine, Nanjing, Jiangsu, China

Metformin, usually used for treatment of type 2 diabetes, has an anticancer potential. In recent years, the considerable interest has been evoked in the relationship between metformin and cancer stem cells. In our previous work, we established a doxorubicin resistant anaplastic thyroid cancer cell line HTh74Rdox which enriched in thyroid cancer stem cells. Here, we investigated the effects of metformin on HTh74Rdox and explored the underline mechanisms. MTT assay was used to detect the proliferation of HTh74Rdox cells and flow cytometry was used to detect the cell apoptosis. We detected the expression levels of these tumor suppressor miRNAs by real-time PCR. The expression levels of proteins were detected by Western Blotting. And we down-regulated the tumor suppressor miRNAs by transfecting miRNA inhibitors.

We found that metformin suppressed cell viability and promoted cell apoptosis of HTh74Rdox cells. The expression of tumor suppressor miRNAs miRNA-34a, miRNA-101, miRNA-125b and miRNA-138 were upregulated, meanwhile the AMPK/mTOR sig- naling pathway was activated in HTh74Rdox cells by metformin. However, after co-transfecting inhibitors of these four miRNAs to HTh74Rdox cells, the cell viability suppression and apoptosis promotion effect of metformin were blocked, furthermore, the activation of AMPK/mTOR signaling pathway was reserved either.

Our results demonstrated that metformin induces miRNA-34a, miRNA-101, miRNA-125b and miRNA-138 to activate AMPK/mTOR signaling pathway, leading to cell growth inhibition of HTh74Rdox which enriched in thyroid cancer stem cells. Therefore, this drug may be used as adjuvant treatment for anaplastic thyroid cancer in clinical.

\section{Poster 222 \\ Thyroid Cancer Friday Poster Basic}

\section{CDK4/6 INHIBITORS AND ANAPLASTIC THYROID CANCER: AN INVESTIGATION INTO THE RETINOBLASTOMA CANCER PATHWAY AND POTENTIAL THERAPEUTIC TARGETS}

K.A. Wong ${ }^{2,1}$, A. Di Cristofano ${ }^{1}$

${ }^{1}$ Molecular Biology, Albert Einstein College of Medicine, Bronx, NY; ${ }^{2}$ Surgery, Montefiore Medical Center, New York, NY

Cyclin-dependent kinase (CDK) 4/6 inhibitors increase progressionfree survival in certain types of stage IV breast cancer. We aim to assess whether Palbociclib, a CDK4/6 inhibitor, prevents tumor cell growth in vitro and in vivo in anaplastic thyroid cancer (ATC).

Retinoblastoma $(\mathrm{Rb})$ is a tumor suppressor protein that blocks cell entry into $S$ phase, preventing DNA synthesis and cell proliferation. Hyper-phosphorylation of $\mathrm{Rb}$ by CDK4/6 causes the release of transcription factors and induces cell division. By blocking CDK4/6 the tumor suppressor function of Rb stays activated. ATC carries a 6 month survival prognosis and there has been little advancement in chemotherapeutic options, making it a good disease target for this new class of drug. We characterized our cultured thyroid cancer cell lines by mRNA and proteins involved in the Rb pathway using qPCR and western blot. We then performed 72-hour dose-dependent cell growth assays using viable cell counts with differing concentrations of Palbociclib to determine EC50 values. The same cell counting technique was used to test Palbociclib in combination with other targeted chemotherapy agents. Finally, we have developed a new protocol for an orthotopic, syngeneic mouse model of ATC to test our drug results in vivo that involves injecting dissociated tumor cells from genetically engineered mice into one lobe of the thyroid. Our human lines show higher sensitivity to Palbociclib, compared to murine lines. Interestingly, the RAS and PI3K mutated lines required the highest relative doses of Palbociclib, followed by BRAF mutated cells. The $\mathrm{Rb}$ negative cells showed no growth inhibition when treated. Preliminary experiments show synergistic effects between Palbociclib and Omipalisib, a PI3K inhibitor. In vitro testing has shown that Palbociclib causes dose-dependent growth inhibition on $\mathrm{Rb}$ positive ATC cells, and genetic mutations may predict response to the drug. $\mathrm{Rb}$ protein is necessary for the drug to have effect. Current work is focused on designing a novel mouse model for in vivo study and to further investigate related pathways for targeted therapies, namely those whose over-activation associated with the least sensitivity to Palbociclib - PI3K/AKT and RAS pathways.

\section{Poster 223}

Thyroid Cancer Friday Poster Basic

EXPRESSION OF GP78/AMFR AND ENDOCYTOSIS OF AUTOCRINE MOTILITY FACTOR IN HUMAN THYROID CANCER CELLS

S.M. Wiseman ${ }^{1,2}$, L.D. Kojic ${ }^{3}$, K. Kasaian ${ }^{4}$, S.J. Jones ${ }^{4}$, B. Joshi ${ }^{3}$ I.R. $\mathrm{Nabi}^{3}$ 
${ }^{1}$ Surgery, University of British Columbia, Vancouver, British Columbia, Canada; ${ }^{2}$ Surgery, St. Paul's Hospital, Vancouver, British Columbia, Canada; ${ }^{3}$ Cellular and Physiological Sciences, Life Sciences Institute, niversity of British Columbia, Vancouver, British Columbia, Canada; ${ }^{4}$ Michael Smith Genome Sciences Center, British Columbia Cancer Agency, Vancouver, British Columbia, Canada

Gp78/autocrine motility factor receptor (Gp78/AMFR) is a cancerassociated endoplasmic reticulum-localized E3 ubiquitin ligase and also the cell surface receptor for autocrine motility factor (AMF). In this study our objective was to determine the association between Gp78/ AMFR and AMF endocytosis in thyroid cancer. Human thyroid tumor cell lines, tissue microarrays and fresh tissues procured at the time of thyroidectomy were utilized to evaluate Gp78/AMFR expression and AMF endocytosis. Tissue microarray analysis shows significantly elevated expression of Gp78/AMFR in differentiated thyroid cancers (DTC) relative to benign tumors or anaplastic thyroid cancer (ATC) specimens. Gp78/AMFR total and surface expression and AMF internalization were elevated in DTC compared to ATC cell lines and in freshly resected human papillary thyroid cancers (PTC) relative to benign goiters and collateral tissue. Culture of enzymatically digested explants from cancer, goiter and collateral thyroid tissue in low serum medium generated loosely attached spheroid-like aggregates after 5-7 days. These cells showed elevated mRNA expression of Gp78/ AMFR and the tumor stem cell gene marker Oct-4 relative to primary thyroid-derived fibroblasts and lacked mRNA expression of thyroid differentiation markers. Gp78/AMFR, Oct-4 and Sox-2 protein expression, Gp78/AMFR surface expression and AMF internalization were elevated in PTC-derived aggregates relative to fibroblasts. Gp78/ AMFR expression and AMF internalization are elevated in PTC and associated with expression of cancer stem cell markers.

\section{Poster 224}

Thyroid Cancer Friday Poster Clinical

OLDER DIFFERENTIATED THYROID CANCER PATIENTS EXHIBIT INCREASED CANCER INVASIVENESS AND NODAL METASTASES: A POSSIBLE EXPLANATION FOR THEIR WORSE PROGNOSIS

\section{J. Johar ${ }^{3}$, H.M. Britton ${ }^{3}$, K. Liu ${ }^{2}$, S.M. Wiseman ${ }^{1,2}$}

${ }^{1}$ Surgery, University of British Columbia, Vancouver, British Columbia, Canada; ${ }^{2}$ Surgery, St. Paul's Hospital, Vancouver, British Columbia, Canada; ${ }^{3}$ Medicine, University of British Columbia, Vancouver, British Columbia, Canada

The objective of this study was to investigate the clinicopathological variables that underlie observed age-related differences in DTC behavior, and may have the potential to improve the tailoring of surgical treatment and adjuvant therapy. Medical records from 941 sequential DTC patients who underwent thyroid surgery at a tertiary care Endocrine Surgical Center (St. Paul's Hospital, Vancouver, BC, Canada) between January 2005 and April 2017 were reviewed. DTC patient sex and pathological characteristics (cancer size, focality, extra-thyroidal cancer extension (ETE), vascular invasion, histological subtype, completeness of cancer resection), and presence (LNM) and extent of nodal or distant metastases, were evaluated for their relationship with patient age. A multivariate analysis of variance (using JMP 13.0 and R 2.12.0) was performed for dichotomous (gender, extra-thyroidal extension, multifocality, and vascular invasion), trichotomous (lymph node metastasis) and continuous (tumor size) variables. After exclusion of cases with incomplete data and papillary microcarcinomas, which generally are incidentally diagnosed and have an excellent prognosis, 622 patients made up the final study population. In the study population patient age was nor- mally distributed (mean 47.0, $1^{\text {st }}$ quartile 37.0 and $3^{\text {rd }}$ quartile 57.0), and the mean age of women was 46.9 years and 50.2 years for men. The correlations between mean age and ETE $\left(\mathrm{P}<10^{-5}\right)$, LNM $\left(\mathrm{P}<10^{-5}\right)$, and sex $(\mathrm{P}=0.028)$ were statistically significant. After splitting the study population into four age groups according to the mean age distribution and quartiles $(<37,37-47,48-57$, and $>57$ years of age), the correlation between mean age and LNM was significant $(\mathrm{P}=0.011)$ in the $48-57$ age group, and the correlation between mean age and ETE was significant $(\mathrm{P}=0.0024)$ in the over 57 age group. The worse prognosis observed in older DTC patients is due to increased cancer invasiveness (ETE) and metastatic behavior (LNM). Further study of the underlying molecular basis for these differences is important, and could lead to more tailored treatment, and improved outcomes for this common human cancer type.

\section{Poster 225}

Thyroid Cancer Friday Poster Basic

\section{EVALUATION OF VANDETANIB ACTIVITY} IN ANAPLASTIC THYROID CANCER IN VITRO AND IN VIVO

A. Antonelli ${ }^{1}$, G. Bocci $^{1}$, P. Fallahi ${ }^{1}$, S. Ferrari ${ }^{1}$, T. Di Desidero ${ }^{1}$, A. Corti ${ }^{2}$, S. Piaggi ${ }^{2}$, G. Materazzi ${ }^{3}$, R. Danesi ${ }^{1}$, P. Miccoli ${ }^{3}$

${ }^{1}$ Department of Clinical and Experimental Medicine, University of Pisa, Pisa, Italy; ${ }^{2}$ Department of Translational Research and of New Technologies in Medicine and Surgery, University of Pisa, Pisa, Italy; ${ }^{3}$ Department of Surgical, Medical, Molecular Pathology and Critical Area, University of Pisa, Pisa, Italy

The antitumor activity of vandetanib, a multiple signal transduction tyrosine kinase inhibitor (TKI), was evaluated in primary anaplastic thyroid cancer (ATC) cells, in the human cell line 8305C (an undifferentiated thyroid cancer), and in an ATC-cell line (AF). The antitumor activity of vandetanib was tested in vitro in primary ATC cells, in $8305 \mathrm{C}$ cells, and in $\mathrm{AF}$ cells, at the concentrations of $1 \mathrm{nM}$, $100 \mathrm{nM}, 1 \mu \mathrm{M}, 10 \mu \mathrm{M}, 25 \mu \mathrm{M}, 50 \mu \mathrm{M}$; in addition it was evaluated in vivo in $8305 \mathrm{C}$ cells injected sc in $\mathrm{CD}$ nu/nu mice. We obtained a significant inhibition of the proliferation and an induction of apoptosis in $8305 \mathrm{C}$ and AF cells treated with vandetanib. In ATC cells vandetanib was able to reduce the proliferation $(P<0.01$, ANOVA), to increase the percentage of apoptosis dose-dependently $(P<0.001$, ANOVA $)$ and to inhibit migration $(P<0.01)$ and invasion $(P<0.001)$. Vandetanib was tested in vivo also, in $8305 \mathrm{C}$ cells injected sc in CD $\mathrm{nu} / \mathrm{nu}$ mice. The treatment ( 25 or $12.5 \mathrm{mg} / \mathrm{kg} /$ day i.p. for 29 days) was initiated after 30 days from cell inoculation, when the average tumor volume was around $100 \mathrm{~mm}^{3}$. We observed a significant inhibition of tumor growth after the treatment with vandetanib $(25 \mathrm{mg}$ / $\mathrm{kg}$ /day). In addition, it was able to decrease significantly the $V E G F-A$ expression and microvessel density in $8305 \mathrm{C}$ tumor tissues. In this study we highlighted the antitumor and antiangiogenic activity of vandetanib as very promising in the treatment of anaplastic thyroid cancer.

\section{Poster 226}

Thyroid Cancer Friday Poster Clinical

EARLY DIAGNOSIS OF LYMPH NODE METASTASES AND LONG TERM FOLLOW UP AFTER RADIOIODINE AND/OR SURGICAL TREATMENT IN PATIENTS WITH PAPILLARY OR FOLLICULAR THYROID CANCER A. Antonelli ${ }^{1}$, P. Fallahi ${ }^{1}$, S. Ferrari $^{1}$, G. Elia $^{1}$, I. Ruffilli ${ }^{1}$, G. Materazzi ${ }^{2}$, P. Miccoli ${ }^{2}$

${ }^{1}$ Department of Clinical and Experimental Medicine, University of Pisa, Pisa, Italy; ${ }^{2}$ Department of Surgical, Medical, Molecular Pathology and Critical Area, University of Pisa, Pisa, Italy 
The early detection of metastatic lateral neck lymph nodes (LNL) was evaluated by assessing thyroglobulin (Tg) measurement and neck ultrasonography (NU) in 673 patients with papillary or follicular differentiated thyroid cancer (DTC), and long-term follow-up after $I^{131}$ and/or surgery. After near-total thyroidectomy and $\mathrm{I}^{131}$ ablation of residual tissue, $\mathrm{Tg}, \mathrm{NU}$, diagnostic and post-therapy whole body scan (WBS), alone or in combination, were performed in DTC-patients.

TSH-stimulated Tg alone had a diagnostic sensitivity of $86 \%$ for detecting LNL and a negative predictive value (NPV) of $83 \%$, while NU had a sensitivity of $68 \%$, and NPV of $69 \%$. The combination of $\mathrm{Tg}$ with NU increased the sensitivity to $98 \%$, and NPV to $97 \%$, respectively.

Patients with LNL (LNL-p) uptaking radioiodine were treated with $\mathrm{I}^{131}$, with the following outcomes: $49 \%$ responded after a median of 4 cycles (median $354 \mathrm{mCi}$, cumulative dose; 7.4 years median follow-up) with "disappearence of uptake at post-therapy WBS and $\mathrm{Tg}<1 \mathrm{ng} / \mathrm{ml}$ " (complete remission, CR); $12 \%$ of not responding cases had CR with other treatments.

Nine\% of LNL-p not uptaking or not responder to $I^{131}$ were treated with surgery, and subsequent $\mathrm{I}^{131}$ readministration with CR in $23 \%$ of cases (6.5 years median follow-up). Early detection of LNL in DTCpatients could lead to a CR by $\mathrm{I}^{131}$ in $49 \%$ after 4 or less $\mathrm{I}^{131}$ treatments. Not responding/not uptaking $I^{131}$ patients can achieve a $C R$ in $23 \%$ of cases combining different therapies.

\section{Poster 227 \\ Thyroid Cancer Friday Poster Translational \\ LYSYL OXIDASE (LOX) IS REGULATED BY BRAF AND IS A PROGNOSTIC MARKER FOR THYROID CANCER}

M. Boufraqech ${ }^{1}$, D. Patel ${ }^{1}$, N. Nilubol ${ }^{1}$, A.S. Powers ${ }^{2}$, T. King ${ }^{1}$, J. Shell ${ }^{1}$, L. Zhang ${ }^{1}$, S.K. Gara ${ }^{1}$, V. Gunda ${ }^{3}$, S.M. Kumar ${ }^{1}$, J. Fagin ${ }^{4}$, J.A. Knauf $^{4}$, S. Parangi ${ }^{3}$, D. Venzon ${ }^{5}$, M. Quezado ${ }^{2}$, E. Kebebew ${ }^{1}$

${ }^{1}$ Endocrine Oncology Branch, NCI/NIH, Bethesda, MD;

${ }^{2}$ Department of Pathology, NIH/NCI, Bethesda, MD; ${ }^{3}$ Surgery, Massachusetts General Hospital, Boston, MA; ${ }^{4}$ Human Oncology and Pathogenesis Program, Memorial Sloan-Kettering Cancer Center, New York, NY; ${ }^{5}$ Biostatistics and Data Management Section, NCI/NIH, Bethesda, MD

LOX is a mediator of cancer progression and is overexpressed in thyroid cancer. BRAF V600E mutation is common in thyroid cancer and is associated with aggressive disease. The aim of this study was to determine if LOX mediates the effect of BRAF and its utility as prognostic marker in thyroid cancer. We studied the effect of BRAF on LOX in vitro and in vivo. The prognostic utility of LOX and $B R A F$ mutation status was analyzed in the TCGA cohort and an independent cohort from our institution. Inhibition of BRAF V600E using PLX-4720 decreased LOX expression and inducible overexpression of BRAF V600E or BRAF E586K increased LOX expression. Moreover, induction of both mutants showed an increase in cell migration/invasion that was inhibited with LOX depletion. In a transgenic mouse model with thyroid-specific BRAF V600E, strong LOX and p-ERK expression was observed in cancer tissue. Treatment with PLX-4720 in mouse models of metastatic thyroid cancer and orthotopic xenograft of thyroid cancer using $B R A F$ V600E cells, showed reduced LOX and p-ERK expression in cancer tissue. In the TCGA cohort, we found higher $L O X$ expression in $B R A F$ V600E mutant tumors as compared to wild type $(\mathrm{p}<0.0001)$. In patients with $B R A F$ V600E mutant tumors, high $L O X$ expression was associated with significantly shorter diseasefree survival as compared to low $L O X$ expression $(\mathrm{p}=0.012$, $\mathrm{HR}=2.5)$. On multivariate analysis, stage III-IV $(\mathrm{HR}=2.3, \mathrm{CI}=1.3$ -
4.1) and high $\operatorname{LOX}(\mathrm{HR}=1.9, \mathrm{CI}=1.03-3.4)$ were the only significant independent variables associated with shorter disease-free survival but not $B R A F$ mutation status. We next assessed the prognostic value of LOX on disease-specific mortality, in an independent cohort of aggressive thyroid cancer cases treated at our institution. On multivariate analysis, positive LOX staining was the only independent prognostic factor $(\mathrm{p}=0.001, \mathrm{HR}=9.9, \mathrm{CI}=2.6-37.81)$ associated with higher disease-specific mortality. Our data suggests that activating mutations in BRAF regulate LOX expression. LOX is a prognostic marker for disease-free survival and disease-specific mortality in patients with thyroid cancer.

\section{Poster 228}

Thyroid Cancer Friday Poster Translational

\section{BRAF MUTANT PAPILLARY THYROID CANCER CELLS} WITH CONCURRENT MUTATIONS ALONG THE KRAS-RAC1 AXIS DEVELOP RESISTANCE TO BRAF(V600E)-INHIBITORS AND ARE ASSOCIATED WITH INCREASES IN EMT MARKERS

B.P. Danysh ${ }^{2}$, N. Busaidy ${ }^{2}$, D. Sinha ${ }^{2}$, K.W. Evans ${ }^{1}$, A. Akcakanat ${ }^{3}$, F. Meric-Bernstam ${ }^{1}$, M.E. Cabanillas ${ }^{2}$, M.D. Ringel ${ }^{4}$, M.H. Shah ${ }^{5}$, M. Hofmann ${ }^{2}$

${ }^{1}$ Investigative Cancer Therapeutics, University of Texas MD Anderson Cancer Center, Houston, TX; ${ }^{2}$ Department of Endocrine Neoplasia and Hormonal Disorders, University of Texas MD Anderson Cancer Center, Houston, TX; ${ }^{3}$ Investigative Cancer Therapeutics, University of Texas MD Anderson Cancer Center, Houston, TX; ${ }^{4}$ Division of Endocrinology, Diabetes, and Metabolism, The Ohio State University and Arthur G. James Comprehensive Cancer Center, Columbus, OH; ${ }^{5}$ Department of Internal Medicine, The Ohio State University and Arthur G. James Comprehensive Cancer Center, Columbus, $\mathrm{OH}$

Targeted BRAF inhibitors have been used to treat patients with advanced BRAF-mutated PTC; however, acquired resistance is common. Recently, we demonstrated inhibitor resistance in BRAF (V600E) PTC cells through de novo acquisition of a KRAS (G12D) activating mutation during long-term in vitro vemurafenib treatment. Our research investigates novel drivers of BRAF inhibitor resistance and the role of downstream effectors in recurrent disease aggressiveness. Two BRAF inhibitor-resistant cell lines were derived and used in this study. 1) A BRAF (V600E)/KRAS (G12D) cell line was derived following longterm in vitro treatment of KTC1 cells with vemurafenib. 2) A cis BRAF (K601N)/(T599del)/RAC1 (P34R) cell line was established from a FNA/PDX taken from a patient who developed dabrafenibresistant PTC during treatment. qPCR and WB assays were used to determine EMT/motility marker expression and pathway activation. Cell growth and motility was assessed using cell counting and transwell assays. A novel clonal RAC1 (P34R) mutation was identified as a possible BRAF inhibitor resistance mutation that is similar to the known resistance mutation in melanoma (P29S). BRAF inhibitor resistance was confirmed in the BRAF/KRAS and BRAF/RAC1 mutant lines relative to BRAF mutant-only lines despite persistent inhibition of MAPK signaling. Both cell lines showed a significant increase in MAPK pathway activation and EMT/cell motility gene expression patterns. However, increased motility was only observed in the BRAF/ KRAS mutant. Modest increased PAK1/2 activation was detected in the $\mathrm{BRAF} / \mathrm{RACl}$ mutant along with a $>$ two order magnitude increase in total RAC1 expression. Although primary PTC tumors often present with single driver mutations, concurrent acquisition of a secondary mutations can cause inhibitor resistance. Our results suggest an association between acquired BRAF (V600E) inhibitor resistance through acquired secondary mutation and gene expression patterns implicated in EMT and cell motility. These results support KRAS and RAC1 
mutations as a potential genetic mechanism of spontaneously acquired secondary BRAF inhibitor resistance in BRAF-mutated thyroid cancer cells.

\section{Poster 229}

Thyroid Cancer Friday Poster Translational ANALYSIS OF GENE AND MIRNA EXPRESSION PROFILES IN FOLLICULAR VARIANT OF PAPILLARY THYROID CARCINOMAS WITH AND WITHOUT DISTANT METASTASES

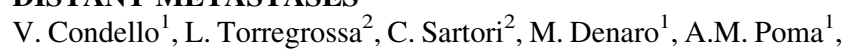

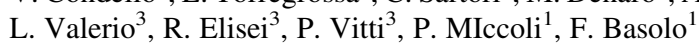

${ }^{1}$ Department of Surgical, Medical, and Molecular Pathology and Critical Care, University of Pisa, Pisa, Italy; ${ }^{2}$ Division of Surgical Pathology, University Hospital of Pisa, Pisa, Italy; ${ }^{3}$ Department of Clinical and Experimental Medicine (Endocrine Unit), University Hospital of Pisa, Pisa, Italy

Follicular variant (FV) is the most frequent and fastest growing subset of papillary thyroid carcinoma (PTC), accounting for 15 to $30 \%$ of all PTC cases. Usually FVPTC is associated with a good outcome, nevertheless in rare cases it can give distant metastases (1-9\%). In this study we retrospectively investigated the molecular bases underlying a different ability to metastasize of FVPTCs. We selected 12 primary FVPTCs with distant metastasis (Met) and 12 primary non-metastatic FVPTCs (NonMet) with similar clinicopathological features in terms of patients' age, tumor size, histotype, vascular and extrathyroidal invasion, multifocality and bilaterality. The mean follow-up duration was 9.3 years for NonMet and 5.1 for Met group. Total RNA, including miRNA, was purified from formalin-fixed paraffin-embedded tumor tissues. All tumors were investigated for gene and miRNA expression by using two panels, the first one included 740 genes involved in cancer progression and the second one was composed of 780 miRNAs. Data were evaluated by NanoString nSolver 3.0 software and unsupervised hierarchical clustering analyses were performed by using Pearson correlation. Twenty-three out of 24 samples resulted adequate for the mRNA expression analysis. Forty-seven out of 740 genes resulted differentially expressed (DE) between Met and NonMet lesions. Considering the $47 \mathrm{DE}$ genes, two distinct clusters were observed. Interestingly, 82\% (9 out of 11) of carcinomas included in the first cluster belonged to the Met group, whereas in the second 9 out of 12 (75\%) were NonMet FVPTCs. In the same way, 22 out of 24 samples resulted adequate for the miRNA expression analysis. Thirty-five out of 798 miRNAs were DE between the two groups and the clustering analysis performed by using these 35 miRNAs formed two main groups: the first one included Met FVPTCs only (9), whereas the second one included all the NonMet (11) and 2 Met FVPTCs. Our results indicate that FVPTCs with metastatic abilities show different gene and miRNA expression profiles compared to the nonmetastatic. A prospective validation is needed to evaluate the usefulness of this molecular approach in the early identification of high-risk FVPTCs.

\section{Poster 230}

Thyroid Cancer Friday Poster Translational

\section{EXPLORING BEYOND BRAF: UPSTREAM} AND DOWNSTREAM MOLECULAR BIOMARKER EXPRESSION IN PAPILLARY THYROID CANCER A. Lin $^{1}$, L. Henke ${ }^{1}$, T. DeWees ${ }^{1}$, J. Schwarz ${ }^{1}$, J. Pfeiffer ${ }^{2}$, P. Grigsby ${ }^{1}$, S. Markovina ${ }^{1}$

${ }^{1}$ Radiation Oncology, Washington University in St. Louis, St. Louis, MO; ${ }^{2}$ Pathology, Washington University, St. Louis, MO
Given the mixed data on BRAF V600E mutation as an independent biomarker for recurrence, we sought to study the expression of five proteins in the BRAF and Ras proliferative pathways in papillary thyroid tumor specimens. A prior published clinical database of differentiated thyroid cancer patients treated at a single institution from 1959-2009 was used. BRAF V600E mutation status was determined by restriction fragment length polymorphism analysis. $1.5 \mathrm{~mm}$ cores were taken from areas of cancer on paraffin embedded blocks and placed in triplicate onto tissue microarrays (TMA). Immunohistochemical stains for RET, phospho-MEK (pMEK), MAPK, PPARy, and phospho-AKT (p-AKT) were scored independently and blindly by two investigators on a scale of $0-3$ for staining intensity. An average score of $\geq 2$ by one or both investigators was considered positive. Kaplan-Meier and Cox regression analyses were performed to evaluate association of these markers with thyroid cancer recurrence. TMAs were scored for 224 patients with mean follow up of 9.3 years (range 1.5-30 years). All patients received surgery and 217 $(97 \%)$ received adjuvant $\mathrm{I}-131$, mean initial dose of $187 \mathrm{mCi}$ (range 0-980 mCi). Baseline patient characteristics included mean age at diagnosis of 45 years (range 6-82 years), female sex (73\%), Caucasian race $(81 \%)$, classic papillary vs. follicular variant $(73 \%$ vs. $27 \%$, respectively), and AJCC stage I (70\%), stage II (9\%), stage III (19\%), and stage IV (2\%). Positive scoring of molecular markers was found for BRAF V600E (70\%), RET (57\%), pMEK (20\%), MAPK (19\%), PPARy (29\%), and p-AKT (40\%). There were $23(14 \%)$ recurrences in classic papillary histology, while follicular variant histology only had one recurrence $(1.6 \%), p=0.01$. Within classic papillary histology, RET staining was protective against recurrence $(\mathrm{HR}=0.34$, 95\% CI 0.14-0.85). No other molecular biomarkers were independent predictors of recurrence on univariate or multivariate analysis. Recurrence was predominantly seen in classic papillary histology, and RET expression was surprisingly protective of recurrence in patients treated with adjuvant I-131. Further effort to understand these molecular marker interactions and create a prognostic signature is ongoing.

\section{Poster 231}

Thyroid Cancer Friday Poster Translational MOUSE MODEL OF RAI THERAPY AND USE OF SOD MIMIC BMX-001 FOR RADIO-PROTECTION

\section{A. Patel ${ }^{1}$, E. Kosmacek ${ }^{2}$, W. Goldner ${ }^{1}$, R. Oberley-Deegan ${ }^{2}$}

${ }^{1}$ DEM, UNMC, Omaha, NE; ${ }^{2}$ Biochemistry and Molecular Biology, UNMC, Omaha, NE

Treatment of thyroid cancer often involves administration of radioactive iodine (RAI) for remnant ablation or adjuvant therapy. However, there is morbidity associated with RAI therapy, which can result in both acute and chronic complications. It is well known that the damaging effects of ionizing radiation are mediated by the formation of free radicals, and cancer cells display increased levels of oxidative stress. In vivo changes after RAI administration in appropriate animal models are not well described in the literature. We aim to study changes in normal tissue that can occur after RAI administration in a murine model and hypothesize that the systemic administration of SOD mimic, BMX-001, will selectively ameliorate RAI induced damage to normal tissue while simultaneously leaving the thyroid tumors vulnerable to RAI ablation. Four-week-old female C57BL/6 mice were divided into 4 groups - control (unexposed group \pm BMX-001) and RAI-treated group that received RAI orally at different doses $(0.005-0.01 \mathrm{mCi} / \mathrm{g}$ of body weight $) \pm \mathrm{BMX}-001$. We measured acute external damage score in RAI group, salivary flow rates, collected blood samples for blood cell counts and collected thyroid tissue and salivary glands for histological examination. We have shown that oral administration of RAI to mice causes an acute decrease in platelets and white blood cells, causes facial 
swelling, and loss of thyroid tissues. We have further shown that when BMX-001 is given during and after RAI administration, the blood cell numbers remain normal, there is less facial swelling, and the thyroid tissues are protected from radiation-induced killing. At 9 months-post RAI administration, mice have significantly reduced salivary production as compared to non-irradiated mice and that the administration of BMX-001 significantly prevented this loss of salivary function. We are describing mouse model to study the effects of RAI therapy using different doses of RAI. Currently there are no approved radio-protector agents that can be used in conjunction with RAI to reduce complications with RAI therapy. These data indicate that BMX-001 may be a potent radio-protector of salivary glands in thyroid cancer patients receiving RAI therapy.

\section{Poster 232 \\ Thyroid Cancer Friday Poster Translational \\ DOWN-REGULATED EXPRESSION OF UNC5D IN PAPILLARY THYROID CARCINOMA AND ITS TUMOR SUPPRESSIVE FUNCTION \\ B. Han, M. Zhang, F. Sun, H. Song \\ shanghai ninth people's hospital, Shanghai, China}

Studies showed an association of UNC5D gene with cancers in kidney, bladder and neuroblastoma. We investigated whether the novel identified $U N C 5 D$ acts as a tumor suppressor in papillary thyroid carcinoma (PTC). Primary PTC tumors and matched normal thyroid tissues were obtained from 112 patients for detection of UNC5D mRNA by real time PCR. Genomic DNA sequencing was performed to detect $B R A F$ mutation in PTC tumors. The association between $U N C 5 D$ expression and clinicopathological data of PTC patients were reviewed retrospectively. PTC cell lines TPC-1 and K1 with stable transfection of $U N C 5 D$ was used to investigate the functions of $U N C 5 D$. Flow cytometry, CCK-8, transwell assay and scratch tests were used to examine cell cycle distribution, proliferation and migration, respectively. The expression of UNC5D was significantly down-regulated in PTC compared with adjacent normal thyroid tissues. Lower UNC5D expression was significantly associated with aggressive tumor behaviors, such as lymph node metastasis and $B R A F$ mutation. Overexpression of $U N C 5 D$ significantly suppressed cell malignant behaviors, including cell proliferation and migration, as well as tumor growth in vivo.

These findings suggest a potential tumor suppressor role of $U N C 5 D$ in PTC progression, and provide insight into potential clinical relevance for the prognosis of PTC.

\section{Poster 233}

\section{Thyroid Cancer Friday Poster Translational}

\section{CORRELATION OF MITOCHONDRIAL PROCESS EXPRESSION AND MACIS SCORE IN PAPILLARY THYROID CANCER}

C.L. Brett ${ }^{1}$, E. Thompson ${ }^{2}$, X. Wang ${ }^{2}$, A. Chindris ${ }^{2}$, R. Smallridge ${ }^{2}$ ${ }^{1}$ Internal Medicine, Mayo Clinic Jacksonville, Ponte Vedra Beach, FL; ${ }^{2}$ Mayo Clinic Florida, Jacksonville, FL

Papillary thyroid cancer (PTC) can have a wide range of presentations, from indolent to aggressive. As such, efforts have been made to identify markers of aggressive disease to better inform treatment decisions and prognostication. To that end, we examined differential gene expression within samples of The Cancer Genome Atlas (TCGA) papillary thyroid cancer database for factors associated with increased risk. RNA expression data and MACIS scores were obtained from the NIH Genomic Data Commons and cBioPortal respectively. Spearman correlation was then run to identify genes with significant positive correlation with MACIS score. These genes were then mapped to Gene Ontology (GO) biological processes to assess for enrichment. RNA expression data was available on 573 samples, out of which MACIS score was also available on 440 samples. 57,233 transcripts reached the lower limit of detection, with Spearman correlation coefficients from -0.34 to +0.25 . Of these, 154 genes had a positive correlation and $\mathrm{p}<0.001$. GO enrichment analysis of this set yielded 11 processes with greater than 10 fold enrichment, 7 of which related to mitochondrial processes- Mitochondrial ATP synthesis coupled electron transport (ET) (GO:0042775); ATP synthesis coupled ET (GO:0042773); respiratory ET chain(GO:0022904); oxidative phosphorylation (GO:0006119); ET chain(GO:0022900); ATP metabolic process (GO:0046034); and cellular respiration (GO:0045333). The other four pathways dealt with nucleoside and ribonucleoside metabolic processes (GO:0009205; 0009199; 0009144; 0009167). Further analysis of the above 154 genes demonstrated 14 of the 37 genes in the mitochondrial genome, including 10 of the 13 mitochondrial polypeptides in the electron transport chain. These results suggest a correlation between the overexpression of mitochondrial related processes and the MACIS risk score in PTC. Further investigation will be required to determine the mechanism behind this observation. It is noted that mutations in mitochondrial DNA have been associated with PTC, and compensatory upregulation of poorly functioning pathways or increased number of mitochondria could represent potential mechanisms for this association and possible markers of increased risk.

\section{Poster 234}

Thyroid Cancer Friday Poster Translational

\section{GENETIC ANALYSIS USING NEXT GENERATION} SEQUENCING IN 46 PEDIATRIC THYROID CANCER SUBJECTS: GENOTYPE-PHENOTYPE CORRELATIONS S. Kadakia ${ }^{1}$, W. Jiang ${ }^{2}$, M. Nikita ${ }^{3}$, S. Cheng ${ }^{4}$, R.E. Reitz ${ }^{4}$, K. Qu ${ }^{4}$, H.X. Li ${ }^{4}$, R.O. Newbury ${ }^{5}$, F.M. Hantash ${ }^{4}$, R. Newfield ${ }^{1}$

${ }^{1}$ Pediatric Endocrinology, University of California, San Diego, San Diego, CA; ${ }^{2}$ Department of Otolaryngology, University of California, San Diego, San Diego, CA; ${ }^{3}$ Department of Pediatrics, University of Maryland School of Medicine, Baltimore, MD;

${ }^{4}$ Hematology and Oncology, Quest Diagnostics, Nichols Institute, San Juan Capistrano, Ca, San Diego, CA; ${ }^{5}$ Department of Pathology, University of California, San Diego, San Diego, CA

Thyroid cancer is the most common endocrine malignancy and the third most common pediatric solid tumor. The objective of this study was to assess the prevalence of mutations, fusions, and rearrangements in pediatric well differentiated thyroid cancer (WDTC) and to correlate the genotype with clinical phenotype. In this single-center retrospective study thyroid tissue blocks from pediatric WDTC cases from 2001 to 2015 were analyzed at Quest Diagnostics for BRAF, RAS (N, K, H), RET/PTC, and PAX8/PPARG mutations using validated molecular methods which was previously reported by Nikita, et al. We provide an update after samples were reanalyzed via next generation sequencing (NGS) to assess for additional fusions. Fortysix samples (36 female, 10 male) with WDTC including papillary thyroid cancer (PTC) (63\%), follicular thyroid cancer (FTC) (15.3\%), and follicular variant of papillary thyroid cancer (FVPTC) $(21.7 \%)$ were analyzed. Mutations prior to NGS were noted in 24/46 subjects (12 BRAF, 2 RAS, 6 RET/PTC, 4 PAX8/PPARG). Analysis for additional mutations via thyroid-specific NGS test is in process and will be verified by an alternate NGS method. Fusions were found in an additional 6 subjects with NGS (Two with ETV6-NTRK3, and one each of TPR-NTRK1, EML4 -ALK, STRA-ALK, NCOA4-RET). All of the latter occurred in PTC and 5/6 were associated with positive lymph nodes. Metastases to lymph nodes were also noted in 4/6 RET/ 
PTC and 9/12 BRAF cases. In terms of ethnicity, in RET/PTC and NGS fusions $5 / 12$ were Hispanic as compared to $10 / 12$ in BRAF $(\mathrm{p}=0.035)$. BRAF is the most common mutation in PTC (37.9\%). All fusions as a group account for $41.4 \%$ of PTC cases. In $82.8 \%$ of PTC we were able to find a genetic alteration. Adding the NGS helped to identify a genetic cause in $82.8 \%$ of PTC cases, which may be higher once we have full mutation analysis via NGS completed. We have found that mutations and fusions that fall within the BRAF-like BRS score (Agrawal, Cell 2014) help predict lymph node metastases, which are common in pediatric WDTC. A larger cohort would help determine whether ethnicity is correlated with specific genetic alterations.

\section{Poster 235}

Thyroid Cancer Friday Poster Translational

\section{HIGHLY ACCURATE DIAGNOSIS OF CANCER IN THYROID NODULES WITH THYROINOW NEXT-GENERATION SEQUENCING ASSAY}

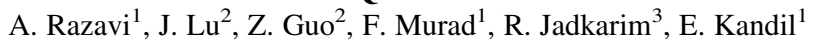
${ }^{1}$ Tulane University School of Medicine, New Orleans, LA; ${ }^{2}$ GoPath Laboratories, Buffalo Grove, IL; ${ }^{3}$ Tulane University School of Public Health and Tropical Medicine, New Orleans, LA

Despite the high reliability of thyroid fine needle aspiration cytology, indeterminate cytology is reported in up to $30 \%$ of aspirations. Indeterminate cytology becomes a clinical gray zone and poses challenges in surgical decision-making. Currently, a plethora of gene panels has been proposed to help with managing patients with indeterminate nodules. Herein, we report a novel concise three-gene panel, BRAF, KRAS, and NRAS, to diagnose thyroid nodules and overcome FNA indeterminate status. In total, 132 thyroid nodules were resected from 93 patients, and surgically pathology was used to determine final diagnosis. Patient demographics, nodule size, and Bethesda cytological classification were retrospectively evaluated. Of the 132 nodules, 89 were benign and 42 were malignant per surgical pathology. The gene panel accurately diagnosed $80.65 \%$ of cases with positive and negative predictive values of $87 \%$ and $79.2 \%$ nodules respectively. With respect to only indeterminate nodules, the three-gene panel accurately diagnosed $81.31 \%$ of cases with a positive predictive value of $84.2 \%$ and a negative predictive value of $80.7 \%$. According to the Chi-square test, a significant relationship was found between nodule diagnosis and BRAF $(p<0.001)$ as well NRAS $(p<0.001)$, but not KRAS $(p=0.543)$. Likewise, the nodule type was significantly related to age $(p=0.0072)$, race $(p=0.017)$, and body mass index $(\mathrm{p}=0.005)$. Our results suggest that focused gene testing is a valuable tool in diagnosing thyroid nodules. More specifically, genetic testing can be used to supplement surgical decision-making when nodule cytopathology is indeterminate. The precise selection of molecular markers used in thyroid nodule screening warrants further study, as the genetic landscape of each nodule may vary according to disease etiology.

\section{Poster 236}

Thyroid Cancer Friday Poster Translational

\section{THE INJECTABLE PEG400-SF HYDROGEL LOADED WITH NGF AND GDNF AS A BIOACTIVE FILLER OF NERVE CONDUIT TO PROMOTE RECURRENT LARYNGEAL NERVE REGENERATION}

D. Ma, W. Dai

The first affiliated hospital of Harbin Medical University, Harbin, China

Damage to the recurrent laryngeal nerve (RLN) causing vocal cord paralysis profoundly decreasing quality of life often occurs during thyroidectomy. RLN is one of the most difficult peripheral nerves in which to achieve functional regeneration, especially when it is severed although awareness of the pathophysiology of nerve damage has improved. Nowadays there is no effective method to repair RLN functionally. In this study, we tested whether the injectable PEG400SF hydrogel loaded with NGF and GDNF could serve as a filler of nerve conduits to promote RLN repair. We developed an injectable hydrogel via mixing PEG400 with silk fibroin solution. In vitro, we evaluated its biomechanics and cyto-compatibility by co-culturing with Schwann cells. Besides we loaded this hydrogel with NGF and GDNF in different ratios, and then investigated release profiles of growth factors and their bioactivities. Additionally, PEG400-SF hydrogels were implanted in the intermuscular space of neck of rats to test biocompatibility and biodegradability in vivo. In the present study, we used sonicated silk gel as the control group.8\% PEG400SF hydrogel revealed well biomechanics (compressive strength: $103.63 \pm 4.28 \mathrm{kPa}$; elastic modulus: $10.78 \pm 0.87 \mathrm{kPa}$ ) as a biofiller. SEM images showed that PEG400-SF hydrofel had higher porosity and better interconnectivity than sonicated SF gels, which was helpful for tissue regrowth. MTT assay and HE staining both demonstrated this gel was biocompatible. For release profiles of NGF and GDNF were sustained for 30 days $(37.22 \pm 2.12 \%$ and $33.72 \pm 2.76 \%$ respectively) guaranteeing the prolonged retention of growth factors at the injured site, of which in terms PEG400-SF hydrogel was superior to sonicated SF gels. Finally, growth factors released from PEG400-SF hydrogels enhanced the axon growth from PC12 cells, which revealed NGF and GDNF remained their bioactivities. When the ratio of NGF to GDNF was 2:3, the enhancement was most obviously. The study shows that the injectable hydrogel provides a favorable bio-molecule environment for RLN regeneration and that it has therapeutic potential for repairing RLN damage.

\section{Poster 237}

Thyroid Cancer Friday Poster Case Report

\section{FROM THYROID ADENOMA TO METASTATIC CARCINOMA: NOT ALL BRAF MUTATIONS ARE CREATED EQUAL}

T.D. Cubb ${ }^{1,2}$, E.Y. Ibrahim ${ }^{2}$, S.I. Sherman ${ }^{2}$, M.D. Williams ${ }^{3}$, N. Busaidy ${ }^{2}$, S.P. Weitzman ${ }^{2}$

${ }^{1}$ Endocrinology, Baylor College of Medicine, Houston, TX;

${ }^{2}$ Endocrine Neoplasia and Hormonal Disorders, University of Texas MD Anderson Cancer Center, Houston, TX; ${ }^{3}$ Pathology, University of Texas MD Anderson Cancer Center, Houston, TX

Cytology from thyroid nodule fine-needle aspiration (FNA) coupled with molecular testing facilitates prognostication and treatment planning. $B R A F$ mutations are present in about half of all papillary thyroid cancers (PTC) and $>95 \%$ are $B R A F^{V 600 E}$. The 2nd most common $B R A F$ mutation is $B R A F^{K 601 E}$. Reports exist of $B R A F^{K 601 E}$ in follicular adenomas, follicular variant PTC, and follicular cancer. It has been asserted that $B R A F^{K 601 E}$ is associated with more benign behavior and thus may influence treatment decisions. We describe 2 cases highlighting the spectrum of pathology and outcomes associated with this less common BRAF mutation. Case 1: A 52yo woman was incidentally discovered to have a $1.6 \mathrm{~cm}$ thyroid nodule. FNA showed a follicular lesion of undetermined significance and an 8 gene mutational panel identified a $B R A F^{K 601 E}$ mutation. A 10 miRNA gene expression classifier was negative (suggesting benignity). After discussing the risks/benefits she underwent a diagnostic lobectomy. Pathology revealed an adenoma.

Case 2: A 65yo man had a thyroidectomy for a $4.5 \mathrm{~cm}$ poorly differentiated follicular carcinoma with lymphovascular invasion and extrathyroidal extension. He received 2 doses of I-131 but 
subsequently presented with rising thyroglobulin levels. He was diagnosed with radioactive iodine refractory (RAIR) lung metastases that were initially stable and observed for 2 years, after which they began to progress. Molecular testing of his tumor revealed only a $B R A F^{K 601 E}$ mutation. He then developed rapidly progressive disease in the lungs and extensive skeletal metastases, whereupon he was treated with dabrafenib/trametinib. He has had a durable oncologic response for $>1$ year. Although current literature indicates a $B R A F^{K 601 E}$ mutation in thyroid cancer is associated with a less aggressive phenotype, these cases illustrate that this mutation may be seen with disease at either end of the spectrum - from a thyroid adenoma to progressive RAIR thyroid cancer requiring systemic treatment. In the latter case, response was obtained with combined $B R A F / M E K$ inhibitor therapy. Given very different clinical courses in these patients, we suggest caution when using the presence of a $B R A F^{K 601 E}$ mutation in patient care decisionmaking.

\section{Poster 238}

Thyroid Cancer Friday Poster Case Report

A CASE OF COEXISTING GRAVES' DISEASE AND MALIGNANT STRUMA OVARII IN WHICH A SOMATIC LOSS-OF-FUNCTION MUTATION OF THE TSH RECEPTOR WAS DEMONSTRATED

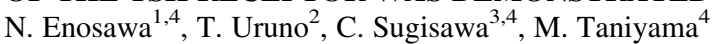

${ }^{1}$ Goto clinic, Mishima, Shizuoka, Japan; ${ }^{2}$ Department of Surgery, Ito Hospital, Shibuya-ku, Tokyo, Japan; ${ }^{3}$ Department of Pediatrics, Keio University School of Medicine, Tokyo, Japan; ${ }^{4}$ Department of Internal Medicine, Division of Endocrinology and Metabolism, Showa-University Fujigaoka Hospital, Kanagawa, Japan

Malignant struma ovarii is a rare condition, and there have only been a few reports of cases also complicated by hyperthyroidism. We encountered an extremely rare case of coexisting malignant struma ovarii and Graves' disease. We were also able to analyze a TSH receptor gene mutation of a malignant struma ovarii lesion. A 39-year-old woman presented with a right ovarian tumor that was detected incidentally. She had been diagnosed with Graves' disease 2 years before with typical thyrotoxic manifestations, a diffuse goiter, bilateral Graves' ophthalmopathy and positive TRAb. She was euthyroid under methimazole. Abdominal imaging revealed a $7-\mathrm{cm}$ complex right ovarian mass and scattered solid tumors in the pouch of Douglas, the anterior wall of the uterus and peritoneal. She underwent a bilateral salpingo-oophorectomy and partial resection of the metastatic lesions. The histopathological findings showed thyroid-type follicular carcinoma arising from struma ovarii. After total thyroidectomy we performed radioiodine therapy on metastatic tumors, which was effective. During 3-years follow-up, her thyroglobulin levels significantly decreased. Furthermore, we detected a heterozygous loss-of-function mutation (p. A204V) in the tumor TSH receptor gene, which was absent in the peripheral leucocytes. Struma ovarii sometimes produce thyroid hormone autonomously. When struma ovarii coexists with Graves' disease, thyroid hormone production was complicated with autonomous production and stimulation by thyroid-stimulating antibodies. The stimulating antibodies may also be involved in tumor proliferation. We examined TSH receptor gene to explore gain-of function mutation and unexpectedly found a loss-of-function mutation. Decreased TSH receptor function may have been involved in both decreased autonomous secretory ability and decreased responsiveness to TSH receptor antibodies. To our knowledge, this is the first case of somatic loss-of-function mutations of the TSH receptor. Although TSH receptor function seems to be relatively impaired with mutated receptor, high-dose radioiodine therapy to metastatic struma ovarii was effective.

\section{Poster 239}

Thyroid Cancer Friday Poster Case Report

INFLAMMATORY RESPONSE AFTER IMMUNOTHERAPY WITH A YEAST-CEA THERAPEUTIC CANCER VACCINE IN METASTATIC MEDULLARY THYROID CANCER

J. Del Rivero, M. Rausckhorst, M. Bilusic, F. Karzai, J. Strauss,

L. Cordes, J. Marte, A. Gramza, W. Dahut, J. Schlom, J. Gulley, R. Madan

National Cancer Institute, National Institutes of Health, Bethesda, MD

Medullary thyroid cancer (MTC) accounts for approximately 4\% of thyroid carcinomas. Metastatic MTC can be treated approved agents vandetanib or cabozantinib, but toxicity limits their use. There are ongoing trials exploring less toxic immunotherapy in patients with MTC including a phase II study at the NCI with a yeast-based therapeutic cancer vaccine targeting CEA (GI-6207; NCT01856920). A 66-year-old female with MEN2A with metastatic MTC initially treated with surgeries and radiation enrolled on the clinical trial with yeast-based therapeutic cancer vaccine. Per protocol the vaccine was administered biweekly for 3 months, then monthly for 9 months, then every 3 months for a year. She completed vaccine in March of 2015 and has not had additional therapy since. One year and five months after completing vaccine, the patient reported a rapidly enlarging lymph node at a known site of metastatic disease confirmed by CT scan. A biopsy of the lymph node was negative for TTF-1 and calcitonin with abundant CD3+ and CD20+ lymphocytes present. Within 1 week, the inflammation had largely resolved without treatment. Her calcitonin increased from a baseline $11618 \mathrm{pg} / \mathrm{mL}$ to $14523 \mathrm{pg} / \mathrm{mL}$ during the episode with a subsequent nadir of $8682 \mathrm{pg} / \mathrm{mL}$ two months later. A patient with metastatic MTC who was treated with a cancer vaccine had an apparent inflammatory event 17 months after completing immunotherpy. Inflammatory events at sites of disease in MTC are rarely seen but have now been seen in multiple MTC patients treated with this vaccine, including the phase I trial (Bilusic et al. Cancer Immunol Immunother 2014). The patient in the phase I study was found to have corresponding antigen-specific immune responses and immune analysis on this patient is pending. This case highlights the potential of immunotherapy in MTC and the potential for this vaccine to initiate an immune response that may be sustained more than one year after treatment. Additional immunotherapy trials in MTC are planned at the NCI including an ongoing study with anti-PD1 therapy (pembrolizumab) [NCT03072160)].

\section{Poster 240}

Thyroid Cancer Friday Poster Case Report

\section{A CASE OF METASTATIC PAPILLARY THYROID CANCER WITH FAVORABLE OUTCOME: A-TEN-YEAR-FOLLOW-UP}

A.F. Malik, A. Rivas Mejia, R. Smallridge

Endocrinology, Mayo Clinic, Jacksonville, FL

Patients with metastatic differentiated thyroid cancer older than sixty have a poorer prognosis than younger patients. We report an elderly woman who presented with a pulmonary nodule as the first finding of metastatic papillary thyroid cancer (PTC) who after subsequent treatment has not had recurrent disease in over a decade. The patient is a 73-year-old woman with a history of hypothyroidism who was found to have a new 2-cm nodule in the left lung on routine chest xray. A CT scan confirmed the finding. Two PET scans were normal. A year later, there was an interval increase in the nodule by 2 to $3 \mathrm{~mm}$. She underwent left axillary thoracotomy and left upper lobectomy. The specimen revealed 3 foci of metastatic papillary 
thyroid carcinoma with predominant follicular architecture and focal papillae, nuclear grooves, intranuclear pseudo-inclusions, and crowding. Several follicles contained colloid. The tumor invaded the visceral pleura but not the pleural surfaces. Two small foci were noted in the lymphatic distribution near bronchioles. Immuno-histochemical stains were positive for thyroglobulin in the colloid and TTF-1 in the follicular cells supporting the diagnosis of papillary thyroid carcinoma (PTC).

Thyroidectomy in 2006 showed follicular variant PTC $(1.0 \mathrm{~cm})$ in the left lobe with metastases to 1 lymph node. Margins were uninvolved. She was staged at IVc (T1N1bM1). She received $153 \mathrm{mCi}$ of Iodine-131 and post-therapy scan showed uptake in the thyroid bed and perihilar regions of both lungs. Subsequent diagnostic radioactive iodine (RAI) scan was unrevealing. Her thyroglobulins have remained stable at 0.2 to $0.3 \mathrm{ng} / \mathrm{ml}$ for ten years while on L-T4 suppression. She has been followed annually with no evidence of clinical recurrence on thyroid ultrasound or CT of the chest or abdomen. Molecular testing of her thyroid is pending. Distant metastases as the initial presentation of DTC, is unusual with a reported $50 \%$ mortality of patients with pulmonary metastases who underwent total thyroidectomy, metastatectomy, and RAI. Our patient had an atypical presentation of metastatic papillary thyroid cancer. Ten years after undergoing a metastectomy, thyroidectomy and RAI, she has had a favorable outcome.

\section{Poster 241}

Thyroid Cancer Friday Poster Case Report

MEDULLARY THYROID CANCER AS A LATE PRESENTING FEATURE IN A MEN2A PATIENT WITH RET C620G MUTATION

K. Bux ${ }^{1}$, k.F. $\mathrm{Ali}^{2}$, L. Olansky ${ }^{2}$

${ }^{1}$ Internal Medicine, Cleveland Clinic, Lakewood, OH;

${ }^{2}$ Endocrinology and Diabetes Institute, Cleveland Clinic, Cleveland, $\mathrm{OH}$

Multiple endocrine neoplasia 2A (MEN2A) syndrome is a familial disorder characterized by medullary thyroid carcinoma (MTC), pheochromocytoma, and primary parathyroid hyperplasia. MEN2A patients with RET C620G mutation typically present with MTC proceeding the development of pheochromocytomas by 10 years on average. Here we report a case of MEN2A with RET C620G mutation presenting initially with bilateral pheochromocytomas. A previously healthy 29-year-old woman presented in early 2014 with asymptomatic hematuria. She underwent a renal ultrasound which showed bilateral adrenal masses. She had no family history of thyroid, adrenal, or parathyroid malignancies. Her 24-hour urine metanephrines and normetanephrine levels measured $1081 \mathrm{mcg} / 24 \mathrm{~h}$ (ref 25-222 mcg/24h) and $2076 \mathrm{mcg} / 24 \mathrm{~h}$ (ref 40-412 mcg/24h) respectively, confirming a diagnosis of pheochromocytoma. A PET/CT scan demonstrated bilateral adrenal masses (right $4.0 \times 3.2 \mathrm{~cm}$ and left $1.8 \times 1.3 \mathrm{~cm}$ ), but no thyroid masses or focal FDG uptake in the thyroid or neck lymph nodes. Subsequent genetic analysis revealed a RET C620G mutation pointing to a MEN2A diagnosis. The patient underwent successful bilateral adrenalectomies. Final pathology confirmed bilateral pheochromocytomas. A subsequent thyroid surveillance ultrasound in late 2015 revealed an indistinct left hypoechoic area with micro-calcifications measuring $1.26 \times 1.18 \times 1.64 \mathrm{~cm}$. Fine needle aspiration was positive for medullary thyroid malignant cells. In 2016, patient underwent total thyroidectomy with bilateral central neck dissections. Final pathology showed bilateral, multifocal MTC $(1.3 \mathrm{~cm}$ focus in the left lobe and $0.5 \mathrm{~cm}$ focus in the right lobe). Four of the 24 lymph nodes dissected were positive for MTC. Cases of bilateral pheochromocytomas may proceed the development of MTC in MEN2A patients with RET C620G mutation. A low threshold should be present to screen patients with bilateral pheochromocytomas for concomitant MTC.

\section{Poster 242}

Thyroid Cancer Friday Poster Case Report

\section{UNUSUAL PRESENTATION OF THYROID CARCINOMA}

S. Akbar, P. Kadambi, J. Cohen

Endocrinology, George Washington University, Arlington, VA

We present a highly unusual case of pelvic mass being a metastatic thyroid cancer in a 65-year female from Ethiopia. Patient first presented to an outside hospital with progressive right hip pain associated with weight loss, fatigue, loss of appetite and night sweats for three months. She described right lower abdominal and pelvic pain. She had a history of thyroid nodule remotely and had fine needle aspiration done in Ethiopia and records were unavailable. On exam, she had antalgic gait and was mostly wheelchair bound and appeared frail. Her vitals were stable and neck exam showed a palpable right thyroid nodule with no lymphadenopathy. Initial work up with routine labs and infectious work up was unremarkable. A CT scan of abdomen and pelvis revealed a large $15 \mathrm{~cm}$ lytic right sacroiliac soft tissue mass. Patient underwent a successful ultrasound guided biopsy of the sacroiliac mass. Pathology revealed carcinoma of thyroid origin with follicular architecture and enlarged nuclei. Thyroglobulin and TTF-1 were strongly positive. Further labs showed a thyroglobulin level of 37582, Anti-Thyroglobulin antibody $<0.1$, TSH of $0.008 \mathrm{uIU} / \mathrm{ml}(0.450-4.50)$, Free T4 of $1.51 \mathrm{ng} / \mathrm{ml}(0.82-1.77)$ and $\mathrm{T} 3$ of $258 \mathrm{ng} / \mathrm{ml}(71-180)$

With these unexpected new findings, patient underwent bilateral thyroid nodule biopsy and results are currently pending. She has also been scheduled for PET scans and CT chest/abdomen/pelvis. Final treatment plan will be delineated after thyroid nodule pathology and imaging results are available. Follicular carcinoma is an aggressive form of thyroid cancer with risk for distant metastasis. We hereby present an unusual case of metastatic thyroid cancer to the pelvis which is an uncommon site for metastasis. Subtle clues like prior history of thyroid nodule and FNA are of extreme importance in our case which sometimes get overlooked in clinical practice. This case highlights the importance of considering thyroid cancer in the differential diagnosis and workup when a patient presents with a suspicious lesion at an unusual site, especially in the presence of thyroid nodules.

\section{Poster 243}

Thyroid Cancer Friday Poster Case Report

FOUR CASES OF THYROGLOSSAL DUCT CYST WITH PAPILLARY CARCINOMA AT GUILLERMO ALMENARA IRIGOYEN NATIONAL HOSPITAL (LIMA, PERU) DURING THE PERIOD OF 1999 TO 2016

D.L. Aranda, M. Valcarcel, J.B. Cruz

Head and Neck Surgery, Hospital Nacional Guillermo Almenara

Irigoyen, Lima, Lima, Peru

Thyroglossal Duct Cyst is the most frequent congenital anomaly of the cervical region. In about 1-2\% contains malignant elements; the most reported primary tumor has been papillary carcinoma (75$85 \%$ ), the definitive diagnosis is post-surgical. The recommended treatment is controversial and it should be applied according to each specific case. We report four cases of Papillary carcinoma on a Thyroglossal Duct Cyst and we discuss their diagnosis and management. Retrospective analysis of medical records from patients diagnosed and treated with Papillary Carcinoma at Guillermo Almenara Irigoyen National Hospital (Lima, Peru) between 1999-2016. 
The four cases reported had histopathological diagnosis of Papillary Thyroid Carcinoma in Thyroglossal Duct Cyst. Case 1: A 26-yearold woman diagnosed after Sistrunk operation; tumor located in the cyst capsule with invasion of the hyoid bone. Case 2: A 36-year-old woman diagnosed by fine needle aspiration biopsy of the cyst, Sistrunk operation was performed; tumor was located in the cyst capsule. In both postoperative and follow-up ultrasound controls revealed normallity in the thyroid gland, they remained under observation. Eighteen years after surgery, there is no recurrence or thyroid tumor. Case 3: A 35-year-old woman diagnosed after Sistrunk procedure, tumor was located in the capsule with skin and peripheral lymph nodes infiltration. Case 4: A 64-year-old woman diagnosed after Sistrunk operation, tumor was located in the cyst capsule. In both cases, Subtotal Thyroidectomy and Total Thyroidectomy were performed respectively, the histopathological finding was Multinodular Goiter; then they received Radioactive Iodine therapy and suppressive Levothyroxine therapy. Eight years after surgery, there is no recurrence or thyroid tumor. Papillary Thyroid Carcinoma in Thyroglossal Duct Cyst is uncommon, it's difficult to identify at ultrasound, $\mathrm{I}^{131}$ scan, and Fine Needle Aspiration Biopsy. There is controversy not yet been resolved between conservative approach (only Sistrunk's operation) and aggressive approach (plus Total Thyroidectomy, Radioactive Iodine therapy and suppressive Levothyroxine therapy). The treatment to be chosen must be individualized and selective.

\section{Poster 244}

Thyroid Cancer Friday Poster Case Report

\section{MULTIPLE PULMONARY NODULES DETECTED AFTER} RIGHT HEMITHYROIDECTOMY DUE TO PAPILLARY MICROCARCINOMA: A CASE REPORT

J. Kim ${ }^{1}$, E. Song ${ }^{2}$

${ }^{1}$ Surgery, Jeju National University Hospital, Jeju-si, Jeju-do, Korea (the Republic of); ${ }^{2}$ Gyeongsang National University Hospital, Jinju, Korea (the Republic of)

Papillary thyroid microcarcinoma (PTMC) is well-known as a common cancer with a good prognosis and low rate of recurrence and distant metastasis from PTMC is extremely rare. However, when multiple lung nodules with mediastinal lymphadenopathy are detected during the follow-up after hemithyroidectomy, clinician should consider the possibility of metastasis. Here we describe an interesting case which makes clinicians very confusing. A 65 -year-old woman found out a $0.7 \mathrm{~cm}$-sized nodule in right thyroid gland from screening, and it was turned out as papillary thyroid carcinoma after fine needle aspiration. She has a past history of breast cancer without recurrence during 14 years. We performed right hemithyroidectomy without any complication, and she returned to her normal life. After 2 months later, she performed the chest CT scan for other reason, and multiple lung nodules with mediastinal lymphadenopathy were identified with suspicion for metastasis of unknown origin. She had two different malignancies but both of them had very low risk of distant metastasis in current situation. At first, we performed PET/CT scan to differentiate between malignant and benign nodules. In both lung and mediastinal space, multiple and focal FDG uptake (SUVmax 11.2) was observed with more suggestion of metastatic lesions. As a next step, we performed Endobronchial ultrasound-guided transbronchial needle aspirate at subcarinal lymph node for tissue confirmation, and the result was reported as chronic granulomatous inflammation. As a final step, we did Video-assisted thoracoscopic surgery for lung biopsy, and concluded that the lung lesion was sarcoidosis as a final diagnosis. The distribution of pulmonary nodules shown on chest CT scan is very important factor in making an accurate diagnosis. However, it is difficult to distinguish malignancy from benign nodules sometimes, es- pecially patient has a known cancer. In case, very careful assessment and multidisciplinary approach are absolutely necessary. Multiple pulmonary lung nodules in patient with malignancy can be misled as metastatic lesions even though it's papillary thyroid microcarcinoma.

\section{Poster 245}

Thyroid Cancer Friday Poster Case Report

\section{HURTHLE CELL ADENOMA IN A THYROGLOSSAL} CYST - A HISTOLOGICAL SURPRISE

V. Singh ${ }^{1}$, P. Jaiswal ${ }^{2}$

${ }^{1}$ Department of Surgery, Command Hospital, Kolkata, Kolkata, West Bengal, India; ${ }^{2}$ Department of Surgical Oncology, Army Hospital (R\&R), Delhi Cantt, Delhi Cantt, Delhi, India

Thyroglossal duct cyst (TDC) is most common developmental anomaly of the thyroid gland and most common midline neck mass in childhood and adolescence. Malignant tumors in TDC arising either from ectopic thyroid follicular cells or ductal epithelium are reported in approx $1.5 \%$ of surgically treated TDC and are usually diagnosed postoperatively. Being rare, diagnostic and therapeutic recommendations are based on small series and single case reports 38-year-old male, noted to have midline neck mass. Persistence of the mass for a period of a year prompted referral. He denied any symptoms The mass had remained constant in size. No history of radiation exposure or family history of thyroid disease. Physical examination $-2 \times 2 \mathrm{~cm}$ firm, nontender midline neck mass at the level of the criciod. The mass moved with protrusion of tongue and on swallowing as well. USG neck showed an ill defined $0.6 \times 1.0 \times 1.3 \mathrm{cms}$ hypoechoic lesion in the region of Isthmus predominantly on Left side. No vascularity/internal calcification on CDFI. FNAC -Hurthle cell neoplasm.

With a diagnosis of Hurthle cell neoplasm in a Thyroglossal cyst, he underwent exploration. Intraoperatively, a small solid mass was found to lie $2 \mathrm{~cm}$ superior to an atrophic thyroid isthmus. Thyroid gland was otherwise unremarkable. Thyroglossal duct tract was identified and followed till base of tongue. Sistrunk operation was performed. Histopathology report - Hurthle cell Adenoma. Review after 13 months patient is asymptomatic. Thyroglossal ductassociated neoplasms are rare and constitute less than $1 \%$ of thyroid cancer. Definitive surgical management requires excision not only of the cyst but also of the path's tracts and branches. Our patient has no lesion in thyroid gland on pre operative evaluation hence we opted for sistrunk's operation without addressing thyroid gland. The decision was ratified intraoperatively with Per operative finding of normal thyroid gland. Our case represents rare case of of a hurthle cell adenoma arising in thyroglossal cyst duct reported in world literatureWe report a 38 -year-old male patient referred for a midline neck mass diagnosed as an atypical TDC after initial evaluation turned out to be Hurthle cell adenoma on final histology.

\section{Poster 246}

Thyroid Cancer Friday Poster Case Report THYROIDECTOMY UNMASKING TUBULAR NEPHROPATHY

S. Samuels ${ }^{1}$, D. Levy Erez ${ }^{2}$, K. Meyers ${ }^{2}$, A. Bauer ${ }^{1}$

${ }^{1}$ Division of Endocrinology and Diabetes, The Children's Hospital of Philadelphia, Philadelphia, PA; ${ }^{2}$ Division of Nephrology, The

Children's Hospital of Philadelphia, Philadelphia, PA

Hypoparathyroidism is a common complication after thyroidectomy. The use of perioperative intact parathyroid hormone (iPTH) and/or phosphorus measurements can help predict which patients are at increased risk of developing hypocalcemia. Here we describe a patient with papillary thyroid cancer (PTC) whose post-operative 
course was complicated by recalcitrant hypocalcemia, hypomagnesemia, and hypokalemia. A 17-year-old male presented with a thyroid nodule measuring $1.8 \times 1.5 \times 1.7 \mathrm{~cm}$ on US. FNA was consistent with a follicular neoplasm. A total thyroidectomy was performed. Pathology revealed follicular variant PTC. Intraoperative iPTH level at completion of surgery was undetectable, and calcitriol and calcium supplementation were initiated. The patient subsequently developed symptomatic hypocalcemia requiring increasing doses of calcium carbonate and calcitriol. Additional laboratory evaluation showed hypomagnesemia $(0.6 \mathrm{mg} /$ $\mathrm{dL}$, normal range $1.5-2.5 \mathrm{mg} / \mathrm{dL})$ and hypokalemia $(2.6 \mathrm{mmol} / \mathrm{L}$, normal range $3.8-5.4 \mathrm{mmol} / \mathrm{L}$ ). Urine electrolytes showed magnesium and potassium wasting. Further review showed that hypomagnesemia and hypokalemia associated with undetectable iPTH were present prior to surgery. The patient denied any previous symptoms associated with hypocalcemia. However, a sibling and other family members had a history of fatigue and muscle spasms temporally associated with illness. Genetic studies done on the patient were positive for two heterozygous variants in the SLC12A3 gene, pathogenic for Gitelman syndrome (GS). Serum calcium, phosphorus, and iPTH levels are commonly measured after thyroidectomy to surveil for hypoparathyroidism. GS is an autosomal recessive disorder associated with loss of function of the thiazide-sensitive sodium chloride co-transporter. Although hypocalcemia is uncommon in GS, it may be present secondary to hypomagnesemia associated decreased synthesis and secretion of PTH. The presence of persistent post-operative electrolyte abnormalities should prompt a broader differential diagnosis and evaluation for an underlying renal or gastrointestinal disorder. While GS is rare, having baseline laboratory studies allowed for more efficient diagnosis of our patient's underlying nephropathy.

\section{Poster 247}

Thyroid Cancer Friday Poster Case Report

HEAD AND NECK COLLISION TUMOR IN A PATIENT WITH LI-FRAUMENI SYNDROME: A CASE REPORT

J.W. Lee, D. Farwell

Otolaryngology, University of California Davis, Sacramento, CA

Li-Fraumeni Syndrome is a rare genetic disorder characterized by a greatly increased risk of developing multiple types of cancers in both childhood and adulthood. Collision tumors are extremely rare, but when they occur they may complicate the diagnosis and treatment of malignancies. We present the case of a 39-year-old woman with a known history of Li-Fraumeni Syndrome who presented with recurrent cervical lymphadenopathy one year after total thyroidectomy, central neck dissection, and right lateral neck dissection for tall-cell variant papillary thyroid carcinoma. She also had a history of left breast carcinoma which was treated with chemotherapy followed by bilateral mastectomy and left axillary node dissection less than one year prior to her total thyroidectomy. Due to her history of Li-Fraumeni Syndrome, the patient elected to forgo radiation for her breast cancer or radioactive iodine for her thyroid carcinoma given the concern for radiation-induced cancer. Workup of her recurrent cervical lymphadenopathy revealed masses in left level VI, left level IV, and right level IV. Fine needle aspiration of the left level VI and right level IV masses confirmed the diagnosis of recurrent papillary thyroid carcinoma, but biopsy of the left level IV mass showed carcinoma which was not consistent with papillary thyroid carcinoma. She was taken for a revision bilateral neck dissection and right lateral neck dissection. Multiple intraoperative frozen sections were taken from various levels of the neck. These revealed a cervical collision tumor of metastatic papillary thyroid carcinoma and breast carcinoma meeting in the inferior aspect of left level VI. After extensive review of the literature, this appears to be the first case report of a collision tumor in a patient with Li-Fraumeni Syndrome. The diagnosis and treatment of this patient depended largely upon having a high clinical suspicion for a collision tumor. In patients at increased risk for malignancy, collision tumors should be kept on the differential whenever inconsistencies become apparent during the workup of new tumors.

\section{Poster 248}

Thyroid Cancer Friday Poster Case Report AN UNCOMMON CASE OF COWDEN SYNDROME L. Kleess ${ }^{1,2}$, P. Kadambi ${ }^{3}$, K.D. Burman ${ }^{1,2}$

${ }^{1}$ Endocrinology, Washington Hospital Center, Alexandria, VA; ${ }^{2}$ Endocrinology, Georgetown University Hospital, District of Columbia; ${ }^{3}$ Endocrinology, GW University Hospital,

District of Columbia

Cowden Syndrome is a rare autosomal dominant syndrome usually caused by a mutation of the tumor suppressor gene PTEN. It is characterized by hamartomoatous changes and an increased risk of thyroid disease, breast, endometrial, kidney and colorectal cancers. There is a high prevalence of early benign and malignant thyroid disease. Patients require close surveillance.18-year-old $\mathrm{F}$ diagnosed with follicular thyroid cancer at age 11 and Cowden syndrome with genetic testing which revealed a mutation in the PTEN (c.585dupT) gene. At age 11, a thyroid nodule was palpated on routine annual health exam. Thyroid ultrasound revealed a rightsided thyroid nodule measuring $2.3 \times 1.8 \times 3.6 \mathrm{~cm}$ with both solid and cystic components. She underwent FNA and cytology was inconclusive for follicular adenomatoid nodule versus follicular neoplasia. She underwent a right lobectomy at age 12. Pathology revealed $3.5 \mathrm{~cm}$ encapsulated tumor with capsular and vascular invasion. The tumor was confined to the thyroid gland with multiple sections showing microfollicular morphology, suspicious for intraglandular spread. Patient then underwent a completion thyroidectomy. There was no malignancy on pathology of the left thyroid lobe. Using dosimetric analysis, she was treated with $63.2 \mathrm{mCi}$ of I131 under levothyroxine withdrawal. Thyroglobulin and thyroglobulin antibodies were monitored closely and remained undetectable. Follow-up chest imaging and neck ultrasounds revealed no disease recurrence. Patients with Cowden syndrome have a high risk of thyroid cancer, specifically $35-38 \%$. Patients should undergo thyroid/neck ultrasound at the time of diagnosis and annually thereafter with neck examinations and serial thyroglobulin levels. These patients should see a Medical Geneticist for recommendations regarding screening for colon cancer. In this patient, recommendations for screening included annual mammogram and breast MRI beginning at age 30 to 35 ; colonoscopy every 5 years starting at age 35; consideration of annual endometrial biopsy at age 30 ; and biannual renal ultrasound beginning at age 40. Patients with Cowden syndrome have a high risk of cancer, often multifocal and bilateral, and requires close surveillance.

\section{Poster 249}

Thyroid Cancer Friday Poster Case Report GENE EXPRESSION TESTING RESULTING IN EXPEDITED DIAGNOSIS OF MEDULLARY THYROID CARCINOMA IN A THYROID NODULE OF ATYPIA OF UNDETERMINED SIGNIFICANCE

J. Kim ${ }^{1}$, D.S. Cheung ${ }^{1}$, M. Yeh ${ }^{2}$, J. Goldstein ${ }^{3}$

${ }^{1}$ Department of Medicine - Division of Endocrinology, Diabetes, and Metabolism, UCLA David Geffen School of Medicine, Los Angeles, 
CA; ${ }^{2}$ Section of Endocrine Surgery UCLA David Geffen School of Medicine, UCLA, Los Angeles, CA; ${ }^{3}$ Pathology and Laboratory Medicine, UCLA David Geffen School of Medicine, Los Angeles, CA

Medullary thyroid carcinoma (MTC) is an uncommon but generally aggressive thyroid cancer. Early diagnosis can lead to improved outcomes and prognosis. However, MTC can be difficult to diagnose on cytology due to variable appearance. Fine needle aspiration (FNA) has been estimated to detect only $56 \%$ of MTC lesions; lesions were otherwise categorized as benign, indeterminate, or other. There are no strict guidelines on when to perform calcitonin immunohistochemistry staining on FNA samples or when to obtain serum calcitonin or carcinoembryonic antigen (CEA) levels. Gene expression testing with Veracyte Afirma can help diagnosis MTC in cytologically-indeterminate nodules and has recently been shown to have a PPV of $97.9 \%$ and NPV of $99.8 \%$ in this context. A 49-year-old female presented for evaluation of a thyroid nodule. Thyroid ultrasound showed multiple thyroid nodules throughout the gland. The most notable nodule was a $1.2 \times 0.9 \times 0.9 \mathrm{~cm}$ hypoechoic, complex but predominantly solid nodule in the right thyroid gland with internal vascular flow and without calcifications. FNA of the nodule resulted as atypia of undetermined significance (AUS). Calcitonin was not stained on initial cytological evaluation given lack of suspicion for MTC. Subsequently, per institution protocol of obtaining gene expression testing on AUS specimens, Veracyte Afirma testing returned positive for MTC. Due to this result, calcitonin staining was performed and was positive. Serum calcitonin and CEA levels were obtained, both of which were elevated at $102 \mathrm{pg} / \mathrm{ml}$ (ref range $<5 \mathrm{pg} / \mathrm{ml}$ ) and $7.9 \mathrm{ng} / \mathrm{ml}$ (ref range $<3.1 \mathrm{ng} / \mathrm{ml}$ ) respectively. After negative evaluation for pheochromocytoma and hyperparathyroidism, patient underwent total thyroidectomy and central neck dissection. Surgical pathology resulted as $1.1 \mathrm{~cm}$ medullary thyroid carcinoma without thyroidal extension, lymphovascular invasion, or lymph node involvement. MTC is difficult to diagnose on cytology, which can lead to delay in diagnosis and need for reoperation. Gene expression testing can provide crucial management altering information. This is a case where gene expression testing resulted in an expedited diagnosis of medullary thyroid carcinoma in an AUS nodule.

\section{Poster 250 \\ Thyroid Cancer Friday Poster Case Report \\ MINIMALLY INVASIVE FOLLICULAR THYROID CARCINOMA: NOT AN OMINOUS PATHOLOGY BUT SHOULD BE MONITORED CLOSELY}

D.S. Assad, S. Ali, J. Samantray

Endocrinology, Detroit Medical Center, Farmington Hills, MI

Follicular Thyroid Cancer (FTC) accounts for $12 \%$ of thyroid cancers in iodine sufficient areas, peaking in the age of $40-60$ years. FTC is classified into 1 of 3 groups based on the type and extent of invasion. Minimally invasive follicular thyroid cancer (MIFTC), demonstrating only invasion of the capsule of the tumor without vascular invasion, is considered a low risk tumor. Distant metastases occur in 10-15\% of patients with FTC and are rare with MIFTC. We present a case of MIFTC with extensive metastasis seven years later. A 41-year-old woman with history of asthma, hypertension and a left thyroid nodule with suspicious cytology underwent a left hemithyroidectomy in 2008. Final pathology showed MIFTC $9 \mathrm{~cm}$, unifocal, with partial capsular and limited vascular invasion $(<4)$.

She presents seven years later with a headache and behavioral issues. Head CT showed a $7 \mathrm{~cm}$ left frontal mass. She underwent a bifrontal craniotomy, final path showed metastatic thyroid cancer. Follow up labs revealed a thyroglobulin level by LCMS of 1,920 ng/ $\mathrm{ml}$. Restaging scans showed macronodular bilateral pulmonary metastatic disease, mediastinal lymph nodes and lytic bone lesions involving the clavicle, femur and spine.

She underwent a completion thyroidectomy with level 6 lymph node dissection as well as excision of a left clavicular mass and resection of a $7 \mathrm{~cm}$ posterior mediastinal mass. She had a thyroxine withdrawal ablation of metastasis with $208 \mathrm{mCi}$. The post therapy scan showed uptake in the right thyroid bed, left superior thyroid bed, right clavicle, right lower thigh, left femur neck and bilateral lungs. She was started on lenvatinib and zoledronate and is currently maintained on levothyroxine $150 \mathrm{mcg}$ daily. Generally, the prognosis of MIFTC is excellent with many patients undergoing conservative surgical excision and still having excellent long-term outcomes. However, clinical follow up is suggested, often for a prolonged period, because the tumors are slow growing and may recur after many years. Despite great outcomes in MIFTC, clinical monitoring for years is suggested, as patients still have a risk of recurrence and development of metastatic disease to the brain, lungs, and bone.

\section{Poster 251 \\ Thyroid Cancer Friday Poster Case Report \\ A RARE CASE OF ECTOPIC THYROID TISSUE REVEALED AFTER RESECTION OF FOLLICULAR VARIANT OF PAPILLARY THYROID CANCER}

D.S. Assad, W. Taha

Endocrinology, Detroit Medical Center, Farmington Hills, MI

Ectopic thyroid tissue (ETT) is an uncommon entity that may be found anywhere along the line of the obliterated thyroglossal duct. With a prevalence of $0.9 \%$, the most common anatomical sites are the lateral cervical $(21.4 \%)$, along the thyroglossal duct $(21.4 \%)$, mediastinum (17.9\%), lingual (17.9\%) and submandibular $(10.7 \%)$. We report a rare case of ETT anterior to the right strap muscles. A 27-year-old African American female presents with weight loss, palpitations and dysphagia. On exam, blood pressure was $116 / 67$ with a heart rate of 106 beats per minute. Labs showed a TSH of $0.008 \mathrm{uIU} / \mathrm{ml}$ and a FT4 of $2.4 \mathrm{ng} / \mathrm{dl}$. A thyroid ultrasound showed a diffusely enlarged thyroid gland with a one $\mathrm{cm}$ right sided nodule. A 24-hour thyroid iodine uptake was $71 \%$, consistent with Graves' disease. Despite benign FNA results, she elected to have a thyroidectomy due to worsening dysphagia. Pathology results revealed follicular variant of papillary thyroid cancer without invasion. During a follow-up visit, a thyroglobulin (TG) level was elevated at $126.1 \mathrm{ng} / \mathrm{ml}(\mathrm{nl} 1.3-31.8 \mathrm{ng} / \mathrm{ml})$. A whole-body scan showed residual thyroid tissue in the neck region without metastasis. Subsequently, a neck CT showed a soft tissue mass along the anterior aspect of the right strap muscles measuring 1.5 by 1.0 by $1.6 \mathrm{~cm}$. FNA of this mass showed atypical thyroid follicular cells. Final pathology revealed nodular thyroid tissue with marked diffuse hyperplasia and focal lymphocytic thyroiditis. A repeat thyroglobulin level a few months later was normal $(0.75 \mathrm{ng} / \mathrm{ml})$. ETT is most commonly seen in Asian females. Possible explanations for this ectopy are displacement during embryonal development, spread of tissue during surgery on the thyroid gland, and metastasis of a highly differentiated papillary thyroid carcinoma. Diagnosis requires clinical imaging with neck CT or MRI. Developmental defects occurring at an early stage of embryogenesis leads to the formation of ETT. Given its malignant potential, physicians should consider ETT in the differential diagnosis of neck masses. 


\section{Poster 252}

Thyroid Cancer Friday Poster Clinical

\section{OUTCOME PREDICTION WITH THE REVISED AJCC/ UICC STAGING SYSTEM AND ATA GUIDELINES} FOR THYROID CANCER

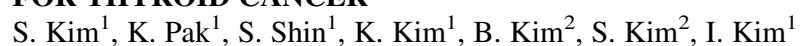

${ }^{1}$ Nuclear medicine, Pusan national university hospital, Busan, Korea (the Republic of); ${ }^{2}$ Yangsan Pusan national university hospital, Yangsan, Korea (the Republic of)

Several staging systems have been developed to predict the risk of mortality in patients with differentiated thyroid cancer (DTC). However, none of them have been shown to be clearly superior to the other. We compared the patient outcome predictability of recently revised staging systems predictability of patient outcome using data from The Cancer Genome Atlas. To set a comparison among American Joint Committee on Cancer (AJCC)/Union for International Cancer Control (UICC) staging 7th, 8th editions, American Thyroid Association guidelines 2009 and 2015, concordance index (c-index), Akaike information criterion (AIC), Bayesian information criterion (BIC), and Brier score were applied to quantify the predictive ability of a survival model, to select the statistical model, and to measure the accuracy of probabilistic predictions. A total of 457 patients with papillary thyroid cancer having a mean age of 45.9 years were included in this study (120 males, 337 females). Among these patients, 43 (9.4\%) experienced recurrence/progression during the follow-up (591.2 \pm 833.5 months). Among the models used, the AJCC/UICC 8th edition, which showed the highest c-index and lowest AIC, BIC, and Brier score, was identified as the best among the models used. AJCC/UICC 8th edition predicted patient outcome more accurately than the other staging systems.

\section{Poster 253}

Thyroid Cancer Friday Poster Clinical

BRAIN METASTASES FROM DIFFERENTIATED THYROID CARCINOMA: CURRENT THERAPIES AND OUTCOMES

C.J. Gomes-Lima ${ }^{1}$, D. Wu ${ }^{1}$, R. Hritani ${ }^{2}$, S. Punukollu ${ }^{2}$, S. Rao ${ }^{2}$, A. Zeymo ${ }^{1}$, H.N. Deeb ${ }^{1}$, M. Mete ${ }^{1}$, D. Van Nostrand ${ }^{1,2}$, J. Jonklaas ${ }^{3}$, K.D. Burman ${ }^{2,3}$

${ }^{1}$ Medicine, MedStar Health Research Institute, Washington, DC;

${ }^{2}$ Medicine, MedStar Washington Hospital Center, Washington, DC;

${ }^{3}$ Medicine, Georgetown University, Washington, DC

The brain is an unusual site for distant metastases of differentiated thyroid carcinoma (DTC), occurring in about $0.15-1.3 \%$ of all cases. Given the rarity of brain metastases (BM) in the course of DTC, little is known about its overall impact on prognosis and how new therapies may affect the course of the disease. The goal of this study was to analyze the current therapies and the outcomes of patients with BM from DTC in follow-up at MedStar Health Institutions. We performed a retrospective analysis of patients with BM from DTC evaluated at MedStar affiliated hospitals $(\mathrm{n}=10)$. The study was approved by MedStar Health Research Institutional Review Board. Among the 24 patients that met our inclusion criteria, $14(58.3 \%)$ were female and $10(41.7 \%)$ were male. The mean age at diagnosis of DTC was $46.3 \pm 15.2$ years. Fifteen patients had papillary TC $(62.5 \%), 7$ patients had follicular TC $(29.2 \%), 1$ patient had poorly differentiated $\mathrm{TC}$, and 1 patient had no report from the thyroidectomy but had confirmed BM from PTC. All but one patient had other sites of distant metastases. BM were diagnosed 0 to 30 years after the initial diagnosis of thyroid cancer, with a mean age at diagnosis of BM of $57.4 \pm 11.2$ years. Only 2 out of 24 patients $(8.3 \%)$ had radioiodine uptake corresponding to the BM. Patients were treated with surgery, stereotatic radiosurgery, and/or external beam radiotherapy, according to the number and sites of the BM. Thirteen patients (54.2\%) were treated with tyrosine kinase inhibitors (TKI), which correlated with better survival (median survival time, 4.3 years vs. 0.6 years, $p<0.05$ ).

The median survival time of the entire group after diagnosis of DTC was 14.3 years. The median survival time after diagnosis of BM was 1.6 years. The brain is an uncommon site of metastases of DTC, associated with a high mortality rate. In our population, the use of TKI improved the survival.

\section{Poster 254}

Thyroid Cancer Friday Poster Clinical

\section{PROMINENT RACIAL DIFFERENCES IN PAPILLARY} THYROID CANCER VARIANTS AND BEHAVIOR

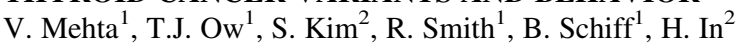
${ }^{1}$ Otorhinolaryngology, Montefiore Medical Center, Bronx, NY; ${ }^{2}$ Surgery, Montefiore Medical Center, Bronx, NY

Recently, the rising incidence of papillary thyroid cancer (PTC) has been recognized to be due to intensified surveillance. This increase in detection has subsequently led to the overtreatment of largely indolent cancers. This trend is especially true in the follicular variant of PTC, which has risen 4 fold over the past two and three decades, and for which a nomenclature change has been proposed to no longer refer to encapsulated fvPTC as a carcinoma. Identification of factors that can help guide surgery preoperatively will be useful to avoid "overtreatment" of these non-aggressive lesions. We sought to investigate whether a patient's race demonstrated predilections for certain PTC variants and tumor behavior. Data was collected from the National Cancer Data Base (NCDB) on all patients ages 18-90 diagnosed with papillary thyroid cancer from 2004 to 2014. Data on demographic and clinical information including histologic variants, grade, lymph node metastases and extrathyroidal extension were collected and analyzed using univariate and multivariate methods. A subgroup analysis was performed on patients with fvPTC. There were 258,973 patients that met the inclusion criteria. When looking at histologic variant by race, Caucasian patients had the classic variant (cv) and fv of PTC in $67.8 \%$ and $29.9 \%$ of cases, respectively. Conversely, black patients had proportionally much higher incidence of fvPTC than cvPTC ( $40 \%$ and $60 \%$, respectively). When looking at factors associated with having fvPTC, black patients had an OR of 1.32 (95\% CI 1.245-1.4) compared to Caucasians. The opposite was observed for Hispanic and Asian patients with an OR of 0.94 and 0.77 , respectively $(\mathrm{p}<0.001)$. African-American patients were also less likely to have extrathyroidal extension (ETE) of fvPTC $(\mathrm{OR}=0.85$, 95\% CI 0.73-0.99) than white patients and had equivalent risk for lymph node metastases (LNM). Hispanic and Asian patients had a higher odds of LNM and ETE than Caucasians $(p<0.001)$. Significant racial differences are seen within the variants of PTC in both incidence and behavior. These disparities should be accounted for in preoperative decision-making, especially in the era of de-escalating therapy.

\section{Poster 255}

Thyroid Cancer Friday Poster Clinical

INTERIM BASELINE CHARACTERISTICS OF PATIENTS FROM THE UNITED STATES ENROLLED IN RIFTOS MKI, A GLOBAL NON-INTERVENTIONAL STUDY ASSESSING THE USE OF MULTIKINASE INHIBITORS (MKIS) IN THE TREATMENT OF PATIENTS WITH ASYMPTOMATIC RADIOACTIVE IODINE-REFRACTORY DIFFERENTIATED THYROID CANCER (RAI-R DTC) M.S. Brose ${ }^{1}$, F. Worden ${ }^{2}$, D.W. Bowles ${ }^{3}$, L.J. Wirth ${ }^{4}$, T.K. Owonikoko ${ }^{5}$, A. Gianoukakis ${ }^{6}$, S.Y. Morita ${ }^{7}$, F. Pitoia ${ }^{8}$, J. Smit ${ }^{9}$, C. Lin $^{10}$, M. Fellous ${ }^{11}$, Y. De Sanctis ${ }^{11}$, I. Sugitani ${ }^{12}$, M. Schlumberger ${ }^{13}$ 
${ }^{1}$ Abramson Cancer Center of the University of Pennsylvania, Philadelphia, PA; ${ }^{2}$ Comprehensive Cancer Center University of Michigan, Ann Arbor, MI; ${ }^{3}$ Division of Medical Oncology University of Colorado, Aurora, CO; ${ }^{4}$ Massachusetts General Hospital, Boston, MA; ${ }^{5}$ Winship Cancer Institute of Emory University, Atlanta, GA; ${ }^{6}$ Harbor-UCLA Medical Center, Torrance, CA; ${ }^{7}$ The Queen's Medical Center, John A. Burns School of Medicine, University of Hawaii, Honolulu, HI; ${ }^{8}$ Universidad de Buenos Aires, Buenos Aires, Argentina; ${ }^{9}$ Radboud University Nijmegen Medical Center, Nijmegen, Netherlands; ${ }^{10}$ National Taiwan University Hospital, Taipei, Taiwan; ${ }^{11}$ Bayer Healthcare Pharmaceuticals, Whippany, NJ; ${ }^{12}$ Nippon Medical School Graduate School of Medicine, Tokyo, Japan; ${ }^{13}$ Gustave Roussy, Villejuif, France

RIFTOS MKI was designed to compare time to symptomatic progression from study entry in asymptomatic patients with RAI-R DTC for whom there was a decision to treat or not to treat with an MKI in the real-life setting. We report interim baseline characteristics for a subgroup of patients from the USA. RIFTOS MKI is a noninterventional study enrolling patients with asymptomatic RAI-R DTC from the USA, Japan, Europe, and the rest of the world. Patients must have radiologic progression, preferably per RECIST 1.1 . The decision to initiate MKIs at study entry was at the discretion of the treating physician. Final analysis will be performed once 700 patients have been enrolled and the last enrolled patient has been followed for 24 months. Of the 74 patients enrolled from the USA, the median duration of observation was 124 days; $53 \%$ were male and the median age was 69 years. Most patients had an Eastern Cooperative Oncology Group performance status of 0 or $1(97 \%)$ and distant metastases at initial visit (69\%); $32 \%$ of patients had prior thyroid disease and $62 \%$ had a family history of cancer. The most frequent histology was papillary (77\%) and 35\% of patients had an intermediate or high initial risk of recurrence according to American Thyroid Association guidelines. The median time from initial diagnosis of DTC to study entry was 7.3 years. RAI refractoriness was mainly due to lack of RAI uptake in at least one lesion (57\%) and median time from RAI-R classification to initial visit was 16 months. The average dose per RAI treatment and median cumulative activity of RAI were $5.5 \mathrm{GBq}$ and $7.8 \mathrm{GBq}$, respectively. The RIFTOS MKI study is the largest non-interventional study in RAI-R DTC. The interim baseline characteristics results here are similar to those previously reported in phase 3 studies and provide insight on real-life treatment practices of RAI-R DTC in the USA. The study is ongoing.

\section{Poster 256}

Thyroid Cancer Friday Poster Clinical

\section{IMPACT OF MOLECULAR TESTING ON SURGICAL MANAGEMENT OF INDETERMINATE THYROID NODULES}

S. Parajuli ${ }^{1}$, R. Jug ${ }^{1}$, S. Ahmadi ${ }^{2}$, X. Jiang ${ }^{1}$

${ }^{1}$ Pathology, Duke University Medical Center, Chapel Hill, NC;

${ }^{2}$ Endocrinology, Duke University Medical Center, Durham, NC

Molecular tests such as the Afirma Gene Expression Classifier (GEC) and mutational panels (such as Thyroseq) have been used to help risk stratify the cytologically-indeterminate thyroid nodule, with the aim to reduce unnecessary surgery.

We reviewed cases of cytologically-indeterminate (Bethesda III/ IV) thyroid nodules with molecular testing from 6/2012 through 8/2016. Demographics, cytology diagnoses, molecular test results, and histopathologic diagnoses were collected.

293 nodules met inclusion criteria; 208 nodules tested with GEC and 85 tested with ThyroSeq. In the GEC cohort, 103/208 (50\%) of nodules yielded "suspicious" and 105/208 (50\%) "benign" results, with an overall resection rate of 84/103 (82\%) in "suspicious" versus $13 / 105(12 \%)$ in "benign" nodules. Of these, 179/208 (86\%) were Bethesda Group III (BIII) and 23/208 (11\%) were Bethesda Group IV (BIV). For BIII nodules, the resection rate was 68/86 (79\%) in GEC "suspicious" versus 7/93 (8\%) in GEC "benign" nodules. For BIV nodules, the resection rates were $12 / 13(92 \%)$ and 6/10 (60\%) in GEC "suspicious" versus "benign" nodules.

In the Thyroseq cohort, 14/85 (16\%) of nodules yielded a "high risk mutation" and 71/85 (84\%) of nodules yielded "no high risk mutation," with overall resection rates of 11/14 (79\%) and 30/71 (42\%), respectively. Of these, 71/85 (84\%) were BIII and 14/85 $(16 \%)$ were BIV. For BIII, the resection rate was $9 / 12(75 \%)$ in "high risk mutation" versus 23/59 (39\%) in "no high risk mutations" nodules. For BIV nodules, the resection rates were $2 / 2(100 \%)$ and $7 / 12(58 \%)$ in "high risk mutation" and "no high risk mutation" nodules, respectively.

Conclusions: For both the GEC and mutational panel, molecular test results seemed to influence rate of resection at our institution. Indeterminate thyroid nodules with positive results on molecular testing undergo resection at a higher rate than those with negative results. Rates of resection for nodules that were GEC "benign" appeared to differ from those that were "negative for high risk mutation" on molecular panel. Rates of resection were higher at our institution for BIV versus BIII nodules, regardless of molecular testing results.

\section{Poster 257}

Thyroid Cancer Friday Poster Clinical

\section{A PHASE II MULTICENTER TRIAL OF THE} MULTITARGETED KINASE INHIBITOR SULFATINIB IN ADVANCED MEDULLARY THYROID CANCER (MTC) AND RADIOIODINE (RAI)-REFRACTORY

\section{DIFFERENTIATED THYROID CANCER (DTC)}

\section{J. Chen ${ }^{1}$, Q. $\mathrm{Ji}^{1}$, J. $\mathrm{Cao}^{2}$, D. Ji ${ }^{2}$, C. $\mathrm{Bai}^{3}$, Y. $\mathrm{Lin}^{3}$}

${ }^{1}$ head and neck surgrey, Fudan university shanghai cancer center, Shanghai, Shanghai, China; ${ }^{2}$ Phase I Unit, Department of Medical Oncology, Fudan university shanghai cancer center, Shanghai, Shanghai, China; ${ }^{3}$ Peking Union Medical College Hospital, Beijing, Beijing, China

Sulfatinib is an oral tyrosine kinase inhibitor targeting Vascular Endothelial Growth Factor Receptor (VEGFR), Fibroblast Growth Factor Receptor 1 (FGFR 1), and Colony Stimulating Factor 1 Receptor (CSF1R). In a proof of concept (PoC) phase II study, sulfatinib showed promising efficacy in patients (pts) with neuroendocrine tumors (NETs). This is an open label, two cohorts phase II study using Simon's two-stage design. In stage I, 15 pts will be enrolled in each cohort (advanced MTC or iodine-refractory DTC), and 10 more pts will be enrolled in a cohort in stage II if at least 2 PR observed in that cohort in stage I. Pts are required to have progressive disease in the past 12 months, but could not have received $>1$ prior antiangiogenesis therapy. Pts are treated with oral sulfatinib $300 \mathrm{mg}$ once daily until disease progression, death, or intolerable toxicity. Primary endpoint is Objective Response Rate (ORR) by investigator per RECIST 1.1. As of Apr 30 2017, the study enrolled 21 pts (MTC: 8, DTC: 13), amongst whom 16 pts were efficacy evaluable. There were a total of 4 confirmed PRs, 1 in the MTC cohort and 3 in the DTC cohort, respectively. The others best response was stable disease (SD). 11 pts had dose interruption due to adverse events (AEs) and 5 pts had dose reduction. A total of eight patients had to discontinue sulfatinib due to disease progression (4 patients), unacceptable toxicities ( 2 patients), or patient decision ( 2 patients). The most commonly reported AEs were proteinuria $71.4 \%$, hypertriglyceridemia $57.1 \%$, hypertension $57.1 \%$, blood bilirubin increased $52.4 \%$, and diarrhea 
$42.9 \%$. No Grade $5 \mathrm{AE}$ was reported by the time of data cut-off. Sulfatinib appears to be well tolerated in the pts with advanced MTC and RAI refractory DTC. Safety profile seems to be consistent to previous report, with mostly manageable AEs. Efficacy is encouraging in both indications. Further investigation is warranted. Clinical trial information: NCT02614495

\section{Poster 258}

Thyroid Cancer Friday Poster Clinical

${ }^{68}$ GA-NOTA-3PRGD2 CAN BE USED AS A PREDICTOR AND EVALUATOR IN RADIOACTIVE IODINE-REFRACTORY DIFFERENTIATED THYROID CANCER TREATED WITH APATINIB

C. Wang, R. Cui, Z. Zhu, Y. Lin

Nuclear Medicine, Peking Union Medical College Hospital, Beijing, China

Tyrosine kinase inhibitors (TKI) targeting vascular endothelial growth factor (VEGF) and its receptor (VEGFR) boomed these years, while the response to TKI varied a lot among different patients. This work was designed to assess the premiere predictive and evaluative value of ${ }^{68} \mathrm{Ga}$-NOTA-3PRGD2 PET/CT in patients with radioactive iodine refractory differentiated thyroid cancer (RAIRDTC) treated by apatinib, a novel TKI targeting VEGFR. After the study protocol was approved by the institute review board of Peking Union Medical College Hospital, ten patients ( 4 men, 6 women, age 27-68 y), with pulmonary metastasis RAIR-DTC were enrolled in this study with written informed consent. All the patients underwent whole-body PET/CT at 45 minutes after intravenous injection of 111 to $148 \mathrm{MBq}$ of 68Ga-NOTA-3PRGD2. 68Ga-NOTA-3PRGD2 PET/ CT was performed within 3 days before apatinib administrated (baseline) and 8 weeks after the apatinib treatment (post-therapy). Two experienced nuclear medicine physicians evaluated the images in blind method. Target lesions were selected and evaluated by RECIST 1.1 criteria. The maximum standardized uptake value (SUVmax) and SUVmean of target lesions and background were measured. The SUVmax and SUVmean of target lesions were 5.80 \pm 3.62 , and $3.39 \pm 2.06$ at baseline, respectively. After 8 weeks' therapy, the SUVmax and SUVmean decrease to $2.05 \pm 0.76$ and $1.42 \pm 0.46$, respectively. Totally 8 patients achieved partial response (PR) at 8 weeks after apatinib treatment, 1 of stable disease (SD), and 1 of progression disease (PD) according to RECIST 1.1 criteria, respectively. In PR group, the mean SUVmax decrease to $2.00 \pm 0.69$ from 5.99 \pm 3.58 . Nevertheless, the SUVmax of PD patient presented limit decrease (from 1.38 to 1.31). Furthermore, there was a significant liner correlation between the SUVmax ${ }_{\text {post-therapy }} / \mathrm{SUVmax}$ baseline and target lesions diameter $(P=0.027)$, as well as the target lesions diameter and SUVmax at baseline $(P=0.027)$. This study indicated the promising value of ${ }^{68} \mathrm{Ga}-\mathrm{NOTA}-3 \mathrm{PRGD} 2$ in predicting and evaluating the response of RAIR-DTC treated with apatinib. Apatinib was effective in high-uptake of ${ }^{68}$ Ga-NOTA-3PRGD2 in RAIR-DTC.

\section{Poster 259-WITHDRAWN}

\section{Poster 260}

Thyroid Cancer Friday Poster Clinical

CAN ULTRASONOGRAPHY PREDICT FINAL STAGE OF PAPILLARY THYROID CARCINOMA BASED ON THE $8^{\text {TH }}$ EDITION OF THE AMERICAN JOINT COMMITTEE ON CANCER STAGING SYSTEM?

C. SEONG, S. MIN, W. PARK

surgery, Kyung Hee University, Seoul, Korea (the Republic of)
Compared with the 7th edition of the American Joint Committee on Cancer (AJCC)/TNM cancer staging system, the $8^{\text {th }}$ edition has many changes, such as raising the age cut off to 55 years of age, removing regional lymph node metastasis and microscopic extrathyroidal extension (ETE) from the T3 disease. It has not been studied whether ultrasonography (USG) can predict the newly updated staging system. The aim of this study identifies that preoperative USG can predict the recently updated staging system. This study is a retrospective single center study from January 2008 to May 2017. Patients who underwent surgery for papillary thyroid carcinoma (PTC) were included. Of these, we excluded the patients less than 55 years of age at diagnosis. Preoperative stage was measured by using USG and the postoperative pathologic reports was recorded as $7^{\text {th }}$ AJCC staging system. Based on these records, we replaced the stage with the revised $8^{\text {th }}$ AJCC staging system. Total 548 PTC patients who underwent thyroid surgery were enrolled. The mean age was $63.0 \pm 6.1$ years of age at diagnosis, and the mean tumor size was $1.2 \pm 0.9 \mathrm{~cm}$. Cervical lymph node metastasis was found 152 patients (26.0\%). Gross and microscopic ETE was $13(2.4 \%)$ and $252(46.0 \%)$, respectively. In preoperative USG stage, stage I was 427 patients $(77.9 \%)$, stage II was 121 patients $(22.1 \%)$. Stage I was $338(70.8 \%)$, stage II was 145 $(26.6 \%)$, stage III was $12(2.2 \%)$, stage IVb was $2(0.4 \%)$ in pathologic stage based on $8^{\text {th }}$ edition of AJCC. The concordance rate between preoperative USG stage and postoperative pathologic stage was $68.1 \%$. The preoperative USG stage of PTC patients 55 years of age or more didn't had a very high concordance with the pathologic stage based on the updated $8^{\text {th }}$ edition of AJCC staging system. Preoperative stage based on USG was generally downstaged than pathologic stage. Therefore, although preoperative ultrasonography is useful for the staging evaluation, it may be necessary to establish a more careful surgical strategy in 55 years or more in patient with PTC.

\section{Poster 261 \\ Thyroid Cancer Friday Poster Clinical}

\section{PRETREATMENT WITH LITHIUM MAY BE ASSOCIATED WITH IMPROVED OVERALL SURVIVAL IN PATIENTS UNDERGOING DOSIMETRY-BASED RADIOACTIVE IODINE TREATMENT FOR METASTATIC DIFFERENTIATED THYROID CANCER}

H. Luo ${ }^{1}$, A. Tobey ${ }^{1}$, S. Auh ${ }^{1}$, C. Cochran ${ }^{1}$, M. Zemskova ${ }^{2}$, C. Gomes Lima $^{3}$, D. Van Nostrand ${ }^{3}$, K.D. Burman ${ }^{3}$, L. Wartofsky ${ }^{3}$, M. Skarulis ${ }^{1}$, E. Kebebew ${ }^{1}$, J. Klubo-Gwiezdzinska ${ }^{1}$ ${ }^{1} \mathrm{NIH}$, Bethesda, MD; ${ }^{2} \mathrm{FDA}$, Bethesda, MD; ${ }^{3}$ Medstar health research institute, Washington, DC

It is known that pretreatment with lithium (Li) maximizes the uptake and residence time of RAI in metastatic lesions, without an established effect on outcome. The goal of our study was to compare the efficacy of three methods of preparation for dosimetry-based radioactive iodine (RAI) therapy of metastatic differentiated thyroid cancer (DTC): recombinant human TSH (rhTSH), thyroid hormone withdrawal (THW), and THW with Lithium pre-treatment for 7 days (THW+Li). We performed a retrospective analysis of outcomes in metastatic DTC patients treated with dosimetry-based RAI activity in our institutions, divided into 3 groups: rhTSH, THW and THW+Li. The primary outcome was overall survival (OS) and progression free survival (PFS). Kaplan-Meier survival analyses were performed to compare time to progression (PFS) and death (OS) between the groups. Cox proportional hazards regression model was performed to study the contribution of age, TNM status and location of distant metastases. The study cohort consisted of 135 patients (82 women, 53 men) treated with average cumulative RAI activity of $550 \pm 393$ 
$\mathrm{mCi}$ and prepared for RAI with either $\operatorname{rhTSH}(\mathrm{n}=42)$, THW $(\mathrm{n}=52)$ or THW+Li $(\mathrm{n}=41)$.

During the follow up of $7.1 \pm 7.6$ years, $52.2 \%$ of patients (70/ $135)$ had disease pogression and $12.6 \%$ of patients $(n=17 / 135)$ died. THW+Li group was characterized by the longest OS $(\mathrm{p}=0.007)$, but after adjusting by the factors affecting the OS, only age (HR 1.05, CI $1.02-1.11, \mathrm{p}=0.005)$ and widespread disease (HR 4.8, CI 1.4-16.0, $\mathrm{p}=0.01$ ) remained statistically significant, while the method of preparation for RAI showed a trend towards improved OS in THW+Li vs rhTSH group (HR 0.193, CI 0.02-1.6, $\mathrm{p}=0.1)$.

There was no difference in PFS between the groups $(\mathrm{p}=0.79)$. The factors independently associated with decreased PFS were older age at diagnosis (HR 1.04, CI 1.02-1.06, p < 0.001) and widespread disease (HR 2.3, CI 1.3-4.2, p=0.005). Although pretreatment with Lithium is associated with improved OS in patients with metastatic DTC, the contribution of age and presence of widespread disease with metastases to the brain, spine, kidney and skin were important factors associated with decreased OS and PFS.

\section{Poster 262 \\ Thyroid Cancer Friday Poster Clinical \\ ROUTINE UTILIZATION OF INTRAOPERATIVE RECURRENT LARYNGEAL NERVE MONITORING DURING THYROID- AND PARATHYROIDECTOMY \\ R. Damle ${ }^{1}$, K.H. Dinh ${ }^{1}$, A.L. Moore ${ }^{2}$, A.C. Larkin ${ }^{1}$, \\ R.M. Quinlan ${ }^{1}$, G.F. Whalen ${ }^{1}$ \\ ${ }^{1}$ Surgery, University of Massachusetts Medical School, Worcester, MA; ${ }^{2}$ Surgery, Brigham and Women's Hospital, Boston, MA}

The added value of intraoperative recurrent laryngeal nerve monitoring (IONM) during routine thyroidectomy and parathyroidectomy is not clear. We reviewed the outcomes of patients undergoing thyroidectomy or parathyroidectomy during a three-year period (20072010) when routine IONM was adopted by a group of high volume endocrine surgeons ( $>75$ procedures per year) who had previously relied only on visual identification of the recurrent laryngeal nerve (RLN). Outcomes evaluated included RLN signal presence before and after dissection, post-operative dysphonia, vocal cord dysmotility on laryngoscopy, and IONM costs. After initial wash-in period for adoption of routine use, a total of 673 patients met inclusion criteria. The majority of patients were female $(76 \%)$. The indications for surgery were thyroid nodule (32\%), thyroid cancer (24\%), multinodular goiter $(19 \%)$, parathyroid adenoma (13\%), Grave's disease $(6.9 \%)$, hyperparathyroidism $(3 \%)$, and other $(<1 \%)$. The majority of patients had a total thyroidectomy $(55 \%), 31 \%$ had thyroid lobectomy, $8 \%$ had thyroid lobectomy, and $18 \%$ had a parathyroidectomy. RLN Signal loss occurred in 27 cases following initial dissection, prompting abandonment of planned contralateral dissection in two cases. Problems with IONM function prompted a change in surgical management in 4 cases, with intraoperative consultation to a second surgeon in 2 cases, and abandonment in contralateral dissection in 2 cases. All changes in operative plan were during planned total thyroidectomies. Laryngoscopy was performed in 21 patients with post-operative dysphonia. Cord dysmotility was identified in in 3 patients, one of which had visual identification of RLN injury during the procedure. IONM had a one-time cost for reusable equipment (monitor) of $\$ 29,536$, and an average disposable cost of $\$ 433$ per case, for an overall direct hospital cost of $\$ 291,409$ for the 673 patients who underwent IONM. IONM did not reliably predict post-operative dysphonia, seldom resulted in a change in the operative plan, and cost nearly $\$ 450$ per patient. The authors advocate for selective use of IONM based on these findings.

\section{Poster 263}

Thyroid Cancer Friday Poster Clinical

\section{EFFECTIVENESS OF RECOMBINANT HUMAN} THYROTROPIN IN THE HIGH-DOSE RADIOIODINE ABLATION THERAPY OF DIFFERENTIATED THYROID CANCER

J. Yoon, Y. An, S. Lee, E. So, J. Lee, Y. Chung

Nuclear Medicine \& Molecular Imaging, Ajou University Medical School, Suwon, Korea (the Republic of)

Recombinant Human TSH (rhTSH) is increasingly used for the ablation and the follow-up of differentiated thyroid cancer after thyroidectomy. In this study, we compared the effectiveness between rhTSH and thyroid hormone withdrawal (THW) in the radioiodine ablation of differentiated thyroid cancer (DTC) in Korean population.

One hundred and fifty-eight DTC patients without distant metastasis after thyroidectomy (male:female $=46: 112$; age $=46 \pm 12$, rhTSH $=64$, THW $=94)$ received the first high-dose radioiodine therapy $(370 \mathrm{MBq} \mathrm{I}-131)$. All patients underwent the laboratory tests for serum stimulated thyroglobulin $(\mathrm{sTg})$ and spot urine iodine concentration on the day of treatment. Six months after treatment, I131 whole body scintigraphy (WBS) and serum sTg measurements were performed to evaluate the effectiveness of therapy. Therapeutic response was divided into 2 categories; complete ablation or incomplete ablation (abnormal WBS and/or $\mathrm{sTg}>2 \mathrm{ng} / \mathrm{mL}$ ).

Overall success rate was $74.1 \%$ (117/158). There were 22 patients with abnormal WBS and 25 patients with abnormal sTg. The success rate was significantly lower in rhTSH group than in THW group $(57.8 \%$ vs. $85.1 \%, \mathrm{p}<0.001, \mathrm{OR}=4.902)$. However, age, sex, tumor size, TSH (137.3 \pm 70.6 vs. $119.2 \pm 54.9, \mathrm{p}=0.126)$ and $\mathrm{sTg}(2.2 \pm 6.4$ vs. $5.2 \pm 13.6, p=0.134)$ on the day of treatment were not significantly different. Urine iodine concentration was higher in rhTSH group than in THW group $(72.1 \pm 77.5$ vs. $41.3 \pm 98.2, \mathrm{p}=0.022)$, while $\mathrm{I} / \mathrm{Cr}$ ratio was not different $(89.4 \pm 155.3$ vs. $74.9 \pm 235.1$, $\mathrm{p}=0.662$ ). On multiple regression analysis, the use of rhTSH was the only independent predictor of incomplete response for the radioiodine ablation in patients with DTC $(\mathrm{p}<0.0001)$. rhTSH was inferior to the conventional THW in the high-dose radioiodine ablation therapy for patients with DTC.

\section{Poster 264}

Thyroid Cancer Friday Poster Clinical TARGETED NEXT GENERATION SEQUENCING AS A TOOL TO HELP DIAGNOSIS OF THYROID LESIONS L. Lebrun, N. D’Haene, M. Le Mercier, N. De Nève, O. Blanchard, I. salmon

Department of Pathology, Erasme University Hospital - Université Libre de Bruxelles (ULB), Brussels, Belgium

Numerous challenges exist regarding the management of patients with thyroid lesions. Firstly, the management of patients with indeterminate cytology still remains problematic. Secondly, the differential diagnosis of thyroid tumors with follicular patterns on surgical specimens (histological diagnosis) is a major problem for the pathologist.

Many biomarkers applied to fine needle aspiration (FNA) or surgical specimens have been proposed to characterize the benign versus malignant nature of thyroid lesions. The recent progress in understanding the molecular pathogenesis of thyroid cancer has identified different potential molecular markers that could help to solve these problems. Gene panel sequencing by NGS is a promising tool.

In the present study, our goal is to share a successful 4 years-old clinical experience using NGS to help diagnosis of thyroid FNA and thyroid histology. The Ion Torrent AmpliSeq cancer panel which 
allows mutation detection in 50 cancer-related genes was prospectively used in clinical practice (BELAC ISO 15189 accredited method). The DNA of 437 patients including 226 (51.7\%) surgical resection and $211(48.3 \%)$ FNA samples from formalin fixed paraffin embedded material and smears was subjected to targeted NGS using the Ion Torrent Personal Genome Machine. Among the 437 tested samples, $223(98.7 \%)$ surgical resection and 204 (96.6\%) FNA samples were successfully sequenced. For malignant lesions, NGS allowed us to detect mutations in $72.5 \%$ of FNA and $56.6 \%$ of histological samples. The most frequent mutations found in cytological and surgical samples concerned BRAF in $22.5 \%$ and $24.9 \%$, RAS in $21.6 \%$ and $18 \%$, TP53 in $0 \%$ and $2.1 \%$, and PTEN in $0 \%$ and $1.7 \%$ respectively. In the surgical specimens, we observed BRAF mutation in 3 adenomatous/FTUMP (follicular tumor with of uncertain malignant potential) (5.2\%) lesions and in 55 malignant lesions $(94.8 \%)$ which only concerned papillary carcinoma. These frequencies detected by NGS were consistent with these reported in literature. Overall, the AmpliSeq cancer panel can be applied in daily practice and provide reliable clinically relevant information for thyroid cancer patients.

\section{Poster 265}

Thyroid Cancer Friday Poster Clinical

\section{INDIVIDUALIZING SURGERY FOR PAPILLARY THYROID CARCINOMA BASED ON DETAILED SONOGRAPHIC ASSESSMENT OF EXTRATHYROIDAL EXTENSION}

E.J. Kuo, F. Zheng, W.J. Thi, K.A. Zanocco, M.J. Livhits, M. Yeh Endocrine Surgery, UCLA, Los Angeles, CA

Based on the 2015 American Thyroid Association guidelines, lobectomy may be sufficient for patients with PTC between 1 to $4 \mathrm{~cm}$ without evidence of ETE or nodal metastasis. Our purpose was to define the role of a detailed sonographic assessment of extrathyroidal extension (ETE) in identifying candidates for lobectomy in papillary thyroid carcinoma (PTC). We retrospectively reviewed patients with Bethesda V or VI cytology who underwent surgery for PTC (January 2016 to May 2017). A detailed sonographic assessment of ETE entailed surgeon-performed evaluation of thyroid capsular distortion, a long interface between tumor and thyroid capsule, irregular or indistinct tumor margins abutting the thyroid capsule, or a long tracheal footprint. Of 139 patients with PTC, 105 patients (75\%) were not candidates for lobectomy: 46 patients for indications unrelated to tumor characteristics (history of hypothyroidism or radiation exposure) and 59 patients for higher-risk tumor features (tumor size $>4 \mathrm{~cm}$, bilateral disease, or cervical metastases). Thirty-six patients $(25 \%)$ were candidates for lobectomy (unilateral tumors $<4 \mathrm{~cm}$ with no evidence of cervical metastases). Total thyroidectomy was performed in 24 patients and was indicated based on final histopathology in 15 patients (ETE, aggressive histology, vascular invasion, or cervical metastasis). Histopathologic ETE was present in 13 of 15 patients and was the only indication for total thyroidectomy in 7 . Twenty-seven of 36 patients had sonographic ETE on detailed assessment. The prevalence of pathologic ETE was 36\%. Positive and negative predictive values for the prediction of ETE based on detailed sonographic assessment were $48 \%$ and $100 \%$, respectively. In comparison to a strategy of routine total thyroidectomy, a detailed sonographic assessment of ETE reduced the rate of potentially avoidable total thyroidectomy from $58.3 \%$ to $33.3 \%$. Patients with PTC who are potential candidates for lobectomy often require total thyroidectomy based on microscopic ETE detected on surgical pathology. A detailed sonographic assessment of ETE can reliably rule out microscopic ETE, reducing the rate of potentially avoidable total thyroidectomy.

\section{Poster 266}

Thyroid Cancer Friday Poster Clinical

THE VALUE OF SALIVARY SCINTIGRAPHY FOR THE DIAGNOSIS OF CHRONIC SIALADENITIS FOLLOWING RADIOIODINE THERAPY

Y. Chung, J. Oh, E. Choi, H.S. Jeong

Department of Nuclear medicine, Incheon St. Mary's Hospital, Incheon, Korea (the Republic of)

To investigate the value of salivary scintigraphy following high-dose radioiodine therapy for the diagnosis of salivary gland dysfunction in thyroid cancer patients. In total, 75 consecutive patients (11 males, 64 females; mean age $50 \pm 13$ years) who underwent post-operative radioiodine therapy $(3,700-5,550 \mathrm{MBq})$ were included. Salivary scintigraphy was performed 6-12 months after radioiodine therapy and the subjective symptoms related to sialadenitis were assessed by questionnaires. The uptake and excretion function of salivary glands were obtained from salivary scintigraphy and correlated with the clinical outcome of chronic sialadenitis. Eighteen of the 75 patients (24\%) complained of symptomatic sialadenitis. Xerostomia (10 patients) was the most common symptom, followed by pain or swelling of salivary glands ( 9 patients) and taste change or loss ( 2 patients). Among these 18 patients with symptomatic sialadenitis, 9 patients (50\%) showed abnormal salivary scintigraphic findings and 9 patients $(50 \%)$ showed normal scintigraphy. The patterns of abnormal finding were impaired excretory function (6 patients) and uptake function (3 patients). Patients with abnormal salivary scintigraphy had significantly higher frequency of chronic sialadenitis compared with patients with normal salivary scintigraphy $(\mathrm{P}=0.001 ; \mathrm{OR}=8.5$; 95\% CI: 2.429-29.748). Salivary scintigraphy has a diagnostic value of chronic sialadenits as a predictor of functional impairment of the salivary glands after high-dose radioiodine therapy.

\section{Poster 267}

Thyroid Cancer Friday Poster Clinical CERVICAL NECK RECURRENCE IN PATIENTS
WITH UNILATERAL PAPILLARY THYROID CANCER
AND IPSILATERAL LATERAL NECK METASTASIS

J. Seong

Endocrine Surgery, Samsung Medical Center, Seoul, Korea (the Republic of)

In patients with papillary thyroid cancer (PTC) and lateral neck metastasis, the incidence of regional lymph node metastasis in ipsilateral central neck has been reported about $80 \%$ and in contralateral central neck has been reported between $8.9 \%$ and $16 \%$.

This study sought to investigate the patterns of regional recurrence in patients who underwent total thyroidectomy and ipsilateral mRND and to determine whether a routine bilateral central compartment neck dissection (CCND) could prevent the chance of recurrence. We retrospectively studied 853 patients who underwent total thyroidectomy with ipsilateral mRND for unilateral PTC and ipsilateral lateral neck metastasis from Jan 1, 1997 to Dec 31, 2015 at Thyroid cancer center of SMC. Of the patients, 574 underwent bilateral CCND and 279 underwent unilateral CCND.

$\mathrm{cNO}$ in bilateral CCND group is $400(69.8 \%)$ and the incidence of contralateral central lymph node metastasis was $20.9 \%$. cN1 in bilateral CCND group is 174(30.3\%) and the incidence of contralateral central lymph node metastasis was $0.9 \%$. The median follow-up period was 76.2 months and rate of recurrence was $8.7 \%$. Median recurrence free survival was 24.7 months. Fifty-five patients $(9.6 \%)$ recurred in bilateral CCND group and nineteen patients $(6.8 \%)$ recurred in unilateral CCND group. Recurrence in contralateral central 
neck is $2.3 \%$ in bilateral CCND group and there was no recurrence in unilateral CCND group. The rate of transient hypoparathyroidism was higher in bilateral CCND group than unilateral CCND group. A rate of pathologic metastasis at contralateral central neck in central $\mathrm{cN} 0$ patients is quite high. (20.9\%). However, an actual recurrence in contralateral central neck is very rare $(0.2 \%)$. The incidence of hypoparathyroidism was a little higher in bilateral CCND group than unilateral CCND group.

Therefore, a prophylactic bilateral CCND might be unnecessary in patients with unilateral PTC and ipsilateral lateral neck metastasis.

\section{Poster 268 \\ Thyroid Cancer Friday Poster Clinical \\ SURGEON-PERFORMED ULTRASOUND IS BENEFICIAL IN THE POSTOPERATIVE SURVEILLANCE OF THYROID CANCER PATIENTS}

L. Muraveika ${ }^{2}$, V. Barratta ${ }^{3}$, P.J. Mazzaglia ${ }^{1}$

${ }^{1}$ Surgery, Rhode Island Hospital, Providence, RI; ${ }^{2}$ Surgery, Rutgers Robert Wood Johnson Medican School, Piscataway Township, NJ;

${ }^{3}$ Surgery, Yale New Haven Medical Center, New Haven, CT

Methods for detecting thyroid cancer recurrence include physical exam, rising thyroglobulin, neck ultrasound and radioiodine uptake (RIU) scanning. This study examines the relative value of surgeon performed ultrasound (SPUS) amongst these options. All patients operated on for thyroid cancer by a single endocrine surgeon between 2006 and 2010 were followed annually with SPUS for at least 5 years. Sensitivity of SPUS for pre-operative detection of lymph node metastases was calculated. In patients who developed recurrent disease the relative value of each surveillance method was calculated. There were 105 patients, 79 women, and 26 men, with a mean age of 48.2 years. Mean primary tumor size was $2.7 \mathrm{~cm}$. 22 patients had suspicion of lateral cervical lymph node metastases by SPUS prior to thyroidectomy, and ultrasound-guided fine needle aspiration biopsy was performed in all of them. 10 biopsies were benign, 11 biopsies were positive for papillary cancer and 1 was non-diagnostic. At the initial operation, 23 patients had a central compartment lymph node dissection and 12 patients had a lateral compartment dissection. Sensitivity of SPUS was $56 \%$ for central compartment metastases, and $100 \%$ for lateral compartment disease. The negative predictive value of a normal surveillance SPUS was $98.7 \%$. Pathology was papillary in 84 , follicular in 19 and medullary in 2.73 had Stage I, 8 Stage II, 22 Stage III, and 2 Stage IV disease. During the follow-up period, recurrent disease was detected in 11 patients, 1 in the central compartment and 10 in the lateral compartment. Mean time to recurrence was 20.7 months. The initial test suggesting disease recurrence was physical exam in 1, rising thyroglobulin in 1 , RIU in 1 and SPUS in 8. After confirmatory biopsy, recurrent disease was treated operatively in 10 and with radio-iodine ablation in 1 . SPUS is highly sensitive for the pre-operative detection of thyroid cancer metastatic to the lateral neck, and was the most sensitive method for detecting recurrent disease. In the post-operative surveillance of thyroid cancer patients, annual ultrasound surveillance by an endocrine surgeon improves care and is more sensitive than RIU, PE, and TG for detecting recurrent disease.

\section{Poster 269}

Thyroid Cancer Friday Poster Clinical

RISK FACTOR ANALYSIS FOR LATERAL LYMPH NODE METASTASIS IN PAPILLARY THYROID MICROCARCINOMA

K. Back, S. Kim, J. Kim, J. Kim, J. Choe

Samsung Medical Center, Deajeon, Korea (the Republic of)
Although there is a controversy over the assertion that lateral lymph node metastasis (LLNM) in papillary thyroid microcarcinoma (PTMC) is associated with regional tumor recurrence and bad prognosis, it is important to identify the prognostic factors for LLNM because it determines the extent of surgery. This study was conducted in retrospective, single center study, and 5081 PTMC patients who underwent total thyroidectomy and central neck dissection and/ or lateral neck dissection(uni- or bilateral) between January 1997 and June 2015 at Samsung medical Center were included. Upper lesion (adjusted odds ratio [OR] 2.859), Male gender (adjusted odds ratio [OR] 1.43), Age under 41 (adjusted [OR] 1.54), Central lymph node metastasis(CLNM) (adjusted [OR] 4.059), Tumor size $>0.5 \mathrm{~cm}$ (adjusted [OR] 1.386) were independent predictors for high prevalence of LLNM. Also, Upper lesion (adjusted odds ratio [OR] 2.174), CLNM (adjusted [OR] 5.06) were statistically significant risk factors for locoregional recurrence (LRR). Based on propensity score analysis using matched data of 2520 patients, Upper lesion (adjusted odds ratio [OR] 2.663), Male gender (adjusted odds ratio [OR] 1.479), Age under 41 (adjusted [OR] 1.389), CLNM (adjusted [OR] 5.045) were independent predictors for high prevalence of LLNM, and CLNM (adjusted [OR] 5.929) was the only independent predictor for LRR. In PTMC patients, the probability of lateral neck metastasis and LRR is higher when the lesion is located in superior pole. Furthermore, we got an unfamiliar age cutoff value, which is age 41 , in this study since the targeted population are different from previous studies. As the lesion is located upwards in preoperative ultrasound images, further evaluation and thorough surgical plan by skilled physicians are needed, especially for patients under age 41 .

\section{Poster 270}

Thyroid Cancer Friday Poster Clinical

EFFICACY OF LOW DOSE SORAFENIB IN ADVANCED DIFFERENTIATED NON-IODINE

\section{CONCENTRATINGTHYROID CANCER}

\section{S. Barai ${ }^{1}$, M. Ora ${ }^{1}$, S. Gambhir ${ }^{2}$}

${ }^{1}$ Nuclear Medicine, Sanjay Gandhi Postgraduate Institute of Medical Sciences, Lucknow, Please Select, India; ${ }^{2}$ NUCLEAR MEDICINE, SGPGIMS, Lucknow, UP, India

Sorafenib is an antivascular endothelial growth factor tyrosine kinase inhibitor. It has been used recently in the treatment of advanced differentiated thyroid cancer (DTC), however many patient cannot tolerate the high incidence of adverse effect. Aim of the study was to evaluate patient tolerability and effectiveness of a low dose sorafenib therapy protocol.

Patients with non-Iodine concentrating advanced DTC having elevated and rising thyroglobulin level while on suppressive dose of lenothyroxine therapy were enrolled in the study. After excluding contraindications for sorafenib therapy patients were put on a low dose sorafenib(400mg/day) as against the standard dose of $800 \mathrm{mg} /$ day. Clinical evaluations were done at 3,21 days and 6 month. Thyroglobulin levels were repeated at $6^{\text {th }}$ month. A patient demonstrating any increase in thyroglobulin level on sorafenib was considered as non-responder.

Fourteen patients ( 9 males and 5 females) were enrolled in this study having a mean age of $47 \pm 13.1$ years (range: $28-63$ years). Histopathological variants were follicular $[n]=10$; hurthle cell variant $\mathrm{n}=2$, tall cell variant $\mathrm{n}=2$. Drug was discontinued in 1 patient due to development of gastro-intestinal bleeding. Two additional patients developed itching which responded well to conservative therapy. Fall in thyroglogulin level was noted in $11(78.5 \%)$ patients with pre-therapy and post-therapy thyroglobulin levels were $1967 \pm 642 \mathrm{ng} / \mathrm{mL}$, and $497 \pm 113 \mathrm{ng} / \mathrm{mL}$ respectively $(\mathrm{p}<0.01)$. However there was no complete response. Stable 
level was noted in one patient, and where an increase in thyroglobulin was noted in only one patient.

Sorafenib is an effective and reasonably well-tolerated treatment in advanced thyroid cancers even in reduced dose of $400 \mathrm{mg} /$ day. This also signifcantly decreases economic burden. A head to head comparison with full dose protocol will help to define the exact place of low dose sorafenib therapy in the management of iodine refractory thyroid cancer.

\section{Poster 271}

Thyroid Cancer Friday Poster Clinical THE ROLE OF PERIOPERATIVE VOCAL CORD
EVALUATION WITH INDIRECT LARYNGOSCOPY

N. Hur, J. Seong, J. Choe, J. Kim, J. Kim

Endocrine Surgery, Samsung Medical Center, Seoul, Korea (the

Republic of)

Voice alteration is one of major postoperative complication after thyroidectomy. It mainly caused by injury to the recurrent laryngeal nerve although some patients experience voice change without nerve damages.

The aim of this study is to evaluate the additive role of perioperative laryngeal exam and to investigate the risk factors of vocal cord palsy. We routinely performed pre- and postoperative laryngeal exam with rigid laryngoscopy for patients who admitted for thyroid surgery. When vocal cord movement abnormality is detected on postoperative one day, we made follow up laryngeal exam within 3 weeks after discharge. If vocal cord palsy or voice change is persistent, we sequentially evaluated vocal cord until postoperative 6 month. Overall 2128 patients from July 2013 to November 2016 were enrolled in this study. The thyroid surgeries were performed by a single experienced surgeon.

Postoperative vocal cord palsies were detected in 162 patients. Clinical characteristics, such as sex, age and body mass index were not different whether having vocal cord palsy or not.

Incidence of postoperative vocal cord movement abnormality was dependent on thyroidectomy extent, lymph node dissection, tumor invasion to recurrent laryngeal nerves, and preoperative vocal cord palsy.

In cases of postoperative bilateral vocal cord palsy, Graves' disease and preoperative vocal cord palsy were statistically significant risk factors.

Clinical courses of vocal cord palsy were noticeable. Most patients recovered within 6 months but 10 patients remained permanent vocal cord paralysis. Surprisingly, three patients had no symptoms even with evident vocal cord palsy. 40 patients out of 969 cases of unilateral total thyroidectomy experienced vocal cord palsy; interestingly 3 patients were found a paralyzed vocal fold in contralateral side.

Among 7 cases of bilateral vocal cord, even though 2 patients underwent tracheostomy, the others recovered with conservative management. Perioperative laryngeal exam enable the surgeon to identify the vocal cord status exactly and to assess its clinical courses. Routine use of perioperative laryngoscopy can be a confident tool to inform patients the risk factors of postoperative vocal cord palsy.

\section{Poster 272}

Thyroid Cancer Friday Poster Clinical

\section{LONG-TERM FOLLOW UP OF NON-INVASIVE} FOLLICULAR THYROID NEOPLASM WITH PAPILLARY-LIKE NUCLEAR FEATURES, A SINGLE CENTER EXPERIENCE

J. Yang ${ }^{1}$, L. Rochon ${ }^{2}$, M. Tamilia ${ }^{2}$

${ }^{1}$ McGill University Health Center, Montreal, Quebec, Canada;

${ }^{2}$ Jewish General Hospital, Montreal, Quebec, Canada
The recent reclassification of encapsulated follicular variant of papillary thyroid cancer (EFVPTC) as non-invasive follicular thyroid neoplasm with papillary-like nuclear features (NIFTP) was adopted in recognition of the benign nature of these lesions. The objective of our study is to determine the prevalence of NIFTP and its long-term outcomes at our center. We retrospectively identified cases diagnosed as EFVPTC between 2007 and 2009 from our thyroid cancer registry. Pathology specimens of all patients with EFVPTC are currently undergoing revision by dedicated thyroid pathologists and reclassification as either NIFTP or non-NIFTP. Data on patient demographics, tumor characteristics and response to primary therapy were collected. Response to primary therapy was assessed after 1 to 3 years using the 2015 ATA guidelines criteria. Patients were followed for evidence of persistent/recurrent disease for at least 5 years. Cases with insufficient data and follow up duration $<5$ years were excluded. Between 2007 and 2009, 118 patients were diagnosed with EFVPTC. So far, 60 cases have undergone reclassification, yielding 10 cases of NIFTP (16.7\%) and 50 cases of non-NIFTP $(83.3 \%)$. Baseline characteristics showed mean age 49 years and $100 \%$ women in the NIFTP group, compared to mean age 47.1 years and $76 \%$ women in the non-NIFTP group. The mean maximal tumor diameter was $2.3 \mathrm{~cm}$ and $2.8 \mathrm{~cm}$ in the NIFTP and non-NIFTP groups respectively. All patients in the NIFTP group were deemed low risk according to the ATA risk stratification system, compared to $88 \%$ in the non-NIFTP group. Response to primary treatment was excellent in $80 \%$ of NIFTP patients with no cases of persistent/recurrent disease after a mean follow up of 8.5 years. In the non-NIFTP group, $12 \%$ of patients were deemed intermediate risk, and $10 \%$ of patients experienced persistent/recurrent disease during a mean follow up of 7.3 years. These preliminary results confirm the favourable outcome of NIFTP lesions. NIFTP is a benign neoplasm of the thyroid gland. Recognizing its indolent behavior could significantly impact patient management. We predict absence of persistent/recurrence disease during long-term follow up in our cohort of patients with NIFTP.

\section{Poster 273}

Thyroid Cancer Friday Poster Clinical

THYROID CANCER RISK AMONGST MEN AND WOMEN PRESENTING FOR CARE AT AN INNER CITY HOSPITAL WITH A PREDOMINATELY MINORITY POPULATION

M. Singh, S. Shakya, R. Trousale

Medicine - Endocrinology, Harlem Hospital Center, New York, NY

Large population studies of thyroid cancer report that women have a higher incidence of thryoid cancer compared to men. Analysis of the SEER-9 registry database (1974-2013) reported 77,276 total new cases of thyroid CA: 58,213 (75.3\%), of the new cases were diagnosed in women and 19,063 (24.7\%) were men. We reviewed thyroid $\mathrm{CA}$ rates between men and women at a inner city hospital with a predominatly minority patient population. Retrospective chart review of patients at Harlem Hospital. The Pathology Department identified patients with a thyroid FNA and/or thyroid surgery from 2006-2015. Gender, thyroid cytology and surgical pathology was collected. Thirty-five total patients were identified with thyroid CA: 28 females (80\%) and 7 males (20\%). This percentage was similar to the previously reported $75 \%$ female and $25 \%$ male.

396 women underwent FNA; 37 had suspicious cytology. 30 of the women with suspicious cytology underwent surgery.15 (50\%) were found to have thyroid CA.

51 men underwent FNA; 8 had a suspicious cytology. 5 men with suspicious cytology underwent surgery. $3(60 \%)$ were found to have thyroid CA. For patients with suspicious cytology, the prevalence of CA was similar between men and women. 
104 women decided to have thyroid surgery even though they had a cytology that was benign or non-diagnostic. 13 women were diagnosed with thyroid CA (12.5\%). In contrast, 9 men decided to have thyroid surgery even though they had a cytology that was benign or non-diagnostic. 4 were diagnosed with thyroid CA (44.5\%). For patients with non-suspicious cytology, the prevalence of thyroid CA was much higher in men compared to women.

The prevalence of thyroid CA is similar in men and women who are found to have a suspicious cytology on FNA. The prevalence of thyroid CA is actually much higher in men with benign or nondiagnostic cytology who undergo surgery compared to women.

In an inner city hospital with a minority population, a higher number of women are diagnosed with thyroid CA compared to men. However, more women undergo evaluation for thryoid nodules including FNA and surgery. Due to the high risk of thyroid CA amongst men with thryoid nodules, physicians should be more vigilant in encouraging men to undergo evaluation.

\section{Poster 274}

\section{Thyroid Cancer Friday Poster Clinical \\ ANTICIPATED RATES OF COMPLETION THYROIDECTOMY DIFFER FROM BETHEDSA CATEGORY 5 TO 6 AMONG CASES MEETING PRE-OPERATIVE ATA CRITERIA FOR LOW RISK CANCER}

A. Flagg, L. Rooper, P. Santhanam, M. Olson, S. Sheth, J. Bishop, J. Mammen

Johns Hopkins University School of Medicine, Baltimore, MD

According to 2015 American Thyroid Association (ATA) guidelines, lobectomy is an option for low-risk thyroid cancers. However, postoperative features can both increase and decrease risk compared to pre-operative findings. Currently the benefit of lobectomy remains controversial especially for Bethesda categories 5 and 6 . We therefore determined the rates at which patients preoperatively identified as low-risk would have required completion thyroidectomy. We evaluated whether additional characteristics improved prediction. Chart review was performed for all thyroid surgeries at Johns Hopkins Hospital since 2005 to identify those who would have been eligible for lobectomy based on pre-operative evaluation. Eligible patients had single nodules $<4 \mathrm{~cm}$ and no evidence of metastasis or extra-thyroidal extension. Surgical pathology was reviewed for high risk variants, significant lymphadenopathy ( $>5 \mathrm{LN}>2 \mathrm{~mm}$ ), ETE and vascular invasion. The rates of additional surgery were calculated stratified by nodule size and Bethesda category. Logistic regression was used to assess pre-operative predictors of completion thyroidectomy. Twenty-three percent of cases reviewed to date (87/380) met ATA criteria for lobectomy. Of these, 21 were microcarcinoma, 47 were Bethesda 6 and $76 \%$ had total thyroidectomy. Among 37 Bethesda 6 cases $1-4 \mathrm{~cm}, 5(13 \%)$ were upstaged intra-operatively, $8(22 \%)$ required completion thyroidectomy, and 24 (65\%) were low-risk, including one NIFTP. In contrast for Bethesda 4 and 5, only 8-9\% required completion thyroidectomy, with 2 NIFTP cases. One micro-carcinomas had a high risk feature (ETE). Age and sex were not significant predictors. Occult microscopic contralateral disease was present in 7 cases, 4 of whom who would not have had additional surgery. Using ATA criteria, overall $26 \%$ of cases thought to be low risk required additional surgery. Although this is an interim analysis with limited power, Bethesda 5 was more similar to 4 than 6 with lower rates of completion thyroidectomy. Occult disease will be missed in $5 \%$ of cases, which might impact recurrence rates. These data are critical to the clinician and patient weighing pre-operative information in order to chose the best surgical option.

\section{Poster 275}

Thyroid Cancer Friday Poster Clinical

THE DIFFICULTY TO COMMUNICATE WITH CHILDREN REGARDING THYROID CANCER SCREENING AFTER RADIATION DISASTER

S. Midorikawa ${ }^{1}$, M. Sekine ${ }^{4}$, M. Ohishi ${ }^{5}$, Y. Hino $^{3}$, M. MUrakami $^{2}$, A. Ohtsuru ${ }^{1}$

${ }^{1}$ Department of Radiation Health Management, Fukushima Medical University, Fukushima, Japan; ${ }^{2}$ Department of Health risk communication, Fukushima Medical University, Fukushima, Japan; ${ }^{3}$ Fukushima Medical University, Fukushima, Japan; ${ }^{4}$ Fukushima Medical University, Fukushma, Japan; ${ }^{5}$ Fukushima Medical University, Fukushima, Japan

Although thyroid cancer screening is generally not recommended in adults due to overdiagnosis issue, thyroid ultrasound examination was lunched in response to anxiety and concerns regarding radiation exposure and thyroid cancer in Fukushima. In the confused situation, it is difficult to communicate with children and their families on the meanings of thyroid cancer screening. In this study, we evaluated the responses of children who participated in class dialogue regarding thyroid cancer screening. We held class dialogue for communication with children in junior high school. We conducted anonymous questionnaires on understanding and enjoyment of children after class dialogue and investigated their interests and what they want to know regarding radiation exposure and thyroid cancer by analyzing their description on the response cards. Before class dialogue, children have hardly had very little understanding of the thyroid examination. After them, questionnaire results (response rate 99\%, $\mathrm{n}=2202$ ) revealed that junior high school students well understood (well understandable and understandable: $92 \%$ ) the medical or scientific topics and enjoyed the class dialogue (very enjoyable and enjoyable: $83 \%$ ). Students who enjoyed classes tend to fully understand, but students who said "not so enjoyable" or "not enjoyable" did not necessarily understand it well. Some students commented in the response card that this class should not be for enjoying, be more serious and important. Most children were interested in thyroid cysts among various medical topics and seemed to want to know their health issue related radiation exposure. There are lots of difficulties and problems psychosocially to conduct thyroid cancer screening after radiation disaster in spite of careful consideration of the screening program. We should continue striving to communicate with residents to provide relief and meanings of thyroid examination for them.

\section{Poster 276}

Thyroid Cancer Friday Poster Clinical

\section{SURVIVAL AFTER TRACHEOLARYNGEAL SURGERY FOR ANAPLASTIC/UNDIFFERENTIATED, INSULAR AND SQUAMOUS CELL THYROID CANCER}

T. Semrad ${ }^{1}$, M. Campbell ${ }^{2}$, T. Keegan ${ }^{2}$, A. Brunson ${ }^{2}$, A. Semrad ${ }^{2}$, D. Farwell ${ }^{2}$

${ }^{1}$ Gene Upshaw Memorial Tahoe Forest Cancer Center, Truckee, CA; ${ }^{2}$ University of California Davis, Sacramento, CA

Although airway invasion is a frequent finding among aggressive variants of thyroid cancer, the role of tracheolaryngeal surgery (TLS) in these patients is not well defined. We evaluated the sociodemographic and tumor characteristics of patients with anaplastic/ undifferentiated, insular, and squamous cell (SC) thyroid cancer to determine predictors of TLS and its impact on survival. Using the California Cancer Registry linked to the California Office of Statewide Health Planning and Development (OSHPD) hospitalization 
data, we identified patients with initial diagnosis of anaplastic/ undifferentiated, insular, and SC thyroid cancer during 1991-2012. We defined TLS from OSHPD using tracheostomy and laryngeal surgery procedure codes. We performed multivariable logistic regression to identify sociodemographic and tumor characteristics associated with TLS and Cox Proportional Hazards regression to determine the impact of TLS on thyroid cancer-specific survival, adjusting for baseline characteristics. Results are presented as adjusted odds ratios (OR) or hazard ratios (HR) and 95\% confidence intervals. Of 532 patients, $33.6 \%$ underwent tracheostomy and/or laryngeal surgery. In unadjusted analysis, TLS was more common among Hispanics, more extensive tumors, and those with SC cancers (50\%). In multivariable analysis, the odds of TLS increased with extrathyroidal extension (OR $1.87 ; 1.07-3.26)$ and was less in insular cancers versus undifferentiated/anaplastic tumors (OR 0.25; $0.09-0.71)$. Survival was worse among those who underwent TLS (HR 2.46; 1.97 - 3.08), were older, with extrathyroidal extension and with greater comorbidity. Survival was better in insular (HR 0.19; $0.11-0.33$ ) and SC (HR 0.48; $0.33-0.69)$ versus anaplastic/undifferentiated histology. Radiation therapy (57.9\%) strongly associated with improved survival (HR 0.53; $0.43-0.67$ ). Among patients with anaplastic/undifferentiated, insular, and SC thyroid cancer, TLS is common, especially with more locally advanced disease and SC tumors. In adjusted analyses, TLS is associated with a worse outcome, reflecting more aggressive disease. The impact of radiation therapy in aggressive thyroid cancer variants warrants further study.

\section{Poster 277}

Thyroid Cancer Friday Poster Clinical

\section{MANAGEMENT OF THYROGLOSSAL DUCT CYST CARCINOMA IN CHILDREN}

K. Prickett ${ }^{2,1}$, B. Patterson ${ }^{3,4}$, A. Alazraki ${ }^{5,6}$, A. Landry ${ }^{2,1}$, L. Rapkin ${ }^{7,8}$

${ }^{1}$ Pediatric Otolaryngology, Children's Healthcare of Atlanta, Atlanta, GA; ${ }^{2}$ Otolaryngology, Emory University, Atlanta, GA; ${ }^{3}$ Endocrinology \& Metabolism, Emory University, Atlanta, GA; ${ }^{4}$ Endocrinology and Metabolism, Children's Healthcare of Atlanta, Atlanta, GA; ${ }^{5}$ Radiology, Emory University, Atlanta, GA; ${ }^{6}$ Radiology, Children's Healthcare of Atlanta, Atlanta, GA; ${ }^{7}$ Pediatrics, University of Pittsburgh Medical Center, Pittsburgh, PA; ${ }^{8}$ Oncology, Children's Hospital of PIttsburgh of UPMC,

Pittsburgh, PA

Thyroglossal duct cyst (TGDC) carcinoma is rare and most frequently diagnosed in adults. We describe treatment of TGDC carcinoma in a series of pediatric patients and propose a measured treatment approach as recommended by current guidelines. Retrospective reviewFour patients between 11-16 years of age were diagnosed with TGDC carcinoma between 2008-2017. Pathology was consistent with follicular carcinoma in 1 and with papillary carcinoma in 3 ( 1 conventional, 1 follicular, and 1 solid type). Three were diagnosed incidentally after routine Sistrunk procedure. One had fine needle aspiration of a concerning submental lesion prior to surgery. All underwent removal of the primary lesion, total thyroidectomy, and $\mathrm{I}^{131}$ ablation. All surgically removed lymph nodes were negative for disease. One patient had coincident malignancy in the thyroid gland, which was suspected based on pre-operative imaging. No patient with normal thyroid imaging was found to have disease in the thyroid. One patient had a subsequent cervical lymphadenectomy based on concerning imaging and mildly elevated thyroglobulin with no malignancy found. No patient had recurrence of disease, with median follow up 48 months (range 3-78 months). TGDC carcinoma in children is rare and has been aggressively treated in the past. Careful preoperative evaluation may allow for more individualized use of total thyroidectomy and $\mathrm{I}^{131}$ ablation after TGDC carcinoma resection, and may help avoid overtreatment in patients without concerning findings.

\section{Poster 278}

Thyroid Cancer Friday Poster Clinical

\section{PHASE 2 STUDY OF PEMBROLIZUMAB COMBINED WITH MULTIMODAL THERAPY IN ANAPLASTIC THYROID CANCER}

A. Chintakuntlawar ${ }^{1}$, J. Yin ${ }^{2}$, R. Foote ${ }^{2}$, J. Kasperbauer ${ }^{2}$, M. Rivera ${ }^{2}$, M. Ryder ${ }^{2}$, J. Morris ${ }^{2}$, K. Bible ${ }^{1}$

${ }^{1}$ Medical Oncology, Mayo clinic, Rochester, MN; ${ }^{2}$ Mayo Clinic, Rochester, MN

Anaplastic thyroid cancer (ATC) is universally fatal and has extremely poor prognosis. Our standard clinical practice is to approach locally advanced ATC with Multimodal Therapy (MMT) which includes concurrent chemotherapy and intensity modulated radiation therapy with or without surgery. Recent data from multiple studies demonstrates that majority of patients succumb to systemic disease after treatment with MMT. We have previously demonstrated that ATC tumor cells have high expression of PD-L1 and it is associated with poor prognosis. MMT could result in increased antigen exposure. Therefore, we postulate incorporating immunotherapy in MMT will improve outcomes by potentially decreasing systemic recurrence. A phase 2 trial with a Simon's optimal two-stage design is proposed in ATC. Patients who are appropriate for surgery followed by chemoradiation (cohort A) or chemoradiation alone (cohort B) are eligible. Pembrolizumab will be administered before surgical resection (cohort A) or initiation of chemo-radiation (cohort B) and continued throughout every 3 weeks for a total of 2 years or until progressive disease, whichever is first. Primary endpoint is overall survival (OS) at 6 months. Secondary endpoint is to determine safety and tolerability of pembrolizumab in combination with MMT. We plan to enroll total of 22 patients. Six month OS based on intention to treat will be reported. Patients with loco-regional recurrence and progression to distant metastasis will be reported. Safety of incorporating pembrolizumab with chemoradiation and surgery will be assessed using all patients who received at least one dose of the study drug. Exploratory analyses include biomarker analysis. We hope to demonstrate that incorporating immunotherapy in a MMT protocol is safe and could improve the 6 month OS compared to historical controls.

\section{Poster 279}

Thyroid Cancer Friday Poster Clinical

QUALITY OF LIFE IN THYROID CANCER SURVIVORS: NEED FOR RECOGNITION OF DISTRESSFUL FACTORS AND ITS AMELIORATION

A. Chekavar, A. Agarwal, S. Mayilvaganan

SGPGIMS, Lucknow, India

The prevalence of thyroid cancer survivors is rising rapidly due to increasing incidence, high survival rates, and a young age at diagnosis. This study was undertaken to assess which aspects of treatment caused maximum anxiety and distress. Divided patients in to 2 groups: group 1 diagnosed patients of differentiated thyroid carcinoma (DTC) awaiting treatment and group 2 DTC survivors (6 months after completion of treatment). Study participants completed a validated $\mathrm{QoL}$ assessment tool that measures four morbidity domains: physical, psychological, social, and spiritual effects. Data were also collected on participant demographics, medical co morbidities, tumour characteristics, and treatment modalities. Unpaired $\mathrm{t}$ 
test and Mann Whitney u test applied for statistical significance. A total of 80 participants (32 group1 and 48 groups 2) with thyroid cancer were recruited. Of these, $61 \%$ were female, with an average age of 38 years, and 32 patients assessed after diagnosis (before treatment) i.e. group 1 and 48 (6 months after completion of treatment). The mean overall QoL, sub-domains for physical, psychological, social, and spiritual well-being was comparable in both group 1 and 2 but survivors had statistically significant worsening of quality of life in change in appearance (p .03), lack of motivation in work (p.04) and time away from work (p.036). Even though there was negative correlation between thyroglobulin, physical, psychological and total quality of life mean rank it was not statistically significant. Distress due to surgery is among 62\% in group 1 (mean rank score 4.7), $58 \%$ in group 2 (mean rank score 4.6), distress due to radioactive iodine scan is $18 \%$ among group 1 cases (mean rank score 0.8 ), $75 \%$ of group 2 cases (mean rank score 4.1 ) distress due to thyroglobulin testing in $25 \%$ group 1 cases (mean rank score 1.12), $60 \%$ of group 2 (mean rank score 3.21). Survivors had worsening of quality of life in individual parameters like distress after completion

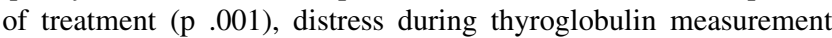
(.003)and whole body radioiodine scan(.03)Thyroxine withdrawal and Radio-active Iodine therapy can result in a decreased quality of life which does not improve 6 months after treatment.

\section{Poster 280}

Thyroid Cancer Friday Poster Clinical

\section{DIAGNOSTIC AND MANAGEMENT CHALLENGES OF THYROID LYMPHOMA IN A TERTIARY REFERRAL CENTRE}

A. Chekavar, A. Agarwal, N. Kumari, S. Nithyanand,

S. Mayilvaganan, A. Verma

SGPGIMS, Lucknow, India

Thyroid lymphoma is a relatively rare disease and often poses a diagnostic and management dilemma. All cases of NHL of thyroid treated at SGPGI between January 1999 and July 2016 were identified. Patient clinical details, investigations and treatment details were collected from case sheet and computerised data entry system were retrospectively reviewed. We analysed 35 cases of thyroid lymphoma from Jan 1999 to July 2016. There were twice as many females as males with mean age of 56.4yrs. Majority were hypothyroid $(\mathrm{n}=13) .24$ patients presented with stridor/dyspnoea and 7 required emergency intubation or tracheostomy. History of rapid enlargement of pre-existing goitre was observed in 16 patients( 10 patientshashimoto's thyroiditis). Fine needle aspiration cytology was lymphoma $(n=20)$, anaplastic carcinoma $(n=3)$, hashimotos thyroiditis $(n=8)$, hashimoto's thyroiditis with suspicion of lymphoma $(n=4)$ or tuberculosis $(\mathrm{n}=1)$. Different subtypes of lymphoma were observed:, DLBCL( $\mathrm{n}=13), \operatorname{DBCL}(\mathrm{N}=3)$ maltoma( $(\mathrm{n}=3), \mathrm{LBC}(\mathrm{n}=8)$, $\operatorname{LCL}(\mathrm{N}=4), \operatorname{BCL}(\mathrm{n}=3)$ and T cell lymphoma $(\mathrm{n}=1)$; those are high grade $(n=25)$, intermediate grade $(n=3)$ and low grade $(n=5)$ lymphoma. In hashimoto's patients final histopathology was High grade lymphoma $(n=8)$, Intermediate grade $(n=1)$ and low grade $(n=3)$. Among all lymphoma patients included treatment modalities used were chemotherapy $(n=22$,$) , Radiotherapy (n=4)$ and surgery $(\mathrm{n}=18) .20$ patients were found operable and operative procedures included lobectomy $(n=1)$, hemithyroidectomy $(n=8)$, total thyroidectomy $(n=9)$ and debulking $(n=2)$. Adjuvant treatment included radiotherapy $(n=4)$, chemotherapy $(\mathrm{RCHOP}(\mathrm{n}=11)$, CHOP $(\mathrm{n}=8)$, methotrexate $(\mathrm{n}=1)$ and COPP $(\mathrm{n}=2)$. Death occurred early in 3 cases (2 -chemotoxicity, 1 - respiratory obstruction). Among chemotherapy 13 were primary chemotherapy and 9 were adjuvant chemotherapy. Outcome survival ranged from 1 day to 84 months (mean-15 months), mainly due to chemotherapy was 14.17 months.
A rapidly enlarging neck mass in the setting of hashimoto's thyroiditis should raise the suspicion of thyroid lymphoma. Fine needle aspiration cytology without any adjunct can be misleading leading to delay in diagnosis and treatment and so core biopsy is recommended.

\section{Poster 281}

Thyroid Cancer Friday Poster Clinical

\section{CHARACTERISTICS AND OUTCOMES OF CHILDREN AND ADOLESCENTS WITH LOW-RISK PAPILLARY THYROID CARCINOMA IN THE SEER DATABASE} P. Mahajan ${ }^{1}$, H. Danysh ${ }^{2}$, D.C. Chelius ${ }^{3}$, R. Venkatramani ${ }^{1}$

${ }^{1}$ Pediatric Hematology-Oncology, Baylor College of Medicine, Houston, TX; ${ }^{2}$ Epidemiology, Baylor College of Medicine, Houston, TX; ${ }^{3}$ Baylor College of Medicine, Houston, TX

Papillary thyroid carcinoma (PTC) accounts for $90 \%$ of childhood thyroid cancer cases. Proper risk classification of children with PTC is important to optimize treatment and avoid long-term side effects. In 2015, the American Thyroid Association (ATA) published guidelines for the management of children with PTC. Data from the Surveillance, Epidemiology, and End Results (SEER) populationbased program were used to describe the characteristics and outcomes of children and adolescents with PTC who were classified as low-risk per the ATA guidelines. Using SEER 18, we analyzed patients less than 20 years of age diagnosed with PTC between 2004 and 2014. TNM staging was used to categorize patients into the ATA pediatric low-risk group, which was defined as T1, T2, T3, or Tx with N0, Nx, or N1a in situ, and no distant metastases. Of 1861 patients with PTC, 887 (48\%) were classified as low-risk. Of these, 748 (84\%) were female, and $717(81 \%)$ were between 15 and 19 years of age at diagnosis. Sixty-one percent of patients were non-Hispanic White, $23 \%$ were Hispanic, and 6\% were non-Hispanic Black. Of the 875 patients who had surgery, $81 \%$ underwent a total thyroidectomy and $11 \%$ had a lobectomy and/or isthmectomy. A total of $409(46 \%)$ patients received irradiation. Eight patients $(0.9 \%)$ developed a second malignancy. Six patients $(0.6 \%)$ died from causes other than their thyroid malignancy. The 5-year disease-specific survival was $100 \%$. Pediatric and adolescent patients with low-risk PTC per ATA classification have an excellent prognosis. A major limitation of this study is the lack of recurrence data in the SEER registry. Nonetheless, favorable outcomes support minimizing therapy for low-risk patients. Prospective studies are warranted to assess radiation avoidance and subsequent outcomes in this group.

\section{Poster 282}

Thyroid Cancer Friday Poster Clinical

\section{CHARACTERISTICS AND OUTCOMES OF CHILDREN AND ADOLESCENTS WITH HIGH-RISK PAPILLARY THYROID CARCINOMA IN THE SEER DATABASE} P. Mahajan ${ }^{1}$, H. Danysh ${ }^{2}$, D.C. Chelius ${ }^{3}$, R. Venkatramani ${ }^{1}$ ${ }^{1}$ Pediatric Hematology-Oncology, Baylor College of Medicine, Houston, TX; ${ }^{2}$ Epidemiology, Baylor College of Medicine, Houston, TX; ${ }^{3}$ Otolaryngology-Head and Neck Surgery, Baylor College of Medicine, Houston, TX

Approximately $10 \%$ to $20 \%$ of pediatric patients with papillary thyroid carcinoma (PTC) will have distant metastases. Proper risk stratification of children with PTC is important to optimize treatment. In 2015, the American Thyroid Association (ATA) published guidelines for the management of children with PTC. Data from the Surveillance, Epidemiology, and End Results (SEER) populationbased database were used to describe the characteristics and outcomes of children and adolescents with PTC who were classified as 
high-risk per the ATA guidelines. Using SEER 18, we analyzed patients less than 20 years of age with PTC diagnosed between 2004 and 2014. TNM staging was used to categorize patients into the ATA pediatric high-risk group, which included patients with either locally invasive tumors (T4) or distant metastatic disease (M1). Of 1861 patients with PTC, $110(6 \%)$ were identified as high-risk. Seventy-six patients had T4 disease and 54 patients had M1 disease; 20 of those patients had both. Eighty (73\%) patients were female. Sixty-two (56\%) patients were between 15 and 19 years old at diagnosis and 39 (35\%) were between 10 and 14 years. Fifty-nine (54\%) patients were non-Hispanic White, $33(30 \%)$ were Hispanic, $6(5 \%)$ were nonHispanic Black, and $12(11 \%)$ were other. Ninety percent of the patients underwent a total thyroidectomy and $6 \%$ underwent a near total thyroidrectomy. A total of $96(87 \%)$ patients received irradiation. Two patients died secondary to their thyroid malignancy within 5 years after diagnosis. Despite having widespread disease, pediatric and adolescent patients with high-risk PTC per ATA classification have an excellent prognosis. Major limitations of this study are the lack of recurrence data and inability to distinguish high-risk nodal disease in the SEER database. Prospective research is warranted to study long-term outcomes and side effects of radiation.

\section{Poster 283}

Iodine Uptake \& Metabolism Friday Poster Clinical PAINFUL NECK EDEMA AFTER RADIOIODINE THERAPY FOR GRAVES' DISEASE

D.S. Rosenthal, S.C. Kumar, A.E. Joseph, A. Lee

Endocrinology, Nassau University Medical Center, East Meadow, NY

Radioactive Iodine (RAI) with $131 \mathrm{I}$ is an effective treatment for Hyperthyroidism. RAI causes radiation-induced injury with necrosis of thyroid follicular cells and associated inflammation. Surrounding soft tissue edema of neck is unusual. A 47 year old woman with Graves' disease was treated initially with methimazole for 19 months and subsequently with RAI $14.5 \mathrm{mCi}$ in August 2015. However, in May 2016 thyroid function tests confirmed treatment failure. She was restarted onto methimazole but developed absolute neutropenia and presented with sepsis, fever and recurrent hyperthyroidism. Her Absolute Neutrophil Count was 220, ESR 98 and CRP 10.9. She received cholestyramine and atenolol for hyperthyroidism as well as antibiotics and Neupogen with resolution of symptoms.

24 hour 123I uptake was $61.1 \%$. She received $30.2 \mathrm{mCi}$ of $131 \mathrm{I}$ on November 29, 2016. 2 days later she presented with acute neck edema and dysphagia. Exam showed tender soft tissue swelling in the anterior neck associated with erythema and warmth. There was no increase in thyroid hormone levels. Sonography showed diffuse stable heterogeneity of both thyroid lobes. CT showed Lobulated and enlarged thyroid with surrounding edema with extension into the antero superior mediastinum and para esophageal region. She had no laryngeal edema or tracheal involvement. Swelling was contained to cutaneous and subcutaneous tissue. She was treated with Ibuprofen and Prednisone $20 \mathrm{mg}$ that was tapered off with complete resolution of symptoms in 4 weeks. Three months later she developed symptoms of hypothyroidism and Levothyroxine was initiated. Soft tissue swelling of neck after RAI therapy of Graves' disease is a rare complication. This is thought to be a radiation dose related phenomenon. Neck pain and tenderness are usually mild and subside spontaneously within two to six weeks. Treatment with nonsteroidal anti-inflammatory drugs is usually sufficient for analgesia, but steroids may be required in severe cases. Soft tissue edema of the neck following RAI thyroid therapy is unusual and clinicians should be aware of this complication and its management.

\section{Poster 284}

Thyroid Cancer Friday Poster Clinical

\section{THYROID LYMPHOMA, ENLARGING ANTERIOR} NECK MASS AND AIRWAY COMPROMISE

D.S. Rosenthal, A.E. Joseph, S.C. Kumar

Endocrinology, Nassau University Medical Center, East Meadow, NY

A rapidly enlarging anterior neck mass poses a diagnostic and management dilemma. Differentiation between Anaplastic Thyroid Carcinoma (ATC) and Thyroid Lymphoma (TL) is imperative as their treatment and prognosis differ. Although Fine Needle Aspiration Biopsy (FNAB) is the diagnostic tool of choice for thyroid masses, core or incisional biopsy may be necessary for subtype confirmation. A 76 yo Female presented with acute respiratory failure. She had dyspnea, dysphonia, dysphagia and a rapidly enlarging anterior neck mass of 3 wks duration. She had no symptoms of thyroid dysfunction and had no history of head or neck radiation. Examination was significant for stridor, tachycardia and respiratory distress. The thyroid was mobile, non-tender and firm. Thyroid was enlarged 5 times normal with the lower border impalpable. Pemberton sign was positive, indicating compression of the jugular veins. She was biochemically euthyroid and had positive antithyroid antibodies. Laryngoscopy demonstrated left vocal cord paresis.

Neck CT revealed $8 \times 7 \times 5.6 \mathrm{~cm}$ heterogeneous mass arising at the level of the thyroid and tracking into the mediastinum. There was significant rightward tracheal deviation and critical narrowing of airway. Chest CT showed $4.6 \times 4.4 \mathrm{~cm}$ lymph node. Acute respiratory failure responded to BiPAP and intravenous Solumedrol. FNAB and flow cytometry of the thyroid mass were suggestive of a lymphoproliferative disorder. She underwent endotracheal stent placement and core biopsy revealing large areas of necrosis and sheets of large atypical cells. Immunohistochemistry staining was positive for B cell markers. Fluorescent In Situ Hybridization was negative for gene rearrangements. These data confirmed diffuse large B cell lymphoma of non-germinal immune phenotype. Thyroid ultrasound and fine needle aspiration cytology, using flow cytometry and immunohistochemistry, remain the main modalities used to confirm the presence of lymphoma. Multimodal therapy with monoclonal antibodies such as Rituximab, chemotherapy with $\mathrm{CHOP}$ and local radiation provides the highest overall survival rates of $91 \%$. A rapidly enlarging neck mass in the setting of Hashimoto's thyroiditis should raise suspicion for thyroid lymphoma.

\section{Poster 285}

Thyroid Hormone Action Friday Poster Basic

LONG-TERM SUSTAINED DELIVERY

OF LIOTHYRONINE (L-T3) WITH SUBDERMAL PRONEURA $^{\text {TM }}$ IMPLANTS

S. Sreedharan, R. Patel

Titan Pharmaceuticals, Inc., South San Francisco, CA

Tetraiodothyronine (T4) and triiodothyronine (T3) are important for development and normal function of many organ systems. T3 is the active hormone, largely produced in the body by peripheral conversion of T4. While treatment with daily oral levothyroxine (L-T4) alone is effective in most hypothyroid patients, about $15 \%$ report feeling not adequately treated and are often prescribed a combination of liothyronine (L-T3) with L-T4. Due to its short half-life and narrow therapeutic window, L-T3 alone is infrequently prescribed because wide peak-to-trough variations in 
serum T3 levels from oral dosing can result in adverse effects. Therapeutic substitution of L-T3 for L-T4 has been evaluated in hypothyroid subjects in a blinded crossover study with a multiple daily dosing scheme, and comparison of L-T3 and L-T4 doses that produce equivalent steady-state thyroid-stimulating hormone (TSH) levels additionally resulted in L-T3-mediated weight loss and positive changes to lipid profiles without appreciable sideeffects (Celi et al., 2010, 2011). A sustained-release formulation of L-T3 could avoid issues of noncompliance to a multiple daily dosing regimen. The ProNeura implant platform for sustained, continuous, non-fluctuating release of medications following subdermal implantation has been validated in multiple clinical trials of Probuphine ${ }^{\circledR}$, the first commercial product approved for maintenance treatment of opioid dependence that is designed to release continuous non-fluctuating serum levels of buprenorphine for 6 months. ProNeura-based L-T3 implants were formulated and tested for release characteristics in normal and thyroidectomized/ parathyroidectomized Sprague Dawley rats, and beagle dogs. Pharmacokinetics of T3, T4, and TSH levels were assessed for varying implant doses in serum samples taken pre- and postimplantation. Results showed these L-T3 implants continuously released non-fluctuating levels of T3 dose-dependently for over 6 months in both rats and dogs. This successful demonstration of a long-term sustained-release formulation for L-T3 provides initial data that support further studies to evaluate the potential therapeutic substitution of, or combination with L-T4 for the treatment of hypothyroidism.

\section{Poster 286}

Thyroid Hormone Action Friday Poster Basic

IN VIVO EVIDENCES TOWARDS A PRO-INFLAMMATORY IMMUNE RESPONSE GENERATED BY TRIIODOTHYRONINE-STIMULATED DENDRITIC CELLS (DCS)

V.A. Alamino ${ }^{1}$, M. Montesinos ${ }^{1}$, F. Soler ${ }^{1}$, L. Giusiano ${ }^{1}$, N. Gigena ${ }^{1}$, G.A. Rabinovich ${ }^{2}$, C. Pellizas ${ }^{1}$

${ }^{1}$ Centro de investigaciones en Bioquímica Clínica e Inmunología (CIBICI-CONICET). Departamento de Bioquímica clínica, Facultad de Ciencias Químicas, Universidad Nacional de Córdoba (UNC), Córdoba, Córdoba, Argentina; ${ }^{2}$ Laboratorio de Inmunopatología, Instituto de Biología y Medicina Experimental (IBYME-CONICET). Departamento de Química Biológica, Facultad de Ciencias Exactas y Naturales, Universidad de Buenos Aires, Buenos Aires, Argentina

The immune and endocrine systems are in constant communication to maintain homeostasis and orchestrate coordinated responses to imbalances and pathologies. In this sense, we reported that mice DCs, the main antigen-presenting cells, express thyroid hormone receptor $\beta 1$ and that physiological levels of $\mathrm{T} 3$ stimulate the maturation of DCs and their ability to direct a Th1-type immune response in vitro, as well as antitumoral effects in an in vivo model of melanoma. Furthermore, in vitro, T3 stimulated DC's production of the Th17-skewing cytokines and reduced the expression of programmed death-ligand 1 and 2 (PD-L1 and PD-L2). In addition, T3-matured DCs increased the production of IL-17 and decreased the frequency of regulatory $\mathrm{T}$ (Treg) cells in allogenic splenocytes. Regarding the in vitro findings, the aim of this study was to analyze the adaptive immune response induced by T3stimulated DCs in vivo. For this purpose, mice bone marrow derived DCs treated with ovalbumin (OVA) and $5 \mathrm{nM}$ T3 (OVA+T3DCs) for $18 \mathrm{~h}$, were injected i.v. into OTII transgenic mice, which own an increase in CD4/CD8 peripheral $\mathrm{T}$ cell ratio that primarily recognize OVA peptide $\left(\mathrm{OVA}_{323}\right)$ when presented by the $\mathrm{MHC}$ class
II molecule. One week later, splenocytes were restimulated ex vivo with $\mathrm{OVA}_{323}$, and proliferation, IL-17 and IFN- $\gamma$ releases, and $\mathrm{CD} 4{ }^{+} \mathrm{CD} 25^{+} \mathrm{FoxP}^{+}$(Tregs) and programmed death-1 protein (PD$1)^{+}$cells were determined 4 days later by MTT assay, ELISA and FACS, respectively. Statistics: ANOVA/SNK test. We observed an increase in proliferation and IL-17 and IFN- $\gamma$ production in splenocytes from OVA+T3-DCs vs OVA-DCs treated mice. In contrast, spleen cells from OVA+T3-DCs group exhibited a reduction of Treg population and expression of the inhibitory molecule PD-1, compared to those from OVA+DCs-treated mice. These results reinforce the critical role of $\mathrm{T} 3$ in the regulation and maintenance of immune homeostasis since T3-exposed DCs favor the promotion of adaptive immunity towards a pro-inflammatory profile in vivo. Our findings have therapeutic implications for the manipulation of the immunogenic potential of DCs to positively regulate the development of protective immunity or negatively control the generation of autoimmune diseases.

\section{Poster 287}

Thyroid Cancer Friday Poster Basic

\section{TUMOR-STROMA INTERACTIONS REPROGRAM METABOLISM IN HUMAN ANAPLASTIC THYROID CANCER CELLS}

L. Fozzatti, V. Alamino, L. Giusiano, C. Stempin, A. Donadio, C. Pellizas CIBICI-CONICET, Córdoba, Argentina

Thyroid cancer is the most common endocrine malignancy, with rising incidence. Anaplastic thyroid cancer (ATC) is one of the most aggressive tumors. Characterized by its undifferentiated cells, it spreads quickly to distant organs and does not respond well to therapy. It is well known that genetic abnormalities in oncogenes and/or tumor suppressor genes promote tumorigenesis. Emerging evidences, however, have shown that tumor stroma has a crucial influence on cancer development and progression as well. Dysregulated metabolism within the tumor stroma is critical to the process of tumorigenesis, however studies analyzing the role of multicompartment metabolism in ATC are lackingTo investigate whether the interaction of ATC cells-fibroblasts (main component of tumor stroma) reprograms their cellular metabolism, we used an in vitro ATC cell (8505c and KTC-2 cells)-fibroblast (MRC-5 cells) transwell co-culture system and measured a variety of metabolic parameters by flow cytometry, RT-qPCR, ELISA and Western blot analysis. We showed that during co-cultures, fibroblasts increased reactive oxygen species (ROS) production, the transcript and secretion of the inflammatory cytokine IL6, the mRNA expression of two glycolytic enzymes: lactate dehydrogenase A (LDHA) and enolase 1 (ENO1) and the mRNA and protein levels of glucose transporter 1 (GLUT1). Conversely, co-cultured ATC cells showed reduction in GLUT1, ENO1 and LDHA expression. High ROS levels induce oxidative stress that may trigger the activation of hypoxiainducible factor $1 \alpha(\mathrm{HIF}-1 \alpha)$, leading to inflammation and glycolysis. Therefore, we analyzed its mRNA expression in the cocultures. Interestingly, we registered an increase of mRNA levels of HIF- $1 \alpha$ in fibroblasts co-cultured with thyroid cells. Accordingly, we observed a significant rise of this transcription factor in the tumor thyroid cells co-cultured with fibroblasts. Our findings provide in vitro evidences of a reprogrammed metabolism by stromal-thyroid tumor cells interactions, suggesting their participation in ATC development and progression. An improved understanding of the molecular basis of tumor-stroma communications may enable identification and targeting of tumorsupportive mechanisms. 


\section{Poster 288}

Thyroid Hormone Metabolism \& Regulation Friday Poster Basic

\section{GENDER-SPECIFIC GENETIC INFLUENCE ON TSH} AND FT4 CONCENTRATIONS AND INTERACTIONS BETWEEN MEASUREMENTS: KNHANES 2013-2015

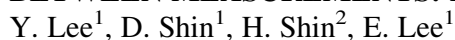

${ }^{1}$ Division of Endocrinology and Metabolism, Department of Internal Medicine, Yonsei University College of Medicine, Seoul, Kora, Seoul, Korea (the Republic of); ${ }^{2}$ Biostatistics Collaboration Unit, Medical Research Center, Yonsei University College of Medicine, Seoul, Korea (the Republic of)

Despite wide range of genetic influences on TSH and fT4 levels have been reported, gender-differences or interaction between traits were not well known. This is a cross-sectional study based on the data from the Korea National Health and Nutrition Examination Surveys (KNHANES) conducted from 2013 to 2015 . We assessed heritability and genotype-by-sex or environment-by-sex interaction with various environmental co-determinants in 2250 subjects without positive TPO antibody. Narrow-sense heritability for TSH and fT4 were 54\% (41\% in males and $75 \%$ in females) and 56\% (62\% in males and 52\% in females), respectively. Genetic variance was significantly higher in females for TSH levels, indicating that genes account for more variance of TSH levels in females than in males. Environmental variance was significantly higher in males for TSH levels. These genderdifferences were insignificant in selected euthyroid subgroup. Genderspecific genetic or environmental effects were not significant for fT4 levels. TSH and fT4 levels showed negative genetic correlation in females $(\rho \mathrm{g}=-0.347, \mathrm{P}=0.040)$ after regressing out the influences of confirmed environmental covariates, ant it was still evident in female euthyroid subgroup, but not in males $(\rho \mathrm{g}=-0.160, \mathrm{P}=0.391)$. TSH levels were positively related to urine iodine-to-creatinine ratio (UICR) and negatively with age and smoking, but not with gender or BMI. FT4 levels were positively related to menopause in female and negatively related to age, female gender, BMI, and UICR, but not with smoking. Our findings confirm the strong familial association of TSH and fT4 levels and its gender differences with female-specific pleiotropy in Korean population with negative TPO antibody.

\section{Poster 289 \\ Thyroid Hormone Metabolism \& Regulation Friday Poster Clinical}

\section{THYROID FUNCTION IS ASSOCIATED WITH BODY} MASS INDEX AND FASTING PLASMA GLUCOSE IN THAI EUTHYROID POPULATION

A. Lertrit, L. Chailurkit, B. Ongphiphadhanakul, W. Aekplakorn, C. Sriphrapradang

Faculty of Medicine Ramathibodi Hospital, Mahidol University, Bangkok, Thailand

The associations between overt thyroid dysfunction and body weight are well established. Several population-based studies have shown that serum thyroid hormone and TSH levels, even within the normal reference range, are associated with body weight. In addition, these thyroid functions are related with insulin resistance and plasma glucose concentrations. However, the data are inconsistent among various populations. Therefore, we investigated whether thyroid functions and thyroid autoimmunity would affect metabolic parameters in Thai euthyroid population. This cross-sectional study is part of the fourth Thai National Health Examination Survey 2009. A total of 2,631 participants were measured for $\mathrm{TSH}, \mathrm{FT}_{4}$, anti-thyroperoxidase (antiTPO) and anti-thyroglobulin (anti-Tg). Euthyroidism was defined by
TSH 0.27-4.20 mIU/L and $\mathrm{FT}_{4}$ 0.93-1.71 ng/dL. A total of 2,242 euthyroid participants were included. Fifty-one percent were female. Mean age, fasting plasma glucose (FPG), body mass index (BMI) were $55 \pm 21$ years, $93 \pm 29 \mathrm{mg} / \mathrm{dL}$ and $23.4 \pm 4.6 \mathrm{~kg} / \mathrm{m}^{2}$, respectively. Multivariate regression analysis after age and sex adjustment showed a negative association of serum FT4 with BMI $(\beta=-0.07, \mathrm{p}=0.001)$ and the relationship was still significant after subjects with positive antiTPO were excluded $(\beta=-0.068, \mathrm{p}=0.003)$. In contrast, serum TSH was positively associated with BMI $(\beta=0.052, \mathrm{p}=0.012)$. Individuals who had TSH levels in the highest quartile had significantly higher BMI than those in the lowest quartile of TSH (mean BMI, $23.7 \pm 4.9$ vs $22.9 \pm 4.3 \mathrm{~kg} / \mathrm{m}^{2}$ ). Moreover, serum FT4 was positively associated with FPG levels $(\beta=0.097, \mathrm{p}<0.001)$. In euthyroid subjects, small variations in serum TSH and $\mathrm{FT}_{4}$ may contribute to the differences in metabolic indexes such as BMI and FPG. These findings suggest that differences in thyroid function within the population reference range could influence the prevalence of obesity and diabetes mellitus.

\section{Poster 290}

Autoimmunity Friday Poster Case Report

\section{A SWINGING PENDULUM: HASHIMOTO'S} TO GRAVE'S DISEASE FOLLOWING R-CHOP

K.N. Vu, T. Church, T.D. Hoang, H. Baloch, M. Shakir

Endocrinology, Walter Reed National Military medical Center, Bethesda, MD

Radiation-induced thyroid dysfunction following oncologic treatment is not uncommon, however limited literature data has been found on patients that underwent chemotherapy only. We present a case of newly diagnosis Grave's thyrotoxicosis following a successful R-CHOP therapy in a patient with concurrent abdominal and thyroid diffuse large B-cell lymphoma (DLBCL). A 66-year-old female was initially diagnosed with concurrent abdominal and thyroid DLBCL two years prior and underwent six cycles of R-CHOP. Initial TSH $4.5 \mathrm{mIU} / \mathrm{mL}$; FT4 $1.2 \mathrm{ng} / \mathrm{dL}$; and TPOAb $18290 \mathrm{IU} / \mathrm{ml}$. Following chemotherapy, PET CT showed resolution of FDG-avid thyroid nodule along with normalized TFT and no evidence of thyroid mass on ultrasound. Repeat thyroid FNA was negative. During follow-up visit 2 years later, patient reported significant weight loss and fatigue. Repeat testing revealed TSH $0.005 \mathrm{mIU} / \mathrm{mL}$, FT4 $6.73 \mathrm{ng} / \mathrm{dL}$ and TSI 535\%. Graves' disease was diagnosed and she was started on methimazole followed by I-131 therapy. The risk of developing thyroid abnormality 20 years after neck irradiation is approximately 52 percent $^{(1)}$. It is unknown whether thyroid function is being affected by R-CHOP therapy. A few case reports on diminishing thyroid autoantibody levels in Hashimoto's thyroiditis after rituximab monotherapy for thyroid MALT lymphoma were found ${ }^{(2)}$. However, the finding of Grave's disease following chemotherapy for DLBCL is limited in our literature research. This unique case of Grave's disease following R-CHOP treatment in patients with known Hashimoto's and thyroid lymphoma is one of the first to be reported in the literature. Continued monitoring for thyroid dysfunction in this subset of population is necessary.

\section{Poster 291}

Thyroid Hormone Metabolism \& Regulation Friday Poster Clinical

\section{A NOVEL MOUSE PARADIGM TO MEASURE} BIOAVAILABILITY OF FREE T3 AND T4 SERUM LEVELS IN OTC THYROID HEALTH SUPPLEMENTS K. Nadolsky ${ }^{2}$, L. Pangaro ${ }^{1}$, B. Reinhardt ${ }^{3}$, I. Robbins ${ }^{2}$, K.N. Vu ${ }^{2}$, D. Brooks ${ }^{3}$, H. Burch ${ }^{2}$ 
${ }^{1}$ Department of Medicine, Uniformed Services University, Bethesda, MD; ${ }^{2}$ Department of Endocrinology, Diabetes \& Metabolism, Walter Reed National Military Medical Center, Bethesda, MD; ${ }^{3}$ Department of Research Programs, Walter Reed National Military Medical Center, Bethesda, MD

Among those attempting to lose weight, nearly $34 \%$ have reported using dietary supplements to aid weight loss. Case reports describe thyrotoxicosis in individuals associated with their use. Conflicting results on whether dietary supplements contain detectable amounts of thyroxine and triiodothyronine have been reported. We created a study to analyze Free T3 (fT3) and Free T4 (fT4) bioavailability in C57BL6 Mice serum levels via radioimmunoassay after three days of daily gavage feeding with dietary supplements. Six groups of six mice each were compared: 1 negative control, 2 positive controls with liothyronine and levothyroxine, and three supplements ("No. 1", "No. 2", and "No. 3") were administered to the last 3 groups via daily gavage. Serum fT3 and fT4 was obtained four hours after gavage feeding on the third day at sacrifice. Descriptive statistics are reported as the medians and the range. Differences in T3 and T4 levels between the groups were assessed using the Wilcoxon rank sum test between the negative control and the experimental supplement groups, with alpha controlled via Bonferroni correction for three comparisons. Negative controls had little measurable fT3 $(4.60 \mathrm{pg} / \mathrm{ml})$ or fT4 $(.31 \mathrm{ng} / \mathrm{dl})$, whereas medicated controls had elevated fT3 $(9.91 \mathrm{pg} /$ $\mathrm{ml})$ and fT4 $(3.78 \mathrm{ng} / \mathrm{dl})$. Although both No. $1(6.28 \mathrm{pg} / \mathrm{ml})$ and No. $2(5.45 \mathrm{pg} / \mathrm{ml})$ had numerically elevated fT3 levels, neither was statistically different when compared to the negative control (No. 1: $p=.69$, No. $2: \mathrm{p}=.94)$. No. 2 had statistically elevated fT4 $(1.03 \mathrm{ng} / \mathrm{dl})$ when compared to the negative controls $(p=.01)$ though below the medicated control fT4 levels $(p<.01)$. This study indicates that thyroid hormones in dietary thyroid supplements are bioavailable when tested in a novel animal paradigm. Mice consuming both No. 1 and No. 2 have numerically increased levels of fT3, and mice consuming No. 2 have statistically increased levels of fT4. The obtainability of these supplements with bioavailable thyroid hormone may pose a risk of thyrotoxicosis to consumers, who may be unaware of the hazard. This study further supports the possibility that thyroid-containing supplements may impact a patient's health.

\section{Poster 292 \\ Thyroid Hormone Metabolism \& Regulation Friday Poster Clinical}

\section{USE OF SERUM PARATHYROID HORMONE (PTH) AND IONIZED CALCIUM (ICA) TREND AS A PREDICTOR OF EARLY NEXT DAY DISCHARGE AFTER TOTAL THYROIDECTOMY}

S. Goh, A. Rao, R. Singaporewalla

Surgery, National Health Group, Singapore, Singapore

Increasing demand and waiting times for acute hospital beds dictate a need to reduce hospitalization stay after elective surgery. Traditionally, patients undergoing total thyroidectomy in Singapore are hospitalized for 48-72 hours to monitor for symptoms of hypocalcemia. Our study explores the predictive value of serum intact PTH (iPTH) and ionized calcium (iCa) levels in determining early discharge on POD1. A prospective study conducted in Khoo Teck Puat Hospital, Singapore between Dec 16-Mar 17 included subjects undergoing total or completion total thyroidectomy. Subjects who underwent cervical nodal dissection or redo thyroid surgery were excluded. Samples of iPTH and iCa immediate post surgery and POD1 were evaluated along with symptoms of hypocalcemia. Cri- teria for early safe discharge on POD1 were asymptomatic patients with iPTH above $0.9 \mathrm{pmol} / \mathrm{L}$ or iCa levels above $0.9 \mathrm{mmol} / \mathrm{L}$. Subjects with vitamin $\mathrm{D}$ deficiency received calcium and vitamin $\mathrm{D}$ supplementation prior to surgery. All patients were reviewed on POD3 in the outpatient clinic to remove drains and review symptoms. Of 30 eligible subjects, majority $(86.7 \%)$ were females and mean age was 41.7 years old. Indications for surgery were multinodular goiter $(63.3 \%)$, malignancy $(13.3 \%)$, Graves $(16.7 \%)$ and Hashimoto's (6.7\%). Mean immediate and POD1 iPTH levels were 3.29 and $3.25 \mathrm{pmol} / \mathrm{L}$ respectively (normal $=1.6-6.9 \mathrm{pmol} / \mathrm{l}$ ). Mean immediate and POD1 iCa levels were 1.10 and $1.01 \mathrm{mmol} / \mathrm{L}$ respectively (normal $=1.13-1.32 \mathrm{mmol} / \mathrm{L}$ ). There was a significant downward trend between iCa levels immediate post-op and POD1; $\beta=-0.76$, pvalue $<0.001$. However no patients experienced any symptoms of hypocalcemia above the cut-off point of $0.9 \mathrm{mmol} / \mathrm{L} .24$ of 30 patients went home POD1 after meeting the discharge criteria. 5 patients met the discharge criteria but went home on POD2 due to caregiver issues. 1 patient requested to stay till neck drain was removed. There were no unscheduled readmissions after early discharge. Majority of patients can be safely discharged the next morning after total thyroidectomy if discharge criteria are met. iPTH and $\mathrm{iCa}$ are independent predictors of hypocalcemia and symptomatic hypocalcemia did not occur above the cut-off values in this study.

\section{Poster 293}

Thyroid Nodules \& Goiter Friday Poster Clinical

THE LATERAL "BACKDOOR" APPROACH TO OPEN THYROID SURGERY: A COMPARATIVE STUDY

S. Goh, R. Singaporewalla, A. Rao

Surgery, National Health Group, Singapore, Singapore

The traditionally taught technique for conventional open thyroidectomy is via a mid-line splitting of the strap muscles following a skin crease neck incision - mid-line approach (MA). The lateral "backdoor" approach (LA) uses the same central neck incision but approaches the thyroid gland between the anterior border of sternocleidomastoid (SCM) and strap muscles. This technique is usually reserved for re-do thyroid surgery. We compared the results of these two approaches in patients undergoing conventional thyroidectomy for the first time. A casecontrol study was performed on 90 patients undergoing conventional open thyroidectomy from 2012 to 2014 . The first 45 patients underwent MA and subsequent 45 patients underwent LA. All patients were given $10 \mathrm{ml}$ of $1 \%$ Marcaine infiltration into the neck incision before closure. Basic demographic data, operative time, incision length, weight of gland, need for transection of strap muscles and complications were recorded. The pain score was measured using the visual analogue score (VAS) on a scale of 0 10. Revision thyroid surgery and minimally invasive thyroid operations were excluded. Patient demographics, operative timing, gland weight and incisional length showed no significant difference. Operative time was 137 minutes $(137 \pm 34.1)$ for MA group versus $124(124 \pm 20.2)$ in LA group $(p=0.984)$. Incision length was $4.9 \pm 1.1 \mathrm{~cm}$ and $5.2 \pm 0.8 \mathrm{~cm}$ for MA and LA group respectively, $(\mathrm{p}=0.071)$. Post-operative pain was significantly lower in the LA group. Five patients $(11 \%)$ in MA group required horizontal transection of strap muscles for extraction of large goitres compared to 1 patient $(2.2 \%)$ in the LA group. No major complications occurred in either group. The LA method is as safe as the midline technique with comparable operative time and significantly lower pain scores. It avoids midline separation and suturing of strap muscles and reduces the need for strap muscle transection to removal large goitres. 


\section{Poster 294}

Thyroid Imaging Friday Poster Case Report

METASTATIC PAPILLARY THYROID CANCER TO VOCAL CORD? WHOLE BODY SCANS DON'T LIE

S.L. Brown

Endocrinology, Diabetes, and Metabolism, University of Arizona

College of Medicine-Phoenix, Gilbert, AZ

Thyroid cancer is the $11^{\text {th }}$ most common cancer in the United States with an incidence of 14.2 cases per 100,000 individuals (1). Despite increasing incidence, the 5-year survival rate has increased to $98 \%$ (1). Total thyroidectomy remains the mainstay of treatment for thyroid cancer. As radioactive iodine ablation is being utilized less in low-risk disease, removing all thyroid tissue is important for thyroglobulin measurements to monitor disease recurrence during followup period (2). However, removal of all thyroid tissue may not be feasible for many reasons. It is imperative for clinicians to recognize common areas that harbor remnant thyroid tissue while treating postsurgical patients with thyroid cancer. We present a case of papillary thyroid cancer to emphasize this point.

A 34-year-old man with no past medical history was found to have an incidental thyroid nodule. Thyroid ultrasound confirmed the nodule and fine-needle aspiration biopsy diagnosed papillary thyroid carcinoma. He underwent total thyroidectomy without significant postoperative events. Pathology revealed minimal capsular invasion with central lymph node involvement for which patient underwent radioactive iodine ablation. Post-ablative whole body scan report showed intense signal in the left vocal cord concerning for metastasis, which caused distress to patient and clinician. Laryngoscopy was negative for vocal cord involvement, and after discussion with the surgeon, the uptake in the vocal cord on WBS was determined to be remnant pyramidal lobe, which was left behind during surgery. Thyroglobulin antibodies were positive, but patient was considered disease free.

The two most common sites for remnant thyroid tissue after total thyroidectomy are surrounding vital neurological structures and in areas problematic for removal: pyramidal lobe, thyroglossal cyst tract elements, and in the tracheo-esophageal groove (2). Recognizing common areas of remnant thyroid tissue is important when assessing post-operative scans to avoid unnecessary procedures and treatment as well as undue stress for the patient.

Recognizing common areas of remnant thyroid tissue following thyroidectomy is important in managment of thyroid cancer patients.

\section{Poster 295}

Thyroid Imaging Friday Poster Clinical ULTRASOUND CHARACTERISTICS OF THYROID NODULES WITH INDETERMINATE CYTOLOGY AND CORRELATION WITH MOLECULAR MARKER TESTING RESULTS

P. Goundan, S.L. Lee

Endocrinology, Boston University School of Medicine, Boston, MA

Thyroid cancer risk assessment based on ultrasound characteristics, fine needle biopsy with cytology and molecular testing has emerged as the optimal method of identifying clinically relevant cancer while at the same time reducing the number of unnecessary surgeries. The objective of this study is to determine if sonographic characteristics of thyroid nodules with an indeterminate cytology (Bethesda III, IV, V) correlate with ThyroSeq V.2 molecular testing (gene mutation, fusion or overexpression). A retrospective review of thyroid nodule biopsies with indeterminate cytology from a single institution during an 18 month period was performed. Two endocrinologists experienced in thyroid ultrasound reviewed the images of the nodules and described the nodule characteristics. These results were correlated with molecular markers using ThyroSeq V.2 and surgical pathology reports. The ultrasound images of 214 thyroid nodules with indeterminate cytology were reviewed. 77 had positive ThyroSeq V.2 results. The insufficiency rate for molecular testing was around $7 \%$. The most common mutations were RAS and BRAF followed by PTEN and EIF1AX. The majority of nodules had a heterogeneous echotexture. Of those that had a homogeneous echotexture, 11 of 26 had positive ThyroSeq V.2 results (RAS, EIF1AX PAX8/PPARG). The three nodules that were homogeneous and very hypoechoic had either negative ThyroSeq V.2 results or was positive for a low risk mutation (EIF1AX). Microlobulated or infiltrative margins were seen in 12 nodules for which ThyroSeq was performed and only one had a negative result. Of the 35 nodules with either micro or macrocalcification, 19 had positive ThyroSeq results. Six out of seven nodules with macrocalcification and a taller than wide configuration had positive ThyroSeq results. Additional characterization of nodule imaging features based on each Bethesda category was also performed. This preliminary study suggests that there may be individual or combinations of sonographic features that correlate with molecular testing in cytologically indeterminate nodules. Larger prospective studies will need to be performed to determine if such correlations can reduce the need for expensive molecular marker testing.

\section{Poster 296}

Thyroid Nodules \& Goiter Friday Poster Basic

NEEDLE GAUGE AND CAPILLARY ACTION VS. ASPIRATION IN FINE NEEDLE BIOPSY OF THE THYROID: A SYSTEMATIC REVIEW

W. Moss, K. Brumund

Head and Neck Surgery, UC San Diego, San Diego, CA

Background: Fine needle biopsy has been a widely utilized technique in the workup of thyroid nodules for many years. Nonetheless, there remains controversy regarding the optimal fine needle biopsy technique. The optimal needle size and the implementation of capillary action vs. aspiration remain topics of debate.

Methods: A systematic review of the Medline database was performed in accordance with PRISMA guidelines. Articles evaluating the effect of needle gauge and capillary action vs. aspiration on success rates of fine needle biopsy of thyroid were assessed for inclusion. The primary outcome of interest was the rate of non-diagnostic cytopathology.

Results: Fifteen articles were ultimately included in the review. All but one of the articles were prospective and many were randomized, blinded trials. Seven articles evaluated the effect of needle gauge on diagnostic accuracy of fine needle biopsy of the thyroid and nine evaluated the effect of capillary action vs. aspiration. Two articles evaluated both. Of the articles evaluating needle gauge, the majority were unable to demonstrate superiority of one needle size over another. Of the studies evaluating capillary action vs. aspiration, six demonstrated superiority of capillary action, three were equivalent and two demonstrated improved success with aspiration.

Conclusion: There are a number of prospective studies evaluating the effect of needle gauge and capillary action vs. aspiration on the diagnostic accuracy of fine needle biopsy of the thyroid. Larger needle gauges (21-23G) do not provide improved accuracy. There is growing evidence that capillary action provides a lower rate of nondiagnostic cytopathology relative to aspiration.

\section{Poster 297}

Thyroid Nodules \& Goiter Friday Poster Case Report A RARE CASE OF THYROID GLAND INFILTRATION FROM CHRONIC LYMPHOCYTIC LEUKEMIA/SMALL LYMPHOCYTIC LYMPHOMA (CLL/SLL)

Y. Khan, S. Kadiyala, G. Dhir 
Departiment of Internal Medicine, Division of Endocrinology \& Metabolism, University of Florida and the Malcolm Randall VA Medical Center, Gainesville, FL

Thyroid lymphoma is a rare medical condition that often presents with a rapidly enlarging goiter. Secondary involvement due to a systemic disease is rare. We present a unique case of chronic lymphocytic leukemia/small lymphocytic lymphoma (CLL/SLL) with secondary involvement of the thyroid gland.60 year old male presented with a rapidly enlarging thyroid gland on a background history of Hashimoto's and CLL. During CLL staging, he was found to have diffuse lymphadenopathy and findings concerning for involvement of the thyroid gland on CT scan. Clinically, he described an enlarging, firm thyroid gland size over the past year. Labs were consistent with Hashimoto's (TPO Ab 683, ref range <9 IU/mL; thyroglobulin Ab 3, ref range $<=1 \mathrm{IU} / \mathrm{mL}$ ) and subclinical hypothyroidism (TSH 10.93, ref range 0.27-4.2 uIU/mL; free T4 1.31, ref range $0.8-1.7 \mathrm{ng} / \mathrm{dL}$ ). Neck ultrasound showed a profoundly hypoechoic thyroid gland with heterogeneous echotexture and two suspicious right and left cervical lymph nodes. After normalizing thyroid function with levothyroxine, fine needle aspiration biopsy (FNAB) of the thyroid parenchyma and both lymph nodes with flow cytometry was performed. Flow cytometric analysis of the lymph nodes demonsted CD5+ B-cell lymphoproliferative disorder with a phenotype characteristic of CLL/SLL. Specimen from the thyroid was not received for flow cytometry, however cytologic analysis demonstrated numerous small lymphocytes with clumped chromatin, consistent with CLL/SLL. Thyroid gland infiltration secondary to systemic disease is rare, with CLL/SLL being an uncommon histiologic subtype. Case reports have described CLL/SLL being incidentally diagnosed in a thyroid nodule, however our patient had sonographic features consistent with Hashimoto's without nodules. Thyroid lymphoma may therefore have similar sonographic features to Hashimoto's due to lymphoid cells, and the presence of a thyroid nodule is not a necessary criteria to perform FNAB. Flow cytometry of the thyroid specimen should also be performed when there is suspicion for thyroid lymphoma. Lymphoma of the thyroid gland should be considered in a patient with a rapidly enlarging thyroid gland and Hashimoto's thyroiditis.

\section{Poster 298}

Thyroid Nodules \& Goiter Friday Poster Clinical

UTILIZATION, OUTCOMES, AND COSTS ASSOCIATED WITH THE ADOPTION OF RECOMBINANT HUMAN TSH IN THE UNITED STATES FOR MEDICARE PATIENTS M.A. Dinan ${ }^{1,2}$, Y. Zhang ${ }^{3}$, S. Reed ${ }^{3}$, J. Sosa $^{3}$

${ }^{1}$ Medical Oncology, Duke Cancer Institute, Durham, NC;

${ }^{2}$ Population Health Sciences, Duke University, Durham, NC; ${ }^{3}$ Duke Clinical Research Insitute, Durham, NC

Recombinant TSH (rhTSH) has long been approved for both diagnostic (1998) and therapeutic (2008) indications in conjunction with radioactive iodine (RAI) administration following thyroidectomy. However, its real world utilization and associated outcomes and costs have not been characterized in a nationally representative patient population. Retrospective SEER-Medicare analysis of beneficiaries $>65$ diagnosed with differentiated thyroid cancer with respect to: 1) rhTSH utilization for patients diagnosed between 1996 and 2011 who underwent thyroidectomy $(\mathrm{N}=6,842)$; and 2) comparison of resource utilization among patients who did vs. did not receive rhTSH as part of therapeutic RAI treatment between 2008 and $2011(\mathrm{~N}=1,444)$. All costs were adjusted to reflect 2013 dollars. By 2011, rhTSH was used in approximately $25 \%$ of all Medicare patients with surgically resected thyroid cancer. In multivariable analysis, decreased receipt of rhTSH was observed in patients residing in the Midwest vs. Northeast region (OR 0.48, $0.37-0.62)$ and census tracts with the lowest quartile of high school education rates (OR $0.69,0.56-0.84)$. No association with rhTSH receipt was seen with sex, age, comorbidities, or stage. Receipt of rhTSH was not significantly associated with total duration of inpatient hospitalizations or number of outpatient and emergency department visits. Multivariable analyses showed similar overall costs among patients who did vs. did not receive rhTSH (cost ratio [CR] 0.91, 0.80-1.04). Increased mean outpatient costs $(\$ 5,213$ vs. $\$ 4,190)$ were offset by lower inpatient costs $(\$ 3,493$ vs. $\$ 6,143)$. Mean total costs within the year following first therapeutic RAI were about $\$ 1,900$ less for patients who received rhTSH vs. those who did not $(\$ 14,087$ vs. $\$ 15,991, p=0.160)$. Patients who received rhTSH had similar total Medicare payments as patients who did not receive rhTSH in conjunction with RAI due to higher outpatient rhTSH costs being balanced by lower inpatient payments for patients receiving rhTSH. Further research focusing on specific patient populations may better demonstrate differential impact of rhTSH utilization on overall resource utilization and outcomes.

\section{Poster 299}

Thyroid Nodules \& Goiter Friday Poster Clinical

\section{THYROTROPIN AND RISK OF MALIGNANCY}

\section{IN FOLLICULAR THYROID NEOPLASM}

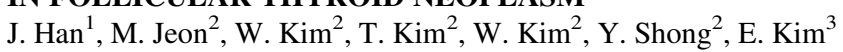

${ }^{1}$ Endocrinology and Metabolism, Samsung Changwon Hospital,

Changwon, Gyeongsangnam-do, Korea (the Republic of); ${ }^{2}$ Asan

Medical Center, Seoul, Korea (the Republic of); ${ }^{3}$ Ulsan University

Hospital, Ulsan, Korea (the Republic of)

TSH suppression therapy is associated with decreased recurrence rates in differentiated thyroid cancer patients, but the pathogenic role of TSH in oncogenesis is not clear. Previous studies showed that the likelihood malignancy increases with higher serum TSH concentration, but preoperative diagnoses on FNA was very heterogeneous. The aim of this study is to evaluate the association between preoperative TSH and thyroid cancer in patients with FNA diagnosis of follicular neoplasm. Between Feb 1998 and May 2012, total 530 patients with follicular neoplasm on FNA underwent surgery in Asan Medical Center and Ulsan University Hospital. Data from the 304 euthyroid patients with preoperative serum TSH were reviewed. $38 \%$ of patients (115 of 304) had malignancy on final pathology. They were 70 follicular, 7 Hürthle cell, 12 papillary, 13 follicular variant papillary, and 13 poorly differentiated carcinoma. There were no significant differences in TSH between malignancy and benign $(p=0.429)$. In binary logistic regression, the risk of malignancy was not associated with preoperative TSH, male gender and age. However, the tumor size was associated with increased risk of malignancy (OR 1.17, $\mathrm{p}=0.045)$. And there was a significant negative correlation between tumor size and preoperative TSH (Spearman's rho $=0.12$, $\mathrm{p}=0.037$ ). In patients with an FNA diagnosis of follicular neoplasm, the likelihood thyroid cancer does not increase with serum TSH within normal ranges. The tumor size is associated with increased malignancy risk, but low TSH does not guarantee of reduction in tumor size.

\section{Poster 300}

Thyroid Nodules \& Goiter Friday Poster Clinical

EFFICACY OF RADIOFREQUENCY THERMAL ABLATION FOR THE TREATMENT OF BENIGN THYROID NODULES ACCORDING TO DIFFERENT US PATTERN: RESULTS FROM AN ITALIAN MULTICENTER STUDY

M. Deandrea ${ }^{1}$, F. Garino ${ }^{1}$, A. Mormile ${ }^{1}$, P. Limone ${ }^{1}$,

R. Garberoglio ${ }^{2}$, R. Rossetto ${ }^{2}$, N. Bonelli ${ }^{2}$, S. Spiezia ${ }^{3}$, M. De Santis ${ }^{3}$, S. Monti ${ }^{4}$, M. Deiana ${ }^{4}$, C. Cugini ${ }^{5}$, G. El Dalati ${ }^{6}$ 
${ }^{1}$ Endocrinology and Metabolism, Mauriziano Hospital Umberto I, Torino, Torino, Italy; ${ }^{2}$ Molinette Hospital, Endocrinology and Metabolism, Turin, Turin, Italy; ${ }^{3}$ UOSSD Chirurgia del Collo, Ospedale Incurabili ASLNA1Centro, Naples, Italy; ${ }^{4}$ UOC

Endocrinologia, Azienda Ospedaliera Sant'Andrea, Rome, Italy; ${ }^{5}$ Dipartimento di Radiologia, Ospedale di Treviso, Treviso, Italy;

${ }^{6}$ Departimento di Radiologia, Policlinico G.B. Rossi, Verona, Italy

The purpose of this study is to validate the generalizability of the efficacy and safety of radiofrequency (RF) ablation for treating benign thyroid nodules (BTN) in a large population multicenter study and to find US-findings that can predict nodules' shrinkage. This study included 337 patients with solid cold thyroid nodules from 6 Italian institutions. The age of the patient was 28 to $82 \mathrm{yrs}$ (median 55), 257 were females (76\%) and 80 were males (24\%). RF ablation was performed with moving-shot technique, using a 18-gauge, internally cooled electrode. Nodule volume and US pattern, thyroid function, symptom/ cosmetic scores, and complications were evaluated before treatment and during each follow-up visit according to a specific reference protocol (before treatment, 6 and 12 months). The median basal nodule volume was $20 \mathrm{~mL}$ and significantly decreased after treatment at 6 months $(7,3 \mathrm{ml}(-63.5 \%), \mathrm{p}<0,001)$; further significant volume reduction was found at 12 months $(6 \mathrm{ml}(-70 \%)$, p vs 6 months $=0,009)$. A correlation was tested between nodules shrinkage and US-structure, vascularity, presence of macrocalcifications and nodule volume at basal, showing significant results for US-structure (pattern 2 spongiform showing a $76 \%$ reduction vs $67 \%$ and $66 \%$ of pattern 1 e 3 respectively, $\mathrm{p}<0,01$ ) and vascularity (pattern 2 showing a $73 \%$ vs $67 \%$ and $59 \%$ of pattern 1 e 3 respectively, $p<0,03$ ). A slight inverse correlation was found between volume reduction and nodule basal volume (spearman $-0,23$ ), while macrocalcifications seemed not to affect at all the treatment result. The mean symptom and cosmetic scores were significantly reduced at the last follow-up. No major complications were encountered. This multicenter study validated the efficacy and safety of RF ablation for treating BTN and showed a positive correlation between final shrinkage and some US-findings easily detectable before treatment. RF ablation can be considered a safe and effective alternative to surgery for cold BTN.

\section{Poster 301}

Thyroid Nodules \& Goiter Friday Poster Clinical

DIAGNOSTIC UTILITY OF MOLECULAR AND IMAGING BIOMARKERS IN CYTOLOGICALLY INDETERMINATE THYROID NODULES: SYSTEMATIC REVIEW AND META-ANALYSIS

E.J. de Koster ${ }^{1}$, L. de Geus-Oei ${ }^{8,1}$, O. Dekkers ${ }^{3}$, I. van Engen-van Grunsven $^{2}$, J. Hamming ${ }^{4}$, E. van der Kleij-Corrsmit ${ }^{3}$, H. Morreau ${ }^{5}$, A. Schepers ${ }^{4}$, J. Smit ${ }^{6}$, W.J. Oyen ${ }^{7}$, D. Vriens ${ }^{8}$

${ }^{1}$ Radiology and Nuclear Medicine, Radboud University Medical Center, Nijmegen, Netherlands; ${ }^{2}$ Pathology, Radboud University Medical Center, Nijmegen, Netherlands; ${ }^{3}$ Endocrinology, Leiden University Medical Center, Leiden, Netherlands; ${ }^{4}$ Surgery, Leiden University Medical Center, Leiden, Netherlands; ${ }^{5}$ Pathology, Leiden University Medical Center, Leiden, Netherlands; ' ${ }^{6}$ ndocrinology, Radboud University Medical Center, Nijmegen, Netherlands;

${ }^{7}$ Nuclear Medicine, Institute of Cancer Research, London, United Kingdom; ${ }^{8}$ Radiology and Nuclear Medicine, Leiden University Medical Center, Leiden, Netherlands

Indeterminate thyroid cytology (Bethesda III and IV) corresponds to follicular-patterned benign and malignant lesions, which are particularly difficult to differentiate on cytology alone. As approximately $25 \%$ of these nodules harbor malignancy, diagnostic hemithyroidectomy is still custom. However, advanced preoperative diagnostics are rapidly evolving. This systematic review and metaanalysis included 164 original articles and provides an overview of the value of additional molecular and imaging diagnostics for indeterminate thyroid nodules, including considerations regarding costeffectiveness, availability, and feasibility of combining techniques. Addressed diagnostics include gene mutation analysis, microRNA, immunocytochemistry (ICC), ultrasonography, elastosonography (USE), Tc-99m sestamibi ( $\left.{ }^{99 \mathrm{~m}} \mathrm{Tc}-\mathrm{MIBI}\right)$ scintigraphy, fluorodeoxyglucose positron emission tomography (FDG-PET) and diffusionweighted magnetic resonance imaging (DW-MRI). The best rule-out tests for thyroid carcinoma were combined galectin-3/HBME-1 ICC (estimated pooled $96.6 \%$ sensitivity, 0.04 negative likelihood-ratio (LR)), semi-quantitative USE $(92.1 \%, 0.08)$, qualitative USE using an alternative threshold score of 1 (98.5\%, 0.08), FDG-PET $(94.2 \%$, 0.12), and the Afirma ${ }^{\circledR}$ GEC $(97.3 \%, 0.18)$. The best rule-in tests were BRAF mutation analysis (estimated pooled $99.9 \%$ specificity, 195 positive LR), semi-quantitative USE (93.4\%, 14.04), combined galectin-3/HBME-1 ICC $(94.3 \%, 13.47)$, combined BRAF/RAS mutation analysis $(94.5 \%, 8.69)$ and galectin-3 ICC $(90.7 \%, 7.07)$. Geographical variations in tumor genetics or cytology (e.g. Hürthle cell neoplasms) strongly influence several tests. None of the diagnostics had near-perfect sensitivity, near-perfect specificity and costeffectiveness. With the techniques currently clinically available, we propose that a multimodal stepwise approach using a sensitive rule-out and a specific rule-in test might offer the most conclusive diagnosis for indeterminate thyroid nodules. There is currently insufficient evidence to reliably interpret sequentially used tests, especially combinations of (molecular) imaging and genetics. The testing sequence and costs of the selected tests and therapeutic procedures are pivotal.

\section{Poster 302}

Thyroid Nodules \& Goiter Friday Poster Clinical

\section{INCIDENTAL HYPOPARATHYROIDISM AFTER} THYROID SURGERY: OUTCOME, FOLLOW-UP AND MANAGEMENT OF 672 PATIENTS WITH POSTOPERATIVE HYPOPARATHYROIDISM TREATED IN 3 ENDOCRINE CENTERS IN GERMANY

M. Grussendorf ${ }^{1}$, B. Stamm ${ }^{2}$, N. Stamm ${ }^{3}$, H. Siggelkow ${ }^{2}$

${ }^{1}$ Endocrine Center, Halblech, Germany; ${ }^{2}$ MVZ Endocrinology,

Göttingen, Germany; ${ }^{3}$ Med. University Clinic, Mainz, Germany

Hypoparathyroidism (HypoPT) is (beside recurrent nerve palsy) one of the most frequent complications after thyroid surgery. We were interested in symptom control of patients with HypoPT presenting for the first time after thyroid surgery and after optimization of the therapy.

We retrospectively evaluated the data of 672 pts. (female: $85 \%$ ) with HypoPT after thyroid surgery in 3 endocrine centers in Germany over the last 10-20 years. $72 \%$ of these patients underwent subtotal or hemi-thyroidectomy (ST) and $28 \%$ total thyroidectomy (TT). The following diagnoses have been the reasons for surgery: goiter with or without thyroid nodules $(n=489(n=375$ ST, $n=94$ TT)); Graves disease $(n=61(n=50 S T, n=11$ TT) ), preoperatively diagnosed or incidental thyroid cancer $(n=122(n=46 S T, n=76$ TT $))$.

Patients were investigated for the first time postoperatively in the endocrine centers $79.6+123.8$ months after thyroid surgery $(=$ mean, median 23). We calculated the percent of pts. in 3 ranges of serum-Ca $(\mathrm{sCa}, \mathrm{mmol} / \mathrm{l})$ (too low <2.1, good 2.1-2.3, too high $>2.3$ ) and percent of patients and complaints (paresthesia (par) and cramps (cr)) under different therapiesWhen presenting for the first time only $34.2 \%$ of patients were in the target range of 2.1 to $2.3,68.2 \%$ of sCa values were either to low or to high. Change of medication (mostly to long acting vitamin D-analogs) was followed by $54.6 \%$ in the target range. Symptoms decreased from $31.6 \%$ (par) and $36.8 \%$ (cr) to $17.4 \%$ (par) and $14.2 \%$ (cr) respectively although $45.4 \%$ of patients were still not 
in the target range of sCa. Interestingly, the complaints did not differ between the different medications at first visit. In contrast, symptoms seemed to be best controlled when using long acting vitamin Danalogs after adaptation of medication by the endocrinologist.

In conclusion, we are showing that treatment adaptation by endocrinologists clearly reduced severe symptoms of hypocalcemia. However, even in experienced hands therapy of HypoPT after thyroid surgery is not satisfying. Long acting vitamin D-analogs should preferably be favoured to reduce symptoms. Nevertheless, the best therapy would be the prevention by avoiding unnecessary operations.

\section{Poster 303 \\ Thyroid Nodules \& Goiter Friday Poster Clinical \\ TREATMENT PREFERENCES AND DECISION MAKING IN PATIENTS DIAGNOSED WITH INDETERMINATE THYROID NODULES}

L.E. Stahl ${ }^{1}$, M. Carr ${ }^{3}$, D. Goldenberg ${ }^{1}$, J.R. Schubart ${ }^{2}$

${ }^{1}$ Surgery/Otolaryngology, Penn State College of Medicine, Palmyra, PA; ${ }^{2}$ Surgery, Penn State College of Medicine, Hershey, PA;

${ }^{3}$ Surgery/Otolaryngology, West Virginia University School of Medicine, Morgantown, WV

Thyroid nodules are a common clinical problem and are evaluated to assess for thyroid cancer risk. Nodules classified as cytologically "indeterminate" require further evaluation to obtain a definitive diagnosis. There is not always a clear consensus for the treatment of indeterminate thyroid nodules (ITN). The optimal choice often depends on the patient's perception of benefits and risks of the options they are offered. Patients desire increasing involvement in treatment decisions, and it is known that informed decisions that fit a patient's personal preferences correlate with higher satisfaction with the overall treatment process. Thus, the objective of this study was to qualitatively assess decision making and treatment choices by patients diagnosed with ITN. Adult patients with a diagnostic FNA testing result of ITN who underwent molecular testing with Thyroseq (CBLPath) were included. Patient sample was seen by a single surgeon at a single academic medical center between May 2016 and January 2017. The analytic framework was a qualitative descriptive study to provide a fundamental description of patient decision making and treatment choices. Transcripts from audio-taped telephone interviews were analyzed. Thirteen patients completed the study; 8 patients opted to undergo surgery and 5 patients decided to wait and monitor their ITN. Risk perception and risk tolerance impacted patients' decision making when faced with two choices for treatment. Additional factors that influenced decision making included trust in the physician, presence of a support network, additional information sources, religion, and concurrent comorbidities. Patients' preferences for treatment of ITN are influenced by their perceptions of risk, yet how patients interpret the percent risk associated with FNA results has not been studied. Our findings suggest a need to understand how physicians communicate risk associated with treatment options to their patients. Because patients lacking numeracy skills may be disadvantaged in understanding the benefits/ risks of treatment choices, physicians need to consider numeracy skills and ability to accurately assess risks of treatment choices in order to provide care aligned with patient preferences.

\section{Poster 304}

Thyroid Nodules \& Goiter Friday Poster Clinical ENDOSCOPIC TRANSAXILLARY THYROIDECTOMY USING A MODIFIED 4-PORT TECHNIQUE

R. Singaporewalla, S. Goh, A. Rao

Surgery, National Health Group, Singapore, Singapore
Transaxillary approach to thyroidectomy is gaining popularity as it avoids the cosmetic impact of an obvious neck scar. Conventional endoscopic transaxillary thyroidectomy via the breast and axilla approach utilizes 1 camera and 2 working ports. We describe a modified technique using 3 working ports, which facilitates retraction and dissection without added morbidity or impairment of cosmesis. Our technique involves the insertion of an additional $5 \mathrm{~mm}$ working port inferior to the axillary camera port after creation of the skin flap and exposure of goiter. The third working port allows the surgeon better control of his dissection and enables the assistant to retract the goiter for visualization of key structures. As shown in our video, it also facilitates the use of intra-operative nerve monitoring. The use of this modified 4-port technique allows successful extraction of large thyroid nodules, significantly improves the operative field with better retraction of the thyroid thereby enabling safe dissection of the key structures and allowing ease of use of intraoperative nerve monitors. The modified 4 port endoscopic technique is useful especially for larger, challenging goiters as it provides the surgeon with added flexibility in port usage to augment the operative field.

\section{Poster 305}

Thyroid Nodules \& Goiter Friday Poster Clinical

RETROSPECTIVE APPLICATION OF THE 2015 AMERICAN THYROID ASSOCIATION GUIDELINES FOR ULTRASOUND CLASSIFICATION, BIOPSY INDICATIONS AND FOLLOW UP IMAGING OF THYROID NODULES: CAN IMPROVED REPORTING DECREASE TESTING?

M. Mohammadi ${ }^{1}$, C. Betel ${ }^{2}$, K. Burton ${ }^{2}$, K.M. Higgins ${ }^{3}$, Z. Ghorab ${ }^{4}$, I.J. Halperin ${ }^{5}$

${ }^{1}$ General Internal Medicine, University of Toronto, Thornhill, Ontario, Canada; ${ }^{2}$ medical imaging, Sunny brook Health Science Center, Toronto, Ontario, Canada; ${ }^{3} \mathrm{Head}$ and Neck Surgery, Sunny brook Health Science Center, Toronto, Ontario, Canada; ${ }^{4}$ Pathology, Sunny brook Health Science Center, Toronto, Ontario, Canada; ${ }^{5}$ Endocrinology, Sunny brook Health Science Center, Toronto, Ontario, Canada

Thyroid ultrasound has been widely used to determine which nodules need further work up. The goal of this study is to determine if using an Ultrasonographic features checklist based on 2015 ATA guidelines can improve reporting and decrease unnecessary further testing. In a retrospective study, ultrasonographic images of the all nodules biopsied in 2015 were reviewed by radiologists, blinded to fine needle aspiration (FNA) biopsy result, using a checklist to report the image. The checklist was prepared based on 2015 ATA guideline. The ultrasonographic characteristics of thyroid nodules were compared with the result of biopsy to determine positive predictive value (PPV), negative predictive value (NPV), sensitivity and specificity of checklist in predicting malignancy. Radiologists also made an overall recommendation on need for FNA.425 thyroid nodule ultrasound images were reviewed by radiologists. Biopsy results of 31 nodules were malignant and 394 were non-malignant. Malignant nodules showed higher frequency of solid, hypoechoechogenicity, and lymph node involvement compare to benign nodules. Solid nodule had the highest PPV (13\%) and NPV (94.7\%). Extra thyroid extension had the highest specificity $(90.1 \%)$. Vascularity has the highest sensitivity $(83.8 \%)$ followed by hypoechogenicity $(65.6 \%)$. Considering all US features of thyroid nodule showed that the checklist has positive predictive value of $9 \%$, negative predictive value of $97.5 \%$, sensitivity of $96.8 \%$ and specificity of $11.14 \%$. Radiologist determined that $10 \%$ of the nodules were very low risk on US and did not require FNA. Using checklists based on 2015 ATA 
guideline thyroid nodule ultrasonographic features is a sensitive tool with high NPV to predict benign thyroid nodule.

\section{Poster 306}

Thyroid Nodules \& Goiter Friday Poster Clinical

\section{FREQUENCY OF FOLLOW UP OF BENIGN THYROID NODULES- CAN WE DO LESS?}

M. Mohammadi ${ }^{1}$, Z. Ghorab ${ }^{2}$, K.M. Higgins ${ }^{3}$, I.J. Halperin ${ }^{4}$

${ }^{1}$ General Internal Medicine, University of Toronto, Thornhill, Ontario, Canada; ${ }^{2}$ Pathology, Sunny Brook Health Science Center, Toronto, Ontario, Canada; ${ }^{3} \mathrm{Head}$ and Neck Surgery, Sunny brook Health Science Center, Toronto, Ontario, Canada; ${ }^{4}$ Endocrinology, Sunny brook Health Science Center, Toronto, Ontario, Canada

Objectives: To determine the frequency of malignancy, follow up ultrasound and repeat fine needle aspiration (FNA) in initially benign thyroid nodules.

Methods: In a retrospective study, the medical records of benign thyroid nodules between 2010-2011 were reviewed. We checked the frequency of follow up ultrasound and Fine needle aspiration (FNA), mean years of follow up, interval between follow up ultrasound, change in nodule size, the reason for repeat FNA, frequency of thyroidectomy and thyroid malignancy during 5 years of follow up.

Results: 750 benign thyroid nodules were reviewed. 643 (80.2\%) of thyroid nodules were in female and $103(13.8 \%)$ were in male patients. Mean years of follow up was $3.47 \pm 1.66$ years; 280 (37.4\%) had no follow up US, 113 (15.1\%) one follow up US, 98 (13.1\%) two follow up US, 257 (34.2\%) three and more follow up US. Review of thyroid nodule size showed $215(28.8 \%)$ nodules with decrease in size, $145(19.4 \%)$ with less than $50 \%$ increase in size, and $91(12.1 \%)$ with more than $50 \%$ increase in size. Among 750 nodules, 31 (4.1\%) nodules underwent thyroidectomy which pathology result of $21(2.8 \%)$ showed malignancy. $109(14.5 \%)$ thyroid nodules underwent repeat FNA (rFNA). The main reason for rFNA was nodule size growth. The cytology result of $70 \%$ of rFNA was benign.

Conclusion: In our sample of initial benign thyroid nodules only $2.8 \%$ of patients had malignancy after a mean follow up of 3.5 years. Over $30 \%$ had more than 3 repeat ultrasounds. Consideration to decreased interval and frequency of repeat US should be given in future thyroid nodules guidelines.

\section{Poster 307}

Thyroid Nodules \& Goiter Friday Poster Clinical

\section{A NOVEL TECHNIQUE OF INTRAOPERATIVE NERVE MONITORING DURING FULLY ENDOSCOPIC TRANSAXILLARY THYROIDECTOMY}

A.D. Rao, R. Singaporewalla, S. Goh, W. Sim

Surgery, Khoo Teck Puat Hospital, Singapore, Singapore

The feasibility of Intraoperative Nerve Monitoring (IONM) during endoscopic thyroidectomy has not been well studied. The currently available IONM devices are dedicated to conventional thyroidectomy and are not suitable for remote access endoscopic approaches. Most centers performing endoscopic thyroid surgery do not regularly attempt dissection for visual documentation of the recurrent laryngeal nerve due to technical difficulty and many surgeons safeguard the recurrent laryngeal nerve by leaving behind a rim of thyroid tissue along the suspensory ligament. We have described a novel technique of IONM using a modified metallic probe that can be used via any working port during remote access endoscopic thyroidectomy. Usual intubation with EMG endotracheal tube was performed in eligible patients during transaxillary endoscopic thyroidectomy. The steps involved initial division of the gland from the isthmus following which the inferior pole of the gland was meticulously freed. This was followed by dissection of the lateral side and the superior pole of the gland. Nerve stimulation was carried out by using the modified long metallic probe passed via a working $5 \mathrm{~mm}$ axillary port. This probe was adapted externally to make direct contact with the conventional nerve monitoring probe. At this point visual documentation was achieved by focused dissection in the tracheo-oesophageal groove. Six patients (M-2, F-4) underwent transaxillary endoscopic hemithyroidectomy with IONM. There were no major complications or conversions. One patient experienced transient voice change which recovered fully. Using this technique 4 out of 6 recurrent laryngeal nerves were exposed with ease $(66.66 \%)$. The overall recurrent laryngeal nerve visualization rate in our endoscopic series was $5 / 13(38.46 \%), p<0.01$. The recurrent laryngeal nerve may be exposed to more conducted heat during endoscopic thyroidectomy due to the exclusive use of energy devices for dissection. Our novel technique allows ease of visual identification

\section{Poster 308}

Thyroid Nodules \& Goiter Friday Poster Clinical

INITIAL LEARNING CURVE EXPERIENCE WITH TRANSAXILLARY ENDOSCOPIC THYROIDECTOMY : COMPARABLE RESULTS TO CONVENTIONAL TECHNIQUE

A.D. Rao, R.M. Singaporewalla, S. Goh, W. Sim

Surgery, Khoo Teck Puat Hospital, Singapore, Singapore

Endoscopic thyroidectomy has excellent cosmetic results. It has been reported to have a significant learning curve of 50-75 cases and is understood to be minimal access but maximally invasive. Eligible patients with unilateral disease (benign tumours $<6 \mathrm{~cm}$ and differentiated microcarcinoma with low risk) were offered endoscopic hemithyroidectomy via the unilateral axillary breast approach. Nodules larger than $6 \mathrm{~cm}$, substernal goiter, previous neck surgery, malignancy and hyperthyroidism were excluded. We judged our progress based on port insertion and flap raising times, visualization of the recurrent laryngeal nerve, operating time, pain scores, length of stay and complications. Results were compared with parallel data from conventional hemithyroidectomy in our unit. From Jan to Dec 2016, fifteen consecutive eligible patients (M-2, F-13) underwent endoscopic hemithyroidectomy. There were no conversions or major complications. One patient experienced transient voice change which recovered fully. All patients were discharged on POD1. Mean operating time was 133.38 minutes $(85-180)$ as against 75 minutes $(65-90)$ in our conventional group $(p<0.01)$. Port insertion and flap raising times showed a progressive improvement, with the second half of the series showing a statistically significant difference. $(p<0.01)$. Recurrent laryngeal nerve visualization rate was $6 / 15(40 \%)$ overall and $4 / 6(66 \%)$ with intraoperative nerve monitoring. Pain scores at recovery, 6 hours and on first postoperative day were 2, 1.8 and 2.3 (range 1-5, 1-3 and 1-4 respectively). Average pain score at clinic follow up was $1.6(0-4)$ with complete resolution of any residual chest wall paraesthesia and numbness. Cosmetic results and overall patient satisfaction were rated as excellent-12, good-2, unsatisfactory-1. Endoscopic thyroidectomy shows results comparable to conventional thyroidectomy in terms of complications and postoperative pain with superior cosmetic and patient satisfaction outcomes even during the initial learning curve period. Individual performance parameters show a steady improvement after the first six cases while operative times may require longer learning curves to reach that of conventional techniques. 


\section{Poster 309}

Thyroid Nodules \& Goiter Friday Poster Clinical

DECODING THYROID NODULES: GENETIC TESTING AND ARFI ELASTOGRAPHY

K. Mohsin, F. Murad, D. Bu Ali, D. Monlezun, E. Kandil

Surgery, Tulane University School of Medicine, New Orleans, LA

Thyroid nodules with indeterminate cytology on fine-needle aspiration pose a significant management challenge. In these patients, genetic testing with the Afirma gene-expression classifier (GEC) is commonly used to screen for thyroid cancer. We have previously shown that use of Afirma GEC in screening for malignant nodules is not reliable due to variability in the NPV. Another diagnostic modality, ultrasound elastography, can identify thyroid nodules that are stiff through acoustic radiation force impulse (ARFI) technology. We assess the use of Afirma GEC and ultrasound elastography in predicting malignancy in indeterminate thyroid nodules.

This is a retrospective review of all patients who underwent surgery for thyroid nodules by a single surgeon for an 18-month period. Demographic and pathologic data was collected, including age, gender, type of surgical procedure performed and pathology results. Afirma GEC testing results were also collected. Color intensity was used to determine the stiffness of nodules on elastography. Nodules that were indeterminate on cytology were further analyzed to assess the role of Afirma GEC and ultrasound elastography. A total of 217 nodules were included, of which 79 (36.4\%) had indeterminate cytology.

$44(55.7 \%)$ of the indeterminate nodules were stiff on elastography and $35(44.3 \%)$ of indeterminate nodules were malignant. Mean patient age was 50.8 and $61(77.2 \%)$ patients were female. Nodules that were suspicious on Afirma GEC were associated with malignant pathology (sensitivity: $87.0 \%$, specificity: $50 \%$, NPV: $83.3 \%$, OR: 6.7, $p=.009)$. Stiffness on elastography was not associated with malignant pathology $(p=0.817)$. In combination, suspicious Afirma GEC and stiffness on elastography were not associated with malignancy, although specificity was high (sensitivity: $31.4 \%$, specificity: 81.8\%, NPV: $60 \%$, OR: $2.06, p=0.171$ ). Stiffness on elastography in indeterminate thyroid nodules is not a strong predictor of malignant disease. Combined use of ultrasound elastography and Afirma GEC is also not helpful in evaluation of indeterminate thyroid nodules. Further larger scale studies are warranted.

\section{Poster 310}

Thyroid Cancer Friday Poster Clinical ASSOCIATION OF TSH LEVELS AND RISK OF THYROID CANCER

K. Mohsin, F. Murad, D. Bu Ali, T. Tran, T. Mallik, R.M. Kholmatov, D. Monlezun, E. Kandil

Surgery, Tulane University School of Medicine, New Orleans, LA

Preoperative TSH levels have been linked to the development of thyroid cancer. Multiple studies have analyzed TSH levels for this association while excluding cases where TSH levels were affected by thyroid medications or previous thyroid surgery. We evaluate TSH levels for association with thyroid cancer in all patients undergoing thyroid surgery without application of such exclusion criteria. This is a retrospective study of all patients who underwent thyroid surgery at an academic institution by a single surgeon. Thyroid malignancy in general, as well as papillary thyroid carcinoma (PTC) subtype, and papillary thyroid microcarcinoma (PTMC) subtype, were analyzed for association with TSH levels. Cases were examined for demo- graphic data, preoperative TSH levels, pathologic findings including malignancy status, extrathyroidal extension, perineural/vascular invasion, lymph node metastasis, and ultrasound nodule size. A total of 896 thyroidectomy cases were reviewed, $33.2 \%$ of them had malignant disease of which $13.9 \%$ were PTMC. Median TSH levels were higher in patients with malignant disease [1.3 (0.7-2.3) mIU/L] as compared to benign cases [1.1 $(0.5-1.9) \mathrm{mIU} / \mathrm{L}],(\mathrm{p}=0.009)$. Median TSH levels were also higher in PTMC [1.4 (0.7-2.4) mIU/L] compared to benign cases [1.1 $(0.5-1.9) \mathrm{mIU} / \mathrm{L}],(\mathrm{p}=0.011)$. Malignancy was more likely with TSH levels above $2.58 \mathrm{mIU} / \mathrm{L}$ [OR:1.6 $(1.0-2.5),(p=0.045)]$. Similarly, malignancy was more likely with TSH levels above the mean TSH level for this study population, $1.8 \pm 3.8 \mathrm{mIU} / \mathrm{L}$ [OR:1.4 $(1.0-2.0) ;(\mathrm{p}=0.029)]$. There was also increased likelihood of PTMC with TSH levels greater than $2.58 \mathrm{mIU} / \mathrm{L}$ [OR: $2.2(1.3-3.8) ;(\mathrm{p}=0.006)]$ and with TSH levels greater than the mean TSH for this study population [OR: $1.7(1.1-2) ;.(\mathrm{p}=0.022)]$. Interestingly, TSH levels did not show association with presence of extrathyroidal extension, perineural/vascular invasion, lymph node metastasis, or increased T-stage. Patients with higher levels of TSH are more likely to develop thyroid cancer, as well as PTMC, but there is no association with aggressiveness of disease. TSH levels influence the development of cancer regardless of others factors affecting TSH, including medication and/or previous thyroid surgery.

\section{Poster 311}

Thyroid Nodules \& Goiter Friday Poster Clinical

\section{COMPLICATIONS OF TOTAL THYROIDECTOMY IN GRAVES DISEASE PATIENTS}

\section{K. Mohsin, E. Kandil}

Surgery, Tulane University School of Medicine, New Orleans, LA

Graves' disease, an autoimmune disease, is the most common cause of hyperthyroidism. Persistent disease requires total thyroidectomy for definitive cure. Total thyroidectomy is an operation with higher risks of complications than unilateral lobectomy due to the involvement of bilateral recurrent laryngeal nerves. We aim to assess if thyroidectomy in Graves' disease patients has a higher rate of complications compared to total thyroidectomy in patients without Graves' disease when performed by high-volume surgeons.

This is a retrospective review of all patients undergoing surgery for Graves' disease and total thyroidectomy at an academic institution by a single-high volume surgeon over a three-year period. Demographic data including age and gender and the type of surgical procedure performed are collected. Pathology results, as well operative complication information is gathered. Rates of complications are compared between cases with and without Graves' disease. A total of 119 thyroidectomies were included. Average patient age was 47.8 and $104(87.4 \%)$ patients were female. 26 $(21.8 \%)$ patients had Graves' disease. One (3.9\%) complication was seen in patients with Graves'disease compared to $2(2.2 \%)$ in patients without Graves' disease $(p=0.632)$. Of the patients without Graves' disease, one patient had anaplastic carcinoma and a very difficult surgery that required a tracheostomy. The single complication in Graves' disease patients was a temporary stretch injury of the recurrent laryngeal nerve that required staging of the operation, and the patient later recovered and received a contralateral left lobectomy. For high-volume surgeons, Graves' disease my not have any effect on intraoperative complications when performing total thyroidectomy. However, the rarity of thyroidectomy complications warrants large-scale studies to further assess the effect of Graves' disease on the safety of thyroid surgery. 


\section{Saturday, October 21, 2017}

\author{
Basic/Translational Oral 23 \\ Thyroid Hormone Action Saturday Basic \\ THYROMIMETICS LOWER CEREBRAL LEVELS \\ OF VERY LONG CHAIN FATTY ACIDS IN MOUSE MODEL \\ OF X-LINKED ADRENOLEUKODYSTROPHY \\ M.D. Hartley, L.L. Kirkemo, T. Banerji, T. Scanlan \\ Physiology and Pharmacology, Oregon Health \& Science University, \\ Portland, OR
}

X-linked adrenoleukodystrophy (X-ALD) is an inborn error of metabolism occurring with a frequency of $1: 17,000$. X-ALD is characterized by adrenal gland dysfunction and central nervous system demyelination. Patients with X-ALD have mutations in $A B C D 1$, a transporter in the peroxisome involved in uptake of very long chain fatty acids (VLCFAs) for degradation. Mutations in $A B C D 1$ block VLCFA degradation resulting in the toxic accumulation of VLCFAs, which leads to the disease symptoms. Thyromimetics represent a promising class of drugs for targeting X-ALD, as thyroid hormone positively regulates $A B C D 2$, a second peroxisomal transporter that can compensate for the genetic defect in $A B C D 1$ to increase VLCFA degradation. A mouse model of X-ALD ( $b b c d l \mathrm{KO})$ with elevated levels of VLCFAs in tissue and plasma was treated with thyroid hormone or sobetirome, a potent thyromimetic, for 1,4 , or 12 weeks. $A b c d 2$ transcript levels were measured using quantitative-PCR. The total levels of C22, C24 and C26 were measured in serum, brain, adrenal glands and testes using GC-MS. In addition, the levels of C26-lysophosphatidylcholine (C26-LPC) were measured in serum and brain using LC-MS/MS. After 7 days of treatment with thyroid hormone or sobetirome, increases in $A b c d 2$ transcript were observed in mouse brain. Both thyroid hormone and sobetirome lowered VCLFA levels in serum and adrenal glands after short term treatment (7 or 28 days), however, the VLCFA levels in brain were unaffected. Chronic treatment (12 weeks) with thyroid hormone or sobetirome was required to induce lowering in brain tissue, which suggested that VLCFAs in the central nervous system have longer half-lives than VLCFAs in the periphery. Thyromimetics lowered both peripheral and cerebral VLCFAs in a mouse model of X-ALD. The data supports a thyromimetic-based strategy for the treatment of X-ALD.

\section{Basic/Translational Oral 24}

Thyroid \& Development Saturday Basic

\section{MCT8-DEPENDENT THYROID HORMONE UPTAKE PROMOTES NEUROGENESIS AND RADIAL CELL MIGRATION CRUCIAL FOR PROPER CORTICAL DEVELOPMENT}

P. Vancamp, V.M. Darras

Biology Department, KU Leuven, Leuven, Belgium

The developing cerebral cortex relies on thyroid hormones (THs) to ensure correct gene transcription via binding to nuclear receptors. Loss of function of the TH transporter monocarboxylate transporter 8 (MCT8) in humans consequently results in an underdeveloped cortex and severe psychomotor disability. The precise origin of these lesions as well as the developmental time window in which MCT8 deficiency causes this phenotype are only partly understood. We focused on the role of MCT8 for neurogenesis and radial cell migration, responsible for creating a layered cortex via an inside-out gradient, two hallmarks of corticogenesis. The chicken optic tectum was used as a model since it develops in a similar way. An MCT8-RNAi vector (or empty vector for controls) was electroporated into the tectal neuro-epithelium on embryonic day 3 (E3) inducing local MCT8 knockdown until E12 when formation of the layered structure is completed. Electroporation in combination with a TH activity reporter construct confirmed decreased in vivo $\mathrm{TH}$ signalling as tested at E6. Early-born type I neuroblasts were labelled at E4 with the S-phase marker EdU. MCT8-RNAi-transfected cells within the EdU-positive population showed an impaired migration from early stages onwards while all EdU-positive cells in the control condition migrated normally. Furthermore, neurogenesis in the MCT8-RNAitransfected population was 3-fold lower than in the untransfected EdU-positive population. Late-born type II neuroblasts were labelled at E6 and MCT8-RNAi-transfected cells initially migrated normally. However, they ultimately failed to reach their proper tectal destination indicating migration was affected at a later stage. They additionally displayed strongly reduced neurogenesis similar to type I neuroblasts. These cytoarchitectural defects due to impaired neurogenesis and migration are compatible with the incapability to form layers and a loss of the normal inside-out gradient. In conclusion, our data suggest that the observed cellular defects are a consequence of reduced TH activity, and trace the origin of cortical damage caused by a dysfunctional MCT8 back to very early stages of development.

\section{Basic/Translational Oral 25}

Thyroid \& Development Saturday Basic

MOLECULAR AND GENETIC ANALYSES OF THE FUNCTION OF THYROID HORMONE RECEPTOR COACTIVATOR SRC3 DURING XENOPUS POSTEMBRYONIC DEVELOPMENT

L. Bao ${ }^{1,2}$, Y. Shibata ${ }^{1}$, L. Fu ${ }^{1}$, Y. Shi ${ }^{1}$

${ }^{1}$ NICHD, National Institutes of Health, Bethesda, MD; ${ }^{2}$ Xian

Jiaotong University, Xi'an, Shanxi, China

Thyroid hormone (T3) is critical for organ metabolism and development. Its effect is mediated by thyroid hormone receptor (TR), which activates gene transcription by recruiting the specific coactivators to T3-inducible promoters when T3 is present. We have been investigating the role of coactivators during vertebrate development by using Xenopus metamorphosis as a model system. We have previously discovered that the steroid receptor coactivator 3 (SRC3) is induced by $\mathrm{T} 3$ treatment of premetamorphic tadpoles, suggesting that T3 induces SRC3, which in turns functions as a TR coactivator to further enhance T3 function during metamorphosis. We have been using transgenic and gene editing tools to test this. Animals and Treatments.

Wild type and transgenic animals expressing a Flag-tagged dominant negative SRC3 (Fdn-SRC3) were treated with the indicated amount of T3. RNA was isolated for quantitative RT-PCR analysis of SRC3 expression. Tissues were isolated for chromatin immunoprecipitation (ChIP) assays with Anti-Xenopus TR, antiacetylated histone H4, anti-Flag M2-agarose, anti-Xenopus SRC3 antibodies. CRISPR/Cas9 system was used to knock out endogenous SRC3 gene.1. SRC3 expression is upregulated in the intestine during natural metamorphosis (stages 54-66).

2. ChIP assay showed tissue- and gene-specific recruitment of SRC3 during T3-induced and natural metamorphosis: SRC3 was recruited to both TR $\beta$ A and TH/bZIP in the intestine but only TH/ bZIP in the tail in a T3-dependent manner.

3. Transgenic expression of F-dnSRC3 inhibits T3-induced and natural metamorphosis as well as T3 activation of gene expression by displacing the endogenous SRC3. 
4. Microinjecting mRNAs for a CRISPR/Cas9 system against SRC3 into fertilized eggs led to the formation of tadpoles with mosaic mutations in the endogenous SRC3 gene. Our molecular analyses showed that SRC3 is activated by T3 and recruited by liganded TR to target genes, and our transgenic studies indicate that coactivator recruitment by TRs is required for gene regulation by T3 and Xenopus metamorphosis. Our Crispr/Cas9 system can mutate the endogenous SRC3 gene and should allow us to generate total knockout animals to determine the role of endogenous SRC3 during metamorphosis.

\section{Basic/Translational Oral 26}

Thyroid Hormone Action Saturday Basic

\section{THYROID HORMONE REGULATES KISS1 GENE EXPRESSION THROUGH PI3K/AKT-MTOR SIGNALING PATHWAY AND SPECIFICITY PROTEIN-1}

G. Chen, J. Wen, L. Cai, L. Wen, Y. Su, Y. Zheng

Endocrinology, Fujian Provincial Hospital, Fuzhou, China

Thyroid function is closely related to reproduction, Thyroid dysfunctions often cause reproductive health problems. Our goal was to investigate whether T3 affects the expression of KiSS1 gene through mTOR regulating the translocation of $\mathrm{Sp} 1$ protein.1. The first part of this study focused on whether T3 regulates KiSS1 gene expression by TR (thyroid hormone receptor) at the level of KiSS1 gene promoter. 2. The second part of this study mainly discussed whether PI3K (phosphatidylinositol 3-kinase)/Akt-mTOR (the mammalian target of rapamycin) signaling pathway is involved in T3-induced KiSS1 Gene Expression. 3. The third part of this study mainly discussed whether PI3K/Akt-mTOR signaling pathway is mediated by Sp1 in T3-induced KiSS1 Gene Expression.1. We showed that $\operatorname{TR} \beta$ siRNA1259 inhibited the T3-induced up-regulation of Kisspeptin protein expression. 2. Wortmannin, LY294002 and Rapamycin results in a decrease of T3-induced KiSS1 Gene expreion. Co-IP studies showed a direct interaction of cytosol-localized TR $\beta 1$ and the p85 $\alpha$ subunit of PI3K in a ligand-independent manner. T3 treatment rapidly increased PI3K activity, which resulted in increased phosphorylation of downstream kinases Akt and mTOR. 3. Deletion analysis of KiSS1 promoter indicates that the T3-regulated increase in promoter activity depends on the Sp1 sites of the proximal promoter $-191 \mathrm{bp} \sim-100 \mathrm{bp}$ region. Overexpression of Sp1 enhanced the activity of KiSS1 gene promoter. The induction of KiSS1 gene promoter activation by Sp1 and T3 was inhibited by Mithramycin A. T3 increased the nuclear proportion of Sp1, whereas LY294002 and rapamycin decreased it. The nuclear $\mathrm{Sp} 1$ abundance was positively correlated with total phospho-serine Sp1. Using both EMSA and ChIP analysis, we confirmed that Sp1 directly binds to proximal $-191 b p \sim-100 b p$ region of the human KiSS1 gene promoter which contains two Sp1 binding site, whereas T3 enhanced the binding, while rapamycin and LY294002 had the opposite effect. PI3K/AktmTOR Signaling pathway was involved in T3-induced KiSS1 Gene Expression. T3-dependent KiSS1 Gene Expression was mediated by PI3K/Akt-mTOR signaling pathway through the interaction of Sp1 protein with the GC-rich motifs of KiSS1 gene promoter.

\section{Basic/Translational Oral 27}

Thyroid Hormone Action Saturday Basic

NON-GENOMIC EFFECTS OF THYROID HORMONES ON MESENCHYMAL STEM CELLS AND ANGIOGENESIS K. Schmohl, M. Dohmann, A. Wechselberger, P. Nelson, C. Spitzweg University Hospital of Munich, LMU Munich, Munich, Germany
Tumour stroma formation is associated with angiogenesis and requires interactions of various different cell types, including endothelial cells (ECs) and mesenchymal stem cells (MSCs). Thyroid hormones (TH) act as non-classical proangiogenic modulators mediated by non-genomic mechanisms via cell surface receptor integrin $\alpha v \beta 3$. The aim of this study was to evaluate the effects of T3 and T4 versus tetrac, a specific inhibitor of $\alpha \mathrm{v} \beta 3$-mediated action of $\mathrm{TH}$, on MSCs in the context of angiogenesis. Primary human umbilical vein ECs (HUVECs) were seeded on Matrigel and tube formation was analysed microscopically. Compared to untreated HUVECs, treatment with primary human bone marrow-derived MSC-conditioned medium stimulated tube formation. Treatment of MSCs with T3 or $\mathrm{T} 4 \pm$ tetrac in the presence of tumour cell-conditioned medium resulted in stimulation of expression of genes associated with angiogenesis as determined by qPCR. After adding supernatant from these prestimulated MSCs to HUVECs in the tube formation assay, significantly enhanced tube formation and, most strikingly, potent inhibition by tetrac were observed. As the vascular endothelial growth factor (VEGF) is a critical angiogenesis mediator, we established a reporter gene system by placing the sodium iodide symporter (NIS) gene under control of the VEGF promoter. MSCs transfected with this construct showed enhanced perchloratesensitive NIS-mediated iodide uptake activity after stimulation with either T3 or T4 that was blocked by tetrac. T3 effects were additionally blocked both by the PI3K pathway inhibitor LY294002 and the ERK1/2 pathway inhibitor RAF265, while T4 effects were only blocked upon RAF265 treatment, supporting integrin $\alpha \mathrm{v} \beta 3$ dependency. Using the NIS reporter gene system, we are currently evaluating tetrac-dependent effects of TH on VEGF expression in MSCs in vivo in an orthotopic hepatocellular carcinoma xenograft mouse model by iodide-124 PET.

Our data suggest that TH stimulate effects of MSCs on angiogenesis in an integrin $\alpha \mathrm{v} \beta 3$-dependent manner, providing further evidence of the critical role of TH in the regulation of angiogenesis and the anti-angiogenic activity of tetrac in the context of tumour stroma formation.

\section{Basic/Translational Oral 28}

Thyroid Hormone Metabolism \& Regulation Saturday

Translational

\section{PROBING THE THYROID PHENOTYPE OF SBP2} DEFICIENCY

H. Fujisawa, J. Fu, X. Liao, A. Dumitrescu

Medicine, The University of Chicago, Chicago, IL

Selenocysteine insertion sequence binding protein 2, SBP2, is required for selenoprotein synthesis. Partial SBP2 deficiency in humans causes selenoproteins deficiencies manifested as a multiorgan syndrome in addition to characteristic thyroid function tests (TFTs). The high serum T4, rT3 and TSH were replicated in our mouse model of induced Sbp2 deficiency, Sbp2 iCKO. Studies on the deiodinase (D) selenoenzymes demonstrate decreased D1 enzymatic activity (EA) in liver and decreased D2 EA and Dio3 expression in cerebrum. We studied the thyroid gland and the metabolism of exogenous L-T4 and L-T3 after chemical suppression of endogenous thyroid hormone (TH) production. Male mice are studied 4 weeks after induction at P30. Serum TFTs, thyroid gland TH content and gene expression are measured. Water containing MMI and perchlorate and low iodine diet are used to suppress the endogenous TH production. After 2 weeks, physiological doses of L-T4 or L-T3 are given for 7 days. Baseline total and free T4 and T3 content of Sbp 2 iCKO thyroids are significantly increased compared to $W t$ in response to the higher TSH levels. While the high thyroidal T4 content is reflected in serum T4, the high thyroidal T3 content is in contrast to the normal circulating T3 levels. 
Diol and other selenoprotein mRNAs are significantly decreased in Sbp 2 iCKO thyroids. L-T4 is able to normalize TSH in $W t$ mice, while TSH is 18 fold higher in Sbp2 iCKO, indicating increased pituitary resistance. Baseline serum T3/T4 ratio of 12.7 and 10 in $W t$ and Sbp2 $i C K O$ decreased to 7.3 and 5.4, respectively, indicating less T3 availability from L-T4 metabolism when endogenous production is suppressed. Exogenous T3 is equally able to suppress TSH in both genotypes at 6 and 16h after last injection. However T3 serum levels are significantly higher in $S b p 2$ iCKO mice $6 \mathrm{~h}$ after last T3 injection, likely due to impaired clearance through 5-deiodination. This study demonstrates high T4 and T3 thyroid gland content in Sbp2 deficiency. The impaired metabolism of L-T4 and L-T3 administered after chemical suppression of endogenous $\mathrm{TH}$ production provides additional information on the thyroid pathophysiology of Sbp2 deficiency.

\section{Clinical Oral 29}

Thyroid Nodules \& Goiter Saturday Clinical

\section{CLINICAL VALIDATION OF THE AFIRMA GENOMIC SEQUENCING CLASSIFIER FOR MEDULLARY THYROID CANCER}

G. Randolph ${ }^{1}$, T.E. Angell ${ }^{2}$, J. Babiarz ${ }^{3}$, N. Barth ${ }^{4,5}$, T. Blevins ${ }^{6}$,

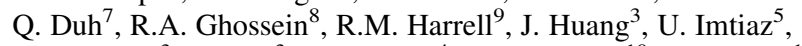
G. Kennedy ${ }^{3}$, S. Kim ${ }^{3}$, R.T. Kloos ${ }^{4}$, V.A. LiVolsi ${ }^{10}$, K.N. Patel ${ }^{17}$, P.M. Sadow ${ }^{11}$, M.H. Shanik ${ }^{12}$, J. Sosa ${ }^{13}$, S.T. Traweek ${ }^{14}$, P.S. Walsh ${ }^{3}$, D. Whitney ${ }^{3}$, M. Yeh ${ }^{15}$, P.W. Ladenson ${ }^{16}$

${ }^{1}$ Department of Otolaryngology, Division of Thyroid and Parathyroid Endocrine Surgery, Massachusetts Eye and Ear Infirmary and Harvard Medical School, Boston, MA; ${ }^{2}$ Department of Medicine, Division of Endocrinology, Diabetes, and Hypertension, Brigham and Women's Hospital and Harvard Medical School, Boston, MA; ${ }^{3}$ Department of Research \& Development, Veracyte, Inc., South San Francisco, CA; ${ }^{4}$ Department of Medical Affairs, Veracyte, Inc., South San Francisco, CA; ${ }^{5}$ Department of Clinical Affairs, Veracyte, Inc., South San Francisco, CA; ${ }^{6}$ Texas Diabetes and Endocrinology, Austin, TX; ${ }^{7}$ Department of Surgery, Section of Endocrine Surgery, University of California San Francisco, San Francisco, CA; ${ }^{8}$ Department of Pathology, Division of Head and Neck Pathology, Memorial Sloan-Kettering Cancer Center, New York, NY; ${ }^{9}$ The Memorial Center for Integrative Endocrine Surgery, Boca Raton, FL; ${ }^{10}$ Department of Pathology and Laboratory Medicine, Anatomic Pathology Division, University of Pennsylvania School of Medicine, Philadelphia, PA; ${ }^{11}$ Department of Pathology, Head and Neck Pathology Subspecialty, Massachusetts General Hospital and Harvard Medical School, Boston, MA; ${ }^{12}$ Endocrine Associates of Long Island, Smithtown, NY; ${ }^{13}$ Department of Surgery, Section of Endocrine Surgery, Duke University Medical Center, Durham, NC; ${ }^{14}$ Thyroid Cytopathology Partners, Austin, TX; ${ }^{15}$ Department of Surgery, Endocrine Surgery Program, UCLA David Geffen School of Medicine, Los Angeles, CA; ${ }^{16}$ Department of Medicine; Division of Endocrinology, Diabetes and Metabolism, Johns Hopkins University, Baltimore, MD; ${ }^{17}$ Department of Otolaryngology-Head and Neck Surgery, Division of Endocrine Surgery, NYU Langone Medical Center, New York, NY

Cytopathological evaluation of thyroid fine-needle aspiration biopsy (FNAB) specimens can fail to raise preoperative suspicion of medullary thyroid cancer (MTC), missing more than one-half of these uncommon, yet aggressive malignancies. Serum calcitonin screening for MTC in thyroid nodule patients is controversial because this has a high false-positive rate. The Afirma Genomic Sequencing Classifier (GSC) identifies, by using RNA sequencing and machine learning algorithms, genomically benign thyroid nodules among those with indeterminate FNAB to prevent unnecessary diagnostic surgery. Additional cassettes are used to detect the molecular signatures of specific neoplasms that further alter patient care. An MTC classifier cassette is included in the GSC to provide additional pre-operative clinical information in a single test. Here we report the clinical performance of the MTC classifier integrated into the GSC. Algorithm training was performed with a set of 483 FNAs (21 MTC and 462 non-MTC). An additional 97 tissues were used in feature selection, but not model training. A support vector machine (SVM) classifier was developed using 108 differentially expressed genes, which includes the five genes in the Afirma GEC medullary thyroid cassette. The final classifier was blindly tested on 211 independent FNAs, which included 21 MTC and 190 non-MTC from benign and malignant neoplasms. The classifier had 100\% sensitivity [21/21 MTC FNAs correctly called positive; CI $=83.9-100 \%]$ and $100 \%$ specificity [190/190 non-MTC FNAs correctly called negative; $\mathrm{CI}=98.1-100 \%$ ]. All positive samples had clinical/surgical confirmation of MTC, while all negative samples were negative for MTC on surgical pathology. The accurate preoperative genomic identification of MTC usually alters patient care by solidifying the need for timely, more thorough surgery, and necessitating MTC specific preoperative evaluations, including screening for concomitant pheochromocytoma and primary hyperparathyroidism.

\section{Clinical Oral 30}

\section{Thyroid Cancer Saturday Clinical \\ ULTRASOUND-GUIDED RADIOFREQUENCY ABLATION OF LOW-RISK PAPILLARY THYROID MICROCARCINOMA: A PROSPECTIVE STUDY ON 421 PATIENTS}

Y. Luo, M. Zhang

General Hospital of Chinese PLA, Beijing, China

Low risk papillary thyroid microcarcinoma (PTMC) has a high incidence and a good prognosis. Surgical operation to all PTMC has been questioned as over treatment and active surveillance is recommended as an alternative. However, some patients have heavy mental burden and minimally invasive treatment could be used to eliminate PTMC and reduce the tumor related risk of growth and metastasis. The objective of this study was to evaluate the efficacy and safety of ultrasound (US)-guided radiofrequency ablation (RFA) of low-risk PTMC on a large scale of patients with over 1 year follow-up.

421 patients with 440 low risk PTMC were included in this study. US and contrast-enhanced ultrasound (CEUS) examinations were performed before ablation. RFA was performed using the movingshot technique. The ablation area exceeded the tumor edge to prevent marginal residue and recurrence. Patients were followed at 1, 3, 6, and 12 months and every six months thereafter. US and CEUS examinations were used to evaluate the ablation area and cervical lymph nodes. The mean volume reduction ratio (VRR) was $0.58 \pm 0.09,0.79 \pm 0.07,0.87 \pm 0.05,0.94 \pm 0.04$, and $0.96 \pm 0.01$ at 1 , $3,6,12$, and 18 months after RFA, respectively. Of all the nodules, $172(39.1 \%)$ resolved in six months, and $415(94.3 \%)$ resolved in 12 months. No residual lesion was detected in ablation area. 82 patients were followed up for more than 3 years and the rest patients were followed up for at least 1 year. During follow up, 4 patients had biopsy proved recurrent PTMC in other parts of thyroid tissue, which had diameter of $2 \mathrm{~mm}-3 \mathrm{~mm}$ and received a second RFA. 5 patients had suspicious thyroid lesions less than $2 \mathrm{~mm}$ and continued follow up. 4 patients had biopsy proved cervical lymph node metastasis and received RFA of the lymph nodes. No distant metastasis was detected in these patients. And no major complications were encountered.

RFA can effectively eliminate low risk PTMC with a very small complication rate. Very low recurrent PTMC and cervical lymph nodes were detected, which could be treated by second RFA. RFA may be an alternative strategy for the treatment of low risk PTMC, especially in patients with heavy mental burden. 


\section{Clinical Oral 31}

Thyroid Cancer Saturday Clinical

THYROGLOBULIN DOES NOT CORRELATE WITH RESPONSE IN RADIOACTIVE IODINE REFRACTORY (RAIR) BRAF MUTATED PAPILLARY THYROID CANCER (PTC) TREATED WITH DABRAFENIB ALONE OR IN COMBINATION WITH TRAMETINIB

B. Konda ${ }^{1}$, F. Nabhan ${ }^{1}$, L. Wei ${ }^{1}$, N. Busaidy ${ }^{2}$, L.J. Wirth ${ }^{3}$, G.A. Daniels ${ }^{4}$, J.A. De Souza ${ }^{5}$, C.D. Timmers ${ }^{1}$, J.L. Sexton ${ }^{1}$, M. Beshara ${ }^{1}$, D. Nichols ${ }^{2}$, N. Snyder ${ }^{3}$, C.E. Devine ${ }^{2}$, M.H. Shah ${ }^{1}$ ${ }^{1}$ The Ohio State University Comprehensive Cancer Center, Columbus, $\mathrm{OH} ;{ }^{2} \mathrm{MD}$ Anderson Cancer Center, Houston, TX; ${ }^{3}$ Massachusetts General Hospital, Boston, MA; ${ }^{4}$ University of California, San Diego, Moores Cancer Center, La Jolla, CA; ${ }^{5}$ The University of Chicago, Chicago, IL

Serum thyroglobulin (Tg) is a commonly used biomarker in PTC that correlates with response to several antiangiogenic agents. We evaluated the correlation between $\mathrm{Tg}$ and treatment response in patients (pts) treated with BRAF inhibitor (BRAFi) dabrafenib \pm MEK inhibitor (MEKi) trametinib in RAIR PTC, as part of a randomized multicenter phase II study of dabrafenib ( $\operatorname{arm}$ A) versus dabrafenib + trametinib (arm B) in BRAF mutated PTC. Tg, anti-Tg antibodies (Abs), TSH, and CT or MRI of neck, chest, and abdomen were done at baseline and every 8 weeks on study. Treatment response was assessed using RECIST v1.1. Minor response (MR) was defined as a $20-29 \%$ decrease in the sum of target lesion diameters compared to baseline. Complete response (CR)+ partial response (PR)+ MR constituted objective response (OR). Intra-pt $\mathrm{Tg}$ levels were considered evaluable when the corresponding TSH levels had a variation no greater than $0.5 \mathrm{mIU} / \mathrm{L}$ and anti-Tg Abs were undetectable. The association between median $\% \mathrm{Tg}$ change from baseline and response was studied using Kruskal-Wallis test. Of 53 enrolled pts, high OR rates of 50\% in Arm A and 54\% in Arm B were observed. Twenty-one ( 8 in $\operatorname{arm~A,~} 13$ in am B) of 53 pts were evaluable for $\% \mathrm{Tg}$ change. The first evaluable response (FER) was defined as the first best response [CR, PR, MR, stable disease (SD), or progressive disease (PD)] for which evaluable intra-pt Tg levels were available. Of 21 pts with FER, 7 (33\%), 13 (62\%), and $1(5 \%)$ had OR (PR+MR), SD, and PD respectively. Of 7 pts with OR, $5(71 \%)$ had increase [\% median (range): 170 (126-673)] while only $2(29 \%)$ had decrease [\%median (range): 81 (67-94)] in Tg from baseline. Of 13 pts with SD, $8(62 \%)$ had increase [\% median (range: $233(23-1571)$ ] while $5(38 \%)$ had decrease [\% median (range): 51 (21-93)] in Tg from baseline. The median \% Tg change was not significantly different between the two groups [p,0.97]. In summary, \% Tg change from baseline does not correlate with radiologic response in BRAFi \pm MEKi treated RAIR PTC, and cannot be used as a reliable therapeutic biomarker in these pts. The differentiating nature of BRAFi/MEKi might explain the increase in $\mathrm{Tg}$ even in the setting of significant tumor shrinkage with dabrafenib \pm trametinib treatment.

\section{Clinical Oral 32}

Thyroid Cancer Saturday Clinical

AGE AND GENDER DISTRIBUTION OF THYROID NODULAR LESIONS FOUND IN THYROID ULTRASOUND EXAMINATION WITHIN THREE YEARS AFTER THE NUCLEAR ACCIDENT: THE FUKUSHIMA HEALTH MANAGEMENT SURVEY

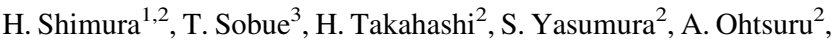
S. Midorikawa ${ }^{2}$, S. Suzuki ${ }^{2}$, T. Fukushima ${ }^{4}$, S. Suzuki ${ }^{4}$, H. Ohto ${ }^{2}$, S. Yamashita ${ }^{2}$
${ }^{1}$ Department of Laboratory Medicine, Fukushima Medical University, Fukushima, Fukushima, Japan; ${ }^{2}$ Radiation Medical Science Center for the Fukushima Health Management Survey, Fukushima Medical University, Fukushima, Fukushima, Japan; ${ }^{3}$ Department of Environmental Medicine and Population Sciences, Osaka University, Suita, Osaka, Japan; ${ }^{4}$ Department of Thyroid and Endocrinology, Fukushima Medical University, Fukushima, Fukushima, Japan

Although childhood thyroid cancer is of great concern after the Fukushima Nuclear Power Plant accident, analytical data on thyroid ultrasound examination in children is quite limited. We analyzed the precise age and sex distribution of findings from the thyroid ultrasound examinations of children and adolescents, which were conducted as the first round examination of the Fukushima Health Management Survey (FHMS). From October 2011 through March 2014, 294,905 participants aged 18 years or younger voluntarily attended a thyroid ultrasound examination in the first round of the FHMS. A secondary confirmatory examination was performed in 2,032 subjects. Age, sex, and size distribution of findings from the firstround examinations were analyzed. The detection rates of thyroid nodules were $1.0 \%$ and $1.7 \%$ in males and females, respectively. A proportional increment in detection rate of thyroid nodules with age was observed in either gender. An evident gender difference was observed in subjects aged 10 years or older. There were age-dependent increases in the median of nodule diameters in both genders, in which no gender differences were evident. 746 male and 1478 female participants were recommended to have a secondary confirmatory examination. In total, 112 cases (38 in males and 74 in females) were cytologically diagnosed as malignancy or suspected malignancy. This detection rate was 0 up to the age of 13 years for males and 8 years for females, and then increased with age, constantly higher in females than males. There was an age-dependent increase in the adjusted detection rates of malignant nodules in three categories $(5.1-10.0 \mathrm{~mm}, 10.1-$ $20.0 \mathrm{~mm}$, and $\geq 20 \mathrm{~mm}$ ), but that in the $5.1-10.0 \mathrm{~mm}$ category reached a plateau in the $\geq 20$ group. The thyroid cancer detection rate was clarified for the first time and the proportion of those with thyroid nodules and cysts varied substantially by age group under a quality-controlled ultrasound examination. The results of this study will make a valid contribution to future epidemiological researches on nodular thyroid diseases in children, adolescents, and young adults.

\section{Clinical Oral 33}

\section{Thyroid Cancer Saturday Translational}

\section{US PREDICTION OF HIGHLY AGGRESSIVE TERT} PROMOTER-MUTATED PAPILLARY THYROID CANCER T. Kim, C. Ki, S. Hahn, Y. Oh, S. Kim, J. Chung, J. Shin Samsung Medical Center, Seoul, Korea (the Republic of)

Telomerase reverse transcriptase (TERT) promoter mutations are found in highly aggressive thyroid malignancies. Our aim was to define the ultrasonographic (US) features of TERT promoter-mutated papillary thyroid cancer (PTC) and to evaluate their predictive performances. US findings were reviewed for 185 patients with surgically confirmed PTC between 1994 and 2004. Genomic DNA to identify TERT promoter mutations was extracted from archived surgical specimens. Logistic regression analysis was performed to compare clinical factors and US findings between TERT promotermutated and wild-type PTCs. A TERT promoter mutation was detected in $8.1 \%$ (15 of 185) of specimens from PTC patients with a strong trend toward increasing age. Nonparallel orientation and microlobulated margin were independent US findings for predicting TERT promoter-mutated PTC in patients over 50 years (odds ratio [OR] 5.898, 95\% confidence interval [95\% CI] 1.092-31.851, 
$P=0.039$ for orientation; OR 5.813, 95\% CI 1.320-25.602, $P=0.020$ for margin). Prevalence of TERT promoter mutations increased to $50.0 \%$ in PTC patients older than 50 years with both US findings and was $0 \%$ in patients without either finding. For identifying TERT promoter-mutated PTC, US had $64.3 \%$ sensitivity, $80.8 \%$ specificity, $50.0 \%$ positive predictive value and $88.4 \%$ negative predictive value. TERT promoter-mutated PTC could be suggested by the US features of nonparallel orientation and microlobulated margin in patients older than 50 years. Prebiopsy recognition of this unique US pattern would mandate the nodule fall into a "biopsy suggested" category.

\section{Clinical Oral 34}

Thyroid Cancer Saturday Clinical

\section{PATIENT AGE-ASSOCIATED MORTALITY RISK IS DIFFERENTIATED BY THE BRAF V600E STATUS IN PAPILLARY THYROID CANCER}

\section{Shen, G. Zhu, R. Liu, M. Xing, T. Group}

Laboratory for Cellular and Molecular Thyroid Research, Division of Endocrinology, Diabetes \& Metabolism, Department of Medicine, Johns Hopkins University School of Medicine, Baltimore, MD

For the last 65 years, patient age has been treated as a major mortality risk factor in papillary thyroid cancer (PTC), which is emphasized again in the recent 8th edtion of AJCC staging system, but whether it is applicable in all patients is untested. This issue was addressed in this large multicenter study by investigating the differentiating role of $B R A F \mathrm{~V} 600 \mathrm{E}$ mutation in patient age-associated mortality risk of PTC. Comparative study of the relationship between patient age at the diagnosis of PTC and PTC-specific mortality with respect to $B R A F$ status in 2,638 patients (623 males and 2,015 females) with a median age of 46 years (interquartile range [IQR], 35-58 years) at diagnosis and a median clinical follow-up time of 58 months (IQR, 26-107 months) at 11 medical centers in 6 countries. There was a linear association between patient age and mortality in patients with $B R A F$ V600E, but not in patients with wild-type $B R A F$ where mortality remained low and flat as patient age increased. There was a rapid progressive decline in Kaplan Meier survival curves with increasing patient age in patients with $B R A F$ V600E, particularly rapidly after 45 years, being sharpest in patients $\geq 65$ years old, but the curves remained un-declined in patients with wild-type $B R A F$, even beyond age 65 years. With adjustment for classical clinicopathological characteristics of patient sex, tumor size, extrathyroidal extension, lymph node metastasis, distant metastasis, and radioactive iodine treatment, there was an independent linear association between age and mortality in patients with $B R A F$ V600E, but not in patients with wild-type $B R A F$. Similar results were obtained when only patients with conventional variant of PTC were analyzed. The long-observed patient age-associated mortality risk in PTC is $B R A F$ V600E-dependent; patient age is a strong, continuous and independent mortality risk only in patients with $B R A F \mathrm{~V} 600 \mathrm{E}$ but not in the more commonly seen patients with wild-type $B R A F$. These results question the conventional use of old patient age as a general high-risk factor and call for differentiation between $B R A F$ V600E and wildtype $B R A F$ when applying it to risk stratification and management of PTC.

\section{Poster \& Trainee Short Oral 312}

Thyroid Hormone Metabolism \& Regulation Saturday Highlighted Trainee Poster \& Short Oral Basic

\section{DE NOVO TRI-IODOTHYRONINE (T3) FORMATION}

C.E. Citterio, B. Veluswamy, P. Arvan

Division of Metabolism, Endocrinology \& Diabetes. Department of Internal Medicine, University of Michigan, Ann Arbor, MI
De novo thyroid hormonogenesis requires iodination and coupling of two Tyr residues within Thyroglobulin (TG) - a dimeric $660 \mathrm{kDa}$ secretory glycoprotein. The acceptor hormonogenic site is a diiodotyrosine (DIT) and the donor is a monoiodotyrosine (MIT) or DIT for T3 and T4 synthesis, respectively. In mouse TG (mTG), Y2519 and Y2552 have been proposed as T3 formation sites, and based on evolutionary conservation, Y2744 is also an expected T3 hormonogenic site. Y2744 is located close to the helices within the Cholinesterase-like (ChEL) domain involved in noncovalent TG homodimerization. We hypothesize that de novo $\mathrm{T} 3$ formation involves not only intra-monomer MIT-DIT coupling, but also inter-monomer coupling within the TG homodimer. To confirm sites involved in T3 formation, we bioengineered recombinant $\mathrm{Y}>\mathrm{F}$ mutants, performed non-radioactive enzymatic iodinations of TG, and monitored de novo T3 formation by immunoblotting with $\mathrm{mAb}$ anti-T3 in parallel with anti-TG $\mathrm{Ab}$. To examine the potential for inter-monomer side-chain coupling, we created $\mathrm{Y}>\mathrm{C}$ mutants and an $\mathrm{N}$-terminally tagged version of the TG ChEL region (Flag-ChEL). A triple Y $>$ F TG mutant (2519/2552/ $2744)$ and a double mutant (2552/2744) significantly decreased de novo T3 formation in TG, while a control substitution TG-Y2566F was without effect. Interestingly, Flag-ChEL by itself did not exhibit efficient $\mathrm{T} 3$ formation, suggesting that structural features provided by upstream regions of TG facilitate this process. TG-Y2744C formed a novel intermolecular coupling (disulfide bond), indicating intimate interaction of the 2744 side chains from opposing monomers within the TG homodimer, whereas TG-Y2519C and TG-Y2552C (or even double mutants) were unable to form disulfide-linked dimers. Even the 3D structure of TG remains unknown, these studies suggest that the most evolutionarily conserved T3 formation site involves an MIT-DIT coupling of Y2744 by closely related TG monomers within the TG homodimer. Upstream regions also stabilize the TG homodimer, and this may account for how these regions assist in the process of new T3 formation, which occurs primarily within and around the TG ChEL domain.

\section{Poster \& Trainee Short Oral 313}

Thyroid Nodules \& Goiter Saturday Highlighted Trainee Poster \& Short Oral Clinical

\section{DIFFERENTIAL GROWTH RATES OF BENIGN VERSUS MALIGNANT THYROID NODULES} C.M. Vyas ${ }^{1}$, Z. Wang ${ }^{1}$, M. Medici ${ }^{2}$, E. Marqusee ${ }^{1}$, M.I. Kim ${ }^{1}$, P. Larsen ${ }^{1}$, E.K. Alexander ${ }^{1}$, T.E. Angell ${ }^{1}$

${ }^{1}$ Thyroid Section, Endocrinology, Diabetes and Hypertension, Brigham and Women's Hospital, Boston, MA; ${ }^{2}$ Erasmus Medical Center, Rotterdam, Netherlands

Thyroid nodule growth was once considered concerning for malignancy, but data demonstrating benign nodule growth questioned the utility of this parameter in clinical practice. To date, no prospective data have sonographically analyzed if growth in malignant nodules differs from that in benign nodules. If so, growth may again prove an important parameter to help guide nodule evaluation. We performed a prospective cohort analysis of patients with tissue proven diagnoses of benign or malignant disease, who had received repeated preoperative ultrasound assessments that were $>6$ months apart. We compared the change in largest dimension per year $(\mathrm{mm} / \mathrm{yr})$ between benign and malignant nodules. Regression models were used to evaluate predictors of nodule growth.135 malignant nodules met inclusion and were compared to 1397 benign nodules, with a median duration of pre-operative ultrasound follow-up of 21.5 and 21.9 months, respectively. On average, malignant nodules grew faster than benign nodules $(1.3 \pm 3.5$ vs. $-0.1 \pm 2.6 \mathrm{~mm} / \mathrm{yr}, \mathrm{p}<0.001)$, and two-thirds $(66 \%)$ of malignant nodules grew while $57 \%$ of benign nodules decreased in size $(\mathrm{p}<0.001)$. Malignant nodules were 
significantly more likely to grow by $>2 \mathrm{~mm} / \mathrm{yr}(\mathrm{RR}=2.2,95 \% \mathrm{CI}$ [1.6-3.0], $\mathrm{p}<0.001)$. For benign nodules, advancing age $(\mathrm{p}=0.002)$ and solid parenchyma $(\mathrm{p}<0.001)$ were significant predictors of growth. For malignant nodules, the only clinical factor associated with growth was TSH, for which each unit increase raised the odds of growing $>2 \mathrm{~mm} / \mathrm{yr}(\mathrm{OR}=1.9,95 \% \mathrm{CI}[1.1-3.2], \mathrm{p}=0.015)$. Notably, compared to the follicular variant of PTC, the classical variant of PTC showed similar growth, while aggressive PTC variants and non-PTC thyroid cancers were more likely to grow $>2 \mathrm{~mm} / \mathrm{yr}(\mathrm{OR}=16.1,95 \% \mathrm{CI}[3.0-87.0]$, $\mathrm{p}=0.001 ; \mathrm{OR}=3.9,95 \%$ CI [1.04-14.4], $\mathrm{p}=0.043$, respectively). Malignant nodules, especially higher risk phenotypes, grow faster than benign nodules. As growth $>2 \mathrm{~mm} / \mathrm{yr}$ significantly predicts malignant compared to benign disease, this clinical parameter should be re-introduced as an additional factor contributing to pre-operatively risk assessment. Conversely, nodules unchanged or smaller over time are likely benign or lower-risk carcinoma.

\section{Poster \& Trainee Short Oral 314}

Thyroid Hormone Metabolism \& Regulation Saturday Highlighted Trainee Poster \& Short Oral Case Report

\section{THE FIRST REPORT OF A HOMOZYGOUS HSA GENE MUTATION CAUSING FAMILIAL DYSALBUMINEMIC HYPERTHYROXINEMIA}

M. Mimoto ${ }^{1}$, N. Scherberg ${ }^{1}$, A. Karaca ${ }^{2}$, A. Dumitrescu ${ }^{1}$, S. Refetoff ${ }^{1}$

${ }^{1}$ Endocrinology, Diabetes and Metabolism, University of Chicago, Chicago, IL; ${ }^{2}$ Endocrine Unit-Thier Bldg. 1050, Massachusetts General Hospital, Boston, MA

Background: FDH is an autosomal dominant condition that causes euthyroid hyperthyroxinemia. It is due to gain-of-function mutations in the human serum albumin (HSA) gene. It is important to consider FDH in the evaluation of patients who present with abnormal thyroid function tests to avoid unnecessary treatment in these individuals.

Objective: To identify the etiology of abnormal thyroid function tests in a large consanguineous family of Anatolian Turkish descent. Comprehensive thyroid function testing, sequencing of candidate genes in 14 family members, and thyroid hormone-binding analysis of affected individuals. The proband, a 23 year-old woman, referred for evaluation of hirsutism, was found to have asymptomatic high free T4 and normal TSH, suggestive of resistance to thyroid hormone, however no mutations were found in the THRB gene. Eight of the 14 family members had similar abnormal thyroid function tests and were found to harbor a missense mutation in the HSA gene resulting in a single amino acid substitution (R218H). This is the most commonly identified HSA variant causing FDH. Affected individuals had elevated serum total T4 and rT3 due to increased binding affinity of the mutant HSA to these iodothyronines, but normal dialyzable free T4, total T3, and TSH. One the 8 affected subjects was found to be homozygous for the R218H mutation. Thyroid function tests from this individual showed abnormalities similar in magnitude to those of the heterozygous family members. Isoelectric focusing of serum identified the mutant HSA protein, which bound all $\mathrm{T} 4$ present in exclusion to the normal HSA in heterozygotes. To our knowledge, this is the first report of a homozygous HSA mutation causing FDH. Interestingly, the magnitude of iodothyronine elevation in the homozygous individual was similar to that observed in heterozygous family members. This can be explained by the observation that T4 exclusively binds the R218H HSA molecule, even in heterozygotes with one wildtype HSA allele. As albumin is present in serum in 2,000- and 800,000-fold molar excess relative to T4 and rT3, respectively, the mutant HSA functionally supersedes wildtype HSA in heterozygotes, even though it represents only half of the total HSA.

\section{Poster 315}

Disorders of Thyroid Function Saturday Trainee Poster Contest Basic

\section{THYROCYTES FROM PATIENTS WITH GRAVES' DISEASE SHOW DECREASED TSH RESPONSIVENESS DESPITE NORMAL TSHR EXPRESSION}

S.J. Morgan, C.C. Krieger, S. Neumann, M. Gershengorn

NIDDK, National Institutes of Health, Bethesda, MD

Activation of the G protein-coupled TSH receptor (TSHR) on thyrocytes stimulates cAMP production and induces expression of thyroid-specific genes (thyroglobulin [TG], thyroperoxidase [TPO], sodium-iodide symporter [NIS], and type II iodothyronine deiodinase [DIO2]) to produce thyroid hormones. TSHR is the main target of thyroid-stimulating auto-antibodies (TSAbs) in patients with Graves' disease (GD) in whom persistent, unregulated TSAb stimulation of TSHR leads to hyperthyroidism. Previous reports do not indicate functional differences between human thyrocytes from GD patients (GThy) and normal tissue (NThy). Human thyrocytes were isolated from normal thyroid tissue from patients undergoing thyroidectomy for thyroid cancer or GD at the National Institutes of Health Clinical Center. Cells were treated with TSH \pm IGF- 1 and examined for various signaling and functional outcomes. TSH responses are significantly altered in GD. GThy produce little or no TSH-stimulated cAMP, although the adenylyl cyclase activator forskolin and betaadrenoceptor agonist isoproterenol responses are not different from NThy. Despite a lack of cAMP, TSH does induce thyroid-specific gene expression in GThy; however, the efficacy and potency of TSH are significantly reduced. Interestingly, insulin-like growth factor 1 (IGF-1) does not affect cAMP generation but has a synergistic effect on TSH stimulation of all four genes in GThy and normalizes GThy gene expression (all genes) and TSH potencies (TG, TPO, and NIS) to NThy levels. These differences between NThy and GThy are present during continuous culturing of cells through 10 passages even though no TSAbs are present. No differences are seen between NThy and GThy in mRNA expression of any $\mathrm{G}$ proteins, major $A D C Y$ isoforms, bARR1/2, EPAC1/2, or IGF-1R. Although TSHR mRNA is reduced, total cell expression of TSHR protein is not different. Surface expression of TSHR and TSH binding determined by flow cytometry are also equivalent between cell types. The pathophysiology of GD alters TSHR-regulated function of human thyrocytes in a heritable manner, leading to reduced TSH responsiveness that is normalized for gene expression by concomitant IGF-1 treatment.

\section{Poster 316}

Iodine Uptake \& Metabolism Saturday Trainee Poster Contest Basic

\section{THE ROLE OF THE RENAL NA ${ }^{+} / I^{-}$SYMPORTER (NIS)} IN IODIDE METABOLISM AND THYROID FUNCTION

A. Reyna-Neyra, G. Ferrandino, R.R. Kaspari, N. Carrasco

Cellular and Molecular Physiology, Yale University, New Haven, CT

The sodium/iodide symporter (NIS), a member of solute carrier family 5 , mediates active iodide $\left(\mathrm{I}^{-}\right)$uptake in the thyroid, the first step in the biosynthesis of the thyroid hormones (THs), of which iodine is an essential constituent. $\mathrm{I}^{-}$is a highly scarce nutrient supplied only in the diet, and $\mathrm{I}^{-}$deficiency is associated with thyroid disorders, including hypothyroidism and, in some cases, severe mental retardation. The THs regulate intermediary metabolism at all ages. The excretion and reabsorption of $\mathrm{I}^{-}$by the kidney, where NIS may play a role, are crucial for adequate $\mathrm{TH}$ secretion and for maintaining euthyroid conditions. To investigate the role of NIS in 
the renal proximal tubule, we first generated a conditional knock-out mouse model in which exons 6 and 7 of the Slc5a5 gene (which encodes NIS) were flanked by LoxP sites (Slc5a $\left.5^{\text {flox } / f l o x}\right)$. Slc $5 a 5^{\text {flox/flox }}$ mice were then crossed with $\gamma$ GT-Cre mice, in which CRE recombinase expression is driven by the gamma-glutamyl transpeptidase promoter $\left(\gamma G T\right.$-CRE). The result was NIS ${ }^{\text {flox/flox }} \gamma$ GT-CRE mice. Both strains of mice were fed either a regular chow diet (CD, $6 \mu \mathrm{g} \mathrm{I} \mathrm{I}^{-} / \mathrm{g}$ food) or a diet supplying the minimum amount of $\mathrm{I}^{-}$required daily (MID, $0.15 \mu \mathrm{g} \mathrm{I}^{-} / \mathrm{g}$ food) for 30 days. In the MID condition, the immunohistochemical analysis showed basolateral NIS staining in the follicular thyroid cells of Slc5a $5^{\text {flox/flox }}$ and Slc5a $5^{\text {flox/flox }} \gamma G T$ Cre mice, and apical NIS staining in the renal proximal tubules of Slc5a $5^{\text {flox/flox }}$ mice only. Serum $\mathrm{T}_{3}$ and $\mathrm{T}_{4}$ levels were significantly lower in Slc5a5 flox/flox $\gamma G T$-Cre mice $(2.1 \pm 0.5 \mu \mathrm{g} / \mathrm{dL})(p<0.05)$ than in $S l c 5 a 5^{\text {flox/flox }}$ mice $(3.6 \pm 0.2 \mu \mathrm{g} / \mathrm{dL})$. Thirty percent more $\mathrm{I}^{-}$was excreted in the urine of Slc5a5 floxfflox $\gamma G T$-Cre mice than in that of control mice. In contrast, the $\mathrm{I}^{-}$concentration in serum from Slc5a $5^{\text {floxflox }} \gamma G T$-Cre mice was less than that in serum from control mice. These results suggest that NIS plays a key role in $\mathrm{I}^{-}$reabsorption in the kidney and may thus be critically important for $\mathrm{I}^{-}$homeostasis.

\section{Poster 317}

Iodine Uptake \& Metabolism Saturday Trainee Poster Contest Basic

REGIONAL HYPERTHERMIA FOR OPTIMIZATION OF EFFICACY AND TUMOR SELECTIVITY OF MESENCHYMAL STEM CELL (MSC)-MEDIATED SODIUM IODIDE SYMPORTER (NIS) GENE THERAPY M. Tutter ${ }^{1}$, C. Schug ${ }^{1}$, K. Schmohl ${ }^{1}$, S. Urnauer ${ }^{1}$, N. Schwenk ${ }^{1}$, W. Lokerse ${ }^{2}$, L. Lindner ${ }^{2}$, P. Nelson ${ }^{1}$, C. Spitzweg ${ }^{1}$

${ }^{1}$ Medical Clinic and Policlinic IV, Klinikum Großhadern, LMU

Munich, Munich, Germany; ${ }^{2}$ Medical Clinic III, Klinikum

Großhadern, LMU Munich, Munich, Germany

The tumor homing capacity of mesenchymal stem cells (MSCs) provides the basis for a "Trojan horse" therapy approach in which MSCs act as non-viral gene transfer vectors to transport a therapeutic agent specifically into the tumor stroma. Due to its dual role as reporter and therapy gene, the sodium iodide symporter (NIS) allows detailed noninvasive imaging of transgene expression and highly effective application of therapeutic radionuclides. To improve the effectiveness and tumor selectivity of MSC-mediated gene therapy, we are investigating the combination of regional hyperthermia with NIS gene therapy. As hyperthermia is able to induce the secretion of immunomodulatory chemokines and cytokines that are well-known attractants of MSCs, we aimed to enhance the selective migration of MSCs to the tumor stroma and thereby trigger targeted delivery of the NIS gene to the tumor with the help of heat treatment. Human hepatocellular carcinoma cells (Huh7) were heat-treated at $41^{\circ} \mathrm{C}$ for 60 minutes, followed by incubation at $37^{\circ} \mathrm{C}$ for $0-48 \mathrm{~h}$. Chemokine mRNA levels were then analyzed by quantitative real-time PCR (qPCR). In an in vivo Huh7 subcutaneous xenograft mouse model we applied hyperthermia locally to the tumor for 1 hour at $41^{\circ} \mathrm{C}$ or as control at $37^{\circ} \mathrm{C}$. Following thermostimulation we systemically injected NIS-transfected MSCs driven by the unspecific CMV-promoter (CMV-NIS-MSCs) and assessed tumoral iodide accumulation by ${ }^{123} \mathrm{I}-$ scintigraphy. Preliminary results indicate a substantial increase in expression levels of chemokines and growth factors by Huh7 cells after heat exposure, such as VEGF, PDGF- $\beta$, HGF, EGF and FGF, which have been shown to be involved in MSC migration. Following application of regional hyperthermia, we observed a significantly increased uptake of ${ }^{123} \mathrm{I}$ in tumors of heat-treated animals $\left(41^{\circ} \mathrm{C}\right)$ compared to control animals $\left(37^{\circ} \mathrm{C}\right)$, demonstrating a hyperthermia-enhanced stimulation of MSC recruitment to the tumor stroma. In summary, we have demonstrated heat-induced stimulation of selective MSC migration towards tumors. The combination of MSC-mediated NIS gene therapy with regional hyperthermia therefore opens the exciting prospect of maximizing the therapeutic efficacy of the NIS gene therapy.

\section{Poster 318}

Iodine Uptake \& Metabolism Saturday Trainee Poster Contest Basic

AN INVESTIGATION INTO SODIUM-IODIDE SYMPORTER (NIS) DIMERIZATION AND ITS IMPACT ON RADIOIODIDE UPTAKE IN THYROID CANCER R.J. Thompson ${ }^{1,2}$, A. Fletcher ${ }^{1,2}$, H. Nieto ${ }^{1,2}$, M. Alshahrani ${ }^{1,2}$, K. Baker ${ }^{1,2}$, J.W. Mueller ${ }^{1,2}$, N.H. Fine ${ }^{1,2}$, D.J. Hodson ${ }^{1,2}$, M.L. Read ${ }^{1,2}$, K. Boelaert ${ }^{1,2}$, V.E. Smith ${ }^{1,2}$, C.J. McCabe ${ }^{1,2}$ ${ }^{1}$ Institute of Metabolism and Systems Research, University of Birmingham, Birmingham, United Kingdom; ${ }^{2}$ Centre for Endocrinology, Diabetes and Metabolism, Birmingham Health Partners, Birmingham, United Kingdom

The thyroid's ability to accumulate iodide via the sodium-iodide symporter (NIS) is used to successfully treat most thyroid cancers with radioiodide, although a subset of patients lose functional NIS activity and become unresponsive to radioiodide therapy. Our knowledge of NIS regulation is limited, but as dimerization of NIS has been proposed, we sought to investigate NIS dimerization and its impact on radioiodide uptake. NIS dimerization was assessed using proximity ligation assays (PLA) in a thyroid (SW1736) and nonthyroid (HeLa) cancer cell line. Constructs conjugating the fluorophore citrine (YFP) or cerulean (CFP) to NIS enabled quantification of dimerization using Förster resonance energy transfer (FRET). To identify residues potentially involved in dimerization, a homology model of NIS structure based on the crystal structure of the bacterial protein vSGLT was built using the modeling platform Phyre2. Residues of interest were mutated using site-directed mutagenesis. PLA revealed that NIS dimerization occurred in both cell lines. This was confirmed using FRET, as the YFP/CFP ratio of the NIS-fluorophore constructs increased compared to the fluorophores alone $(1.71 \pm 0.10$ vs $1.09 \pm 0.16, \mathrm{P}<0.05$ in SW1736 cells, $\mathrm{n}=3$ and $1.73 \pm 0.10$ vs $1.13 \pm 0.04, \mathrm{P}<0.01$ in HeLa cells, $\mathrm{n}=3$ ). To identify residues involved in dimerization, we mutated five residues identified from our homology model (D237A, Y242A, T243A, Q471A and A525F) and two putative dimerization motifs identified in the literature: a glycinezipper motif in transmembrane domain (TMD) 12 (key glycine residues mutated to valine) and a leucine-zipper in TMD6 (key leucine residues mutated to alanine). PLA suggested that all mutants retained the ability to dimerize, indicating that multiple, or as yet undiscovered, residues are involved. NIS dimerization has been conclusively demonstrated using two discreet methodologies. Further work is ongoing to determine the critical residues, cellular localization and regulation of NIS dimerization and its impact on radioiodide uptake.

\section{Poster 319}

Thyroid \& Development Saturday Trainee Poster Contest Basic

FUNCTION OF THE HISTONE METHYLTRANSFERASE PRMT1 DURING POST-EMBRYONIC DEVELOPMENT AND ADULT INTESTINAL STEM CELL FORMATION IN XENOPUS TROPICALIS

Y. Shibata, Y. Shi

NIH/NICHD, Bethesda, MD

Anuran amphibians such as Xenopus tropicalis offer a unique opportunity to study how the adult organs and their organ-specific stem 
cells are formed in vertebrates during the so-called postembryonic development. The postembryonic events such as the maturation/ remodeling of many organs are totally regulated by thyroid hormone (TH). Of particularly interest among TH target genes is protein arginine methyltransferase1 (PRMT1), which can function as a TR co-activator. We have shown that PRMT1 mRNA and protein expression peaks at the climax of metamorphosis in the intestine when adult stem cells are forming de novo and proliferating, and PRMT1 overexpression enhanced the expression of TH target genes and increased the number of adult intestinal stem cells during metamorphosis, suggesting a critical role during the formation of adult stem cells in the intestine. To determine the function of the endogenous PRMT1 gene during intestinal stem cell development, we adapted the gene editing technology to knockout PRMT1 gene in X. tropicalis. Transcriptional activator-like effector nuclease (TALEN) technology was used to generate PRMT1 knockdown frogs (F0). After generating PRMT1 heterozygous frogs (F1) with out of frame mutations, we tried to produce PRMT1 homozygous frogs by intercross the F1 frogs. We measured the survival, animal size, and the developmental stage of the resulting tadpoles, including wild type, heterozygous and homozygous animals. We successfully generated PRMT1 heterozygous (F1) frogs including out frame mutation, and PRMT1 homozygous (F2) animals by intercross with F1 frogs. Surprisingly, all PRMT1 homozygous tadpoles died within 14 day after fertilization although they completed embryogenesis to form apparently normal tadpoles. In addition, morphological analysis showed that PRMT1 knockout tadpoles delayed animal development and growth. PRMT1 knockout tadpoles completed embryogenesis normally but had delayed development and tadpole lethality. The availability of knockout tadpoles will allow us to study the role of PRMT1 during T3 -induced metamorphosis, thus overcoming the difficulty to study PRMT1 during postembryonic development in mammals due to the lethal phenotype in PRMT1 knockout mice.

\section{Poster 320}

Thyroid \& Development Saturday Trainee Poster Contest

Basic

INSIDE THYROID DYSGENESIS THROUGH EXPANDED EXOME ANALYSIS

E. Brust ${ }^{1,3}$, G. Thezolin ${ }^{1}$, F. Koyama ${ }^{2}$, A. Camargo ${ }^{2}$, F.O. Rego ${ }^{2}$, P. Galante ${ }^{2}$, T. Oliveira ${ }^{4}$, T. Amorim ${ }^{5}$, H. Ramos ${ }^{4}$, C. Friguglietti ${ }^{6}$, G. Medeiros-Neto ${ }^{7}$, I.G. Rubio ${ }^{8,9}$

${ }^{1}$ Laboratório de Ciências Moleculares da Tireoide (LCMT), Universidade Federal de São Paulo (UNIFESP), São Paulo, São Paulo, Brazil; ${ }^{2}$ Centro de Oncologia Molecular (MOC), Hospital Sírio-Libanês - Instituto de Ensino e Pesquisa (HSL-IEP), São Paulo, São Paulo, Brazil; ${ }^{3}$ Programa de Pós-graduação em Biotecnologia, Universidade Federal de São Paulo (UNIFESP), São Paulo, São Paulo, Brazil; ${ }^{4}$ Department of Biorregulation, Thyroid Study Laboratory, Health \& Science Institute, Federal University of Bahia, Salvador, Salvador, Bahia, Brazil; ${ }^{5}$ Association of Parents and Friends of Exceptional Children - APAE-Salvador, Salvador, Bahia, Brazil; ${ }^{6} \mathrm{Head}$ and Neck Surgery, Santa Catarina Hospital, São Paulo, São Paulo, Brazil; ${ }^{7}$ Thyroid Unit, Cellular and Molecular Endocrine Laboratory, LIM-25, Faculdade de Medicina da Universidade de São Paulo (FMUSP), São Paulo, São Paulo, Brazil; ${ }^{8}$ Programa de Pósgraduação em biologia estrutural e funcional, Universidade Federal de São Paulo (UNIFESP), São Paulo, São Paulo, Brazil;

${ }^{9}$ Departamento de Ciências Biológicas, Universidade Federal de São Paulo (UNIFESP), Diadema, São Paulo, Brazil

Thyroid dysgenesis (TD) is the most common cause of congenital hypothyroidism $(\mathrm{CH})$ (85\% of cases). However, genomic variants in candidate genes, involved since the early stages of thyroid devel- opment, have been identified in only $3 \%$ of cases. The aim of this study was to investigate genomic and somatic variants associated to TD. This study included blood DNA samples from $33 \mathrm{CH}$ children and 2 tissue DNA samples of adult ectopic thyroid. The expanded whole exome sequencing was performed using Illumina NextSeq 500 platform. The candidate gene approach was adopted in this analysis, SNVs and indels selection criteria were: non synonymous, not described in databases nor present in 6 controls individuals exome, present in exonic region or 3'UTR target sites of miRNAs of 178 candidate genes. Condel platform were used to predict a possible protein deleterious effect, miRNA target sites were analyzed with PolyMIRts, miRanda and TargetScan databases. Up to now 19 samples were investigated and 27 exonic SNVs in 19 candidate genes were identified. These SNVs were present in 13 blood samples and 2 tissues samples genomes. While $6 \mathrm{SNVs}$ were observed in more than one patient no correlation between the presence of this recurrent SNVs and patients clinical and phenotype characteristics was observed. Condel plataform indicated a possible deleterious action of exonic SNVs in HADHA, TBX1 and FCGBP genes. HADHA gene is highly expressed in thyroid and participates in mitochondrial function on T3-mediated ATP production HADHA dependent. TBX1 transcription fator is involved since early thyroid embryogenesis, determining gland's correct size and position. FCGBP is involved in thyroid cell division and migration and found in autosomal monoallelic expression in TD. Moreover, 2 SNVs were identified, in 4 and 6 patients respectively, in the target site of miR-17-3p of HLA-DQB1 gene, with an important role in immune system and autoimmune thyroid disease. In conclusion we identified 3 new exonic SNVs in TD patients genome. Only functional analysis will confirm the association of these SNVs with TD. None of the 3'UTRs alterations was associated with TD. A complete study are underway to determine alterations involved in the genetics of TD.

\section{Poster 321}

Thyroid Cancer Saturday Trainee Poster Contest Basic

THE METABOLIC PROFILE AND EXPRESSION OF MITOCHONDRIAL GLYCEROPHOSPHATE DEHYDROGENASE (MGPDH) IN THYROID CANCER PREDICTS THE SENSITIVITY TO METFORMIN

\section{TREATMENT IN VIVO}

S. Thakur ${ }^{1}$, B. Daley ${ }^{1}$, K. Gaskins ${ }^{2}$, V.V. Vasko ${ }^{3}$, M. Boufraqech ${ }^{2}$, D. Patel $^{2}$, C. Sourbier ${ }^{4}$, E. Kebebew ${ }^{2}$, J. Klubo-Gwiezdzinska ${ }^{1}$ ${ }^{1}$ Metabolic Diseases Branch, National Institute of Diabetes and Digestive and Kidney Diseases, National Institutes of Health, Bethesda, MD; ${ }^{2}$ Endocrine Oncology Branch, National Cancer Institute, National Institutes of Health, Bethesda, MD; ${ }^{3}$ Department of Pediatrics, Uniformed Services University of the Health Sciences, Bethesda, MD; ${ }^{4}$ Urology Oncology Branch, National Cancer Institute, National Institutes of Health, Bethesda, MD

Targeting cancer cells oxidative phosphorylation (OXPHOS) and glycolysis is a promising strategy against cancer. The mGPDH is the key enzyme connecting OXPHOS and glycolysis via the glycerol-3phosphate shuttle. mGPDH is the target of metformin (MF) in the liver. There are no data on the role of $\mathrm{mGPDH}$ in thyroid cancer (TC). We analyzed mGPDH expression in $253 \mathrm{TC}$ and normal tissues by immunostaining and examined its expression in TC-derived cell lines (FTC133, BCPAP) by confocal microscopy. The effects of MF on mGPDH expression were determined by qRT-PCR and Western blot. We used Seahorse analyzer to assess the effects of MF on OXPHOS and glycolysis in TC cells. The effects of MF on tumor growth and mGPDH expression were examined in metastatic TC mouse models. The mGPDH expression was significantly higher in human TC tissues compared with normal thyroid $(\mathrm{p}<0.001)$. mGPDH was 
expressed in mitochondria at significantly higher level in FTC133 than BCPAP cells and MF decreased mGPDH expression more prominently in FTC133 than BCPAP cells. FTC133 cells were characterized by higher baseline OXPHOS rate $(p=0.02)$ and larger inhibition of OXPHOS after MF treatment $(p=0.048)$. There was no difference in glycolysis between FTC133 and BCPAP cells at baseline and after MF. Silencing of mGPDH led to the inhibition of OXPHOS $(\mathrm{p}=0.002)$ and mGPDH overexpression resulted in increased OXPHOS $(\mathrm{p}=0.007)$ in both cell lines. FTC 133 and BCPAP mGPDH-silenced cells were more resistant to OXPHOSinhibitory effects of MF $(\mathrm{P}<0.001)$. Overexpression of $\mathrm{mGPDH}$ sensitized TC cells to OXPHOS-inhibitory effects of metformin $(\mathrm{P}=0.01$ in $\mathrm{FTC} 133$ and $\mathrm{P}=0.02$ in BCPAP cells $)$

Therapeutic concentration of MF inhibited growth $(\mathrm{p}=0.04)$, and mGPDH expression $(\mathrm{p}=0.01)$ in vivo in $\mathrm{mGPDH}$-rich FTC133 metastatic mouse model, but not in BCPAP model $(\mathrm{p}=0.8)$ characterized by low mGPDH expression $(\mathrm{p}=0.6)$.

mGPDH is overexpressed in TC compared with normal thyroid. Cells with higher level of mGPDH and OXPHOS are more sensitive to metabolic effects of MF in vitro and growth inhibitory effects of MF in vivo, suggesting that mGPDH may serve as biomarker of response to MF therapy.

\section{Poster 322}

Thyroid Cancer Saturday Trainee Poster Contest Basic

DUAL RECEPTOR TARGETING FOR IMPROVED SYSTEMIC TUMOR-SPECIFIC DELIVERY OF THE SODIUM IODIDE SYMPORTER (NIS) GENE

S. Urnauer ${ }^{1}$, K. Schmohl ${ }^{1}$, S. Morys ${ }^{2}$, M. Tutter ${ }^{1}$, N. Schwenk ${ }^{1}$, C. Schug ${ }^{1}$, R. Oos ${ }^{3}$, P. Bartenstein ${ }^{3}$, D. Clevert ${ }^{4}$, E. Wagner ${ }^{2}$, C. Spitzweg ${ }^{1}$

${ }^{1}$ Department of Internal Medicine IV, University Hospital of Munich, LMU Munich, Munich, Germany; ${ }^{2}$ Department of Pharmacy, Center of Drug Research, Pharmaceutical Biotechnology, LMU Munich, Munich, Germany; ${ }^{3}$ Department of Nuclear Medicine, University Hospital Munich, LMU Munich, Munich, Germany; ${ }^{4}$ Department of Clinical Radiology, University Hospital of Munich, Munich, Germany

Tumor heterogeneity displays a major problem in cancer therapy, not only between patients, but even between primary tumor and metastases and within the tumor itself. To address the variable tumor characteristics and improve gene delivery for cancer therapy of hepatocellular cancer, a dual targeting approach was performed. For tumor targeted gene delivery, synthetic LPEI-based polymer backbones were coupled to two tumor-specific peptide ligands. GE11 was used for EGFR-targeting and cMBP served for cMET-targeting. The dual targeting approach was applied for the delivery of the sodium iodide symporter (NIS) gene. NIS as well characterized theranosic gene allows diagnostic analysis of functional NIS gene expression by noninvasive imaging modalities as well as effective anticancer therapy after application of radioiodide. Tumor specificity and transduction efficiency of dually targeted polyplexes (complex polymer/DNA) were examined in comparison to single targeted polyplexes by non-invasive PET-imaging using ${ }^{124}$ I as NIS-specific tracer. Mice bearing orthotopic $\mathrm{HuH} 7$ liver tumors were injected intravenously (i.v.) with polyplexes and $48 \mathrm{~h}$ later, high levels of tumor-specific NIS-mediated radionuclide uptake were detected with a trend towards higher levels of iodide uptake in the intrahepatic tumor tissue after application of dually targeted polyplexes. Subsequently, the therapeutic efficacy of this promising dual-targeting concept was evaluated. To this end, 3 cycles of i.v. injections of dually targeted NIS polyplexes followed by therapeutic application of $55.5 \mathrm{MBq}{ }^{131} \mathrm{I}$ or saline $48 \mathrm{~h}$ later were conducted. Therapy mon- itoring of tumor growth and tumor perfusion was performed by contrast-enhanced ultrasound showing significantly reduced tumor growth along with prolonged survival in ${ }^{131}$ I treated animals. In conclusion, the dually targeted NIS gene therapy approach resulted in high tumor-specific radioiodine accumulation and significant therapeutic efficacy in an advanced orthotopic tumor model. The bifunctional strategy enhances the applicability even in heterogeneic tumors with variable receptor expression level.

\section{Poster 323}

Thyroid Cancer Saturday Trainee Poster Contest

Translational

\section{NELFINAVIR (NFV) IS AN EFFECTIVE ANTICANCER AGENT FOR THYROID CANCER AND HAS} DIFFERENTIAL ANTICANCER ACTIVITY BY CELL TYPE

Y. Kushchayeva ${ }^{1}$, K. Gaskins ${ }^{4}$, D.R. Donahue ${ }^{2}$, M. Boufraqech ${ }^{3}$, D. Wei ${ }^{4}$, S. Mukherjee ${ }^{3}$, S.M. Kumar ${ }^{3}$, C. Sourbier ${ }^{4}$, J. Shell ${ }^{3}$, S. Kushchayev ${ }^{5}$, S. Tella ${ }^{6}$, J. Klubo-Gwiezdzinska ${ }^{7}$, V. Vasko ${ }^{8}$, E. Kebebew ${ }^{3}$

${ }^{1}$ NIDDK/NIH, Bethesda, MD; ${ }^{2}$ NINDS/NIH, Bethesda, MD; ${ }^{3}$ Endocrine Oncology Branch, NCI/NIH, Bethesda, MD; ${ }^{4} \mathrm{NCI} / \mathrm{NIH}$, Bethesda, MD; ${ }^{5}$ Radiology, Mercy Catholic Medical Center, Philadelphia, PA; ${ }^{6} \mathrm{NICHD} / \mathrm{NIH}$, Bethesda, MD; ${ }^{7} \mathrm{MDB}$, NIDDK/ $\mathrm{NIH}$, Bethesda, MD; ${ }^{8}$ Pediatrics, Uniformed Services University, Bethesda, MD

Treatment of radioiodine-refractory metastatic thyroid cancer (TC) is challenging with limited options. Nelfinavir (NFV) is a protease inhibitor that is safe, well-tolerated and an FDA approved treatment for HIV. Repurposing NFV for cancer therapy has generated significant interest. The aim of this study was to investigate the antitumor activity and mechanism of NFV action in TC using in vitro and in vivo models.

BCPAP, FTC-133, and XTC-UC1 cell lines derived from human papillary, follicular, and hürthle cell carcinoma tumors were treated with NFV at $2.5-20 \mu \mathrm{M}$ for $24-72 \mathrm{~h}$. Western blot analysis, Flow Cytometry, caspase-Glo 3/7 assay, Comet assay and Seahorse analysis were performed to determine the mechanism of NFV action. In vivo, BCPAP-luc 2 cells were injected via tail vein into gNOD. $\mathrm{Cg}$ $\mathrm{Prkdc}^{\text {scid }} \mathrm{Il} 2 \mathrm{rg}^{\mathrm{tm} 1 \mathrm{Wjl}} / \mathrm{SzJ}$ mice with disease status assessed by bioluminescence and CT with contrast with and without NFV $(200 \mathrm{mg} / \mathrm{kg}$ by gavage)

NFV $(20 \mu \mathrm{M})$ treatment decreased cell viability $(76 \%, 51 \%$, and $22 \%$ at $48 \mathrm{~h}$, and $89 \%, 50 \%$ and $24 \%$ at $72 \mathrm{~h}$ in BCPAP, XTC-UC1 and FTC-133). DNA damage was measured as mean olive tail moment after $48 \mathrm{~h}$ of NFV $(20 \mu \mathrm{M})$ treatment and were $17.7 \pm 1.6$, $80.5 \pm 2.7$ and $80.3 \pm 7.6$ in BCPAP, XTC-UC1 and FTC-133 cells with increased apoptosis in BCPAP and XTC-UC1, and necrosis in FTC-133 cells. Cell signaling pathway analysis revealed inhibition of HSP60. Furthermore, NFV decreased oxygen consumption by $57.5 \%, 69 \%$, and $57 \%$ in BCPAP, XTC-UC1 and FTC-133 at $48 \mathrm{~h}$ of NFV treatment $(20 \mu \mathrm{M})$. BCPAP cells had the lowest level of maximal respiration and spare respiratory capacity under effect of mitochondrial respiration modulators.

In vivo, 3-5 wks NFV treatment of BCPAP-derived metastases resulted in lower number of pulmonary metastases per animal $(\mathrm{p}<0.05)$, size of the hepatic $(\mathrm{p}<0.05)$ and pulmonary $(\mathrm{p}<0.01)$ metastases with normalized bioluminescence values $4.3 \pm 1.8$ vs $1.6 \pm 0.4$ at 3 wks and $34.8 \pm 20.3$ vs $12.4 \pm 7.7$ at 5 wks.

NFV has significant and differential anticancer activity in vitro and in vivo in TC cells. The mechanism of action NFV-mediated anticancer activity is cell line specific and analysis of dysregulated pathways in patient tumors can help determine the likelihood of NFV treatment response. 
Poster 324

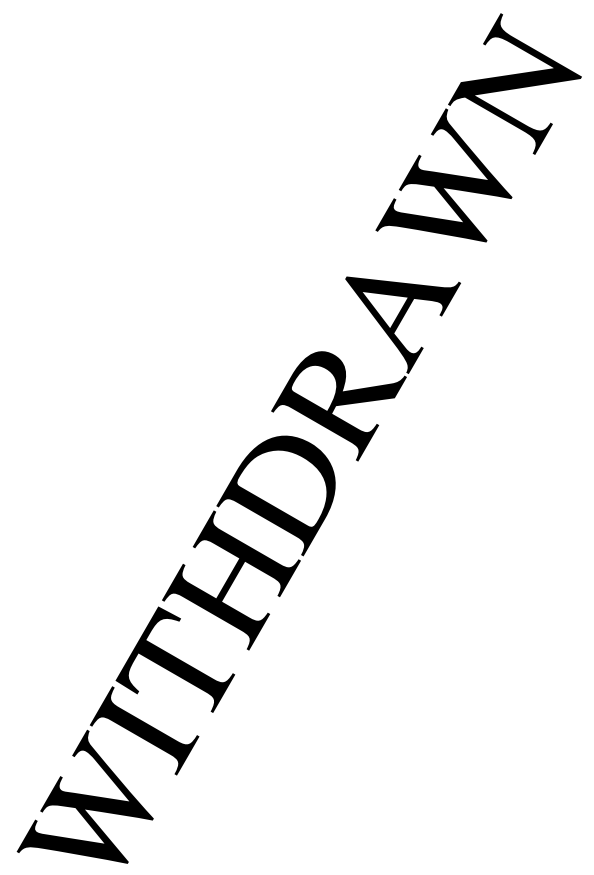

Poster 325

Disorders of Thyroid Function Saturday Trainee Poster Contest Clinical

\section{EFFECTS OF HYPOTHYROIDISM ON SURGICAL} OUTCOMES

R. Villavicencio ${ }^{1}$, C.N. Mariash ${ }^{2}$

${ }^{1}$ School of Medicine, Indiana University, Indianapolis, IN; ${ }^{2}$ Indiana University, Indianapolis, IN

Each year 45 million surgical procedures are performed in the United States with the prevalence of overt hypothyroidism being $0.3 \%$ to $0.4 \%$. With new surgical techniques and modern anesthesia, we hypothesized there would be no difference in outcome between hypothyroid and euthyroid surgical patients. Thus, we conducted a retrospective observational study of hypothyroid patients with primary outcomes of length of stay (LOS), death, and post-operative cardiac complications. Surgical databases were queried for patients who underwent an operative procedure between January 1, 2010 and December 31, 2015. Twenty-nine adult patients with a TSH $>10$ $\mathrm{mcU} / \mathrm{mL}$ by itself or a TSH at the upper limit of normal along with a free $\mathrm{T} 4<0.6 \mathrm{ng} / \mathrm{dL}$ were evaluated by measurement against the predicted length of stay using the American College of Surgeons National Surgical Quality Improvement Program (ACS NSQIP) surgical risk calculator. Average LOS was significantly longer for the hypothyroid group (14.4 days) compared to 6.6 days predicted by the NSQIP calculator. We then evaluated the LOS in an age, sex, and surgical procedure matched euthyroid group and found the LOS in this group was significantly less (9.2 days) than the hypothyroid group and not different from that predicted by the NSQIP calculator. One patient in the hypothyroid group died compared with none in the euthyroid group. Two patients in the hypothyroid group experienced atrial fibrillation and one in each group had PEA arrest. Other complications including rates of hypotension, bradycardia, and hyponatremia did not differ between groups. However, degree of hypotension was slightly more pronounced with an average low mean arterial pressure in the hypothyroid group of $51 \mathrm{mmHg}$ compared to $56 \mathrm{mmHg}$ in the controls. In contrast to our initial hypothesis, hypothyroidism is associated with a markedly increased LOS. However, absolute incidence of hypotension, bradycardia, and hyponatremia did not significantly differ. The study was not sufficiently powered to discern differences in mortality or post-operative cardiac complications but does show that even with modern techniques and anesthesia there remains a significant increased risk when operating on a hypothyroid patient.

\section{Poster 326}

Disorders of Thyroid Function Saturday Trainee Poster

Contest Clinical

THYROID FUNCTION, EPICARDIAL ADIPOSE TISSUE AND ATRIAL FIBRILLATION, A POPULATION BASED COHORT STUDY

L. Chaker ${ }^{1,2}$, D. Bos ${ }^{2,1}$, A. Bano ${ }^{1}$, A. Hofman ${ }^{2,1}$, T. VanderWeele ${ }^{2}$, M. Kavousi ${ }^{1}$, O. Franco ${ }^{1}$, R. Peeters ${ }^{1}$, A. Ikram ${ }^{1}$

${ }^{1}$ Erasmus Medical Center, Rotterdam, Zuid-Holland, Netherlands;

${ }^{2}$ Harvard T.H. Chan School of Publich Health, Boston, MA

The underlying mechanism of the association between thyroid function and atrial fibrillation (AF) is not completely understood, but epicardial fat tissue (EAT) could be a promising mediator. Therefore our objective is to determine the association thyroid-stimulating hormone (TSH) and free thyroxine (FT4) with EAT and investigate whether EAT has a mediating role in the association of thyroid function with $\mathrm{AF}$.

TSH and FT4 were measured in 1995 participants (mean age 64.5 years) with CT-determined EAT volumes and AF incidence information, in the Rotterdam Study, a population-based cohort study. Main outcome measures were EAT and AF risk as well as the mediated effect of EAT on the association of thyroid function and AF. The mediation analysis was based on the approach of a four-way decomposition that unifies within a single framework the methods assessing mediation and interaction.

Results: Higher FT4 levels are associated with larger EAT volumes in persons with a large waist circumference (WC), defined by sex-specific cut-offs, (Beta 0.08 per 1 pmol/L FT4, 95\% CI 0.02, 0.14 ), but not in persons with a small WC. In persons with a large WC, higher FT4 levels were associated with a higher AF risk (Hazard Ratio $1.50,95 \%$ CI 1.22, 1.83). We report no evidence of a mediating role of EAT in the association of thyroid function with AF (mediated interaction $1.6 \%$, pure indirect effect $3.2 \%$ ), but some evidence for reference interaction of EAT with thyroid function on AF risk $(10.8 \%)$. 
Conclusions: Higher FT4 levels are associated with larger EAT volumes in persons with abdominal obesity. We report no mediating role of EAT in the association of thyroid function with AF. There is a suggested interaction of FT4 with EAT volumes on AF risk.

\section{Poster 327}

Disorders of Thyroid Function Saturday Trainee Poster Contest Clinical

\section{INFLUENCE OF SOCIODEMOGRAPHIC FACTORS ON RATE OF EMERGENCY HOSPITALIZATIONS AND SURGICAL MANAGEMENT OF GRAVES' DISEASE}

T.M. Vaghaiwalla, G. Rubio, J. Farra, J.I. Lew

Department of Surgery, University of Miami, Miami, FL

Management of Graves' disease (GD) includes anti-thyroid medication, radioiodine ablation, and total thyroidectomy (TTx). While disease acuity and presentation determine management, demographic and socioeconomic factors may also affect access to definitive treatment and influence the decision for surgical management in patients with GD. This study examines the association of sociodemographic factors to emergent hospitalizations and use of TTx for GD. A crosssectional analysis was performed using the Nationwide Inpatient Sample (2006-2011) to identify hospitalizations for GD. Univariate and logistic regression analyses were performed to evaluate factors associated with the use of TTx during emergent and elective hospitalizations for GD. of 33, 279 patients hospitalized for GD, 60.3\% were admitted emergently. Patients admitted non-electively were older (mean 44 vs. 43 years, $\mathrm{p}<0.01$ ), with a higher proportion of men $(25.0 \%$ vs. $17.2 \%, \mathrm{p}<0.01)$ and non-white $(59.6 \%$ vs $41.3 \%, \mathrm{p}<0.01)$ patients compared to those admitted electively. Patients admitted emergently were also more likely to be uninsured (55.2\% vs. $27.4 \%$, $\mathrm{p}<0.01)$ and from the two lowerest income quartiles $(61.8 \%$ vs. $48.9 \%, \mathrm{p}<0.01)$ compared to the elective hospitalization group. TTx was performed in 10,434 (31.4\%) patients during hospitalization. On multivariate analysis, men (OR 1.26; 95\% CI 1.14-1.39), non-whites (OR 1.49; 95\% CI 1.38-1.62), Medicaid/uninsured (OR 2.53; 95\% CI 2.32-2.75), and lowest income quartile (OR 1.30; 95\% CI 1.16-1.50) had higher odds of emergency hospitalizations for GD. Alternatively, women (OR 1.52; 95\% CI 1.37-1.69), whites (OR 1.27; 95\% CI 1.171.39), Medicare/insured (OR 1.23; 95\% CI 1.12-1.35), and highest income quartile (OR 1.28; 95\% CI 1.14-1.45) were more likely to undergo TTx. Race, sex, income, and insurance status affect rate of emergent hospitalizations for GD, and such factors may influence the utilization of TTx. Increased access to definitive care for GD may improve outcomes and reduce emergency healthcare utilization.

\section{Poster 328}

Disorders of Thyroid Function Saturday Trainee Poster Contest Clinical

\section{SUBCLINICAL HYPOTHYROIDISM AND ITS ASSOCIATION WITH GESTATIONAL DIABETES MELLITUS}

F. Sharifi-Ghorveh ${ }^{1}$, I.J. Halperin ${ }^{2}$, M. Diba ${ }^{3}$

${ }^{1}$ Internal Medicine, University of Toronto, Thornhill, Ontario, Canada; ${ }^{2}$ Internal Medicine/Endocrinology, University of Toronto, Sunnybrook Health Science Center, Toronto, Ontario, Canada; ${ }^{3}$ Metabolic Diseases Research Center, Zanjan University of Medical Sciences, Zanjan, Iran (the Islamic Republic of)

Both gestational diabetes mellitus (GDM) and hypothyroidism are prevalent during pregnancy and compromise maternal and fetal health. The aim of the present study was to compare thyroid function tests and antithyroid antibodies of pregnant women with and without
GDM. We also aimed to determine the association between thyroid dysfunction with insulin resistance in pregnant women. Serum free triiodothyronine $\left(\mathrm{FT}_{3}\right)$, free thyroxine $\left(\mathrm{FT}_{4}\right)$, thyroid-stimulating hormone (TSH), antiTPO antibody and insulin level were measured in 142 pregnant women who underwent universal screening for Gestational diabetes mellitus(GDM) at the third trimester of pregnancy between weeks 20-24 of gestational age. GDM was confirmed by a 75 -g oral glucose tolerance test using World Health Organization criteria. HOMA-IR was calculated and used as an index of insulin resistance. The results were analyzed using T-test, chi-squared, and multivariable regression analysis. Sixty eight women with GDM and 74 pregnant women without GDM were included in the study. No significant association was found between GDM and having subclinical or clinical hypothyroidism. However, the rate of thyroid autoimmunity was higher in women with GDM. Anti TPO antibodies were positive in $14.1 \%$ of women with GDM versus $8.6 \%$ of those without GDM (P: 0.01). Although there was no significant correlation between serum TSH and serum insulin or HOMA-IR, anti TPO antibody level was significantly correlated with both HOMA-IR index (p: 0.01) and insulin levels (P: 0.003). This study shows more thyroid autoimmunity in women with GDM as well as a positive relationship of anti TPO antibody with insulin resistance in pregnancy. The importance of this finding and any suggestion to incorporate routine testing of pregnant women for thyroid antibodies as a risk factor of GDM remains controversial.

\section{Poster 329}

\section{Thyroid Cancer Saturday Case Report}

\section{PAPILLARY THYROID CANCER WITH UNUSUAL LATE ONSET OF PITUITARY AND CHOROIDAL METASTASES}

N. Bin Hareez, H. Lochnan

Endocrinology, University of Ottawa, Ottawa, Ontario, Canada

We report a very unusual case of late onset of pituitary and choroidal metastasis from thyroid cancer that began as a fairly routine Papillary Thyroid Cancer (PTC).

Case Report: The case is a 59-year-old man presented with multifocal PTC size $2.5 \mathrm{~cm}$. and $0.1 \mathrm{~cm}$, intrathyroidal cancer. T2 N1 MX with one cervical lymph node involvement. After treatment, his iodine 131 whole bode scan was negative with no evidence of functional thyroid tissue in the neck or distant metastases. Five years later mediastinum lymph nodes metastases were identified; pathology now described a poorly differentiated component. Patient went on to develop multiple recurrences to lymph node, lung and liver. Treated with multiple neck dissection and high doses radioactive iodine (RAI). Fourteen years later with new onset headache, his imaging revealed pituitary, choroidal (ocular) and temporal bone metastases. He was treated by CyberKnife surgery, radiation, Lenvatinib, laser, intraocular injections, suppressive levothyroxine and required replacement with glucocorticoids for secondary hypoadrenalism.

Methods: We reviewed all clinical data available for this patient, including whole-body scan, computed tomography images, MRI, thyroglobulin measurements, RAI doses and pathology samples. A literature search for pituitary and choroidal metastases was performed in PubMed, for English-language articles and case reports using the terms "pituitary metastases," "choroidal metastases," and "thyroid cancer." Relevant references were selected and reviewed. Twenty papers reporting 21 cases of pituitary metastases were found. 9 cases reporting choroidal metastases ( 7 cases PTC, 1 insular and 1 PTC with follicular variant). But no any case report found have both pituitary and choroidal metastases in the same patient.

This case highlights the importance of clinicians to be aware of the clinical and biochemical manifestations to detect a rare occurrence as pituitary metastases which can lead to hypopituitarism that can be 
life threatening. The rarity of pituitary and choroidal metastases in patients with thyroid cancer warrants clinicians to consider pituitary involvement when symptoms such as headache or new fatigue and weakness present.

\section{Poster 330 \\ Disorders of Thyroid Function Saturday Trainee Poster Contest Clinical}

THE IMPACT OF 2016 ATA GUIDELINE FOR DIAGNOSIS AND MANAGEMENT OF HYPERTHYROIDISM ON THE FREQUENCY OF TRAB MEASUREMENT

N. Bin Hareez ${ }^{1}$, J. Shaw ${ }^{1}$, E. Leung, ${ }^{2}$, H. Lochnan ${ }^{1}$

${ }^{1}$ Endocrinology, University of Ottawa, Ottawa, Ontario, Canada;

${ }^{2}$ Nuclear Medicine, Ottawa, Ontario, Canada

Objectives: The 2016 American Thyroid Association (ATA) Guidelines for Diagnosis and Management of Hyperthyroidism and other causes of Thyrotoxicosis, published in 2016 endorsed the use of Thyrotropin Receptor Antibody (TRAb) testing for diagnosis and decision-making. citing high sensitivity, specificity for diagnosis of Graves' Disease and potential for cost saving by avoiding thyroid scan and RAIU by $\sim 47 \%$. This was shift from the ATA 2011 guidelines which emphasized using RAI uptake. The new 2016 guidelines also suggest measuring TRAb levels measurement prior to stopping ATD therapy and as an indicator of response to therapy for the management of patients with Graves' disease. In this study, we sought to determine whether the guidelines resulted in practice change in regard to use of TRAb testing.

The frequency of TRAb, TSH, FT4 and FT3 at a large Academic Medical Centre was analyzed over the past 14 months in parallel with scanning and radioactive iodine uptake testing (Seven months before and after the ATA meeting in Oct.2016).

A dramatic increase in testing was observed beginning in November 2016 (ATA guidelines were published in the October 2016 issue of Thyroid). The number of TRAb measurements by 3.7 fold with no significant change in the rate of testing for TSH, freeT4 and free $\mathrm{T} 3$ over the same time period. Rates of testing are remarkably consistent per month (on average 8 per month until October 2016 and $\sim 30$ per month since then. TSH testing remained consistent over this time at $\sim 5000$ per month. Rates of thyroid scanning and RAIU have not changed dramatically and usually correspond with requests for therapyThe significant increase in the number of TRAb measurements performed coincides with the publication and dissemination of new ATA guidelines. This appears to reflect evidence of impact and effective dissemination of ATA guidelines. The effect on patient care requires more in-depth assessment and longer term follow up in regards to impact on use of radioactive iodine as a treatment.

\section{Poster 331}

Iodine Uptake \& Metabolism Saturday Trainee Poster Contest Clinical

\section{IODINE NUTRITION IN U.S. WEANING INFANTS}

R. Fallah ${ }^{5}$, L. Du ${ }^{1}$, L. Braverman ${ }^{2}$, X. $\mathrm{He}^{2}$, M. Segura-Harrison ${ }^{3}$, M. Yeh ${ }^{6}$, H. Chiu ${ }^{5}$, A.M. Leung ${ }^{4}$

${ }^{1}$ UCLA Fielding School of Public Health, University of California Los Angeles, Los Angeles, CA; ${ }^{2}$ Endocrinology, Diabetes, and Nutrition, Boston University School of Medicine, Boston, MA; ${ }^{3}$ Family Medicine, Memorial Hospital of Rhode Island; Brown University, Pawtucket, RI; ${ }^{4}$ Endocrinology, Diabetes, and Metabolism, UCLA David Geffen School of Medicine, Los Angeles, CA; ${ }^{5}$ Pediatric Endocrinology, UCLA David Geffen School of Medicine, Los Angeles, CA; ${ }^{6}$ Endocrine Surgery, UCLA David Geffen School of Medicine, Los Angeles, CA
Iodine is a requisite micronutrient for infant brain development. Infants are at risk for iodine deficiency during the weaning period, when their diet transitions from breast milk or formula to baby food. Only infant formula produced in the U.S. is required to contain iodine. Sources of iodine intake during the weaning period are minimal, as adding table salt to baby food is uncommon and jarred baby food is not required to contain iodine. A median urinary iodine concentration of $>100 \mathrm{mcg} / \mathrm{L}$ indicates adequate iodine nutrition in an infant population. The purpose of this study is to report the current status of iodine nutrition (as measured by urine iodine concentration) and the factors affecting this among weaning infants in the U.S. Subjects were infants $<12$ months of age who were fed any combination of infant formula and/or baby food. Samples of all formula and food consumed in the 24 hours prior to the collection of a spot urine from the infant were obtained. The iodine content of all specimens was measured spectrophotometrically. The ingested quantities of formula and food were summed from a food diary recorded by infants' parents. Mean age of infants $(n=60 ; 50 \%$ Caucasian, $30 \%$ Black) was $6.3 \pm 3.5$ (SD) months. Their mothers were primarily U.S.-born (87\%), had less than a college-degree education (58\%), and were nonsmokers $(80 \%)$. Infants' median urinary iodine concentration $(117 \mathrm{mcg} / \mathrm{L}$; range, $26.9-1302.8 \mathrm{mcg} / \mathrm{L})$ was positively correlated with the median iodine content of each infant's 24-hour total ingested formula and food $(89 \mathrm{mcg} / \mathrm{L}$; range, $0-288 \mathrm{mcg} / \mathrm{L})$ $(\mathrm{r}=0.4, \mathrm{p}<0.001)$, but not with the amount of formula consumed $(\mathrm{p}=0.26)$. Race, infant age, mothers' educational level, and mothers' cigarette smoking status were not predictive of infants' urine iodine concentrations. In this U.S. sample, infants fed a combination of baby formula and food were iodine sufficient. Iodine-containing infant formula and foods are important sources of iodine nutrition during infancy, and further research is needed to determine the specific iodine contributions of each during this important developmental period.

\section{Poster 332}

Thyroid Cancer Saturday Trainee Poster Contest Clinical PEMBROLIZUMAB ADDED TO KINASE INHIBITOR (KI) THERAPY IN ANAPLASTIC THYROID CARCINOMA (ATC): A SINGLE CANCER CENTER'S EXPERIENCE P. Iyer ${ }^{1,2}$, R. Ferrarotto ${ }^{3}$, M. Gule-Monroe ${ }^{4}$, N. Busaidy ${ }^{1}$, R. Dadu ${ }^{1}$, M. Habra ${ }^{1}$, M.D. Williams ${ }^{5}$, M. Hofmann ${ }^{6}$, M. Zafereo ${ }^{7}$, H. Skinner ${ }^{8}$, M.E. Cabanillas ${ }^{1}$

${ }^{1}$ Department of Endocrine Neoplasia and Hormonal Disorders, Division of Internal Medicine, The University of Texas MD Anderson Cancer Center, Houston, TX; ${ }^{2}$ Endocrinology, Diabetes and Metabolism, Baylor College of Medicine, Houston, TX; ${ }^{3}$ Department of Thoracic/Head and Neck Medical Oncology, Division of Cancer Medicine, The University of Texas MD Anderson Cancer Center, Houston, TX; ${ }^{4}$ Department of Diagnostic Radiology, Division of Diagnostic Imaging, The University of Texas MD Anderson Cancer Center, Houston, TX; ${ }^{5}$ Department of Pathology, Division of Pathology/Lab Medicine, The University of Texas MD Anderson Cancer Center, Houston, TX; ${ }^{6}$ Department of Endocrine Neoplasia and Hormonal Disorders - Research, Division of Internal Medicine, The University of Texas MD Anderson Cancer Center, Houston, TX; ${ }^{7}$ Department of Head and Neck Surgery, Division of Surgery, The University of Texas MD Anderson Cancer Center, Houston, TX; ${ }^{8}$ Department of Radiation Oncology, Division of Radiation Oncology, The University of Texas MD Anderson Cancer Center, Houston, TX

ATC has a 1 year mortality of $80 \%$ with a dismal median overall survival (OS) of 5 months. Targeted therapy with KI in ATC has provided substantial clinical benefit but eventually patients develop 
resistance. Pembrolizumab (pembro) is an anti-programmed cell death protein (PD-1) immunotherapy (I/O) agent approved for several cancers. Our objective was to explore the efficacy of adding pembro to KI at the time of progression disease (PD) in ATC patients (pts). We retrospectively reviewed the charts of consecutive ATC pts treated with $\mathrm{KI}+$ pembro. Best overall response (BOR) by RECIST and median OS on KI alone (OS1) and on combined KI + pembro (OS2), and progression free survival (PFS) were calculated. Adverse events (AE) were extracted from the medical record. From 8/2016-3/ 2017, 7 ATC pts were treated with KI + pembro. Median age was 60 years (range $58-84$ years) and $70 \%$ were men. $6 / 7$ pts $(86 \%)$ were stage IVC at diagnosis. All had distant metastases at the time of initiating KI. 4 pts received dabrafenib/trametinib and 3 received lenvatinib. The PD-L1 was checked in 5/7 pts and were 5\%, 10\%, $>10 \%, 30 \%$ and $50 \%$. All 7 pts were started on pembro at the time PD. Median time to initiation of pembro was 5.5 months (range 1.226 months). BOR after initiation of pembro was: 2/7 (29\%) partial response, 2/7 (29\%) stable disease with tumor regression (range 8\%$21 \%$ ) and 3/7 (43\%) PD. Median OS1 was not reached (range 3.332.4 months), at a median follow up (f/u) time of 12.5 months. Median PFS and OS2 were not reached (range 2.3-9.8 months) however median f/u time was only 3.9 months. All but 1 pt are alive. This pt died 12.5 months from start of KI and 4 months from starting $\mathrm{KI}+$ pembro. He was non-compliant with KI due to dysphagia. Grade 1 diarrhea lasting 1-2 days was the most common AE with pembro. One pt had grade 2 colitis, presumably due to pembro, that resolved with treatment. Toxicities for KI were as expected and manageable. The long OS seen in our ATC pts treated with combination pembro + $\mathrm{KI}$ is remarkable. Adding $\mathrm{I} / \mathrm{O}$ to $\mathrm{KI}$ immediately at progression may be beneficial in ATC. A prospective clinical trial exploring frontline $\mathrm{KI}$ and $\mathrm{I} / \mathrm{O}$ is underway (NCT03181100).

\section{Poster 333}

Thyroid Cancer Saturday Clinical

CIRCULATING BRAFV600E CELL-FREE DNA DETECTED BY DROPLET DIGITAL PCR (DDPCR) AS A BIOMARKER IN THE MANAGEMENT OF ANAPLASTIC THYROID CARCINOMA (ATC) PATIENTS

P. Iyer ${ }^{1,2}$, G.J. Cote ${ }^{1}$, R. Dadu ${ }^{1}$, R. Ferrarotto ${ }^{3}$, N. Busaidy ${ }^{1}$, M. Hofmann ${ }^{4}$, M. Zafereo ${ }^{5}$, M.D. Williams ${ }^{6}$, V. Subbiah ${ }^{7}$, M.E. Cabanillas ${ }^{1}$

${ }^{1}$ Department of Endocrine Neoplasia and Hormonal Disorders, Division of Internal Medicine, The University of Texas MD Anderson Cancer Center, Houston, TX; ${ }^{2}$ Endocrinology, Diabetes and Metabolism, Baylor College of Medicine, Houston, TX; ${ }^{3}$ Department of Thoracic/Head and Neck Medical Oncology, Division of Cancer Medicine, The University of Texas MD Anderson Cancer Center, Houston, TX; ${ }^{4}$ Department of Endocrine Neoplasia and Hormonal Disorders - Research, Division of Internal Medicine, The University of Texas MD Anderson Cancer Center, Houston, TX; ${ }^{5}$ Department of Head and Neck Surgery, Division of Surgery, The University of Texas MD Anderson Cancer Center, Houston, TX; ${ }^{6}$ Department of Pathology, Division of Pathology/Lab Medicine, The University of Texas MD Anderson Cancer Center, Houston, TX; ${ }^{7}$ Department of Investigational Cancer Therapeutics, Division of Cancer Medicine, The University of Texas MD Anderson Cancer Center, Houston, TX

$B R A F$ is an actionable mutation in ATC. Combination BRAF + MEK inhibitor therapy has a response rate of $60 \%$ in ATC. Mutation testing on tumor is the gold standard, but there can be delays in acquiring tissue and obtaining results. Guardant360 (G360), a commercial liquid biopsy test provides information on a number of mutations but is expensive and takes a median of 13 days for results. There is a need for a faster liquid biopsy test to identify $B R A F$ mutation and initiate timely treatment. At MD Anderson, ddPCR liquid biopsy results are possible within 3 days. Our objective was to compare $B R A F$ mutation detection by ddPCR to our current standard of care-G360 testing coupled with tumor tissue testing by DNA analysis or immunohistochemistry (IHC). ATC patients (pts) with active disease, tumor mutation testing, and G360 testing were included. For ddPCR analysis of $B R A F$ mutation status DNA was isolated from the plasma and V600E allelic fraction determined in a research setting by the BioRad QX200 ${ }^{\mathrm{TM}}$ Droplet Digital ${ }^{\mathrm{TM}}$ PCR System. Concordance, sensitivity and specificity of ddPCR with tissue and G360 results were calculated. From 3/2016-5/2017, 35 consecutive ATC pts were eligible and included in the study. Median age was 67 years and 29/35 (83\%) were men. Stage at diagnosis: 20/35 pts (57\%) stage IVC, 13/35 pts (37\%) stage IVB and $2 / 35$ pts $(6 \%)$ stage IVA. BRAF mutation analysis on tumor was done by DNA based testing in 32/35 pts (91\%) and IHC in $3 / 35$ pts $(9 \%)$. BRAF mutation on tissue was identified in $17 / 35$ pts (49\%). BRAF mutation detection by ddPCR was $91 \%$ concordant with tissue findings. Sensitivity was $94 \%$ and specificity $89 \%$. G360 was $89 \%$ concordant with tissue. Sensitivity was $72 \%$ and specificity $100 \%$. ddPCR was $85 \%$ concordant with G360 (not significantly different, $\mathrm{p}=0.15$ ). The lowest value of allelic fraction detected by ddPCR was $0.05 \%$. There was one false negative by ddPCR, which was also negative on G360, likely explained by the low disease burden in the pt. ddPCR is a rapid and a reliable test to identify $B R A F$ mutations in ATC. Implementing its use in the clinical setting may help expedite potentially effective treatment- hopefully leading to improved patient outcomes.

\section{Poster 334}

Thyroid Hormone Metabolism \& Regulation Saturday Trainee Poster Contest Clinical

\section{THE VARIATION OF TYPE-2 DEIODINASE CONCENTRATION AND ACTIVITY IN BLOOD AMONG DIFFERENT SURGICAL APPROACHES OF DIFFERENTIATED THYROID CARCINOMA}

X. Zheng, K. Qian, K. Guo, Z.Y. Wang

Head Neck surgery, Fudan University Cancer Center, Shanghai, China

Other recent studies reported that the total thyroidectomy (TT) with supplemental L-T4 had significantly higher free T4 (FT4) and lower free T3 (FT3) levels compared to preoperative levels. Conversion of $\mathrm{T} 4$ to $\mathrm{T} 3$ is catalyzed by type 2 deiodinase (D2). This study aimed to explore the effect of remnant thyroid tissue on D2 concentration and activity in blood through testing D2 concentration and activity in different surgical approaches for papillary thyroid carcinoma (PTC). We performed a prospective cohort study of 180 PTC patients from January to June in 2016. If the patients were treated by TSH suppression therapy, blood sample would be recollected. We classified all patients into two groups including TT and Hemithyroidectomy (HT), according to surgical approaches. By gene sequencing, we detected genotype of D2. And using enzyme linked immunosorbent assay (ELISA), we measured pre- and postoperative concentration of D2. The expression of D2 was obtained by PCR. Finally, we examined the activity of D2 via radioimmunoassay. For the prospective study, there was no genetic mutation of D2 in our 135 patients. After TSH suppression therapy, the level of D2 increased remarkably in HT group $(P=0.022)$. However, there was no statistical significance in TT group $(P=0.928)$. During T4 stimulation, the expression for D2 mRNA increased nearly twice in normal thyroid cell. Moreover, the activity of D2 in HT was higher than TT group $(P=0.006)$. In our study, we found the genetic mutation rate of D2 was low among population. After TSH suppression therapy, the level of D2 increased remarkably in HT 
group, but there was no statistical significance in TT group. Experienced different surgical, the D2 activity in TT group was significantly lower than HT group. In summary, the concentration and activity of D2 in blood decreased with the reducing of thyroid tissue in PTC patients. So we assume that the less presence of residual thyroid lead to D2 decrease, the lower ability of converting T4 to T3.

\section{Poster 335 \\ Thyroid Hormone Metabolism \& Regulation Saturday Trainee Poster Contest Clinical \\ ASSOCIATION OF SUBCLINICAL HYPOTHYROIDISM WITH NAFLD: KOREA NATIONAL HEALTH AND NUTRITION EXAMINATION SURVEY 2013 TO 2015}

J. Lee, J. Ha, D. Lim, M. Kang, B. Cha, M. Kim

Endocrinology and Metabolism, St. Mary's hospital, The Catholic University of Korea, Seoul, Korea, Seoul, Korea (the Republic of)

Non-alcoholic fatty liver disease (NAFLD) is prevalent liver disease encompassing broad spectrum of pathologic changes in liver, from simple steatosis to fatal liver cirrhosis and hepatocellular carcinoma. Metabolic derangements such as metabolic syndrome and insulin resistance are suggested to be main causes of NAFLD. As thyroid hormone is a main regulator of energy metabolism and several studies has shown the association between thyroid function and insulin resistance, a link between NAFLD and thyroid function could be suspected. In previous studies, the associations of NAFLD with thyroid function were not conclusive. The aim of study was to clarify the relationship between NAFLD and thyroid function, focusing on subclinical hypothyroidism, in nationwide survey data which representing Korean population. After excluding subjects with under age of 19, history of thyroid disease and lack of thyroid function tests, data of 3,452 subjects were recruited from the Sixth Korea National Health and Nutrition Examination Survey. Subclinical hypothyroidism was defined as increased thyrotropin (TSH) levels (above 97.5 percentile of TSH levels in the data) with normal range free thyroxine levels. Probability of NAFLD was defined based on hepatic steatosis index (HIS); under 30: low, 30 35; intermediate, 36 or above; high probability. There are no significant differences between euthyroid group $(n=3,324,96.3 \%)$ and subclinical hypothyroidism $(n=128,3.7 \%)$ except for age. Subjects with subclinical hypothyroidism were older than euthyroid subjects. Intermediate to high probability of NAFLD was frequently observed in subclinical hypothyroidism subjects (Odd ratio (OR) $2.81,95 \%$ confidence interval $(\mathrm{CI}) 1.45-5.46, p=0.002)$. Statistical significance was also observed after adjustment for confounding factor including age, sex, waist circumference, smoking status, physical activity and history of diabetes mellitus (OR 2.53, 95\% CI 1.29-4.98, p=0.007). Subclinical hypothyroidism could be related to high risk of NAFLD based on the analysis of nationwide representative data. Therefore, assessment of NAFLD in subjects with subclinical hypothyroidism should be considered in clinical practice.

\section{Poster 336}

Thyroid Imaging Saturday Trainee Poster Contest Clinical

\section{COMPARISON BETWEEN THYROID IMAGING} REPORTING AND DATA SYSTEM AND 2015 AMERICAN THYROID ASSOCIATION MANAGEMENT GUIDELINES IN EVALUATION OF THYROID NODULE WITH ULTRASOUND

R. Liu,. Yang, Y. Jiang, Y. Wang, L. Gao, J. Liu, J. Wang, X. Xi,. Zhu, X. Lai, R. Zhao, X. Zhang, B. Zhang

Chinese Academy of Medical Sciences \& Peking Union Medical College Hospital, Beijing, China
Both 2015 American Thyroid Association (ATA) Management Guidelines for Adult Patients with Thyroid Nodules and Differentiated Thyroid Cancer and the Thyroid Imaging Reporting and Data System (TIRADS) are widely used in clinical. while which one is more effective? To find the answer, We compared their diagnostic values by applying them to thyroid nodules cases. From November 2011 to December 2015, 485 thyroid nodules in 331 patients (mean age, 42.9 years \pm 10.4 ) were included in this study. Characteristics including size, composition, shape, margin, echogenicity, calcifications and extrathyroidal extension of thyroid nodules were evaluated. Every nodule was stratificated by criteria set by TIRADS and ATA guidelines, and malignant rate of eachrisk stratification were calculated and analysed. With pathology as the gold standard, different cutoff were taken to diagnose malignant nodules, and diagnostic values of the two methodologies were calculated at each cutoff. And the two methodologieswere evaluated and measured by ROC curve. Of the 485 thyroid nodules, 96 were benign and 389 were malignant. The malignancy rates under TIRADS category $2,3,4 \mathrm{a}, 4 \mathrm{~b}, 4 \mathrm{c}$, and 5 nodules were $0 \%, 12.0 \%, 22.2 \%, 29.8 \%, 99.2 \%$ and $100 \%$. Malignancy rates under ATA guidelinesof benign, very low, low, intermediate, and high suspicion for malignancy were $0 \%, 12.5 \%, 16.1 \%, 27.7 \%$, and $99.2 \%$. There were significant differences inside each patterns $(\mathrm{P}<0.01)$ respectively and high correlation between risk stratification with TIRADS $(r=0.70)$ and ATA guidelines $(r=0.83)$. Areas under the ROC curve of the TIRADS and ATA guidelines classifications were 0.966 and 0.959 . Best cut-off point for diagnosing malignant by TIRADS and ATA guideline classifications were $\geq 4 \mathrm{c}$ and $\geq$ high suspicion, and at that point, the sensitivity, specificity, PPV, NPV, and accuracy, of TIRADS and ATA guidelineswere nearly the same, and there was no significant differences $(\mathrm{P}>0.05), \mathrm{k}=0.97$. Both TIRADS and the ATA guidelinesprovide effective malignancy risk stratification for thyroid nodules. The diagnosticvalue of TIRADS when considering $\geq 4 \mathrm{c}$ and ATA guidelines when considering $\geq$ high-suspicion nodules as malignant were nearly the same and both high.

\section{Poster 337}

Disorders of Thyroid Function Saturday Trainee Poster Contest Case Report

\section{A NOVEL MUTATION IN THE TG GENE (G2341S)} CAUSING CONGENITAL HYPOTHYROIDISM IN A SUDANESE FAMILY

Y. Watanabe ${ }^{1}$, E. Sharwood ${ }^{2,3}$, B. Goodwin ${ }^{2,3}$, M. Creech $^{1}$, M. Netea ${ }^{4}$, M. Jaeger ${ }^{4}$, S. Refetoff ${ }^{5}$, T. Huynh ${ }^{2,3}$, R.E. Weiss ${ }^{1}$ ${ }^{1}$ Department of Medicine, University of Miami Miller School of Medicine, Miami, FL; ${ }^{2}$ Department of Endocrinology and Diabetes, Lady Cilento Children's Hospital, Brisbane, Queensland, Australia; ${ }^{3}$ School of Medicine, The University of Queensland, Brisbane, Queensland, Australia; ${ }^{4}$ Department of Internal Medicine, Radboud University Medical Center, Nijmegen, Netherlands; ${ }^{5}$ University of Chicago, Chicago, IL

Congenital hypothyroidism $(\mathrm{CH})$ is a condition of thyroid hormone deficiency present from birth with an incidence of 1:1500 to 1:4000 depending on the cut-off TSH value at neonatal screening (NS). Mutations in both alleles of the $T G$ gene lead to permanent $\mathrm{CH}$. A 3week old female presented with a goiter causing upper airway obstruction and stridor. Her TSH on NS was $14 \mathrm{mIU} / \mathrm{L}$, which was flagged for re-testing as it was at the upper limit of normal. No treatment was offered at that time. At age 3 weeks, retesting showed an elevated TSH $[100 \mathrm{mU} / \mathrm{L}(0.7-5.9)]$ with low free T4 $[<3.2 \mathrm{pmol} /$ $\mathrm{L}(8.7-16)]$ and high thyroglobulin $(\mathrm{TG})[101 \mu \mathrm{g} / \mathrm{L}(<38)]$. Urine analysis suggested mild iodine deficiency. Thyroid ultrasound showed a large goiter and Tc-99 $\mathrm{m}$ scan homogeneous and significantly increased radiotracer uptake. One brother was hypothyroid on 
NS and treated without further investigation. The parents and grandparents had normal thyroid function. Both maternal and paternal families are Sudanese (Gogrial and Aweil, respectively) and non-consanguineous. Whole exome sequencing of proposita's DNA identified a novel homozygous missense mutation of the $T G$ gene in exon 40: c.7021G>A, p. Gly2341Ser. Sanger sequencing revealed that the affected brother was homozygous for the same mutation. The unrelated parents were heterozygous for this mutation which on haplotyping suggested an ancient origin. DNA samples from 354 alleles in 4 Sudanese ethnic groups (Nilotes, Darfurians, Nuba, and Halfawien) failed to demonstrate the presence of the mutant allele. The mutation is located in the cholinesterase-like (ChEL) domain of TG and it falls between the first and second disulfide bonds where is considered to be critical for ChEL folding. A novel mutation in the $T G$ gene was identified. Despite both parents being carriers and the region of the DNA being very well conserved over many generations, we did not find this mutation among four ethnic groups in Sudan. Functional predictions and the structure of TG suggest the mutation is deleterious. This area of the $T G$ gene is a mutational hot spot and may be a significant cause of $\mathrm{CH}$ in the Sudan.

\section{Poster 338}

Autoimmunity Saturday Trainee Poster Contest Case Report PEMBROLIZUMAB INDUCED HYPOPHYSITIS

A. Chauhan ${ }^{2}$, P. Shah ${ }^{2}$, B. Tendler ${ }^{3}$, U.P. Hegde ${ }^{1}$

${ }^{1}$ Hematology-Oncology, University of Connecticut, Farmington, CT; ${ }^{2}$ Internal Medicine, University of Connecticut, Farmington, CT; ${ }^{3}$ Endocrinology, University of Connecticut, Farmington, CT

Increased uptake of immune-checkpoint blockers (ICB) in cancer therapy has brought to the forefront their unique spectrum of immune mediated adverse events (irAEs). We present a case of Pembrolizumab (anti-PD1) induced hypophysitis with central hypoadrenalism and hypothyroidism. An 86-year-old gentleman developed metastatic melanoma for which treatment was initiated with Pembrolizumab. After 6 doses, he presented with new onset fatigue, associated with difficulty standing up from a sitting position. Laboratory tests yielded the following: hemoglobin $15.7 \mathrm{~g} / \mathrm{dL}$, hematocrit $43.9 \%$, WBC count $6400 \mu \mathrm{l}^{-1}$, CK 121 , ESR 37 and CRP 4.6. Eosinophils were elevated at $17 \%$ with an absolute count of $1.1 \mu \mathrm{l}^{-1}$. Anti-acetylcholine receptor antibodies were negative. Serum examination revealed significantly decreased basal ACTH and cortisol levels at $<5 \mathrm{pg} / \mathrm{mL}$ and $<1 \mu \mathrm{g} / \mathrm{dL}$, respectively. There was no previous exposure to steroids and a prior serum cortisol was $11 \mu \mathrm{g} /$ dL. TSH was normal at $3.87 \mathrm{uU} / \mathrm{mL}$ but free $\mathrm{T} 3$ was decreased at $1.8 \mathrm{pg} / \mathrm{mL}$. Free T4 was $0.91 \mathrm{ng} / \mathrm{mL}$. The low T3 indicated central hypothyroidism. FSH (7.29 mIU/mL) and LH (4.09 mIU/mL) were preserved. Magnetic resonance imaging (MRI) of the pituitary gland was unremarkable. Oral prednisone was started with resolution of symptoms and return of serum cortisol, TSH, T3 and T4 levels to baseline. Pembrolizumab was not discontinued. Pathogenesis of immune-related hypophysitis remains unclear. Rejuvenation of antigen exhausted T-cells by anti-PD1 agents is likely culpable, supported by the CD8+ T lymphocytic infiltration seen on histological examination of anterior pituitary in proven cases. Pituitary enlargement on MRI is not typically seen in anti-PD1 induced hypophysitis, unlike autoimmune lymphocytic and Ipilimumab (anti-CTLA4) induced disease. In suspected cases, it is imperative to measure T3 and T4 as TSH alone can fail to detect central hypothyroidism. Immunosuppression with corticosteroids usually suffices with thyroxine supplementation being required rarely. irAEs can masquerade as general complaints of malignancy such as fatigue, requiring a high index of suspicion. Prompt diagnosis and management is necessary to allow ICB continuation and successful tumor remission.

\section{Poster 339}

Disorders of Thyroid Function Saturday Trainee Poster Contest Case Report

\section{ANTI-PD-1 MONOCLONAL ANTIBODIES LEAD TO THYROID DYSFUNCTION: A CASE SERIES OF THREE PATIENTS}

A. Alameer, S. Adler, J. Paulson

Endocrinology, George Washington university hospital, Springfield, VA

Anti-PD-1 (programmed cell death protein 1) monoclonal antibodies have shown promising results for patients with metastatic malignancies such as melanoma, NSCLC and RCC. However, there have been multiple associated immune mediated endocrinopathies including thyroid dysfunction.

Anti PD-1 monoclonal $\mathrm{Ab}$ acts as an inhibitor of the checkpoint signal that would have prevented activated $\mathrm{T}$ cells from attacking cancer cells. Here, we present three cases of anti-PD-1 monoclonal antibodies (Nivolumab) leading to hypothyroidism.

Case 1:

A 25 year old woman with refractory adrenocortical carcinoma without history of thyroid disease developed transient thyrotoxicosis after 4 cycles of off-label Nivolumab. Labs were: TSH $0.014 \mu \mathrm{IU} / \mathrm{ml}(0.450$ $4.500 \mu \mathrm{IU} / \mathrm{ml})$, FT4 $4.23 \mathrm{ng} / \mathrm{dl}(0.82-1.77 \mathrm{ng} / \mathrm{dl})$, TPO Ab $44 \mathrm{IU} / \mathrm{ml}$ $(0-34 \mathrm{IU} / \mathrm{ml})$. Prior to treatment TSH was $3.1 \mu \mathrm{IU} / \mathrm{ml}$. Methimazole was started. Repeat labs in 12 days showed TSH $25.440 \mu \mathrm{IU} / \mathrm{mL}$, direct FT4 0.36. Methimazole was discontinued and repeat labs in a few weeks showed TSH $162.400 \mu \mathrm{IU} / \mathrm{mL}$, direct FT4 $0.15 \mathrm{ng} / \mathrm{dL}$. She was started on levothyroxine which is being uptitrated. She continues on Nivolumab.

Case 2:

A 30 year old woman with SCL of the tongue developed hypothyroidism after 2 cycles of nivolumab. TSH prior to treatment was normal. After 2 cycles: TSH $19.740 \mu \mathrm{IU} / \mathrm{ml}$, direct FT4 $0.94 \mathrm{ng} / \mathrm{dL}$ FT3 $1.7 \mathrm{pg} / \mathrm{ml}$. Levothyroxine was started and labs subsequently normalized. Nivolumab was continued.

Case 3:

A 68 year old male with metastatic RCC developed hypothyroidism after his $7^{\text {th }}$ cycle of nivolumab. Labs showed: TSH $68.3 \mu \mathrm{IU} / \mathrm{ml}$ (0.27-4.20 $\mu \mathrm{IU} / \mathrm{ml})$, direct FT4 0.2 ng/dl (0.93-1.7 ng/dl), TPO Ab 27 IU/ $\mathrm{ml}(<9 \mathrm{IU} / \mathrm{ml})$. He is being treated with levothyroxine and Nivolumab.

Thyroid dysfunction is a common immune effect of Anti-PD-1 Ab. Early detection and frequent testing are required to prevent complications. Continuation of immunotherapy seems to be safe, from a thyroidal perspective, among those patients. As mentioned in discussion

\section{Poster 340}

Disorders of Thyroid Function Saturday Trainee Poster Contest Case Report

\section{EARLY IDENTIFICATION OF SEVERE PRIMARY} HYPOTHYROIDISM IN NEONATES EXPOSED TO INTRA-LYMPHATIC IODINATED CONTRAST:

\section{A CASE SERIES}

C. Cherella ${ }^{1}$, D. Breault ${ }^{1}$, V. Thaker ${ }^{2}$, B. Levine ${ }^{1}$, J. Smith ${ }^{1}$

${ }^{1}$ Boston Children's Hospital, Boston, MA; ${ }^{2}$ Division of Molecular Genetics, Columbia University Medical Center, New York, NY

In neonates, iatrogenic hypothyroidism can result from topical or intravenous (IV) iodine exposure. Data on intra-lymphatic iodine exposure, risk factors for disease severity, and timing of hypothyroidism is limited. We report 4 cases of neonatal hypothyroidism after intra-lymphatic contrast.

Cases:

1: 34-week male with lymphatic malformation underwent sclerotherapy on day of life (DOL) 4 with Optiray contrast and 
doxycycline. Thyroid function tests (TFTs) on DOL 7 and 8 demonstrated TSH 16.7 and $18.19 \mathrm{mIU} / \mathrm{mL}$ (range 1.7-9.1) and FT4 1.32 and $1.31 \mathrm{ng} / \mathrm{dL}$ (range 1.6-3.8), respectively. Spot urine iodine was $228,712 \mathrm{mcg} / \mathrm{L}(<200)$. IV levothyroxine (LT4) was initiated; FT4 normalized in 1 week.

2: 30-week female with Trisomy 21, duodenal atresia, and lymphangiectasia had chylous effusions and high-volume chest tube output. Lymphangiogram with Lipiodol contrast was performed on DOL 97. TFTs on DOL 111 demonstrated TSH $335.7 \mathrm{mIU} / \mathrm{mL}$ (range 1.7-9.1) and FT4 0.1 ng/dL (range 0.9-2.3). Spot urine iodine was $49,594.6 \mathrm{mcg} / \mathrm{L}(<200)$. IV LT4 was initiated; TFTs normalized in 5 days.

3: 34-week female with transposition of the great arteries had post-operative chylous effusions and high-volume chest tube output. Lymphangiogram with Lipiodol contrast was performed on DOL 43. TFTs on DOL 68 demonstrated TSH $470.2 \mathrm{mIU} / \mathrm{mL}$ (range 1.7-9.1) and FT4 $0.2 \mathrm{ng} / \mathrm{dL}$ (range 0.9-2.3). Spot urine iodine was 6,307.8 $\mathrm{mcg} / \mathrm{L}(<200)$. IV LT4 was initiated; TFTs normalized in 5 days.

4: 5 month full-term female with lymphatic malformation underwent embolization with Lipiodol and Optiray contrast. 2 weeks later TSH remained normal, though FT4 nadir $0.5 \mathrm{ng} / \mathrm{dL}$ (range 0.8 1.8). Spot urine iodine was $21,814 \mathrm{mcg} / \mathrm{L}(<200)$. Enteral Synthroid was initiated; FT4 normalized in 1 week. In preterm infants (cases 13), rapid, severe primary hypothyroidism developed soon after lymphatic iodine exposure. TFT derangement was less acute in case 4 , suggesting escape from the Wolff-Chaikoff effect. Intra-lymphatic iodine should be considered a major risk factor in the development of iatrogenic primary hypothyroidism, particularly in premature neonates soon after exposure. Close TFT monitoring is imperative to avoid potential long-term adverse outcomes in this population.

\section{Poster 341}

Disorders of Thyroid Function Saturday Trainee Poster Contest Case Report

\section{THYROTOXICOSIS IN TRIPLET PREGNANCY}

M.A. Jara ${ }^{1}$, J.M. Jara Ruiz ${ }^{3}$, E. Jara Ruiz ${ }^{2}$, c. Arevalo ${ }^{2}$, J. Jara Yorg ${ }^{2}$

${ }^{1}$ Endocrinology, University of Miami, Miami, FL; ${ }^{2}$ National University Asuncion, Asuncion, Paraguay; ${ }^{3}$ Endocrine, Federal University of Parana, Curitiba, Parana, Brazil

Overt thyrotoxicosis occurs in $0.2 \%$ to $0.4 \%$ of all pregnancies while the prevalence of fetal hyperthyroidism is 1 to $5 \%$ in pregnant women with Grave's disease. Thyrotoxicosis presenting during pregnancy can be challenging due to limitations in radiologic testing. We report a 29-year-old Hispanic woman in her 12th week of spontaneous pregnancy who was initially seen due to palpitations and abnormal thyroid function tests. On examination, she presented tachycardia, fine tremors of the hands and a moderately diffuse goiter. TSH was $0.03 \mu \mathrm{UI} / \mathrm{ml}(0.30-4.94)$, free T4 $1.57 \mathrm{ng} / \mathrm{dl}(0.70-1.48)$ and total T3 $2.03 \mathrm{ng} / \mathrm{ml}(0.59-1.59)$. Thyroglobulin: $5.80 \mathrm{UI} / \mathrm{ml}(0-4.11 \mathrm{UI} / \mathrm{ml})$ and TPO 208.01UI/ml $(0-30 \mathrm{UI} / \mathrm{ml})$ antibodies were positive while TRAb was negative: $<0.3 \mathrm{UI} / \mathrm{L}(<1.75 \mathrm{UI} / \mathrm{L})$. Thyroid ultrasound showed a solid hypoechoic nodule in the right lobe measuring $27 \times 45 \mathrm{~mm}$. FNA was negative for malignancy and showed moderate cellularity (follicular cells), rounded nuclei, evident nucleolus and cytoplasmic vacuolization. She was treated throughout pregnancy with potassium iodide $(30 \mathrm{mg} /$ day $)$, methimazole $(5 \mathrm{mg} /$ day $)$ and bisoprolol $(5 \mathrm{mg})$ with clinical and biochemical normalization. By 29 weeks, due to threatening preterm labor, the patient was hospitalized and two weeks later she was submitted to cesarean section. The third trimester TSH was $1.59 \mu \mathrm{UI} / \mathrm{ml}$ and TRAb $0.43 \mu \mathrm{UI} / \mathrm{L}$. Newborn A weighed $1,770 \mathrm{~g}$, B weighed $1,500 \mathrm{~g}$ and C $1,490 \mathrm{~g}$ without any complications and stayed in the ICU for monitoring due to low birthweight. Newborns TSH levels were within normal range. In Paraguay, the prevalence of hyperthyroidism in pregnant women has been reported to be in the range of 1 to $2 \%$, and Graves' disease is known to be the major cause of hyperthyroidism in pregnant women. Inadequately treated maternal hyperthyroidism has been associated with a high risk of preeclampsia, heart failure, preterm birth, low birth weight and fetal loss. To our knowledge, the association of thyrotoxicosis and multiple pregnancies is rare. Careful follow-up of pregnant women with thyrotoxicosis is advised as well as close monitoring of newborns after delivery as rapid FT4 elevation during the first postnatal week is predictive of hyperthyroidism and warrants therapy.

\section{Poster 342}

Disorders of Thyroid Function Saturday Trainee Poster

Contest Case Report

\section{A CASE OF SEVERE GESTATIONAL THYROTOXICOSIS}

K. Chen, J. Benmoussa, V. Sharma

Albany Medical Center, Albany, NY

Hyperthyroidism due to hyperemesis gravidarum, a syndrome of nausea, vomiting, and weight loss of $5 \%$ or more is typically mild and self-limiting but may be clinically significant in rare cases. An 18year-old pregnant woman with no medical history was transferred to our medical center for management of severe thyrotoxicosis. She presented to an outside hospital with nausea, vomiting, and $40 \mathrm{lb}$ weight loss. She was found to be pregnant at approximately 13 weeks; BhCG was 361,065. TSH was $0.04(0.45-4.5)$, free T4 was 4.13 (0.6-1.3), total T3 was 197 (55-170), free T3 was 3.3 (2.3-5.0), TSI was 46\% (0-139\%). Creatinine was 2.6, AST 109, and ALT 344. TTE showed global hypokinesis with EF of 35-39\%. She reported onset of severe nausea, vomiting, weight loss, and tremor 2 months prior to hospitalization. She also noted resting heart rate to $160 \mathrm{bpm}$ on her heart monitor. Physical exam was remarkable for tachycardia and tremor. No proptosis, lid lag, or thyromegaly were noted. Empiric treatment with IV fluids, steroids, PTU, and propranolol was initiated. Severe thyrotoxicosis in the setting of elevated BHCG was concerning for possible partial molar pregnancy. She underwent elective termination of pregnancy. Surgical path showed products of conception with rare dysmorphic chorionic villi with mild trophoblast hyperplasia. Chromosomal analysis revealed 46 XX karyotype. Following D\&E, creatinine improved to 0.8, AST to 14 and ALT to 73. She was discharged on propranolol and methimazole, which was titrated off as outpatient. Most recent BhCG was 5 and TSH was 4.5. Repeat TTE showed basal hypokinesis with improved EF of 40$50 \%$. Human chorionic gonadotropin (hCG) is a heterodimeric glycoprotein synthesized by the placenta with peak levels at 10-12 weeks of pregnancy. Hyperemesis gravidarum occurs in $0.1-0.2 \%$ pregnancies and is typically seen in women with higher hCG levels in early pregnancy. Increased free T4 and suppressed TSH are seen due to homology of the hCG alpha subunit to thyrotropin. Overt hyperthyroidism in hyperemesis gravidarum is rare but may be severe as in our patient's case and while typically self-limiting, transient treatment with antithyroidal medication can be considered in severe cases.

\section{Poster 343 \\ Thyroid Cancer Saturday Trainee Poster Contest Case Report \\ A RARE PEDIATRIC PRESENTATION OF HYPEREOSINOPHILIC SYNDROME WITH MEDULLARY THYROID CANCER IN MEN2B}

G. Snoeyenbos, A.J. Recupero

Pediatrics, Tripler Army Medical Center, Tripler AMC, HI 
Hypereosinophilic syndrome (HES) is characterized by chronic severe eosinophilia with organ involvement. It is an uncommon paraneoplastic manifestation, and has only been described in medical literature associated with medullary thryoid cancer (MTC) in two adult cases. An 11-year old male presented for evaluation of new onset rapidly enlarging neck mass. Thyroid function tests were normal. Thyroid ultrasound revealed bilateral nodules with intranodal blood flow and calcifications. History and exam revealed classic MEN2B presentation with absent tears as an infant, reoccurring colonic obstruction, chronic constipation and failure to thrive. Physical exam revealed a marfanoid body habitus and facial mucosal neuromas. Abdominal imaging was noted for severe megacolon. Eye exam did not show corneal nerve thickening. Eosinophilic esophagitis (EoE) was diagnosed after having new onset dysphagia at age seven. Bloodwork revealed persistent elevated absolute eosinophil counts between 840-1909/mcL (40-350), with most recent being 962 . Screening calcitonin level was $1491 \mathrm{pg} / \mathrm{mL}$ (0-8.4) and CEA 55.7ng/ml (0-5). RET gene analysis was heterozygous for $\mathrm{p}$. Met918Thr. HES as a paraneoplatic phenomena involving MTC is rare and there are no known cases involving MEN2B. There are two known adult cases of esophageal involvment in HES with severely elevated eosinophilic counts well above the typical mild counts seen in EoE, and not paraneoplasic. There are a few cases of HES treated with tyrosine kinase inhibitors. Interestingly our patient's diagnosis of EoE, better fits with HES with esophageal involvement in the context of MTC as part of MEN2B. In retrospect, the many features presented can all be explained by MEN2B, including the more recent diagnosis of EoE as part of HES. Effective treatment for MTC includes prompt recognition and total thyroidectomy before metasasis. This case highlights the need to have cancer and HES as part of the differential for persistent high eosinophilia, is the only known pediatric case of esophogeal HES and HES as part of MTC in MEN2B, and the need for research on the role for TKIs in HES as part of MTC. Better treatment outcomes maybe achieved with prompt recognition of HES in MTC.

\section{Poster 344}

Thyroid Hormone Action Saturday Poster Case Report

\section{TRANSIENT CENTRAL DIABETES INSIPIDUS IN} UNCONTROLLED GRAVES' DISEASE

I. Unal ${ }^{1}$, S. Shikdar ${ }^{1}$, R. Mundada ${ }^{1}$, R. Plotzker ${ }^{2}$

${ }^{1}$ Internal Medicine, Mercy Catholic Medical Center, Darby, PA;

${ }^{2}$ Endocrinology, Mercy Philadelphia Hospital, Philadelphia, PA

Polyuria is an uncommon presentation of Graves' disease and multiple etiologies play a role including increased solute excretion and autoimmune Diabetes Insipidus (DI).

We report a case of a patient with an uncontrolled Graves' disease who presented with a transient central DI. A 68 year-old-man with a history of Graves' disease who is never been compliant with methimazole treatment presented to hospital with abdominal pain, nausea and vomiting. Over years, his TSH remained below 0.01 $\mu \mathrm{IU} / \mathrm{mL}$ and serum FT4 was ranging between $1.80-5.30 \mathrm{ng} / \mathrm{dL}$. Given elevated lactate of $5 \mathrm{mmol} / \mathrm{L}$, he underwent CTA abdomen \& pelvis, which showed only upper retroperitoneal edema. His vitals were stable, and did not meet any systemic inflammatory response syndrome (SIRS) criteria. He was made nothing by mouth (NPO) due to persistent abdominal pain, treated with intravenous normal saline and pain medications. After 18 hours past, his mentation became altered, and increased urine output was noted overnight (6.9 $\mathrm{L}$ in $24 \mathrm{~h}$ and $600-800 \mathrm{ml} / \mathrm{hr}$ ). His serum sodium (Na) rose from $139 \mathrm{mEq} / \mathrm{L}$ to $175 \mathrm{mEq} / \mathrm{L}$, stayed high for a day despite hypotonic maintenance fluids and normal kidney function. Serum and urine osmolality (Osm) was 355 uosm/ $\mathrm{kg}$ and 192 uosm/ $\mathrm{kg}$ respectively. Urine studies revealed specific gravity of $<1.005$, Na of $42 \mathrm{mEq} / \mathrm{L}$ and creatinine of $1.0 \mathrm{mg} / \mathrm{dL}$. CT brain was unremarkable. Blood glucose was $321 \mathrm{mg} / \mathrm{dL}$ with $\mathrm{HbA} 1 \mathrm{c}$ of $3.5 \%$. After receiving $1 \mathrm{mcg}$ Desmopressin (DDAVP) subquatenosly, his urine output significantly dropped to $30-50 \mathrm{ml} / \mathrm{hr}$. On next day, his mentation improved and he was started on oral hydration. Serum Na level trended down to $\sim 150 \mathrm{mEq} / \mathrm{L}$, which later dropped further. Following day, he was prepared for water deprivation test, Arginine vasopressin level after $12 \mathrm{~h}$ was $<1.0 \mathrm{pg} / \mathrm{mL}(\mathrm{N} 1-13 \mathrm{pg} / \mathrm{mL})$ and serum $\mathrm{Na}$ of $152 \mathrm{mEq} / \mathrm{L}$ reported. His urine Osm was up to $915 \mathrm{mOsm} / \mathrm{kg}$ with serum Osm of $321 \mathrm{mOsm} / \mathrm{kg}$ ruling in diabetes insipidus. He was diagnosed with transient central DI of unclear etiology. He responded to DDAVP, and recovered with no evidence of renal impairment. This case highlights a rare and largely under recognized complication of Graves' disease in the context of severe hypernatremia from Central DI.

\section{Poster 345}

Thyroid Hormone Action Saturday Trainee Poster Contest Case Report

\section{BRADYCARDIA AND HYPOTHYROIDISM -} MANAGEMENT CONSIDERATIONS

O. Oraibi, F. Chaudhry

Endocrinology, University of Toledo, Toledo, $\mathrm{OH}$

Hypothyroidism can manifest with cardiac abnormalities. Low voltage and sinus bradycardia are usually found on EKG. Correction of free hormone levels improves conduction abnormalities. A $79 \mathrm{y} / \mathrm{o}$ female presented with fatigue and vomiting. Prior to presentation she had been feeling progressively tired, more sluggish and forgetful. Her medical history revealed hypothyroidism, dementia, GERD and hyperlipidemia. The patient was not taking any medications due to forgetfulness.

On physical exam, she was conscious; with BP 149/66 mmHg and heart rate 37 beats $/ \mathrm{min}$. She was confused, oriented to self only.

The EKG showed marked sinus bradycardia with $\mathrm{T}$ wave inversions.

Lab revealed TSH level $>161 \mathrm{uIU} / \mathrm{ML}(0.34-5.60)$ and a free T4 of $0.75 \mathrm{ng} / \mathrm{dl}$ (0.71-1.85). Cortisol was 58.7 and TPO $1.85 \mathrm{dl}(0.0-35.0)$. Serial troponins were negative. Chest X-ray and TTE were normal.

The patient was treated with IV levothyroxine (LT4) $75 \mathrm{mcq}$ for three days. Due to lack of improvement in heart rate, liothyronine 25 mcg was added for an additional 4 days. Due to her frail condition, she was kept in the hospital until HR improved to $>50 \mathrm{bpm}$. Cardiac pacing was not pursued due to overall clinical stability and presumed improvement with thyroid replacement.

Based on comorbidities and weight, she was discharged on PO LT4 112 mcg daily. Also, Aricept and Ranexa were held secondary to potential bradycardic effect. Bradycardia is a known manifestation of hypothyroidism. The major effects of thyroid hormones on the heart are mediated by triiodothyronine (T3) and its binding to the nuclear receptor. In situations where more immediate response is needed, T3 replacement is can be used cautiously. Although it is known that $\mathrm{T} 3$ replacement can have a more immediate effect on end organ function than T4, there is a lack of guidelines regarding dosage type, strength, and duration. Also, our patient remained admitted soley for observation whilst thyroid hormone levels were raised given persistent bradycardia. No other cardiac intervention was considered due to expectation of improvement with hormone replacement. Further guidelines are needed to improve care in these frail patients while hoping to reduce medical costs and ensure their safe recovery. 


\section{Poster 346 \\ Thyroid Hormone Metabolism \& Regulation Saturday Trainee Poster Contest Case Report}

\section{EFFECT OF RIFAMPIN ON THYROID HORMONE METABOLISM IN A PATIENT ON LEVOTHYROXINE}

P. Mejia Osuna, R. Robbins

Endocrinology, Metabolism and Diabetes, Houston Methodist Hospital, Houston, TX

Rifampin has known effects on thyroid function tests, which include increase in thyroxine (T4) and reverse triiodothyronine (rT3) clearance through induction of hepatic oxygenases and increased iodothyronine conjugates biliary excretion. This is usually compensated in individuals with normal thyroid by increasing thyroid hormone production, resulting in unchanged thyrotropin (TSH) levels. However, patients with preexistent hypothyroidism on thyroid replacement can present remarkable increase in TSH, requiring adjustment of levothyroxine doses. We report a 57 year old female with history of stage III papillary thyroid cancer following a total thyroidectomy. One year ago she developed persistent dry cough and intermittent shortness of breath. Based on abnormal CT scan of chest and bronchoscopy, she was diagnosed with Mycobacterium AviumIntracellulare (MAI) infection. Treatment was initiated with ethambutol, clarithromycin and rifampin. Previous management of her thyroid malignancy included radioactive iodine ablation and suppressive therapy with levothyroxine. Surveillance with serial thyroglobulin (Tgb) levels and thyroid ultrasound showed undetectable Tgb levels and no ultrasound evidence of recurrence. Most recently, she was on replacement therapy with levothyroxine $137 \mathrm{mcg}$ six days per week, resulting in TSH and free T4 within normal range (baseline values: TSH of $1.0 \mu \mathrm{IU} / \mathrm{mL}$ and free T4 $1.6 \mathrm{ng} / \mathrm{dl}$ ). After initiation of rifampin, despite strict compliance, TSH increased gradually to 6.0 $\mu \mathrm{IU} / \mathrm{mL}$ at three months, and to $19.4 \mu \mathrm{IU} / \mathrm{mL}$ ten months after (unchanged Tgb level). This was accompanied by fatigue, dry skin and weight gain, requiring increase of levothyroxine dose. Our case illustrates the pharmacological effect of rifampin on thyroid hormone metabolism in a thyroid cancer survivor on previously stable doses of levothyroxine. The effect of this drug interaction can be clinically significant on hypothyroid patients on replacement therapy, especially without remnant thyroid gland. As the incidence of thyroid cancer is rapidly increasing, any patient on levothyroxine treatment, including thyroid cancer survivors, should be closely monitored for significant drug interactions

\section{Poster 347}

Autoimmunity Saturday Poster Basic

THE ROLES OF DIFFERENT TYPES OF CD19 ${ }^{+}$B CELLS IN THE DISEASE PROGRESSION IN IODINE-INDUCED AUTOIMMUNE THYROIDITIS OF NOD. $\mathrm{H}-2^{\mathrm{H} 4}$ MICE

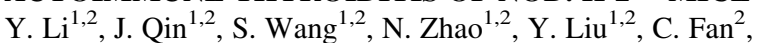
G. Wang ${ }^{3}$, X. Cui ${ }^{1,2}$, M. Huang ${ }^{1,2}$, Z. Shan ${ }^{1,2}$, W. Teng ${ }^{2}$

${ }^{1}$ Department of Endocrinology and Metabolism, The First Affiliated Hospital of China Medical University, Shenyang, Liaoning, China; ${ }^{2}$ Institute of Endocrinology, Liaoning Provincial Key Laboratory of Endocrine Diseases, Shenyang, Liaoning, China; ${ }^{3}$ Department of Laboratory Animal Center, China Medical University, Shenyang, Liaoning, China

B cells display a variety of characteristics in autoimmunity. It has been reported the $\mathrm{CD}^{+}$and $\mathrm{CD} 1 \mathrm{~d}^{\text {hi }} \mathrm{B}$ cells may be potential regulators while other B cells are contributors to autoimmune disorders. NOD. H-2 ${ }^{\text {h4 }}$ mice spontaneously develop autoimmune thyroiditis(AIT) after administration of $\mathrm{NaI}$ water for weeks, which is a classical mice model for studying AIT. Here, we explored for the first time the roles of $\mathrm{CD}^{+}$,

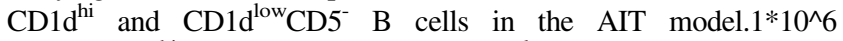
$\mathrm{CD} 19^{+} \mathrm{CD} 1 \mathrm{~d}^{\text {hi }}, \mathrm{CD} 19^{+} \mathrm{CD}^{+}$or $\mathrm{CD} 19^{+} \mathrm{CD} 1 \mathrm{~d}^{\text {low }} \mathrm{CD}^{-} \mathrm{B}$ cells isolated by FACS were immediately transferred intravenously into recipient NOD. $\mathrm{H}-2^{\mathrm{h} 4}$ mice at the time they were given $0.05 \% \mathrm{NaI}$ water. A control group of age-matched mice were injected with PBS. The thyroiditis were scored by HE staining and the anti-thyroglobulin antibody $(\mathrm{TgAb})$ were detected by ELISA after 12 weeks of $\mathrm{NaI}$ intaking in all groups of mice. The percentage of Th1, Th2, Th17 and CD $4{ }^{+} \mathrm{CD} 25^{+}$Treg cells in splenocytes were observed by flow cytometry. Besides, The intracellular IL-10 synthesis of B cells in general NOD. H-2 ${ }^{\mathrm{h} 4}$ mice was detected by flow analysis and the total RNA from stimulated CD1d $\mathrm{d}^{\text {hi }}$, $\mathrm{CD}^{+}$and $\mathrm{CD} 1 \mathrm{~d}^{\text {low }} \mathrm{CD}^{-} \mathrm{B}$ cells were extracted for real-time RT-PCR. The thyroiditis scores were significantly higher in $\mathrm{CD} 1 \mathrm{~d}^{\text {low }} \mathrm{CD} 5$ transfer group compared with other 3 groups and no significant differences among the $\mathrm{CD5}^{+}, \mathrm{CD} 1 \mathrm{~d}^{\text {hi }}$ and PBS transfer groups. The concentration of serum $\mathrm{TgAb}$ was significantly lower in CD1 $\mathrm{d}^{\text {hi }}$ transfer group than other 3 groups. The spleen Th17 cell proportion was significantly reduced in $\mathrm{CD} 1 \mathrm{~d}^{\text {hi }}$ transfer group than the PBS and $\mathrm{CD} 1 \mathrm{~d}^{\text {low }} \mathrm{CD}^{-}$transfer groups. In the flow cytometry analysis for intracellular IL-10 secretion of B cells in general NOD. H- $2^{\mathrm{h} 4}$ mice, both $\mathrm{CD} 1 \mathrm{~d}^{\mathrm{hi}}$ and $\mathrm{CD}^{+} \mathrm{B}$ cells secret significantly higher IL-10 protein than CD1d ${ }^{\text {low }} \mathrm{CD}^{-} \mathrm{B}$ cells. Besides, the IL-10 gene expression was significantly increased in $\mathrm{CD}^{\mathrm{hi}}$ and $\mathrm{CD}^{+} \mathrm{B}$ cells compared with CD1d ${ }^{\text {low }} \mathrm{CD}^{-} \mathrm{B}$ cells after stimulation. The $\mathrm{CD} 1 \mathrm{~d}^{\text {hi }}$ and $\mathrm{CD}^{+} \mathrm{B}$ cells may possess regulatory capacity for the competence to secret IL-10. Unlike the $\mathrm{CD} 1 \mathrm{~d}^{\text {low }} \mathrm{CD}^{-} \mathrm{B}$ cells promoting AIT progression, the CD1d ${ }^{\text {hi }}$ B cells may limit AIT by decreasing autoantibodies and Th17 response while the $\mathrm{CD}^{+} \mathrm{B}$ cells limit disease through other ways.

\section{Poster 348}

Autoimmunity Saturday Poster Clinical

\section{ELEVATED SEMAPHORIN5A IN PATIENTS WITH} HASHIMOTO'S THYROIDITIS: A CASE CONTROL STUDY

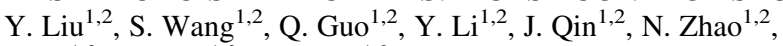

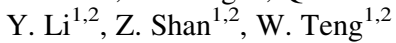

${ }^{1}$ Endocrinology and Metabolism, The First Affiliated Hospital of China Medical University, Shenyang, Liaoning, China; ${ }^{2}$ Institute of Endocrinology, Liaoning Provincial Key Laboratory of Endocrine Diseases, Shenyang, China

Hashimoto's thyroiditis (HT) is the most common chronic autoimmune thyroid disease. It is characterized with elevated specific autoantibodies, including $\mathrm{TgAb}$ (antithyrogloblin antibody) and TPOAb (thyroperoxidase antibody). HT has been traditionally thought as Th1 cells mediated disease, but increasing evidence reported the essential role of Th17 cells in HT recent years. However, the underlying mechanism is not yet fully comprehended. Semaphorin5A (Seman5A) has been shown to be involved in several autoimmune diseases through regulation of immune cells. The aim of the study was to explore the role of Sema5A in HT. We measured the serum Sema5A level in HT $(n=92)$ and healthy controls $(n=111)$ by enzyme-linked immunesorbent assay (ELISA). The expressions in RNA levels of Sema5A and their receptors (plexin-A1 and plexinB3), as well as several cytokines (including IFN- $\gamma$, IL-4 and IL-17) in peripheral blood mononuclear cells (PBMCs) from 23 patients with HT and 31 healthy controls, were also detected by real-time polymerase chain reaction (RT-PCR). In addition, we further investigated the relationship between serum Sema5A and HT. Serum Sema5A in HT increased significantly compared with healthy controls $(P<0.001)$. Moreover, serum Sema5A levels were positively correlated with $\mathrm{TgAb}(\mathrm{r}=0.511, P<0.001)$, TPOAb $(\mathrm{r}=0.423$, $P<0.001)$, TSH $(\mathrm{r}=0.349, P<0.001)$, and IL-17 mRNA expression $(\mathrm{r}=0.442, P<0.001)$. We also detected higher RNA expression of 
Sema5A $(P=0.041)$ in HT compared with control group. In receiver operating characteristic (ROC) analysis, serum Sema5A predicted HT with a sensitivity of $79.35 \%$, and specificity of $96.40 \%$, and the area under the curve (AUC) of the ROC curve was 0.836 (95\% CI: $0.778-0.884, P<0.001)$. These data demonstrated the elevated serum Sema5A in HT patients for the first time. Serum Sema5A levels were correlated with thyroid autoantibodies, and IL-17 mRNA expression. It is plausible that Sema5A may be of importance in the process of immune response of HT patients.

\section{Poster 349}

Autoimmunity Saturday Poster Translational

MACRO TSH IN FERTILE WOMEN: THE NATURE AND EFFECTS ON COMMERCIAL TSH ASSAY PLATFORMS

N. Hattori ${ }^{1}$, K. Aisaka ${ }^{2}$, A. Shimatsu ${ }^{3}$

${ }^{1}$ Pharmaceutical Sciences, Ritsumeikan University, Kusatsu, Japan; ${ }^{2}$ Department of Obstetrics and Gynecology, Hanada Hospital, Tokyo, Japan; ${ }^{3}$ Clinical Research Institute, National Hospital Organization Kyoto Medical Center, Kyoto, Japan

Macro TSH has a molecular mass of $>150 \mathrm{kDa}$ and is mainly a complex of TSH with anti-TSH autoantibodies. Serum thyroid hormone levels are normal despite high serum TSH concentrations in patients with macro TSH, mimicking subclinical hypothyroidism.

The aim of this study was to examine the prevalence of macro TSH in fertile women and the effects of macro TSH on commercial TSH assay platforms. Serum TSH levels were measured in 1794 women who visited an obstetrics and gynecology hospital, and 305 women (aged 15-39 yr) who had serum $\mathrm{TSH}>2.5 \mu \mathrm{U} / \mathrm{mL}$ were enrolled in this study. Macro TSH was examined by polyethylene glycol (PEG) precipitation, gel filtration chromatography, human anti-mouse antibody (HAMA) blockers, and ${ }^{125}$ I-TSH binding study. PEG precipitation ratio of TSH was greater than $75 \%$ (mean + SD in controls) in 54 serum samples. Gel filtration chromatography showed that TSH was eluted at the molecular mass greater than $150 \mathrm{kDa}$ in 27 of the 54 sera. Addition of HAMA blockers to the sera significantly reduced TSH levels in 24 of the 27 sera, suggesting that HAMA interfered to the immunoassay and produced spuriously high TSH values. Significant amount of ${ }^{125}$ I-TSH bound to the serum component and the binding was displaced by an excess amount of unlabeled TSH in one of the three sera without HAMA, indicating the presence of anti-TSH autoantibodies in this patient. TSH levels in these three sera were all higher than those predicted by the non-PEG precipitable TSH levels in three commercially available TSH assay systems (Elecsys, Centaur, and Architect). Macro TSH was found in three of 305 fertile women $(0.98 \%)$ whose serum TSH was greater than $2.5 \mu \mathrm{U} / \mathrm{mL}$, the level at which thyroid hormone replacement therapy may be considered. One of them was found to have anti-TSH autoantibodies and the other two patients had macro TSH with unknown origin. HAMA was found in 24 of 305 patients (7.87\%), mimicking macro TSH.

Macro TSH should be examined when thyroid hormone replacement therapy is considered in fertile women with subclinical hypothyroidism.

\section{Poster 350}

Autoimmunity Saturday Poster Clinical

PREVENTION OF OVERT HYPOTHYROIDISM FOLLOWING RADIOACTIVE IODINE THERAPY FOR GRAVES' DISEASE BY EARLY INITIATION OF LEVOTHYROXINE THERAPY

S. Maraka ${ }^{1,2}$, M. Castro ${ }^{3}$, D. Dean ${ }^{2}$, A. Sharma ${ }^{2}$, V. Fatourechi ${ }^{2}$,

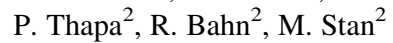

${ }^{1}$ University of Arkansas for Medical Sciences and Central Arkansas Veterans Healthcare System, Little Rock, AR; ${ }^{2}$ Mayo Clinic, Rochester, MN; ${ }^{3}$ Mayo Clinic, Rochester, MN

Radioactive iodine (RAI) therapy is a common method of treating Graves' disease (GD). The majority of patients are hypothyroid 10 weeks thereafter. We aimed to determine whether early treatment with levothyroxine (LT4) after RAI therapy will prevent overt hypothyroidism $(\mathrm{OH})$ and improve quality of life (QOL). Patients (18$70 \mathrm{yr}$ ) with GD treated with RAI were randomized 4 weeks later, in double-blinded manner, to start LT4 $25 \mathrm{mcg}$ /day or placebo. The LT4 dose was increased to $50 \mathrm{mcg} / \mathrm{day}, 2$ weeks later. At 8 weeks all patients were evaluated clinically. Incidence of $\mathrm{OH}$ at 8 weeks, defined as TSH $>4.2 \mathrm{mIU} / \mathrm{L}$ or $\mathrm{fT} 4<0.9 \mathrm{ng} / \mathrm{dL}$ or TT3 $<80 \mathrm{ng} / \mathrm{dL}$ was the primary endpoint. Hyperthyroidism was defined as fT4 $>1.7 \mathrm{ng} /$ dL. The hypothyroid-Health Related Quality of Life (HRQL) and the Thyroid Specific Questionnaire (TSQ) were completed by patients to assess QOL. Higher scores are associated with lower QOL. Thyroid tests and QOL were checked at 4, 6, and 8 weeks. Data is reported as mean (SD). Thirty patients were randomized to LT4 and 31 patients to placebo. There was no difference at baseline between patients in the LT4 and placebo arm regarding age (47 (15) vs. 48 (13) yr), gender (female 76.7 vs. $83.9 \%$ ), thyroid size (32.7 (10.8) vs. 31.4 (13.9) g), and fT4 (3.0 (1.5) vs. 2.3 (1.4) ng/dL). At 8 weeks, OH was found in $11 / 28$ patients $(39.3 \%)$ on LT4 compared to $17 / 31$ patients $(54.8 \%)$ on placebo $(\mathrm{P}=0.23)$. Hyperthyroidism persisted in $8 / 28$ patients $(28.6 \%)$ on LT4 compared to $6 / 31$ patients $(19.4 \%)$ on placebo $(\mathrm{P}=0.41)$. Between 4 and 8 weeks, the TSQ change was -2 (12.8) for the LT4 group and -3.8 (8.7) for the placebo group $(\mathrm{P}=0.33)$. The HRQL change was 12 (28.4) for the LT4 group and 19.8 (25.0) for the placebo group $(\mathrm{P}=0.41)$. Palpitations (3 patients) and atrial fibrillation (1 patient, resolved after holding the study drug) occurred only in the LT4 group. OH develops early post RAI for GD. Early LT4 intervention by this regimen has not significantly diminished the incidence of $\mathrm{OH}$ or altered QOL, but an encouraging trend is present. The intervention can be associated with symptoms of hyperthyroidism, though mostly mild.

\section{Poster 351}

Autoimmunity Saturday Poster Clinical THE RELEVANCE OF THYROID AUTOIMMUNITY IN HYPERHOMOCYSTEINEMIA DURING ACUTE IATROGENIC HYPOTHYROIDISM

F. Cicone ${ }^{2}$, M. Santaguida ${ }^{1}$, C. Virili ${ }^{1}$, A. Papa ${ }^{2}$, A. Tofani ${ }^{2}$, F. Scopinaro ${ }^{2}$, M. Centanni ${ }^{1}$

${ }^{1}$ Medico-Surgical Sciences and Biotechnologies, "Sapienza" University of Rome, Latina, Italy; ${ }^{2}$ Department of Surgical and Medical Sciences and Translational Medicine, "Sapienza" University of Rome, Rome, Italy

Hyperhomocysteinemia is an independent cardiovascular risk factor, producing endothelial dysfunction and atherogenesis, and it is emerging as a key player in the inflammatory activation of autoimmune diseases. Hashimoto's thyroiditis (HT) is the leading cause of hypothyroidism and overt hypothyroidism has been associated to a significant raise of Hcy levels. However, the role of the concurrent presence of autoimmune thyroid disease and overt hypothyroidism on plasma Hcy levels has not been previously assessed. The present study was designed to assess the impact of thyroid autoimmunity on plasma Hcy levels in patients with acute post-surgical hypothyroidism.

A total of 121 patients (Mean age $=46 \pm 12.5 ; \mathrm{F} / \mathrm{M}=102 / 19$ ) were enrolled in the present study. All patients had overt hypothyroidism following total thyroidectomy and radioiodine remnant ablation for thyroid cancer. They were divided into three groups: 1) non- 
autoimmune patients $(\mathrm{n}=53) ; 2)$ inflammatory non autoimmune patients (negative thyroid autoantibodies but presence of lymphocytic infiltration; $n=32$ ); 3) autoimmune patients; $n=29$ ). Serum homocysteine was determined by chemiluminescence immunoassay. Hcy values were above the normal ranges in $98(81 \%)$ patients and within the normal limits in $23(19 \%)$ patients. The resulting median concentrations of Hcy in the whole sample were therefore above the normal range $(16.8 \mu \mathrm{mol} / \mathrm{L})$. Among the patients' characteristics and available blood test parameters, only Hcy levels were significantly different between HT and non-HT patients (median Hcy $=19.7$ vs $16.2 \mu \mathrm{mol} / \mathrm{L}$, respectively; $p=0.018$ ). Serum TSH was not significantly associated with Hcy levels (slope $=0.00005, t=0.071$, $p=0.943$ ). So far, the strongest predictor of Hcy levels (coefficient $=0.25534, t=3.181, p=0.001)$ was the presence of autoimmune thyroiditis. In patients with overt iatrogenic hypothyroidism, those with autoimmune thyroiditis show significantly higher serum Hcy levels than those without HT. The increase of Hcy levels appears to be mainly determined by the HT-related immune-inflammatory condition rather than by the degree of hypothyroidism.

\section{Poster 352}

Autoimmunity Saturday Poster Clinical ANALYSIS OF EARLY THYROIDECTOMY TO INDUCE QUIESCENCE OF MODERATE TO SEVERE THYROID EYE DISEASE

\author{
L. Bodily ${ }^{1}$, S. Zhou ${ }^{1}$, L. Umfrey ${ }^{1}$, K. Duncan ${ }^{1}$, S. Stefko ${ }^{1}$, \\ U. Duvvuri ${ }^{2}$ \\ ${ }^{1}$ Ophthalmology, Univeristy of Pittsburgh Medical Center, \\ Pittsubrgh, PA; ${ }^{2}$ Otolaryngology, UPMC, Pittsburgh, PA
}

Graves disease and other autoimmune conditions of the thyroid can cross react with orbital antigens, leading to an inflammatory condition of the orbit resulting in chronic orbital inflammation, proptosis, diplopia, and dysfunction of the eyelids, cornea, and optic nerve, known as thyroid eye disease (TED). This condition is debilitating, disfiguring, and potentially blinding. The disease course is often protracted. Our empiric observation has been that thyroidectomy leads to faster quiescence of TED. We set out to examine patients with moderate to severe disease, to examine the effect of thyroidectomy on time to quiescence of the disease. TED patients with Clinical Activity Score (CAS) of 4-7 from 2011-2016 at one institution were retrospectively reviewed, all of which were offered IV corticosteroids and thyroidectomy. Quiescence (CAS of 2 or less) at first follow up visit after the primary treatment was the primary outcome. Total time of disease was also examined. We excluded patients with previous thyroidectomy, thyroid cancer, or incomplete records. 26 patients, 13 with thyroidectomy, 13 with steroid infusion only met criteria for inclusion. Baseline features and follow up times were similar. 9/13 thyroidectomy patients had a CAS of 2 or less at first follow up. 4/13 steroid infusion only patients had a CAS of 2 or less $(\mathrm{P}=0.0472)$. There was a non-significant trend to shorter total disease time for thyroidectomy patients. Our results suggest that early thyroidectomy can lead to more rapid resolution of active thyroid disease.

\section{Poster 353}

Autoimmunity Saturday Poster Clinical

MEAN PLATELET VOLUME IS A GOOD MAKER TO MAKE DIAGNOSIS OF THYROID-ASSOCIATED OPHTHALMOPATHY AND TO MAKE A PREDICTION OF ITS PROGNOSIS

J. Tani, H. Eguchi, Y. Hiromatsu, M. Nomura

Endocrinology and Metabolism, Kurume University, Kurume, Fukuoka, Japan
Thyroid-associated ophthalmopathy (TAO) is an autoimmune disease associated with autoimmune thyroid disease, mainly Graves' disease (GD). It has inflammatory conditions in the retro-orbital tissues, which are not fully understood yet. Mean platelet volume (MPV) is recently reported as a good marker of cancer, sepsis, cardiovascular diseases and so on. However, the pathological association between such diseases and MPV is less well understood. Some immunological mechanisms are believed to be involved in it. We here show that MPV of patients with TAO is significantly lower than that of patients with GD without TAO. We recruited 79 TAO patients who had to undergo intravenous steroid therapy because of active and moderate to severe TAO. And we also recruited 77 patients with GD without clinically TAO findings, who were matched FT4 titer and the duration of therapy with anti-thyroid drug with these TAO patients. The MPV of patients with TAO was $9.54 \pm 1.45$ and that of patients with GD without TAO was $9.83 \pm 1.55(\mathrm{p}=0.0176)$. Furthermore, MPV of refractory TAO patients $(n=12)$ decreased at the moment of 6 months after intravenous steroid therapy in spite that MPV of curable TAO patients $(n=48)$ remained unchanged. These findings indicated TAO had some immunological mechanisms different from GD without TAO, and it might involve in refractory nature of TAO. And MPV may be help us to estimate the development of TAO among patients with autoimmune thyroid diseases and to make a prediction of response of intravenous steroid therapy on TAO patients.

\section{Poster 354}

Disorders of Thyroid Function Saturday Poster Case Report

A RARE CASE OF ACQUIRED CENTRAL HYPOTHYROIDISM ASSOCIATED WITH AIP GENE MUTATION

M. Suzuki, J. Klubo-Gwiezdzinska, F.R. Faucz, A. Delaney, C. Stratakis

Endocrinology, National Institutes of Health, Bethesda, MD

23 year old male with history of acquired central hypothyroidism, which has resolved. Genetic testing revealed a rare synonymous variant in the AIP gene (c.807C $>$ T, p. F239=).

The patient was initially seen at age 16 (in 2010), for cold intolerance, growth deceleration, polyuria, fatigue and stalled puberty. Labs notable for:

TSH $0.29 \mathrm{mcIU} / \mathrm{ml}(0.4-4)$

Free T4 $0.9 \mathrm{ng} / \mathrm{dl}(0.8-1.5)$

Prolactin $9.3 \mathrm{mcg} / \mathrm{L}(2-25)$

FSH $4.4 \mathrm{U} / \mathrm{L}$

LH $2.3 \mathrm{U} / \mathrm{L}$

Alpha subunit $0.3 \mathrm{ng} / \mathrm{ml}(\leq 1.2)$

Total testosterone $103 \mathrm{ng} / \mathrm{dl}$

Free testosterone $1.6 \mathrm{ng} / \mathrm{dl}$ (7.4-22.6)

As growth hormone stimulation test, ACTH stimulation test, pituitary and brain MRI were unremarkable, he was commenced on levothyroxine $100 \mathrm{mcg}$ daily. Subsequently, his growth and pubertal development resumed and constitutional symptoms resolved. He was taken off levothyroxine at age 18; no subsequent findings of pituitary hormone deficiency.

Initial pituitary MRI was notable for a possible microadenoma, but follow-up MRI brain and pituitary in 2012 was with no findings of lesions to explain central hypothyroidism.

Genetic testing revealed a rare synonymous variant in the AIP gene $(\mathrm{c} .807 \mathrm{C}>\mathrm{T}, \mathrm{p} . \mathrm{F} 239=)$.

The germline loss of function AIP gene mutations are associated with familial isolated pituitary adenomas (FIPA), in particular somatotropinomas in $78 \%$ of patients, prolactinomas, non-secreting pituitary adenomas and very rarely $\mathrm{TSH}$-omas 
The reported variant is predicted to affect splicing in silico. It has been shown to reduce mRNA levels in vitro, suggesting reduced expression of AIP. Thus, there is a high likelihood of pathogenicity, though penetrance of FIPA in patients with AIP mutations is incomplete (30-40\%).

We do not have evidence to suggest that the diagnosis of central hypothyroidism in our patient was related to the germline pathogenic AIP mutation, as there is no familial history nor was there a sizable adenoma evident on MRI, but ongoing surveillance was recommended. Our patient's presentation confirms the important role of appropriate diagnosis and treatment of central hypothyroidism in patients with stalled puberty.

In central hypothyroidism, normal growth, puberty and fertility can be anticipated, with treatment.

\section{Poster 355}

Disorders of Thyroid Function Saturday Poster Case Report EFFECT OF TWO CONSECUTIVE ANTI-TUMOR MEDICATION ON THYROID FUNCTION

Y. Malan, D. Bellabarba, N. Van Rossum

Endocrinology, CIUSSSE-CHUS, Sherbrooke, Quebec, Canada

Nivolumab, is a novel agent used as second line therapy for progressive diseases in certain neoplasms including renal carcinoma. It is a monoclonal anti programmed cell death-1 (PD-1) antibody and can cause auto-immune effects, including thyroid diseases. Sunitinib, a tyrosine kinase inhibitor, is also used for advanced renal cell carcinoma and also can cause auto-immune thyroid disorders. We report the case of a patient, with progressive renal cell carcinoma, who received Sunitinib followed by Nivolumab and developed thyrotoxicosis followed by severe hypothyroidism. A 62-year old man had resection of a renal cell carcinoma with clear cell and papillary features, pT4N2M0. Six months after surgery, he had progressive loco-regional recurrence and was started on Sunitinib. He developed hypothyroidism $(\mathrm{TSH}=17,3 \mathrm{mUI} / \mathrm{L}, \mathrm{N}=0,35-3,5$; and fT4 $=14 \mathrm{pmol} /$ $\mathrm{L}, \mathrm{N}=11-22$ ) after 3 months of treatment. No replacement therapy was started since he was switched from Sunitinib to Nivolumab, $3 \mathrm{mg} / \mathrm{kg}$ because of tumor progression. 10 weeks later, he developed thyrotoxicosis $(\mathrm{TSH}=0.02 \mathrm{mUI} / \mathrm{L}, \mathrm{fT} 4=40 \mathrm{pmol} / \mathrm{L}, \mathrm{fT} 3=9 \mathrm{pmol} / \mathrm{L})$. TPO antibodies were slightly elevated at $51,3 \mathrm{UI} / \mathrm{mL}(\mathrm{N}=0-34)$ and the TSH receptor antibodies were negative. He was treated with methimazole $5 \mathrm{mg}$ BID and thyrotoxicosis resolved after about 5 weeks, followed by significant hypothyroidism ( $\mathrm{fT} 4=<0.3 \mathrm{pmol} / \mathrm{L}, \mathrm{TSH}=$ $74.4 \mathrm{mUi} / \mathrm{L}$ ), needing replacement therapy. After 10 weeks of thyroxine, at $1,2 \mu \mathrm{g} / \mathrm{kg}$, his TSH is still at $15,6 \mathrm{mUI} / \mathrm{L}$ with a normal $\mathrm{fT} 4=17,7 \mathrm{nmol} / \mathrm{L}$. He received no glucocorticoids. This case is particulalrly interesting because the patient had a normal thyroid function before using Sunitinib. He developed sub-clinical hypothyroidism on that treatment and then a painless thyroiditis on Nivolumab. Compared to other reports, the appearance of thyrotoxicosis occurred somewhat later after the initiation of Nivolumab (10 weeks vs 4). The hypothyroidism was more severe than previously observed. Also the TSH remained elevated with a mid-normal fT4 while on replacement therapy. This may depend on a longer delay in the TSH normalization or on a more marked effect because he received two cancer treatments differently affecting the thyroid function.

\section{Poster 356}

Disorders of Thyroid Function Saturday Poster Case Report NEONATAL GRAVES' DISEASE: IMPORTANCE OF A TEAM COMMUNICATION

A. Manea, S.H. LaFranchi

Pediatric Endocrinology, OHSU, Portland, OR
Neonatal Graves' disease results from transplacental passage of maternal TSHR antibodies in the context of maternal history of Graves': active disease or an autoimmune process persisting despite radioactive iodine ablation or thyroidectomy. Maternal thyroid stimulating immunoglobulin (TSI) level during the 3rd trimester predicts the risk for fetal and neonatal Graves'. Prompt diagnosis and treatment is important, given the significant morbidity and mortality. A 5 day old female presented to emergency room with tachycardia and tachypnea. It was a documented maternal history of Graves' disease, treated with radioablation 4 months prior to pregnancy and levothyroxine. The baby was admitted with concerns for congenital heart disease. Echocardiogram showed moderate pulmonary hypertension. Elevated free T4 $11.9 \mathrm{ng} / \mathrm{dL}(0.96-4.08)$ and undetectable $\mathrm{TSH}<0.008 \mathrm{uIU} / \mathrm{mL}(0.58-5.58)$ were noted, consistent with neonatal Graves' disease. Pediatric endocrinology recommended treatment with propanolol $1 \mathrm{mg}$ PO TID and methimazole $1 \mathrm{mg} / \mathrm{kg} / \mathrm{day}$. The on-call endocrinologist had been consulted after birth, maternal TSI was unknown, thyroid function studies were recommended at day of life (DOL) 4, but not obtained. On further discussion with mother and chart review, it was discovered that: TSI $>500 \%$ at 26 weeks gestation, fetal goiter and tachycardia were noted prenatally, diagnostic for fetal Graves', and methimazole was started at the end of pregnancy. At DOL 7 ECHO showed worsening pulmonary hypertension with heart failure, consistent with persistent pulmonary hypertension (PPHN). The baby was transferred to pediatric ICU, intubated for 9 days, treated with Sildenafil, Hydrocortisone and Milrinone. Free T4 level normalized at DOL 10, TSH remained undetectable until DOL 12. Infant was discharged at DOL 23, with resolved PPHN and normal thyroid function on Methimazole 1mg/ $\mathrm{kg} / \mathrm{day}$. The diagnosis and initial treatment was delayed in this neonate until DOL 5. This case shows the importance of communication between physicians caring for the mother with a history of Graves' disease and physicians caring for a newborn at risk for neonatal Graves'. It is unknown if earlier diagnosis and treatment might have prevented or ameliorated the PPHN.

\section{Poster 357}

\section{Disorders of Thyroid Function Saturday Poster Case Report WHEN THE THYROID IS TO BLAME; A RARE CAUSE OF RHABDOMYOLYSIS}

R. Milyani

Internal Medicine, Bay State Medical Center, Ellington, CT

Hypothyroidism must be considered as a cause for rhabdomyolysis in patients without a known cause. Having this as a differential will allow for early diagnosis and management of rhabdomyolysis with standard therapies, and quick initiation of thyroid replacement therapies to prevent further damage. A 31 year old female with a history of hypothyroidism, cocaine abuse and medication nonadherence presented to the hospital with a month of worsening diffuse myalgias. She had significant pain on palpation of her muscles, and diffuse non-pitting edema. A creatinine kinase (CK) level was checked and returned at 1452 U/L (normal 0-190), diagnosing rhabdomyolysis. Given her history of medication non-compliance, a thyroid-stimulating-hormone (TSH) level was checked returning at $267 \mathrm{mIU} / \mathrm{ml}$ (normal 0.4-4.0). Cocaine use was considered as a differential, but the time-frame from her last drug use to the onset of current symptoms did not overlap. Other causes were ruled out including statin use, exercise, trauma or seizures. Aggressive IV fluids and levothyroxine replacement were started, which rapidly normalized her free T4 and CK levels. Rhabdomyolysis is a potentially life threatening condition caused by damage to musculoskeletal cells. This damage leads to loss of cellular integrity and the release of intracellular components, presenting as myalgias, edema and 
pigmenturia. Common causes include trauma, dehydration and drugs. When a source is unidentified, rarer etiologies such as hypothyroidism must be considered. Hypothyroidism induced rhabdomyolysis is rare with only a few cases reported. The mechanism is unclear, but an impaired state of mitochondrial oxidative metabolism and deterioration of glycogenolysis in severe hypothyroid states, leading to muscle breakdown is theorized. The usual treatment of rhabdomyolysis is aggressive hydration. However, in patients with hypothyroid induced rhabdomyolysis, it is important to start immediate thyroid replacement therapy to prevent further damage. It is important to recognize hypothyroidism as a rare cause of rhabdomyolsis, as thyroid replacement therapy is an important additional therapy in hypothyroidism induced rhabdomyolysis, to prevent further damage and complications.

\section{Poster 358}

Disorders of Thyroid Function Saturday Poster Case Report

\section{FROM FEARING WEIGHT GAIN TO TYROID STORM}

L. Rodriguez, L. Arzeno

Internal Medicine, Morristown Medical Center, Morristown, NJ

Hyperthyroidism is a common condition that occurs in 1.3 percent of the population. Many achieve euthyroid state with medications and/ or ablation and in some cases surgery. Thyroid storm is a feared complication of the hyperthyroid state. A 36-year-old woman who presented to the hospital complaining of dysuria, palpitations and tremors. She had a history of hyperthyroidism and medication noncompliance due to fear of weight gain. Her physical exam disclosed tachycardia, fever and tremors. Laboratory tests included a urinalysis with $>50 \mathrm{wbc} / \mathrm{hpf}$ and many bacteria, free thyroxine level of $7.9 \mathrm{ng} /$ $\mathrm{dL}$ and a thyroid stimulating hormone level of $<0.01 \mu \mathrm{IU} / \mathrm{ml}$. An electrocardiogram showed atrial fibrillation with a rate of 160 beats per minute. A diagnosis of thyroid storm and urinary tract infection was made and she was started on ceftriaxone, esmolol, propylthiouracil (PTU), a solution of potassium iodide and methylprednisolone. Her heart rate was controlled and the thyroid markers trended to normal levels. She completed antibiotics and was eventually discharged on PTU and propranolol. Unfortunately due to recurrence of hyperthyroid state a thyroidectomy had to be performed. Thyroid storm is a rare and life-threatening condition where severe signs and symptoms of thyrotoxicosis are present. It is often precipitated by an acute event such as trauma or infection. Manifestations include arrhythmias, hyperpyrexia, anxiety, nausea, vomiting, and abdominal pain. Lid lag, hand tremor and warm skin may be present. The diagnosis is made when the above mentioned lifethreatening signs and symptoms are combined with biochemical markers of hyperthyroidism. The treatment is complex and should take place in an intensive care unit due to the high mortality rate; it includes betabloquers, thionamides like propylthiouracil and methimazole, a saturated solution of potassium iodide, glucocorticoids, bile acid sequestrants as well as the prompt identification and management of the precipitating event. This case highlights the importance of the early identification and treatment of patients with hyperthyroidism to avoid development of complications such as thyroid storm and as well as the need for thyroidectomy.

\section{Poster 359}

Disorders of Thyroid Function Saturday Poster Case Report FACTITIOUS THYROTOXICOSIS: HOW TO FIND IT P. Vorasart, C. Sriphrapradang Department of Medicine, Faculty of Medicine Ramathibodi Hospital, Mahidol University, Bangkok, Thailand
Diagnosis of surreptitious ingestion of thyroid hormones or thyrotoxicosis factitia, often presents a difficult challenge especially in a patient with underlying Graves' disease. A 35-year-old female presented with frequent syncope and fatigue for 1 month. Graves' disease was diagnosed since 9 years ago. She had severe thyrotoxicosis that needed high dose of anti-thyroid drugs followed by radioactive iodine (RAI) treatment. Thyroid uptake was very high. After the third dose of RAI, sight-threatening Graves' orbitopathy was developed that needed pulse methylprednisolone and orbital radiation. Finally, she underwent thyroidectomy and had postoperative hypothyroidism treated with levothyroxine (LT4) $100 \mathrm{mcg} / \mathrm{day}(1.8 \mu \mathrm{g} / \mathrm{kg} / \mathrm{day})$. At the first visit, she had hypothyroidism due to self-discontinuation of LT4. After resumption of LT4, she developed intractable thyrotoxicosis. LT4 was stopped but thyrotoxicosis was not improved. TSH receptor antibody was $3.93 \mathrm{IU} / \mathrm{L}(<1.75)$. The provisional diagnosis was relapsed Graves' disease. Methimazole was initiated. During 4 years, mean FT3 and FT4 levels were $3.72 \pm 1.38 \mathrm{pg} / \mathrm{mL}$ and $2.16 \pm 0.68 \mathrm{ng} /$ $\mathrm{dL}$, respectively. Ratios of FT3/FT4 were $1.74 \pm 0.29\left(10^{-2} . \mathrm{pg} / \mathrm{ng}\right)$.

We decided to treat relapsed Graves' disease with RAI. However, RAI uptake showed very low uptake. Repeated RAI uptake showed very low uptake after restricted high iodine diet and discontinuation of antithyroid drugs. Total body scan also showed no uptake. Computed tomography (CT) of neck, chest and abdomen were normal. CT reported no visualized thyroid gland. Thyroglobulin level was $0.7 \mathrm{ng} /$ $\mathrm{mL}$ (normal, 3.5-77) while antibody to thyroglobulin was negative. After repetitive measurement, thyroglobulin levels remained low. She refused any medication or substance use. Factitious thyrotoxicosis was confirmed by using bile acid sequestrant, cholestyramine $4 \mathrm{~g}$ four times daily. The thyroid hormone levels returned to normal within 1 month. Thyrotoxicosis factitia was suspected because of absence of goiter, suppressed serum thyroglobulin level, decreased RAI uptake and excellent response after cholestyramine treatment. This report demonstrates various approaches to support the diagnosis of thyrotoxicosis factitia.

\section{Poster 360}

Disorders of Thyroid Function Saturday Poster Case Report COEXISTENCE OF PRIMARY HYPERPARATHYROIDISM DUE TO MEN1 IN A HYPERCALCEMIC PATIENT WITH GRAVES' DISEASE

B.H. Kimura ${ }^{3}$, S.S. Davis ${ }^{1}$, S. Bhat ${ }^{1,2}$

${ }^{1}$ Division of Endocrinology, Diabetes and Hypertension, David Geffen School of Medicine at UCLA, Los Angeles, CA, CA; ${ }^{2} \mathrm{VA}$ Greater Los Angeles Healthcare System, Los Angeles, CA; ${ }^{3}$ Cedars Sinai Medical Center, Los Angeles, CA

Hypercalcemia may occur in up to $20 \%$ of patients with Graves' (GD) disease cases ${ }^{1}$. The mechanism is thought to be enhanced bone resorption, unrelated to PTH levels, due to activation of IL-6 and T3 induced hypercalcemia. More unusual causes of hypercalcemia such as coexistent primary hyperparathyroidism (PHP) due to adenomas occurs infrequently in less than $1 \%$ of GD patients, and even more rare is multigland hyperplasia ${ }^{2}$. We report a patient with thyrotoxicosis and PHP with hypercalcemia due to hyperplasia. She was found to have undiagnosed MEN1, and underwent simultaneous thyroidectomy and parathyroidectomy. The occurrence of GD and PHP with known MEN1 was described in one other case report in the literature $^{3}$. A 36-year-old female with history of recurrent peptic ulcers presented with anorexia, nausea, and ten pound weight loss. Initial labs showed hyperthyroidism and hypercalcemia. FH revealed father, paternal grandfather, sister and brother required parathyroidectomies for PHP before age 50. Mother had Hashimoto's thyroiditis. Exam was significant for sinus tachycardia and diffuse goiter. Calcium and PTH were elevated. GD was confirmed by diffuse uptake 
on scan and positive antibodies. The diagnosis of MEN1 was confirmed genetically. She underwent simultaneous thyroidectomy and parathyroidectomy with autotransplantation of parathyroid to the forearm. Pathology revealed GD and multigland parathyroid hyperplasia. Hypercalcemia in GD is usually due to increased bone resorption, but PHP must be considered. In this case, a rare presentation of PHP due to MEN1 was found. This emphasizes the value of FH and the PTH level. PTH levels are necessary as an elevation defines coexisting PHP in a patient with thyrotoxicosis and hypercalcemia, and broadens the differential diagnosis to include the rare coexistence of MEN1 with GD only found in one other case in the literature. Clinicians should be aware of the rare occurrence of PHP due to MEN1 in a GD patient presenting with hypercalcemia.

\section{Poster 361}

Thyroid Cancer Saturday Poster Case Report

VOCAL CORD PARALYSIS AND OVERT HYPOTHYROIDISM DUE TO METASTATIC COLON ADENOCARCINOMA TO THE THYROID GLAND B.H. Kimura ${ }^{1}$, A. Mita ${ }^{3}$, A. Ho ${ }^{4}$, W.L. Sacks ${ }^{2}$

${ }^{1}$ Internal Medicine, Cedars Sinai, Los Angeles, CA; ${ }^{2}$ Endocrinology, Cedars Sinai, Los Angeles, CA; ${ }^{3}$ Hematology Oncology, Cedars

Sinai, Los Angeles, CA; ${ }^{4}$ Otolaryngology, Cedars Sinai, Los Angeles, CA

Metastatic cancer to the thyroid is a rare occurrence. It is not known why metastasis to the thyroid is rare but some postulate that the high blood flow or high iodine environment inhibit the development of metastatic cancer cells. The estimated occurrence of metastasis to the thyroid is highly variable ranging from $1-3 \%$ in patients who underwent surgery for suspected malignancy, while others report up to a rate of $24 \%$ on autopsy of patients who died of cancer. Colon cancer metastasis to the thyroid is estimated to be only $10-14 \%$ of all reports of cancer metastasis to the thyroid. We report a case of a 67 year old female with colon adenocarcinoma who was found to have metastatic disease to the thyroid. At the time of presentation to the endocrinologist, she had stage IV colon adenocarcinoma with metastasis to the liver and lung. She presented to the thyroid clinic after developing sudden hoarseness and was noted to have right vocal cord paralysis by laryngoscopy. On physical examination, she had a firm asymmetric goiter three times normal in size, normal heart rate and temperature, however brachiocephalic reflexes showed slowed relaxation phase. Neck ultrasound revealed a diffusely hypoechoic vascular thyroid gland without definitive nodules and lab testing revealed hypothyroidism (TSH $90 \mathrm{mcu} / \mathrm{mL}$ ). Fine needle aspiration of the right thyroid obtained cells that were CDX-2 positive and morphologically consistent with colon adenocarcinoma. There are very few cases reporting colon cancer metastasis to the thyroid, and the majority are found incidentally on imaging. Our patient presented with symptoms that led to the diagnosis of metastatic disease to the thyroid. The metastasis consumed the thyroid parenchyma causing significant hypothyroidism and rapid enlargement of the gland resulting in recurrent laryngeal nerve compression and vocal cord paralysis. Though a rare occurrence, metastatic disease to the thyroid should be considered in patients with thyroid nodules or goiter who have a known primary malignancy.

\section{Poster 362}

Disorders of Thyroid Function Saturday Poster Clinical

MATERNAL AND NEONATAL OUTCOMES OF PREGNANCIES AFFECTED BY HYPOTHYROIDISM

Z. Kiran ${ }^{1}$, A. Sheikh ${ }^{1}$, S. Malik ${ }^{2}$, A. Meraj ${ }^{3}$, M. Masood ${ }^{4}$, S. Ismail ${ }^{3}$, O. Rashid ${ }^{1}$, N. Majeed ${ }^{5}$, L. Sheikh ${ }^{6}$, N. Islam ${ }^{1}$
${ }^{1}$ Endocrinology, Aga Khan University Hospital, KARACHI, Pakistan; ${ }^{2}$ Endocrinology, Polyclinc, Islamabad, Punjab, Pakistan; ${ }^{3}$ Dow University of Health Sciences, Karachi, Pakistan; ${ }^{4}$ Karachi Medical \& Dental College, Karachi, Sindh, Pakistan; ${ }^{5}$ Armed Force Institute of Pathology, Rawalpindi, Punjab, Pakistan; ${ }^{6}$ Obstetrics \& Gynecology, Aga Khan University Hospital, Karachi, Sindh, Pakistan

The effects of hypothyroidism on the women of reproductive age and pregnant population is currently an area of ongoing research and controversies. Studies have described abortion, anemia, pregnancyinduced hypertension, postpartum hemorrhage, premature birth and neurodevelopmental dysfunction to be associated with overt hypothyroidism during pregnancy. Overt hypothyroidism has an incidence of about 0.25 to $0.91 \%$ in pregnancies whereas subclinical hypothyroidism is even more prevalent with a range of 3 to $15 \%$. Subclinical hypothyroidism is associated with pregnancy loss, stillbirth as well as gestational diabetes. Our study aims to study the effect of overt as well as subclinical hypothyroidism on maternal and neonatal outcomes in Pakistani women. We conducted a crosssectional retrospective study on 572 patients in the Aga Khan University Hospital after ethical approval. Data was collected on pregnant females who were a known case of hypothyroidism or were diagnosed during their antenatal visits during the year 2010-2016. We noted the etiology of hypothyroidism as well as other comorbidities. Maternal outcomes were recorded as abortion, pregnancy induced hypertension, pre-eclampsia, postpartum hemorrhage and placental abruption. Neonatal outcomes were noted as premature birth, low birth weight, intrauterine death, neonatal respiratory distress syndrome, sepsis, hyperbilirubinemia, hypocalcemia, need for intensive care admission and neonatal death. Data analysis was performed on Statistical Package for the Social Sciences version 19.0. During the 5 years duration, our eligible cases had $86 \%$ live births and 9\% abortions. Pregnancy induced hypertension occurred in $11 \%$, pre-eclampsia in $4 \%$ and postpartum hemorrhage in $36 \%$. Out of the live births, $15 \%$ were premature and $18 \%$ had low birth weight. Thirty two percent of neonates had issues of hyperbilirubinemia among whom $11.4 \%$ required phototherapy. Ten percent required admission of more than 24 hours in intensive care unit. Successful live births in our patients were complicated by neonatal hyperbilirubinemia and maternal postpartum hemorrhage. We report the maternal and neonatal outcomes of hypothyroid pregnancies for the first time from the region of Pakistan.

\section{Poster 363}

Disorders of Thyroid Function Saturday Poster Clinical

PREVALENCE AND CLINICO-EPIDEMIOLOGY OF THYROID DYSFUNCTION IN PATIENTS WITH METABOLIC SYNDROME- A PAN-INDIA STUDY V. Deshmukh ${ }^{1}$, F. Farishta ${ }^{2}$, R. Hegde ${ }^{3}$

${ }^{1}$ Shree Hospital, Siddhant Mansion, Opp AgaKhan Palace, Kalyani Nagar, Nagar Road, Pune, Pune, Maharashtra, India; ${ }^{2}$ FS Endocrinology \& Diabetic Center 4th Floor, Room No. 404, Opposite Neerus Showroom, King Kothi, - 500 001, Hyderabad, Telangana, India; ${ }^{3}$ Abbott India Limited Floor 16, Godrej BKC, Plot No. C - 68, BKC, Near MCA Club, Bandra (E) Mumbai - 400051 , Mumbai, Maharashtra, India

The objective of this study was to assess the prevalence, clinical and epidemiological factors of thyroid dysfunction (TD), with respect to individual components of metabolic syndrome (MetS), in Indian milieu. In this cross-sectional study, 432 adult patients with an established diagnosis of MetS (ATP III criteria with modified waist) were enrolled across 10 centers in India. Overt hypothyroidism was 
defined as TSH $>4.50 \mu \mathrm{IU} / \mathrm{mL}$ (FT4: $<0.8 \mathrm{ng} / \mathrm{dL}$; FT3: $<1.4 \mathrm{pg} / \mathrm{mL}$ ); subclinical hypothyroidism-TSH $>4.50 \mu \mathrm{IU} / \mathrm{mL}$ (FT4: $0.8-1.8 \mathrm{ng} /$ dL; FT3: $1.4-4.4 \mathrm{pg} / \mathrm{mL}$ ); overt hyperthyroidism-TSH $<0.45 \mu \mathrm{IU} /$ mL (FT4: $>1.8 \mathrm{ng} / \mathrm{dL}$; FT3: $>4.4 \mathrm{pg} / \mathrm{mL}$ ); subclinical hyperthyroidism $-\mathrm{TSH}<0.45 \mu \mathrm{IU} / \mathrm{mL}$ (FT4: $0.8-1.8 \mathrm{ng} / \mathrm{dL}$; FT3: $1.4-4.4 \mathrm{pg} / \mathrm{mL}$ ). Descriptive statistics and logistic regression analysis was used for statistical analysis. Out of 432 enrolled patients with MetS, 121 (28\%; 95\% CI: $23.83 \%, 32.32 \%$ ) were diagnosed with TD (mean age: 47.9 [10.96] years; mean BMI: $30 \pm 4.94 \mathrm{~kg} / \mathrm{m}$ ), with higher prevalence in women [91 (75\%)] compared to men [30 (25\%)]. Of these 121 TD cases, 39 (32\%) were known cases compared to 82 (68\%) newly diagnosed cases. Most of patients were in $>45$ years' age group versus $\leq 45$ years (men: $63 \%$ vs. $37 \%$; women: $59 \%$ vs. $41 \%)$. The MetS components observed in patients diagnosed with TD were reduced HDL-C (88\%), followed by raised HOMA-IR (87\%), systolic blood pressure (78\%), diastolic blood pressure $(60 \%)$, fasting glucose $(59 \%)$, triglycerides $(33 \%)$ and waist circumference (greater than cut-off values [99\%]). Of 432 MetS patients, overt hypothyroidism was reported in $18 \%$ patients; subclinical hypothyroidism in $8 \%$; overt hyperthyroidism in $2 \%$ and subclinical hyperthyroidism in $1 \%$ patients. Of the 111 patients with hypothyroidism (26\%), 90 patients $(74 \%)$ were on levothyroxine treatment. Patients with MetS have considerably high prevalence of TD of which hypothyroidism was the most commonly reported TD. Age and gender represent significant risk factors for TD in MetS patients, prompting a detailed clinical and laboratory evaluation in these groups. This study highlights the need for high degree of clinical suspicion for early diagnosis and management of TD for better outcomes in MetS patients.

\section{Poster 364}

Disorders of Thyroid Function Saturday Poster Clinical

HEART RATES IN TYHYROTOXIC FETUSES OF WOMEN IN REMISSION AFTER ABLATIVE THERAPY FOR GRAVES' DISEASE

N. Momotani

Endocrinology, Tokyo Health Service Association, Tokyo, Japan

Background: Fetal hyperthyroidism (FH) could develop in women in remission after ablative therapy for Graves' disease. A definitive method for diagnosing FH is the examination of free T4 and TSH concentrations by sampling fetal blood, but this method may be discouraged because of possible serious complications. A fetal heart rate (FHR) above 170 is known as one of the potential sings of FH. However, this may be applicable only to severe FH since FHR physiologically decreases as pregnancy progresses and it is not until around 20 weeks of gestation that fetal thyroid responds to the stimulation of TSH receptor antibodies.

Objectives: To know FHRs that indicate FH.

Methods: Cut-off levels of maternal TBII and TSAb that indicate FH were determined based on TBII and TSAb in the third trimester in women treated for Graves' disease whose neonates had hyperthyroidism. When FH was estimated by the cut-off levels and also by FHRs, iodine was given to the mothers. Thionamides were added when free T4 was increased after a transient fall during iodine administration. L-T4 was also given to prevent maternal hypothyroidism. Gestational time was taken into account in assessing the FHRs. The reason for using iodine was that it has few side effects and exposes the fetus to a lower risk of hypothyroidism than thionamides (JCEM 75: 738-744, 1992). FHRs in relation to gestational time at the initiation of treatment were examined.

Results: FH was estimated in 10 women between 20 and 33 weeks of gestation and iodine was started. The highest FHR (180 bpm) was seen in a woman at 22 weeks of gestation. In another 5 of the 9 whose
FHRs were $163-169 \mathrm{bpm}$ were at $20-27^{\text {th }}$ gestational week and FHRs of the other 4 were $151-158 \mathrm{bpm}$ at $31-33^{\text {th }}$ gestational week. Overt neonatal hyperthyroidism developed in 9 of the 10 .

Conclusions: Gestational time needs to be taken into account in the evaluation of FHRs.

\section{Poster 365}

Iodine Uptake \& Metabolism Saturday Poster Clinical IODINE NUTRITIONAL STATUS IN MEXICAN PREGNANT AND LACTATING WOMEN, THEIR NURSING INFANTS AND SCHOOL AGED CHILDREN FROM AN INDIGENOUS POPULATION IN CENTRAL MEXICO

A.M. Estardante ${ }^{1}$, O. Cuenca ${ }^{2}$, P. García Solis ${ }^{2}$, A. Aguilera Barreyro $^{1}$, J.C. Solís-Sáinz ${ }^{2}$, X. He ${ }^{3}$, E.N. Pearce ${ }^{3}$, L. Braverman ${ }^{3}$

${ }^{1}$ Faculty of Natural Sciences, Autonomous University of Queretaro, Queretaro, Mexico; ${ }^{2}$ Laboratory of Endocrinology and Nutrition, Department of Biomedical Research, Autonomous University of Queretaro, Queretaro, Mexico; ${ }^{3}$ Section of Endocrinology, Diabetes, \& Nutrition, Boston University School of Medicine, Boston, MA

Iodine is required for normal neurodevelopment and is an essential component of the thyroid hormones. The most vulnerable stage for iodine deficiency occurs during pregnancy and early childhood. The objective was assess the iodine nutritional status of pregnant (PW) and lactating women (LW), nursing infants (NI), and school-aged children (SAC) in Amealco, Querétaro, Mexico.46 PW provided spot urine samples (SUS), of whom 36 also provided SUS during lactation at $7.6 \pm 4.5$ weeks postpartum. Women provided 34 household table salt samples. 24 of the LW each provided 6 breast milk (BM) samples (morning, noon, and afternoon on two consecutive days). 31 of the women's NI provided SUS and 38 unrelated SAC provided SUS. Samples of local dietary food (bread, eggs, quelites and corntortillas) were collected for iodine measurement. Iodine was measured by standard procedures. Socioeconomic status was mainly very low $(27.8 \%)$ or low $(66.7 \%)$. The median urine iodine concentration was $171 \mu \mathrm{g} / \mathrm{l}$ in PW, $134 \mu \mathrm{g} / \mathrm{l}$ in LW, $295 \mu \mathrm{g} / \mathrm{l}$ in NI and $471 \mu \mathrm{g} / \mathrm{l}$ in $\mathrm{SAC}$. The median BM iodine concentration was $178 \mu \mathrm{g} / \mathrm{l}$. The mean iodide concentration (IC) in household salt was $28 \pm 7 \mathrm{ppm} ; 85.0 \%$ of salt samples were adequately iodized according to Mexican regulations (20-40 ppm), and only $6.0 \%$ were below the $15 \mathrm{ppm}$ WHO threshold. The IC per portion in food samples was $27 \pm \mu \mathrm{g}, 12 \pm \mu \mathrm{g}$, $7 \pm \mu \mathrm{g}$ and $1 \pm \mu \mathrm{g}$ for bread, eggs, quelites and corn-tortillas, respectively. Iodine nutritional status in pregnant and lactating women from central México are adequate and those in nursing infants and school age children more than adequate, despite the low socioeconomic level, and low levels of iodine in staple foods.

\section{Poster 366}

Iodine Uptake \& Metabolism Saturday Poster Basic

\section{INVESTIGATING IODINE NUTRITION IN NAGORNO} KARABAKH

N. Hutchings ${ }^{1,2}$, S. Baghdasaryan ${ }^{3}$, M. Qefoyan ${ }^{4}$, M. Manoukian ${ }^{5}$, K. Atayan ${ }^{6}$, J.P. Bilezikian ${ }^{1}$

${ }^{1}$ College of Physicians \& Surgeons, Columbia University, New York, NY; ${ }^{2}$ Endocrinology, Yerevan State Medical University, Yerevan, Armenia; ${ }^{3}$ Management Mix, Yerevan, Armenia; ${ }^{4}$ National Institute of Health, Ministry of Health, Yerevan, Armenia; ${ }^{5}$ Manoukian Medical Group, Stanford University Healthcare Alliance, Mountain View, CA; ${ }^{6}$ Ministry of Health, Stepanakert, Nagorno Karabakh, Armenia

Iodine is a required nutrient for thyroid function. The mountainous terrain of the Republic of Nagorno Karabakh in the south Caucasus 
suggests that it is likely to be endogenously deficient in iodine. The region is heavily dependent on the Republic of Armenia for food imports including iodized salt. Since 2005, iodine is a governmentsanctioned compulsory supplemental ingredient in salt in Armenia. However, there has never been a study of iodine nutrition in Nagorno Karabakh. We, therefore, sought to determine in the level of iodine nutrition in Nagorno Karabakh as well as the iodine content of table salt in the region. The study was cross-sectional, using a modified cluster model to measure urine and salt iodine concentrations from a representative sample of children living in Karabakh. Urine and table salt samples from 772 children aged 8-12 were obtained from 30 schools throughout the country. Repeat urine samples from $19 \%$ of participants were obtained. Urine and salt samples were analysed for iodine content, and repeat samples were used to reduce intraindividual variability and provide adjusted urine iodine concentrations (UICs). The median adjusted UIC was $206 \mathrm{mcg} / \mathrm{L}$, with $89 \%$ of samples within the normal range of $100-299 \mathrm{mcg} / \mathrm{L}$. There were small but statistically significant differences within the groups between the adjusted UIC values of 8 administrative regions ( $p=0.015$ ), but there was no difference between individual sites. A random sampling of 294 salt samples representing all study sites indicated that all were iodized, with a median iodine content of $36.2 \mathrm{mg} / \mathrm{kg}$, and $95 \%$ of samples within $25-55 \mathrm{mg} / \mathrm{kg}$, the level considered to be sufficient to ensure nutritional iodine adequacy. We have shown that among school-age children in Karabakh, iodine nutrition is adequate and the salt is appropriately iodized. These results illustrate the remarkable success of the salt iodization program in Armenia providing sufficient but not excessive iodine to the mountainous region of Nagorno Karabakh.

\section{Poster 367}

Iodine Uptake \& Metabolism Saturday Poster Basic

\section{IODINE NUTRITION IN ARMENIA: A MODEL} OF REPRESENTATIVE SURVEILLANCE

N. Hutchings ${ }^{1,3}$, Y. Aghajanova ${ }^{2,3}$, S. Baghdasaryan ${ }^{4}$, M. Qefoyan ${ }^{5}$, C.A. Sullivan ${ }^{6}, \mathrm{X} . \mathrm{He}^{6}$, M. Manoukian ${ }^{7}$, G. Gerasimov ${ }^{8}$,

L. Braverman ${ }^{6}$, J.P. Bilezikian ${ }^{1}$

${ }^{1}$ College of Physicians \& Surgeons, Columbia University, New

York, NY; ${ }^{2}$ Endocrinology, Muratsan University Hospital, Yerevan, Armenia; ${ }^{3}$ Endocrinology, Yerevan State Medical University, Yerevan, Armenia; ${ }^{4}$ Management Mix, Yerevan, Armenia; ${ }^{5}$ National Institute of Health, Ministry of Health, Yerevan, Armenia;

${ }^{6}$ Endocrinology, Diabetes, and Nutrition, Boston University, Boston, MA; ${ }^{7}$ Manoukian Medical Group, Stanford University Healthcare Alliance, Mountain View, CA; ${ }^{8}$ Iodine Global Network, Ottawa, Ontario, Canada

In the mid-1990s, researchers identified high rates of endemic goiter in Armenia. As a result, in 2004 the government implemented a universal salt iodization program. In the past 12 years, however, there has been no formal study of iodine nutrition in the country despite continued observation of high rates of goiter. This study sought to determine the prevalence of iodine deficiency in Armenia 12 years after universal salt iodization was implemented. We conducted a cross-sectional study to measure urine and salt iodine concentrations from a representative sample of children and women living in Armenia. Urine and table salt samples from 357 schoolaged children (SAC) (mean age 10.5 yrs, $46 \%$ female), 353 pregnant women (PW, mean age $26 \mathrm{yrs}$ ) and 353 women of reproductive age (WRA, mean age 36 yrs) were obtained from 13 towns throughout the country. Repeat urine samples from $18 \%$ of participants were obtained. Urine and salt samples were analysed for iodine content, and repeat samples were used to reduce intraindividual variability and provide adjusted urine iodine concen- trations (UICs) which were weighted based on population and geographic distribution. The median UICs were $242 \mathrm{mcg} / \mathrm{L}$ for $\mathrm{SAC}, 227 \mathrm{mcg} / \mathrm{L}$ for PW, and $302 \mathrm{mcg} / \mathrm{L}$ for WRA. Within each of the three groups, there were no differences found between the 13 study sites. There was no effect of sex or age on the adjusted mean UIC for SAC, or of age on the adjusted mean UIC of PW. There was a small but significant negative effect of age on the adjusted mean UIC of WRA ( $\left.p=0.003, R^{2}=0.0287\right)$. PW taking iodine supplements showed a higher mean UIC (237 mgl/L) than those not taking supplements $(222 \mathrm{mcg} / \mathrm{L}, p=0.01)$. The median iodine content of salt from a subset of samples was $31.6 \mathrm{mg} / \mathrm{kg}$. In conclusion, the median UIC for SAC and PW are within the WHO ranges indicative of sufficient iodine nutrition, while that of WRA indicates slightly excessive intake. The results reveal that 12 years after iodine supplementation was initiated, but without regular surveillance, Armenia continues to be iodine sufficient, nationwide, in children, pregnant women, and women of reproductive age.

\section{Poster 368}

Thyroid \& Development Saturday Poster Clinical

\section{RELATION BETWEEN ADHD, AUTISM AND} CONGENITAL HYPOTHYROIDISM

J.J. Bongers-Schokking ${ }^{2}$, Y.B. de Rijke ${ }^{1}$, S.M. de Muinck KeizerSchrama

${ }^{1}$ Department of Clinical Chemistry, Erasmus MC, University Medical Center Rotterdam, Rotterdam, Netherlands; ${ }^{2}$ Pediatrics, Sophia Children's Hospital Erasmus MC, Rotterdam, Zuid-Holland, Netherlands

Background: For patients with congenital hypothyroidism $(\mathrm{CH})$ not only a normal cognitive outcome is important, but also a normal behavioral development. We analyzed to what extent behavioral problems in $\mathrm{CH}$ at ages 6 and 11 years are related to early over and undertreatment.

Subjects and methods: $55 \mathrm{CH}$ patients were followed from birth until 11 years. At 6 and 11 years parents rated the Child Behavior Checklist (CBCL), whereas teachers rated the Teacher's Report Form (TRF). The aggregated scores for the domains Attention problems, Delinquency and Aggression, addressed as ADA scores, as well as those for the domains Withdrawn, Anxious, Social and Thought problems, addressed as WAST scores, were related to the occurrence of over and undertreatment in five periods, from one month to 11 years. Over and undertreatment were defined as free thyroxine ( $\mathrm{fT}_{4}$ ) concentrations above/below the range of the patient's individual $\mathrm{fT}_{4}$ steady state concentration (SSC) (Bongers-Schokking et al., Thyroid 2013;23:160-165).

Results: Parental and teachers' ratings at 6 and 11 years of $\mathrm{CH}$ patients, overtreated in period 1-3 months after birth, showed 4.00 (0.87) $[2.30 ; 5.71]$ points higher ADA scores (mean (SEM) $[95 \%$ $\mathrm{CI}])$ than those not-overtreated $(\mathrm{p}<0.001)$; patients undertreated in period 3-6 month showed $6.19(0.99)$ [4.23; 8.16] points higher WAST scores than those not-undertreated $(\mathrm{p}<0.001)$. In no other period such significant positive relations were found. ADA and WAST scores at ages 6 and 11 were comparable $(\mathrm{p}=0.788$ and $\mathrm{p}=0.818$, respectively) and correlated strongly: parents $\mathrm{r}=0.686$ and $r=0.617$, respectively, teachers $r=0.818$ and $r=0.575$, respectively, $<0.001$ for all. Patients with severe and mild $\mathrm{CH}$ were equally affected, pleading for a postnatal cause.

Conclusions: Our $\mathrm{CH}$ patients showed behavioral problems. Overtreatment in period 1-3 months postnatally led to ADHD-like behavior, whereas undertreatment in period 3-6 months led autistiform behavior. These problems, assessed at 6 and 11 years, seem permanent and possibly avoidable by adequate treatment. 


\section{Poster 369}

Thyroid Cancer Saturday Poster Basic

\section{THIOREDOXIN INTERACTING PROTEIN AS A TUMOR SUPPRESSOR IN THYROID CANCER}

L.J. Hostetter ${ }^{1}$, R. Mackie ${ }^{1}$, V. Sharma ${ }^{1}$, H. Somerset ${ }^{2}$, J. Morrison ${ }^{1}$, B. Haugen ${ }^{1}$

${ }^{1}$ Department of Medicine, Division of Endocrinology \& Metabolism, University of Colorado School of Medicine, Aurora, CO;

${ }^{2}$ Department of Pathology, University of Colorado School of

Medicine, Aurora, CO

Thioredoxin interacting protein (TXNIP) is expressed in differentiated thyroid cancer (DTC) and is low to absent in anaplastic thyroid cancer (ATC). We hypothesize that TXNIP acts as a tumor suppressor in thyroid cancer and loss will transform DTC into poorlydifferentiated thyroid cancer (PDTC) or ATC. We used in vitro and in vivo models to explore this hypothesis. CRISPR/Cas-9 was used to create a TXNIP knock-out (KO) TPC-1 (DTC, expresses TXNIP) cell line. TPC-1 TXNIP-KO cells were injected into the flanks of nude mice $(n=8)$ to analyze tumor growth.

A complementary transgenic model, TPO-Cre, LSL-BrafV600E mice were bred with TXNIP fl/fl mice to generate mice with PTC harboring WT (BRAF) or KO TXNIP (BRAF/TXNIP KO). Euthanized at 8 weeks, mouse weight, thyroid weight, serum T4, TXNIP RNA, and H\&E tumor stains were obtained from the experimental TXNIP KO mice to compare to TXNIP WT/BRAF littermates. TXNIP-KO was confirmed by western blot in TXNIP KO TPC1 cells. Invasion capability of TXNIP KO TPC1 cells compared to TXNIP WT cells was unchanged. TPC1 cells do not grow in nude mice, and TXNIP KO TPC1 cells did not grow in nude mice after 8 weeks.

Transgenic BRAF $(n=19)$ and BRAF/TXNIP KO $(n=25)$ littermates were generated and followed for 8 weeks. Growth rates were similar in the two groups. As previously shown, serum T4 levels were lower in BRAF versus non-BRAF mice. T4 levels were similar in BRAF and BRAF/TXNIP KO mice. Examination of the thyroid tumor showed a 5-fold lower TXNIP RNA levels in the BRAF/TXNIP KO mice compared with BRAF mice. There was no difference in thyroid tumor weight at 8 weeks between the two groups. $63 \%$ of the BRAF and $64 \%$ of the BRAF/TXNIP KO mice had extrathyroid extension of the tumor. There was no histologic evidence of PDTC (STI growth, increased mitoses, necrosis) or gross LN involvement in either group. Low levels of TXNIP appear be a marker of tumor aggressiveness in human thyroid cancer, but this putative tumor suppressor does not induce tumor aggressiveness in our in vitro and in vivo models. Allowing the transgenic mice longer follow-up (6-12 months), may show differences, but may be subtle.

\section{Poster 370}

Thyroid Cancer Saturday Poster Basic

\section{ETV5, AN ETS FAMILY TRANSCRIPTION FACTOR,} DRIVES EPITHELIAL-TO MESENCHYMAL TRANSITION THROUGH EXPRESSION OF TWIST1 IN PAPILLARY THYROID CANCER

O. Puli ${ }^{1,3}$, B.P. Danysh ${ }^{1}$, D. Sinha ${ }^{1,2}$, G.J. Cote ${ }^{1}$, M.E. Cabanillas ${ }^{1}$, M. Hofmann ${ }^{1}$

${ }^{1}$ Endocrine Neoplasia and Hormonal Disorders, MD Anderson Cancer Center, Houston, TX; ${ }^{2}$ The University of Houston, Houston, TX; ${ }^{3}$ Moffitt Cancer Center, Tampa, FL

Oncogenic mutations in RET, RAS, and BRAF genes serve as primary drivers of thyroid cancer. In papillary thyroid cancers (PTC), BRAF V600E is the most prevalent mutation and thus a key thera- peutic target. Analysis of PTC samples data sets (NCBI GEO and TCGA) indicated that ETV5, a member of the PEA3 subfamily of ETS transcription factors, is overexpressed in these tumors in comparison to normal thyroid samples. Our preliminary data using a PTC cell line also showed that ETV5 is a downstream effector of the MAPK pathway, but not the PI3K pathway. While ETV5 seems critical for PTC cell proliferation, it might have other functions. Our objectives were to identify specific ETV5-regulated genes/proteins and investigate whether ETV5 drives epithelial-to-mesenchymal transition (EMT) in these cells in the hope to establish this molecule as a marker of progression to advanced disease. We used the KTC1 cell line (human PTC), which harbors a BRAF V600E mutation, as well as a vemurafenib-resistant KTC1 subclone with concurrent BRAF V600E and KRAS G12D mutations. ETV5 was downregulated in KTC1 cells by shRNA or upregulated by lentiviral transduction. PCR arrays were used to probe for EMT or cell motility markers and results confirmed by qPCR. Finally we performed ChIPPCR to demonstrate binding of ETV5 to the promoter of selected genes found with the PCR arrays. Our studies revealed that decreased ETV5 expression in PTC cells caused significant downregulation in SNAI1 and TWIST1 transcript expression. ChIP-PCR analysis indicated that ETV5 directly binds to the TWIST1 promoter to activate its expression and EMT. However, SNAI1 expression appeared not to be directly dependent on ETV5. Increase in ETV5 expression caused a concomitant increase of TWIST1 and SNAI1 expression, a loss of markers of cell-cell and cell-extracellular matrix interaction, and promoted cell migration. ETV5 acts downstream of the MAPK pathway and plays a crucial role in PTC proliferation and EMT. ETV5 level of expression could therefore serve as a predictive biomarker of tumor aggressiveness linked to RAS mutations or MAPK pathway alterations. Inhibitors of the PEA3 sub-family of transcription factors are presently being tested.

\section{Poster 371}

Thyroid Cancer Saturday Poster Basic

\section{DETAILED CHARACTERIZATION OF HUMAN} TELOMERASE REVERSE TRANSCRIPTASE (HTERT) PROMOTER METHYLATION AND ALTERNATIVE SPLICING IN THYROID CANCER

\section{B. Avin ${ }^{1,3}$, Y. Wang ${ }^{1}$, R.E. Workman ${ }^{2}$, I. Lee ${ }^{2}$, L. Florea ${ }^{4,5}$,} W. Timp ${ }^{2}$, C.B. Umbricht ${ }^{1,6}$, M.A. Zeiger ${ }^{1,6}$

${ }^{1}$ Surgery, Johns Hopkins University School of Medicine, Baltimore, MD; ${ }^{2}$ Biomedical Engineering, Johns Hopkins University, Baltimore, MD; ${ }^{3}$ Graduate Program of Biochemistry Cellular and Molecular Biology, Johns Hopkins University School of Medicine, Baltimore, MD; ${ }^{4}$ Computer Science, Johns Hopkins University, Baltimore, MD; ${ }^{5}$ McKusick-Nathans Institute of Genetic Medicine, Johns Hopkins University School of Medicine, Baltimore, MD;

${ }^{6}$ Oncology, Johns Hopkins University School of Medicine, Baltimore, MD

Addition of telomeres by telomerase to chromosomal ends opposes cellular senescence and allows continued proliferation. The catalytic subunit of telomerase, hTERT, is upregulated in the majority of human malignancies through multiple mechanisms, two of which include promoter $\mathrm{CpG}$ methylation and alternative splicing. However, hTERT promoter methylation and alternative splicing have not been characterized fully in thyroid cancer of follicular origin. Previously, we demonstrated through Methylation Specific PCR (MSP) that methylation perturbation in thyroid cancer cell lines resulted in changes in hTERT splicing pattern. To better understand the methylation status of hTERT in thyroid cancer, we have now developed a method to bisulfite sequence the hTERT promoter to determine methylation status at $\mathrm{CpG}-$ level resolution and correlate these 
findings with splice pattern variation in thyroid cancer cell lines and FNA samples. hTERT promoter methylation was analyzed by tiled amplicon bisulfite sequencing in six thyroid cancer cell lines of PTC, ATC, and FTC origin, as well as in thyroid FNA samples (benign to malignant). Targeted RNA-Seq was utilized to determine hTERT splice isoforms. To perturb methylation status, cells were treated with a DNA methylase inhibitor, DAC, and the resulting changes in splice isoforms were characterized. Consistent with other telomerase positive cancers, hTERT promoter methylation in thyroid cancer exhibits a departure from normal thyroid cells. Bisulfite sequencing in thyroid cancer cell lines shows hypermethylation of the upstream region of hTERT, while the TSS is largely unmethylated. This pattern correlated with cancer cells exhibiting higher levels of the full length, active isoform. hTERT promoter bisulfite sequencing reveals alterations in methylation patterns in thyroid cancer. This transformed methylation correlates with changes in transcript splice patterns, and demonstrates the potential link between these two important regulatory mechanisms of telomerase in thyroid cancer.

\section{Poster 372}

Thyroid Cancer Saturday Poster Translational

\section{OVERCOMING ATF4 DEFICIENCY FOR TREATMENT OF MEDULLARY THYROID CANCER}

R. Yarmand ${ }^{3}$, K. Sinha ${ }^{4}$, L. Li ${ }^{3}$, Y. Lu ${ }^{1}$, M.D. Williams ${ }^{2}$, E.G. Grubbs ${ }^{5}$, G.J. Cote ${ }^{3}$, R. Gagel ${ }^{3}$

${ }^{1}$ Molecular Carcinogenesis, The University of Texas MD Anderson Cancer Center, Houston, TX; ${ }^{2}$ Pathology, The University of Texas MD Anderson Cancer Center, Houston, TX; ${ }^{3}$ Endocrine Neoplasia \& HD, The University of Texas MD ANderson Cancer Center,

Houston, TX; ${ }^{4}$ Orthopaedic Surgery, The University of Texas Health Science Center of Houston, Houston, TX; ${ }^{5}$ Surgical Oncology, The University of Texas MD Anderson Cancer Center, Houston, TX

Medullary thyroid carcinomas (MTCs) arise from the thyroidal C cells. Researchers have identified somatic gain-of-function point mutations of RET, a receptor tyrosine kinase, in $50 \%$ of sporadic MTCs. Treatment with tyrosine kinase inhibitors (TKIs) can delay MTC progression, but responses are incomplete, and resistance occurs. Therefore, understanding of MTC biology is needed to develop improved therapies. Activating transcription factor 4 (ATF4) plays a central role in regulation of the integrated stress response, which activates cell death pathways during cellular stress. ATF4 promotes RET degradation and inhibits RET signaling pathways. We performed comparative genomic hybridization, qPCR copy number, immunohistochemistry using tumors from 52 patients with MTC. We examined the effect of ATF4 inducers alone or in combination with TKIs on survival of MTC cells. We identified loss of heterozygosity on chromosome 22q in MTCs at ATF4 locus. This ATF4 loss of function occurs at a frequency similar to that of RET mutation in sporadic MTCs and is associated with poor overall survival and distant metastasis. Two drugs that induce ATF4 expression were evaluated to overcome ATF4 deficiency: eeyarestatin, endoplasmic reticulum associated protein degradation inhibitor, ONC201, a member of imipridones. Both drugs induce apoptotic cell death of MTC cells through induction of ATF4 expression and proapoptotic ATF4 target genes. These compounds downregulate expression of RET, VEGFR2 and MCL1. Flow cytometry analysis demonstrated an increase in the sub G1 population of the cells treated with ONC201, indicating increased cell death. When we treated MTC cells with eeyarestatin or ONC201 in the presence or absence of TKIs, the combination synergistically caused apoptosis. We applied genome-wide chromatin immunoprecipation sequencing to identify genes regulated by ATF4 in eeyarestatin-treated MTC cells. Real time RT-PCR analysis demonstrated that ATF4 inducers upregulated expression of ATF4 targets with tumor suppressor functions and act in synergy with TKIs to regulate these genes.

These results suggest that induction of ATF4 alone or in combination with TKIs could be a promising therapeutic strategy to treat patients with MTC.

\section{Poster 373}

Thyroid Cancer Saturday Poster Translational A DIFFERENTIAL SUBCELLULAR EXPRESSION PATTERN OF A GENE IN WELL-DIFFERENTIATED THYROID CANCER TO ANAPLASTIC THYROID CANCER C. $\mathrm{Kim}^{2,3}$, M.C. Perez ${ }^{4,2}$, A.C. Simental ${ }^{2,3}$, I. Munir ${ }^{5,6}$, A.A. Firek ${ }^{5,6}$, S. Khan ${ }^{1,2}$

${ }^{1}$ Biochemistry, Loma Linda University, Loma Linda, CA; ${ }^{2}$ Otolaryngology, Loma Linda University, Loma Linda, CA; ${ }^{3}$ Surgery, Loma Linda University, Loma Linda, CA; ${ }^{4}$ Pathology \& Human Anatomy, Loma Linda University, Loma Linda, CA; ${ }^{5}$ Endocrinology, Riverside University Health System, Moreno Valley, CA; ${ }^{6}$ Internal Medicine, University of California, Riverside, Riverside, CA

Anaplastic thyroid carcinoma (ATC) is one of the most aggressive solid tumors known to affect humans [1]. There is an unmet to discover novel and innovative strategies to combat this uniformly lethal disease. ATC is thought to arise de novo or from preexisting welldifferentiated thyroid cancer [2]. The PDZ-LIM scaffold protein Enigma has recently been discovered as an oncogene in cancer cells [3]. We, therefore, hypothesize that Enigma pays a role in the initiation and progression of thyroid cancer. In this study, we demonstrate that Enigma and its known binding partners are expressed in a series of different subtypes of human of thyroid cancer. We have analyzed the differential expression of Enigma, the subcellular distribution of Enigma and its binding partners in human ATC, follicular cancer (FTC), poorly differentiated cancer (PDTC), and papillary thyroid cancer (PTC) by immunohistochemistry and confocal microscopy. Enigma was highly expressed in ATC compared to no or minimal within benign thyroid tissue. Despite this lack of difference in expression in the subtypes there was there was a distinct mixed type of subcellular expression in both the nuclear and cytoplasm in ATC compared to other cancers. Compared to ATC, Enigma expression in PTC, was within the cytoplasm and in the nuclei within FTC samples. Dual staining showed that Enigma co-localized with BMP-1 in PTC but with RET within FTC. Enigma co-localized with RET and RAS in PDTC and with RAS/myc protein in ATC. Our study for the first time demonstrates the possible role of Enigma in the different subtypes of thyroid cancer and suggests that Enigma may have a specific role in the progression to ATC. These results may lead to a better understanding of the process by which ATC develops and may lead to specific targeting focusing on disrupting Enigma expression and co-localization.

\section{Poster 374}

Thyroid Cancer Saturday Poster Translational COSEGREGATION OF MSN VARIANT IDENTIFIED BY WHOLE-EXOME SEQUENCING IN A BRAZILIAN FAMILY SUSPECTED OF FAMILIAL NON-MEDULLARY THYROID CANCER

M. Teixeira Rodrigues ${ }^{1,2}$, F. Koyama ${ }^{3}$, A. Camargo ${ }^{3}$, F.O. Rego ${ }^{3}$, P. Galante ${ }^{3}$, L. de Lima Feitoza ${ }^{5}$, G.M. Griz Carvalheira ${ }^{6}$, J.M. Cerutti ${ }^{6,2}$, I.G. Rubió ${ }^{1,4}$ 
${ }^{1}$ Laboratório de Ciências Moleculares da Tireoide (LCMT), Universidade Federal de São Paulo (UNIFESP), São Paulo, São Paulo, Brazil; ${ }^{2}$ Programa de pós-graduação em Biologia Estrutural e Funcional - Universidade Federal de São Paulo (UNIFESP), São Paulo, Brazil; ${ }^{3}$ Centro de Oncologia Molecular (MOC), Hospital Sírio-Libanês - Instituto de Ensino e Pesquisa (HSL-IEP), São Paulo, Brazil; ${ }^{4}$ Departamento de Ciências Biológicas, Universidade Federal de São Paulo (UNIFESP), São Paulo, Brazil; ${ }^{5}$ Departamento de Biologia, CCN, Universidade Federal do Pauí, Teresina, Brazil; ${ }^{6}$ Laboratório de Bases Genéticas dos Tumores da Tireoide, Departamento de Morfologia e genética, Universidade Federal de São Paulo (UNIFESP), São Paulo, Brazil

Familial non-medullary thyroid cancer (FNMTC) corresponds to $3-6 \%$ follicular thyroid epithelium carcinomas and is considered syndromic when patients present tumor syndromes with low preponderance of thyroid tumors, such as Cowden's Syndrome, Gardner's Syndrome, Carney's Complex, and Werner's Syndrome. Their clinical manifestations may vary between families. The new sequencing techniques, among them the Whole-Exome Sequencing (WES), have been used in studies of rare genetic diseases caused by single genes. The objective of this study was to investigate genetic alterations in a family with suspicious of FNMTC. The family from Brazil has two cases of thyroid cancer (papillary and follicular) and one case thyroid hypoplasia. Moreover, 10 members were diagnosed with, skin, breast, lung, gastric, oral or bone cancer. Peripheral blood DNA from three cancer patients was sequenced by expanded-WES using the SureSelectXT Human All Exon V6 + UTR Capture Library kit and the Illumina ${ }^{\circledR}$ NextSeq ${ }^{\mathrm{TM}} 500$ platform. WES generated 3.69$4.85 \mathrm{~Gb}$ of data/sample with coverage greater than $30 \mathrm{x}$. Variations were confirmed by Sanger's sequencing. The single nucleotide variants (SNVs) and insertion/deletion (indels) selection criteria were: present in the genome of the three sequenced cancer patients, not described in databases, non synonymous, not present in the exome of 18 controls individuals, present in exonic and UTRs regions. A candidate gene approach was first adopted, which investigated variants in 550 genes related to familial and sporadic thyroid cancer, familial cancer and familial syndromes. However, no alterations were associated with the disease. Further analysis of all data identified 6 variants (SNVs and Indels) with high potential to be mutations. All of them were investigated in the family and only one variation in MSN gene segregated with the disease, except in a breast cancer patient, which could be considered a sporadic case. This gene has been linked to proteoglycan pathway in cancer. We conclude that no genomic variations in genes previously described as related to thyroid cancer or other syndromes were identified in this family. On the other hand, a new MSN variant segregates with the disease in the studied family.

\section{Poster 375}

Thyroid Cancer Saturday Poster Translational

\section{POTENTIAL OF HMGA2 IMMUNO-HISTOCHEMICAL} STAINING AS A PROGNOSTIC MARKER OF METASTATIC RECURRENCE IN FOLLICULAR THYROID CANCER

T. Kogai ${ }^{1}$, J. Sakumoto ${ }^{1}$, W. Konno ${ }^{2}$, H. Kanaya ${ }^{2}$, H. Hirabayashi ${ }^{2}$, A. Hishinuma ${ }^{1}$

${ }^{1}$ Department of Infection Control and Clinical Laboratory Medicine, Dokkyo Medical University, Mibu, Tochigi, Japan; ${ }^{2}$ Department of Otorhinolaryngology and Head and Neck Surgery, Dokkyo Medical University, Mibu, Tochigi, Japan

Distant metastasis often confers a poor prognosis in follicular thyroid cancer (FTC), even in cases with tumor(s) pathologically diagnosed as minimal invasion. High Mobility Group AT- Hook 2 (HMGA2), reported to be expressed in some of FTC, have a critical role in cancer metastasis. We therefore retrospectively investigated if positive immuno-histochemical staining of HMGA2 in the primary tumor(s) was correlated with metastatic recurrence in FTC. The 28 patients of FTC, whose electronic records available 5 years or more after thyroidectomy in our university hospital, were investigated. Their mean follow-up duration was 10.5 years (median 8 years; range, 5-30 years). Immunohistochemical staining of HMGA2 was performed on $4-\mu$ m-thick sections of formalin-fixed, paraffin-embedded tissue blocks of the primary tumor(s) from our archive of residual specimens. Approval of the clinical research ethics committee was obtained at the Dokkyo Medical University. Positive staining of HMGA2 was observed in 18 of 28 cases of primary tumors (64\%), but negative in unaffected surrounding thyroid tissues. Overall, the staining was observed focally in tumor tissues. Metastatic recurrence was detected in 4 patients 1 to 12 years after the primary surgery, while no recurrence or persistent disease was described within the first year from the surgery in all patients. All of the patients with metastatic recurrence (4 of 4) had HMGA-positive primary tumor(s) removed previously, while the other HMGA2-positive patients (14 of 18) were still without recurrence for 5 to 21 years after the surgery. Notably, all of patients previously with HMGA2-negative tumors (10 of 10) had no recurrence for 5 to 31 years. The sensitivity of HMGA2 staining of the primary tumor(s) for subsequent metastatic recurrence were 100\%; specificity, 42\%; positive predictive value, $22 \%$; and negative predictive value, $100 \%$. Negative staining of HMGA2 in primary tumor(s) of FTC may predict a good long-term prognosis after thyroidectomy.

\section{Poster 376}

Thyroid Cancer Saturday Poster Translational

\section{TUMORSPHERES OF PAPILLARY THYROID CANCER} HAVE CANCER STEM CELL PROPERTIES

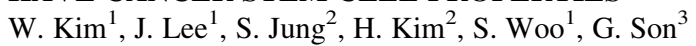

${ }^{1}$ Surgery, Korea University Guro Hospital, Seoul, Korea (the Republic of); ${ }^{2}$ Korea University Hospital, Seoul, Korea (the Republic of); ${ }^{3}$ Korea University Ansan Hospital, Ansan, Korea (the Republic of)

It was known that $3 \mathrm{D}$ cell structures, tumorspheres, might have the cancer stemness in various carcinomas. We performed to identify cancer stem cell properties in tumorspheres from papillary thyroid carcinoma (PTC), a majority of thyroid carcinomas. PTC tumorsphere was made by culturing cell line SNU 790 in the sphere medium and passages was performed for next generations. To identifying the cancer stemness, the expression of stem cell marker SOX2, OCT4, NANOG and thyroid specific genes thyroglobulin (TG), thyroid stimulating hormone receptor (TSHR) was investigated in PTC tumorspheres and cell line by western blot and RTPCR. Tumorspheres had the high clonogenic potential of cancer progenitor cells, the high expressions of stem cell marker octamerbinding transcription factor 4 (OCT4), sex-determining region $\mathrm{Y}$ (SRY)-box2 (SOX2), and NANOG and the lower expressions of thyroid specific genes thyroglobulin $(\mathrm{Tg})$ and thyroid stimulating hormone receptor (TSHR). E-cadherin, signal transducers and activators of transcription 3 (STAT3) expression were downregulated and Yes-associated protein 1 (YAP1) were similar compared with those expressions of PTC cells. Tumorspheres had tumorigenic PTC cells and cancer stem cell (CSC)-like properties. Our findings confirm the presence of CSCs in PTC cells, and suggest that tumorsphere culture may be a suitable option for future research on PTC stem cells. 


\section{Poster 377}

Thyroid Cancer Saturday Poster Case Report

N-RAS MUTATION IN CODON 61 ASSOCIATED WITH AGGRESSIVE FOLLICULAR VARIANT PAPILLARY THYROID CANCER PRESENTING WITH BONE METASTASIS

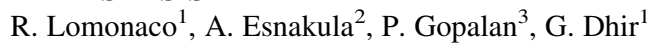

${ }^{1}$ Medicine/Division of Endocrinology, Diabetes \& Metabolism, University of Florida, Gainesville, FL; ${ }^{2}$ Pathology, University of Florida, Gainesville, FL; ${ }^{3}$ Medicine/Oncology, University of Florida, Gainesville, FL

$R A S$ mutations are the second most common genetic alteration in thyroid cancer, after $B R A F$ V600E mutation. Histologically $R A S$ mutations can be found in up to $57 \%$ of FTC and $21 \%$ of PTC mostly follicular-variant-PTC (FV-PTC), anaplastic and poorly differentiated thyroid cancer. A 56 year-old woman with history of breast cancer presented to the hospital with progressive back pain and right lower extremity weakness for three months. MRI spine showed $8.8 \mathrm{~cm}$ destructive bony lesion in the right sacral ala/ilium. Sacral biopsy was consistent with metastatic thyroid carcinoma. Patient underwent total thyroidectomy. Pathology confirmed multifocal encapsulated FV-PTC with capsular invasion (T1N0M1, Stage IV C, ATA initial risk category: high risk). She received multiple RAI treatments with over $500 \mathrm{mCi}$ and external beam radiation/ cyberknife for spinal metastasis. Thyroglobulin levels remained consistently $>1000 \mathrm{ng} / \mathrm{mL}$. FDG-uptake was positive for skeletal metastasis (L4-L5). Given persistent and progressive structural disease (RAI refractory), a TKI Lenvatinib was initiated. Patient subsequently developed a large bowel perforation requiring colectomy/colostomy, thus medication was discontinued, and she was felt to be ineligible for other VEGF-inhibiting TKI. Next generation sequencing revealed an $N-R A S$ mutation (c.182A $>\mathrm{G}$ p. Q61R) and immunohistochemistry was low for PD-1 expression, therefore patient was evaluated for $N-R A S$ directed clinical trial. $R A S$ gene encodes a family of three proto-oncogenes: $N$-RAS, $K$-RAS and $H$ $R A S$. RAS mutation results in aberrant constitutive activation of critical pathways in thyroid cancers. $N$-RAS mutation in codon 61 accounts for $67-88 \%$ of all $R A S$ mutations and is frequently present in malignant nodules associated with dedifferentiation and poor prognosis. Aggressive tumor behavior and bone metastasis have been associated with $N$-RAS mutation in FTC. Our case represents a clinical challenge in thyroid cancer and the difficulty in identifying a subset of thyroid cancers with aggressive biological behavior and negative impact on morbidity and mortality. Future studies are needed in order to determine prognostic utility of $R A S$ testing and other molecular markers in the management of thyroid cancer.

\section{Poster 378}

Thyroid Cancer Saturday Poster Case Report FROM THE COLONOSCOPY SUITE TO THYROID FNA:
CONNECTED THROUGH DIARRHEA

H. Baloch, T. Church, K.N. Vu, T.D. Hoang, M. Shakir

Endocrinology, Walter Reed National Military Medical Center, Rockville, MD

Medullary thyroid cancer (MTC) can initially present with a spectrum of symptoms, one of which is diarrhea. Diarrhea in MTC is believed to be caused by calcitonin-induced intestinal fluid secretion and decreased absorption. Despite higher calcitonin levels in virtually all cases of MTC, only $28-39 \%$ of these patients develop watery diarrhea. We present two contrasting cases of MTC with diarrhea.
Case 1: A 27 year old male was evaluated by gastroenterology for non-bloody diarrhea and $17 \mathrm{lb}$ weight loss with negative workup to include colonoscopy, detailed blood work and stool studies. Two years later, patient was noted to have left neck mass incidentally found during sinusitis workup and patient was referred to endocrinology for evaluation. Subsequent FNA was significant for MTC with initial calcitonin of 52, $894 \mathrm{pg} / \mathrm{mL}$. Patient underwent thyroidectomy with cervical lymph node dissection with pathology confirming Stage IVc MTC. Following surgery, patient had $>80 \%$ reduction in calcitonin levels with a minimal improvement in diarrhea.

Case 2: 28 year-old female presented to endocrinology for evaluation of thyroid nodules. Patient endorsed diarrhea and $8 \mathrm{lb}$ weight loss of one-month duration. Thyroid US showed a left $1.6 \mathrm{~cm}$ left and a $1.8 \mathrm{~cm}$ right thyroid nodules. FNA of thyroid nodule and lymph node was consistent with MTC. Patient's initial calcitonin was $2,890 \mathrm{pg} / \mathrm{mL}$, which improved to $490 \mathrm{pg} / \mathrm{mL}$ after extensive neck dissection. Post-operatively her diarrhea resolved and she required treatment for constipation. If calcitonin levels were the sole and primary etiological factor of diarrhea in MTC, diarrhea would be seen in all cases of MTC with elevated calcitonin levels. However, the above two cases demonstrate that decline in calcitonin levels may or may not result in resolution of diarrhea. There are likely other factors involved in the manifestation of diarrhea in MTC. A broad differential including MTC with unexplained diarrhea could make the difference in early detection and treatment for these patients. Further studies are needed to investigate this process further and identify the etiology.

\section{Poster 379}

\section{Thyroid Cancer Saturday Poster Case Report}

\section{METASTATIC HURTHLE CELL CARCINOMA} OF THE THYROID, A UNIQUE CASE REPORT

\section{P. Ohri ${ }^{1}$, P. Labinson ${ }^{2}$}

${ }^{1}$ Endocrinology and Metabolism, UConn Health, Farmington, CT;

${ }^{2}$ Endocrinology and Metabolism, Hartford Healthcare, Hartford, CT

Hurthle cell carcinoma of the thyroid is relatively rare, accounting for only $3-10 \%$ of all differentiated thyroid cancers. We present a rare case of Hurthle cell carcinoma of the thyroid with a large metastatic lesion to the pelvis. A 67 year old male with no significant past medical history presented to his PCP with abrupt onset sharp left hip pain with difficult ambulation. Plain radiograph revealed a large osteolytic lesion of the left acetabulum suspicious for metastatic disease. Full metastatic workup with CT of the chest, abdomen and pelvis revealed a large multinodular goiter with associated cervical lymphadenopathy and multiple bilateral pulmonary nodules concerning for metastatic thyroid cancer. Biochemical analysis revealed TSH of $3.87 \mathrm{mIU} / \mathrm{L}$ (N 0.4-4.5), Thyroglobulin level $14219.0 \mathrm{ng} / \mathrm{mL}$ (N 2.8-40.9) and undetectable thyroglobulin antibodies. He underwent radical resection of the left hemipelvic lesion with placement of left total hip arthroplasty. Surgical pathology revealed necrotic carcinoma with histologic and immunophenotypic features consistent with metastasis from a thyroid primary. He subsequently underwent total thyroidectomy, which revealed a right-sided $2.8 \mathrm{~cm}$ focus of Follicular Hurthle Cell Carcinoma with poorly differentiated areas. Vascular invasion and extra thyroidal extension were present, as well as positive margins. No lymph nodes were assessed. This was classified as pT4B, pNX, cM1, and stage IVC with an age of $67 . \mathrm{He}$ received treatment with $235 \mathrm{mCi}$ of I-131. A post therapy I-131 metastatic survey revealed increased abnormal uptake throughout the thyroid bed, multiple foci of abnormal uptake in both lungs and a focus of abnormal uptake in the mid abdomen corresponding to the left adrenal gland. Hurthle cell carcinoma of the thyroid has the 
highest incidence of metastasis among differentiated thyroid cancers, with metastatic disease reported at the time of initial diagnosis in 10 $20 \%$ of cases. Metastasis often occurs to the lungs, bones and CNS, but large metastases to the bone are rare. This case highlights the metastatic potential of Hurthle cell carcinoma and the need for diligent surveillance of these patients for metastasis and recurrence.

\section{Poster 380}

Thyroid Cancer Saturday Poster Case Report

RARE CASE OF MIXED MEDULLARY-FOLLICULAR CARCINOMA AND MICRO-PAPILLARY CARCINOMA IN A SINGLE THYROID

T. Swiss, W. McIlwain, K. Groom

Otolaryngology, Head and Neck Surgery, Madigan Army Medical Center, Tacoma, WA

Mixed medullary-follicular thyroid carcinomas of the thyroid gland are an extremely rare entity. We present the case of a 72 year old female who was diagnosed with a mixed medullary-follicular thyroid carcinoma. A 72 year old female underwent a thyroid ultrasound which demonstrated 3 distinct nodules. The largest underwent biopsy demonstrating atypia of undetermined significance. The patient underwent a total thyroidectomy. Intra-operative frozen specimen demonstrated suspicion for malignancy. Final pathology demonstrated mixed medullary and follicular derived carcinoma with an incidental finding of micro-papillary carcinoma in a separate nodule. This was confirmed by immunohistochemical staining for CEA, Calcitonin, and thyroglobulin. The patient underwent baseline CEA and Calcitonin labs post-operatively which were normal. A neck ultrasound did not demonstrate any evidence of central neck lymphadenopathy. She underwent radioactive iodine treatment after surgery Post treatment scans and labs to date have not demonstrated any further disease. Mixed medullary-follicular-derived carcinomas are an extremely rare entity with less than 50 case reports reported in the literature. Both tumors arise from different embryologic origins. Theories regarding how these tumors arise include separate tumors, collision tumors, and mixed tumors. There is little data regarding management of these tumors and it has been suggested that they be treated as separate entities. Management of medullary and follicular carcinomas are well established. These tumors appear to follow traditional medullary thyroid carcinoma patterns of increased metastatic rates, recurrence rates, and long term prognosis. Incidental findings of papillary thyroid carcinoma can be as high as $20 \%$ in patients with medullary thyroid carcinoma. Mixed medullaryfollicular carcinomas are an extremely rare entity. There are no current guidelines regarding their treatment. Further studies are necessary to better outline their prognosis and if treatment regimens differ from currently established practice guidelines.

\section{Poster 381}

Thyroid Cancer Saturday Poster Case Report

HYALINIZING TRABECULAR TUMOR IMPERSONATING AS PAPILLARY THYROID CANCER IN A PATIENT WITH POSSIBLE RADIATION EXPOSURE

\section{H. Bakeri, H. Goel, B. Tendler}

Medicine, University of Connecticut Health Center, West Hartford, CT

Hyalinizing trabecular tumor (HTT) is an infrequent thyroid neoplasm of follicular cell origin with peculiar features that allow recognition on surgical specimen. On fine-needle aspiration (FNA), it is often confused with papillary (PTC) and medullary thyroid carcinoma (MTC). Clinically, HTT is usually benign and should be treated conservatively, however rare invasive cases have been reported. A 55-year old lady from Poland presents with an incidentally found $1.7 \mathrm{~cm}$ right thyroid lobe nodule. FNA showed atypical follicular epithelium with pale chromatin, nuclear grooves and rare papillary cluster, suspicious for PTC. Laboratory TSH was $0.96 \mu \mathrm{U} /$ $\mathrm{mL}$ and calcitonin was $<2 \mathrm{pg} / \mathrm{mL}$, and the patient underwent a total thyroidectomy. Surgical pathology revealed hyalinizing trabecular tumor of the thyroid gland. Thyroglobulin stain was diffusely positive. Stains for calcitonin and Congo red were negative and MIB-1 showed a proliferation index $<1 \%$. Of note, the patient was in Poland at the time of the Chernobyl nuclear accident in 1986 and remembers being given iodine after that. Given her proximity to radiation fall out, we had a discussion regarding remnant ablation with 30 millicuries. But, after review of literature, the patient and the medical team opted not to undergo that. She is monitored closely and has undetectable thyroglobulin and no thyroid antibodies 1 year after surgery. She is maintained on thyroid replacement with good symptomatic control. Hyalinizing trabecular tumors are rare neoplasms often mistaken for thyroid carcinomas on FNA. On FNA, both HTT and PTC can demonstrate hypercellularity, psammoma bodies, cytoplasmic and nuclear inclusions and nuclear grooves. However, its clinical behavior remains unclear. While they usually have low malignancy potential, malignant variants exist. In a case series by Carney et al., 118 of 119 HTTs met criteria for benign neoplasms. Given its primarily benign nature, it can be managed conservatively with precautionary follow-up. Total or hemithyroidectomy is usually adequate treatment. As HTTs are rare, lowmalignant-potential neoplasms that are often mistaken for thyroid carcinomas on FNA, an awareness of their characteristics is valuable for recognition and management.

\section{Poster 382}

Thyroid Cancer Saturday Poster Case Report

\section{PREGNANCY AFTER CONSERVATIVE TREATMENT FOR MALIGNANT STRUMA OVARII}

J. Chen, C. Dosiou

Stanford University, Stanford, CA

Malignant struma ovarii (MSO) accounts for only 5\% of struma ovarii cases. There is no current standard of care on the optimal surgical and post-operative management. Aggressive surgical treatment entails hysterectomy with bilateral salpingo-oopherectomy and thyroidectomy to facilitate use of radioactive iodine. However, survival rates, regardless of type of surgery, are excellent with low rates of recurrence. Therefore, some women may be candidates for conservative management. We discuss a case of a woman who was able to achieve a successful pregnancy after conservative treatment of MSO.29yo woman presented with abdominal cramping and found to have bilateral ovarian cysts. She underwent left cystectomy given an increase in size of the left ovarian cyst. Pathology notable for MSO with follicular carcinoma with vascular invasion as well as papillary carcinoma. Her thyroid ultrasound was normal. Her thyrogen I-123 scan and MRI abdomen/pelvis were negative for metastatic disease. The patient opted for conservative treatment to preserve fertility. She was started on levothyroxine to suppress her TSH to $0.1-0.5 \mathrm{mIU} / \mathrm{L}$. Subsequent imaging has remained negative and thyroglobulin has remained stable. She was able to spontaneously conceive 4 years after her initial diagnosis and delivered successfully. The natural history of MSO is unclear, given the rarity of this disease. Overall, the long term prognosis appears to be good. Risk stratification can be a tool to minimize surgery but there are little data on which factors affect survival and recurrence. This is the first case report of a successful pregnancy in a patient with MSO treated conservatively, with only cystectomy and TSH suppression. Interestingly, this patient's 
pathology findings of vascular invasion and mixed histology type might not have necessarily placed her in a low risk caregory. The lack of evidence regarding the management of MSO continues to pose a challenge. Conservative management may be appropriate for low risk patients to improve long term quality of life and maintain fertility. More studies are needed on predictors of aggressive behavior to better risk stratify women with this disease.

\section{Poster 383}

Thyroid Cancer Saturday Poster Case Report

METASTATIC ANAPLASTIC THYROID CARCINOMA: CURED BY SURGERY?

P.M. Specenier ${ }^{1,2}$, M. Twickler ${ }^{5}$, P. Van Schil ${ }^{3,2}$, M. Lammens ${ }^{2,4}$, K. De Greef ${ }^{2,6}$, D. Ysebaert ${ }^{6,2}$

${ }^{1}$ Oncology, Antwerp University Hospital, Edegem, Belgium;

${ }^{2}$ Faculty of Medicine and Health Sciences, University of Antwerp, Wilrijk, Belgium; ${ }^{3}$ Thoracic Surgery, Antwerp University Hospital, Edegem, Belgium; ${ }^{4}$ Pathology, Antwerp University Hospital,

Edegem, Belgium; ${ }^{5}$ Endocrinology, Antwerp University Hospital, Edegem, Belgium; ${ }^{6}$ Endocrine Surgery, Antwerp University Hospital, Edegem, Belgium

Anaplastic thyroid cancer has a dismal prognosis with a median overall survival of less than 6 months. Treatment options in patients with metastatic disease include participation in a clinical trial best supportive care, palliative chemotherapy, and/or palliative radiotherapy.

There are no clear guidelines for patients with oligo-metastasis or solitary metastasis. We here describe a case of a patient who developed a solitary metastasis from an anaplastic thyroid carcinoma. A 53-year old lady underwent a right hemi-thyroidectomy for what initially was considered a multinodular goiter. However, pathology revealed that the entire resection specimen was invaded by anaplastic thyroid carcinoma. The diameter of the tumor was $5.9 \mathrm{~cm}$ and there was extracapsular spread and invasion of the surrounding muscles. Patient was referred to our hospital and underwent a resection of the residual thyroid tissue, which was free of tumor, and a lymphadenectomy. Four lymph nodes were invaded by macroscopic tumor (diameter up to $2.1 \mathrm{~cm}$ ) without extracapsular spread. No BRAF mutation was detected. Surgery was followed by chemoradiation up to 60 Gy in 30 fractions over six weeks in combination with weekly carboplatin at an area under the curve of $2 \mathrm{mg} / \mathrm{ml} / \mathrm{min}$ and paclitaxel $60 \mathrm{mg}$ per meter square.

PET-CAT 15 months after the initial diagnosis and 12 months after the end of chemoradiation showed a solitary FDG PET positive lung nodule (diameter $24 \mathrm{~mm}$ ) in the left lower lobe. A video assisted wedge resection was performed. The resection specimen showed presence of anaplastic thyroid tumor with a diameter of $3 \mathrm{~cm}$. Resected lymph nodes were free of tumor.

So far, further follow up is uneventful and the latest PET-CAT 30 months after initial diagnosis and 15 months after lung surgery showed no evidence of disease. Our case reports illustrates that aggressive treatment of anaplastic thyroid cancer, including multimodality treatment for the primary tumor and surgical resection of a solitary metastasis can be associated with a prolonged recurrencefree survival and potential cure.

\section{Poster 384}

Thyroid Cancer Saturday Poster Case Report

A RARE COMBINATION OF PAPILLARY THYROID CANCER AND PANCREATIC NEUROENDOCRINE TUMOR IN CERVICAL LYMPH NODES

L. Gondin, J.L. Gonzalez, G. Kabbach, T. Bright
Internal Medicine, Texas Tech University Health Sciences Center, El Paso, TX

Pancreatic neuroendocrine tumors (PNET) are rare with an incidence of $\leq 1$ case per 100,000 individuals per year and account for 1 to $2 \%$ of all pancreatic tumors. The incidence of papillary thyroid carcinoma (PTC) has increased from 4.8 to 14.9 per 100,000 individuals between 1997 and 2012. An association between these two entities outside of MEN1 has not been previously reported. A 58 year old female had a pancreatectomy and splenectomy followed by radiotherapy for a $3.5 \mathrm{~cm}$, Grade 1 , PNET in 2009. CT scanning every 6 months showed stable periaortic nodes. 2013 PET scan, in addition to the known periaortic nodes, discovered focal activity in the left thyroid, FNA of which was positive for PTC. Total thyroidectomy revealed a left lobe $3.0 \times 1.7 \times 1.1 \mathrm{~cm}$ and a right lobe $0.3 \mathrm{~cm}$ PTC, tall cell variant, with angio-lymphatic and capsular invasion with skeletal muscle extension. Stimulated thyroglobulin (TG) was $2.4 \mathrm{ng} /$ $\mathrm{mL}$ with negative TG ab prior to $150 \mathrm{mCi}^{131}$. Post treatment whole body scan (WBS) showed only thyroid bed uptake and suppressive levothyroxine therapy was given. One year WBS was negative, but stimulated TG $5.2 \mathrm{ng} / \mathrm{mL}$ and US showed 2 left neck vascular nodules, $1.6 \times 1 \mathrm{~cm}$ at level 3 and $4 \times 4 \mathrm{~mm}$ level 4 . FNA of the larger lesion positive PTC. Patient underwent a left modified radical dissection and 5/17 nodes positive for neuroendocrine tumor and 2/17 positive for PTC; $99 \%$ of tumor was neuroendocrine with similar histologic features compared to her pancreatic tumor. Postop stimulated TG $5.8 \mathrm{ng} / \mathrm{mL}$ so received $150 \mathrm{mCi}$ of $\mathrm{I}^{131}$. CT scan of the neck 6 months later was negative, abdominal periaortic nodes showed decrease in $5 / 7$ but increase in $2 / 7$, and suppressed TG $0.5 \mathrm{ng} / \mathrm{mL}$. We presented a case of a PNET with metastatic disease 7 years post diagnosis to neck lymph nodes, and concomitant PTC metastatic to the same regional lymph nodes. To our knowledge, there are no previous case reports or association in the literature of sporadic PNET with the occurrence of a PTC, particularly with metastasis to the same region. Further research needs to be done to determine whether a similar mutation, particularly BRAF, exists between these two entities or if this was just fortuity.

\section{Poster 385}

Thyroid Cancer Saturday Poster Case Report THYROID LYMPHOMA CASE SERIES

R. Singarayer, D. Morrison

Endocrinology, Western University, London, Ontario, Canada

Primary thyroid lymphoma (PTL) are usually non-Hodgkin's type, with Hodgkin's lymphoma being rare. Pre-existing chronic lymphocytic thyroiditis (Hashimoto's thyroiditis or HT) is the only risk factor for PTL (60 times higher risk) and is present in about $50 \%$ of patients. Diffuse large B cell lymphoma (DLBCL) accounts for up to $70 \%$ of all PTLs, followed by up to $30 \%$ due to extranodal marginal zone lymphoma of MALT subtype.

Thyroid malignancy is the most common endocrine malignancy. PTL comprises $5 \%$ of all thyroid malignancies. The annual incidence is 1-2 per million. PTL typically presents with compressive symptoms from a rapidly enlarging goitre. B symptoms only occur in $20 \%$ of patients. Roughly 30-60\% are biochemically euthyroid. Diagnosis can be confirmed by fine needle aspiration biopsy, cytology with the addition of flow cytometry, or excisional biopsy, which may be necessary for subtyping. Treatment consists of chemotherapy and radiation, rarely surgery; which reinforces the need for differentiating PTL from more common thyroid neoplasias. We present a series of six primary thyroid lymphoma (PTL) cases between 2010-2016. Three stage II DLBCL, one stage IV DLBCL on a background of chronic lymphocytic leukemia/small lymphocytic lymphoma 
(Richter transformation), one Hodgkin's lymphoma, one MALT lymphoma.

DLBCL is the most aggressive type, with $60 \%$ presenting with metastasis (three of the four cases of DLBCL metastasized). MALT lymphoma is typically more indolent and presents at an earlier stage (only one case presented as stage I disease). Hodgkin's lymphoma accounts for only $2 \%$ of PTL and tends to occur in younger females (only case, in a 33-year-old lady). Roughly $50 \%$ of PTL presents with thyroid-limited disease (only one was limited to the thyroid), and 5\% have either stage III disease with involvement of both sides ofthe diaphragm, or stage IV disease with distant organ involvement (only one case was stage III or more). PTL is rare, with treatment being chemotherapy and radiation as opposed to surgery, which places more importance on clinical suspicion and diagnosis.

\section{Poster 386}

Thyroid Cancer Saturday Poster Clinical

COMPREHENSIVE GENOMIC PROFILING OF PRIMARY, NODAL, OR DISTANT METASTASES IN RECURRENT OR METASTATIC DIFFERENTIATED THYROID CANCER: MUTATIONS, MICROSATELLITE STABILITY STATUS, AND TOTAL MUTATIONAL BURDEN

D.W. Bowles ${ }^{1}$, N. Pozdeyev ${ }^{1}$, B. Haugen ${ }^{1}$, J. Hreno ${ }^{1}$, L.M. Gay ${ }^{2}$, P. Vanden Borre ${ }^{2}$, A.B. Schrock ${ }^{2}$, S.M. Ali ${ }^{2}$, J.M. Chung ${ }^{2}$, J.A. Elvin ${ }^{2}$, J.S. Ross ${ }^{2}$

${ }^{1}$ University of Colorado, Aurora, $\mathrm{CO} ;{ }^{2}$ Foundation Medicine, Cambridge, MA

We explored the comprehensive genomic profiles (CGP) of recurrent/metastatic (R/M) papillary (PTC) and follicular thyroid cancer (FTC), including microsatellite instability (MSI) and total mutational burden (TMB). DNA was extracted from 40 micron FFPE sections from consecutive R/M DTCs submitted for commercial analysis. CGP was performed on hybridization-captured, adaptor ligation based libraries to a mean coverage depth of $>500 \mathrm{X}$ for coding sequences of 315 cancer-related genes and 37 introns from 28 genes frequently rearranged in cancer. TMB was determined on 1.1 megabases of sequenced DNA.407 PTCs and 76 FTCs were sequenced. The median number of genomic alterations (GA)/sample was low for PTC (2.75) and FTC (3.01). CGP in PTC v FTC was performed on the primary (35\% v 38\%), lymph nodes (25\% v 9\%), head/neck tissue (13\% v 7\%) and distant sites (27\% v $46 \%)$. TERT promoter mutations were common in PTC $(58 \%)$ and FTC $(67 \%)$. The most common actionable GA in PTC were BRAF (73\%), RET (9\%) and PIK3CA (7\%). Actionable GA were uncommon in FTC: PTEN (16\%), BRAF (7\%), PIK3CA (3\%). RAS GA were more common in FTC than PTC: NRAS (35\% v 6\%), GRAS (7\% v 0\%), HRAS (7\% v 1\%), KRAS (7\% v 2\%). RBM10 GAs were seen in PTC $(5 \%)$ and FTC $(10 \%)$ and there was a relatively high frequency of $D N M T 3 A$ GA $(5 \%)$ in PTC. Oncogenic rearrangements of RET, $B R A F, A L K$, and NTRKI were detected in $8 \%, 3 \%, 1 \%$, and $1 \%$ of PTC cases, respectively, but were not identified in FTC cases. In PTC the frequently of BRAF GA was similar between thyroid (63\%) and bone/brain/lung/soft tissue $(68 \%)$ but TERT (33\% v 52\%) and RBM 10 GAs $(9 \%$ v $3 \%$ ) differed. In FTC the frequency of TERT GAs was similar between thyroid and distant metastasis (50-60\%) but RBM10 GA were seen in only $3 \%$ of thyroid samples versus $17 \%$, respectively. Overall the mutational load was low for R/M DTCs, with only $2 \%$ of PTCs having a TMB $>10$ mut/MB compared to $1 \%$ of FTCs. Only 1/49 FTC and no PTC were found to be MSI-high. This large DTC series identified known and novel GA and suggests possible differences in CGP between thyroid and metastatic sites. The low TMB and rare MSI-H raise caution for potential immunotherapy as a monotherapy in DTC.

\section{Poster 387}

Thyroid Cancer Saturday Poster Clinical

\section{GRAVES' DISEASE DOES NOT AFFECT THE PROGNOSIS} OF PAPILLARY THYROID CANCER

H. Kwon, B. Moon, J. Kim

Surgery, Ewha Womans University, Seoul, Korea (the Republic of)

Graves' disease is the most common cause of hyperthyroidism and is a risk factor of thyroid cancer. Incidence of thyroid cancer increased about 2.5 times in patients with Graves' disease compared to the normal population. However, there is a controversy about the prognosis of thyroid cancer associated with Graves' disease. The aim of this study was to investigate the recurrence of papillary thyroid carcinoma (PTC) in patients with Graves' disease. From January 2004 to January 2012, 3,047 patients underwent total thyroidectomy for PTC. Of those, 90 patients had Graves' disease with non-occult PTC (GD group). Recurrence rates were analyzed between GD group and 1:10 matched patients (control group), after propensity score matching according to age, gender, and pathological features including tumor size, extrathyroidal extension, lymphatic invasion, resection margin involvement, and lymph node metastasis. Before propensity score matching, GD group and control group showed comparable clinicopathologic features including age, gender, tumor size, extrathyroidial extension, and lymph node metastasis. Of the 90 patients with GD, $45.6 \%$ had tumors with extrathyroidal extension, and $32.2 \%$ had lymph node involvement; $34.4 \%$ had $\mathrm{T} 1$ tumors without lymph node involvement, $10.0 \%$ had T1 tumors with lymph node involvement, and $6.7 \%$ had $\mathrm{T} 1$ tumors with nodal involvement that could not be assessed; for patients with $\mathrm{T} 3$ tumors, the proportions were $24.4 \%, 20.2 \%$, and $2.2 \%$, respectively. Patients in GD group showed lower rate of resection margin involvement $(1.1 \% \mathrm{vs}$. $6.8 \%, \mathrm{p}=0.032)$ and recieving radioactive iodine ablation $(35.6 \% \mathrm{vs}$. $58.6 \%, \mathrm{p}<0.001)$ than control group.

After matching, there was no difference in any of clinicopathologic features. Patients in GD group experienced no recurrence after median follow-up of 56.2 months. No difference was found on 5-year recurrence-free survival between GD group and control group (100\% vs. $99.6 \%, \mathrm{P}=0.516$ ). The Cox hazard analysis indicated that only gross extrathyroidal extension is a prognostic factor for predicting disease-free survival (hazard ratio 10.6, 95\% CI 1.2-93.4, P=0.033). Our data suggest that Graves' disease does not affect the prognosis of papillary thyroid carcinoma.

\section{Poster 388}

Thyroid Cancer Saturday Poster Clinical

HEALTH RELATED QUALITY OF LIFE IN THYROID CANCER PATIENTS COMPARED TO OTHER CANCERS AND NORMATIVE DATA

S. Goswami ${ }^{1}$, B.J. Peipert ${ }^{1}$, M.N. Mongelli ${ }^{1}$, I. Helenowski ${ }^{3}$, S. Yount ${ }^{2}$, C. Sturgeon ${ }^{1}$

${ }^{1}$ Department of Surgery, Northwestern University, Chicago, IL; ${ }^{2}$ Department of Medical Social Sciences, Northwestern University, Chicago, IL; ${ }^{3}$ Department of Preventive Medicine, Northwestern University, Chicago, IL

Impaired health-related quality of life (HRQOL) in thyroid cancer survivors has been documented but is poorly understood. Large studies utilizing validated metrics with normative data and disease comparators are needed. We hypothesized that HRQOL in thyroid cancer survivors would be lower than that of the general US population but similar to patients with other cancers. Adults $\geq 18$ years recruited from a thyroid cancer support group completed PROMIS29 via online questionnaire $(n=1872)$. The mean age was 50.5 years 
and the mean time since diagnosis was 6.8 years. T-scores \pm standard deviations were compared to normative data using a one-sample ttest. PROMIS scores for non-Hodgkin lymphoma (NHL; $\mathrm{n}=443$ ), breast $(n=1588)$, prostate $(n=1140)$, uterine $(n=388)$, cervical $(n=141)$, colorectal $(n=890)$, and lung $(n=694)$ cancers were obtained from literature review. T-scores for PROMIS domains were compared using Wilcoxon Signed-Rank Test. Individuals with thyroid cancer report greater anxiety, depression, fatigue, and sleep disturbance than patients with NHL, breast, prostate, uterine, colorectal, and lung cancers $(\mathrm{p}<0.05)$. Thyroid cancer patients report less pain and greater physical functioning than all other cancers except prostate cancer $(\mathrm{p}<0.0001)$ and lower social functioning than all other cancers except lung cancer $(\mathrm{p}<0.0001)$. Thyroid cancer patients reported worse anxiety (56.8 \pm 10.9$)$, depression $(53.0 \pm 10.6)$, fatigue $(58.1 \pm 11.5)$, pain interference $(51.8 \pm 10.6)$, sleep disturbance (54.5 \pm 9.1$)$, physical functioning $(47.8 \pm 9.0)$, and social functioning $(48.0 \pm 10.2)$ scores compared to normative data $(50 \pm 10, \mathrm{p}<0.0001)$. HRQOL data are essential for understanding the impact of illness from a patient perspective. This study is the first to use PROMIS to benchmark HRQOL in thyroid cancer patients against other cancers and normative data. Individuals with thyroid cancer report worse HRQOL across all 7 PROMIS-29 domains compared to normative data. Thyroid cancer patients also report greater anxiety, depression, fatigue, and sleep disturbance compared to individuals with several other cancers.

\section{Poster 389}

\section{Thyroid Cancer Saturday Poster Clinical}

\section{A POPULATION-BASED REVIEW OF SALVAGE SURGERY, RADIOACTIVE IODINE, EXTERNAL BEAM RADIATION AND SYSTEMIC THERAPY FOR MANAGEMENT OF RECURRENT DIFFERENTIATED THYROID CANCER}

A. Shokoohi ${ }^{1}$, E. Berthelet ${ }^{2}$, G. Sexsmith ${ }^{3}$, J. $\mathrm{Wu}^{2}$, S. Gill ${ }^{4}$, A. White ${ }^{4}$, E. Prisman ${ }^{5}$, E. Tran ${ }^{2}$, S.M. Wiseman ${ }^{6}$, C. Ho $^{1}$ ${ }^{1}$ Medical Oncology, BC Cancer Agency, Vancouver, British Columbia, Canada; ${ }^{2}$ Radiation Oncology, BC Cancer Agency, Vancouver, British Columbia, Canada; ${ }^{3}$ Nuclear Medicine, St. Paul's Hospital, Vancouver, British Columbia, Canada; ${ }^{4}$ Endocrinology, St. Paul's Hospital, Vancouver, British Columbia, Canada; ${ }^{5}$ Otolaryngology, Vancouver General Hospital, Vancouver, British Columbia, Canada; ${ }^{6}$ General Surgery, St. Paul's Hospital, Vancouver, British Columbia, Canada

Management of recurrent differentiated thyroid cancer (DTC) may include surgery, radioactive iodine (RAI) and/or external beam radiotherapy (XRT). Patients may also be treated with systemic agents such as sorafenib and lenvatinib for RAI refractory DTC. The study objective was to evaluate DTC recurrent disease treatment and utilization of systemic therapy. The British Columbia Cancer Agency (BCCA) provides cancer care for over 4.5 million Canadians. A retrospective review of all DTC patients referred to the BCCA between 2009 and 2013 was conducted. Baseline characteristics, local/ distant recurrence, surgical management, RAI, external beam radiotherapy (XRT) and systemic therapy details were collected. Disease free survival (DFS) and overall survival (OS) were estimated using the Kaplan Meier method.1070 DTC patients were referred to the BCCA with stage I-IVB disease ( $\sim 70 \%$ of all diagnoses in the province). Median follow up $4.1 \mathrm{y}$. Baseline characteristics: female $73 \%$, median age $50 \mathrm{y}$, histology; papillary $90 \%$, follicular $7 \%$, Hurthle cell $3 \%$. Stage at presentation using the AJCC $7^{\text {th }}$ edition: I $60 \%$, II $8 \%$, III 25\%, IVA/IVB 7\%. Local and/or distant recurrence occurred in 159 patients $(15 \%) .120(11 \%)$ of the patients with local recurrence were treated with primary surgery \pm RAI or XRT $51 \%$,
$\mathrm{RAI} \pm \mathrm{XRT} 40 \%$, XRT alone $1 \%$, unknown $8 \% .33$ (3\%) had a second recurrence and were treated with primary surgery \pm RAI or XRT $39 \%$, RAI \pm XRT $27 \%$, XRT alone $3 \%$, and unknown $31 \%$. Of 59 patients who developed distant metastatic disease 20 had prior local recurrence. Common sites of metastases were lung $73 \%$, bone $29 \%$ and liver $10 \%$. Of the entire cohort, $6(0.6 \%)$ received systemic therapy with sorafenib. The 5 y DFS was $85 \%$ and OS was $95 \%$ for all patients. In our population-based cohort, $85 \%$ of patients were cured by primary disease management. Local recurrence was successfully managed with surgery, RAI and/or XRT with no evidence of residual disease in $57 \%$ of patients. Multi-modality treatment of local recurrence facilitates complete disease ablation in the majority of patients, and despite a significant number of metastatic recurrences, only a small fraction of patients require systemic therapy.

\section{Poster 390}

Thyroid Cancer Saturday Poster Clinical

\section{VALIDATION OF THE GENE EXPRESSION CLASSIFIER TO PREDICT MALIGNANCY IN THYROID NODULES WITH INDETERMINATE CYTOLOGY}

E. Oommen, C. Maxwell

Endocrinology, Stony Brook University Hospital,

East Northport, NY

Afirma Gene Expression Classifier (GEC) may be used in thyroid nodules initially noted to be Bethesda Category (BC) III or IV to supplement malignancy risk assessment. The original validation study reported $40 \%$ rate of malignancy in nodules with a "suspicious" GEC result. However, accuracy of the test has been shown to vary depending on the institution and patient population. Our study aimed to assess the accuracy of a suspicious result on the GEC in our patient population. We retrospectively reviewed all thyroid nodules that were biopsied at our institution from 4/2013 through 4/2017 that were indeterminate on cytology (BC III and IV) and sent for GEC testing. Pathology reports were reviewed for those sent for surgery. From a pool of 618 nodules, 76 nodules were identified from 72 patients $(17 \mathrm{M}, 55 \mathrm{~F})$. All were indeterminate on cytology: 56 were BC III and 20 were BC IV, and all were analyzed as suspicious on the GEC. Cytology was read by a single pathology group.

76 nodules reviewed: $51.3 \%$ (39/76) benign on pathology, $30.2 \%$ (23/76) malignant, and $18.4 \%$ (14/76) did not have surgical pathology available.

Of the pathologically benign nodules, $15 \%(5 / 39)$ were NIFTP. NIFTP was seen in $6.5 \%(5 / 76)$ of the total number of nodules.

62 nodules had pathology available: $37 \%$ (23/62) were malignant, $63 \%(39 / 62)$ were benign, and $8 \%(5 / 62)$ were NIFTP. $65 \%(15 / 23)$ of the malignancies were PTC, $17 \%$ (4/23) were follicular carcinoma, $4.3 \%(1 / 23)$ were medullary carcinoma, $4.3 \%(1 / 23)$ were hurthle cell carcinoma, and $8.7 \%(2 / 23)$ were poorly differentiated papillary carcinoma. A four-year review at our institution demonstrated that the rate of malignancy confirms what was originally published regarding GEC performance. As NIFTP is a newer classification, re-categorization of previous malignancies will both decrease the general rates of thyroid malignancy, as well as the performance of the GEC testing. However, our data supports the use of Afirma GEC testing to identify patients who are at a higher risk of having thyroid malignancy.

\section{Poster 391}

Thyroid Cancer Saturday Poster Clinical SOCIOECONOMIC DETERMINANTS OF PEDIATRIC PAPILLARY THYROID CANCER: A SEER DATABASE REVIEW

W. Porter ${ }^{5}$, P. Mahajan ${ }^{3,4}$, H. Danysh ${ }^{3,4}$, D.C. Chelius ${ }^{1,2}$ 
${ }^{1}$ Otolaryngology-Head and Neck Surgery, Baylor College of Medicine, Houston, TX; ${ }^{2}$ Pediatric Otolaryngology - Head and Neck Surgery, Texas Children's Hospital, Houston, TX; ${ }^{3}$ Pediatrics, Baylor College of Medicine, Houston, TX; ${ }^{4}$ Pediatric Oncology, Texas Children's Hospital, Houston, TX; ${ }^{5}$ Baylor College of Medicine, Houston, TX

Socioeconomic determinants are known to impact the severity of various cancers at presentation. A recent Surveillance, Epidemiology, and End Results (SEER) cancer registry review concluded that several demographic factors had no impact on risk of nodal metastasis in pediatric papillary thyroid cancer (PTC). However, this study did not analyze multiple other socioeconomic determinants which we hypothesize may impact disease severity. We analyzed pediatric patients of less than 20 years of age with PTC during 2010-2014 in the SEER database. Patients were stratified both by AJCC summary stage (Local Disease-LD versus Regional/Distant Disease- RDD) and by American Thyroid Association (ATA) pediatric PTC low/high risk classification. Socioeconomic variables analyzed included race, Hispanic origin, insurance, and county-of-origin characteristics (education status, median income, language isolation and poverty level). Considering summary stage data, Hispanics versus Non-Hispanic Caucasians (NHC) had a lower incidence of LD (2.62/million v 4.86/M, RR $=0.54$, $\mathrm{P}<0.00)$ and similar rate of RDD $(4.96 / \mathrm{M} \vee 5.23 / \mathrm{M}, \mathrm{RR}=0.95$, $\mathrm{p}=0.6$ ). Therefore, for Hispanics versus NHC with PTC, there was a higher risk of having RDD rather than $\mathrm{LD}$ disease $(\mathrm{OR}=1.76,95 \% \mathrm{CI}$ $1.30-2.38, \mathrm{p}=0.0002$ ). Among patients with PTC, there was no significant risk for RDD versus LD considering case-based insurance status or county-level education, poverty, income, or language isolation status. Approximate ATA risk-stratification by splitting N1a and $\mathrm{N} 1 \mathrm{~b}$ disease between the low and high risk groups respectively demonstrated no impact of any socioeconomic determinants on the severity of disease presentation. Patients of Hispanic origin with PTC may have a higher risk of advanced disease versus NHC. This may be due to lower recognition of localized disease in the Hispanic population. Other socioeconomic determinants do not seem to affect the severity of PTC in the pediatric population. Major limitations are that the SEER database does not collect nodal data in a mannor that allows for risk straification by the ATA guidelines and does not collect various casebased socioeconomic factors allowing only county/state level analysis.

\section{Poster 392}

Thyroid Cancer Saturday Poster Clinical

ULTRASOUND AND CYTOLOGIC FEATURES CAN PREDICT MALIGNANCY IN INDETERMINATE THYROID NODULES

I. Shafiq ${ }^{1}$, M.H. Ahmad ${ }^{1}$, S. Messing ${ }^{2}$, S. Newlands ${ }^{2}$, E.J. Giampoli ${ }^{2}$, J. Moalem ${ }^{2,1}$

${ }^{1}$ Endocrinology, University of Rochester, Rochester, NY;

${ }^{2}$ University of Rochester, Rochester, NY

Indeterminate cytopathology categories including Atypia of undetermined significance (ATUS) or follicular neoplasm/suspicious for follicular neoplasm (FN) occurs in 10-25\% of all thyroid biopsies, and often prompt surgery for definitive diagnosis. While molecular diagnostics have been helpful, the optimal management of these nodules remains a significant challenge. Final cytopathology reports issued between January 2010 and June 2016 on thyroid biopsies were retrospectively reviewed. Patient demographics, sonographic features (calcifications, vascularity, borders and echogenicity) and cytologic findings (nuclear and architectural atypia) were tabulated. Univariate, and multivariate (with backwards selection process) logistic regressions were carried out to determine predictors of malignancy. Of 4,241 biopsies performed during the study period, 8.1\% (345) had indeterminate results (156 ATUS, 196
FN). Nodules that did not have definitive diagnosis or long term follow up were excluded from our analysis (82 ATUS, $23 \mathrm{FN}$ ), leaving 245 indeterminate nodules for our analysis. Of these, $51 \%$ (126) were found to be benign, and $49 \%$ (119) were malignant.

Univariate analysis revealed the presence of microcalcifications (MC), hypervascularity (HV), and nuclear atypia(NA) as the only significant clinical/cytologic predictors of malignancy (Odds ratio $(\mathrm{OR})=1.82,1.84$, and 2.18; $\mathrm{p}=0.05,0.02$, and 0.01$)$.

Multivariable regression revealed that the presence of any two of these factors greatly increased the predictive nature of the above identified factors: $\mathrm{HV}+\mathrm{NA}$, OR 3.65; MC+HV- OR 4.9, and $\mathrm{MC}+\mathrm{NA}-\mathrm{OR}=36.1 ; \mathrm{p}=0.01$ for all. The presence of all 3 factors when compared to their absence was associated with a 6.5 -fold increase in the odds of cancer; $\mathrm{p}<.01$. Conversely, the absence vs. the presence of all three of these factors was associated with an $85 \%$ reduction in cancer odds. Among patients with indeterminate cytology, clinicopathologic variables can be powerful adjuncts to enhanced risk stratification.

\section{Poster 393}

Thyroid Cancer Saturday Poster Clinical

NO PROGNOSTIC VALUE OF POST-ABLATION THYROGLOBULIN INCREASE AT FIRST RADIOIODINE THERAPY IN PAPILLARY THYROID CANCER

J. Yoon, H. Kim, H. Kang

Department of Internal Medicine, Chonnam National University

Medical School, Gwangju, Korea (the Republic of)

Serum thyroglobulin (Tg) level immediately after radioactive iodine therapy (RAI) is frequently elevated, but the implications of this are controversial. A total of 680 patients with PTC undergoing RAI after total thyroidectomy were included. Serum $\mathrm{Tg}$ was sampled prior to ablation (pre-Tg) and 7 days after RAI (post-Tg). To verify the response to therapy, we classified the patients into excellent response (ER), biochemical incomplete response (BCIR), structural incomplete response (SIR), and indeterminate response (IR) groups using dynamic risk stratification (DRS). After RAI therapy, 514 patients (75.6\%) had an ER, $34(5.0 \%)$ had a BCIR, $13(2.0 \%)$ had an SIR, and $119(17.5 \%)$ had an IR. Pre-Tg level was significantly different among the groups, with the highest level being in the SIR group, followed by the BCIR, IR, and ER groups. However, post-Tg levels were not different among the four groups. In a multivariate analysis, sex, RAI dose, and pre-Tg level affected the dynamic risk stratification. Mean post-Tg level $(13.77 \pm 32.19 \mathrm{ng} / \mathrm{mL})$ increased significantly after RAI therapy compared to the mean pre-Tg level $(2.51 \pm 8.88 \mathrm{ng} / \mathrm{mL})$. In 422 patients whose pre-Tg level was $<1 \mathrm{ng} / \mathrm{mL}, 205$ had post-Tg levels $<1 \mathrm{ng} / \mathrm{mL}$, while 167 patients had increased post-Tg levels of $1-10 \mathrm{ng} / \mathrm{mL}$, and 50 had increased post-Tg levels $>10 \mathrm{ng} / \mathrm{mL}$. No difference was observed in the postoperative risk stratification, response to therapy (DRS), or clinical outcome among the three groups. Pre-Tg was useful as a prognostic factor in patients with PTC. Tg increased in most patients after RAI, but the clinical significance of the increase in post-Tg may reflect remnant thyroid tissue and does not help predict the prognosis.

\section{Poster 394}

Thyroid Cancer Saturday Poster Clinical

MANAGEMENT OF DIFFERENTIATED THYROID CANCER IN ACCORDANCE WITH THE AMERICAN THYROID ASSOCIATION GUIDELINES: IMPACT ON PATIENT DISEASE FREE AND OVERALL SURVIVAL OUTCOMES

E. Berthelet ${ }^{2}$, A. Shokoohi ${ }^{1}$, J. $\mathrm{Wu}^{2}$, S. Gill ${ }^{3}$, A. White ${ }^{3}$, G. Sexsmith ${ }^{4}$, E. Prisman ${ }^{5}$, E. Tran ${ }^{2}$, S.M. Wiseman ${ }^{6}$, C. Ho ${ }^{1}$ 
${ }^{1}$ Medical Oncology, BC Cancer Agency, Vancouver, British Columbia, Canada; ${ }^{2}$ Radiation Oncology, BC Cancer Agency, Vancouver, British Columbia, Canada; ${ }^{3}$ Endocrinology, St. Paul's Hospital, Vancouver, British Columbia, Canada; ${ }^{4}$ Nuclear medicine, St. Paul's Hospital, Vancouver, British Columbia, Canada;

${ }^{5}$ Otolaryngology, Vancouver General Hospital, Vancouver, British Columbia, Canada; ${ }^{6}$ General surgery, St. Paul's Hospital,

Vancouver, British Columbia, Canada

The study objective was to evaluate practice adherence to the upfront management of differentiated thyroid cancer (DTC) in accordance with the ATA guidelines and the impact on outcomes. The British Columbia Cancer Agency (BCCA) provides cancer care for over 4.5 million Canadians. A retrospective review of DTC patients referred to the BCCA between 2009 and 2013 was conducted. Baseline characteristics, upfront surgical management, and adjuvant radioactive iodine (RAI) and external beam radiotherapy (XRT) were evaluated. Disease management was assessed for adherence with the 2009 ATA guidelines. Disease free survival (DFS) and overall survival (OS) were estimated using the Kaplan Meier method and compared with the log rank test.1099 DTC patients were referred to the BCCA ( $\sim 70 \%$ of all diagnoses of DTC in the province). Baseline characteristics: female sex $73 \%$, median age 50, histology; papillary $90 \%$, follicular $7 \%$, Hurthle cell $3 \%$. Stage at presentation using the AJCC $7^{\text {th }}$ edition was: pT1 $45 \%$, pT2 $18 \%$, pT3 $31 \%$, pT4 $5 \%$, unknown T 1\%, pN0 26\%, pN1 36\%, unknown N 38\%, and cM1 3\%. Surgical management for multifocal disease and/or cancer $>1 \mathrm{~cm}$ was; lobectomy $3 \%$, total thyroidectomy $69 \%$ and staged total thyroidectomy $28 \%$. Lymph node sampling was performed for tumors $>4 \mathrm{~cm}$ in $62 \%$. RAI was delivered in $83 \%$ of patients with tumors $>4 \mathrm{~cm}, \mathrm{M} 1$ disease and/or gross extra-thyroidal extension. Curative intent XRT was utilized in $48 \%$ with T4 lesions. The 5 y outcomes for management consistent with guideline recommended primary surgery and/or nodal management versus non-adherence was DFS $82 \%$ versus $84 \%(\mathrm{p}=0.64)$, and OS $94 \%$ versus $90 \%(\mathrm{p}=0.10)$. The 5 year outcomes for guideline recommended adjuvant RAI \pm XRT versus non-adherence was DFS $83 \%$ versus $43 \%(\mathrm{p}<0.001)$, and OS $95 \%$ versus $83 \%(\mathrm{p}=0.057)$. In our population-based cohort, compliance with ATA guideline recommended surgical management did not affect the DFS or OS. The DFS was significantly inferior if patients did not receive the recommended adjuvant $\mathrm{RAI} \pm \mathrm{XRT}$. RAI and XRT are integral components of the management of DTC and should be utilized within the context of ATA guideline treatment recommendations.

\section{Poster 395}

Thyroid Cancer Saturday Poster Clinical

\section{PEMBROLIZUMAB AS A CANDIDATE THERAPEUTIC APPROACH IN METASTATIC, TREATMENT- REFRACTORY THYROID CANCER}

S. Jasim ${ }^{1}$, J. Morris ${ }^{1}$, A. Chintakuntlawar ${ }^{2}$, C. Hilger ${ }^{2}$, C. Dalpiaz ${ }^{2}$, M. Ryder ${ }^{1}$, K. Bible ${ }^{2}$

${ }^{1}$ Endocrinology, Mayo, Rochester, MN; ${ }^{2}$ Medical Oncology, Mayo Clinic, Rochester, MN

We reviewed our initial single institution experience in treating advanced and therapy-refractory thyroid cancer patients with the antiPD-1 monoclonal antibody, pembrolizumab. Institutional Review Board-approved retrospective review of outcomes among thyroid cancer patients treated with pembrolizumab. Nine patients with advanced, imminently threatening, therapy-refractory thyroid cancer (4 anaplastic, 2 poorly differentiated, 1 hürthle cell, 1 medullary, 1 differentiated) were treated with pembrolizumab. Median age at diagnosis was 60 yrs (range 20-69); at therapy, 60 yrs (range 20-75);
$55 \%$ were female. Prior therapies included RAI $(2 / 9,22 \%)$, radiation therapy $(7 / 9,78 \%)$, cytotoxic chemotherapy $(7 / 9,78 \%)$, kinase inhibitors $(6 / 9,67 \%)$. Chemotherapy included: CVD (cyclophosphamide, vincristine, and dacarbazine), paclitaxel, and docetaxel/ doxorubicin. Mutations available in 8/9 patients included TERT promoter (4), TP53 (4), HRAS (1), RET (1), BRAF (1) and DICER1 (1). In anaplastic thyroid cancer patients, tumor mutational burden was low in 3/4 (75\%), with microsatellite status reported as "stable" in those 3 patients. Pembrolizumab was used alone in 5/9 (56\%), with lenvatinib in $2 / 9(22 \%)$, with paclitaxel/radiotherapy in $1 / 9(11 \%)$, and radiotherapy in $1 / 9(11 \%)$. Tolerance was good, with no CTCAE 4.03 toxicities $>$ grade 2 observed, but with one patient incurring prolonged grade 1 pancreatitis requiring therapy interruption. Median follow up duration was 3.6 months (1.4-7.3); median cycles administered was 3 (range 1-7). Discontinuation was triggered by disease progression in $7 / 9 ; 2$ patients remain on therapy. Best RECIST response was stable disease, but disease trajectory appeared to be favorably affected in several patients. Our limited initial experience in treating advanced and heavily pretreated thyroid cancer patients with pembrolizumab has shown some indication of disease modifying activity, but as of yet no RECIST responses. Predictors of response such as genetic mutations, mutational load, microsatellite instability, tumor immune infiltrates and PD-1/PD-L1 expression needs to be further studied in hopes of optimization of patient selection for pembrolizumab mono- versus combination therapies

\section{Poster 396}

\section{Thyroid Cancer Saturday Poster Clinical}

\section{CORE NEEDLE BIOPSY MORE ACCURATELY GUIDES} TREATMENT DECISIONS IN PATIENTS WITH NON-INVASIVE FOLLICULAR THYROID NEOPLASM WITH PAPILLARY-LIKE NUCLEAR FEATURES

M. Kim, J. Lee, J. Ha, S. lee, J. Bae, D. Lim, S. Jung, M. Kang, C. Jung The Catholic University of Korea, Seoul, Korea (the Republic of)

An indolent thyroid tumor, non-invasive encapsulated follicular variant of papillary thyroid carcinoma (EFVPTC) has recently been renamed as "non-invasive follicular thyroid neoplasm with papillary-like nuclear features" (NIFTP). The aim of the study was to compare the diagnostic efficacy of fine needle aspiration (FNA) and core needle biopsy (CNB) for the preoperative assessment of NIFTP. A total of 152 tumors that met the histologic criteria for diagnosis of EFVPTC on surgical specimens were selected from a seven-year period (January 2008 - December 2014). EFVPTCs were further subdivided into invasive EFVPTC and NIFTP according to the consensus diagnostic criteria. After excluding 17 cases without available preoperative FNA and/or CNB results, 135 cases (45 invasive EFVPTCs and 90 NIFTPs) were retrospectively reviewed. FNA was performed in $94(69.6 \%)$ cases, CNB alone in $51(37.7 \%)$ and both FNA and CNB in 10 (7.4\%). FNA and CNB results were categorized into one of six categories based on the Bethesda system. There was no difference in the distribution of cases in the Bethesda categories between NIFTP and invasive EFVPTC. NIFTPs were interpreted as categories II (3.3\%), III (36.1\%), IV (23.0\%), V $(18.0 \%)$ and VI $(19.7 \%)$ in FNA whereas the tumors were categorized into categories II ( $0 \%)$, III (3.1\%), IV $(53.1 \%), \mathrm{V}(9.4 \%)$ and VI (34.4\%) in CNB. CNB yielded more conclusive results (categories IV, V, and VI) than FNA (96.9\% vs. $60.7 \%, \mathrm{p}<0.001)$. In nodules with categories from IV to VI, proportion of category IV by FNA and by CNB was not different $37.8 \%$ vs. $54.8 \%, p=0.161$ ). In the CNBs with results of categories IV, V and VI, the possibility of follicular variant was always mentioned on the report, but not in the FNA specimens. FNA and $\mathrm{CNB}$ cannot preoperatively differentiate NIFTP from invasive EFVPTC. However, CNB has advantage over 
FNA for the preoperative triage of NIFTP patients into surgical candidates preventing overtreatment of NIFTP. Therefore, CNB is a useful method for guiding clinicians to proper management of NIFTP considering that NIFTP is a surgical disease necessitating lobectomy instead of total thyroidectomy.

\section{Poster 397}

Thyroid Cancer Saturday Poster Clinical

\section{BARRIERS AND FACILITATORS OF ACTIVE} SURVEILLANCE: INFORMATIONAL AND EMOTIONAL M.C. Saucke, J.L. Jennings, H.J. Khokhar, C.I. Voils, S.C. Pitt University of Wisconsin, Madison, Madison, WI

The 2015 ATA guidelines include active surveillance (AS) as a management strategy for patients with papillary thyroid microcarcinomas (PTMC). Data are lacking on attitudes and beliefs about the acceptability of this nonsurgical option. The purpose of this study was to determine providers' views on AS. This mixed-methods study used semi-structured interviews and a survey of 12 endocrinologists and 12 surgeons who treat patients with PTMC. We thematically analyzed interview transcripts using content analysis until we reached saturation $(n=15)$. Survey respondents were $70.8 \%$ male and $87.5 \%$ white; $87.5 \%$ practiced at academic institutions. Almost all $(91.7 \%)$ felt they knew enough about treatment alternatives for PTMC, but only $75 \%$ explain AS as an option. The majority $(62.5 \%)$ agreed that treatment decisions about PTMC are hard to make, and $79.2 \%$ would use a decision support tool (DST). Qualitative analysis revealed the following barriers to AS: worry about metastasis, fear of worse outcomes, the instinct to remove cancer, and reassurance provided by surgical removal. Providers believed these barriers were also significant to patients. Other barriers included the assumption that patients do not want AS, lack of resources, ultrasound reliability, skepticism about data on AS, fear of patients not following up, expectation of surgery, and patients getting surgery elsewhere. Facilitators of AS included physician recommendation, patients' fear of surgery, life circumstances, and avoidance of thyroid hormone. When discussing AS, providers who recommend this approach framed the option by providing reassurance and decisional support. They emphasized the low-risk nature of PTMC, gave time to make a decision, educated patients, used analogies to low-risk events, and stressed that surgery remains an option. In contrast, providers who hesitated to offer AS described the approach as "reasonable." The barriers and facilitators to AS of patients with PTMC include practice limitations, but also emotional reactions to a cancer diagnosis and uncertainty about outcomes. Findings suggest that reassurance by providers and/or utilization of a DST may facilitate patients' consideration of AS.

\section{Poster 398}

Thyroid Cancer Saturday Poster Clinical

\section{DOES EXTRATHYROIDAL EXTENSION PREDICT INVASIVE AND PERSISTENT DIFFERENTIATED THYROID CANCER?}

N.K. Jain ${ }^{1,2}$, L. Surrey ${ }^{3,2}$, S. Mostoufi-Moab ${ }^{1,2}$, S. Adzick ${ }^{1,2}$ K. Kazahaya ${ }^{1,2}$, J. Pizza ${ }^{1,2}$, N. Nelson ${ }^{2}$, A. Bauer ${ }^{1,2}$

${ }^{1}$ Pediatric Endocrinology, The Children's Hospital of Philadelphia, Philadelphia, PA; ${ }^{2}$ Perelman School of Medicine at the University of Pennsylvania, Philadelphia, PA; ${ }^{3}$ Pathology and Laboratory Medicine, Children's Hospital of Philadelphia, Philadelphia, PA

There is ongoing debate if the presence of extrathyroidal extension (ETE) in adult patients with differentiated thyroid cancer (DTC) is associated with an increased risk of lymph node metastasis (LMN) as well as persistent and recurrent disease. In pediatrics, only extensive
ETE has been shown to correlate with persistent or recurrent disease. There are no data defining whether microscopic ETE is associated with an increased risk of lymph node metastasis or event-free survival. The aim of our study was to assess whether ETE and tumor size $>4 \mathrm{~cm}$ is associated with an increased risk of lymph node metastasis (LNM) as well as persistent or recurrent disease. Using a retrospective cohort research design, we examined all DTC patients $\leq 19$ years of age at the time of evaluation in the CHOP Thyroid Center. In total, between 2009 and 2016, 152 patient charts were available for review: 53 patients diagnosed with $\mathrm{T} 3$ or $\mathrm{T} 4$ were compared to a randomly selected control group of patients diagnosed with $\mathrm{T} 1$ or $\mathrm{T} 2$ disease. The AJCC staging system was used to classify the thyroid cancer size and metastasis. All pathological findings such as microscopic foci and tumor size were confirmed by single pathologist review. Using a chi square analysis, we determined that there is a strong positive relationship between the degree of tumor staging and LNM (53 T3/T4 vs. $38 \mathrm{~T} 1 / \mathrm{T} 2, p=0.0003)$. ETE $(\mathrm{n}=61)$ was a stronger predictor of persistent disease $(p=0.02)$ than tumor size $(\mathrm{n}=72, p=0.1510)$ and there was a statistically significant correlation between ETE and LNM ( $n=30$ without ETE vs. 34 with ETE, $p<0.001)$. We concluded that ETE is a significant predictor of persistent disease and a superior measure of predictability than tumor size. ETE was also shown to be a statistically strong determinant of LNM. Single pathologist review of the samples is underway to explore if there is a difference in risk of LNM and persistent disease based on the degree of ETE (microscopic vs. macroscopic).

\section{Poster 399}

Thyroid Cancer Saturday Poster Clinical

\section{COMPARISON OF SALIVARY GLAND FUNCTIONS} ON THE SALIVARY GLAND SCAN IN DIFFERENTIATED THYROID CANCER PATIENTS UNDERWENT RADIOACTIVE IODINE ABLATION; RECOMBINANT HUMAN THYROID-STIMULATING HORMONE VERSUS THYROID HORMONE WITHDRAWAL

J. Jeong ${ }^{1}$, S. Lee ${ }^{1}$, B. Ahn ${ }^{1}$, J. Lee ${ }^{1}$, A. Chong ${ }^{2}$, S. Jeong ${ }^{1}$

${ }^{1}$ Department of Nuclear Medicine, Kyungpook National University Hospital, Daegu, Korea (the Republic of); ${ }^{2}$ Department of Nuclear Medicine, Chosun University Hospital, Gwangju, Korea (the Republic of)

Recombinant human thyroid-stimulating hormone (rhTSH) have been widely used for pre-therapeutic stimulation in differentiated thyroid cancer (DTC) patients but there has been no study comparing salivary gland function after radioactive iodine (RAI) ablation according to preparation (rhTSH versus thyroid hormone withdrawal (THW)) on the salivary gland scan (SGS). Thus, we evaluated salivary gland function after RAI therapy with rhTSH and THW preparation using the dynamic SGS.136 DTC patients who underwent RAI therapy with $3.7 \mathrm{GBq}$ from 2012 to 2015 were enrolled. 77 patients with rhTSH and 59 patients with THW performed dynamic SGS at a median follow-up of 16 months after RAI therapy. Using time-activity curves, 5 scintigraphic parameters were calculated for each parotid gland (PG) and submandibular gland (SMG): time at maximum counts (Tmax), time at minimum counts (Tmin), maximum accumulation (MA), maximum secretion (MS), uptake ratio (UR).15 patients (19.48\%) of rhTSH and 13 patients (22.03\%) of THW groups complained of xerostomia or related symptom $(p=0.831)$. On the patient-based analyses using visual assessment of SGS, salivary gland dysfunction was observed in 27 patients $(35.06 \%)$ of rhTSH group and in 22 patients $(37.29 \%)$ of THW group $(\mathrm{p}=0.858)$. There were no significant differences in all PG parameters between rhTSH and THW groups (Tmax, 22.34 \pm 2.89 in rhTSH group vs. $23 \pm 2.52$ in THW group, $p=0.052$; Tmin, $27.30 \pm 1.29$ vs. 
$27.15 \pm 1.66, \quad \mathrm{p}=0.423 ; \quad$ MS, $69.90 \pm 24.74$ vs. $69.96 \pm 25.72$, $\mathrm{p}=0.980$; UR, $2.48 \pm 1.41$ vs. $2.61 \pm 1.53, \mathrm{p}=0.449$ ), except the MA $(69.44 \pm 12.03$ vs. $74.07 \pm 14.44, p=0.004)$. After 21 patients $(15$ patients in rhTSH group, 6 patients in THW group) showing early, unstimulated saliva excretion were excluded from the SMG analysis, 5 parameters were also assessed and there were no significant differences in all SMG parameters between rhTSH and THW groups (Tmax, $21.44 \pm 18.90$ vs. $19.13 \pm 6.09, \mathrm{p}=0.23$; MA, $54.60 \pm 14.77$ vs. $51.93 \pm 15.87, \mathrm{p}=0.19$; MS, $63.57 \pm 12.23$ vs. $60.61 \pm 10.51$, $\mathrm{p}=0.05$; UR, $3.02 \pm 0.92$ vs. $2.80 \pm 0.84, \mathrm{p}=0.06$ ), except the Tmin $(27.36 \pm 1.30$ vs. $26.94 \pm 1.11, p=0.01)$. This study demonstrates comparable salivary gland function in patients prepared for RAI therapy with $3.7 \mathrm{GBq}$ by either administering rhTSH or THW.

\section{Poster 400}

Thyroid Cancer Saturday Poster Clinical

LONG-TERM PROGNOSTIC SIGNIFICANCE OF GROSS
EXTRATHYROIDAL EXTENSION INVADING ONLY
STRAP MUSCLES IN PATIENTS WITH DIFFERENTIATED
THYROID CARCINOMA

S. Park, S. Park, M. Choi, H. Kim, T. Kim, S. Kim, J. Chung

Division of Endocrinology \& Metabolism, Department of Medicine, Thyroid Center, Samsung Medical Center, Sungkyunkwan University School of Medicine, Seoul, Korea (the Republic of)

In the proposed eighth edition of the American Joint Committee on Cancer (AJCC) staging system for differentiated thyroid carcinoma (DTC), microscopic extrathyroidal extension (ETE) is no longer a determinant of T3 category. Instead, gross ETE invading only strap muscles is designated as a new T3b category. We investigated the long-term prognostic significance of gross ETE invading only strap muscles. This study retrospectively enrolled 3,003 DTC patients with T1-T3 disease who underwent thyroidectomy at a tertiary Korean hospital between 1996 and 2005. We assessed the differences in the cancer-specific survival (CSS) and structural recurrence-free survival (RFS) according to the extent of ETE. There were no significant differences in 10-year CSS among the patients with no ETE, microscopic ETE, and gross ETE invading only strap muscles (98.6, 98.2 and $98.9 \%$, respectively; $P=0.332$ ). The 10 -year RFS of patients with gross ETE invading only strap muscles $(89.9 \%)$ was shorter than those having no ETE $(94.5 \% ; P<0.001)$, but was similar to those with microscopic ETE (90.9\%). In univariate analysis, gross ETE invading only strap muscles showed a significantly higher risk for recurrence than those with no ETE [HR (95\% CI) $1.78(1.15-2.78) ; P=0.01]$. In multivariate analysis, however, gross ETE invading only strap muscles was not an independent predictor for recurrence [1.16 (0.73-1.83); $P=0.53)$. Despite the gross ETE invading only strap muscles could have prognostic information for long-term recurrence outcome, it did not affect the mortality of the patients with DTC. Our results called for the alternative use of gross ETE invading only strap muscles as a staging variable to be reconsidered.

\section{Poster 401}

Thyroid Cancer Saturday Poster Clinical

\section{MEDIASTINAL LYMPH NODES METASTASES IN THYROID CANCER: CHARACTERISTICS, PREDICTIVE FACTORS AND PROGNOSIS}

T. Zhang, Q. Ji, N. Qu

Head\&neck, Fudan University Shanghai Cancer Center, Shanghai, China, China

Mediastinal lymph nodes metastases (MLNM) haven't been extensively studied. The aim of this study is to investigate the characteristics, predictive factors and prognosis of mediastinal lymph nodes metastases in thyroid cancer. This is a retrospective analysis based on the data of the thyroid cancer patients with MLNM operated at our institution between 2008-2015. Systematic review about MLNM in thyroid cancer was also performed. Totally 73 thyroid cancer patients with positive mediastinal lymph nodes metastases (MLNM) were included in this study. It contained sixty $(82.2 \%)$ papillary thyroid carcinoma (PTC), twelve $(16.4 \%)$ medullary thyroid carcinoma and one (1.4\%) anaplastic thyroid carcinoma. 48 patients had the surgery as initial treatment, while 25 patients as reoperation due to the recurrence. Fifty-three $(72.6 \%)$ patients remained disease-free and fifteen $(20.5 \%)$ developed a regional recurrence. Distant metastases occurred in four $(5.5 \%)$ patients and five $(6.8 \%)$ patients died. Five-year overall survival rate and disease-free survival (DFS) rate of the PTC patients for initial treatment is $95.4 \%$ and $77.2 \%$. In the analysis of 47 PTC patients who had surgery as the initial treatment and proved with positive mediastinal lymph nodes, extrathyroidal extension $(\mathrm{HR}=8.06,95 \% \mathrm{CI} 1.45-44.87, \mathrm{p}<0.05)$ and multiple lymph nodes involved $(\mathrm{HR}=1.83,95 \% \mathrm{CI} 1.01-3.30, \mathrm{p}<0.05)$ was associated with DFS and RFS of the PTC patients. The incidence of MLNM in thyroid cancer was reported to be ranged from $0.7 \%$ to $48.1 \%$. Bilateral cervical metastasis, distant metastasis and reoperation were proved previously to be the predictive factors of mediastinal metastasis in thyroid cancer. Careful selection of patients at high risk of MLNM remains important. Initial therapeutic control is very important for the patients with thyroid cancer. The thyroid cancer patients with bilateral cervical metastasis, distant metastasis or reoperation would have higher risk of acompanying MLNM. Extrathyroidal extension and multiple mediastinal lymph nodes involved were the main influence factors of prognosis in the thyroid cancer patients with MLNM.

\section{Poster 402}

Thyroid Cancer Saturday Poster Clinical

\section{RECENT PREGNANCY IS NOT ASSOCIATED WITH HIGH- RISK PATHOLOGICAL FEATURES OF DIFFERENTIATED THYROID CANCER}

A. Chen ${ }^{1}$, M.J. Livhits ${ }^{2}$, L. Du ${ }^{3}$, J.X. $\mathrm{Wu}^{2}$, E.J. $\mathrm{Kuo}^{2}$, M. $\mathrm{Yeh}^{2}$, A.M. Leung ${ }^{4,5}$

${ }^{1}$ UCLA David Geffen School of Medicine, Los Angeles, CA; ${ }^{2}$ Section of Endocrine Surgery, Department of Surgery, UCLA David Geffen School of Medicine, Los Angeles, CA; ${ }^{3}$ Department of Biostatistics, UCLA David Geffen School of Medicine, Los Angeles, $\mathrm{CA} ;{ }^{4}$ Division of Endocrinology, Diabetes, and Nutrition;

Department of Medicine, UCLA David Geffen School of Medicine, Los Angeles, CA; ${ }^{5}$ Division of Endocrinology, Diabetes, and Nutrition; Department of Medicine, VA Greater Los Angeles Healthcare System, Los Angeles, CA

Thyroid cancer is commonly diagnosed in the first postpartum year, supporting the theory that high levels of estrogen may stimulate progression of hormone-mediated thyroid cancer. The American Thyroid Association states pregnancy may be a stimulus in women with known structural or biochemical thyroid cancer at the time of conception and thus recommends monitoring throughout gestation. The aim of this study was to assess the impact of recent pregnancy on histopathologic disease characteristics of differentiated thyroid cancer (DTC). Cases of DTC (1999-2012) were identified from the California Cancer Registry and linked to the Office of Statewide Health Planning and Development to allow for longitudinal follow-up. This was a matched-control study; recently pregnant women (pregnancy up to five years before and nine months after a thyroid cancer diagnosis) were compared with nonpregnant controls matched by age (within one year), gender, race, and ethnicity. The main outcome was histopathologic disease characteristics (tumor size, extrathyroidal extension, and nodal metastases) compared between the recently pregnant and non-pregnant groups. 
The study sample of 1,204 women (mean age $30.9 \pm 5.5$ [SD] years, $46.5 \%$ Caucasian and $40.0 \%$ Hispanic) included 301 recently pregnant women matched against 903 non-pregnant controls. Comparing recently pregnant vs. non-pregnant women, no significant differences were observed with respect to tumor size $(2.2 \pm 1.6$ vs. $2.2 \pm 3.9 \mathrm{~cm}$; $\mathrm{p}=0.39)$, extrathyroidal extension $(12.0 \%$ vs $14.1 \% ; \mathrm{p}=0.46)$, distant metastasis $(2.0 \%$ vs. $3.8 \%, p=0.17)$, or disease-specific survival $(\mathrm{p}=0.78)$. In multivariate analyses, after controlling for patient age and ethnicity, recent pregnancy was not a significant predictor of tumor size, extrathyroidal extension, nodal metastases or distant metastases. In this cohort, recent pregnancy was not associated with high-risk pathological features of differentiated thyroid cancer. These findings provide reassurance with regards to the concern that pregnancy may act as a potential stimulus for thyroid cancer growth, as cautioned by the 2017 American Thyroid Association guidelines for thyroid disease in pregnancy and the postpartum period.

\section{Poster 403 \\ Thyroid Cancer Saturday Poster Clinical \\ PREOPERATIVE VITAMIN D LEVEL AS A PREDICTIVE FACTOR FOR POST-THYROIDECTOMY HYPOCALCEMIA}

J. Kim, J. An, S. Baik, H. Kim, S. Paek, J. Woo, J. Lee, H. Kwon, W. Lim, B. Moon, N. Paik

Ewha Womans University Mokdong Hospital, Seoul, Korea (the Republic of)

Postoperative hypocalcemia is a common event following total thyroidectomy and has been reported in up to $35 \%$ of patients. Whether vitamin D deficiency is a risk factor of postoperative hypocalcemia is not clear. In this study, we evaluated the predictive value of preoperative vitamin $\mathrm{D}$ for the hypocalcemia following total thyroidectomy. We retrospectively evaluated the data of 273 patients who underwent total thyroidectomy. Patient demographics, preoperative serum $25-\mathrm{OH}$ vitamin $\mathrm{D}$ level, operative variables, and the development of hypocalcemia were collected and analyzed.

Patients had a mean age of $48.3 \pm 12.2$ years (range, 16 to 95 years) and $232(85.0 \%)$ patients was female and $41(15.0 \%)$ patients was male. Preoperative vitamin D level was $33.3 \pm 13.0 \mathrm{ng} / \mathrm{ml}$. Fifty-three (19.4\%) patients developed Transient hypocalcemia. By using a recursive partitioning procedure or tree classification algorithm, preoperative vitamin D level of $16 \mathrm{ng} / \mathrm{ml}$ was recommended as cut-off value. Of the 273 patients, 26 patients $(9.5 \%)$ was classified as low vitamin D group whereas 247 patients $(90.5 \%)$ was classified as normal vitamin D group. Patient demographics and operative variables were comparable between two groups. However, transient hypocalcemia was found more frequently in low vitamin $\mathrm{D}$ (34.6\% vs $17.8 \%, p=0.039$ )

Preoperative vitamin D level can be used as a predictive factor for postoperative hypocalcemia. In patients with low vitamin D level, caution should be given to prevent injury of parathyroid glands.

\section{Poster 404}

Thyroid Cancer Saturday Poster Clinical

RISK FACTORS FOR NON-SMALL-VOLUME CENTRAL LYMPH NODE METASTASES (MORE THAN FIVE OR LARGER THAN 2MM) IN CLINICALLY NODE-NEGATIVE PAPILLARY THYROID CARCINOMA

J. Wang

Department of Head and Neck Surgery, Sir Run Run Shaw Hospital, School of Medicine, Zhejiang University, Institute of Micro-Invasive Surgery of Zhejiang University, Sir Run Run Shaw Hospital, School of Medicine, Zhejiang University, Hangzhou, Zhejiang, China
The value of prophylactic central neck dissection (pCND) for papillary thyroid carcinoma (PTC) is unclear. However, lymph node (LN) metastasis is known to correlate with local recurrence, especially for metastatic LN more than five or $>2 \mathrm{~mm}$. The purpose of this study was to determine the incidence and risk factors of metastatic LN in the central compartment with more than 5 or $>2 \mathrm{~mm}$, which was defined as non-small-volume central LN metastases (NSVCLNM). A total of 621 patients with cN0 PTC from January 2013 to December 2015 were retrospectively analyzed. Cervical LNs were harvested and the size of metastatic LNs was measured. The prevalence and predictive factors of NSVCLNM in PTC and papillary thyroid microcarcinoma (PTMC) was analyzed. NSVCLNM were detected in $72(11.6 \%)$ patients with PTC, while the incidence was $8.5 \%$ (41 of 484 ) in PTMC. Male gender, age $\leq 36$ years, multifocal lesions, extrathyroidal extension, and tumor size $>0.85 \mathrm{~cm}$ were risk factors of NSVCLNM in PTC. The sensitivity and specificity of having $\geq 3$ risk factors for predicting NSVCLNM in PTC was $45.8 \%$ and $86.7 \%$, respectively. Male gender, age $\leq 37$ years, multifocal lesions, and tumor size $>0.65 \mathrm{~cm}$ were predictive risk factors of NSVCLNM in PTMC. The sensitivity and specificity of having $\geq 3$ risk factors for predicting NSVCLNM in PTMC was $43.6 \%$ and $91.2 \%$, respectively. These findings suggest $\mathrm{pCND}$ as suitable procedure for PTC patients with $\geq 3$ risk factors of NSVCLNM, while immediate surgery and pCND benefitted PTMC patients with $\geq 3$ risk factors.

\section{Poster 405}

Thyroid Cancer Saturday Poster Clinical

WIDESPREAD GENE FUSION ANALYSIS OF FORMALINFIXED, PARAFFIN-EMBEDDED (FFPE) SAMPLES IMPROVES PRIMARY DIAGNOSTICS IN THYROID NEOPLASIA AND STRATIFIES RECURRENT CASES FOR TARGETED THERAPY

K. van der Tuin ${ }^{1}$, M. Ventayol Garcia ${ }^{2}$, W. Corver ${ }^{2}$, D. Ruano Neto ${ }^{2}$ M. Snel ${ }^{3}$, T. Links ${ }^{4}$, H. Kapiteijn ${ }^{5}$, J. Smit ${ }^{6}$, F. Hes ${ }^{1}$, H. Morreau ${ }^{2}$ ${ }^{1}$ Department of Clinical Genetics, Leiden University Medical Centre, Leiden, Netherlands; ${ }^{2}$ Department of Pathology, Leiden University Medical Centre, Leiden, Netherlands; ${ }^{3}$ Department of Medicine, Division of Endocrinology, Leiden University Medical Centre, Leiden, Netherlands; ${ }^{4}$ Department of Endocrinology, University Medical Center Groningen, Groningen, Netherlands; ${ }^{5}$ Department of Oncology, Leiden University Medical Centre, Leiden, Netherlands; ${ }^{6}$ Department of Medicine, Division of Endocrinology, Radboud University Medical Center, Nijmegen, Netherlands

Somatic point mutations and gene fusions leading to the activation of the MAPK and PI3K/AKT signalling pathways are crucial for tumorgenesis and progression of thyroid tumours. The ability to detect several different gene fusions in RNA isolated from formalin-fixed, paraffin-embedded (FFPE) tumour tissues has so far been limited due to technical reasons. Fusion transcripts are of interest for primary diagnostics and targeted therapy in patients with advanced thyroid cancer refractory to radioactive iodine (RAI) treatment. The Archer ${ }^{\circledR}$ FusionPlex ${ }^{\circledR}$ analysis was performed on 69 FFPE thyroid neoplasia samples, that previously tested negative for pathogenic variants in $B R A F$ and $R A S$ genes. Of these, 36 cases have recurrent disease refractory to RAI treatment. We identified 15 known fusions, including CCDC6-RET (PTC1), NCOA4-RET (PTC3), GOLGA5-RET (PTC5), PAX8-PPARG, EML4-ALK and ETV6-NTRK3. The majority was identified in classical papillary carcinoma (PTC) and follicular variant of PTC. Another 19 novel fusion transcripts were identified of which the prognostic and diagnostic value are still unknown. We analysed three cases with multifocal disease and identified independent gene variants and fusions, with possible repercussions for adjuvant 
therapy choice, considering the metastatic potential of the individual lesions. Seven out of 36 recurrent cases showed NTRK or ALK fusions and therefore might benefit from targeted therapeutic approaches with tyrosine kinase inhibitors. Widespread gene fusion analysis of FFPE tissues is effective and feasible. The chosen method can be used for further tumour classification in complex casus (e.g. subtyping, multifocality and metastasis) and might stratify for targeted therapies for advanced thyroid cancer. This method can also be applied to preoperative fine needle aspirations to guide therapeutic choices.

\section{Poster 406}

Thyroid Cancer Saturday Poster Clinical

\section{DO ATA RISK CLASSIFICATIONS ACCURATELY PREDICT MORTALITY IN DIFFERENTIATED THYROID CANCER?}

K. Weaver, M. Roth

Medicine, University of Washington Medical Center, Seattle, WA

The incidence of thyroid cancer has been increasing steadily since 1975, but the mortality rate remains low and stable. The 2015 ATA guidelines for thyroid cancer recommend additional risk stratification for patients based on the risk of thyroid cancer recurrence. The objective of this study is to determine if patients who die from thyroid cancer would have been appropriately identified as high risk for recurrence based on their original risk stratification. This retrospective cohort evaluation includes patients with differentiated thyroid cancer (DTC) seen at the University of Washington Medical Center or Seattle Cancer Care Alliance from 2009 to 2014 and who died of DTC during this time. All deaths were adjudicated by one physician to determine correct primary diagnosis and disease-specific cause of death. Cases were reviewed to determine the ATA Risk Stratification (low, intermediate, high) and patients who died within 1 year of diagnosis or died after a diagnosis before age 45 were further evaluated. Of the 1,602 patients seen with DTC, 38 (2.4\%) died from thyroid cancer. Based on the 2015 ATA Guidelines, $26 \%(n=10)$ were considered low or intermediate risk at diagnosis and $74 \%(n=28)$ were considered high risk for recurrence. Age was significantly lower in the low/int risk group versus the high-risk group ( 44 vs. 57 years, respectively, $p<0.01$ ), and the time from diagnosis to death was longer in the low/int risk group (13 vs. 5 years, $\mathrm{p}<0.05$ ). Distant metastases were found at primary diagnosis in $20 \%$ of the high-risk patients.

Seven (18\%) deaths occurred in patients diagnosed before age 45 years and of these patients, two died within 5 years of diagnosis. Six patients in the study died within 1 year of their diagnosis, all of whom had distant metastatic disease at diagnosis. While death from thyroid cancer remains low, not all patients who die from their disease are identified as high risk for recurrence at diagnosis. This highlights the importance of ongoing dynamic risk stratification and long-term surveillance for differentiated thyroid cancer. Additional research is needed to help identify prognostic factors which may further identify patients at risk for disease-specific mortality from DTC.

\section{Poster 407}

Thyroid Cancer Saturday Poster Clinical

\section{THYROGLOBULIN MEASUREMENT USING MASS SPECTROMETRY IN THYROID CANCER}

L. Collins ${ }^{1}$, M. Roth ${ }^{1}$, A. Hoofnagle ${ }^{2}$

${ }^{1}$ Endocrinology, University of Washington, Seattle, WA;

${ }^{2}$ Laboratory Medicine, University of Washington, Seattle, WA

Thyroglobulin ( $\mathrm{Tg}$ ) measurement is crucial to the management of long term follow up for patients with differentiated thyroid cancer. Two-site immunoassays have high false negative rates in patients with $\mathrm{Tg}$ antibodies ( $\mathrm{Tg}-\mathrm{Ab}$ ) due to antibody interference. Liquid chromatography-tandem mass spectrometry (LCMS) assays may improve long-term management for patients with $\mathrm{Tg}$-Ab. This prospective randomized trial was designed to determine if use of LCMS decreases cost of care and imaging utilization for patients with Tg-Ab. Eligible patients were seen for thyroid cancer surveillance at the University of Washington, Seattle Cancer Care Alliance, or Harborview Medical Center between August 2011December 2015. Patients were invited to enroll if they had a positive $\mathrm{Tg}-\mathrm{Ab}$ or measurable $\mathrm{Tg}>1.0$ by immunoassay. Randomization to LCMS assay in addition to standard of care was based on age, gender, and Tg-Ab status. Tg by LCMS was done at the University of Washington with a lower limit of quantitation of $1.0 \mathrm{ng} /$ ml. Imaging use frequency at first follow-up visit and secondary analysis of the LCMS assay accuracy was evaluated with Fischer's exact T test. Sixty-two patients enrolled with 3 lost to follow up and 58 patients were included in the final analysis. LCMS use did not affect the frequency at which physicians ordered ultrasounds, but physicians ordered a CT scan more often if Tg LCMS was detectable: $2(50 \%)$ versus $1(4 \%)$.

In the 48 patients with $\mathrm{Tg}-\mathrm{Ab}$, the $\mathrm{Tg}$ immunoassay sensitivity and specificity for detecting structural disease were $37 \%$ and $76 \%$. In the 29 patients with $\mathrm{Tg}-\mathrm{Ab}$ randomized to obtain a Tg LCMS assay, the sensitivity and specificity for detecting structural disease were $29 \%$ and $100 \%$. In patients with thyroid cancer with positive $\mathrm{Tg}-\mathrm{Ab}$, use of LCMS did not decrease imaging utilization at the first follow up visit. The Tg LCMS assay was frequently undetectable given functional sensitivity of $1 \mathrm{ng} / \mathrm{ml}$, and did not improve accuracy in patients with $\mathrm{Tg}-\mathrm{Ab}$. Future work to improve analytical sensitivity of the LCMS assay may prove clinically useful.

\section{Poster 408}

\section{Thyroid Cancer Saturday Poster Clinical}

\section{TWENTY-YEAR SURVIVAL ANALYSIS OF PAPILLARY THYROID CANCER PATIENTS WITH COMPUTERIZED CYTOLOGICAL MORPHOLOGICAL AND CHROMATIC FEATURES}

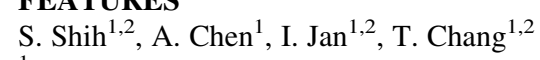

${ }^{1}$ National Taiwan University, Taipei, Taiwan; ${ }^{2}$ National Taiwan

University Hospital, Taipei, Taiwan

Fine needle aspiration cytology (FNAC) is important and simple for preoperative diagnosis of papillary thyroid cancer (PTC). We have reported that the computerized cytological features could be used to predict recurrence of PTCin 10 years. In the present study, we further applied innovative computerized cytological features to 20-year survival analysis.1055 patients diagnosed of thyroid cancers from 1993 to 2016 in National Taiwan University Hospital were reviewed by this study. Among the 40 patients who were known to die within 20 years, 27 patients expired due to unknown reasons or cancers other than PTC and only 13 patients were confirmed to die from PTC. To find patients surviving for more than 20 years, there were 124 patients diagnosed and surgical resected before 1996 and only 60 patients among them were pathologically proven to be PTC and confirmed to be alive for at least 20 years. Digital images of the FNAC specimens by Liu's stain were obtained from these 73 patients ( 13 expired and 60 survived), and their morphological and chromatic features were analysed by the computer. A total of 15 cytological features, including 12 morphological features and 3 chromatic features, were quantified and their values between the survived and the expired cases were compared. Among the 15 quantified features, 2 morphological features, i.e., mean and standard deviation of nucleus size, and 1 chromatic feature, i.e., nuclearcytoplasmic hue ratio, were shown to be statistically significant in distinguishing the expired cases from the survived ones with $p$-values 
equal to $0.0027,0.0017$ and 0.0013 , respectively in the Mann-Whitney U test. Their AUROC's were $0.767,0.767$ and 0.785 and their $p$-values in the Kaplan-Meier survival analysis were 0.0384, 0.0361 and 0.0007, respectively. Our studies showed that PTC patients with larger and irregular nucleus size were more likely to expire while patients with hyperchromatic nuclei had a better chance of 20 -year survival. The visualization and quantification of cytological morphology and chromatic features have the potential of being developed as a computer analysis tool for predicting the long-term survival of PTC.

\section{Poster 409}

Thyroid Cancer Saturday Poster Clinical EVALUATION OF SERUM P53 ANTIBODIES AS A TUMOR MARKER OF ANAPLASTIC THYROID CANCER T. Uruno ${ }^{1}$, T. Kondo ${ }^{2}$, C. Tomoda ${ }^{1}$, A. Suzuki ${ }^{1}$, K. Matsuzu ${ }^{1}$, K. Ohkuwa ${ }^{1}$, W. Kitagawa ${ }^{1}$, M. Nagahama ${ }^{1}$, K. Sugino ${ }^{1}$, K. Ito ${ }^{1}$ ${ }^{1}$ Department of Surgery, Ito Hospital, Tokyo, Japan; ${ }^{2}$ Department of Pathology, University of Yamanashi, Yamanashi, Japan

Mutations in the TP53 gene can lead to the expression of mutant p53 proteins that accumulate in cancer cells and can induce circulating serum p53 antibodies (s-p53Ab) in cancer patients. Inactivating p53 mutation has been considered the hallmark of anaplastic thyroid cancer (ATC), with a cumulative mutation rate of $60-80 \%$. The aims of the present study were to evaluate the usefulness of s-p53Ab as a tumor marker in ATC patients and to investigate their association with p53 expression in tumor tissue. A total of 83 ATC patients were evaluated for serum $\mathrm{p} 53 \mathrm{Ab}$ using enzyme-linked immunosorbent assay, and p53 expression in the tumor tissue was evaluated in 73 patients using immunohistochemistry. Among the 83 patients (28 men, 55 women; median age, 70 years; age range, $44-90$ years), four were classified as stage IVa, 36 as stage IVb, 30 as stage IVc, seven as transformation with local recurrence, and six as transformation with distant metastasis including lung and/or bone. Forty-two (50.6\%) of the 83 patients had autoimmune thyroid disease $(\mathrm{TgAb}(+)$ or $\operatorname{TPOAb}(+))$, while 41 patients did not $(\mathrm{TgAb}(-)$ and $\operatorname{TPOAb}(-))$. At the time of ATC diagnosis, $18(21.7 \%)$ of the 83 patients were positive for s-p53Ab ( $>1.30 \mathrm{U} / \mathrm{ml}$; range, $1.86-2660 \mathrm{U} / \mathrm{ml})$, which is a significantly higher prevalence than healthy populations $(5 \%$, $\mathrm{p}<0.001)$. The prevalence of $\mathrm{s}-\mathrm{p} 53 \mathrm{Ab}(+)$ did not correlate to the coexistence of autoimmune thyroid disease. Although mismatch results between s-p53Ab and high levels of p53 expression in the corresponding tumors were observed in s-p53 $\mathrm{Ab}(-)$ patients, all the patients with s-p53 $\mathrm{Ab}(+)$ represented high levels of $\mathrm{p} 53$ expression in the tumor tissues. All of $17 \mathrm{~s}-\mathrm{p} 53 \mathrm{Ab}(+)$ patients with multiple time series data showed decreased values after receiving treatment such as surgery, chemotherapy, or radiotherapy. Nine of 39 patients showed increased values after disease progression, while $30(77 \%)$ showed no increase in values. The prevalence of s-p53Ab(+) was higher in ATC patients compared with healthy populations, and the values decreased after treatment. However, these findings do not suggest the usefulness of s-p53Ab as a marker for the early detection of disease recurrence or progression.

\section{Poster 410}

Thyroid Cancer Saturday Poster Clinical

PREOPERATIVE CLINICAL AND SONOGRAPHIC CHARACTERISTICS PREDICT THE LATERAL CERVICAL LYMPH NODE METASTASIS IN SPORADIC MEDULLARY THYROID CARCINOMA

H. Oh, H. Kwon, E. Song, M. Jeon, D. Song, T. Kim, J. Baek, S. Hong, W. Kim, Y. Shong, W. Kim

Asan Medical Center, Seoul, Seoul, Korea (the Republic of)
Total thyroidectomy and cervical lymph node (LN) dissection is generally recommended for patients with medullary thyroid carcinoma (MTC). However, there is no clear evidence for deciding the extent of LN dissection. This study aims to evaluate the preoperative characteristics for predicting the lateral cervical LN (LCLN) metastasis of MTC. This study included 26 MTC patients with LCLN metastasis at initial surgery (N1b group) and 45 MTC patients without any LN metastasis or persistent of disease (N0 NED group). We evaluated the association between preoperative clinical and sonographic characteristics (size, location, solid component, shape, margin, echogenicity, calcification and subcapsular location of the tumor) and LCLN metastasis. There was no significant difference in age and sex between N1b and N0 NED group. Preoperative serum calcitonin (CT) level above $100 \mathrm{pg} / \mathrm{mL}$ was associated with LCLN metastasis $(p<0.001)$. In preoperative neck ultrasonogrphy (US), N1b group were more commonly associated with larger tumor size $(>1.5 \mathrm{~cm})$, irregular shape, spiculated margin, and subcapsular location compared with N0 NED group $(p=0.029, p<0.001, p<0.001$, and $p<0.001$, respectively). An increase in the number of these LCLN metastasis related features was significantly associated with a higher risk of LCLN metastasis $(p<0.001)$. Presence of two or more suspicious features presented as an appropriate cut-off point for predicting the LCLN metastasis of MTC with $73.1 \%$ of sensitivity and $95.6 \%$ of specificity, respectively. MTC with high preoperative CT levels $(>100 \mathrm{pg} / \mathrm{ml})$, larger tumor size $(>1.5 \mathrm{~cm})$, irregular shape, spiculated margin, and subcapsular location in neck US has a higher risk of LCLN metastasis. MTC patents less than 1 suspicious feature might be appropriate for treatment without lateral cervical LN dissection.

\section{Poster 411}

Thyroid Cancer Saturday Poster Clinical

OCCULT NODE METASTASES IN PAPILLARY THYROID CARCINOMA CNO PATIENTS: RESULTS OF CENTRAL AND LATERAL LYMPH NODE PROPHYLACTIC NECK DISSECTION

R. Ciuni

Clinica Chirurgica, az. osp. Vittorio-emanuele, Catania, Italy

The percentage of occult node metastases of cervical lymph nodes is high in patients with papillary thyroid carcinoma: $64 \%$ for central lymph nodes and $23 \%$ for lateral ones. Therefore many patients just undergoing thyroidectomies do not benefit from the best postoperative staging which might invalidate any follow-up or radiometabolic therapy. Lymph node and distance metastases frequently lose iodine proclivity rendering them unresponsive to radiometabolic therapy. From May 2014 to May 2016122 patients were recruited who had had thyroidectomies with VI level bilateral lymphadenectomies and III-IV level unilateral tumours with the same skin incision of thyroidectomy. All those patients diagnosed with papillary thyroid carcinoma NO. In cases of clinically manifest cervical metastases therapeutic lymphadenectomies but not prophylactic ones were carried out, and these patients were excluded from the study. There were 34 males (27.9\%) and 88 females (72.1\%). Average tumour sizes after surgery were $1.9 \mathrm{~cm} \pm 0.6$ (range $1,1-3 \mathrm{~cm}$ ). The percentage of metastases at the III-IV levels was $34.42 \%$ (42 patients) of whom 22 were female and 20 male. $75.4 \%$ had central occult metastases, the remaining $24.6 \%$ had N0 or micro-MTS. The average of total lymph nodes removed per patient was 31.66 \pm 13.87 (range 10$71)$. A statistically significant correlation $(p<0,05)$ was found between laterocervical metastases and: thyroiditis $\mathrm{p}=0.000$; tumour infiltration $\mathrm{p}=0.000$; thyroid infiltration $\mathrm{p}=0.024$; vascular invasion $\mathrm{p}=0.008$; lymph vessel invasion $\mathrm{p}=0.0014$; hyperthyroidism $\mathrm{p}=0.02$; female $\mathrm{p}=0.03$; VI level metastases $\mathrm{p}=0,000$. VI level metastases $(75.4 \%)$ is an independent risk factor associated with laterocervical metastases as 
shown by multivariate analysis. During follow-up the plasma levels of $\mathrm{Tg}$ and $\mathrm{Ab}-\mathrm{Tg}$ fell between $<0,01 \mathrm{ng} / \mathrm{ml}$ and $2,5 \mathrm{ng} / \mathrm{ml}$, so pT1 patients were classified as $\mathrm{pN} 0$ at low risk of relapse. The level of occult node metastases in papillary thyroid carcinoma $\mathrm{cNO}$ patients found in this study was $34.42 \%$. Optimising staging provides more reliable patient classification. Our approach of prophylactic lymphadenectomy makes patients whit low thyroglobulin level that simplifying follow-up and reducing ancillary treatments (RAIT).

\section{Poster 412}

Thyroid Cancer Saturday Poster Clinical

\section{RATES OF THYROID FINE NEEDLE ASPIRATION CYTOLOGY BY BETHESDA CATEGORIZATION IN CALGARY, CANADA}

S. Ghaznavi ${ }^{1,2}$, H. Clayton $^{1}$, M. Eszlinger ${ }^{1}$, C.J. Symonds ${ }^{1}$, M. Khalil ${ }^{1}$, R. Paschke ${ }^{1}$

${ }^{1}$ University of Calgary, Calgary, Alberta, Canada; ${ }^{2}$ Memorial Sloan Kettering Cancer Center, New York, NY

After clinical und ultrasound malignancy risk stratification, fine needle aspiration cytology (FNAC) is the gold standard test to distinguish benign from malignant thyroid nodules. FNAC results are communicated as six Bethesda categories, each with an associated risk of malignancy. Over or under-utilization of certain Bethesda categories diminishes the usefulness of FNAC. For example, a large proportion of nondiagnostic samples necessitates repeat biopsy and leads to further downstream tests. Likewise, over-utilization of the "indeterminate" categories also leads to clinical ambiguity and further downstream actions including diagnostic lobectomy. Therefore, the breakdown of Bethesda categories at a local centre is an important process quality indicator for pathologists and clinicians alike. Calgary Laboratory Services (CLS) processes all cytologies for the Calgary region (1.4 million inhabitants). Using administrative health care data, we reviewed all 5921 consecutive thyroid FNAC samples received by Calgary Laboratory Services (CLS) from three tertiary and several community sites in Southern Alberta from 20102013. Thyroid FNAC were evaluated by 14 pathologists. The breakdown of Bethesda categories was as follows: Bethesda $1=19 \%$, Bethesda $2=56 \%$, Bethesda $3=14.7 \%$, Bethesda $4=2.7 \%$, Bethesda $5=1.5 \%$, Bethesda $6=5.6 \% .162 / 1127(14 \%)$ of the Bethesda 1 cytologic results were sub-classified as a cyst. A secondary analysis of the data examining surgical rates and malignancy rates for each Bethesda category is currently underway. Compared to a metaanalysis of thyroid FNAC studies that pooled data largely from single tertiary referral centers, our study reports a higher rate of nondiagnostic samples $(19 \% \text { vs. } 12.9 \%)^{1}$. It is unknown whether this is due to a higher rate of cysts especially at our community sites, or operator dependant factors. We saw a slightly higher rate of Bethesda 3 cytology in our study ( $14.7 \%$ vs. $9.6 \%$ ), which may point to possible benefits for further cytologist training. Despite a combination of patients from tertiary care and community sites and involvement of 14 pathologists, we saw a similar rate of malignancy as Bongiovanni et al. $(5.6 \% \text { vs. } 5.4 \%)^{1}$.

\section{Poster 413}

Thyroid Cancer Saturday Poster Clinical MEDULLARY THYROID CARCINOMA, PARAGANGLIOMA, AND SDHB MUTATION

R. Koshy, J. Kung

Tufts Medical Center, Boston, MA

There is little information to suggest an association of SDHB mutation with the development of medullary thyroid carcinoma.56 year- old developmentally delayed male, with a previously resected nonfunctioning right carotid body paraganglioma in 2006, who presented with a recurrent right carotid body tumor. CT neck incidentally showed a $1.9 \mathrm{~cm}$ solid hypodense left thyroid nodule. FNA of the nodule revealed medullary thyroid carcinoma. Calcitonin was 506 $(\mathrm{nL}<10)$ with CEA of 2.1 ( $\mathrm{nL}<2.5)$. Patient underwent a total thyroidectomy and resection of the right carotid body tumor. Thyroid surgical pathology revealed a $1.6 \mathrm{~cm}$ medullary carcinoma and a $0.4 \mathrm{~cm}$ papillary microcarcinoma. Carotid body tumor pathology revealed a $5 \mathrm{~cm}$ paraganglioma. Genetic screen showed SDHB mutation p. L212F (c.634C $>$ T), considered variant of undetermined significance. Immunohistochemistry (IHC) staining showed an absence of SDHB staining in the paraganglioma, but positive SDHB staining in the medullary thyroid cancer. Succinate dehydrogenase B subunit (SDHB) gene germline mutations predispose to pheochromocytomas, sympathetic paragangliomas, head and neck paragangliomas, and non-paraganglionic tumors (e.g. renal cell carcinoma, gastrointestinal stromal tumor and pituitary neoplasia) (Niemeijer et al., The Phenotype of SDHB Germline Mutation Carriers; a Nationwide Study. European Journal of Endocrinology. May 2017). Papillary thyroid carcinomas have been reported with pathogenic variants in SDHB and SDHD but its significance is unclear. Medullary thyroid carcinoma and its association with a SDHB variant is novel and to date has not been reported in the literature. In this case, because there is positive SDHB IHC staining in the medullary thyroid cancer, indicating functioning SDHB, the SDHB mutation has not contributed to the development of the medullary thyroid cancer, and is a coincidental finding, with two rare tumors in the same patient. Further investigation will be needed to assess the possible role of SDHB mutation with medullary thyroid carcinoma as this may be important for diagnosis, management and genetic counseling.

\section{Poster 414}

Thyroid Cancer Saturday Poster Clinical MITOXANTRONE HYDROCHLORIDE IS A NOVEL LYMPHATIC TRACER THAT MAY HELP PRESERVING PARATHYROID GLANDS IN THYROID SURGERY

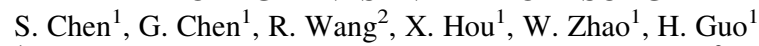

${ }^{1}$ Peking Union Medical College Hospital, Beijing, China; ${ }^{2}$ Shenyang Pharmaceutical University, Shenyang, China

Mitoxantrone hydrochloride is a water-soluble dark blue agent that is widely used as a chemotherapeutic agent. When injected into tissues or organs, mitoxantraone hydrochloride stains the lymphatic tissue into a dark blue color while blood vessels and other unrelated organs are spared. We developed mitoxantrone hydrocholride into a lymphatic tracer and propose that this drug could be safe and effective in visualizing parathyroid glands during thyroid surgeries. Animal studies were conducted to verify the efficacy and pharmacokinetic properties. Mitoxantrone hydrochloride was injected into different tissues and organs in mice, rats and rabbits and observed for staining pattern. Animals were then dissected and send for pathological studies. Drug concentration in blood and other vital organs were measured after injection. After completion of animal studies, a phase 1 clinical trial was designed and conducted in patients who had been planned for thyroidectomy. Mitoxantrone hydrochloride was injected into subcutaneous tissue, mammary gland and stomach. Lymphatic ducts and nodes nearby were stained into blue in less than 10 minutes. Pathology suggests that surrounding connective tissues and blood vessels were spared. Blood concentration was undetectable in all time points $(0 \mathrm{~min}$, $30 \mathrm{~min}, 2 \mathrm{~h}, 12 \mathrm{~h}, 24 \mathrm{~h}$ and $48 \mathrm{~h}$ ) after injection. 15 patients were selected for phase 1 clinical trial. Mitoxantrone hydrochloride was injected into thyroid gland during surgery. Lymphatic tissues near the thyroid gland turned dark blue in 1-3 minute while parathyroid glands were left 
unstained, and thus visualized and preserved in situ. Blood concentrations were not detected in all patients and no drug-related adverse effects were observed. Mitoxantrone hydrochloride is a safe lymphatic tracer that might be helpful in preserving parathyroid glands during thyroidectomy.

\section{Poster 415}

Thyroid Cancer Saturday Poster Clinical CLINICAL RELATIONSHIP BETWEEN HASHIMOTO'S
THYROIDITIS AND BRAFV600E MUTATION STATUS
IN PAPILLARY THYROID CARCINOMA PAIENTS

S. Kang, S. Lee, H. Youn, S. Jung

Surgery, Chonbuk National University Medical School, Jeonju-si, Jeollabuk-do, Korea (the Republic of)

Concomitant papillary thyroid carcinoma (PTC) and Hashimoto's thyroiditis (HT) is a frequent occurrence. Whether these two conditions are linked and whether PTC with concurrent HT has distinct clinicopathological characteristics are still debated issues. Lymphocytic infiltration is abundant in HT and might be relevant in the pathogenesis and progression of PTC. $\mathrm{BRAF}^{\mathrm{V} 600 \mathrm{E}}$ mutation is associated with a more advanced PTC at diagnosis; however, its role in the clinicopathological characteristics of PTC with concurrent HT is unknown. The purpose of this study was to evaluate the potential relationship between Hashimoto's thyroiditis and $\mathrm{BRAF}^{\mathrm{V} 600 \mathrm{E}} \mathrm{mu}-$ tation status in patients with PTC. A total of 198 patients who underwent surgery for PTC between January 2013 and June 2013 were enrolled in this study. $\mathrm{BRAF}^{\mathrm{V} 600 \mathrm{E}}$ mutation analysis was performed using polymerase chain reaction (PCR)-based amplification of DNA extracted from paraffin-embedded tumor specimens. $\mathrm{BRAF}^{\mathrm{V} 600 \mathrm{E}}$ mutation and HT were detected in the numbers of 149 (70.2\%) patients and 73 patients (36.9\%), respectively. BRAFV600E mutation was not correlated with HT $(\mathrm{P}=0.749)$. Lymph node metastasis was more frequent in $\mathrm{BRAF}^{\mathrm{V} 600 \mathrm{E}}$ mutation patients $(\mathrm{OR}=2.04, \mathrm{P}=0.039)$. However, age, tumor size, extrathyroidal extention, and mutifocality were not significantly associatied with the $\mathrm{BRAF}^{\mathrm{V} 600 \mathrm{E}}$. The results of our study suggest that $\mathrm{BRAF}^{\mathrm{V} 600 \mathrm{E}}$ mutation were associated with aggressive PTC. However, there was no clinicopathological association between $\mathrm{BRAF}^{\mathrm{V} 600 \mathrm{E}}$ mutation and $\mathrm{HT}$.

\section{Poster 416}

Thyroid Cancer Saturday Poster Clinical

THYROID LOBECTOMY VERSUS TOTAL THYROIDECTOMY IN THE TREATMENT OF LOW RISK WELL-DIFFERENTIATED THYROID CANCER 1-4 CM IN SIZE: A SYSTEMATIC REVIEW

J.N. Athayde, D. MacNeil

Otolaryngology, Western University, London, Ontario, Canada

Until recently, for low risk well-differentiated thyroid cancer between $1-4 \mathrm{~cm}$ in size, total thyroidectomy (TT) has been the recommended treatment with or without central neck dissection and radioactive iodine ablation. Thyroid lobectomy is associated with fewer complications and reduced requirement for thyroid replacement. Recent literature has shown similar 10-year survival and recurrence rates between total thyroidectomy and thyroid lobectomy (TL) for well differentiated thyroid cancer $>1 \mathrm{~cm}$ and $<4 \mathrm{~cm}$, with no evidence of extrathyroidal extension or nodal disease. The purpose of this meta-analysis is identify relevant literature and compare total thyroidectomy and thyroid lobectomy as treatment options for low risk well differentiated thyroid cancer. Systematic review methods were used to identify literature, both published and unpublished, from MEDLINE, EMBASE, and Scopus as well as the grey literature. Studies regarding well-differentiated primary thyroid cancer, with data on tumors $1-4 \mathrm{~cm}$ in size, were included in the analysis. Two reviewers independently screened all titles, abstracts, and articles to be included based on pre-established criteria. Pooled odds ratios (ORs) and 95\% confidence intervals were calculated using RevMan 5.3 software. Four studies met the inclusion criteria, including tumour size between $1-4 \mathrm{~cm}$, with a total of 122886 pooled patients. There was only one study that had post-operative tumour recurrence data that fit the criteria and so this outcome could not be compared between TT and TL. Three of the studies met the inclusion criteria for unadjusted 10-year survival, where there was no significant difference between the two surgeries (OR 1.04, 95\% CI 0.69, 1.56). Three of the studies had data on adjusted 10-year survival hazard ratios, which also showed a non-significant difference between the two procedures (OR $0.89,95 \%$ CI $0.74,1.06)$. This is the first study to quantify the postoperative outcomes following surgery for well-differentiated thyroid cancer in the size category of $1-4 \mathrm{~cm}$ tumours. Our results suggest that thyroid lobectomy is not significantly different in terms of 10-year survival, although we cannot comment on recurrence data.

\section{Poster 417}



\section{Poster 418}

Thyroid Cancer Saturday Poster Clinical

COMPARING THE QUALITY OF SCAR AFTER CONVENTIONAL THYROIDECTOMY WITH SILICONE GEL, SHEET AND STICK

Y. Bang, Y. Kim

Department of Surgery, Samsung Medical Center, Seoul, Korea (the Republic of)

Especially, Patients who underwent thyroid surgery have a high interest in scar management because the scar is well visible. Recently, 
patients are often using scar management products after surgery. However, research on which products are more effective is not sufficient.

In this study, we evaluate the quality of scar after using scar management products at 6 months after conventional thyroidectomy. To compare the quality of resulting scar, we used Vancouver scar scale (VSS) and patient's self-rating satisfaction.

We compared the efficacy of silicone gel (Dermatics ultra), silicone sheet (Scar clinic, Mepiform) and stick (Remescar). Patients who underwent conventional thyroidectomy from Febrary 2016 were identified and administered the self-scoring patient Questionnaire. We explained each 4 products before discharge, then participants selected maximum 2 products. After 6month from surgery, 105 participants completed questionnaires comprised of VSS score and Patient self-rating satisfaction score. These were evaluated against sex, age, operation type and type of products. Of 319 patients, $35 \mathrm{did}$ not choose the product and 51 choose 2 products. Most people chose silicone gel. Regardless of the product, the most important reason for choosing was convenience. Sex, Age and Operation type did not affect Quality of scar. Patient satisfaction score was statistically associated with Vancouver Scar Scale scores. The higher patient's selfrating satisfaction score is, the lower VSS score. Patients group which used Mepiform had the best objective score, but it is not statistically significant. There was no statistically significant differences in either VSS or questionnaire between the 4 products. Also, there was no better effect in patients with two products. Recently, various products are used in management of postoperative scars. This study shows that there is no significant difference in efficacy between product types.

Scar management is an ongoing process over several months. Patient compliance is therefore essential throughout these periods. Consequently, it is important choosing the product that is easiest to use. For that, doctor should explain the pros and cons to patients in detail.

\section{Poster 419}

Thyroid Cancer Saturday Poster Clinical

\section{LONG TERM OUTCOME OF SURGICAL PREFERENCE IN LOW-RISK THYROID CANCERS AND PAPILLARY THYROID MICROCARCINOMAS}

M. Sahin, R. Emral, i. aydogan, S. Gullu, M. Erdogan, O. Demir, V. Gedik, N. Baskal, D. Corapcioglu

Endocrinology and Metabolic diseases, Ankara University, Ankara, Turkey

Aim of our study is to investigate the long term outcomes of 368 low risk differentiated thyroid cancers (mean age $\pm \mathrm{SD}, 47.7 \pm 12$ years,) and 190 papillary microcarcinomas as a subgroup according to surgical extension. We divided low risk thyroid cancer patients into four groups (Group A 268 patients with total thyroidectomy, group B 60 patients with total thyroidectomy and prophilactic central lymph node dissection, group C 28 patients with subtotal thyroidectomy and group D 14 patients with lobectomy). Non-remission rate was significantly higher in Group C (4/28) according to group A $(10 / 268)(p=0.034)$. Odds ratio was 4.28 for initial non-remission. But longterm reccurence rate was not different significantly among all groups.

For papillary microcarcinomas, seventy-nine males and $111 \mathrm{fe}-$ males were included in our study with a mean age of $47.8 \pm 11.5$ years. We divided into four groups as in whole low risk differantiated thyroid cancers. In group A there are 134 patients had total thyroidectomy, in group B there are 28 patients with total thyroidectomy and prophilactic central lymph node dissection in Group C there are 13 patients had subtotal thyroidectomy and finally in Group D there are 10 patients with lobectomy. Non-remission rates were (3/134) \% 2.23 in group $\mathrm{A},(2 / 28) \% 7.1$ in group $\mathrm{B},(3 / 13) \% 23$ in group $\mathrm{C}$ and all patients were in remission in group $\mathrm{D}$. Reccurence rates were (3/ 134) \% 2.23 in group $A, 1 / 10$ in group $D$, no reccurence were observed in $\mathrm{B}$ and $\mathrm{C}$ groups.

Less than total thyroidectomy may not be suitable in some low risk differantiated thyroid cancer patients.

\section{Poster 420}

Thyroid Cancer Saturday Poster Clinical

\section{CASE REPORT : MEDULLARY THYROID CANCER} WITH NEGATIVE CALCITONIN

N. Bin Swedan, M. Aldawish

PSMMC, Riyadh, Saudi Arabia

Medullary Thyroid Cancer occupy 1-2\% of all Thyroid cancer in US. Calcitonin is secreted from $\mathrm{C}$-Cell in thyroid gland. Undetectable Calcitonin pre-operative was reported in rare cases. ook effect (falsely low) of Calcitonin also has been noted. case reportA 51 year old female presented with neck swelling for one year, six months ago, she had mild dysphagia and dyspnea with weight loss of four KG, No Hx of radiation exposure.

Past history : hypertension 10 years with renal impairment.

On examination : looks well, No goiter. Right Upper Anterior cervical L. N measure $3 \mathrm{~cm}$ ( hard, mobile, non-tender and and no skin changes) and few scattered small L. $\mathrm{N}$ at posterior compartment.

Lab work: FT4 15.2 (12.0-22.0 pmol/L), TSH 0.59 (0.270-4.200 $\mu \mathrm{IU} / \mathrm{mL})$, Creatinine 199 (45-84) $\mu \mathrm{mol} / \mathrm{L}$, Urea $14.1(2.8-8.1 \mathrm{mmol} /$ L), Corrected Calcium 2.32 (2.07-2.46 mmol/L)

US Neck: Enlarged heterogenous right thyroid lobe with multiple enlarged right cervical lymph nodes (largest $2.4 \times 1.5 \mathrm{~cm}$ ) worrisome of neoplastic process. also left thyroid nodules hypoechoic measures $2 \times 1.2 \mathrm{~cm}$.

FNA showed : Right thyroid, Bethesda VI, Right cervical lymph node: Positive for malignancy. Highly suggestive of medullary carcinoma. Left thyroid nodule: Benign

Calcitonin $<2.0 \mathrm{pg} / \mathrm{ml}$ ( up to 5.0), Plasma Meta/Normetanephrines were normal

Total Thyroidectomy showed :

Medullary thyroid carcinoma, $2.5 \mathrm{~cm}$ in Rt lobe with Lymphovascular invasion, No ETE

Positive LN metastases, (pTNM): T2 N1b Mx.

Follow -up: Six months later :Calcitonin $112 \mathrm{pg} / \mathrm{ml}$ (in the same lab) (up to 5) CEA $2.7(0.0-3.4) \mu \mathrm{g} / \mathrm{L}$

RET oncogene : Negative

Neck US: No residual or recurrence, suspecious right cervical lymph node. Negative calcitonin, can be explained by many factors, including heterophilic antibodies. Endocrinologist should be aware of MTC negative Calcitonin pre-Thyroidectomy.

\section{Poster 421}

Thyroid Cancer Saturday Poster Clinical

A CASE OF THYROID LYMPHOMA IN A PATIENT WITH A HISTORY OF MULTINODULAR GOITER AND RENAL CELL CARCINOMA

A. Cannavo ${ }^{1}, \mathrm{C} . \mathrm{Nasr}^{2}$

${ }^{1}$ Internal Medicine, Cleveland Clinic Foundation, Beachwood, $\mathrm{OH}$; ${ }^{2}$ Endocrinology, Cleveland Clinic, Cleveland, $\mathrm{OH}$

Primary lymphoma occurs in the thyroid gland in only $\sim 2 \%$ of cases of lymphoma. Additionally, only $\sim 1 \%$ of thyroid malignancies are cases of thyroid lymphoma. A history of Hashimoto's thyroiditis increases a patient's risk for this condition. A patient will typically 
present with a rapidly enlarging goiter. Prompt evaluation, diagnosis, and treatment are imperative. An 86-year-old female with a history including multinodular goiter, renal cell carcinoma (status post left nephrectomy), and polycythemia vera has been followed by our team for over one decade. In the setting of, then, recently-diagnosed renal cell carcinoma, a right thyroid lobectomy was performed 25 years earlier; pathology was consistent with a MNG. An FNA of a dominant right thyroid nodule performed 10 years earlier was consistent with a colloid nodule.

Subsequently, she was followed with periodic thyroid ultrasounds and thyroid function tests. Three years earlier, she was diagnosed with hypothyroidism and was started on levothyroxine. Regarding her history of renal cell carcinoma and polycythemia vera, there have been no issues of concern.

Two months prior to her presentation, she started to experience dysphagia and noticed an enlargement on the left side of her neck. A thyroid ultrasound showed a new $3.5 \times 3 \times 3 \mathrm{~cm}$ lobulated infiltrating hypoechoic nodule. FNA and flow cytometry were performed. The biopsy findings were consistent with new-onset diffuse large B-cell lymphoma of the thyroid. Based on pan-CT scans, she is thought to have lymphoma limited to the thyroid. She is undergoing radiation treatment and agreed to forego chemotherapy given her age and comorbidities. A patient with a rapidly enlarging goiter should be evaluated promptly. In this particular patient, concern for possible anaplastic thyroid cancer, thyroid lymphoma, and metastatic renal cell carcinoma to the thyroid were considerations. Excellent outcomes have been noted with radiation treatment alone in patients with large B-cell lymphoma limited to the thyroid. This case illustrates not only the importance of accurate diagnosis of thyroid lymphoma, as this will dictate treatment, but also the importance of prompt evaluation in a patient with a rapidly enlarging goiter.

\section{Poster 422}

Thyroid Imaging Saturday Poster Clinical

ANALYZING THYROID IMAGING GUIDELINES: ULTRASOUND CHARACTERIZATION OF NONINVASIVE FOLLICULAR THYROID NEOPLASM WITH PAPILLARYLIKE NUCLEAR FEATURES

A.M. Eckhoff ${ }^{1}$, A. Chen ${ }^{2}$, C.C. Griffith ${ }^{4}$, A.C. Schmitt ${ }^{4}$, D.A. Baumgarten ${ }^{3}$

${ }^{1}$ Emory University School of Medicine, Atlanta, GA;

${ }^{2}$ Otolaryngology - Head \& Neck Surgery, Emory University School of Medicine, Atlanta, GA; ${ }^{3}$ Radiology, Emory University School of Medicine, Atlanta, GA; ${ }^{4}$ Pathology \& Laboratory Medicine, Emory University School of Medicine, Atlanta, GA

Thyroid nodules are a common finding on neck sonography yet most nodules are benign. To address this issue, several groups of investigators have proposed methods to risk stratify on the basis of ultrasound features. Recently, the American College of Radiology Thyroid Imaging, Reporting, and Data System (ACR TI-RADS) proposed a risk classification algorithm in which nodules are awarded points for suspicious features within four categories: composition, echogenicity, shape, and margin. This system differs from the widely used 2015 American Thryoid Association (ATA) Management Guidelines for Adult Patients with Thyroid Nodules and Differentiated Thyroid Cancer which is a pattern-based approach and shown by Yoon et al to be unable to classify $3.4 \%$ of 1,293 nodules. We evaluated whether the 2015 ATA guidelines or the ACR TI-RADS is better at predicting which thyroid nodules are noninvasive follicular thyroid neoplasms with papillary-like nuclear features (NIFTP) vs. encapsulated follicular variant of papillary thyroid carcinoma (EFVPTC). Pathology slides of patients with a diagnosis of EFVPTC were examined to evaluate for NIFTP vs invasive EFVPTC. Ultrasounds from this patient cohort were classified via the 2015 ATA guidelines and via ACR TIRADS. Chi-squared test and Mann-Whitney $U$ test were used to correlate ultrasound findings to surgical pathology. The study cohort consisted of 57 patients. There were $23(40.4 \%)$ NIFTP and $34(59.6 \%)$ EFVPTC. A total of 29 patients (50.9\%) had thyroid ultrasounds prior to surgery. Both the 2015 ATA guidelines $(\mathrm{U}=92.50 \mathrm{P}=0.884$ twotailed) and the ACR TIRADS did not show a statistically significant difference in ultrasound categories between NIFTP vs. EFVPTC $(\mathrm{U}=83.50 \mathrm{P}=0.565$ two-tailed). Neither risk stratification strategy suggested by the 2015 ATA guidelines or by the ACR TIRADS was predictive of NIFTP vs. EFVPTC. Thus, it is not possible to compare which classification system is better at identifying NIFTP tumors. This is most likely because NIFTP's diagnostic criteria are based on pathology examination and include lack of capsule invasion, angio/ lymph invasion, and psammoma bodies, and other higher risk features that cannot be identified by ultrasound.

\section{Poster 423}

Thyroid Cancer Saturday Poster Clinical

\section{SONOGRAPHIC PATTERN IN NONINVASIVE} FOLLICULAR THYROID NEOPLASM WITH PAPILLARYLIKE NUCLEAR FEATURES VS. ENCAPSULATED FOLLICULAR VARIANT PAPILLARY THYROID CANCER A.M. Eckhoff ${ }^{1}$, C.C. Griffith ${ }^{4}$, A.C. Schmitt $^{4}$, D.A. Baumgarten ${ }^{3}$, A. $\mathrm{Chen}^{2}$

${ }^{1}$ Emory University School of Medicine, Atlanta, GA;

${ }^{2}$ Otolaryngology - Head and Neck Surgery, Emory University School of Medicine, Atlanta, GA; ${ }^{3}$ Radiology, Emory University School of Medicine, Atlanta, GA; ${ }^{4}$ Pathology \& Laboratory Medicine, Emory University School of Medicine, Atlanta, GA

A recent international, multidisciplinary study published in JAMA Oncology suggested the reclassification of a subset of encapsulated follicular variant of papillary thyroid carcinoma (EFVPTC) as a separate pathologic diagnosis. These noninvasive follicular thyroid neoplasm with papillary-like nuclear features (NIFTP) have highly indolent behavior and are genetically distinct from infiltrative tumors. NIFTP rarely reoccurs or metastasizes, obviating the need for aggressive treatment and potentially decreasing healthcare costs and morbidity. We evaluated if sonographic patterns are predictive of NIFTP versus EFVPTC on surgical pathology. Pathology slides of patients with a diagnosis of EFVPTC were examined to evaluate for NIFTP vs invasive EFVPTC. Ultrasounds from this patient cohort were classified via the 2015 American Thyroid Association (ATA) Management Guidelines for Adult Patients with Thyroid Nodules and Differentiated Thyroid Cancer. Additionally, demographic factors and past medical history were examined to determine if specific traits correlated more with NIFTP vs. EFVPTC. Chi-squared test and Mann-Whitney U test were used to correlate ultrasound findings to surgical pathology and for demographic analysis. The study cohort consisted of 57 patients. There were $23(40.4 \%)$ NIFTP and 34 $(59.6 \%)$ EFVPTC. A total of 29 patients $(50.9 \%)$ had thyroid ultrasounds prior to surgery. Sonographic pattern was not predictive of final pathology $(\mathrm{U}=92.50 \mathrm{P}=0.884$ two-tailed $)$. Additionally, there were no statistically significant differences in age, race, gender, smoking status, drinking status, family history of thyroid disease, and personal history of cancer distribution between patients with NIFTP vs. EFVPTC. Sonographic pattern characterization according to the 2015 ATA guidelines is not predicative of NIFTP vs. EFVPTC. This supports the fact that NIFTP is a pathological, not clinical, diagnosis. Additionally, we did not find demographic factors or past medical history predictive of patients presenting with NIFTP vs. EFVPTC. Further analysis with large sample sizes is necessary to better preoperatively characterize NIFTP tumors. 


\section{Poster 424}

Thyroid Nodules \& Goiter Saturday Poster Clinical

ENDOSCOPIC THYROID SURGERY IN LARGE GOITRES: EXPERIENCE FROM A TERTIARY CARE CENTRE IN INDIA

G. Johri, G. Chand, A. Mishra, C. Sonthineni, S. Mayilvaganan, A. Agarwal, G. Agarwal, A.K. Verma, S. Mishra

Endocrine Surgery, SGPGIMS, LUCKNOW, UTTAR PRADESH, India

Scarless (in the neck) endoscopic thyroidectomy (ET) has evolved into a cosmetically notable alternative to conventional thyroidectomy. The cut off goitre size for ET procedures usually ranges from 4-6 cm. In India, a developing nation, most patients present with long standing goitres, many of which are larger than the recommended size for ET. This study aims at comparing the differences in outcomes of ET for small $(<6 \mathrm{~cm})$ with those of larger $(\geq 6 \mathrm{~cm})$ goitres.

This is a retrospective analysis (September 2012 to May 2017) of prospectively kept data. Patients undergoing hemithyroidectomy (HT) or total thyroidectomy (TT) by Axillo Breast Approach (ABA) and Bilateral Axillo- Breast Approach (BABA) with FNAC of Bethesda category II-IV and minimum 6 months follow up were included. Cases were divided into groups based on maximum tumor diameter. Group I small $(<6 \mathrm{~cm})$ and Group II large $(\geq 6 \mathrm{~cm})$ goitres. The demographic and clinicopathological profile, approach of ET, operation time, conversion and complication rates and hospital stay were compared between the two groups.

Total 68 patients were included, 44 in Group I and 24 in Group II. Using ABA and BABA approaches, $48 \mathrm{HT}$ and $20 \mathrm{TT}$ were performed.

The mean tumor size (+SD) in Group I and II were $4.47(+0.95) \mathrm{cm}$ and $6.93(+1.38) \mathrm{cm}$ respectively, range $2.5-11 \mathrm{~cm}$. There was no significant difference between mean operating time $(148.43+37.83$ vs $181.31+46.09$ mins, $p$ value $=0.19)$ and mean hospital stay $(2.91+1.48$ vs $3.85+1.77$ days, $\mathrm{p}$ value $=0.36)$ of HT and mean operating time $(204.11+51.3$ vs $248.18+53.62$ mins, $p$ value $=0.84)$ and mean hospital stay $(4.11+1.36$ vs $4.73+0.78$ days, p val$\mathrm{ue}=0.65$ ) of TT in the two groups. Four cases were converted to open surgery without any significant difference in conversion rates amongst groups ( 1 vs 3 , p value $=0.122$ ).

There was no significant difference in the complication rates in the two groups. No patient had prolonged hypocalcaemia or permanent vocal cord palsy.

We conclude that ET can be offered to a select subset of patients with larger goitres with no significant difference in operation time, hospital stay and complication rates in experienced hands.

\section{Poster 425}

Thyroid Cancer Saturday Poster Case Report

CEREBRAL METASTASES AS PRIMARY PRESENTATION OF OCCULT PAPILLARY THYROID CANCER

G. Johri, G. Chand, R.A. Bichoo, A. Mishra, S. Mayilvaganan, G. Agarwal, A. Agarwal, A.K. Verma, S. Mishra

Endocrine Surgery, SGPGIMS, LUCKNOW, UTTAR PRADESH, India

Papillary thyroid carcinoma (PTC) is the most common type of Differentiated thyroid carcinoma (DTC) with an indolent course and good prognosis despite regional lymph node involvement. Cerebral metastasis $(\mathrm{CM})$ in PTC are extremely rare $(\sim 1 \%)$ with a median survival of less than a year. We report a rare case of $\mathrm{CM}$ as the presenting symptom of PTC without a visible goitre in neck or prior diagnosis of primary in thyroid or cervical lymphnodes.
A 59 year old woman, attended the neurosurgery Clinic with complaints of headache, left hemiparesis since 3 months and recent onset blurring of vision. MRI Brain revealed a solid cystic lesion in right parietal lobe with perilesional edema and midline shift. She underwent craniotomy and gross complete resection of the tumor. Squash smear was suggestive of metastatic adenocarcinoma hence a PET/CT was done which showed uptake in a solitary thyoid nodule. Paraffin sectoin histology showed PTC (classical variant). On evaluation patient was hypothyroid $(\mathrm{Se} \mathrm{TSH}=27.3 \mathrm{mIU} / \mathrm{ml}, \mathrm{FT} 4=11.74$ $\mathrm{pmol} / \mathrm{L})$. Further evaluation revealed, bilateral thyroid nodules, cervical lymphadenopathy and pulmonary metastases. Patient underwent total thyroidectomy, central neck dissection and bilateral selective neck dissection. Prior to whole body radioiodine scan, patient developed massive pleural effusion and subsequently succumbed.

PTC presenting with $\mathrm{CM}$ as the sole manifestation of disease with no overt evidence of primary in the neck or other site distant metastases is extremely rare. Only a few cases have been reported till date.

Women are affected more frequently (F: M ratio-1.33:1). Patients may be asymptomatic ( $8 \%$ ) but typically present with site specific CNS symptoms. MRI is the diagnostic investigation of choice. There is no clearly defined management protocol. Surgery, WBRT, stereotactic radiosurgery and RAI therapy are the avaliable options.

Lesions are usually RAI refractory. Surgical excision followed by WBRT offer the highest chance of survival and should be offered to all patients. Reported median survival after diagnosis is 9.4 months.

Though CM in PTC are rare, thorough history taking and clinical examination are paramount in carcinomas with unknown primaries.

\section{Poster 426}

Thyroid Cancer Saturday Poster Clinical

UNFAVORABLE EFFICACY TO RADIOIODINE REMNANT ABLATION IN BRAF V600E MUTANT PAPILLARY THYROID CARCINOMA WITH POSITIVE TGAB

\section{N. Zhang ${ }^{1,2}$, J. Liang ${ }^{3}$, Y. Lin ${ }^{1}$}

${ }^{1}$ Nuclear Medicine, Peking Union Medical College Hospital, Beijing, China; ${ }^{2}$ Oncology, the Affiliated Hospital of Qingdao University, Qingdao, Shandong, China; ${ }^{3}$ Oncology, Beijing Cancer Hospital, Beijing, haidian qu, China

As the most common oncogenic event in papillary thyroid carcinoma (PTC), the BRAF V600E mutation has shown a close relationship of aggressiveness, while it remains unclear about its influence on the therapeutic response of PTC. Hence a retrospective study was designed to explore the cooperative effect of BRAF V600E mutation and positive thyroglobulin antibody ( $\mathrm{TgAb}$ ) on efficacy to radioiodine remnant ablation (RRA) in PTCs. We included 298 consecutive PTC patients without distant metastases who underwent surgery and RRA from June 2012 to June 2016. The TgAb level and BRAF V600E status were measured and patients were divided into four groups according to the combined status: group 1 (both positive in $\mathrm{TgAb}$ and $\mathrm{BRAF}$ ); group 2 (TgAb positive only); group 3 (BRAF mutant only); group 4 (both negative). The imaging information was collected at 6 months after RRA and used to assess ablative efficacy in terms of success or failure. The change of $\mathrm{TgAb}$ levels was followed up to 24 months in $\mathrm{TgAb}$ positive patients. The BRAF V600E mutation rates declined along with increasing $\mathrm{TgAb}$ levels. Older age, more males, extracapsular invasiveness and advanced TNM stage were more likely to harbor BRAF V600E mutation. TgAb positive were more prevalent in younger females with less bilateral tumour lesions. The characteristics had significant differences among the four groups in sex, age (all $P=0.000$ ), group 2 presented more younger females. The success rates were statistically different among four groups $(P=0.009)$, group 1 presented the lowest efficacy. The 
combined status was associated with the poor efficacy to RRA in the multivariate analysis $(P=0.029)$. The effect of RRA in reducing $\mathrm{TgAb}$ level might be weakened in BRAF mutant status. The combined status of BRAF V600E mutation and positive TgAb predicts low efficacy to RRA and might be served as an independent unfavorable prognostic factor for PTC. BRAF mutant might weaken the effect of RRA in reducing TgAb levels in PTCs.

\section{Poster 427 \\ Thyroid Cancer Saturday Poster Clinical \\ 500MG VERSUS 750MG DAILY, PILOT DOSE COMPARISON OF APATINIB IN CHINESE PATIENTS WITH PROGRESSIVE RADIOIODINE REFRACTORY DIFFERENTIATED THYROID CANCER: INITIAL EXPERIENCE OF EFFICACY AND SAFETY \\ X. Zhang, Y. Lin}

Nuclear Medicine, Peking Union Medical College Hospital, Beijing, China

In our previous study, apatinib, a novel tyrosine kinase inhibitor (TKI) of VEGFR-2, has shown its rapid response in terms of biochemistry, structure and glucose metabolism in radioiodine refractory differentiated thyroid cancer (RAIR-DTC) with an initial dose of $750 \mathrm{mg}$ qd. In order to optimize the therapy protocol, the initial dose was regulated to $500 \mathrm{mg}$ qd. We prospectively compared the 8 weeks' efficacy and safety between the two dosed groups. A total of 20 patients (pts) with RAIR-DTC were included in 2016. 10 pts were treated with the initial dose of $750 \mathrm{mg}$ qd ( 5 male, $54 \pm 14 \mathrm{y}$ ) and the others with $500 \mathrm{mg}$ qd $(5 \mathrm{male}, 55 \pm 14 \mathrm{y})$ with disease progression during previous 14 months and at least 1 identifiable lesion above $1 \mathrm{~cm}$. Serum thyroglobulin and thyroglobulin antibody $(\mathrm{Tg} / \mathrm{Tg} \mathrm{Ab})$ and total target lesions were evaluated at least every 4 weeks. All AEs were recorded and graded according to CTCAE 4.0. Study interruption and sequential dose reduction $(500 \mathrm{mg}$ and $250 \mathrm{mg}$ qd)were allowed due to AEs. According to RECIST 1.1, in the 8 weeks' treatment, the disease control rate was $100 \%$. In $750 \mathrm{mg}$ qd group, $9 \mathrm{PR}$ and 1 SD were achieved vs. 5 PR, 4 SD and $1 P D$ in the group $500 \mathrm{mg}$ qd respectively $(p=0.141) .3 / 10$ in $750 \mathrm{mg}$ group and $2 / 10$ in $500 \mathrm{mg}$ group were observed transient $\mathrm{Tg}$ oscillations in initial 2 weeks and descended afterward, except for $1 \mathrm{pt}$ in $750 \mathrm{mg}$ group who had a $102.33 \%$ of Tg fluctuation caused by AE-related drug withdrawal. By the end of 8 weeks, the median reduction of $\mathrm{Tg}$ was $74.78 \%$ (range, $-102.33 \%, 96.37 \%$ ) for $750 \mathrm{mg}$ group and $92.61 \%$ (range, $2.01 \%$, $96.82 \%$ ) for $500 \mathrm{mg}$ group ( $p=0.105$ ). The frequencies of grade 3 and 4 AEs occurred less in $500 \mathrm{mg}$ group than $750 \mathrm{mg}$ ( $12 \mathrm{vs} 22, p=0.032$ ), and the most frequently were hand-foot skin reaction (35\%), hypocalcemia (20\%) among the 20 pts. All of the pts in $750 \mathrm{mg}$ group completed the 8 weeks' treatment without dose regulation, but in $500 \mathrm{mg}$ group, 2 pts' daily dose downregulated to $250 \mathrm{mg}$ due to AEs. All the above results indicated a comparable effective but less sufferable response in pts with $500 \mathrm{mg}$ qd. Within an 8-week short term observation, the initial dose of $500 \mathrm{mg}$ qd presented less sufferable and comparable response as $750 \mathrm{mg}$ qd in pts with progressive RAIR-DTC.

\section{Poster 428}

Thyroid Cancer Saturday Poster Clinical COMPARISON OF $8^{\text {TH }}$ AND $7^{\text {TH }}$ AJCC TNM STAGING SYSTEM: EVALUATION IN TERMS OF RESPONSE TO RAI THERAPY IN 1083 CHINESE DIFFERENTIATED THYROID CANCER PATIENTS

Y. Lin, H. Hu

Nuclear Medicine, Peking Union Medical College Hospital, Beijing, China
The $8^{\text {th }}$ AJCC staging system was published with big modification in October 2016, the objective of this study was to demonstrate how $8^{\text {th }}$ staging made a difference from $7^{\text {th }}$ on Chinese DTC cohort and evaluate the rationality in those patients with a stage shift. A number of 1083 consecutive DTC patients were enrolled in this retrospectively study with a median follow-up of 2 years. Patients were individually stratified based on $7^{\text {th }}$ and $8^{\text {th }}$ TNM staging system. Stage shift from $7^{\text {th }}$ to $8^{\text {th }}$ was observed and evaluated in terms of American Thyroid Association (ATA) response system. Totally, $8^{\text {th }}$ TNM staging leads to a downstage in $28.7 \%$ DTC patients: with $99.3 \%$ of patients in $7^{\text {th }}$ III stage transferred into $8^{\text {th }}$ I $(77.5 \%)$ and II $(21.9 \%)$ stage individually; and $89.3 \%$ of patients in $7^{\text {th }}$ IV stage changed into $8^{\text {th }}$ I $(53.4 \%)$, II $(25.9 \%)$ and III $(10.1 \%)$ stage respectively. Those downstaged patients totally presented a high excellent response (ER) rate which is similar to the rest of $8^{\text {th }}$ I stage (71.4\% VS $70 \%$ ), indicating the feasibility and rationality of $8^{\text {th }}$ staging in this group shifting. The rate of structural incomplete response (SIR) were identified in $6.6 \%, 96 \%, 2.6 \%, 28.1 \%$ of patients with $7^{\text {th }}$ I, II, III, IV stage. Re-staging in $8^{\text {th }}$ TNM stage decreased it to $6.1 \%, 63 \%$ in I and II stage, increased it to $14.3 \%, 100 \%$ in III and IV stage patients which showing a more appropriateness of $8^{\text {th }}$ stratification in differentiating the worse prognosis patients especially in IV stage $(\mathrm{P}=0.00)$. Our data firstly validated the rationality of $8^{\text {th }}$ TNM staging, the information carried by which may facilitate subsequent management in DTC patients.

\section{Poster 429}

Thyroid Cancer Saturday Poster Clinical

QUALITY IMPROVEMENT IN THYROID SURGERY: REDUCTION IN UNPLANNED HOSPITAL READMISSION, EMERGENCY DEPARTMENT VISIT AND REOPERATIONS POST-THYROIDECTOMY

J.F. Margolick ${ }^{3}$, W. Chen ${ }^{2}$, S.M. Wiseman ${ }^{1}$

${ }^{1}$ Surgery, University of British Columbia, VANCOUVER, British

Columbia, Canada; ${ }^{2}$ Pharmaceutical Sciences, University of British Columbia, Vancouver, British Columbia, Canada; ${ }^{3}$ Surgery,

University of British Columbia, VANCOUVER, British Columbia, Canada

Unplanned hospital readmission, reoperation and Emergency Department (ED) visits following thyroid operations cause frustration for both surgeons and patients. With an aim to provide insights required for the development of patient care improvement initiatives, the objective of this review was to systematically evaluate the available literature in order to determine the rates of readmission, reoperation and ED visits following thyroid operations. This systematic review was conducted in accordance with the Preferred Reporting of Items for Systematic Reviews and Meta-Analyses protocols. Twenty-two studies were included in the systematic review. Meta-analysis was performed to obtain the weighted-pooled summary estimates of rates of hospital readmission, ED visits and reoperation. Jackknife sensitivity analyses was performed for each dataset. To detect the risk of publication bias and the small-study effect, a funnel plot analysis was performed. The pooled rate of hospital readmission from 19 studies was $2.7 \%$ (95\% CI: $2.1-3.4 \%$ ) while the pooled rate of ED visits was $8.1 \%$ (95\% CI: $6.5 \%-9.8 \%$ ). The pooled rate estimate for reoperation was very low $(0.6 \%[95 \%$ CI: $0.3-1.1 \%]$ ). This was subject to publication bias because small studies tended to report lower rates of reoperation. Post-operative hypocalcemia was the most common reason for hospital readmission, while neck hematoma was the most common reason for reoperation. Strategies for reducing post-operative readmission and reoperation were identified. For example, routine post-operative calcium and vitamin $\mathrm{D}$ supplementation may reduce rates of post-operative 
hypocalcemia, while avoiding post-operative hypertension may decrease risk of neck hematoma. Older age, thyroid cancer, dependent functional status, higher ASA score, diabetes, COPD, steroid use, hemodialysis and recent weight loss increase the risk of hospital readmission after thyroid surgery. By further identifying risk factors for readmission, reoperation and ED visits, this review may assist practitioners to optimize perioperative care and therefore reduce thyroidectomy complications.

\section{Poster 430}

Thyroid Cancer Saturday Poster Clinical

RE-OPERATION, HOSPITAL READMISSION AND EMERGENCY DEPARTMENT VISITS POST THYROIDECTOMY AND PARATHYROIDECTOMY: INCIDENCE AND RISK FACTORS

J.F. Margolick, W. Chen, S.M. Wiseman

Surgery, University of British Columbia, VANCOUVER, British

Columbia, Canada

Unplanned re-operation, Emergency Department (ED) visits and hospital readmissions after thyroid and parathyroid surgery is a source of frustration for patients and surgeons alike. The primary objective of the study was to review our own experience in order to ascertain rates of reoperation, ED visits, and hospital readmission after thyroid and parathyroid surgery. A secondary objective was to determine if there were any identifiable risk factors for these unplanned events.

Patient records were retrospectively reviewed. All patients undergoing parathyroid and thyroid surgery between 2011 and 2014 were included. Clinical variables including age, gender, surgical indication, type of surgery, anticoagulant status and surgeon were obtained and analyzed. Principle outcome measures were rates of reoperation, ED visits and hospital readmission.

Four hundred and thirty-six patients underwent thyroid and parathyroid operations during the study period. Thirty-day follow up was $97.2 \%$. Rates of re-operations, ED visits and hospital readmissions after thyroid surgery were: $3.4 \%, 0.6 \%$ and $3.0 \%$, respectively. Seventy-Five percent of re-operations after thyroid surgery were for post-operative haemorrhage, and the only identified risk factor for re-operation was the attending surgeon. Rates of reoperation, ED visits and hospital readmission after parathyroid surgery were: $1.4 \%, 0 \%$ and $0.07 \%$, respectively.

Sixty-seven percent of re-operations after thyroid operations for post-operative hemorrhage were due to cases performed by a single surgeon. We identified a low rate of hypocalcemia postoperative $(0.9 \%)$ which may be because all total thyroidectomy patients are routinely given calcium and vitamin D supplementation. Further research into risk factors for post-operative hemorrhage is needed to improve outcomes for individuals undergoing thyroid and parathyroid procedures.

\section{Poster 431}

Thyroid Cancer Saturday Poster Clinical

PROGNOSTIC VALUE OF FDG PET/CT FOR PATIENTS WITH RADIOIODINE NEGATIVE LUNG METASTASES FROM DIFFERENTIATED THYROID CANCER K. Nakada ${ }^{1}$, N. Hattori ${ }^{2}$, H. Sugie ${ }^{2}$, M. Sakurai ${ }^{3}$

${ }^{1}$ Radiology, Hokko Memorial Hospital, Sapporo, Japan; ${ }^{2}$ Radiology, LSI Sapporo Clinic, Sapporo, Japan; ${ }^{3}$ Internal Mwdicie, Hokko Memorial Hospital, Sapporo, Japan

Presence of radioactive iodine negative tumor is a sign for unfavorable prognosis for patients with distant metastasis from differentiated thyroid cancer (DCA). However, there is a considerable variety in the clinical course of radioiodine-negative lung metastases from DCA.

The aim of this study was to determine whether PET/CT with F-18 flourodeoxyglucose (FDG) was valuable in assessing prognosis of radioiodine-negative lung metastases from DCA. This study included 33 patients who had lung metastases from DCA (PCA/FCA 29/4) that did not avid I-131 on the post therapy I-131 scan. All patients underwent total thyroidectomy followed by high-dose radioactive iodine therapy (RAI) 1 to 3 times (cumulative dose;100$400 \mathrm{mCi}$ ). 2 out of 26 patients had bone metastasis and another 16 patients had lymph node metastases in addition to lung metastases. FDG-PET/CT was performed 4 to 12 months after the last RAI. FDG uptake in the lung tumor was visually assessed. When at least one focal lung FDG uptake higher than that in the mediastinum was observed on the whole-body MIP images, FDG uptake was defined as positive. Patients were classified into 2 subgroups.

Group A: positive FDG uptake $(\mathrm{n}=21)$,

Group B): negative FDG uptake $(\mathrm{n}=12)$

Follow up period ranged from 39 to 141 mos (median:87mosa). Patents did not receive further treatment for lung metastases except for TSH suppression. Anatomical imaging and $\mathrm{Tg}$ measurement were performed at a regular interval to evaluate $\mathrm{Tg}$-doubling time ( $\mathrm{TgDT})$ and changes in tumor size (as a rule, based on RECIST1.1) during the follow-u.

Additionally, progression-free survival (PFS) was determined using Kaplan-Meier plot. A short TgDT of less than 1 year was seen more frequently in group A than in group B (68\% vs $0 \%)$. The median PFS (mos.) in group A vs. group B was 40 vs. $79(\mathrm{p}=<0.01)$. Patients with FDG positive lung metastases are at highr risk for progressive disease. On the other hand, patients with FDG negative lung metastases are associated with gentle tumor behavior that favorable prognosis may be expected. FDG-PET CT may be noninvasive and valuable in optimizing the management of radioiodinenegative lung metastases from DTC.

\section{Poster 432}

Thyroid Cancer Saturday Poster Clinical

\section{DOES LOW IODINE CAUSE SEVERE HYPONATREMIA} IN PATIENTS UNDERGOING RH-TSH AIDED REMNANT TISSUE ABLATION FOR THYROID CANCER?

\section{K. nakada ${ }^{1}$, Y. Furuta ${ }^{2}$, M. Tamura ${ }^{3}$, M. Sakurai ${ }^{4}$}

${ }^{1}$ Rdiology, Hokko Memorial Hospital, Sapporo, Japan; ${ }^{2}$ Head and Neck Surgery, Teine Keijinkai Hospital, Sapporo, Japan; ${ }^{3}$ Nutrition, Hokko Memorial Hospital, Sapporo, Japan; ${ }^{4}$ Internal Medicie, Hokko Memorial Hospital, Sapporo, Japan

So far, previous reports have suggested a risk of developing severe hyponatremia by a low iodine diet (LID) for radioactive iodine remnant tissue ablation (RAI) for thyroid cancer (Shakir M. et al. 2005, Krishnamurthy VR. et al. 2007, etc.). We assumed that hyponatremia might have been combined effects of hypothyroidism and restriction of iodized salt. The aim of this study was to investigate association among LID, serum sodium (Na) and daily salt intake (SI) in patients who are to undergo rh-TSH aided RAI for papillary thyroid cancer (PCA). We included 56 sequential patients (M/F 20/36, age 27-78 yrs.) with postsurgical PCA who were to undergo rh-TSH aided RAI using $30 \mathrm{mCi}$ of I-131 and had eGFR of more than $40\left(\mathrm{~mL} / \mathrm{min} / 1.73 \mathrm{~m}^{2}\right)$. The LID plan was designed to restrict dietary iodine intake less than $100 \mu \mathrm{g} /$ day but not to restrict use of non-iodized salt. LID was done from 7 days before to 3 days after RAI. $20-40 \mathrm{mg}$ of furosemide was given to patients from 7 days before to 1 day before RAI.

A spot urine sample was collected to determine urinary iodine concentration (UIC; $\mu \mathrm{g} / \mathrm{gCRE}$ ) before start of LID and on the day of RAI in all. Serum levels of Na (normal range: $135-145 \mathrm{mEq} / \mathrm{l}$ ) were determined before start of LID and on the day of RAI. In addition, 
daily sodium intake (SI: g/day) was estimated in 40 patients according to previous reports (Tanaka T, et 12002, and Kasakaki T, et al 1993). The average value of UIC, SI and Na after LID were about $18 \%$ (121.3 vs. 675.0, p $<0.001), 42 \%$ (4.7 vs. 9.3, p<0.001) and $100 \%$ (140.0 vs. $140.5, \mathrm{~ns})$ of the baseline value, respectively. The average value of SI was decreased after LID. However, it remained above the required minimal daily amount of $1.5 \mathrm{~g}$.

Mild hyponatremia was observed in only $2(0.4 \%)$ of the patients. In the remaining 54 patients, $\mathrm{Na}$ level remained in normal range. Correlation between UIE and SI after a LID $(r=0.34, p=0.09)$ or that between Na and SI after a LID $(r=0.22, p=0,35)$ was not significant. Practice of LID may be associated with reduced salt intake. However, it is unlikely that a one-week LID is responsible for severe hyponatremia in patients undergoing rh-TSH aidwed RAI without hypothyroidism even if the procedure is combined with oral diuretics.

\section{Poster 433}

Thyroid Cancer Saturday Poster Clinical

PROGNOSTIC FACTORS IN THE OVERALL SURVIVAL RATE OF PATIENTS WITH DIFFERENTIATED THYROID CANCER BONE METASTASIS

D. $\mathrm{Wu}^{1,2}$, C. Gomes Lima ${ }^{1,3}$, S. Moreau ${ }^{2}$, K. Kulkarni ${ }^{2}$, A. Zeymo ${ }^{4}$, K.D. Burman ${ }^{3}$, L. Wartofsky ${ }^{3}$, D. Van Nostrand ${ }^{1,2}$

${ }^{1}$ MedStar Health Research Institute, Washington, DC; ${ }^{2}$ Nuclear Medicine, MedStar Washington Hospital Center, Washington, DC; ${ }^{3}$ Endocrinology, MedStar Washington Hospital Center, Washington, DC; ${ }^{4}$ Department of Biostatistics and Biomedical Informatics, MedStar Health Research Institute, Hyattsville, MD

The 5y overall survival (OS) rate of differentiated thyroid cancer (DTC) patients (pt) with bone metastases (BM) was reported at 7 $65 \%$. The objective of this study was to determine the OS for DTC $\mathrm{BM}$ pts treated at MWHC and to determine the variables affecting survival. This retrospective study included DTC pts who had $\geq 1$ dosimetric ${ }^{131}$ I treatment (DosiTx) after BM diagnosis (dx) at MedStar Wash Hosp Ctr (MWHC) between 2001-2017. A group without ${ }^{131}$ I tx after BM dx was identified for comparison ( $\left.\mathrm{No}^{131} \mathrm{ITx}\right)$. Age of DTC $\mathrm{dx}$, gender, initial tx, histology, age at BM dx, extent of disease, and tx modalities were tabulated. Kaplan-Meier survival curve determined the OS and was compared by long-rank test. A total of $91 \mathrm{BM}$ pts were identified of which $62 \mathrm{had} \geq 1$ DosiTx and 12 had $\mathrm{No}^{131}$ ITx after BM $\mathrm{dx}$. The mean follow-up was $4.7 \mathrm{y}$ (range, $0-41 \mathrm{y}$ ). The pt demographics and OS curves will be presented. The mean cumulative ${ }^{131} \mathrm{I}$ dosage after BM dx was $438 \mathrm{mCi}$, range $98.2-1220.2 \mathrm{mCi}$. The 1, 2, 3, 5 and 10y OS of DosiTx were $92 \%, 88 \%, 77 \%, 62 \%$, and $32 \%$, respectively, and the 1,2 and $3 y$ OS of $\mathrm{No}^{131}$ ITx were $92 \%, 71 \%, 43 \%$, respectively. The median OS of DosiTx was significantly higher than No ${ }^{131}$ ITx $(6.5$ vs. $2 \mathrm{y} ; p=0.007)$.

Seventy-four percent of DosiTx pts (46/62) received other tx modalities. The median survival for multiple tx modalities is $7.7 \mathrm{y}$ and for DosiTx only is $3.8 \mathrm{y}$ ( $p=0.064)$. The $5 \mathrm{y}$ and $10 \mathrm{y}$ survival were $68 \%$ and $45 \%$ for DosiTx with other modalities, respectively, versus $45 \%$ and $22 \%$ for DosiTx only.

In the overall cohort, age $\geq 55 \mathrm{y}$ at BM dx had lower median OS (5 vs. 9y; $p=0.006$ ) than those age $<55 \mathrm{y}$. Pts who had undergone either remnant ablation, ${ }^{131} \mathrm{I}$ tx after BM dx, surgical excision, and/or external beam radiotherapy conferred better OS $(p<0.05)$ than those who did not. Sex, histology, synchronous dx, number of BM sites at initial $\mathrm{dx}$, radioiodine avidity, presence of other metastasis or tumor did not significantly influence OS $(p>0.05)$. Combination of dosimetric ${ }^{131} \mathrm{I}$ tx plus other modalities improved the OS of BM pts relative to those who were not treated by ${ }^{131} \mathrm{I}$ tx. However, it is difficult to assess whether the improvement was due to the tx or a distinct difference in prognosis of patient population.

\section{Poster 434}

Thyroid Cancer Saturday Poster Clinical

XEROPHTHALMIA AND EPIPHORA SECONDARY TO ${ }^{131}$ I THERAPY IN PATIENTS WITH DIFFERENTIATED THYROID CANCER: A PROSPECTIVE STUDY

D. $\mathrm{Wu}^{1,6}$, G. Khorjekar ${ }^{2}$, P. Kharazi ${ }^{3}$, A. Zeymo ${ }^{4}$, K.D. Burman ${ }^{5}$, L. Wartofsky ${ }^{5}$, D. Van Nostrand ${ }^{1,6}$

${ }^{1}$ MedStar Health Research Institute, Washington, DC; ${ }^{2}$ The George Washington University Hospital, Washington, DC; ${ }^{3}$ CareNow Urgent Care, Las Vegas, NV; ${ }^{4}$ Department of Biostatistics and Biomedical Informatics, MedStar Health Research Institute, Hyattsville, MD;

${ }^{5}$ Endocrinology, MedStar Washington Hospital Center, Washington, DC; ${ }^{6}$ Nuclear Medicine, Medstar Washington Hospital Center, Washington, DC

Xerophthalmia (XP) and epiphora (EP) are less common side effects of

${ }^{131}$ I therapy (tx) in differentiate thyroid cancer (DTC) patients, and the frequency, time of onset, severity, and duration of XP and EP are less well characterized. The objective of this study is to evaluate the above parameters in DTC pts after ${ }^{131} \mathrm{I}$ tx and the factors influencing these symptoms. Six surveys were designed to prospectively assess the above metrics at pre-tx and post-tx (i.e., 1d, 5-7d, 3mo, 6mo \& 12mo). The inclusion criteria were: $\geq 18 y$, DTC, and ${ }^{131}$ I tx at MedStar Wash Hosp Ctr. Patients were excluded if they had a prior eye disease with or without XP and/or Sjögren's disease. The frequency, time of onset, duration, and severity of XP and EP were tabulated. Ninety-nine patients were consented of which 92 were included in this analysis. Patients were subdivided into prior XP (pXP), no prior XP (nXP), pEP, and nEP.

$\mathrm{XP}$ was reported in 13/79 (16\%) pXP and 8/13 (62\%) nXP. Patients with pXP were mostly female (85\%) and older (mean 53yo vs. 46yo; $p=0.07$ ). The odds of XP were $\sim 8.1$ times higher for $\mathrm{pXP}$ than $\mathrm{nXP}(\mathrm{p}=0.001)$. Peak time of onset was $1 \mathrm{~d}(7 / 13)$. Two patients reported XP lasting $>1 \mathrm{mo}$ with the longest duration of $4 \mathrm{mo}$. On an intensity scale, $2 / 13$ rated pain as severe.

EP was reported in 10/88 (11\%) pEP and 2/4 (50\%) nEP. Patients with $\mathrm{pEP}$ were older (mean age, 57yo vs. 47yo; $\mathrm{p}=0.15$ ). There was no association between $\mathrm{pEP}$ and $\mathrm{EP}(\mathrm{P}=0.14)$. Peak time of onset was on 1d (4/10). Two patients reported EP lasting $>1$ mo with the longest duration of $4 \mathrm{mo}$. On an intensity scale, $1 / 10$ rated EP as severe.

In patients with post-tx XP or EP, there was no significant association between XP or EP onset and age, sex, ${ }^{131}$ I dosage or concern about radiation. However, more females had post-tx XP than males $(28.6 \%$ vs. $10.7 \% ; \mathrm{p}=0.11$ ). For both XP and EP, time of onset and duration are significantly associated; when symptoms appeared 7 days or later, they lasted longer (XP, $p=0.015 ; \mathrm{EP}, \mathrm{p}<0.01)$. This report describes the frequency, duration and severity of ${ }^{131}$ I-induced XP and EP in DTC patients. In this small cohort, both XP and EP duration was associated with time of onset, but not with ${ }^{131}$ I dosage. The odds of XP were higher in those with pXP.

\section{Poster 435}

Thyroid Hormone Action Saturday Poster Basic ACTIVATION OF TUMOR CELL INTEGRIN $\alpha$ V $\beta 3$ BY RADIATION AND REVERSAL OF ACTIVATION BY CHEMICALLY MODIFIED TETRAIODOTHYROACETIC ACID (TETRAC)

J.T. Leith ${ }^{3}$, S. Kenney ${ }^{3}$, A. Hercbergs ${ }^{1}$, S.A. Mousa ${ }^{2}$, P.J. Davis ${ }^{4,2}$ ${ }^{1}$ Radiation Oncology, CLEVELAND CLINIC, Beachwood, OH; ${ }^{2}$ Albany College of Pharmacy and Health Sciences, Rensselaer, NY; ${ }^{3}$ Rhode Island Nuclear Science Center, Narragansett, RI; ${ }^{4}$ Albany Medical College, Albany, NY

Integrin $\alpha \mathrm{v} \beta 3$ is an important structural and signaling protein of the plasma membrane of cancer cells and dividing blood vessel cells. 
The plastic extracellular domain of the protein binds to extracellular matrix proteins and plasma membrane proteins, changing cell-cell interactions and generating intracellular signals that influence cell behavior. $\alpha \mathrm{v} \beta 3$ also contains a receptor for thyroid hormone and derivatives, including tetraiodothyroacetic acid (tetrac). Using an antibody that recognizes unliganded $\beta 3$ monomer in the extended or open (activated) configuration, we exposed human prostate cancer (PC3) cells to X-radiation in varying dosages (1-10 Gy). Radiation significantly increased activated $\beta 3$ within $1 \mathrm{~h}$, a radiation response not previously reported. Incubation of cells with unmodified tetrac or tetrac covalently linked to a nanoparticle (Nanotetrac, NDAT) did not change basal activation state of the integrin monomer, but prevented radiation-induced activation of $\beta 3$. Activation of the integrin in response to radiation is interpreted as a defensive response, perhaps leading to increased intercellular affinity and inhibition of cell division, a radioresistant state. Action of NDAT indicates that pharmacologic interventions in the radiation response of integrin $\beta 3$ monomer and therefore of $\alpha \mathrm{v} \beta 3$ are feasible.

\section{Poster 436 \\ Thyroid Hormone Metabolism \& Regulation Saturday Poster Clinical \\ PRE-OPERATIVE OPTIMIZATION WITH SUPER SATURATED POTASSIUM IODIDE SOLUTION (SSKI) IN PATIENTS WITH GRAVES DISEASE UNDERGOING TOTAL THYROIDECTOMY}

D. Reznick, A. Siperstein

Endocrine and Metabolic Institute, Cleveland Clinic, Cleveland, $\mathrm{OH}$

Iodine containing solutions have been utilized to help control hyperthyroidism for patients with Graves disease. Super saturated potassium iodide solution (SSKI) is traditionally given about 10 days prior to surgery for patients with Graves disease to help render patients euthyroid, in addition to any other medication that they may be taking for symptoms. Despite routine administration, few studies have confirmed the effects of SSKI. The purpose of this study is to review those patients who undergo thyroidectomy for Graves disease with the pre-operative administration of SSKI. Single institution review of all patients undergoing a total thyroidectomy for Graves disease. Demographics and preoperative use of methimazole and propylthiouracil (PTU) were recorded. Perioperative use of SSKI was documentd along with the change in T3 and T4 levels along with any observational changes on pre- and intra-operative ultrasound. Gland texture was noted, and post-operative complications were documented.386 total patients had a total thyroidectomy for Graves disease between 2000-2016. A total of 312 (80\%) received SSKI. No statistical differences were noted between the demographics of the two groups. Of those that received SSKI, there was a significant decreased in both T3 and T4 levels after receiving SSKI $(\mathrm{p}<0.01)$ as well as a decrease in flow on 42 of 152 patient's ultrasound exams $(\mathrm{p}<0.01)$. This study confirms that SSKI assists with rendering patients euthyroid prior to total thyroidectomy for Graves disease as well as decreases the vascularity of their thyroid glands.

\section{Poster 437 \\ Thyroid Hormone Metabolism \& Regulation Saturday Poster Clinical}

THE GASTRIC NEUROENDOCRINE TUMOR CARCINOID AND UNREGULATED HYPOTHYROID STATE

O.M. Boskovic

Endocrinology, Clinical Center of Montenegro, Podgorica, Montenegro
Multiple dose adjustments can sometimes be inefficient in reaching eumetabolic state in patients with hypothyroidism treated by levothyroxine sodium tablets. We report a case of 44-year-old female who has been on levothyroxine sodium tablet replacement therapy since 2004 without ever reaching eumetabolic state. As sellar MRI ruled out pituitary adenoma, investigation targeting malabsorption was initiated. Absorption test with $300 \mathrm{mcg}$ levothyroxine was positive in aspect of presence of selective levothyroxine malabsorption. Initial endoscopies revealed chronic atrophic gastritis without excluding possibility for tumor presence. Serum $\mathrm{CgA}$ level was $521 \mathrm{mcg} / \mathrm{L}$. The final third endoscopy was successful in detecting the gastric submucosal change, confirmed by endoscopic ultrasound as submucosal lesion no larger than $12 \mathrm{~mm}$ located in the upper part of anterior gastric wall. Histological analysis of biopsy specimen confirmed lesion to be neuroendocrine tumor-Carcinoid tumour ventriculi, Chromogranin A+, NSE+; synaptophhisin-, CK $7+$ with $\mathrm{Ki} 67+$ in $2 \%$ tumour cell. Further imaging excluded its metastatic spreading, so the indication for surgical removal was established. Patient could not undergo surgery with TSH levels exceeding $100 \mathrm{mmU} / \mathrm{L}$ and free T4 lower than $5 \mathrm{pmol} / \mathrm{L}$ despite being on $300 \mathrm{mcg}$ of levothyroxine daily substitution regimen. Changing to a gel capsule formulation taken once a day at dose of $100 \mathrm{mcg}$ lead to a rapid TSH decreasement and thyroid hormones normalization, so the patient was surgically treated as eumetabolic. On reviews thereafter she remained euthyroid, without complaints of previously reported fatigue and loss of appetite. This case suggests that gastric chronic inflammatory processes and neuroendocrine tumors as well may affect levothyroxine absorption.

\section{Poster 438}

Thyroid Imaging Saturday Poster Case Report

THE UTILITY OF SHEAR WAVE ELASTOGRAPHY IN THE EVALUATION OF THYROID NODULES AND CORRELATION WITH AGGRESSIVE FEATURES ON MICROSCOPY

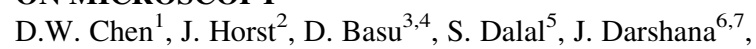
K.A. Hyland ${ }^{8}$

${ }^{1}$ Internal Medicine, Thomas Jefferson University, Philadelphia, PA; ${ }^{2}$ Pathology, Wilmington VA Medical Center, Wilmington, DE; ${ }^{3}$ Head and Neck Surgery, The University of Pennsylvania, Philadelphia, PA; ${ }^{4}$ Head and Neck Surgery, Philadelphia VA Medical center, Philadelphia, PA; ${ }^{5}$ Cytopathology, Clinical Chemistry, and Microbiology, Philadelphia VA Medical Center, Philadelphia, PA; ${ }^{6}$ Clinical Pathology and Laboratory Medicine, University of Pennsylvania Perelman School of Medicine, Philadelphia, PA; ${ }^{7}$ Pathology and Laboratory Services, Philadelphia VA Medical center, Philadelphia, PA; ${ }^{8}$ Department of Medicine, Wilmington VA Medical Center, Wilmington, DE

Thyroid nodules (TNs) have prevalence up to $50 \%$ in the adult population. Most are benign, but approximately 5\% are malignant. Shear wave elastography (SWE) is a relatively new and dynamic technique that quantitatively and qualitatively evaluates tissue elasticity, or stiffness. SWE utilizes an acoustic ultrasound probe to stimulate tissue, then subsequently calculates the speed of the resultant transverse-oriented shear waves through the tissue. The stiffer the tissue, the faster the wave travels, and the higher the measured shear wave velocity (SWV). A 71-year-old male presented for evaluation of an incidental TN. Ultrasound showed a $3.1 \mathrm{~cm}$ ill-defined heterogeneous isoechoic solid nodule with irregular margins, micro-calcifications, a taller-than-wide shape, and increased vascularity in the right lobe; and a $1.3 \mathrm{~cm}$ wellcircumscribed solid nodule with micro-calcifications, a wider-thantall shape, and interval vascularity in the isthmus. SWE showed 
high SWV of $3.88 \mathrm{~m} / \mathrm{s}$ (baseline SWV of surrounding tissue $2.06 \mathrm{~m} /$ s) in the right nodule; and higher SWV of $4.56 \mathrm{~m} / \mathrm{s}$ (baseline SWV $2.46 \mathrm{~m} / \mathrm{s}$ ) in the isthmus nodule. Cytology of the TN fine needle aspirate demonstrated benign follicular cells in the right; and papillary thyroid carcinoma in the isthmus. A total thyroidectomy was performed. Pathology showed a benign right thyroid lobe, but papillary thyroid carcinoma with a small component of aggressive tall cell morphology, focal extrathyroidal extension and perineural invasion without lymphatic or angioinvasion in the isthmus. Sebag et al. first reported on the utility of SWE to evaluate TNs in 2010, demonstrating that the elasticity index of malignant TNs was significantly higher than that of benign TNs and normal thyroid glandular tissue. Azizi et al. $(2013,2015)$ showed that TN stiffness is an independent predictor of thyroid cancer; and that the sensitivity and specificity of predicting thyroid cancer in a TN is higher when accounting for both its elasticity characteristics and sonographic features. Adding to the sparse endocrine literature on SWE, this case demonstrates the clinical utility of SWE as an adjunctive tool to identify TNs with high concern for malignancy; and localize high-yield areas of TNs to biopsy.

\section{Poster 439}

Thyroid Imaging Saturday Poster Clinical

\section{THE EFFECT OF CHEMORADIOTHERAPY FOR} CANCERS, NOT INVOLVING THE HEAD-AND-NECK, ON THYROID GLAND SIZE

M.G. Suarez ${ }^{3,1}$, R. Gavidia-Quezada ${ }^{3}$, E. Juarez-Tello ${ }^{3}$, J. LadoAbeal $^{3,2}$

${ }^{1}$ Endocrinology, Oregon Health Sciences University, Portland, OR; ${ }^{2}$ Endocrinology, Texas Tech University Health Science Center, Lubbock, TX; ${ }^{3}$ Internal Medicine, Texas Tech University Health Science Center, Lubbock, TX

Thyroid gland abnormalities are well described in patients treated with chemoradiotherapy to the head-and-neck. However, it is unknown if chemoradiotherapy causes similar effects when given to treat cancers located in the chest. We aimed to investigative the effect of carboplatin, paclitaxel and external radiation of the chest on thyroid gland size. As it is not common practice to protect the thyroid when giving radiation to the chest. A retrospective study on patients treated at University Medical Center - Lubbock from January 2005 to April 2016 with either radiation therapy to the chest, carboplatin or paclitaxel individually or in combination. Patients with hypothyroidism, thyroid nodules, thyroidectomy, or non-availability of computerized tomography (CT) scan images to measure thyroid gland volume were excluded. CT images obtained before and after treatment were used to measure thyroid gland width, depth, and height, as well as volume with Vitrae ${ }^{\circledR}$ Software. Differences from baseline to the last measure were assessed using paired sample T-test. Days elapsed since treatments were normalized by logarithmic transformation. A linear regression model was used to predict relative differences in each outcome from average doses of paclitaxel, carboplatin, radiation, and log daysForty-two subjects of a total of 318 patients were included. The average time between evaluated images was 353 days (range: 33-1440 days). Radiation delivered to the thyroid ranged from 15 to $6,646 \mathrm{~Gy}$. Thyroid gland volume decreased from $11.62 \pm 6.36 \mathrm{~mm}^{2}$ to $9.49 \pm 5.78 \mathrm{~mm}^{2}(\mathrm{p}<0.001)$ after chemo-radiation. The linear regression models showed a significant decrease in width of $1.33 \mathrm{~mm}$ in patients who received radiation $(\mathrm{p}<0.01)$. Also, there was a significance decrease in thyroid gland volume, depth, and width when analyzed based on the log days elapsed since treatment $(\mathrm{p}<0.05) .1)$ Patients treated with chemoradiation for tumors located outside head-and-neck have a decrease in thyroid gland volume. 2) The estimated radiation delivered to the thyroid has the largest effects on thyroid size reduction. 3) The reduction in thyroid gland size after chemoradiotherapy is time dependent.

\section{Poster 440}

Thyroid Imaging Saturday Poster Clinical COMPARISON BETWEEN 2015 AMERICAN THYROID ASSOCIATION MANAGEMENT GUIDELINES AND THYROID IMAGING REPORTING AND DATA SYSTEM IN DIAGNOSTIC EFFICIENCY OF THYROID NODULE: RESULTS IN SURGICALLY RESECTED THYROID

\section{NODULES}

L. Gao, X. Xi, Y. Jiang, Y. Wang, R. Liu, B. Zhang

Peking Union Medical College Hospital Union Medical College Hospital, Beijing, China

To compare diagnostic efficiency between the thyroid imaging reporting and data system (TIRADS) and 2015 American Thyroid Association (ATA) guidelines and to further evaluate the impact of thyroid nodule sizes and cystic appearance on the two patterns. From January 2015 and December 2015, 2,544 thyroid nodules in 1,758 patients who underwent thyroidectomy at our center were included in this study. A TIRADS category and US pattern based on ATA guidelines were assigned to each thyroid nodule. Nodules were respectively divided into groups according to the cystic appearance and maximal diameter.

Of all the thyroid nodules, $863(33.9 \%)$ were benign and 1681 $(66.1 \%)$ were malignant. TIRADS had a higher specificity $(95.5 \%$ vs $89.4 \%, \mathrm{P}=0.00)$, while ATA ultrasound pattern yielded a higher sensitivity $(77.4 \%$ vs $73.0 \%, \mathrm{P}<0.01)$. TIRADS and ATA guidelines performed better for differentiating nodules greater than or equal to $1 \mathrm{~cm}$ (AUC: 0.92 and 0.91 vs AUC: 0.70 and 0.70 ) and cystic-solid nodules(AUC: 0.96 and 0.95 vs AUC: 0.73 and 0.71 ). TIRADS had better diagnostic efficiency than ATA guidelines for differentiating nodules greater than or equal to $1 \mathrm{~cm}$ (AUC: $0.92 \mathrm{vs}$ $0.91, \mathrm{P}=0.01$ ).

Both TIRADS category and ATA patterns provide effective diagnostic value for thyroid nodules. TIRADS category and ATA patterns performed better for differentiating nodules greater than or equal to $1 \mathrm{~cm}$ and cystic-solid nodules.

\section{Poster 441}

Thyroid Nodules \& Goiter Saturday Poster Case Report

A CASE OF STATUS ASTHMATICUS WITH A LARGE THYROID TUMOR COMPRESSING THE TRACHEA E. Song ${ }^{1}$, J. Kim ${ }^{2}$, I. Kim ${ }^{1}$, J. Kim ${ }^{1}$, E. Jung ${ }^{1}$

${ }^{1}$ Surgery, Gyeongsan National University Hospital, Jinju,

Gyeongsang-nam, Korea (the Republic of); ${ }^{2}$ Surgery, Jeju national university hospital, Jeju, Jeju, Korea (the Republic of)

Case of patients with hyperthyroidism and asthma have often been reported. However, reports of huge goiter and asthma crisis in euthyroid state are rare. An 83-year-old Korean woman was found left huge thyroid mass on a test for health check-up. This woman was diagnosed with hypertension, diabetes mellitus, stroke, and asthma before the thyroid mass was discovered. On pharynx CT performed on first hospital day, approximately $6.2 \mathrm{~cm}$ of left thyroid nodule was observed extending to the mediastinum with trachea deviation to the right side. Ultrasonography revealed a heterogenous but hypoechoic dominant, $6 \mathrm{~cm}$ sized mass with interval vascularity. This mass was observed category 4 according to TIRADS category. And also a $5 \mathrm{~mm}$ lesion of the right thyroid gland was found on ultrasonography. Fine needle aspiration (FNA) was performed on the huge mass of the left 
thyroid gland. This patient showed euthyroid state in the thyroid function test. Fine needle aspiration results were consistent with benign follicular nodules. An asthma attack occurred on the evening of the FNA administration, and symptom improvement was observed after the use of Solu-medrol. On the next day, mild dyspnea was observed. Over time, a severe crisis due to asthma attack occurred. Solu-medrol was administered immediately, but asthma attack remained unchanged. This patient was intubated because of no improvement of asthma attack and ventilator care was performed in intensive care unit. Nevertheless, cardiac arrest occurred after a few minutes. Return of spontaneous circulation was performed after 10 minutes of cardiac pulmonary resuscitation. Status asthmaticus improved after administration of empirical antibiotics, intermittent Solu- Medrol, and Theophylline.

In this case, the surgical resection of benign huge thyroid goiterous mass is controversy. Considering the high risk of general anesthesia, we should consider whether or not to perform the operation. But there are not many cases of huge thyroid mass in the euthyroid state. It is obvious that the number of patients with various co-morbidity will increase in Korean society becoming aging. Therefore, research on this case should be continuously carried out.

\section{Poster 442}

Thyroid Nodules \& Goiter Saturday Poster Case Report

THORACIC OUTLET SYNDROME: A UNIQUE PRESENTATION OF A PRIMARY INTRATHORACIC GOITER

J.M. Tate, D. Beckman, J. Colburn, M. True

Endocrinology, San Antonio Military Medical Center,

San Antonio, TX

Primary and secondary intrathoracic goiters (P-ITGs and S-ITGs, respectively) account for up to $5.8 \%$ of mediastinal masses. Unlike $S$ ITGs, P-ITGs are structurally distinct from the cervical thyroid gland and receive their blood supply from mediastinal vessels while only representing $0.2 \%-1.0 \%$ of all ITGs. Although most remain asymptomatic, symptoms typically arise in relation to compression of the esophagus, trachea, or superior vena cava (SVC). No ITGs have been reported as a source of thoracic outlet syndrome. We report a case of a P-ITG causing right-sided thoracic outlet syndrome.

A 43-year-old female presented with a 1-month history of right arm pain, paresthesia, and weakness with right shoulder pain. A right shoulder x-ray revealed a superior mediastinal mass. Chest CT confirmed a heterogeneous mass within the superior, anterior mediastinum, measuring $12.1 \mathrm{~cm} \times 7.8 \mathrm{~cm} \times 8.8 \mathrm{~cm}$ and extending into the right chest apex. Notably there was severe compression of the SVC, distal trachea, and main-stem bronchus, with mass effect of the right brachiocephalic vein and artery. Fine needle aspiration via endobronchial ultrasound and percutaneous CT-guided biopsy of the mass were consistent with benign thyroid follicles. Given the massive size and significant compression of surrounding structures, a median sternotomy was completed, with intraoperative findings consistent with imaging results. Her thoracic outlet syndrome associated symptoms resolved following surgery.

P-ITGs are known to progress in size over time, and can be relatively large at time of diagnosis due to lack of symptoms until significant compression of adjacent structures occur. The most common symptoms are cough, dyspnea, stridor, dysphagia, and symptoms related to Horner's syndrome and SVC syndrome. To our knowledge this is the first reported case of a P-ITG causing thoracic outlet syndrome. Therefore, it would be reasonable to add P-ITGs to the list of potential causes of this obstructive condition. Case Reportsee above for discussion.

\section{Poster 443}

Thyroid Nodules \& Goiter Saturday Poster Case Report

HUGE ANTERIOR MEDIASTINAL GOITER REMOVED VIA MEDIAN STERNOTOMY: A CASE REPORT AND LITERATURE REVIEW

L. Kim, S. Ko, S. Lee, Y. Suh

Breast and Endocrine Surgery, Hallym University Sacred Heart

Hospital, Anyang, Gyeonggi-do, Korea (the Republic of)

The majority of substernal goiters can be retrieved through the neck. However, sternotomy should be performed in about a $1 \%$ rate. Herein, the authors report a case of huge anterior mediastinal goiter removed via median sternotomy. A 62-year old woman visited our hospital in October 2015, complaining of large mass in the neck. The thyroid function test including antibody measurement was within normal limit. Ultrasonography showed multiple nodules, which were ranged from $1.0 \mathrm{~cm}$ to $4.7 \mathrm{~cm}$ in longest diameter. The possibility of papillary thyroid carcinoma could not be excluded in fine needle aspiration cytology. Furthermore, computed tomography revealed $17 \times 8 \times 6 \mathrm{~cm}$ bulky thyroid gland extending to thoracic cavity. The patient underwent total thyroidectomy via median sternotomy in December 2016. Operation time was 370 minutes. During surgery, $600 \mathrm{cc}$ of red blood cells were transfused and estimated blood loss was $1600 \mathrm{cc}$. The patient with 35 points in SAPS3 admitted to intensive care unit for 2 days immediately after operation. The recovered patient was discharged 11 days following operation. The pathologist reported multinodular hyperplasia, along with stage I (T1aN0) papillary thyroid microcarcinoma. The sternal split is possible in case of difficult retrieval of substernal component or unexpected hemorrhage in the thorax. The patient should be well informed of the sternotomy and its potential complications.

\section{Poster 444}

Thyroid Nodules \& Goiter Saturday Poster Clinical

DEVELOPMENT OF A MALIGNANCY RISK PREDICTOR MODEL FOR CYTOLOGICALLY INDETERMINATE THYROID NODULES THROUGH ARTIFICIAL NEURAL NETWORKS

P. Valderrabano ${ }^{1}$, E. Kim ${ }^{2}$, Z.J. Thompson ${ }^{3}$, R.R. Bravo ${ }^{2}$, X. Wang ${ }^{3}$, C.H. Chung ${ }^{1}$, A. Anderson ${ }^{2}$, B. McIver ${ }^{1}$

${ }^{1}$ Head and Neck-Endocrine Oncology, H. Lee Moffitt Cancer Center and Research Institute, Tampa, FL; ${ }^{2}$ Integrated Mathematical Oncology, H. Lee Moffitt Cancer Center and Research Institute, Tampa, FL; ${ }^{3}$ Biostatistics and Bioinformatics, H. Lee Moffitt Cancer Center, Tampa, FL

Thyroid cytology is indeterminate in $25 \%$ of the biopsied nodules and, despite $70 \%$ of these being benign, most undergo surgery for diagnostic purposes. Artificial neural networks (ANN) are machine learning algorithms that can find patterns from complicated and large datasets. We hypothesized that ANN could be used to predict the cancer risk allowing for individualized management of patients with indeterminate thyroid nodules (ITNs). All consecutively evaluated ITNs between 10/2008 and 04/2016 with clinical, radiological, and cytological information, and known histology were used to develop and validate a model to predict malignancy (malignant versus benign; Model_1). All cancers in this cohort with detailed histological characterization were used to develop and validate a second model to predict the aggressiveness of the cancer (low-risk versus intermediate/high-risk features; Model_2). Low-risk features were T1-T2 differentiated thyroid cancers completely resected without lymphvascular invasion, regional or distant metastases. Cohorts were divided into training and testing sets. The area under the receiver 
operating curves (AUC) in the testing sets of the ANN models were compared to those of standard backward elimination logistic regression models (BELR) using variables with a p-value $<0.10$ on univariate analysis. The same variables fit for the BELR models were used to build the ANN models. Ten different weight sets were calculated with ANN for each model. For Model_1, 536 ITNs were split into training $(\mathrm{n}=375)$ and testing $(\mathrm{n}=161)$ sets. The AUC in the testing set was 0.67 with BELR and 0.82-0.86 with ANN models $(\mathrm{p}<0.05)$. For Model_2, 150 cancers were split into training $(\mathrm{n}=101)$ and testing $(\mathrm{n}=49)$ sets. The AUC in the testing set was 0.75 with BELR and $0.86-0.92$ with ANN models $(\mathrm{p}<0.05)$. This study suggests that the diagnostic ability of ANN models using available clinical, radiological, and cytological information is good, significantly better than for BELR models, and similar or perhaps even better than that of currently available molecular marker tests. ANNs could reduce evaluation costs and allow for personalized management of patients with ITNs.

\section{Poster 445}

Thyroid Nodules \& Goiter Saturday Poster Clinical THYROID NODULE COMPOSITION IS A SENSITIVE PREDICTOR OF THYROID CARCINOMA IN CHILDREN A.W. Gannon ${ }^{1}$, J.E. Langer ${ }^{2}$, R. Bellah ${ }^{3}$, S. Ratcliffe ${ }^{4}$, J. Pizza ${ }^{5}$, S. Mostoufi-Moab ${ }^{5,6}$, A.R. Cappola ${ }^{7}$, A.J. Bauer ${ }^{5}$

${ }^{1}$ Endocrinology, Nemours Alfred I DuPont Hospital for Children, Wilmington, DE; ${ }^{2}$ Radiology, University of Pennsylvania, Philadelphia, PA; ${ }^{3}$ Radiology, Children's Hospital of Philadelphia, Philadelphia, PA; ${ }^{4}$ Center for Clinical Epidemiology and Biostatistics, University of Pennsylvania, Philadelphia, PA; ${ }^{5}$ Endocrinology, Children's Hospital of Philadelphia, Philadelphia, PA; ${ }^{6}$ Oncology, Children's Hospital of Philadelphia, Philadelphia, PA; ${ }^{7}$ Endocrinology, Diabetes and Metabolism, University of Pennsylvania, Philadelphia, PA

Thyroid nodules are increasingly recognized in children and are associated with greater risk for thyroid cancer compared to adults. Thyroid ultrasound is the favored tool for initial evaluation of thyroid nodules; however, there are limited data regarding which features are most predictive of thyroid carcinoma in children. Because of wide variation in the patterns of ultrasound features, it would be useful to find common features that are effective in thyroid cancer risk stratification. We performed a retrospective cohort study including all children in the medical system betwen January 2009 and March 2013 age $\leq 18$ years who had thyroid nodules larger than $5 \mathrm{~mm}$ and sufficient follow up to determine whether the nodule was malignant. Two blinded expert radiologists independently reported ultrasound findings. Ultrasound features were analyzed to determine prevalence, reliability and predictive value for thyroid cancer.417 subjects were found to have thyroid nodules and 152 subjects with 236 nodules had adequate follow-up to determine the diagnosis. 59 subjects (38.8\%) were found to have thyroid cancer in a total of 80 nodules. Thyroid nodule structure was reported in all nodules and $\geq 75 \%$ solid composition was found in $152(64 \%)$ nodules; $45 \%$ of these nodules were malignant and these represented $85 \%$ of thyroid cancer cases. High risk ultrasound features were tightly clustered within the group of $\geq 75 \%$ solid nodules and this finding was $85 \%$ sensitive (95\% CI 75.3, $92 \%$ ) and $46.2 \%$ specific (95\% CI 38.2, 54.2\%) with a negative predictive value of $85.7 \%$ (95\% CI 76.4, 92.4\%); interrater agreement between radiologists for this finding was very good [kap$\mathrm{pa}=0.639, \mathrm{p}<0.001]$. The presence of $\geq 75 \%$ solid composition is a sensitive and reliable independent predictor of thyroid malignancy in children and is very closely correlated with the presence of all other high-risk features. On this basis, children with solid/partially cystic thyroid nodules should be strongly considered for fine needle aspirate biopsy. It is possible that children with $>25 \%$ cystic thyroid nodules are at lower risk for thyroid cancer but further prospective studies are needed to confirm whether more conservative planning would be reasonable for these.

\section{Poster 446}

Thyroid Nodules \& Goiter Saturday Poster Clinical

OFFICE-BASED ULTRASOUND-GUIDED FINE NEEDLE ASPIRATION BIOPSY TECHNIQUE FOR THYROID NODULES IN A CHILDREN

N. Patel, S. Manning

Otolarygology, Seattle Children's Hospital, Seattle, WA

The use of fine needle aspiration (FNA) biopsy in the diagnosis and management of pediatric thyroid nodules presents a unique set of challenges. Children diagnosed with a nodule carry a greater risk of malignancy compared to adults with thyroid nodules. Uncertainty in diagnosis can lead to anxiety in patients and their families. With the release of the 2015 American Thyroid Association (ATA) guidelines, ultrasound-guided FNA biopsies are recommended to help distinguish malignant disease from benign disease in order to counsel families regarding treatment. This study proposes a cost-effective, safe, and well tolerated technique of office-based ultrasound-guided fine needle aspiration biopsy (USFNAB) technique for thyroid nodules in children for which to base clinical decision making in the pediatric population without the use of general anesthesia.

Methods: Retrospective chart review of a technique using "J-tip" administered local anesthesia is proposed in children with thyroid nodules for office-based ultrasound-guided fine needle aspiration. Traditionally it was believed that younger patients may not tolerate the procedure and may require sedation or general anesthesia. Limited data are available describing office-based US-FNAB in children. This is the first study describing a protocol for in office USFNAB exclusively in the pediatric population. 163 patients had a FNA for thyroid disease in our tertiary pediatric institution. Over 10 years, $80 \%$ of the patients had a office-based US-FNAB. This tecqnique is safely tolerated in the pediatric population and avoids the use of general anesthesia. This review of the use of USFNAB in the management of thyroid nodules shows that an office-based biopsy can be safely performed in the pediatric population.

A protocol for in office USFNAB is safely tolerated in the pediatric population and avoids the use of general anesthesia.

\section{Poster 447}

Thyroid Nodules \& Goiter Saturday Poster Clinical

CLINICAL MANAGEMENT OF ATYPIA OF

UNDETERMINED SIGNIFICANCE/FOLLICULAR LESION OF UNDETERMINED SIGNIFICANCE (AUS/FLUS) AMONGST PRIMARY CARE PHYSICIANS AT OUR INSTITUTION

S. Karimi ${ }^{1,3}$, L. Willard ${ }^{2}$

${ }^{1}$ Internal Medicine, UPMC Mercy, Pittsburgh, PA; ${ }^{2}$ Endocrinology, UPMC Mercy, Pittsburgh, PA; ${ }^{3}$ Endocrinology, UTMB,

Galveston, TX

With increased utilization of radiographic studies, the discovery of incidental thyroid nodules in clinical practice has consequently increased as well. With recent updates by both the ATA and AACE on the approaches to thyroid nodules, it has become imperative that not only endocrinologists, but also primary-care physicians, pediatricians, internists, and surgeons be updated on these evidence-based guidelines. Our study was done to determine the clinical management of thyroid follicular-aspirations amongst PCPs in particular at 
our institution. A search of our information system was done which identified 29 thyroid fine needle aspiration (FNA) cases diagnosed AUS/FLUS between the years 2013-2015. A retrospective chart review was subsequently done to characterize the data by cytopathology, patient demographic information, size of thyroid nodule sampled, sonographic characteristics, repeat FNA, surgical procedures, and applicable follow-up. The role and clinical management of thyroid FNAs diagnosed as AUS/FLUS were assessed amongst PCPs in our institution (primary end point). An online survey was also sent out among resident physicians in order to gauge their understanding on the management of thyroid FNAs (secondary end point). Of the 29 reports of AUS/FLUS, 13 were ordered molecular testing to assess with their decision making. Only 3 of all cases had a repeat FNA performed and 8 ultimately underwent surgery. Only 9 of the patients had repeat ultrasounds done with intervals ranging from 3 to 18 months. Our study indicates many discrepancies in the management of these particular nodules between PCPs. A short survey was distributed among our medicine residents with $88.24 \%$ of repsponders stating they were not familiar with molecular testing. Clinical management of thyroid nodules is becoming more complex with the advent of molecular testing. With the incidence of thyroid nodule detection increasing, it is important for all physicians that take part in the management of thyroid nodules to be familiar with recent evidence-based guidelines. Our study showed that many primary care physicians are not prepared nor familiar with the proper management of thyroid nodules, in particular those with AUS/FLUS cytology.

\section{Poster 448}

Thyroid Nodules \& Goiter Saturday Poster Clinical

\section{SAFETY OF SUTURELESS AND DRAINLESS OPEN THYROIDECTOMY USING ULTRASONIC COAGULATOR: PROSPECTIVE STUDY}

S. Lee

Surgery, Yeungnam University College of Medicine, DAE GU, Korea (the Republic of)

The aim of this study was to evaluate the safety and to confirm the feasibility of sutureless and drainless open thyroidectomy using Ultrasonic Coagulator. A prospective study was conducted on 1200 consecutive thyroid surgeries, over a period of 74 months. In case of carcinoma, central lymph node dissection was conducted routinely. Grave's disease, radical neck dissection and endoscopic thyroidectomy cases were excluded. During surgery, bleeding control, vessel ligation, soft tissue dissection were done by monopolar coagulator, bipolar coagulator and mainly untrasonic coagulator (Harmonic ${ }^{\circledR}$ ). Any suture materials and drainage procedure were not used in this period. All patients were observed for the swelling of operative site, dyspnea, neck tightness, vital sign on first 1, 3, 9, 24 hours after thyoroidectomy and USG of neck was done on first postoperative day for monitor the fluid collection or hematoma. The mean age of patients was 48.3 years (ranges, 19-77 years). Numbers of thyroidecotmy of benign tumor was 87 cases (unilateral thyroidecotmy: 67 cases, bilateral thyroidecotmy: 20 cases) and number of malignant tumor was 1113 cases (unilateral thyroidecotmy: 617 cases, bilateral thyroidecotmy: 496 cases). After surgery, total 24 patients complained symptom. 2 patients complained mild dyspnea, 7 patients complained tightness of operative site, 15 patients was examined neck swelling by surgeon. But, on following USG of operative site, only 4 patients were observed significant fluid collection and needed USG-guided aspration. Amount of aspirate fluid was $16 \mathrm{cc}, 20 \mathrm{cc}, 22 \mathrm{cc}, 47 \mathrm{cc}$ and natures of aspirates were all serous nature. No patient required reoperation for bleeding or hematoma.
Sutureless and non-drain open thyroidectomy using ultrasonic coagulator is safe and feasible and has many advantages (minimal skin incision, decreased hospital stay, no problem of suture, granuloma, decreased time of operation, no adverse wound from drainage procedure, no ascending infection from suction drainage).

\section{Poster 449}

Thyroid Nodules \& Goiter Saturday Poster Clinical 3D ENDOSCOPIC THYROID SURGERY FOR LARGE GOITER VIA BILATERAL AXILLARY AND BREAST APPROACH (BABA): INITIAL INSTITUTIONAL EXPERIENCE FROM SUB-HIMALAYAN REGION

G. Chand

Endocrine Surgery, Sanjay Gandhi Post Graduate Institute of Medical Sciences, Lucknow, Lucknow, Uttarpradesh, India

We belong to sub-Himalayan region, which is known for the endemic goiter zone. Here the large goiter is common among the young women. The purpose of this study is to introduce our initial experience of 3D endoscopic thyroid surgery via Bilateral Axillary and Breast Approach (BABA) in large goiter.

We had performed 33 cases of 3D endoscopic thyroid surgery via bilateral axillary and breast approach from Aug. 2015 till April 2017. The following variables were studied: mean age, duration of goiter, preoperative functionality \& fine needle aspiration cytology, mean size \& weight of gland, hospital stay, final histopathology, complications and cosmetic outcome. $\mathrm{M}: \mathrm{F}=1: 4$, Mean age was 32.70 years (20-56 years), mean duration of goiter was 38.66 months ( $<1$ month to 40 years); 31 euthyroid and 02 were toxic MNG; FNAC reveled 27 colloid nodule, 03 follicular lesion, 01 hurthle cell lesion, 01 PTC and 01 FTC. 31 operated successfully and 02 converted to open due to suspicious of malignancy. Mean tumor size was $6.125 \mathrm{~cm}(4-8 \mathrm{~cm}) \&$ mean weight was $54.19 \mathrm{gm}$ (10-223 gm). Mean operating time was 3 hrs \& 56.3 minutes for TT and 2 hrs \& 43.17 minutes for HT. Final histopathology reveled 6 malignancy (4PTC, 1 FTC \& 1HCC) in TT \& 4 malignancy (3PTC \& 1 FTC) in HT and 23 were benign. 02 in HT \& 05 in TT group develop temporary change in voice. 01 required type-2 thyroplasty. 06 patients of TT develop temporary hypocalcaemia and managed with oral calcium \& vit $\mathrm{D}$, however 02 patients required intravenous calcium therapy. The cosmetic outcome was excellent during 06 month follow-up.

$3 \mathrm{D}$ endoscopic thyroid surgery through breast and axillary approach in large goiter is feasible with excellent cosmetic outcome and acceptable complications.

\section{Poster 450}

Thyroid Nodules \& Goiter Saturday Poster Clinical PERCUTANEOUS ETHANOL INJECTION: AN EFFICIENT AND NON-EXPENSIVE TREATMENT FOR SOLID, NONFUNCTIONING BENIGN THYROID ADENOMAS, MEXICAN PILOT STUDY

\section{S. Miracle}

Endocrinology, Hospital Angeles de las Lomas, Huixquilucan, Estado de Mexico, Mexico

Percutaneous ethanol injection therapy (PEIT) has been used for more than 15 years in the treatment of both benign and malignant thyroid lesions. Although the treatment of nodules with radiofrequency ablation (RFA) and laser has been very successful, in countries where this technology is not available or is too expensive (as in Mexico and most all Latin American countries), PEIT is a proven, safe, non-expensive and underutilized alternative. We are not aware of PEIT reports in Latin America for solid, non-functioning 
benign thyroid adenomasThis is a pilot study in Mexico, Prospective, Comparative, Quasi-Experimental, Longitudinal, Historic, Open, with External Controls, of PEIT in solid, non-functioning benign thyroid adenomas. All patients previously underwent an ultrasoundguided fine needle aspiration biopsy (FNAB); all nodules were reported as Class II of the Bethesda system. The PEIT was performed under ultrasound guidance (MINDRAY 5) and $99.77 \%$ ethanol (JT Baker) was used in all procedures. The total volume injected was one $\mathrm{cm} 3$ per $\mathrm{cm} 3$ of thyroid volume, divided in three to five sessions. Nodular volume was calculated using the equation: $\mathrm{V}=\pi \mathrm{abc} / 6$, were "V" is the volume, " $a$ " is the maximum diameter, and " $b$ " y " $c$ ", the other 2 perpendicular diameters. Success was calculated as a $50 \%$ total volume reduction at 6 months. We report the results of our first
10 patients treated. The mean starting nodule volume was $8.2 \mathrm{cc}$ (range: 4.3 to $19 \mathrm{cc}$ ), the average volume reduction at 6 months was $70 \%$ (range: 48 to $94 \%$ ). 9 of 10 patients attained a volume reduction of $50 \%$ or more, with 1 patient only managing a $48 \%$ volume reduction. The most common side effects was pain on injection site that lasted 48 to 72 hours. One patient presented extravasation of ethanol to the ipsilateral ECM muscle, one patient presented with acute thyroiditis, one patient presented with laryngeal spam that had to be treated in the ER with IV steroidsOur first report shows PEIT to be an efficient and non-expensive procedure. We believe that PEIT is a safe, efficient, non-expensive and underused alternative to surgery, radiofrequency ablation (RFA) and laser for the treatment for solid, non-functioning benign thyroid adenomas 